\title{
CONSTANT-FREQUENCY, CLAMPED-MODE RESONANT CONVERTERS
}

\author{
by \\ Fu-Sheng Tsai
}

Dissertation submitted to the Faculty of the

Virginia Polytechnic Institute and State University

in partial fulfillment of the requirements for the degree of

Doctor of Philosophy

in

Electrical Engineering

APPROVISD:

Tired C. Lee, Chairman

Bo II. Cho

Vatché Vórpérian

Kwa S. Tam

Ronald D. Riess

Deccmber 13, 1989

Blackshurg, Virginia 


\title{
CONSTANT-FREQUENCY, CLAMPED-MODE RESONANT CONVERTERS
}

\author{
by \\ Fu-Sheng Tsai \\ Fred C. Lee, Chairman \\ Electrical Engineering \\ ( $\triangle$ BSTRACT)
}

Two novel clamped-mode resonant converters are analyzed. These clamped-mode converters operate at a constant frequency while retaining many desired features of conventional resonant converters such as fast responses, zero-voltage turn-on or zerocurrent turn-off, and low EMI levels, etc. The converters are able to regulate the output from no load to full load and arc particularly suitable for off-line, high-power applications.

To provide insights to the operations and derive design guidelines for the clampedmode resonant converters, a complete dc characterization of both the clamped-mode series-resonant converter and the clamped-mode parallel-resonant converter, operating above and below resonant frequency, is performed. State-plane analysis techniques are employed. By portraying the converters' operation on a state-plane diagram, various circuit operating modes are identified. The boundaries between different operating modes are determined. The regions fin natural and force commutation of the active switches are defined. Important dc characteristics, such as control-to-output transfer ratio, rms inductor current, peak capacitor voltage, rms switch currents, average diode currents, switch turn-on currents, and switch turn-off currents, are derived to facilitate the converter designs.

To illustrate the converter designs in different operating regions, several design examples are given. Finally, three prototype circuits are built to verify the analytical results. 


\section{Acknowledgements}

I would like to express my sincere appreciation to my advisor, Dr. Fred C. Lee, for his guidance, support, and encouragement during the course of this research work. His extensive knowledge, creative thinking, and patience have been invaluable help and are very much appreciated.

I also owe my gratitude to Dr. Bo II. Cho, Dr. Vatché Vorpérian, Dr. Kwa S. Tam, and Dr. Ronald D. Riess for their teaching, suggestions, and discussions, and their kindness to serve as my committee members.

I am thankful to my old colleagues, , , and my fellow student, $\quad$, for their help in obtaining the experimental results used in this thesis.

Thanks also extend to all the VPI: memhers for their friendship which has made my stay at Virginia Tech an enjoyable onc.

Special thanks go to VPEC secretary , who is always kind and ready to help, and , who has done excellent jobs in editing my manuscripts. 
I would like to give my heartfelt appreciation to my parents,

and

, for their never-ending efforts to provide me with good education opportunities, and my sisters, , for helping me taking care of my mother while I was away. Most of all, I would like to thank my beloved wife, , for her continuous support and understanding during the course of my study and my daughter, who has brought so much joyfulness into my life.

Finally, I would like to thank EG\&G MImond Instruments, N $\Lambda$ SA Lewis Research Center, and Rocketdyne Division, Rockwell International for their financial support during my stay. 


\section{TABLE OF CONTENTS}

\section{CHAPTER 1. INTRODUCTION}

1.1 General Background ......................................................................... 1

1.2 Derivation of Clamped-Mode Resonant Converters .................................... 10

1.3 State-Plane Analysis Techniques ....................................................... 14

\section{CHAPTER 2. ANALYSIS OF CLAMPED-MODE SERIES-RESONANT CONVERTER}

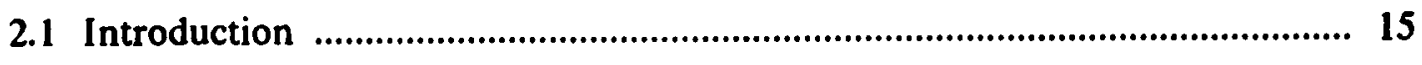

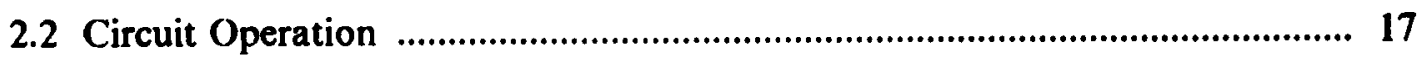

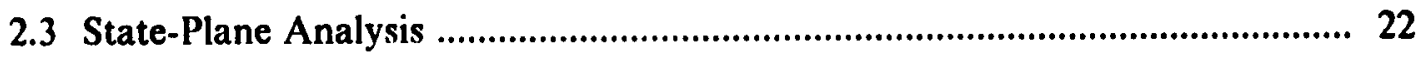

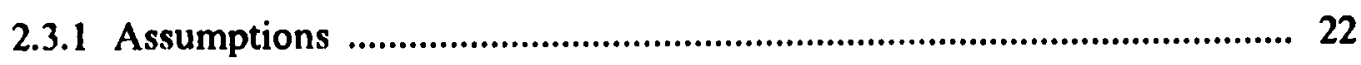

2.3.2 Circuit topological modes .............................................................. 22

2.3.3 State trajectories for circuit topological modes .................................. 25

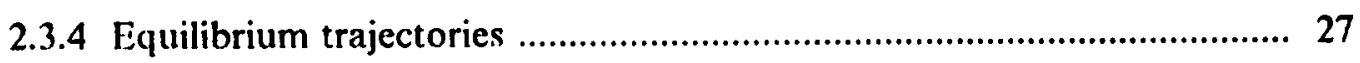

2.3.5 Circuit operating modes ......................................................... 32

2.3.6 Regions of operation ............................................................... 55

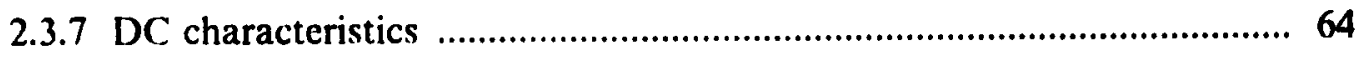

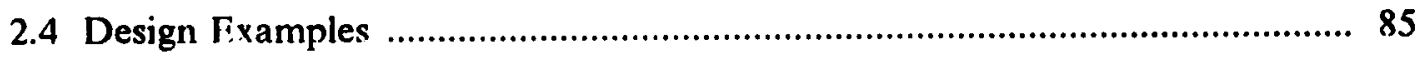

2.4.1 Example 1 - Design in natural-commutation region ........................... 86

2.4.2 Example 2 - Design in nixed-commutation region ............................ 93 
2.4.3 Example 3 - Design in force-commutation region .................................. 101

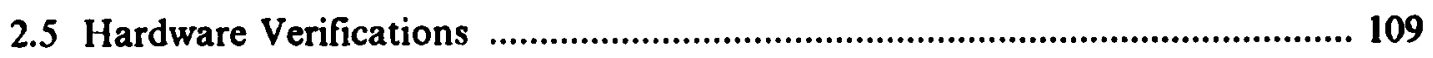

2.5.1 Circuit operating modes below resonant frequency .................................. 109

2.5.2 Circuit operating modes above resonant frequency ............................... 123

2.6 Conclusions ..................................................................................................... 130

\section{CHAPTER 3. ANALYSIS OF CLAMPED-MODE PARALLEL-RESONANT CONVERTER}

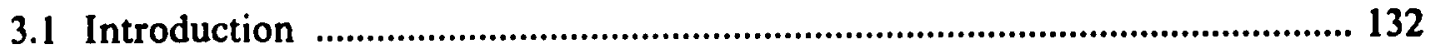

3.2 Circuit Operation ......................................................................................... 134

3.3 State-Plane Analysis .................................................................................... 139

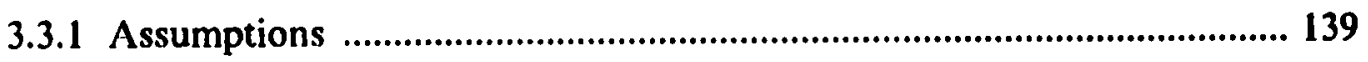

3.3.2 Circuit topological modes ......................................................................... 139

3.3.3 State trajectories for circuit topological modes ....................................... 145

3.3.4 Equilibrium trajectories ........................................................................... 148

3.3.5 Circuit operating modes .......................................................................... 151

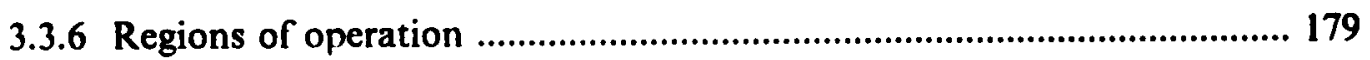

3.3.7 DC characteristics .................................................................................... 183

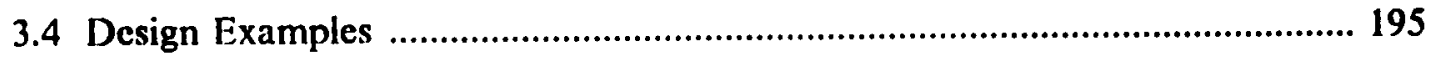

3.4.1 Example 1 - Design in natural-commutation region ............................... 196

3.4.2 Example 2 - Design in mixed-commutation region .................................. 201

3.4.3 Example 3 - Design in force-commutation region ................................... 203

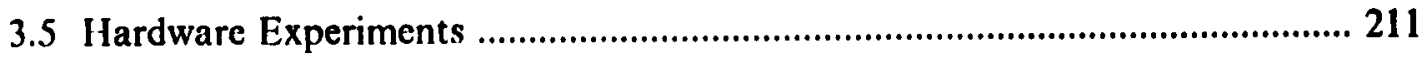

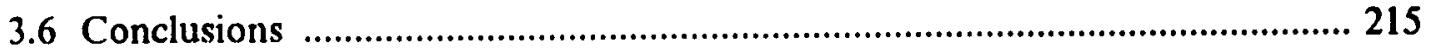

CHAPTER 4. CONCLUSIONS …................................................................................. 217 


\section{APPENDIX A. DERIVATION OF CLAMPED-MODE, PARALLEL-RESONANT CONVERTER}

225

\section{APPENDIX B. SUPPLEMENTS TO CHAPTER 2}

B.1 Rules for Constructing Equilibrium State Trajectories of a CM-SRC 228

B.2 Prediction of Mode Transitions of a CM-SRC Operating Below Resonant Frequency 232

B.3 Prediction of Mode Transitions of a CM-SRC Operating Above Resonant

Frequency 249

B.4 Calculation of Trajectory Parameters of a CM-SRC Operating Below

Resonant Frequency

B.5 Expressions for Circuit Salient Features of a CM-SRC Operating Below Resonant Frequency 271

B.6 DC Characteristics of a CM-SRC Operating Below Resonant Frequency ... 279

B.7 Calculation of Trajectory Parameters of a CM-SRC Operating Above

Resonant Frequency 285

B.8 Expressions for Circuit Salient Features of a CM-SRC Operating Above

Resonant Frequency 294

B.9 DC Characteristics of a CM-SRC: Operating Above Resonant Frequency ... 297

B.10 Generation of Circuit Waveforms of a C.M-SRC 303

\section{APPENDIX C. SUPPLEMENTS TO CHAPTER 3}

C.1 Rules for Constructing Equilibrium State Trajectories of a CM-PRC 
C.2 Prediction of Mode Transitions of a CM-PRC Operating Below Resonant Frequency

C.3 Prediction of Mode Transitions of a CM-PRC Operating Above Resonant Frequency 334

C.4 Calculation of Trajectory Parameters of a CM-PRC 344

C.5 Calculations of Circuit Salient Features of a CM-PRC 377

C.6 DC Characteristics of a CM-PRC Operating Below Resonant Frequency ... 388

C.7 DC Characteristics of a CM-PRC Operating Above Resonant Frequency .. 391

C.8 Generation of Circuit Waveforms of a CM-PRC 397 


\section{CHAPTER 1.}

\section{INTRODUCTION}

\subsection{GENERAL BACKGROUND}

In recent years, resonant power conversion technology has gained much attention in power conversion applications [1-32]. Due to their distinct advantages, such as fast response, high efficiency, reduced switch stresses, and low EMI (electromagnetic interference) levels, resonant power processors have gradually taken over roles previously dominated by the pulse-width-modulated (PWM) converters.

The major advantage of resonant converters, as compared to PWM converters, is their ability to eliminate switching losses by commutating the switches at zero voltage and/or zero current. $\Lambda s$ a result, resonant converters can be operated at much higher frequencies than PWM converters. The increase in operating frequency reduces the size of magnetic components, thus increasing the converters' power density.

Conventional resonant converters have bridge configurations, as illustrated in Figure 1.1. An LC resonant tank is used to transfer energy from input to the load. The two controlled switches are triggered with $50 \%$-duty-cycle gating signals such that a squarewave voltage, $v_{S}$, is generated across the resonant tank. The output is obtained by rectifying and filtering the sinusoidal-like resonant inductor current (series-resonant 


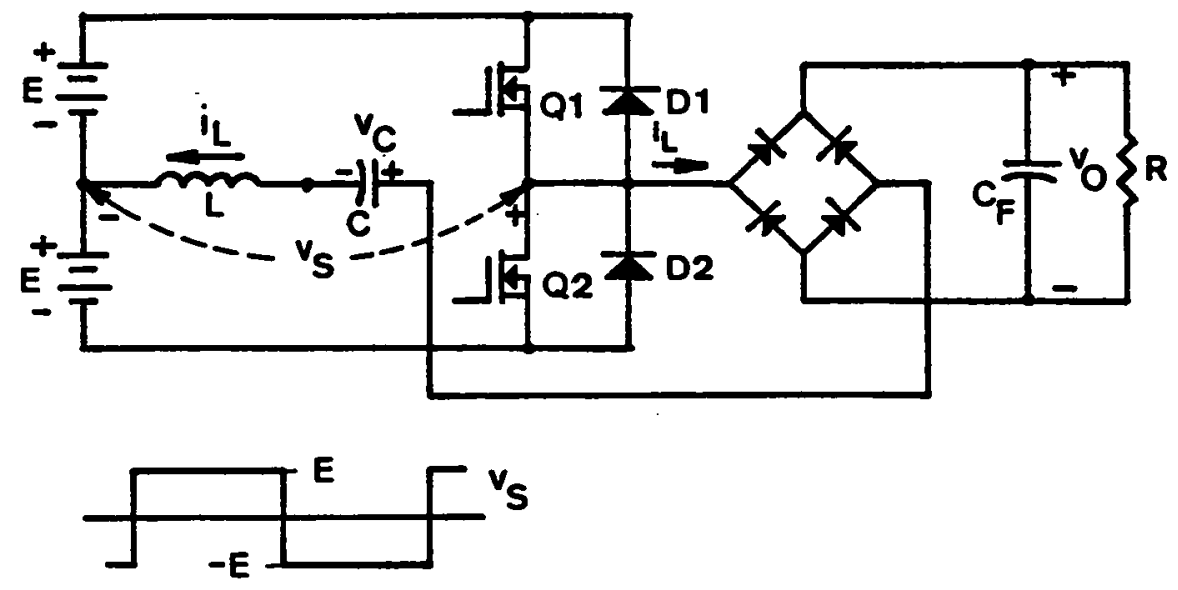

(a) Series-resonant converter (SRC)

Figure 1.1 Conventional Series- and Parallel-Resonant Converters 


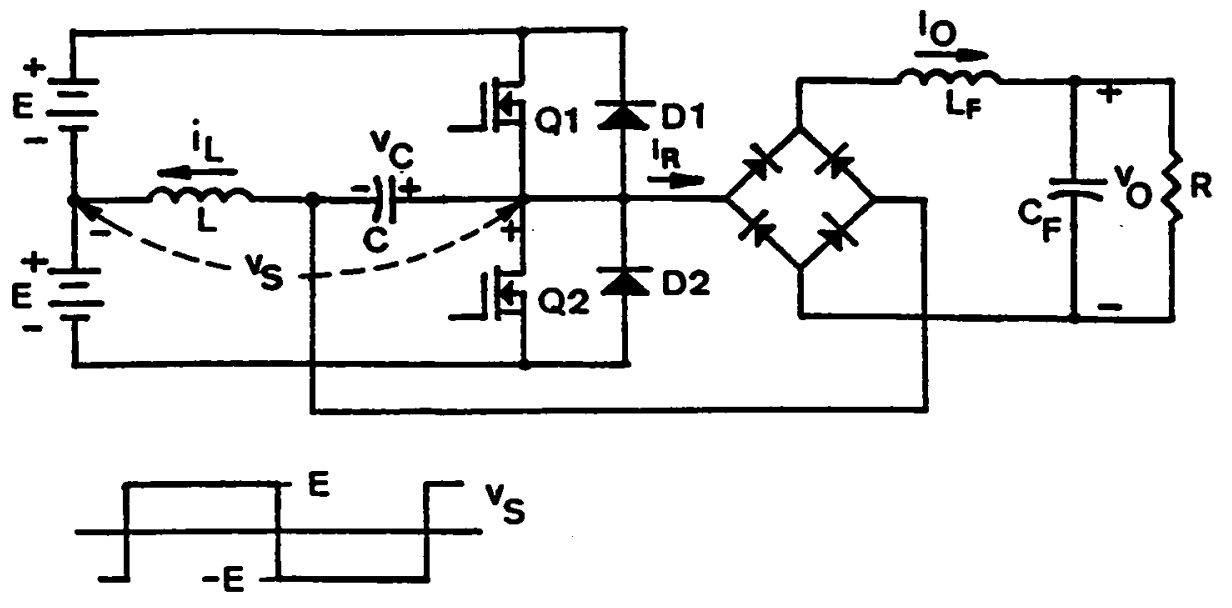

(b) Parallel-resonant converter (PRC)

Figure 1.1 Continued 
converter) or resonant capacitor voltage (parallel-resonant converter). The output voltage regulation is achieved by varying the operating frequency.

Depending upon the operating frequency and the load, the controlled switches in the resonant converters can be naturally commutated or force commutated $[18,19,31]$. In general, natural commutation of the switches occurs when the converter operates below the resonant frequency of the LC tank, while force commutation occurs when the converter operates above the tank's resonant frequency.

When natural commutation is achieved, the switches' turn-off losses are eliminated. However, since the diodes are commutated at high currents fast antiparallel diodes are required to minimize the cross conductions in the totem-pole switch-diode pairs caused by the reverse recovery of the diodes. When force-commutation is achieved, the power switches operate with zero-voltage turn-on and the switches' turn-on losses are eliminated. Slow antiparallel diodes and simple losseless capacitor snubbers can be used for the switches since the diodes are commutated at zero current and the switches always turn on at zero voltage.

Due to the line and/or load variations, the operating frequency of the conventional resonant converters usually has to vary over a wide range to regulate their outputs. This results in a penalty in the magnetics and filter design and lowers the overall conversion efficiency.

To optimize the design of magnetic components and filters, several circuit topologies and control techniques able to operate at a constant frequency were proposed [33-46].

Figure 1.2 shows a parallel-resonant converter (PRC) with a controlled output rectifier. The converter's operating frequency is fixed. Its output is controlled by varying the conduction intervals of the output controlled switches [33,34]. Although the converter is able to regulate its output over a wide load range and is bidirectional, it is only suitable for low-current, high-voltage applications since the output controlled switches introduce additional losses. 


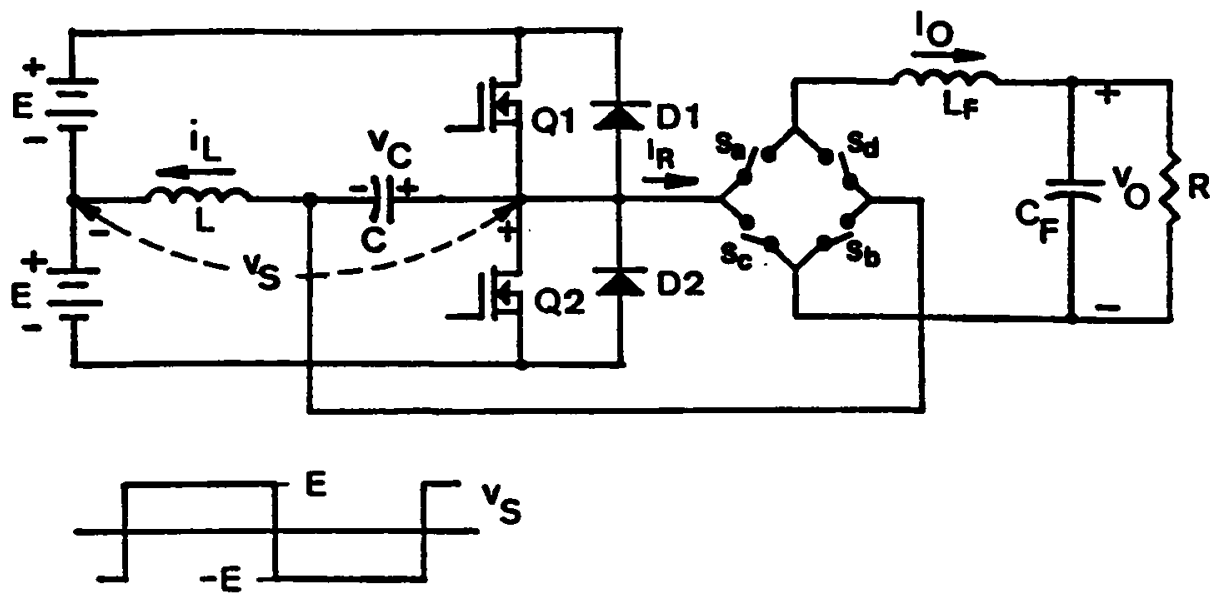

Figure 1.2 A Parallel-Resonant Converter with a Controlled Output Rectifier 
A phase-control scheme was introduced which employs two identical series- or parallel- resonant inverters with their outputs connected in parallel or in series, respectively, to a common load $[35,36,37]$, as shown in Figure 1.3. The two resonant inverters are driven by gating signals which have the same frequency but have different phases. The outputs of the converters are controlled by varying the phase displacement between the gating signals. These phase-controlled converters are able to regulate their outputs from no load to full load. However, due to the phase displacement of the two inverters, the effective load power factor seen by each inverter is different. As a result, the inductor currents and capacitor voltages in the resonant tanks of the inverters are unbalanced. This introduces high component stresses in one of the resonant inverters [37]. Also, high circulating currents exist in the inverters under light load since the exciting frequency of the inverters remains unchanged.

The pseudo-resonant converter proposed in Reference [38] is an analogy to the phase-controlled converters.

To alleviate the current and voltage imbalance and reduce the circulating currents under light load, the two phase-controlled resonant inverters can be combined. This results in two new clamped-mode, resonant converters which are functional equivalence to their phase-controlled counterparts. The circuit topologies of the clamped-mode resonant converters are the same as those of conventional full-bridge resonant converters. Ilowever, they operate under a quite dilferent principle. $\Lambda$ s illustrated in Figure 1.4(d) and 1.4(e), the switched input voltage $v_{S}$ to the resonant tank of a clamped-mode resonant converter is a fixed-frequency, duty-cycle-modulated, quasi-square wave, instead of a frequency-modulated square wave in the conventional resonant converters. The output of the converter is controlled by varying the time interval, $\phi_{S} / \omega_{S}$ as shown in Figure 1.4(e), during which voltage $v_{S}$ is "clamped" at 7.ero volt. These converters are therefore referred in the text as "clamped-mode resonant converters". The fact that clamped-mode resonant converters possess the same desired output characteristics as the 


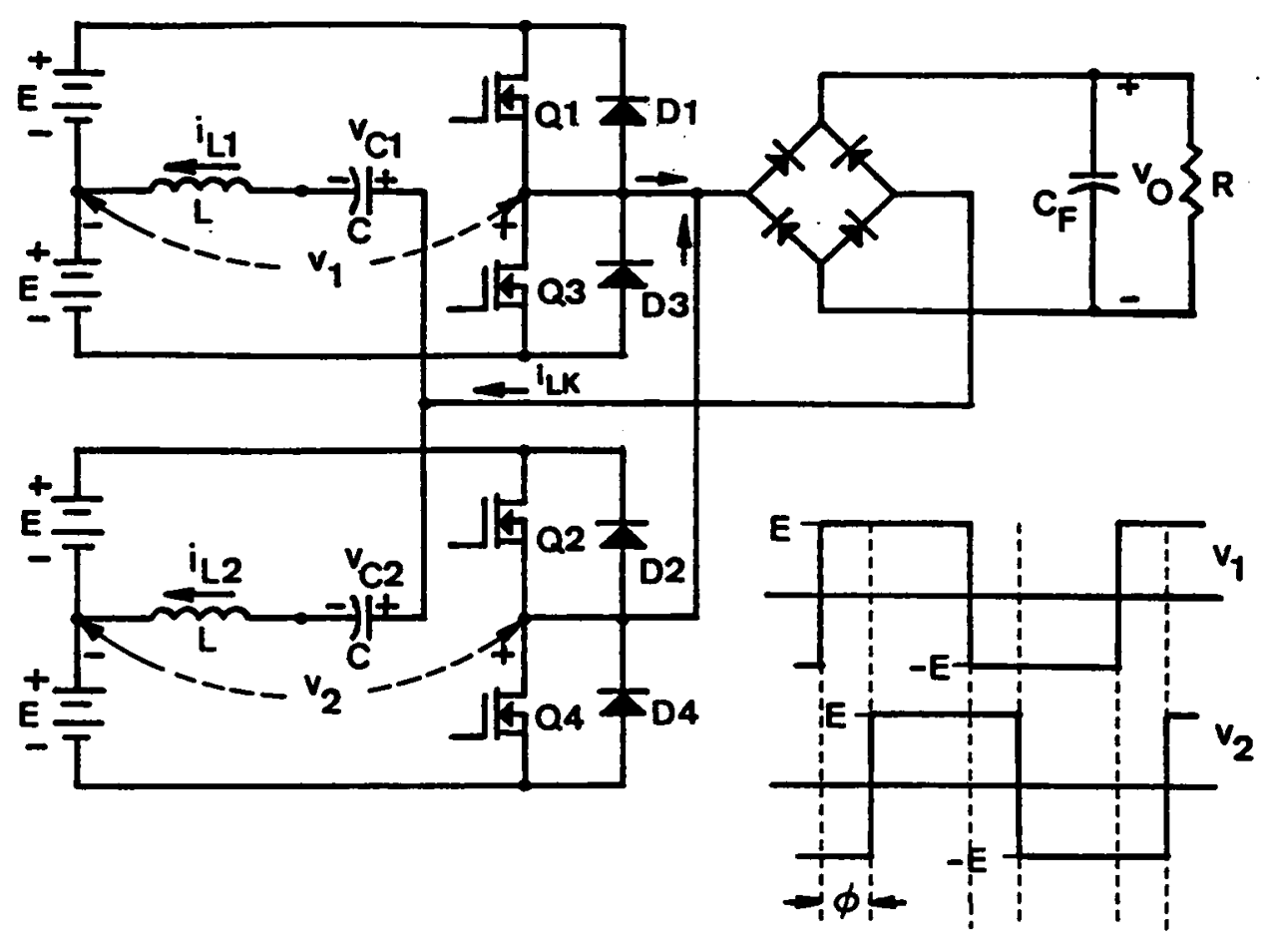

(a) Phase-controlled series-resonant converter

Figure 1.3 Phase-Controlled Resonant Converters 


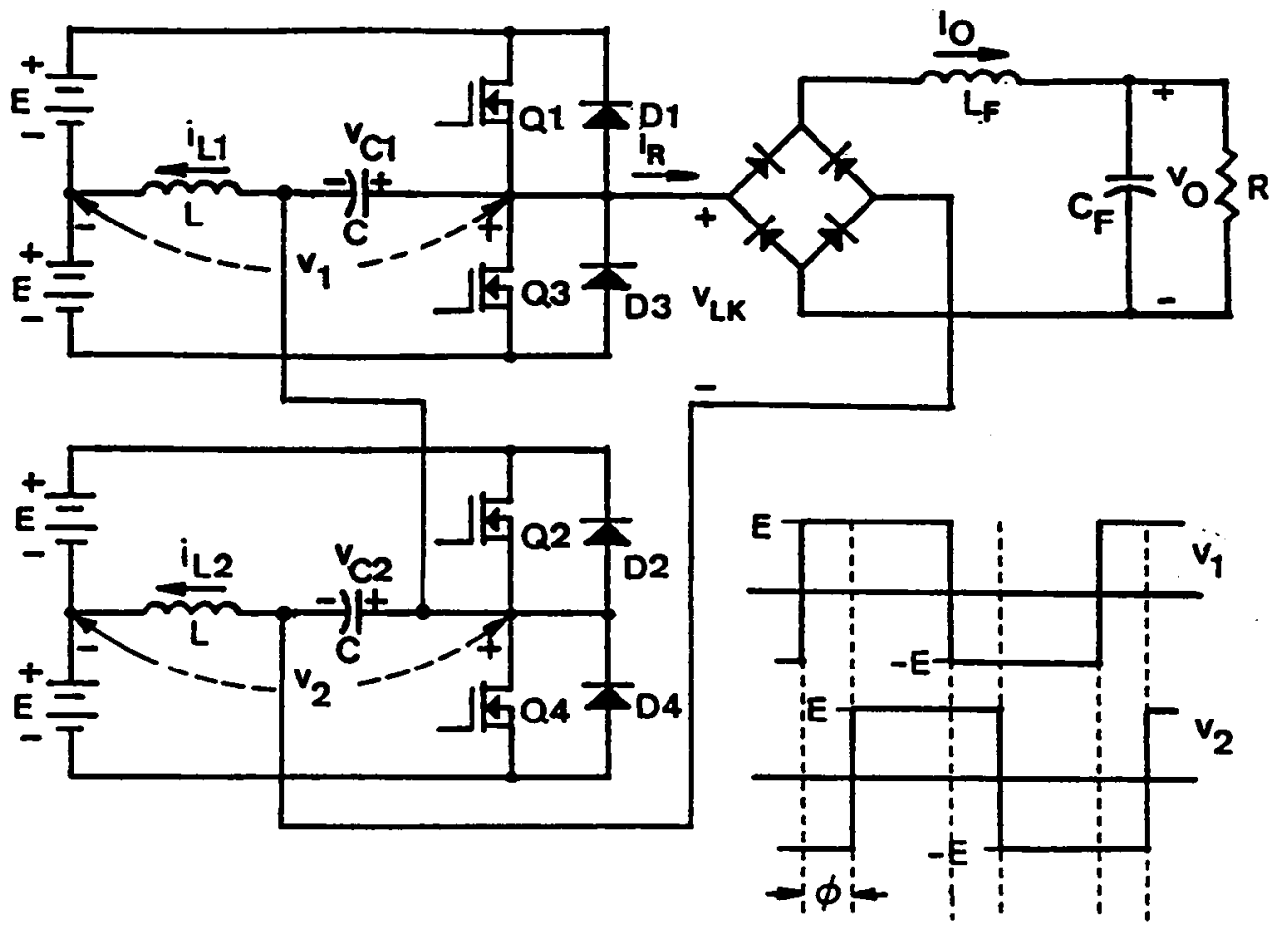

(b) Phase-controlled parallel-resonant converter

Figure 1.3 Continued 
phase-controlled converters while alleviating their aforementioned shortcomings makes these converters very attractive. It is a major goal of this research to fully characterize the dc behaviors of these two clamped-mode resonant converters and provide systematic design guidelines. 


\subsection{DERIVATION OF CLAMPED-MODE RESONANT CONVERTERS}

Figure 1.4 illustrates how the clamped-mode series-resonant converter is derived by combining the two phase-controlled resonant inverters. The outputs of the inverters are connected in parallel. Viewing tank voltages $v_{1}$ and $v_{2}$ as new voltage sources, a simplified equivalent circuit is shown in Figure 1.4(b). The circuit equations for the phasecontrolled, series-resonant converter (PC-SRC) are

$$
\begin{gathered}
v_{1}=v_{O}+v_{C 1}+L \frac{d i_{L 1}}{d t}, \\
i_{L 1}=C \frac{d v_{C 1}}{d t}, \\
v_{2}=v_{O}+v_{C 2}+L \frac{d i_{L 2}}{d t}, \\
i_{L 2}=C \frac{d v_{C 2}}{d t},
\end{gathered}
$$

and

$$
i_{0}=i_{l, 1}+i_{l, 2}
$$

$\Lambda$ dding (1.1) to (1.3) and (1.2) to (1.4), we obtain

$$
\left(v_{1}+v_{2}\right)=2 v_{O}+\left(v_{C 1}+v_{C 2}\right)+L\left(\frac{d i_{L 1}}{d t}+\frac{d i_{L 2}}{d t}\right),
$$

and 


$$
\left(i_{L 1}+i_{L 2}\right)=C\left(\frac{d v_{C 1}}{d t}+\frac{d v_{C 2}}{d t}\right)
$$

Rewriting (1.6) and (1.7), we have

$$
\begin{gathered}
\frac{\left(v_{1}+v_{2}\right)}{2}=v_{O}+\frac{\left(v_{C 1}+v_{C 2}\right)}{2}+\left(\frac{L}{2}\right) \frac{d}{d t}\left(i_{L 1}+i_{L 2}\right), \\
\left(i_{L 1}+i_{L 2}\right)=(2 C) \frac{d}{d t}\left(\frac{v_{C 1}+v_{C 2}}{2}\right) .
\end{gathered}
$$

From Eqs. (1.5),(1.8), and (1.9), viewing $\left(v_{C 1}+v_{C 2}\right) / 2$ and $\left(i_{L 1}+i_{L 2}\right)$ as new state variables, an equivalent circuit can be derived, as shown in Figure 1.4(c), where

$$
\begin{gathered}
v_{C}=\frac{\left(v_{C 1}+v_{C 2}\right)}{2}, \\
i_{L}=\left(i_{L 1}+i_{L 2}\right),
\end{gathered}
$$

and

$$
v_{s}=\frac{\left(v_{1}+v_{2}\right)}{2}
$$

The voltage, $v_{S}$, as shown in Figure 1.4(c), is a quasi-square wave with pulse width, $\beta_{S}$, determined by the phase displacement hetween $v_{1}$ and $v_{2}$. The zero-voltage period of $v_{S}$ is equal to the phase displacement, $\phi_{S}$, hetween $v_{1}$ and $v_{2}$. Such a voltage can be realized using a full-briclge circuit, as shown in lïgure 1.4(d), by operating switches $S 1, \mathbf{S 2 , S 3}$, and S4 in a fashion illustrated in Figure 1.4( () . When switches $\mathrm{S} 1$ and $\mathrm{S} 4$ or S2 and S3 are both on, $v_{s}$ is clamped at zero volts. Otherwise, $v_{s}$ is equal to either $+\mathrm{E}$ or $-\mathrm{E}$. A full- 
bridge, series-resonant converter is referred to as "clamped-mode, series-resonant converter (CM-SRC)" when it is operated in such a fashion.

Following a similar manner, a "clamped-mode, parallel-resonant converter $(C M-P R C)^{\prime \prime}$ can be derived from a phase-controlled, parallel-resonant converter (PC-PRC), as illustrated in $\Lambda$ ppendix A.1. 

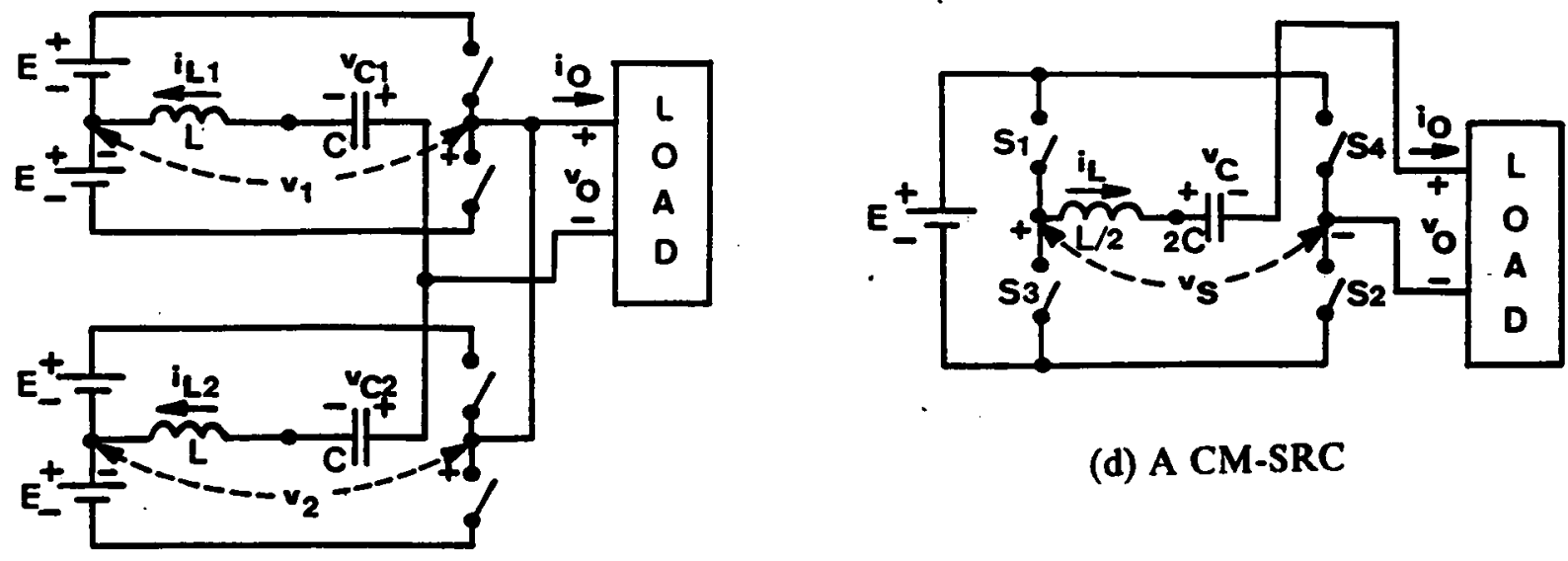

(d) A CM-SRC

(a) A PC-SRC

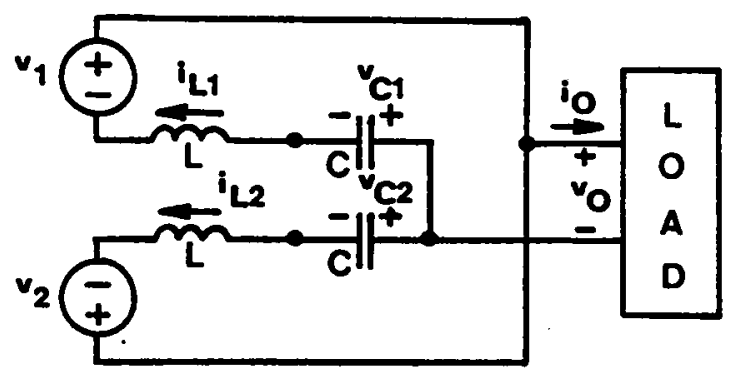

(b) Simplified circuit

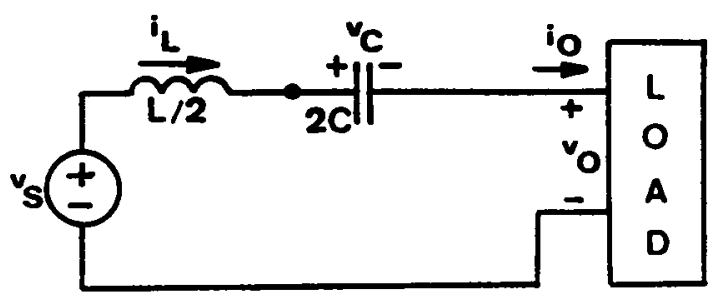

(c) Equivalent circuit

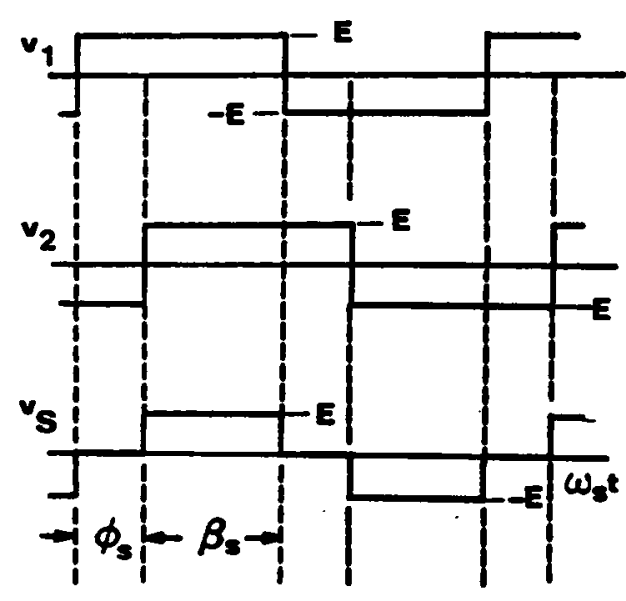

(e) Equivalent source voltage

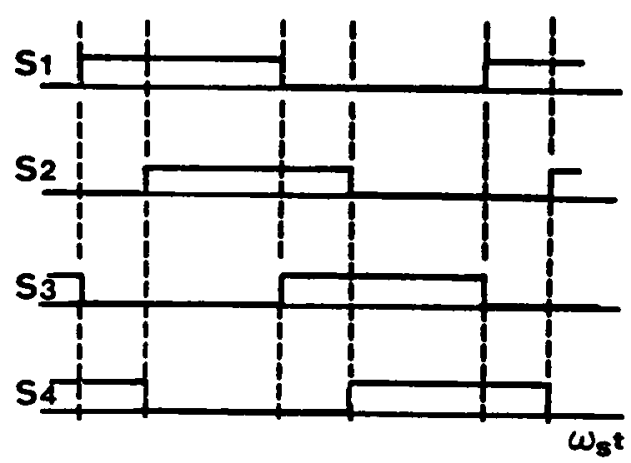

(f) Switch conduction sequence

Figure 1.4 Derivation of Clamped-Mode Series-Resonant Converter (CM-SRC) 


\subsection{STATE-PLANE ANALYSIS TECHNIQUES}

Many different analysis techniques have been used to characterize the dc behaviors of resonant converters [1,14-15,18-19,33-34]. Due to the complexity of the circuit operation, most analytical work presented to date are numerical in nature. In general, a computer method numerically calculates the instantaneous current and voltage in the resonant tank and determines the steady state by comparing the values of inductor current and capacitor voltage a half switching period away [1]. This approach, although simple and straightforward, provides no physical insights to the converter's behaviors.

A piece-wise linear circuit approach divides a steady-state operation into several switching intervals and derive expressions for the current and voltage during each interval. Boundary conditions are matched to determine the steady-state values for the current and voltage $[33,34]$. This approach, however, has to assume the phase relationship between certain circuit waveforms which is sometimes unpredictable.

Graphical techniques have recently been employed in the analysis of resonant converters [14-15,18-19,31]. The advantage of these approaches is their ability to illustrate graphically the converter's behavior on a planar diagram. The complex circuit operation can be easily visualized and many less familiar operating modes uncovered. Important circuit features can be derived from the geometrical relationship easily identified in the diagram.

Among the two graphical approaches, the state-plane analysis technique is more powerful than the output-plane technique. The state-plane technique monitors monitor the instantaneous resonant inductor current and the instantaneous resonant capacitor voltage and provides better insights to the converter operation.

In Chapter 2 and Chapter 3, state-plane techniques are employed to characterize the $\mathrm{dc}$ behaviors of the clamped-mode, scrics-resonant and the clamped-mode, parallelresonant converters. 


\section{CHAPTER 2.}

\section{ANALYSIS OF CLAMPED-MODE SERIES}

\section{RESONANT CONVERTER}

\subsection{INTRODUCTION}

In designing a conventional series-resonant converter(SRC), two operating frequency ranges are usually considered. One is below the resonant frequency (often between $50 \%$ and $100 \%$ of the resonant frequency) where zero-current turn-off(natural commutation) of the controlled switches is achieved, thus eliminating the turn-off losses and stresses of the switches. The other is above resonant frequency where zero-voltage turn-on of the switches is achieved, thus eliminating the turn-on losses of the switches. When an SRC operates below resonant frequency, fast antiparallel diodes are required for the switches since these diodes are commutated at high currents. While, when an SRC operates above the resonant frequency, slower antiparallel diodes can be used for the switches since the diodes turn off at zero current. $\Lambda$ simple lossless capacitor snubber can be employed for the switches to reduce their turn-ofr losses since the switches always turn on at zero voltage. 
Similar to SRC, a clamped-mode series-resonant converter (CM-SRC) can be designed to operate either below or above the resonant frequency to obtain either zerocurrent turn-off or zero-voltage turn-on. The design is, however, more complicated.

For a CM-SRC, three modes of commutation can exist: (1) all the controlled switches are naturally commutated; this mode is referred to as "natural commutation"; (2) two of the switches in one leg of the bridge are naturally commutated and the other two switches are force commutated; the two switches which are force commutated are nevertheless operating with zero-voltage turn-on; this mode is referred to as "mixed commutation"; (3) all the switches are force commutated (or zero-voltage turn on); this mode is referred to as "forced commutation". It should be noticed that in the context of this thesis, the forced commutation mode is always associated with zero-voltage turn-on property. This is, however, not necessurily the case in general When a CM-SRC operates below the resonant frequency, the switches may be operated under natural commutation or mixed commutation. When a CM-SRC operates above the resonant frequency, the switches may be operated under forced commutation or mixed commutation.

To provide insights to the converter's operation and derive guidelines for the converter design, a complete dc characterization of the CM-SRC is presented in this chapter. Graphical state-plane techniques are employed to identify various circuit operating modes for the converter. The boundaries between different operating modes are determined. The regions for natural, mixed, and force commutation are specified. Important dc control-to-output characteristics are derived. Three design examples are given. Finally, the analytical results are verified using two breadboard circuits. 


\subsection{CIRCUIT OPERATION}

Figure 2.1(a) shows a clamped-mode, series-resonant converter with the controlled switches S1,S2,S3,S4 in Figure 1.4(d) implemented by transistors Q1,Q2,Q3,Q4 and their antiparallel diodes D1,D2,D3,D4, respectively. The transistors are triggered in a timing sequence as illustrated in Figure 2.1(b). All the transistors are driven with $50 \%$ dutycycle gating signals. Transistors Q1,Q3 are triggered according to a clock signal whose frequency determines the converter's operating frequency. Transistors Q2,Q4 are triggered with a controllable time delay, $\frac{\phi_{S}}{\omega_{S}}$, with respect to the triggering of $Q 1, Q 3$, respectively. The time delay is the interval during which the resonant tank is clamped as a short circuit. By controlling the time delay, the pulse-width, $\beta_{S}$, of the quasi-squarewave voltage, $v_{S}$, is controlled and the converter's output voltage, $V_{o}$, is regulated.

Depending upon the operating conditions, a CM-SRC can result in different modes of operation. Each mode of operation represents a unique conduction sequence of the switching devices, and is characterized by different circuit waveforms and device commutation requirements.

A typical circuit operation of a CM-SRC operating below the resonant frequency is illustrated in Figure 2.2. Prior to $t=0$, diodes D3,D4 are conducting. At $t=0$, transistor Q1 turns on and diode D3 is commutated and the inductor current, $i_{r}$, resonates through Q1,D4. $\Lambda \mathrm{t} t=\mathrm{t}$, transistor $\mathrm{Q} 2$ is triggered. Diode $\mathrm{D4}$ is commutated and $i_{L}$ resonates through $\mathrm{Q} 1, \mathrm{Q} 2$. $\Lambda \mathrm{t} \mathrm{t}=\mathrm{t} 2$, $i_{l}$, decreases to zero duc to resonance. Transistors $\mathrm{Q} 1, \mathrm{Q} 2$ turn off naturally and diodes D1,D2 conduct subsequently. The inductor current, $i_{L}$, resonates through D1,D2. $\Lambda \mathrm{t}$ the end of the half switching cycle, $\mathrm{t}=\frac{T_{S}}{2}$, transistor $\mathrm{Q} 3$ turns on, commutating diode D1 and a similar process repeats with the roles of Q1,Q2,D1,D2 and Q3,Q4,D3,D4 interchanged, respectively. The reflected load voltage, $v_{R}$, changes polarity whenever $i_{L}$, crosses the 7.cro-axis. 
A typical circuit operation of a CM-SRC operating above the resonant frequency is illustrated in Figure 2.3. $\Lambda \mathrm{t} t=0$, transistor $\mathrm{Q3}$ is forced off and $\mathrm{Q} 1$ is triggered. Since the inductor current $i_{L}$ is negative, Q1 cannot conduct. Instead, diode D1 conducts. The inductor current, $i_{\mathrm{L}}$, resonates through $\mathrm{Q} 4$ and $\mathrm{D} 1$. $\mathrm{At} t=\mathrm{tl}$, transistor $\mathrm{Q} 4$ is forced off and Q2 is triggered. Transistor Q2 cannot conduct since $i_{L}$ is still negative. Instead, diode D2 conducts. The inductor current $i_{L}$ resonates through D1 and D2. At $t=t 2, i_{L}$ increases to zero. Diodes D1 and D2 commutate naturally and transistors Q1 and Q2 conduct subsequently. The inductor current, $i_{L}$, resonates through Q1 and Q2. At the end of the half switching cycle, $\mathrm{t}=\frac{T_{S}}{2}$, transistor $\mathrm{Q} 1$ is forced off. and $\mathrm{Q} 3$ is triggered. A similar process occurs with the roles of Q1,Q2,D1,D2 and Q3,Q4,D3,D4 interchanged, respectively. The reflected load voltage, $v_{R}$, changes polarity whenever $i_{L}$ crosses the zero-axis.

In the examples discussed above, the commutation features of the transistors $\mathrm{Q} 1, \mathrm{Q} 2, \mathrm{Q} 3, \mathrm{Q} 4$, and the diodes D1,D2,D3,D4 are the same as those in a conventional SRC. The commutation feature for the devices, however, can be different if the operating condition of the converter changes, as shall be discussed in later sections. 


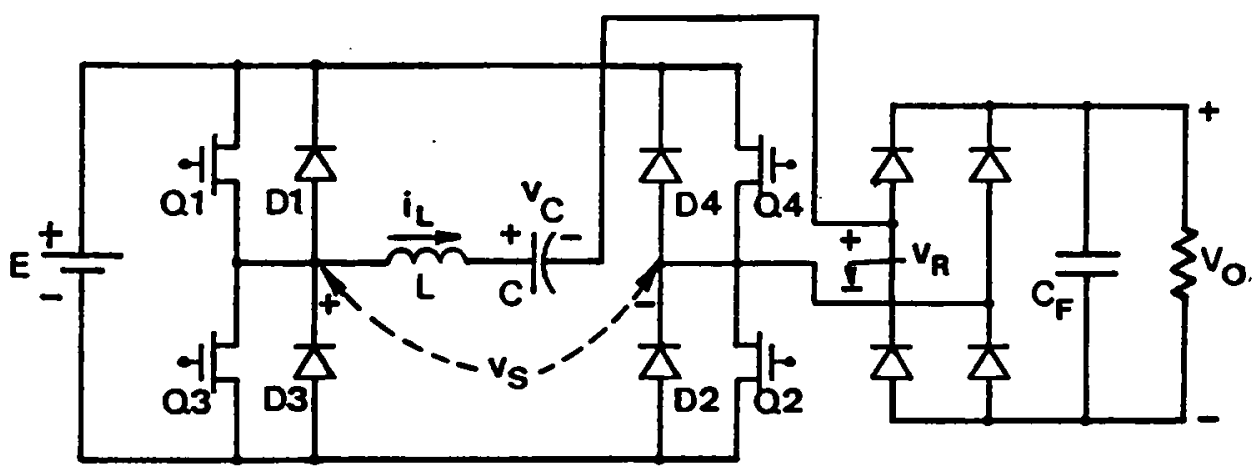

(a) Circuit diagram

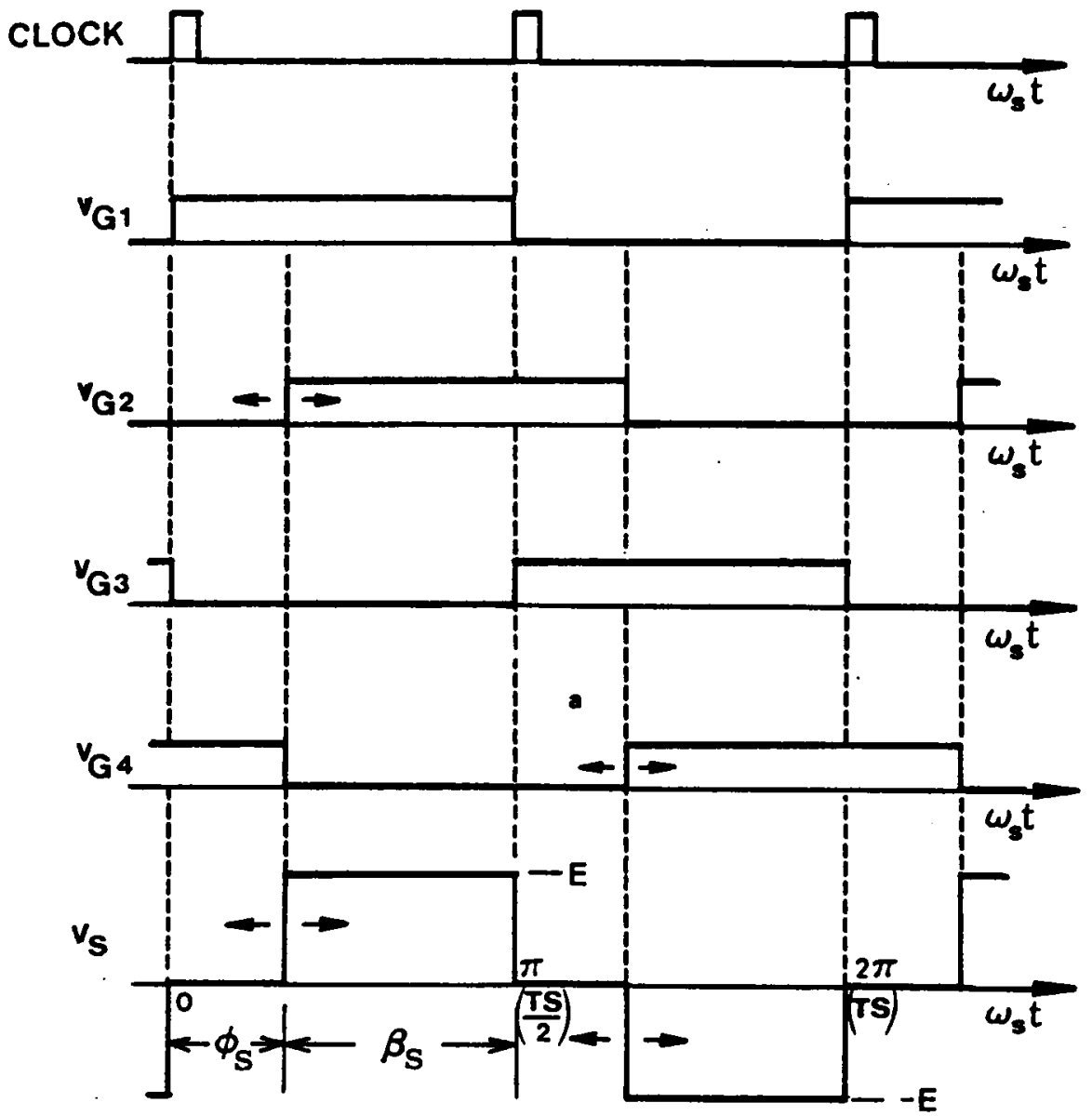

(b) Gating Signals

Figure 2.1 A Clamped-Mode Series-Resonant Converter 

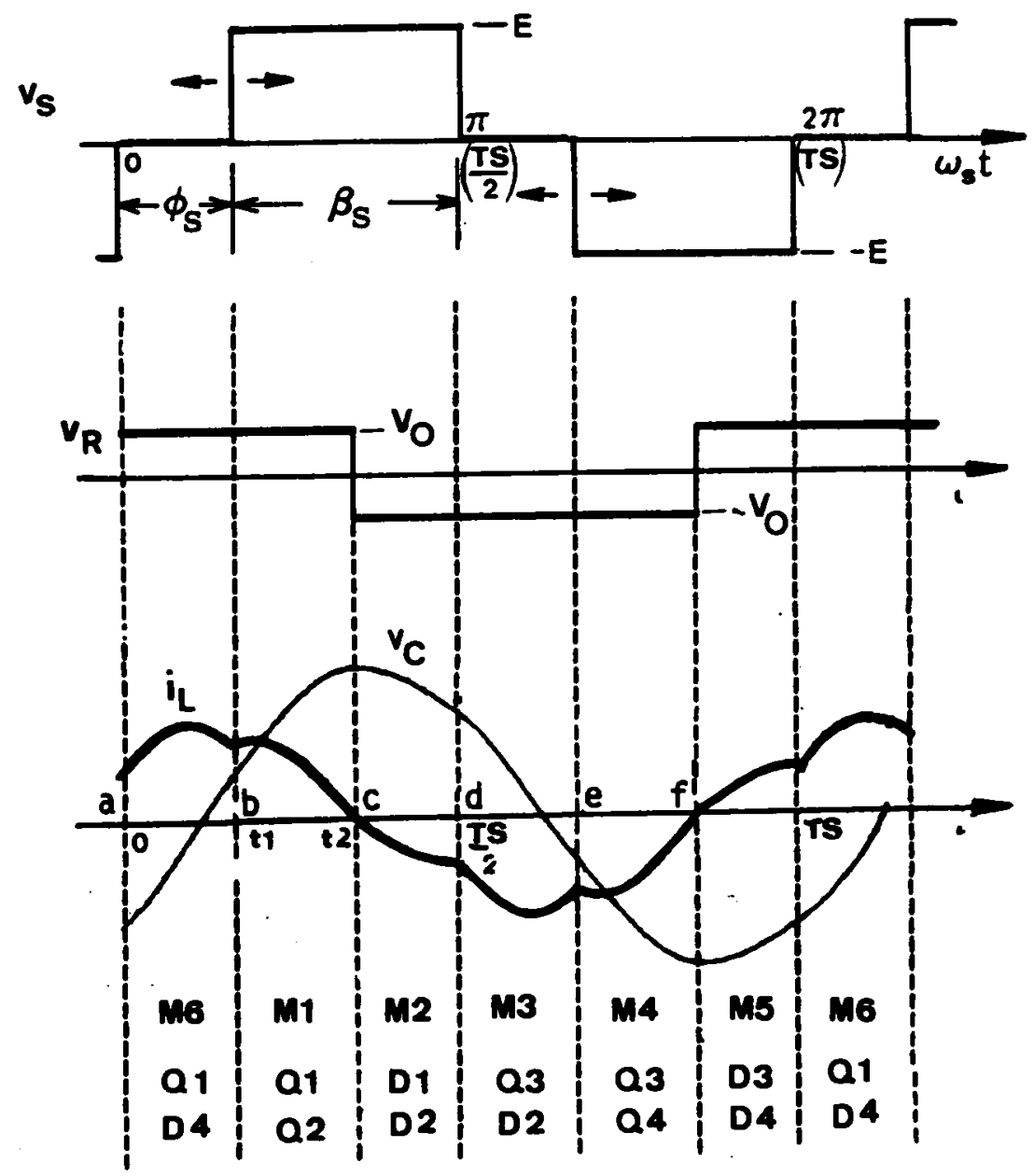

Figure 2.2 A Typical Circuit Operation Below Resonant Frequency 


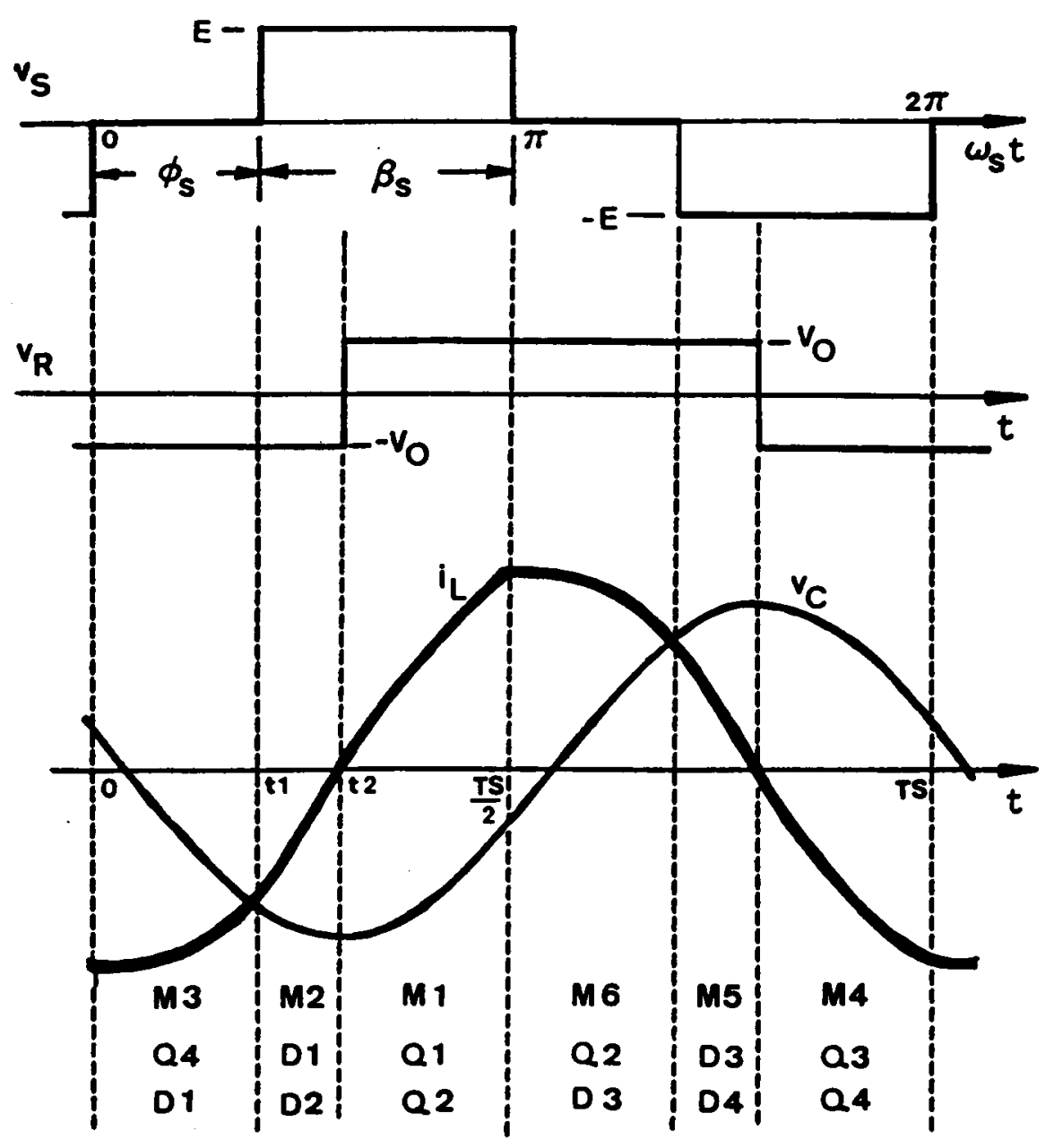

Figure 2.3 A Typical Circuit Operation Above Resonant Frequency 


\subsection{STATE-PLANE ANALYSIS}

In this section, graphical state-plane techniques are employed to characterize the CM-SRC. The state-plane analysis enables one to visualize the converter's complex behavior from a state portrayal and derive various important circuit features such as control-to-output transfer ratio, rms inductor current, peak capacitor voltage, rms switch currents, average diode currents, etc. from the state trajectory, which significantly simplifies the analysis.

\subsubsection{Assumptions}

The following assumptions are made during the analysis:

1. all the transistors are ideal, with zero switching time and no conduction drop;

2. the quality factor of the resonant tank is infinite; in other words, there is no loss in the tank circuit;

3. the output filter is large enough such that the output voltage, $V_{o}$, can be assumed constant during several switching cycles;

4. the transistors are driven with ideal $50 \%$-duty-cycle gating signals;

5. the switching frequency of the converter is greater than $50 \%$ of the resonant frequency.

\subsubsection{Circuit Topological Modes}


As illustrated in Figures 2.2 and 2.3, the one-cycle operation of a CM-SRC is composed of a sequence of linear circuits, each corresponding to a particular switching interval. There are seven linear circuit topologies for a CM-SRC, as shown in Figure 2.4. These circuit topologies are referred to as circuit topological modes of a CM-SRC. The topological modes M1,M2,M3,M4,M5, and M6 are called "resonant modes" and topological mode M0 is called "recess mode". The circuit behaviors of a CM-SRC under each topological mode can be described using the following differential equations.

For resonant modes M1,M2,M3,M4,M5, and M6,

$$
\begin{aligned}
& L \frac{d i_{L}}{d t}+v_{C}=v_{E}, \\
& C \frac{d v_{C}}{d t}=i_{L},
\end{aligned}
$$

where,

$$
v_{E}=\left[\begin{array}{cc}
E-V_{O} & \text { for } M 1 \\
E+V_{O} & \text { for } M 2 \\
V_{O} & \text { for } M 3 \\
-E+V_{O} & \text { for } M 4 \\
-E-V_{O} & \text { for } M 5 \\
-V_{O} & \text { for } M 6
\end{array}\right.
$$

For recess mode M0,

$$
\begin{aligned}
& L \frac{d i_{L}}{d t}=0 \\
& C \frac{d v_{C}}{d t}=0 .
\end{aligned}
$$

By solving these differential equations, expressions for $i_{L}$ and $v_{C}$ in each topological mode can be derived. The expressions are normalized and shown in the following.

For resonant modes M1,M2,M3,M4,M5, and M6, 


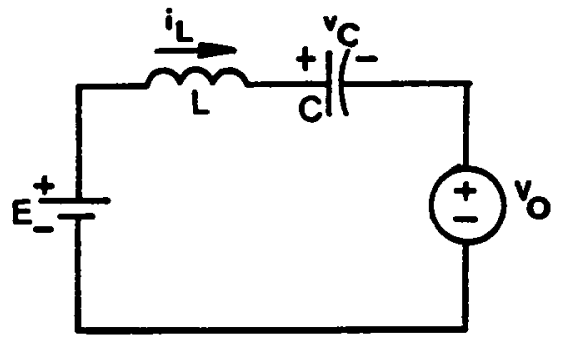

- $M 1: Q 1, Q 2$ on $\left(I_{L}>0\right)$

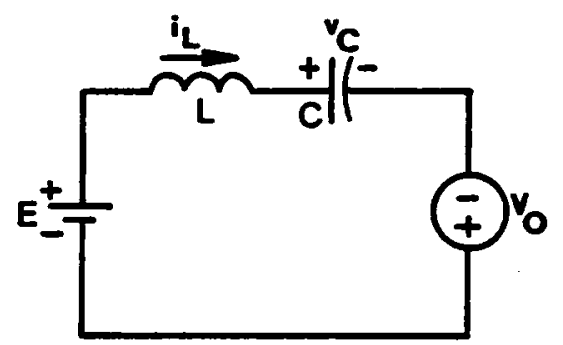

- $M 2: D 1, D 2$ on $\left(i_{L}<0\right)$

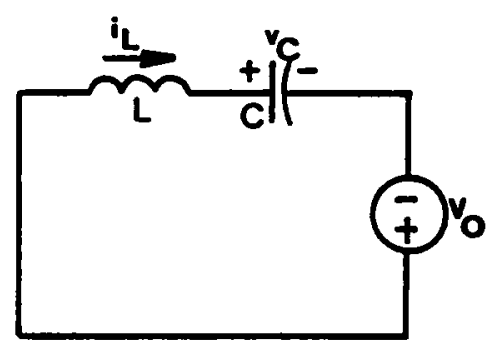

- M3 : Q3,D2 or $Q 4, D 1$ on $\left(i_{L}<0\right)$

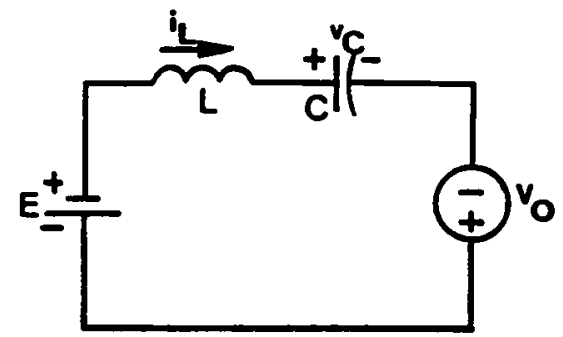

- M4:Q3, Q4 on $\left(I_{L}<0\right)$

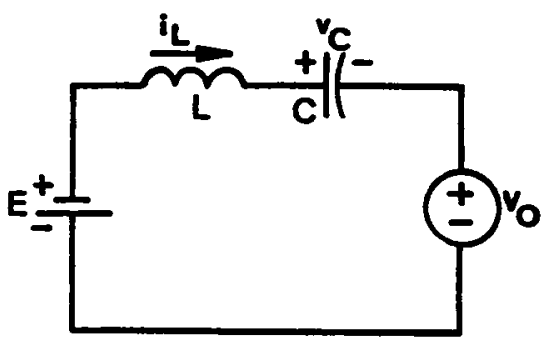

- $M 5: D 3, D 4$ on $\left(i_{L}>0\right)$

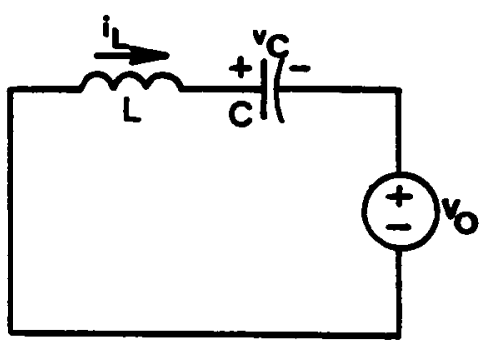

- $M 6: Q 1, D 4$ or $Q 2, D 3$ on $\left(i_{L}>0\right)$

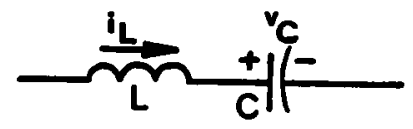

- MO : None of the devices on $\left(i_{L}=0\right)$

Figure 2.4 Circuit Topological Modes of a CM-SRC 


$$
\begin{aligned}
& i_{L N}=-\left(V_{C O N}-v_{E N}\right) \sin \omega_{0}\left(t-t_{0}\right)+I_{I D N} \cos \omega_{0}\left(t-t_{0}\right) \\
& v_{C N}=\left(V_{C O N}-v_{E N}\right) \cos \omega_{0}\left(t-t_{0}\right)+I_{L O N} \sin \omega_{0}\left(t-t_{0}\right)+v_{E N}
\end{aligned}
$$

where, $t_{0}$ is the initial time and

$$
\begin{aligned}
& \omega_{0}=\frac{1}{\sqrt{L C}} \quad \text { is the angular resonant frequency, } \\
& Z_{0}=\sqrt{\frac{L}{C}} \quad \text { is the characteristic impedance, } \\
& v_{C N}=\frac{v_{C}}{E} \quad \text { is the normalized capacitor voltage, } \\
& i_{L N}=\frac{i_{L}}{E / Z_{0}} \quad \text { is the normalized inductor current, } \\
& V_{C D N}=\frac{V_{C O}}{E} \quad \text { is the normalized initial capacitor voltage, } \\
& I_{L O N}=\frac{I_{L 0}}{E / Z_{0}} \quad \text { is the normalized initial inductor current, } \\
& v_{E N}=\frac{v_{E}}{E} .
\end{aligned}
$$

For recess mode M0,

$$
\begin{aligned}
& i_{I . N}=0 \\
& v_{C N}=V_{C O N} .
\end{aligned}
$$

The normalizing factors for the voltages and currents are $E$ and $E / Z_{0}$, respectively.

\subsubsection{State Trajectories for Circuit Topological Modes}


Table 2.1 Trajectory Equations for Various Circuit Topological Modes

\begin{tabular}{|c|l|c|}
\hline $\begin{array}{c}\text { Topo'I } \\
\text { Modes }\end{array}$ & \multicolumn{1}{|c|}{ State Trajectory Equations } & $\begin{array}{c}\text { Trajectory } \\
\text { Centers } \\
\left(V_{C N,} I_{L N}\right)\end{array}$ \\
\hline$M 1$ & $L_{L N}^{2}+\left(V_{C N}-\left(1-V_{O N}\right)\right)^{2}=I_{L O N}^{2}+\left(1-V_{O N}-V_{C O N}\right)^{2}$ & $\left(1-V_{O N}, 0\right)$ \\
\hline$M 2$ & $i_{L N}^{2}+\left(V_{C N}-\left(1+V_{O N}\right)\right)^{2}=I_{L O N}^{2}+\left(1+V_{O N}-V_{C O N}\right)^{2}$ & $\left(1+V_{O N,} 0\right)$ \\
\hline$M 3$ & $i_{L N}^{2}+\left(V_{C N}-V_{O N}\right)^{2}=I_{L O N}^{2}+\left(V_{O N}-V_{C O N}\right)^{2}$ & $\left(V_{O N}, 0\right)$ \\
\hline$M 4$ & $i_{L N}^{2}+\left(V_{C N}+\left(1-V_{O N}\right)\right)^{2}=I_{L O N}^{2}+\left(-1+V_{O N}-V_{C O N}\right)^{2}$ & $\left(-1+V_{O N}, 0\right)$ \\
\hline$M 5$ & $i_{L N}^{2}+\left(V_{C N}+\left(1+V_{O N}\right)\right)^{2}=I_{L O N}^{2}+\left(-1-V_{O N}-V_{C O N}\right)^{2}$ & $\left(-1-V_{O N,}, 0\right)$ \\
\hline$M 6$ & $i_{L N}^{2}+\left(V_{C N}+V_{O N}\right)^{2}=I_{L O N}^{2}+\left(-V_{O N}-V_{C O N}\right)^{2}$ & $\left(-V_{O N}, 0\right)$ \\
\hline$M 0$ & $i_{L N}=0, \quad V_{C N}=V_{C O N}$ & NOne \\
\hline
\end{tabular}


The trajectories for resonant modes M1,M2,M3,M4,M5, and M6, when plotted in the state plane $\left(v_{C N}\right.$ versus $i_{L N}$ plane), are circular arcs with centers located at $\left(v_{E N,}, 0\right)$ and radii $R=\sqrt{\left(v_{E N}-V_{C O N}\right)^{2}+I_{\text {ZON }}}$. Table 2.1 summarizes the trajectory equations. A family of trajectories for each resonant topological mode is plotted in Figure 2.5, where the

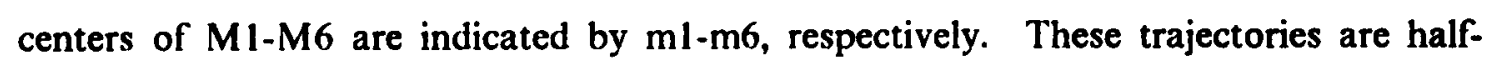
circles since the polarity of $i_{L}$ in each topological mode is either positive or negative. The state trajectory corresponding to a particular switching interval is a segment of these trajectories determined by the topological mode, the initial conditions of the inductor current and the capacitor voltage, and the duration of the interval. Time is implicit in these trajectories. The time elapsed in a trajectory segment is measured by the angle subtended by the segment with respect to its center. For example, the time elapsed from point a to point $b$ in topological mode Ml in Figure 2.5 is $\Delta t=\gamma / \omega_{0}$. As time advances, the state trajectories travel clockwise as indicated by the arrows.

The trajectory for the recess mode M0 is a stationary point lying on the $v_{\mathrm{CN}^{-}}$-axis. The coordinate of the stationary point is either $\left(V_{C O N}, 0\right)$ or $\left(-V_{C O N}, 0\right)$. A finite amount of time elapses at the stationary point since both $i_{I N N}$ and $v_{C N}$ are independent of time. A circle is used in the state plane to indicate such a stationary point, as illustrated in Figure 2.5. This topological mode occurs when none of the switching devices are conducting. In other words, all the switching devices are either reverse-biased or not triggered.

\subsubsection{Equilibrium Trajectory}

A steady-state operation of a CM-SRC can be represented by an equilibrium trajectory in the state plane $[18,19,31]$. $\Lambda \mathrm{n}$ equilibrium trajectory is a closed contour composed of several trajectory segments. It is symmetric with respect to the origin and is constructed via a particular sequence of circuit topological modes determined by the circuit operation. An equilibrium trajectory constructed via a different topological mode 
sequence represents a different device conducting sequence, thus corresponding to a different circuit operating mode.

An equilibrium state trajectory of a CM-SRC can be constructed using a composite diagram generated by overlapping the families of trajectories in Figure 2.5 on the same $i_{L N^{-}}, v_{C N^{-}}$axes, as shown in Figure 2.6. The rules for constructing an equilibrium trajectory on the composite diagram are summarized in $\Lambda$ ppendix B.1. Figure 2.7 shows an example illustrating the construction of an equilibrium trajectory on the composite diagram. The constructed trajectory corresponds to the circuit operation described in Figure 2.2, where $\mathrm{M} 6(\mathrm{M} 3)$ is initiated when $\mathrm{Q} 1(\mathrm{Q} 3)$ is triggered at point a(d), M1(M4) is initiated when $\mathrm{Q} 2(\mathrm{Q} 4)$ is triggered at point $\mathrm{b}(\mathrm{e})$, and $\mathrm{M} 2(\mathrm{M} 5)$ is initiated when $i_{\mathrm{L}}$ reverses polarity at point $c(f)$. 


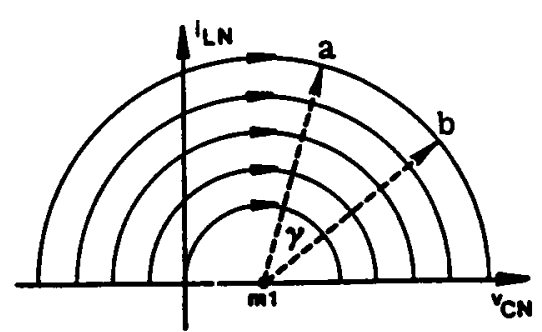

- Mode M1

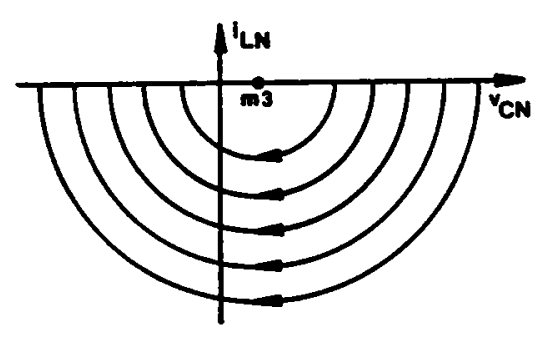

- Mode M3

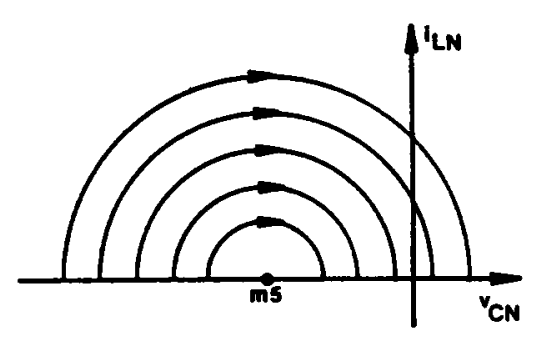

- Mode M5

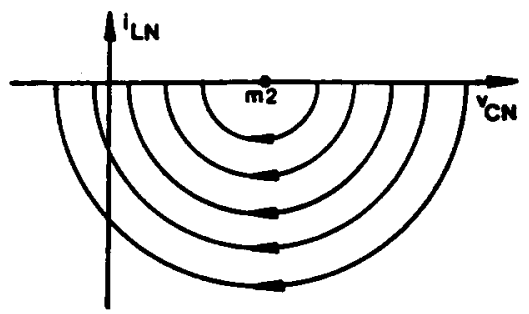

- Mode M2

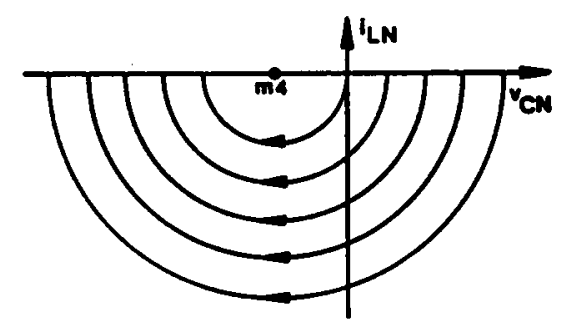

- Mode MA

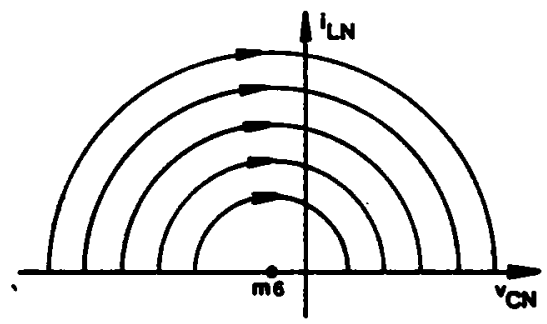

- Mode MG

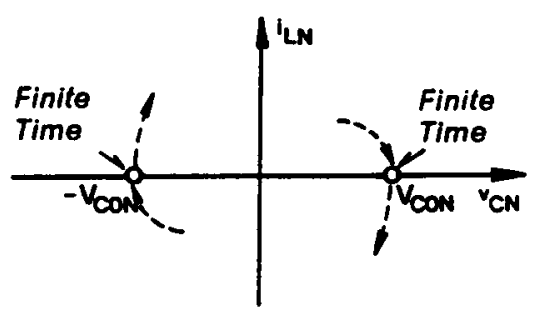

- Mode MO

Figure 2.5 Families of State Trajectory for Various Circuit Topological Modes 


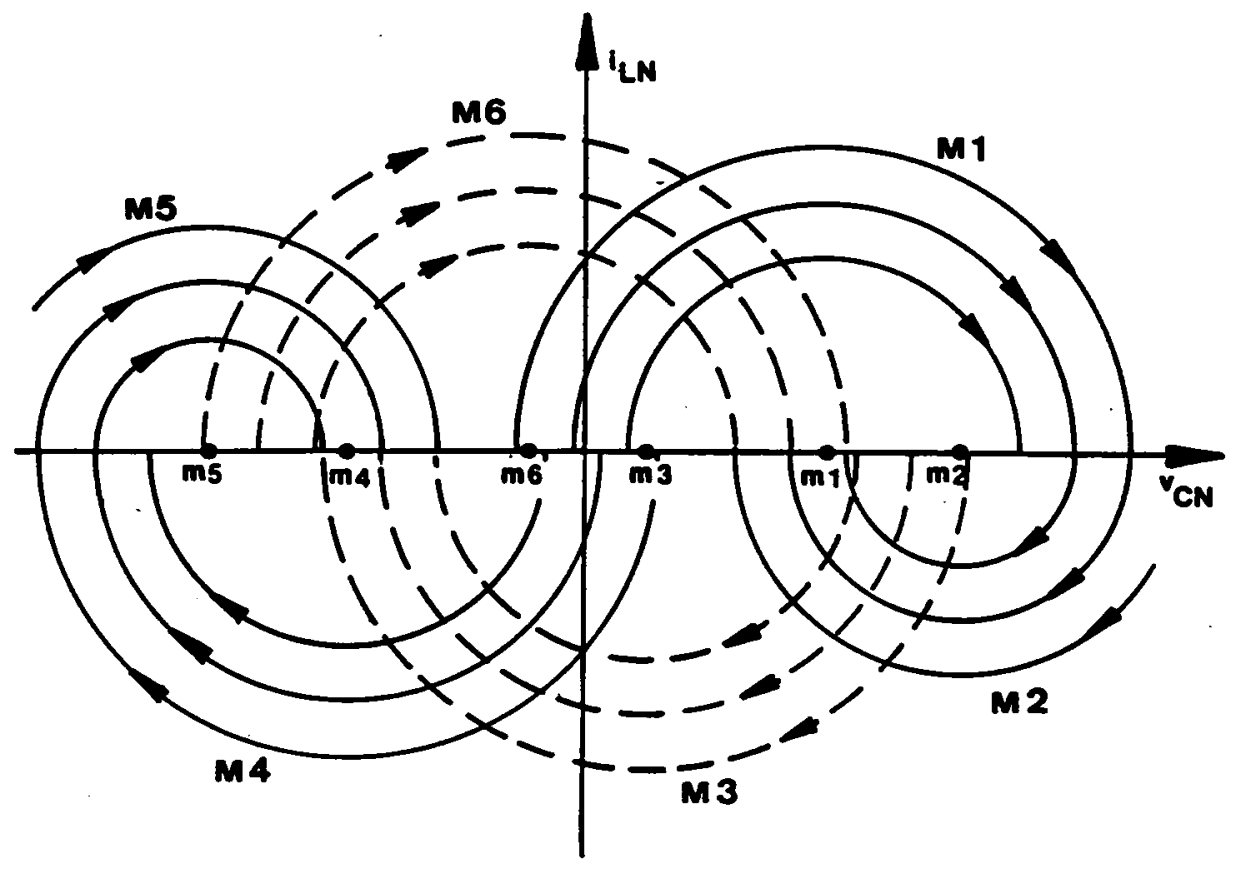

Figure 2.6 A Composite State Diagram for Constructing Equilibrium State Trajectories 


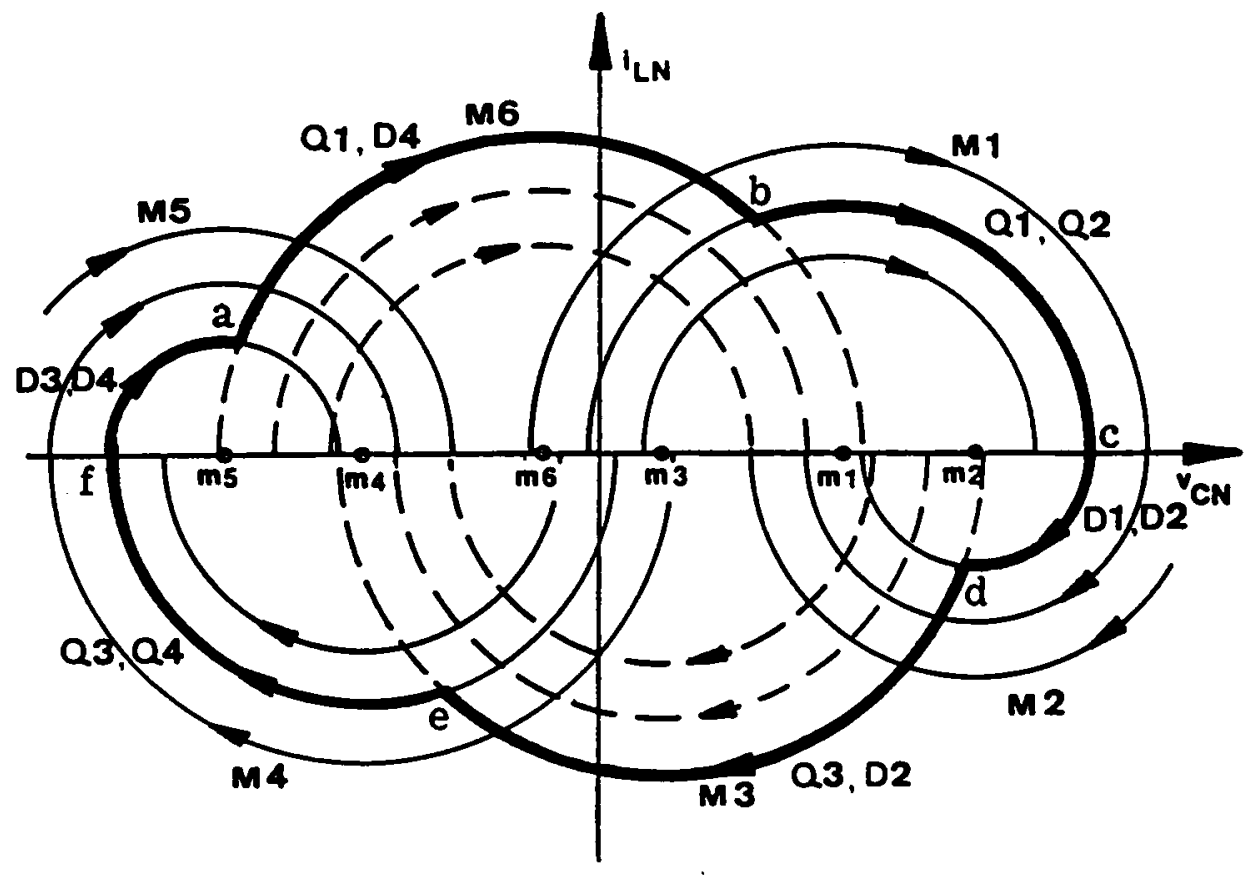

Figure 2.7 An Example Illustrating Construction of an Equilibrium State Trajectory on the Composite Diagram 


\subsubsection{Circuit Operating Modes}

Many equilibrium trajectories, each representing a circuit operating mode, have been constructed. These trajectories are categorized into two frequency ranges and discussed in the following.

\subsubsection{Operating modes below the resonant frequency}

Figure 2.8 shows a series of equilibrium state trajectories which illustrate all the possible operating modes of a CM-SRC operating below the resonant frequency. The corresponding circuit waveforms for the trajectories are also included in the figure to help explain the converter's operation.

Trajectory 0. This trajectory represents the case when the converter operates as a conventional SRC. In other words, no zero-voltage interval exists in voltage $v_{s}$. As shown in Figure 2.8(a), at $t=a$, transistors $\mathrm{Q} 1$ and $\mathrm{Q} 2$ turn on commutating diodes D3 and D4. The inductor current, $i_{L N}$, resonates through $\mathrm{Q} 1$ and $\mathrm{Q} 2(\mathrm{M} 1)$. At $\mathrm{t}=\mathrm{b}, i_{\mathrm{LN}}$ reverses polarity due to resonance. Transistors $Q 1$ and $Q 2$ turn off naturally and diodes D1 and D2 conduct subsequently. The inductor current resonates through DI and D2(M2). At $t=c$, transistors $Q 3$ and $Q 4$ turn on commutating diodes D1 and D2 and a similar process occurs with the roles of Q1,Q2,D1,D2 and Q3,Q4,D3,D4 interchanged, respectively. The topological mode sequence of this trajectory is M1-M2-M4-M5.

Trajectory 1: When a 7,ero-voltage interval is introduced into $v_{s}$, topological modes $\mathrm{M} 3$ and M6 will be present in the trajectory. $\Lambda \mathrm{s}$ shown in Figure $2.8(\mathrm{~b})$, at $\mathrm{t}=\mathrm{a}$, transistor Q1 turns on commutating diode D3. The inductor current, $i_{L N}$, resonates through $\mathrm{Q} 1$ and D4(M6). $\Lambda \mathrm{t} t=\mathrm{b}$, transistor $\mathrm{Q} 2$ turns on commutating diode D4. The inductor current, $i_{L N}$, resonates through $Q 1$ and $Q 2(M 1)$. $\Lambda t t=c, i_{L N}$ reverses polarity. Transistors Q1 and Q2 turn off naturally and diodes D1 and D2 conduct subsequently. The inductor current resonates through $\mathrm{D} /$ and $\mathrm{D} 2(\mathrm{M} 2)$. At $\mathrm{t}=\mathrm{d}$, transistor $\mathrm{Q} 3$ turns on 
commutating diode D1 and a similar process occurs with the roles of Q1,Q2,D1,D2 and Q3,Q4,D3,D4 interchanged, respectively. The topological mode sequence of this trajectory is M6-MI-M2-M3-M4-M5, which is defined as "mode-I operation" of CM-SRC. Obviously, Trajectory 0 is a special case of Trajectory 1.

Trajectory 1': As the zero-voltage interval in $v_{s}$ increases, natural commutation of all the transistors no longer can be achieved. In this trajectory, the natural-commutation boundary for transistors $\mathrm{Q} 1$ and $\mathrm{Q} 3$ is reached. As shown in Figure 2.8(c), transistors Q1,Q3 are triggered at the instants $i_{L N}$ reverses polarity $(\mathrm{t}=\mathrm{a}$ and $\mathrm{c})$. As a result, no D1,D2 or D3,D4 conduction period exists. The topological mode sequence for this trajectory is M6-M1-M3-M4, which is also a special case of Trajectory 1.

Trajectory 2: In Trajectory 1, it is possible that the magnitude of the capacitor voltage, $\left|v_{C N}\right|$, is less than the sum of the input voltage and the output voltage, $1+V_{O N}$, when $i_{L N}$ reverses polarity at $\mathrm{t}=\mathrm{c}$ (or $\mathrm{f}$, as shown in Figure 2.8(d). As a result, diodes D1,D2 (or D3,D4) are reverse biased and none of the switching devices conducts. The circuit stays in a recess mode(M0) until transistor $Q^{3}$ (or $\left.Q 1\right)$ is triggered at $t=d$ (or a) to initiate another resonant mode. The topological mode sequence of this trajectory is M6-MI-M0-M3-M4-MO, which is defined as "mode-II operation".

Trajectory 3: When the zero-voltage interval in $v_{s}$ is further increased, natural commutation of $Q 1$ and $Q 3$ is no longer possible. As illustrated in Figure 2.8(e), at $t=a$, Q3 is forced off and $\mathrm{Q} 1$ is triggered. Diode $\mathrm{DI}$ conducts since $i_{L N}$ is negative. The inductor current resonates through $\mathrm{Dl}$ and $\mathrm{Q} 4(\mathrm{M} 3) . \quad \Lambda \mathrm{t} t=\mathrm{b}, i_{L N}$ reverses polarity. Diode D1, transistor Q4 turn off naturally and transistor Q1, diode D4 conduct subsequently. The inductor current resonates through $Q 1$ and D4(M6). At $t=c$, transistor Q2 turns on commutating diode D4. The inductor current resonates through Q1 and Q2(M1). At $t=d, Q 1$ is forced off and $Q 3$ is triggered. A similar process occurs with the roles of Q1,Q2,D1,D2 and Q3,Q4,D3,D4 interchanged, respectively. The topological 

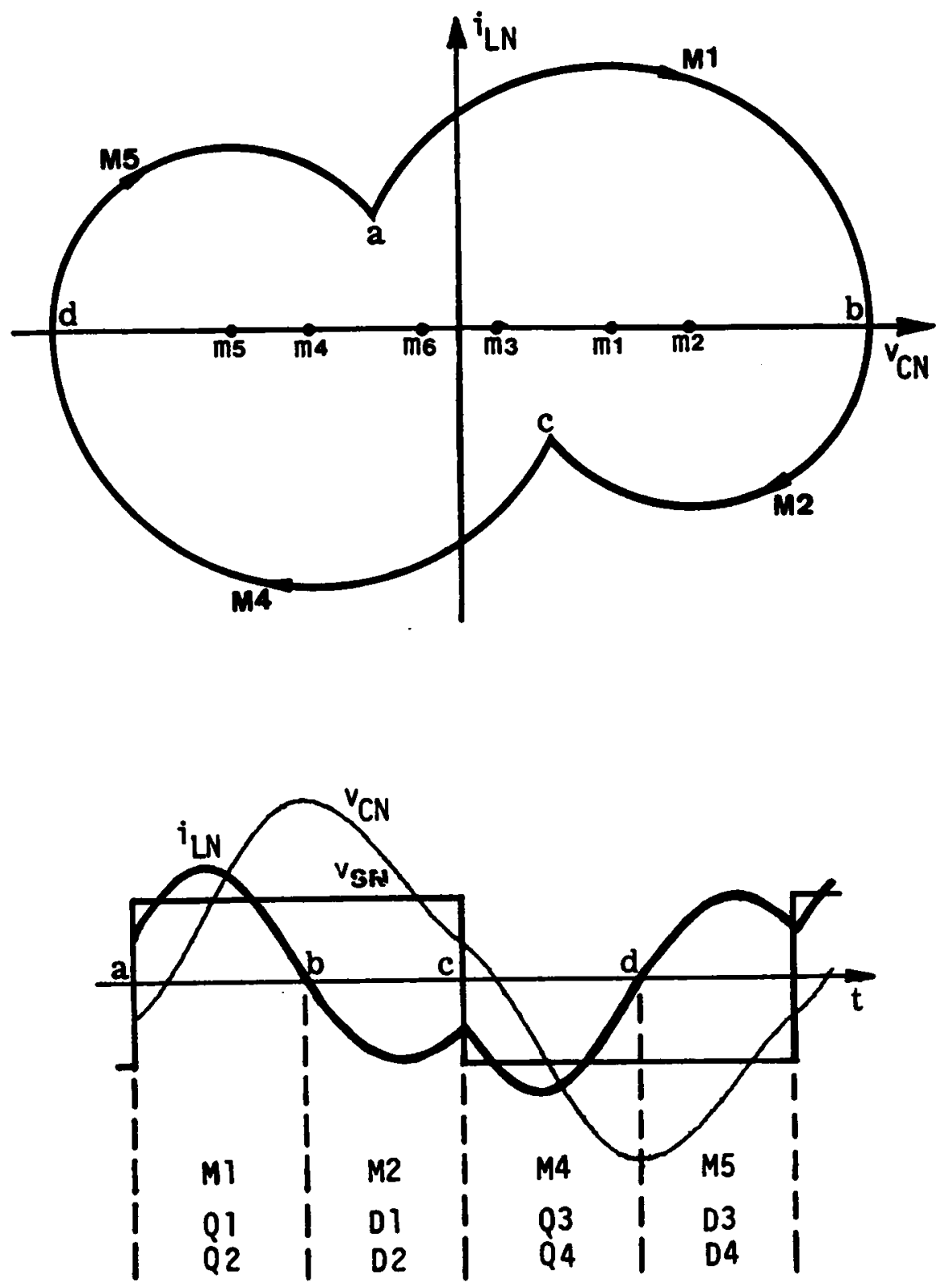

(a) Trajectory 0 and its corresponding circuit waveforms (SRC operation)

Figure 2.8 Equilibrium Trajectories of a CM-SRC Operating Below Resonant Frequency 

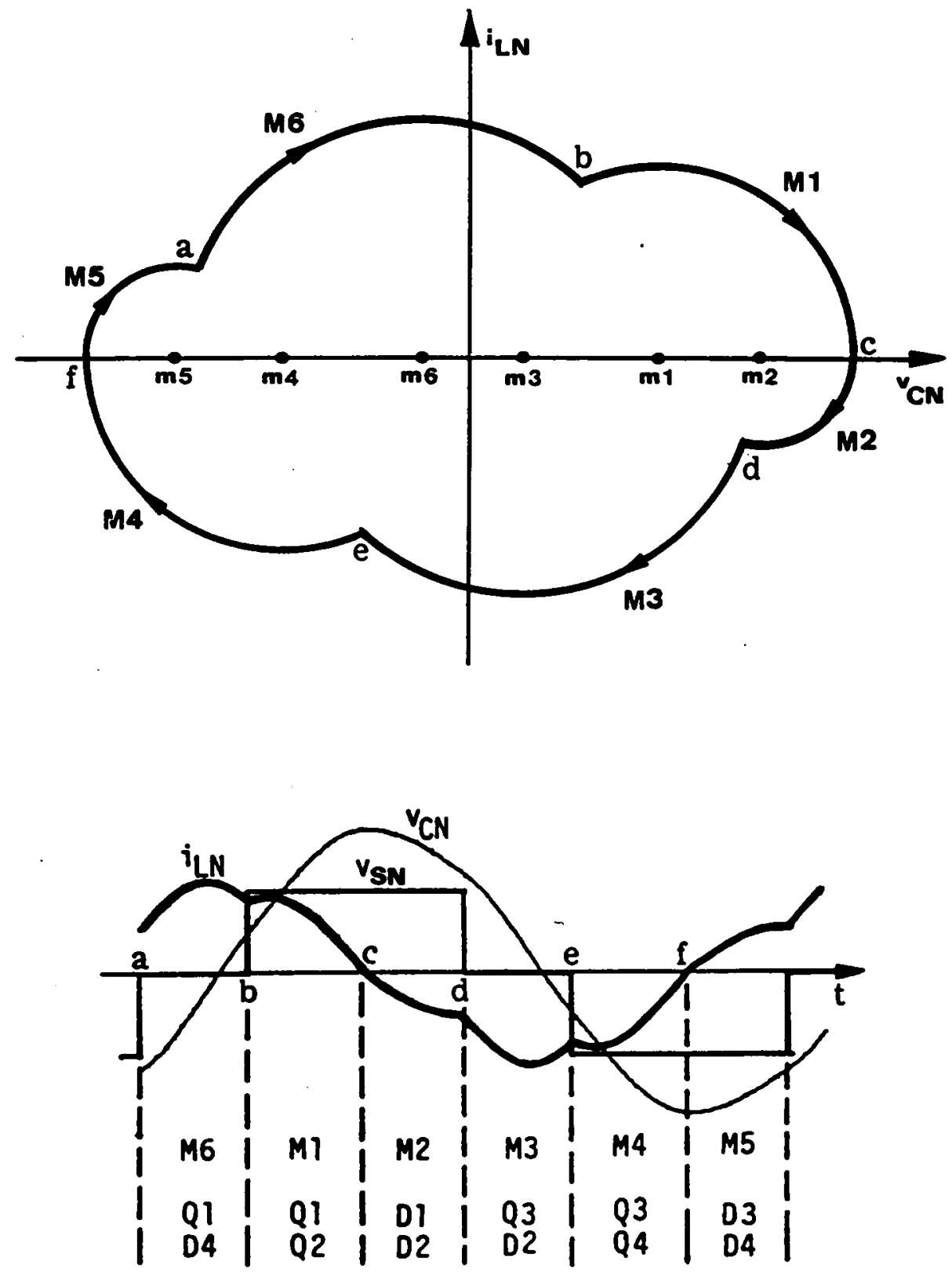

(b) Trajectory $l$ and its corresponding circuit waveforms (Mode-l operation)

Figure 2.8 Continued 

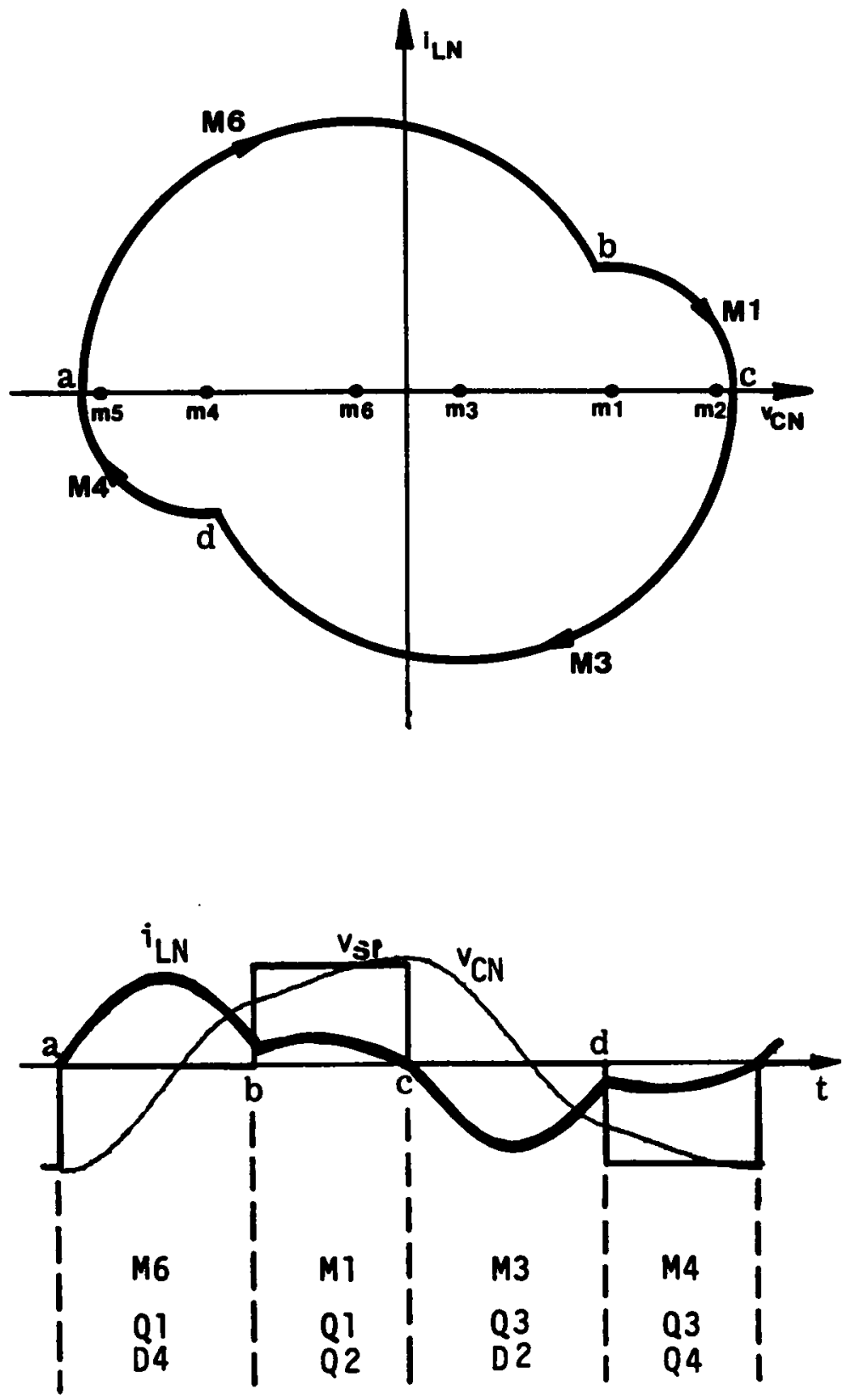

(c) Trajectory $l^{\prime}$ and its corresponding circuit waveforms (Mode-I operation)

Figure 2.8 Continued 

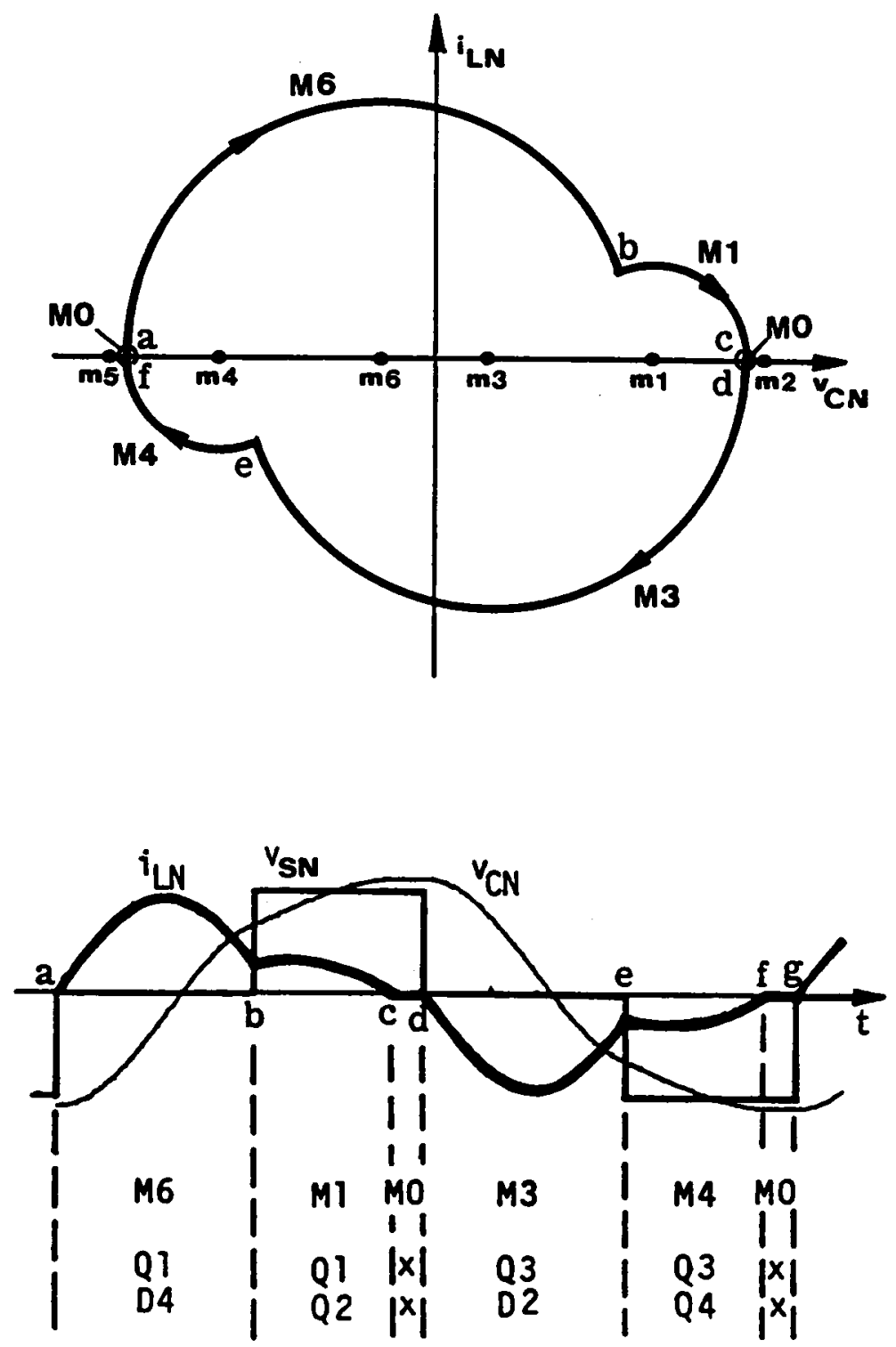

(d) Trajectory 2 and its corresponding circuit waveforms (Mode-II operation)

Figure 2.8 Continued 

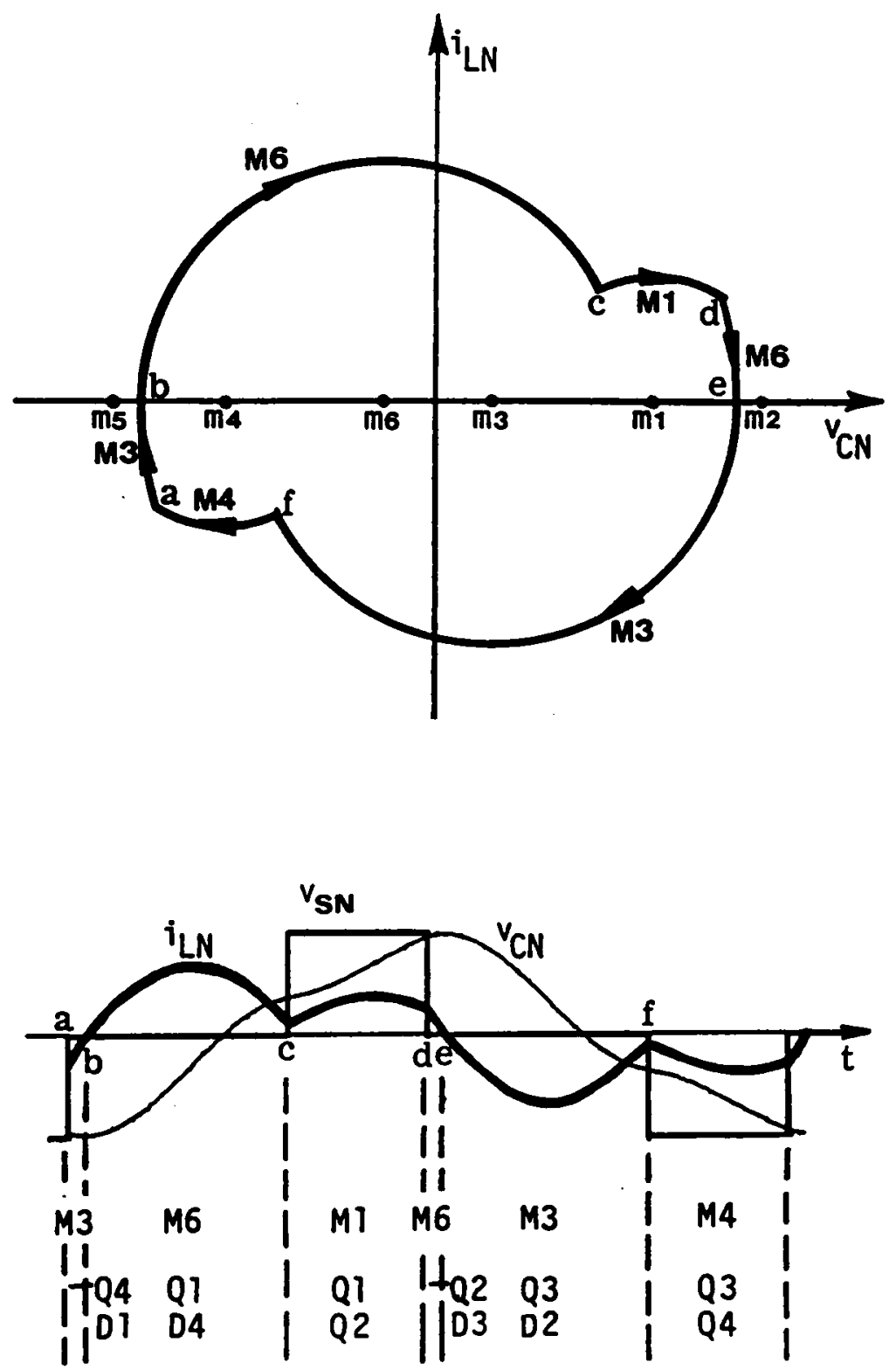

(e) Trajectory 3 and its corresponding circuit waveforms (Mode-III operation)

Figure 2.8 Continued 
mode sequence for this trajectory is $M 3-M 6-M I-M 6-M 3-M 4$, which is defined as "mode-III operation".

Trajectory 4: In Trajectory 3, if $i_{L N}$ reverses polarity for the second time before Q2 (or Q4) is triggered at $\mathrm{t}=\mathrm{c}$ (or $\mathrm{f}$ ) and the magnitude of $v_{C N}$ is greater than $V_{O N}$ at both instants $i_{L N}$ reverses polarity, transistors $\mathrm{Q} 1, \mathrm{Q} 2, \mathrm{Q} 3, \mathrm{Q} 4$ will conduct twice during an operating cycle. As illustrated in Figure 2.8( () , at $\mathrm{t}=\mathrm{a}, \mathrm{Q} 3$ is forced off and $\mathrm{Q} 1$ is triggered. Diode D1 conducts since $i_{L N}$ is negative. The inductor current resonates through DI and Q4(M3). At $\mathrm{t}=\mathrm{b}, i_{L N}$ reverses polarity. Diode D1, transistor Q4 turn off naturally and transistor Q1, diode D4 conduct subsequently. The inductor current resonates through Q1 and D4(M6). At $\mathrm{t}=\mathrm{c}, i_{L N}$ reverses polarity again. Transistor Q1, diode D4 turn off naturally and diode D1, transistor Q4 conduct for the second time. The inductor current resonates through D1 and Q4(M3). At $\mathrm{t}=\mathrm{d}, \mathrm{Q} 4$ is forced off and $\mathrm{Q} 2$ is triggered. Diode D2 conducts since $i_{L N}$ is negative. The inductor current resonates through D1 and $\mathrm{D} 2(\mathrm{M} 2)$. At $\mathrm{t}=\mathrm{e}, i_{L N}$ reverses polarity. Diodes D1,D2 turn off naturally and transistors Q1,Q2 conduct subsequently. The inductor current resonates through $\mathrm{Q} 1$ and $\mathrm{Q} 2(\mathrm{M} 1)$. At $t=f, Q 1$ is forced off and $Q 3$ is triggered. Diode $D 3$ conducts since $i_{L N}$ is positive and a similar process occurs with the roles of Q1,Q2,D1,D2 and Q3,Q4,D3,D4 interchanged, respectively. The topological mode sequence for this trajectory is M3-M6-M3-M2-MI-M6-M3-M6-M5-M4, which is defined as "mode-IV operation".

Trajectory 5: This trajectory evolves either from Trajectory 3 or from Trajectory 4 as the zero-voltage interval in $v_{S}$ is increased. Consider Trajectory 4 . At $t=c($ or $h$ ), if the magnitude of $v_{C N}$ is less than $V_{O N}$, diode DI and transistor Q4 (or D3 and Q2) will be reverse-biased. As a result, the circuit will stay in a recess mode(M0) until transistor Q2 (or Q4) is triggered to initiate another resonant mode. The circuit's operation for this trajectory is illustrated in Figure $2.8(\mathrm{~g})$. The topological mode sequence is M3-M6-MO-MI-M6-M3-MO-M4, which is defined as "mode-V operation". 

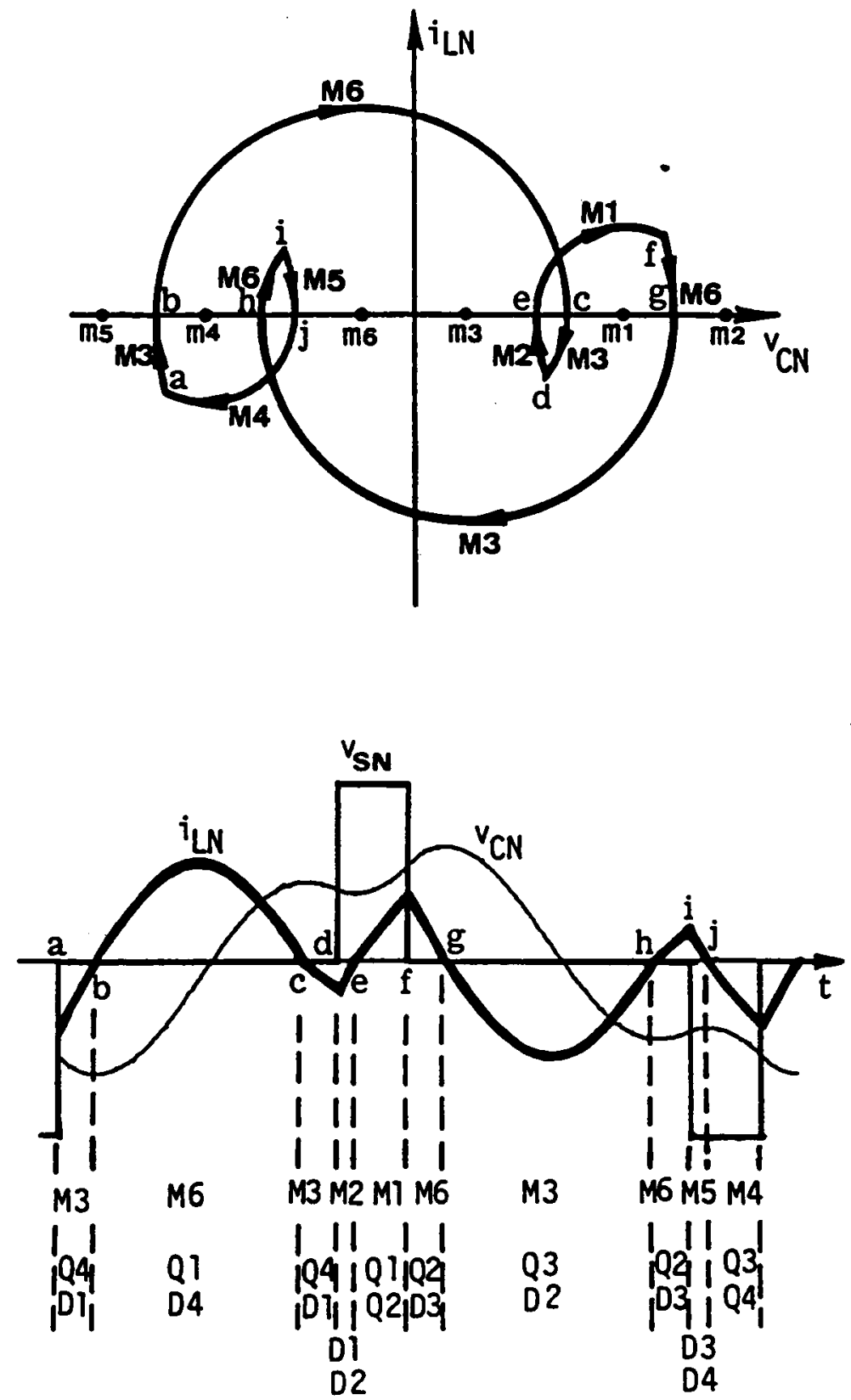

(f) Trajectory 4 and its corresponding circuit waveforms (Mode-IV operation)

Figure 2.8 Continued 

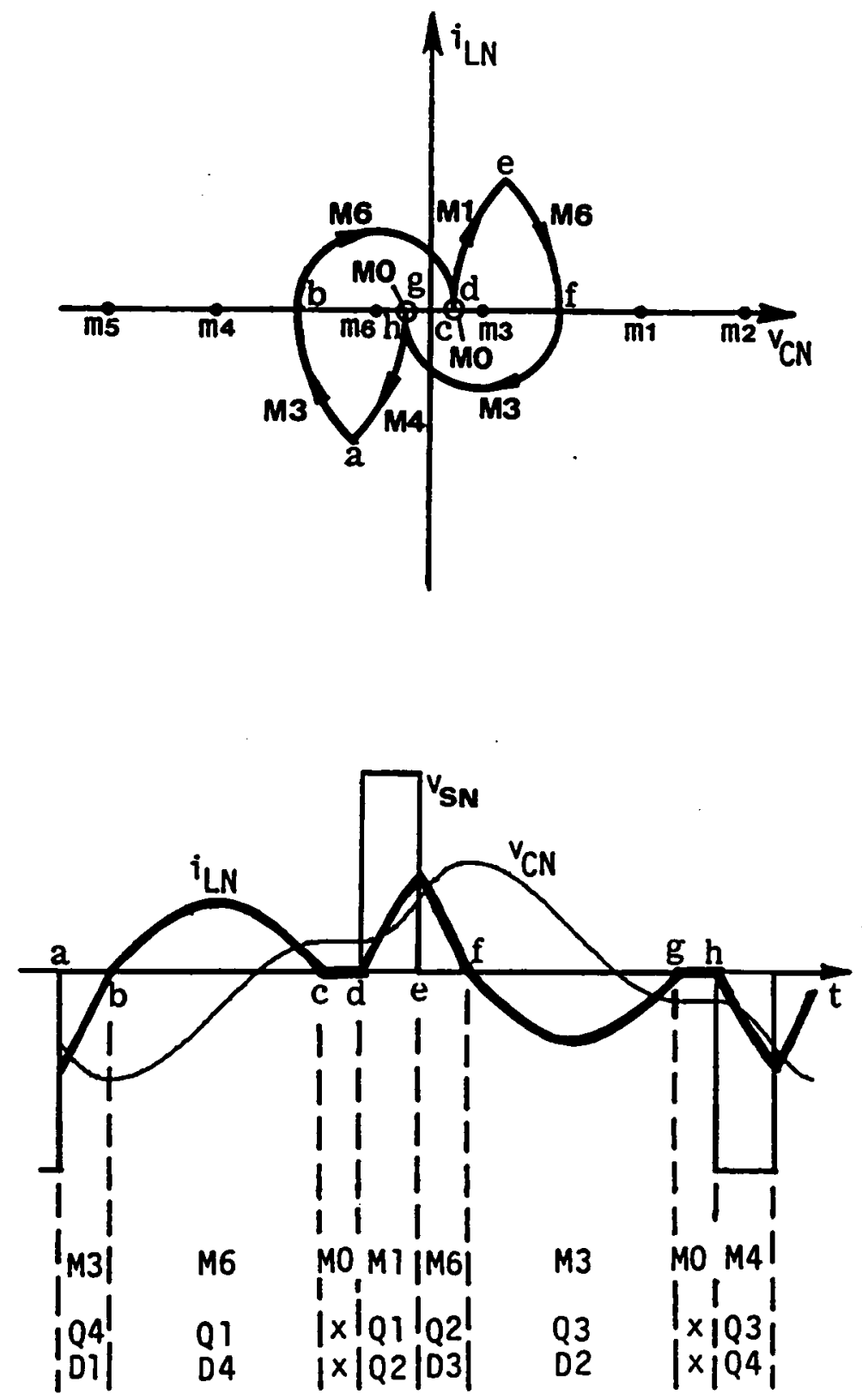

(g) Trajectory 5 and its corresponding circuit waveforms (Mode-V operation)

Figure 2.8 Continued 
Trajectory 6: Further increasing the zero-voltage interval in $v_{s}$, Trajectory 3, 4 or 5 may degenerate into this trajectory. Consider Trajectory 5. If the magnitude of $v_{C N}$ is less than $V_{O N}$ when $i_{L N}$ reverses polarity at $\mathrm{t}=\mathrm{b}$ (or $\mathrm{f}$ ), transistor $\mathrm{Q} 1$ and diode $\mathrm{D} 4$ (or Q3 and D2) will be reverse-biased and none of the switching devices will conduct. The circuit will stay in a recess mode(M0) until transistor Q2 (or Q4) is triggered at $t=c$ (or f) to initiate another resonant mode. The circuit's operation for this trajectory is illustrated in Figure 2.8(h). The topological mode sequence is $M 3-M 0-M I-M 6-M 0-M 4$, which is defined as "mode-VI operation".

Table 2.2 summarizes the topological mode sequences for the six operating modes. When the CM-SRC operates in mode I or mode II, all the transistors, Q1-Q4, are naturally commutated. When the CM-SRC operates in mode III or mode VI, transistors $\mathrm{Q} 1$ and $\mathrm{Q} 3$ are force-commutated while transistors $\mathrm{Q} 2$ and $\mathrm{Q} 4$ are naturally commutated. When the CM-SRC operates in mode IV, transistors Q1-Q4, are naturally commutated for their first conduction and force-commutated for their second conduction. When the CM-SRC operates in mode V, transistors $Q 1, Q 3$ are naturally commutated for their first conduction and force-commutated for their second conduction; transistors Q2 and Q4 are always naturally commutated. Table 2.3 summarizes the commutation features of all the transistors in various operating modes. It should be pointed out that force-commutated transistors usually turn on at zero voltage. As a result, lossless capacitor snubbers can be used to reduce their turn-off losses.

\subsubsection{Operating modes above resonant frequency}

Figure 2.9 shows a series of equilibrium state trajectories which illustrate all the possible operating modes of a CM-SRC operating above resonant frequency. The corresponding circuit waveforms for the trajectories are also included in the figure to help explain the converter's operation. 

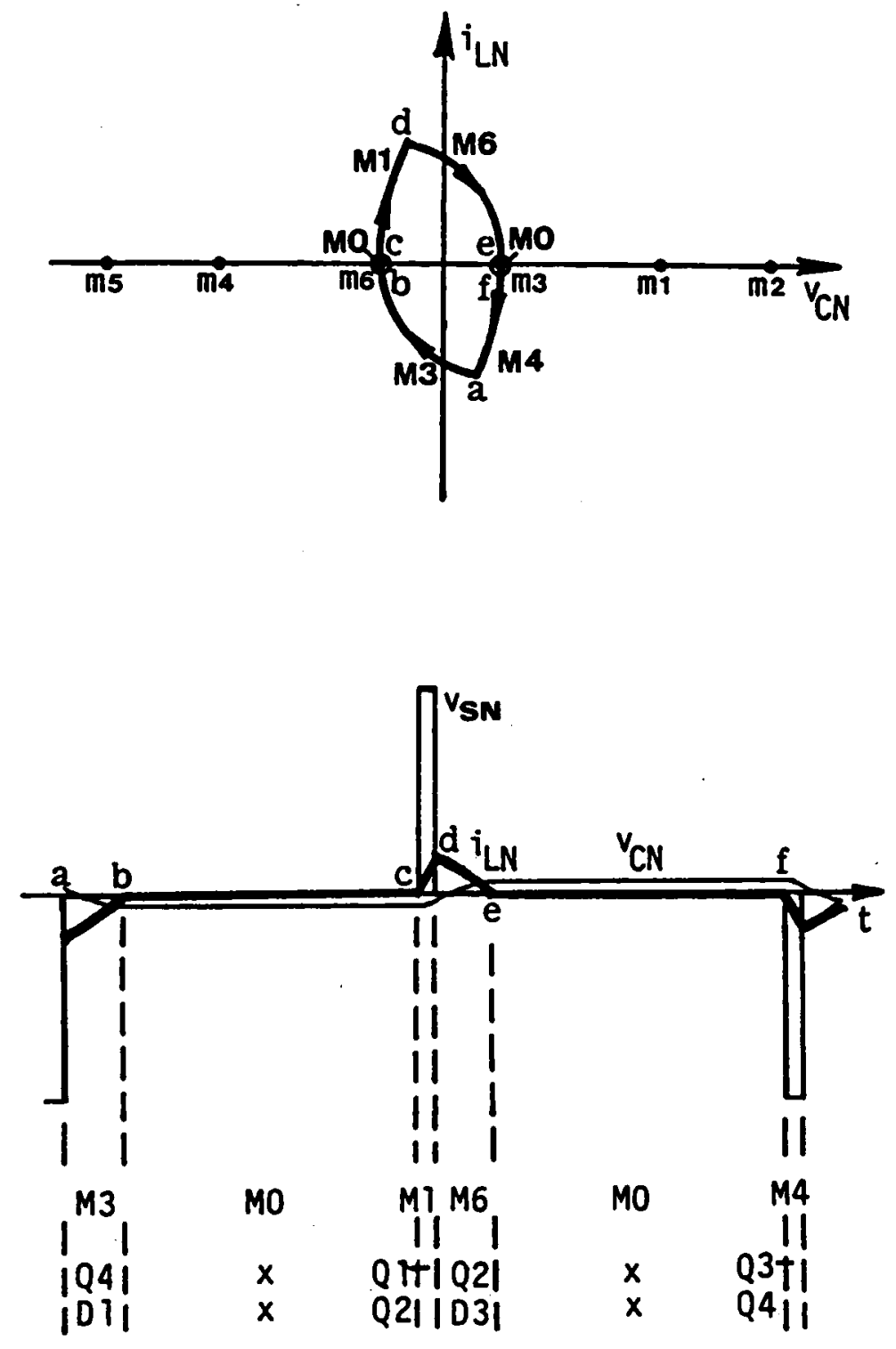

(h) Trajectory 6 and its corresponding circuit waveforms (Mode-VI operation)

Figure 2.8 Continued 
Table 2.2 Topological Mode Sequences for Circuit Operating Modes Below Resonant Frequency

\begin{tabular}{|c|l|}
\hline $\begin{array}{c}\text { Operating } \\
\text { Modes }\end{array}$ & \multicolumn{1}{|c|}{ Topological Mode Sequences } \\
\hline I & M6-M1-M2-M3-M4-M5 \\
\hline II & M6-M1-MO-M3-M4-M0 \\
\hline III & M6-M1-M6-M3-M4-M3 \\
\hline IV & M3-M6-M3-M2-M1-M6-M3-M6-M5-M4 \\
\hline V & M3-M6-MO-M1-M6-M3-M0-M4 \\
\hline VI & M3-MO-M1-M6-MO-M4 \\
\hline
\end{tabular}


Table 2.3 Commutation Features for Transistors in Various Circuit Operating Modes Below Resonant Frequency

\begin{tabular}{|c|c|c|c|c|c|c|}
\hline \multirow{2}{*}{ Mode } & \multirow{2}{*}{$i_{L}$} & \multicolumn{2}{|c|}{ Turn-On } & \multicolumn{2}{|c|}{ Turn-Off } & \multirow{2}{*}{ Comments } \\
\hline & & Q1,Q3 & Q2,Q4 & Q1,Q3 & Q2,Q4 & \\
\hline $\mathbf{I}$ & cont. & n.z.v. & n.z.v. & z.c. & z.c. & $m * * * *$ \\
\hline II & discont. & Z.v. & n.z.v. & z.c. & z.c. & "xm*n \\
\hline III & cont. & z.v. & n.z.v. & n.z.c. & z.c. & "***** \\
\hline IV & cont. & $\begin{array}{l}\text { z.V. } \\
\text { z.v. }\end{array}$ & $\frac{\text { z.v. }}{\text { z.v. }}$ & $\begin{array}{c}\text { z.c. } \\
\text { n.z.c. }\end{array}$ & n.z.c. & $\begin{array}{c}\text { Q1-Q4 } \\
\text { turn on } \\
\text { twice }\end{array}$ \\
\hline $\mathbf{v}$ & discont. & $\begin{array}{l}\text { z.v. } \\
\text { z.v. }\end{array}$ & $\frac{Z . V .}{* * * *}$ & $\frac{\text { z.c. }}{\text { n.z.c. }}$ & $\frac{\text { Z.C. }}{m \ldots+\infty}$ & $\begin{array}{c}\text { Q1,Q3 } \\
\text { turn on } \\
\text { twlce }\end{array}$ \\
\hline VI & discont. & z.v. & z.v. & n.z.c. & z.c. & n**** \\
\hline
\end{tabular}

n.z.c. - turn off at nonzero current

z.c. - turn off at zero current

n.z.v. - turn on at nonzero voltage

z.v. - turn on at zero voltage 
Trajectory $A_{0}$ : This trajectory represents the case when the converter operates as a conventional SRC. As shown in Figure 2.9(a), at $\mathrm{t}=\mathrm{a}$, transistors $\mathrm{Q3}, \mathrm{Q} 4$ are forced off and Q1,Q2 are triggered. Transistors $\mathrm{Q} 1, \mathrm{Q} 2$ cannot conduct since $i_{L N}$ is negative. Instead, diodes D1,D2 conduct. The inductor current resonates through D1 and D2(M2). At $\mathrm{t}=\mathrm{b}, i_{L N}$ reverses polarity due to resonance. Diodes D1,D2 turn off naturally and transistors Q1,Q2 conduct subsequently. The inductor current resonates through Q1 and Q2(M1). At $\mathrm{t}=\mathrm{c}$, transistors $\mathrm{Q} 1, \mathrm{Q} 2$ are forced off and $\mathrm{Q} 3, \mathrm{Q} 4$ are triggered. $\mathrm{A}$ similar process occurs with the roles of Q1,Q2,D1,D2 and Q3,Q4,D3,D4 interchanged, respectively. The topological mode sequence of this trajectory is M2-M1-M5-M4.

Trajectory A: When a zero-voltage interval is introduced into $v_{S}$, topological modes M3 and M6 will be present in the trajectory. As shown in Figure 2.9(b), at $t=a$, transistor Q3 is forced off and Q1 is triggered. Diode D1 conducts instead of Q1 since $i_{L N}$ is negative. The inductor current resonates through Q4 and D1(M3). At $t=b$, transistor Q4 is forced off and Q2 is triggered. Diode D2 conducts instead of Q2 since $i_{L N}$ is still negative. The inductor current resonates through D1 and D2(M1). At $t=c, i_{L N}$ reverses polarity due to resonance. Diodes D1,D2 turn off naturally and transistors Q1,Q2 conduct subsequently. The inductor current resonates through $Q 1$ and $Q 2(M 1)$. At $t=d$, transistor Q1 is forced off and Q3 is triggered. A similar process occurs with the roles of Q1,Q2,D1,D2 and Q3,Q4,D3,D4 interchanged, respectively. The topological mode sequence of this trajectory is M6-MI-M2-M3-M4-MS, which is defined as "mode-A operation" of CM-SRC. Obviously, Trajectory $\Lambda_{0}$ is a special case of Trajectory A.

Trajectory $A^{\prime}: \Lambda s$ the zero-voltage interval in $v_{s}$ increases, the force commutation boundary of Q2 and Q4 is reached. As shown in Figure 2.9(c), Q2 and Q4 are commutated at the instants when $i_{L N}$ reverses polarity ( $t=\mathrm{b}$ and $\mathrm{d}$ ). In other words, Q2 and Q4 no longer turn off with current. The topological mode sequence for this trajectory is M3-M1-M6-M4, also a special case of Trajectory A. 

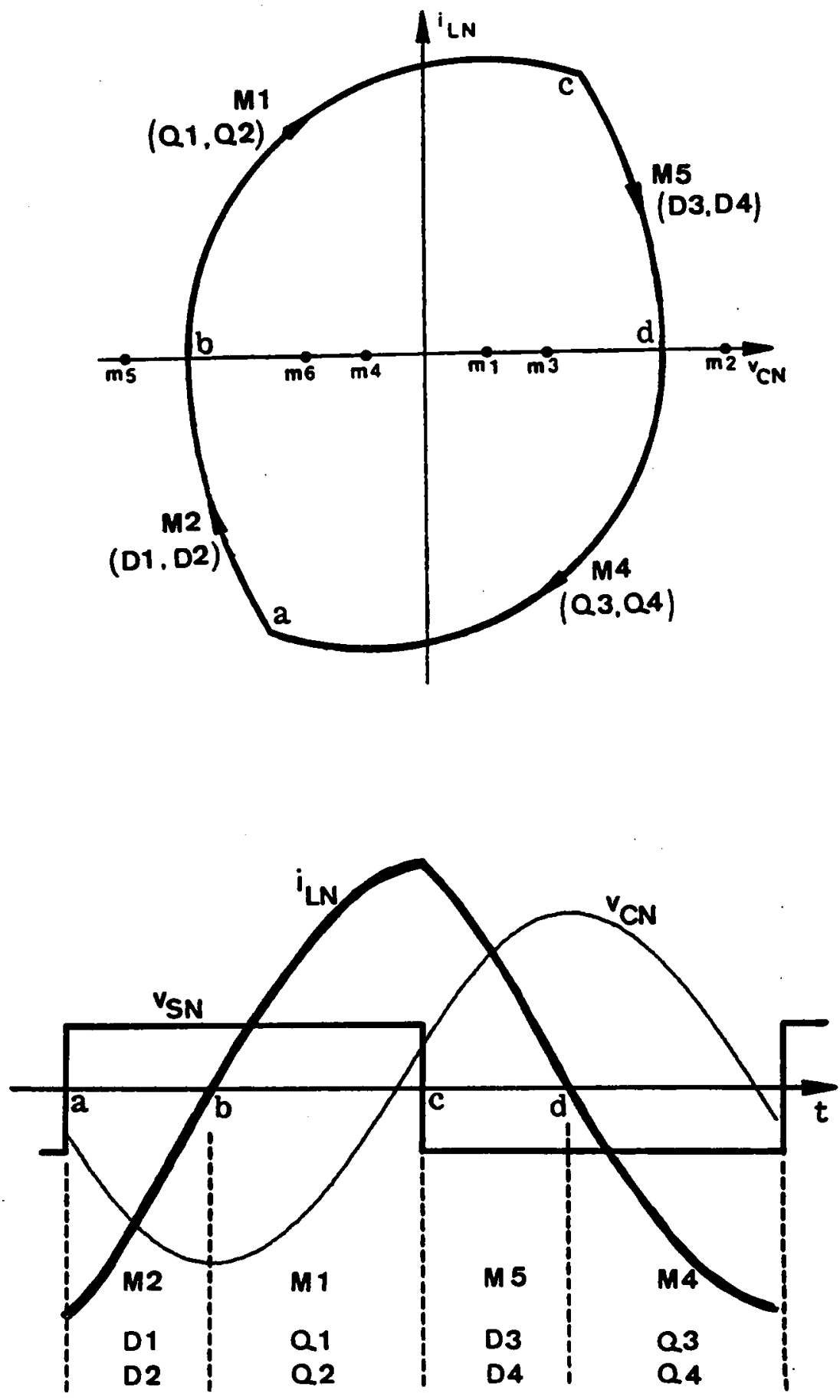

(a) Trajectory $A_{0}$ and its corresponding circuit waveforms (SRC operation)

Figure 2.9 Equilibrium Trajectories of a CM-SRC Operating Above Resonant Frequency 

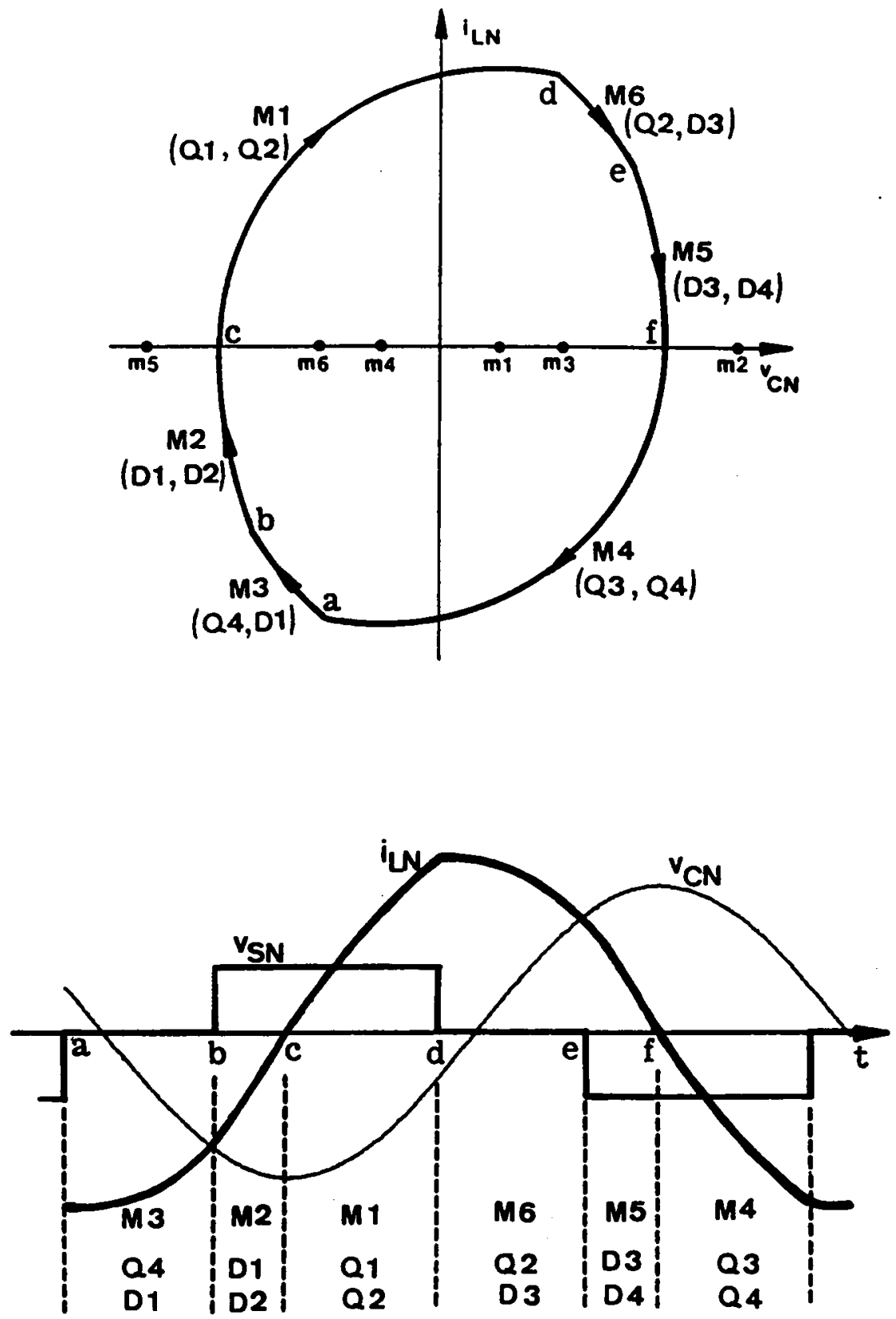

(b) Trajectory $A$ and its corresponding circuit waveforms (Mode-A operation)

Figure 2.9 Continued 

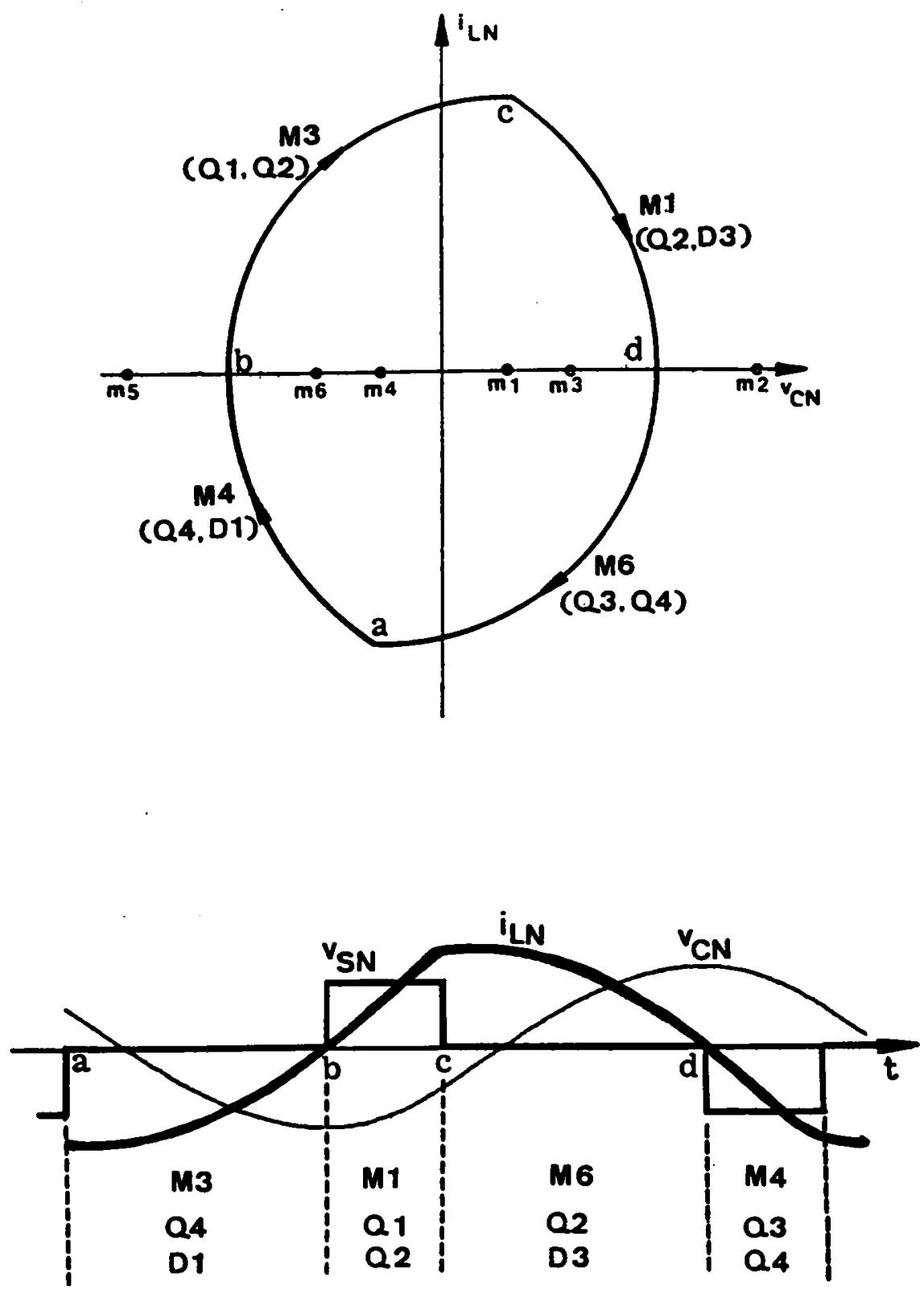

(c) Trajectory $A^{\prime}$ and its corresponding circuit waveforms (Mode-A operation)

Figure 2.9 Continued 
Trajectory B: When the zero-voltage interval in $v_{S}$ is further increased, transistors Q2 and $\mathrm{Q} 4$ become naturally commutated. In this case, the converter's operation is the same as mode-III operation below resonant frequency, as illustrated in Figure 2.9(d). The topological mode sequence for this trajectory is $M 3-M 6-M I-M 6-M 3-M 4$ and is defined as "mode-B operation".

Trajectory C: Further increasing the zero-voltage interval in $v_{S}$, Trajectory B will degenerate into this trajectory if the magnitude of $v_{C N}$ is less than $V_{O N}$ when $i_{L N}$ reverses polarity at $\mathrm{t}=\mathrm{b}$ (or $\mathrm{e})$. The converter's operation is the same as Mode-VI operation below resonant frequency, as illustrated in Figure 2.9(e). The topological mode sequence is M3-M0-MI-M6-M0-M4 and is defined as "mode-C operation".

Table 2.4 summarizes the topological mode sequences for the three operating modes above resonant frequency. The commutation features for the transistors in each operating modes are summarized in Table 2.5.

Note that the trajectories discussed above are used only to qualitatively illustrate the existence of various operating modes and to demonstrate the transitions between different modes. The frequencies corresponding to the trajectories may not be the same. Some trajectories exist only for certain frequency and output-to-input voltage ratio range, as shall be seen in the following section. 

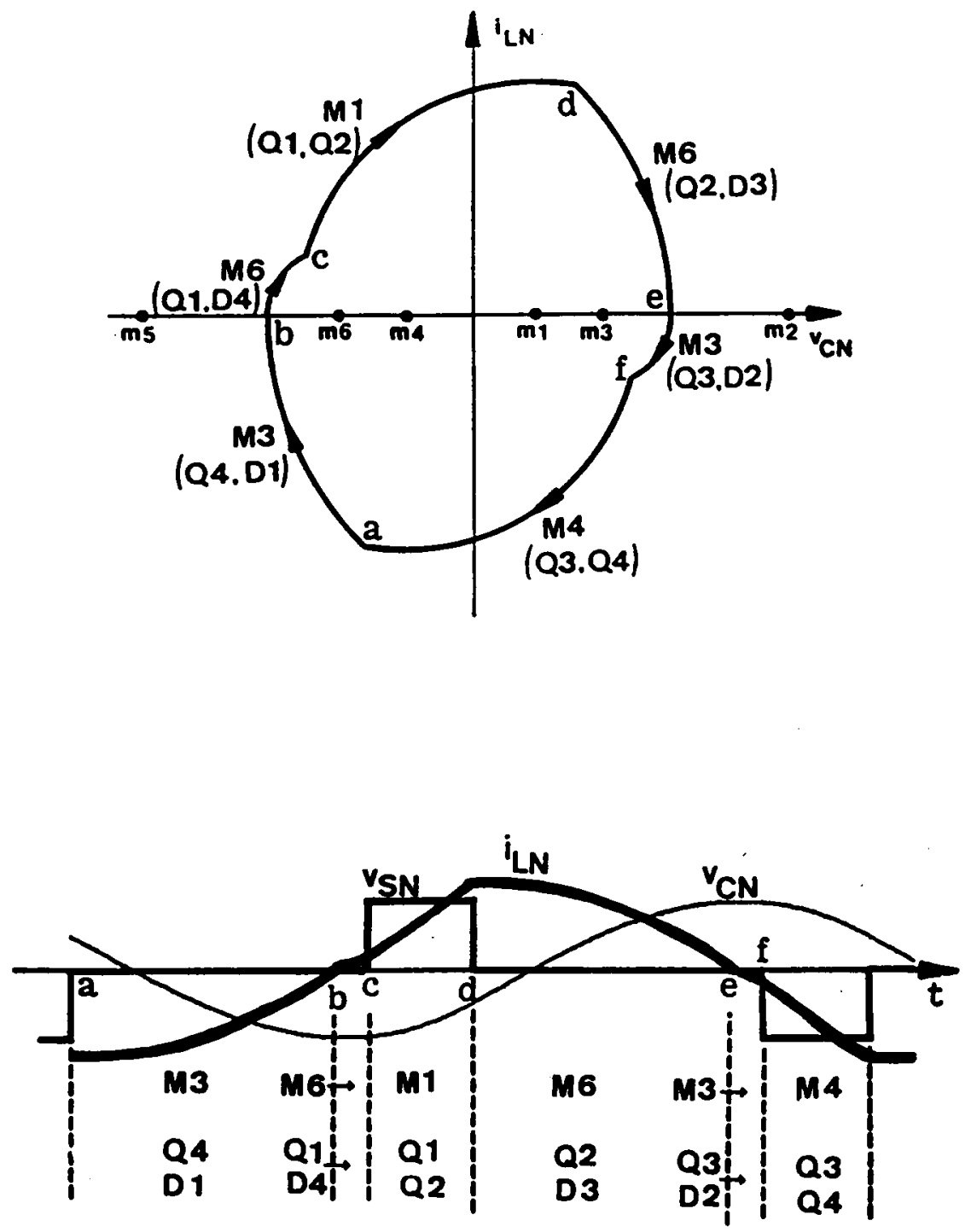

(d) Trajectory $B$ and its corresponding circuit waveforms (Mode-B operation)

Figure 2.9 Continued 

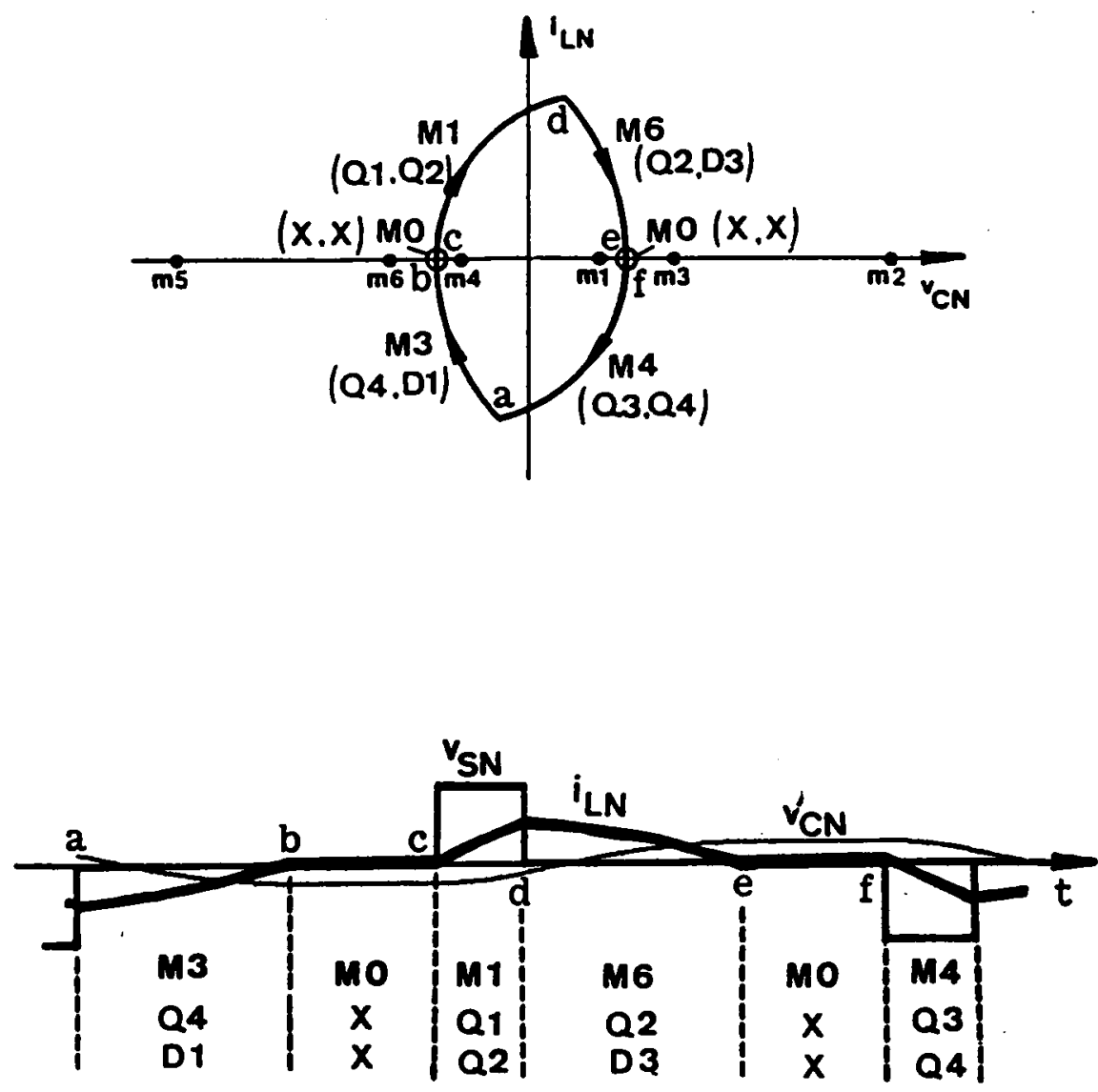

(e) Trajectory $C$ and its corresponding circuit waveforms (Mode-C operation)

Figure 2.9 Continued 
Table 2.4 Topological Mode Sequences for Circult Operating Modes Above Resonant Frequency

\begin{tabular}{|c|c|}
\hline $\begin{array}{c}\text { Operating } \\
\text { Modes }\end{array}$ & Topological Mode Sequences \\
\hline A & M3-M2-M1-M6-M5-M4 \\
\hline B & M3-M6-M1-M6-M3-M4 \\
\hline C & M3-MO-M1-M6-MO-M4 \\
\hline
\end{tabular}


Table 2.5 Commutation Features for Transistors in Various Circuit Operating Modes Above Resonant Frequency

\begin{tabular}{|c|c|c|c|c|c|}
\hline \multirow{2}{*}{ Mode } & \multirow{2}{*}{$i_{L}$} & \multicolumn{2}{|c|}{ Turn-On } & \multicolumn{2}{c|}{ Turn-Off } \\
\cline { 3 - 6 } & & Q1,Q3 & Q2,Q4 & Q1,Q3 & Q2,Q4 \\
\hline A & cont. & z.v. & z.v. & n.z.c. & n.z.c. \\
\hline B & cont. & z.v. & n.z.v. & n.z.c. & z.c. \\
\hline C & discont. & z.v. & z.v. & n.z.c. & z.c. \\
\hline
\end{tabular}

n.z.c. - turn off at nonzero current z.c. - turn off at zero current n.z.v. - turn on at nonzero voltage

z.v. - turn on at zero voltage 


\subsubsection{Regions of Operation}

Since the commutation features of the power switches in each operating mode are different, it is important to determine the converter's modes of operation for a specific design. Knowing the converter's operating mode(s) enables one to choose proper devices for the converter to optimize circuit performance. For example, if a CM-SRC operates in Mode III or Mode VI below resonant frequency, a simple lossless capacitor snubber, $C_{S}$, as shown in Figure 2.10, can be used across transistor $\mathrm{Q} 1$ and $\mathrm{Q} 3$ to reduce their turn-olf losses. This is feasible since $Q 1$ and $Q 3$ always turn on at zero voltage. Slower diodes can be used for D1 and D3 since D1 and D3 always turn off at zero current. The simple lossless capacitor snubbers, however, cannot be used when the converter operates in other operating modes below resonant frequency since Q1 and Q3 no longer turn on at zero voltage. Faster diodes are required for D1 and D3 since these diodes are no longer commutated at zero current.

To determine the converter's operating modes, regions of operation for a CM-SRC must be defined.

\subsubsection{Operating regions below resonant frequency}

Operating regions: Figure 2.11 shows the opcrating regions for a $\mathrm{CM}-\mathrm{SRC}$ at frequencies below the resonant frequency. In the figure, the mode of operation is represented as a function of the pulse width, $\beta_{s}$, of the quasi-squarc-wave voltage $v_{s}$ and the ratio of output to input voltage, $V_{O N}$. The frequencics are normalized to the resonant frequency, $\omega_{S N}, \omega_{S N}=\omega_{S} / \omega_{0}$. Given a range of $V_{O N}, \beta_{S}$, and a specific operating frequency, the converter's modes of operation can be casily determined using these figures. For example, if $\omega_{S N}$ is equal to $0.7, V_{O N}$ is from 0.4 to 0.6 , and $\beta_{S}$ is from $25^{\circ}$ to $75^{\circ}$, the converter will operate in either mode III or mode VI, as indicated by the shaded area in Figure $2.11(\mathrm{~d})$. 


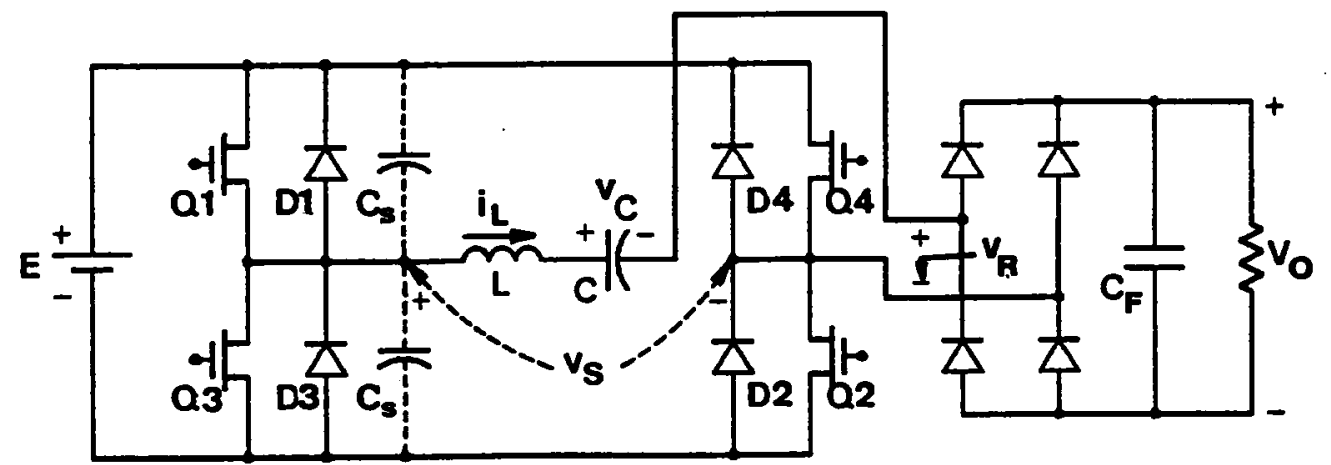

Figure 2.10 Lossless Snubbers Used Across Force-Commutated Transistors Q1 and Q3 


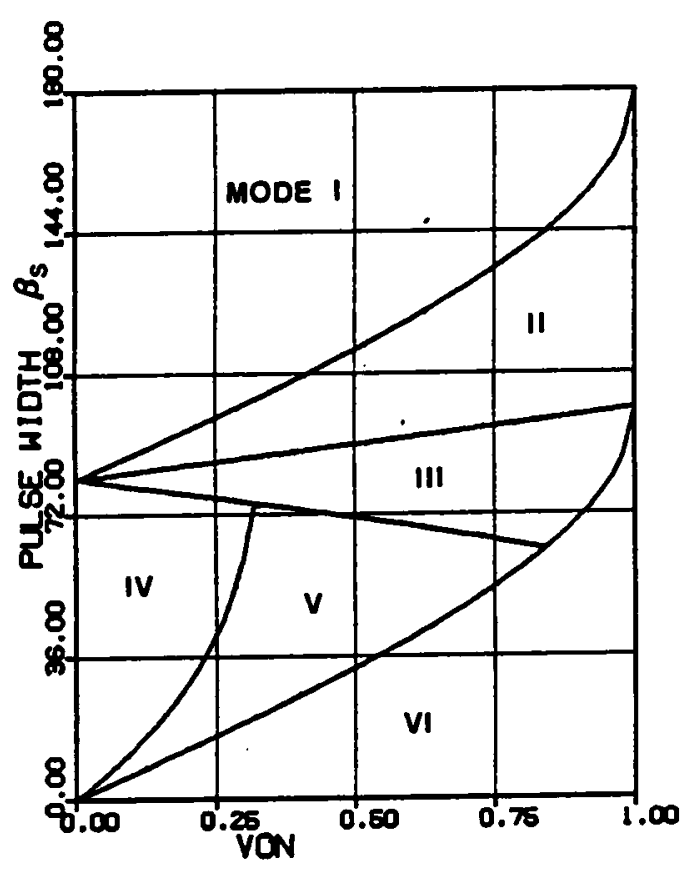

(a) $\omega_{S N}=0.55$

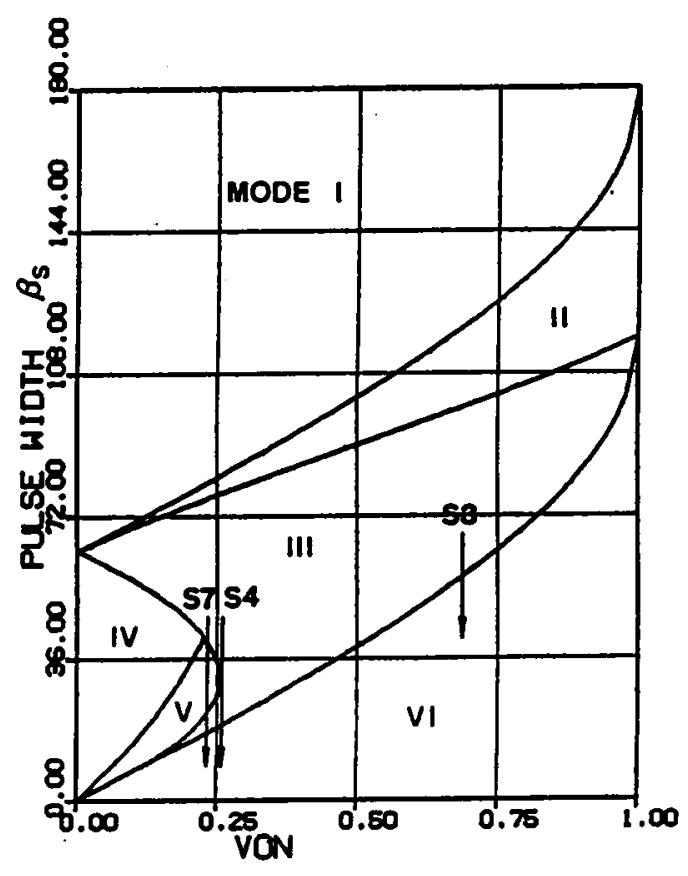

(c) $\omega_{S N}=0.65$

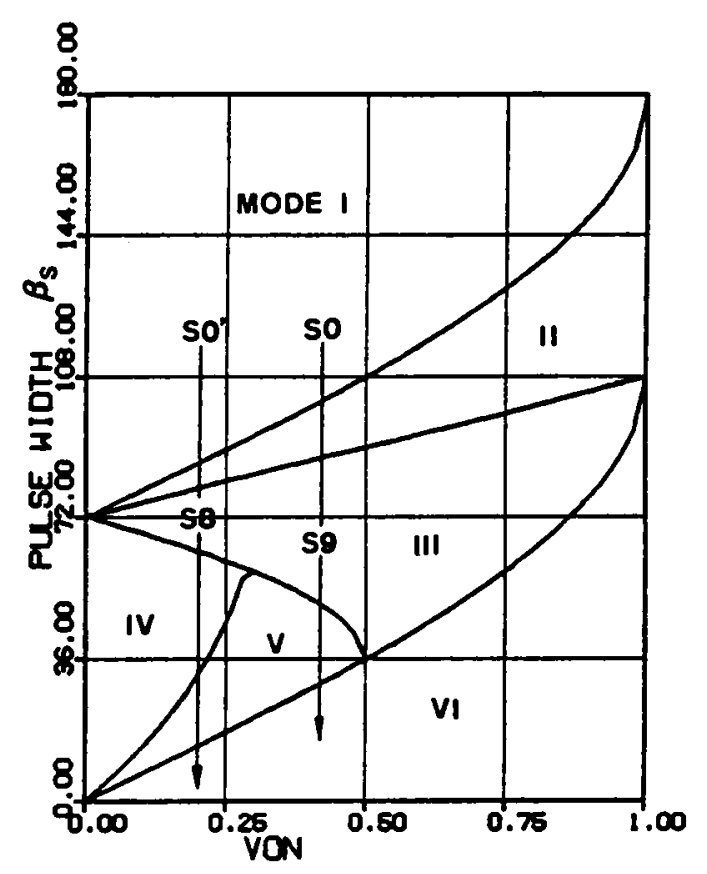

(b) $\omega_{S N}=0.6$

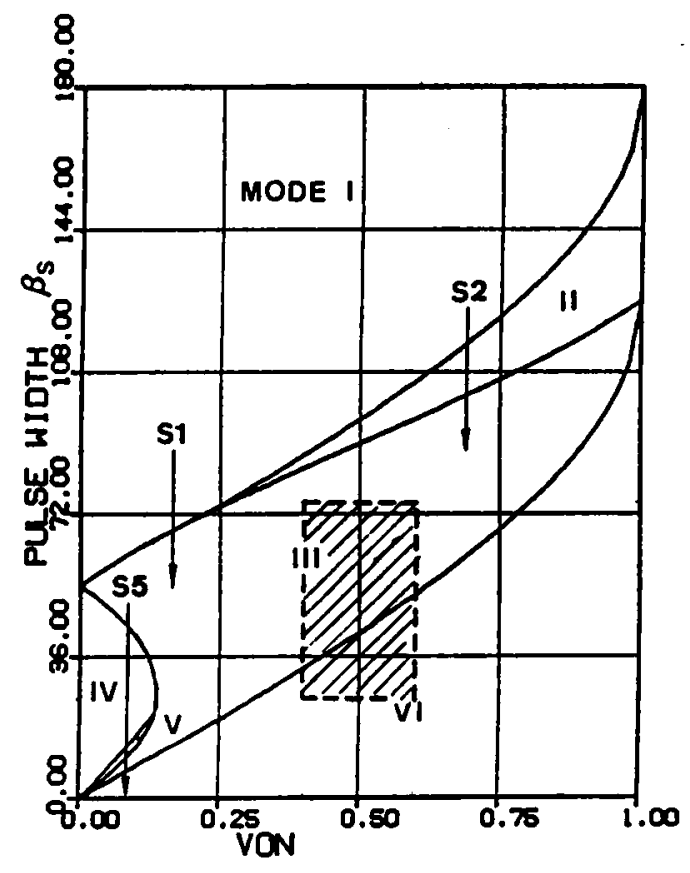

(d) $\omega_{S N}=0.7$

Figure 2.11 Regions of Operation Below Resonant Frequency 


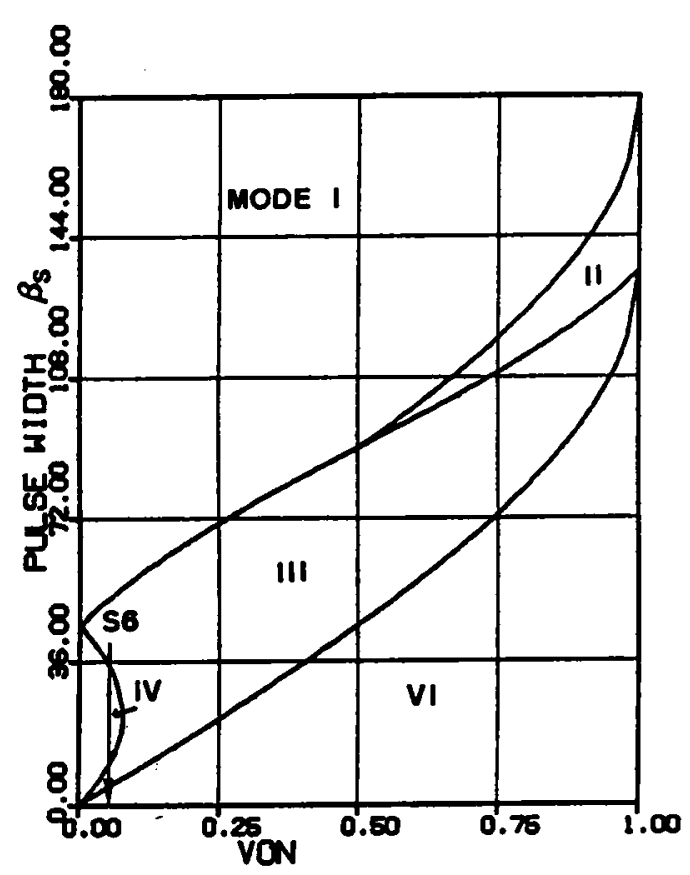

(e) $\omega_{S N}=0.75$

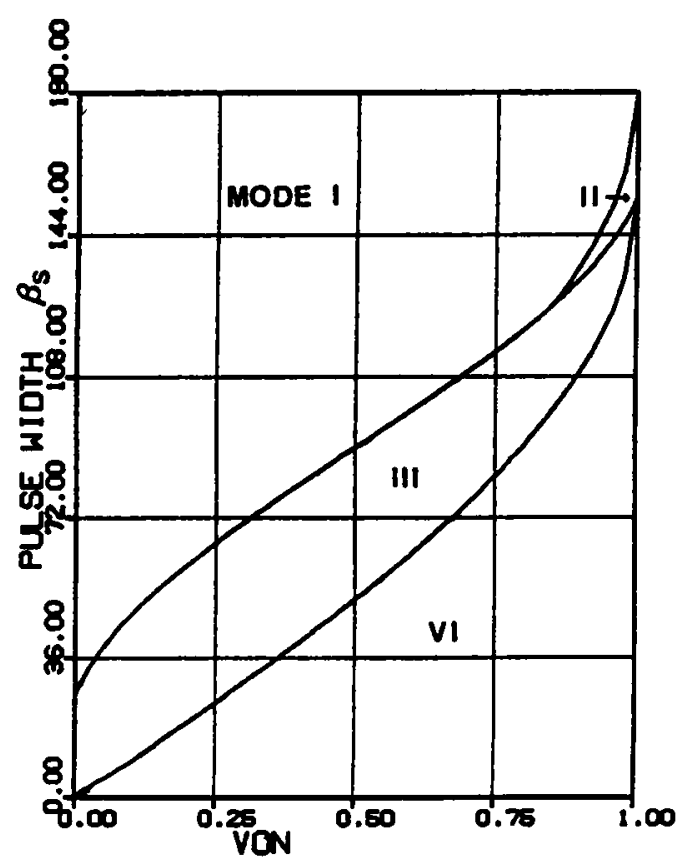

(g) $\omega_{S N}=0.85$

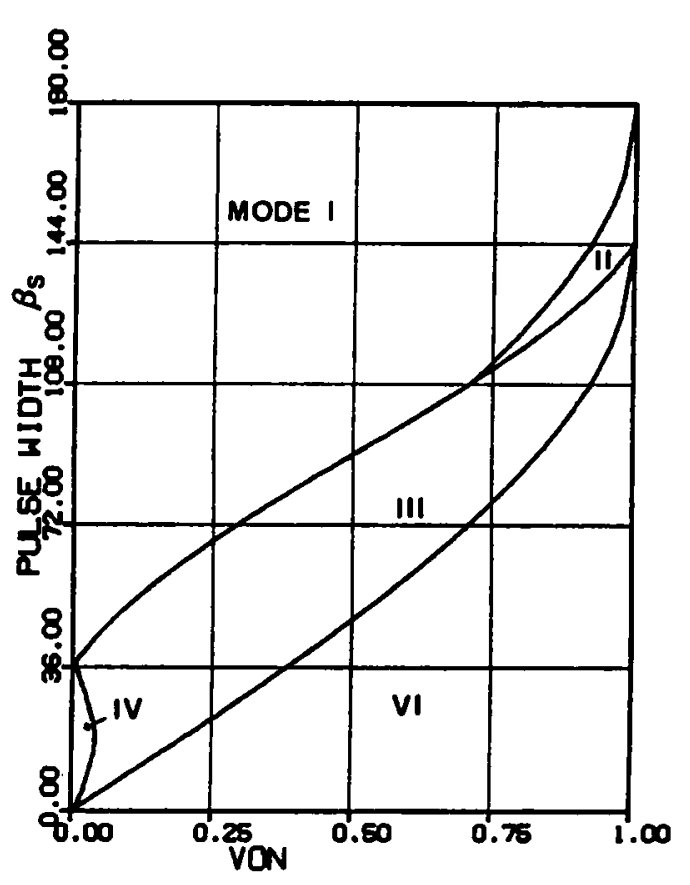

(f) $\omega_{S N}=0.8$

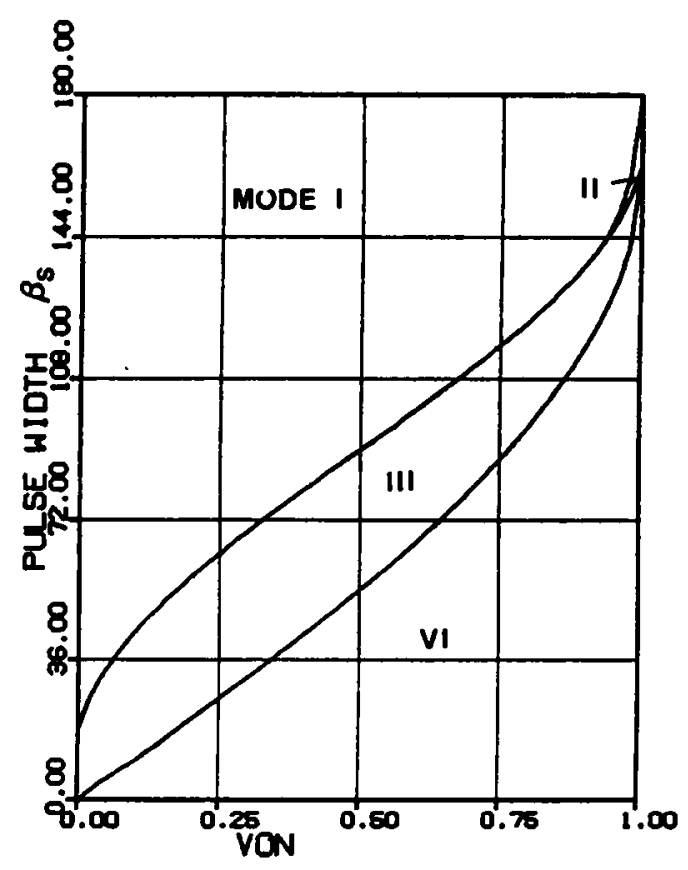

(h) $\omega_{S N}=0.9$

Figure 2.11 Continued 
For other frequencies not shown in Figure 2.11, an algorithm is developed in the following which enahles one to plot a similar graph to those in Figure 2.11.

Mode transitions: The first step in defining the operating regions of an CM-SRC is to determine the existing operating modes for a given $\omega_{S N}$ and $V_{O N}$. The corresponding $\beta_{S}$ ranges to different operating modes can then be determined by solving the mode boundaries.

For a given $V_{O N}$ and $\omega_{S N}$ possible operating modes can be found from the mode transition sequence when $\beta_{S}$ angle is reduced from $180^{\circ}$ to $0^{\circ}$. For example, as indicated by $\mathbf{S O}$ in Figure 2.11(b), the mode transition sequence for $V_{O N}=0.4$ and $\omega_{S N}=0.6$ is Mode I - Mode II - Mode III - Mode V - Mode VI. This implies that all the operating modes except Mode IV exist for $V_{O N}=0.4$ and $\omega_{S N}=0.6$. The boundaries for the different operating modes are found to be $\beta_{\mathrm{s}(\text { I-II })}=100^{\circ}, \beta_{\mathrm{S}(I-I I I)}=87^{\circ}, \beta_{\mathrm{s}(I I-V)}=52^{\circ}$, and $\beta_{S(V . V)}=28^{\circ}$. The $\beta_{S}$ ranges for the operating modes are thus $180^{\circ} \sim 100^{\circ}$ for Mode I, $100^{\circ} \sim 87^{\circ}$ for Mode II, $87^{\circ} \sim 52^{\circ}$ for Mode III, $52^{\circ} \sim 28^{\circ}$ for Mode V, and $28^{\circ} \sim$ $0^{\circ}$ for Mode VI. The transition in the circuit behavior as $\beta_{S}$ varies was illustrated in Figure 2.8, Section 2.3.5.1.

It should be noticed that the mode transition sequence of a CM-SRC is not unique. $\Lambda$ total of twelve mode transition sequences may cxist below resonant frequency, as illustrate in Figure 2.12. The transition of the waveforms in Figure 2.8 corresponds to the case when $V_{O N}=0.2$ and $\omega_{S N}=0.6$, which swceps through all the six operating modes and is indicated by SO $\mathbf{S O}^{\prime}$ in Figure 2.11(b).

In the following, an algorithm is developed which predicts the mode transition sequence (as $\beta_{S}$ decreases) and determines the mode boundaries for any arbitrary $V_{O N}$ and $\omega_{S N}$.

Mode boundaries: $\Lambda s$ shown in Figure 2.12, from Mode I the converter's operation may change to Mode II or Mode III when $\beta_{S}$ is reduced. Consider the boundary trajectory, 


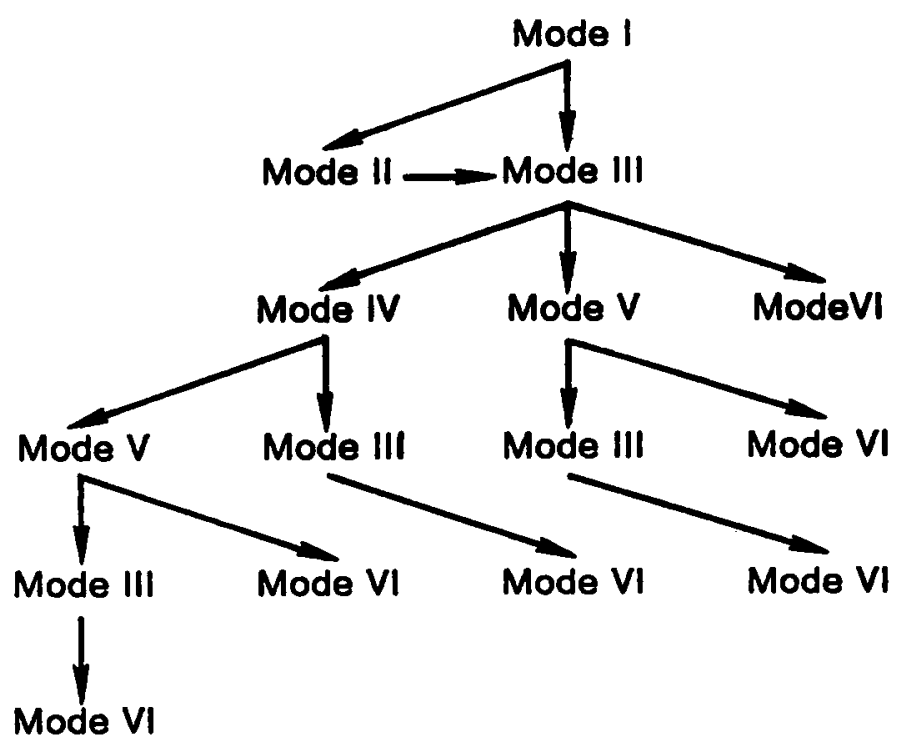

Figure 2.12 Mode Transition Sequences Below Resonant Frequency $\left(\beta_{s}\right.$ decreases from $180^{\circ}$ to $\left.0^{\circ}\right)$

60 
$T_{12}$, between Mode $\mathrm{I}$ and Mode II, as shown in Figure 2.13. The frequency of $T_{12}$ is calculated as

$$
\omega_{12}=\frac{\pi}{\zeta+\delta+\theta}
$$

where $\zeta=\pi-\cos ^{-1}\left(1-2 V_{O N}^{2}\right), \delta=\pi-\cos ^{-1}\left(V_{O N}\right)$, and $\theta \geq 0$ is the corresponding phase angle at stationary points $a$ and $b$.

To construct this boundary trajectory, the maximum possible frequency is $\omega_{12 \max }=\pi /(\zeta+\delta)$. Thus, when the converter operates at a frequency $\omega_{S N}>\omega_{12 \max }$, trajectory $T_{12}$ can not exist. This implies that mode-II operation does not exist and the converter's operation transits from Mode I to Mode III, as indicated by S1 in Figure $2.11(d)$.

The boundary $\beta_{S}$ angle, $\beta_{13}$, separating Mode I and Mode III can be calculated from trajectory $T_{13}$, as shown in Figure 2.14, by solving equations

$$
\begin{aligned}
& \cos (\pi-\zeta)=\frac{1+\left(1+R-2 V_{O N}\right)^{2}-R^{2}}{2\left(1+R-2 V_{O N}\right)}, \\
& \cos (\pi-\delta)=\frac{1+R_{2}-\left(1+R-2 V_{O N}\right)^{2}}{2 R}, \\
& \omega_{S N}=\frac{\pi}{\zeta+\delta} .
\end{aligned}
$$

Angle $\beta_{13}$ is equal to $\omega_{S N} \times \delta$.

When $\omega_{S N} \leq \omega_{12 \max }$, trajectory $T_{12}$ exists. This implics that mode-II operation exists and the circuit's operation transits from Mode I to Mode II to Mode III, as indicated by $\mathbf{S} 2$ in Figure $2.11(\mathrm{~d})$.

The boundary $\beta_{S}$ angle, $\beta_{12}$, separating Mode I and Mode II is calculated from trajectory $T_{12}$, 


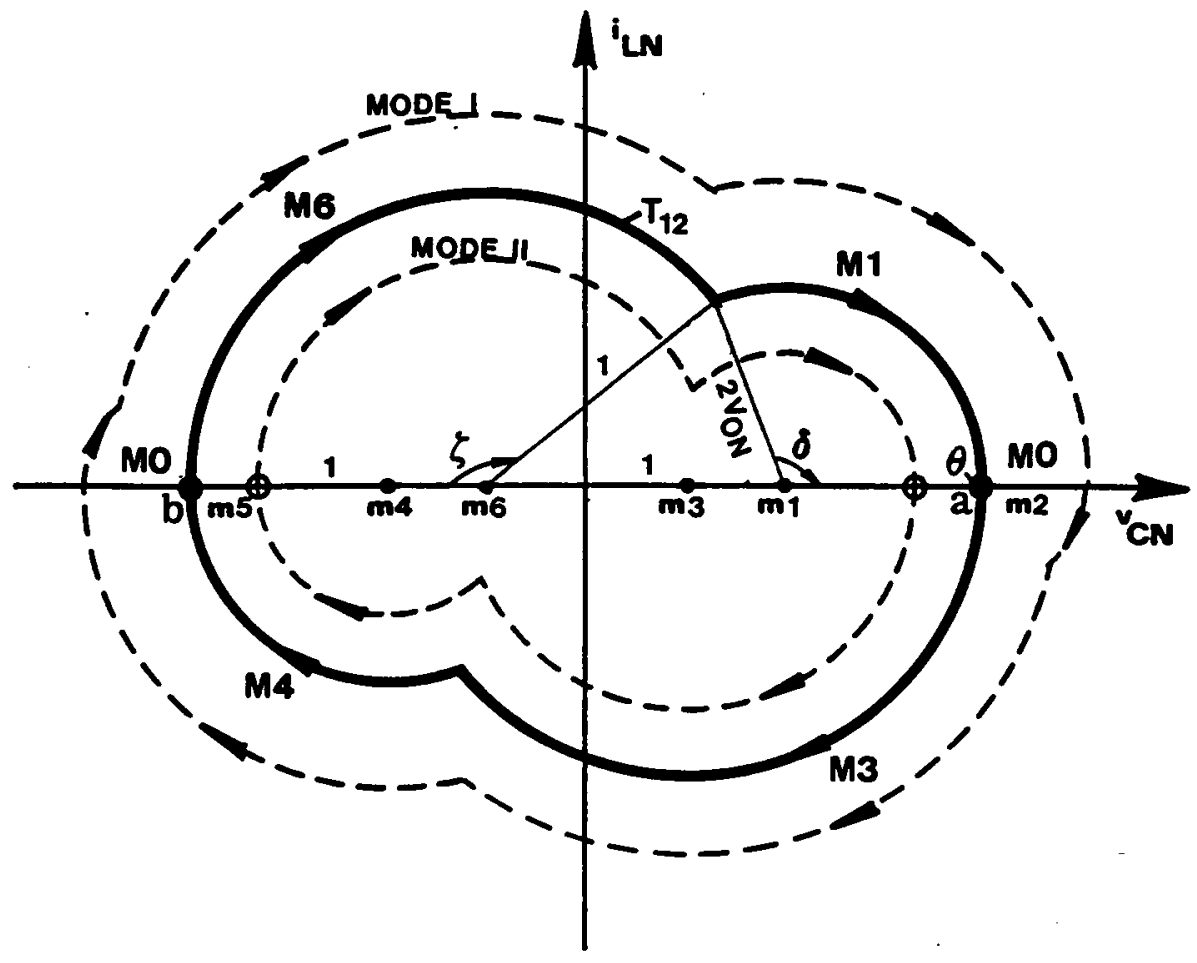

Figure 2.13 Boundary Trajectory Between Mode I and Mode II 


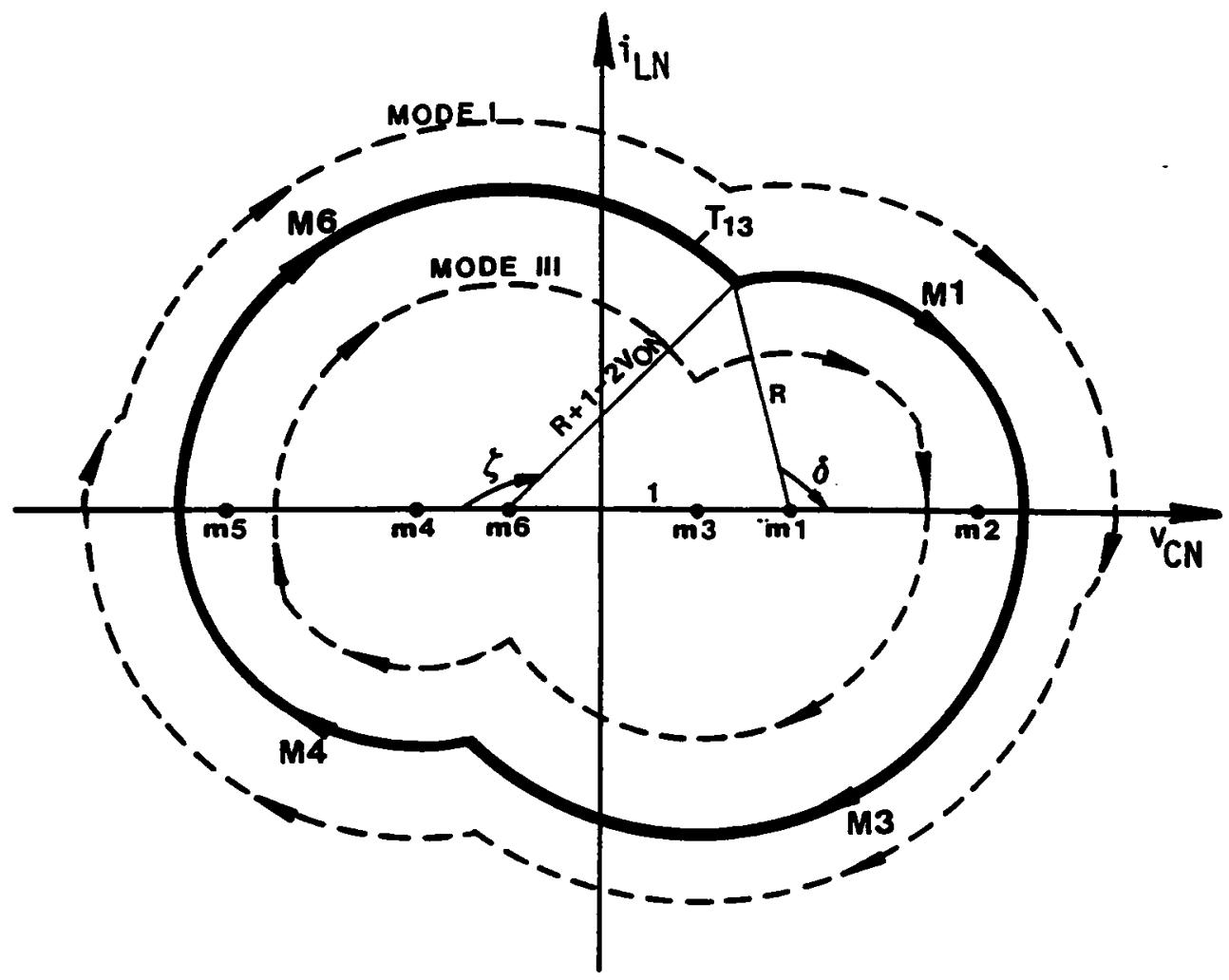

Figure 2.14 Boundary Trajectory Between Mode I and Mode III 


$$
\beta_{12}=(\delta+\theta) \times \omega_{S N}, \quad \theta=\left(\frac{\pi}{\omega_{S N}}-\zeta-\delta\right)
$$

The boundary $\beta_{s}$ angle, $\beta_{23}$, separating Mode II and Mode III can be calculated from trajectory $T_{23}$, as shown in Figure 2.15, by solving the same equations as in (2.2). Angle $\beta_{23}$ is equal to $\omega_{S N} \times \delta$.

From mode-III operation, as $\beta_{S}$ is reduced, the mode transitions and mode boundaries are discussed in Appendix B.2.

Using the algorithm described above, the mode transition sequence and the mode boundaries at various $V_{O N}$ and $\omega_{S N}$ can be easily determined. The operating regions shown in Figure 2.11 were results obtained using the algorithm.

It can be seen from Figure 2.11 that the undesirable Mode IV and Mode V operations tend to occur at low $V_{O N}$ and low $\omega_{S N}$.

\subsubsection{Operating regions above resonant frequency}

Figure 2.16 shows several operating regions at frequencies above the resonant frequency. These regions are derived from a similar process as discussed previously in section 2.3.6.1. There are, however, only two possible mode transition sequences existing, as illustrated in Figure 2.17. An algorithm for predicting the mode transition sequence and determining the mode boundaries in this frequency range is developed in Appendix B.3.

\subsubsection{Characteristics}

Once the mode of operation is determined for a given $V_{O N}, \omega_{S N}$, and $\beta_{S}$, the corresponding equilibrium state trajectory can be defined and employed to derive various circuit salient features such as average inductor current (load current), rms inductor 


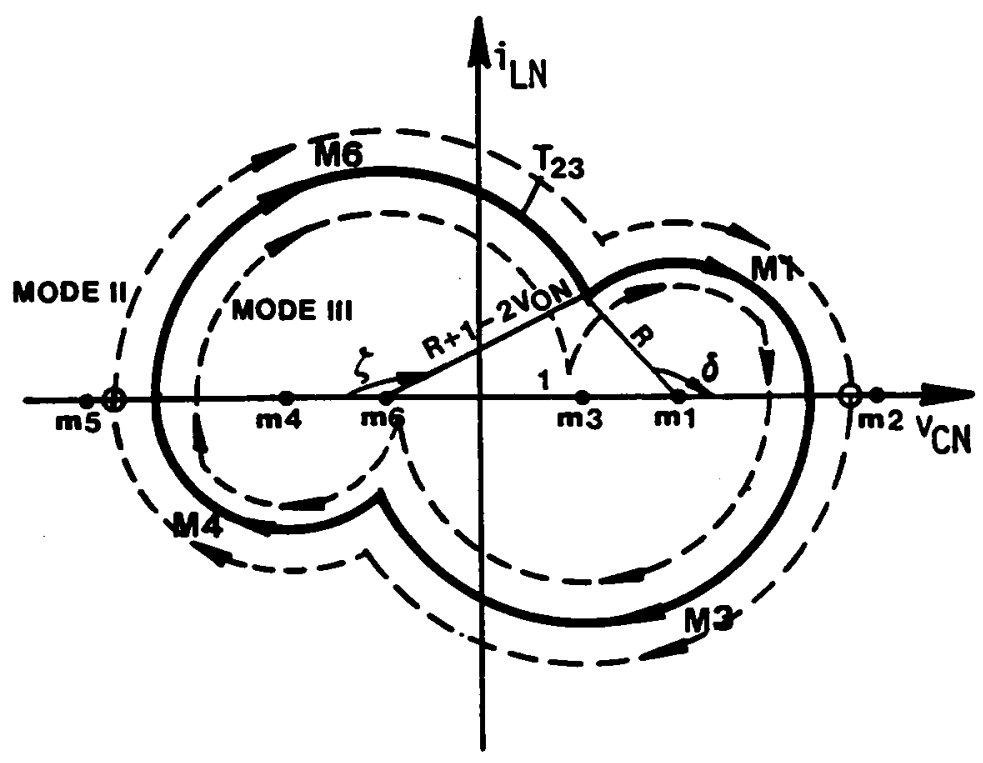

Figure 2.15 Boundary Trajectory Between Mode II and Mode III 


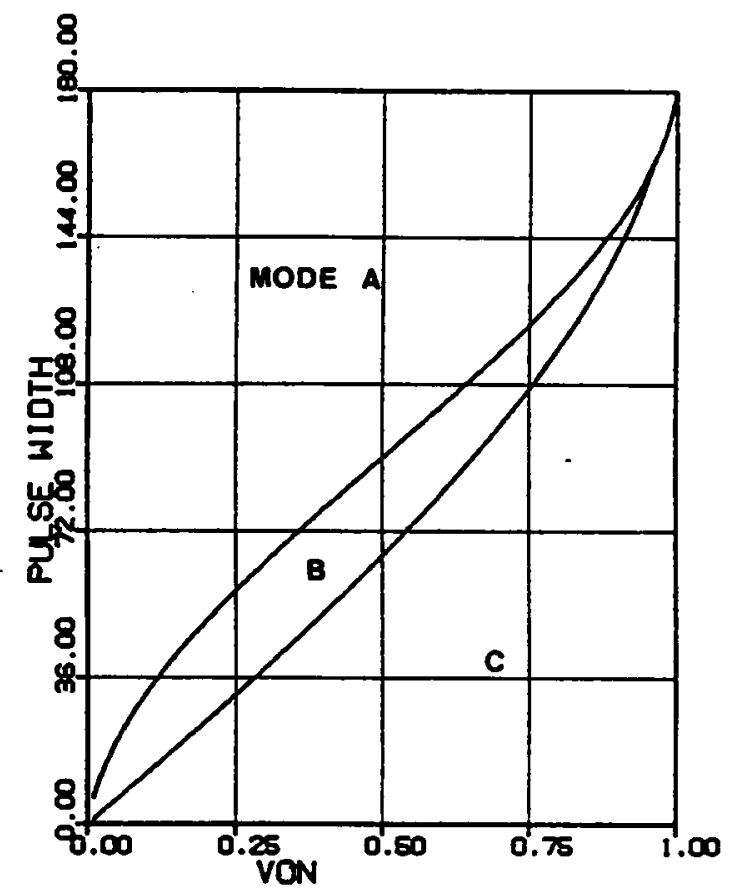

(a) $\omega_{S N}=1.1$

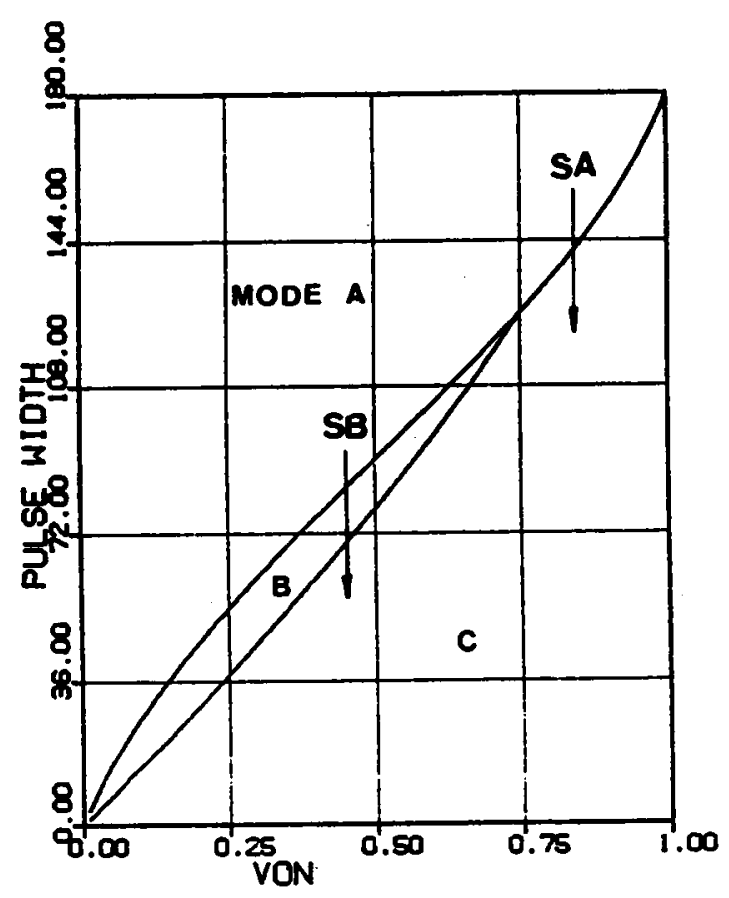

(c) $\omega_{S N}=1.3$

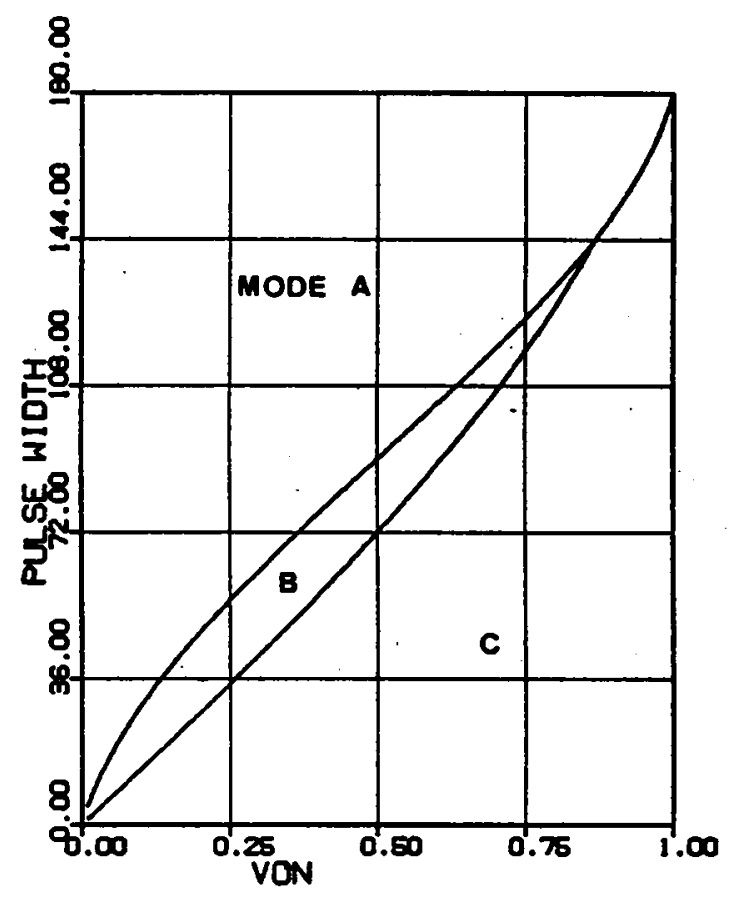

(b) $\omega_{S N}=1.2$

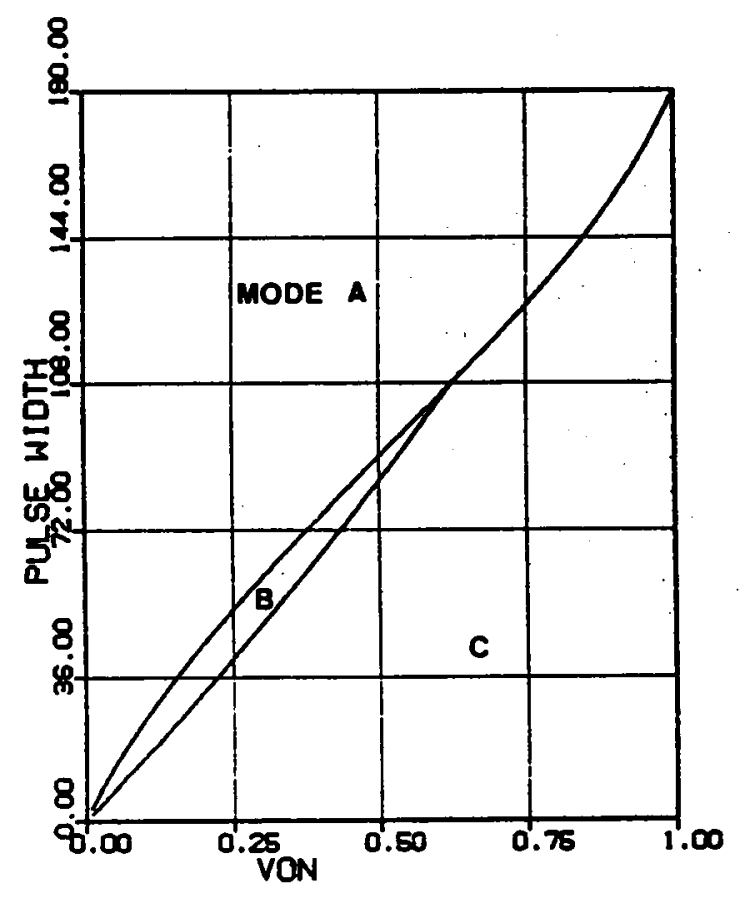

(d) $\omega_{S N}=1.4$

Figure 2.16 Regions of Operation Above Resonant Frequency 


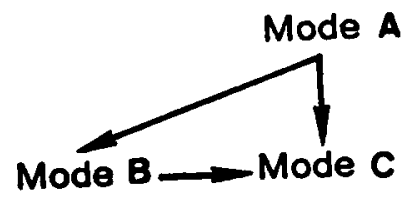

Figure 2.17 Mode Transition Sequences Above Resonant Frequency $\left(\beta_{s}\right.$ decreases from $180^{\circ}$ to $0^{\circ}$ )

67 
current, peak capacitor voltage, rms switch currents, average diode currents, switch turn-on currents, and switch turn-off currents. In this section, various important dc characteristics for the CM-SRC are derived. 


\subsubsection{DC characteristics below resonant frequency}

Trajectory parameters: To employ an equilibrium state trajectory to calculate various circuit salient features, the parameters defining the trajectory must be obtained first. As illustrated in Figure 2.18, an equilibrium state trajectory is uniquely defined by a number of parameters including several radii, $R_{1}, R_{2}, R_{3}$, and angles, $\eta, \zeta, \delta, \gamma$. These parameters can be obtained by solving a set of nonlinear equations derived from the state trajectory. For example, the parameters for the Mode-I trajectory in Figure 2.18 can be obtained by solving equations

$$
\begin{aligned}
& \cos (\pi-\gamma)=\frac{1+R_{1}^{2}-R_{2}^{2}}{2 R_{1}}, \\
& \cos (\pi-\eta)=\frac{1+R_{3}^{2}-R_{2}^{2}}{2 R_{3}}, \\
& \cos \zeta=\frac{1+R_{2}^{2}-R_{3}^{2}}{2 R_{2}}, \\
& \cos \delta=\frac{1+R_{2}^{2}-R_{1}^{2}}{2 R_{2}}, \\
& R_{3}=R_{1}-2 V_{O N}, \\
& \frac{\beta_{S}}{\omega_{S N}}=\eta+\gamma, \\
& \frac{\pi}{\omega_{S N}}=\eta+\gamma+\pi-(\zeta+\delta),
\end{aligned}
$$

where $V_{O N}, \omega_{S N}$, and $\beta_{S}$ are given values.

Since equation (2.8) have multiple solutions, constraints should be imposed on the parameters to obtain the correct one. These constraints can be directly derived from the state trajectory. For example, from Figure 2.18, the parameters for Mode-I trajectory should satisfy 


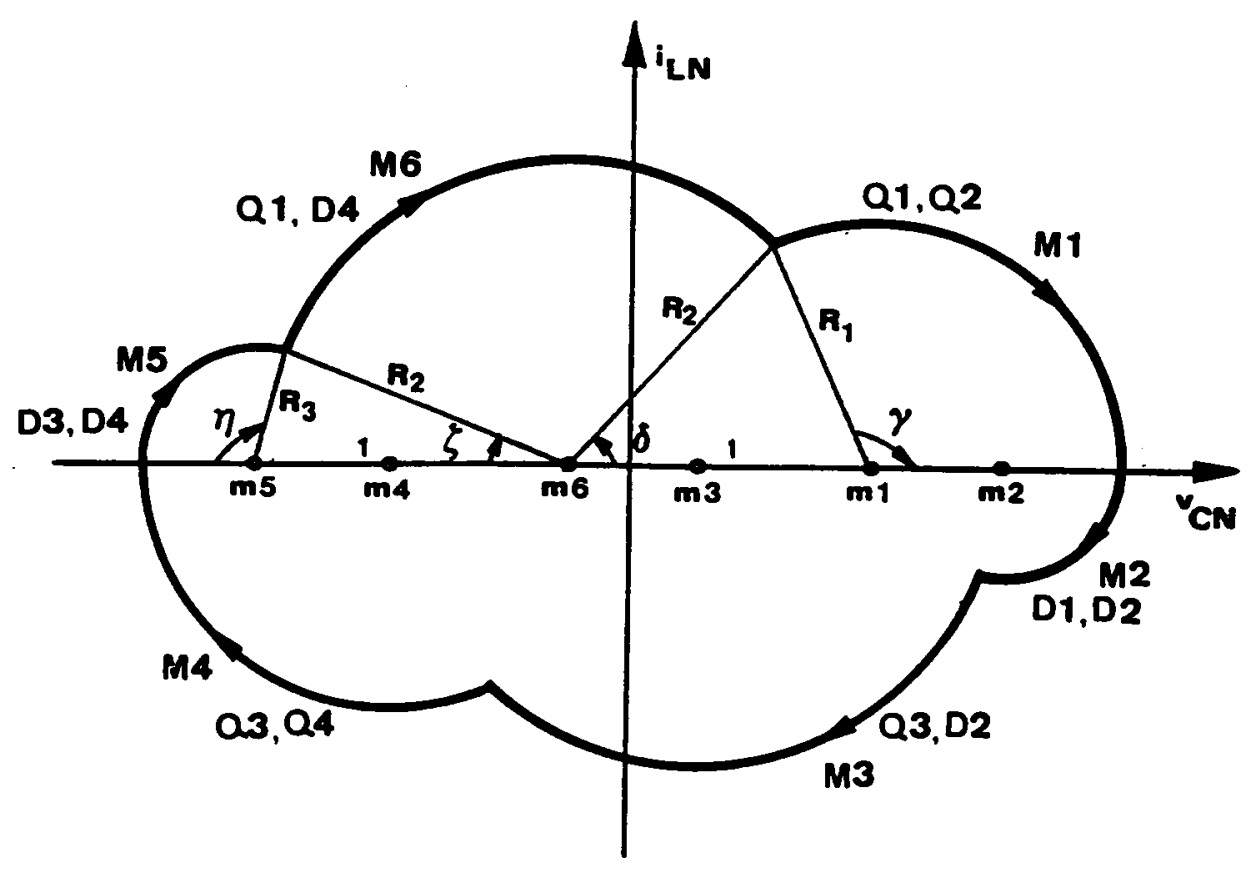

Figure 2.18 Mode-I Trajectory Below Resonant Frequency 


$$
0 \leq \eta, \zeta, \delta, \gamma \leq \pi, 0 \leq R_{2}, R_{3}, 2 V_{O N} \leq R_{1} .
$$

The equations and constraints for calculating trajectory parameters for other operating modes are summarized in Appendix B.4.

Circuit salient features: After the trajectory parameters are determined, various salient features of the circuit can be easily calculated. Consider the Mode-I trajectory in Figure 2.18, the following circuit features can be calculated:

- peak capacitor voltage $V_{C P K}=\left(1-V_{O N}\right)+R_{1}$;

- average inductor current $I_{A V}=\frac{(D A+D B+D C)}{\omega_{0} T_{S}}$, where,

$$
\begin{aligned}
& D A=\int_{0}^{\eta} R_{3} \sin \lambda \mathrm{d} \lambda=R_{3} \times(1-\cos \eta), \\
& D B=\int_{\zeta}^{\pi-\delta} R_{2} \sin \lambda \mathrm{d} \lambda=R_{2} \times(\cos \zeta+\cos \delta), \\
& D C=\int_{\pi-\gamma}^{\pi} R_{1} \sin \lambda \mathrm{d} \lambda=R_{1} \times(1-\cos \gamma) ;
\end{aligned}
$$

- $r m s$ inductor current $I_{L R M S}=\left(\frac{R A+R B+R C}{\omega_{0} T_{S}}\right)^{\frac{1}{2}}$, where,

$$
\begin{aligned}
& R A=\int_{0}^{\eta}\left(R_{3} \sin \lambda\right)^{2} \mathrm{~d} \lambda=\frac{R_{3}^{2}}{2} \times\left(\eta-\frac{\sin 2 \eta}{2}\right), \\
& R B=\int_{\zeta}^{\pi-\delta}\left(R_{2} \sin \lambda\right)^{2} \mathrm{~d} \lambda=\frac{R_{2}^{2}}{2} \times\left(\pi-\zeta-\delta+\frac{\sin 2 \zeta}{2}+\frac{\sin 2 \delta}{2}\right), \\
& R C=\int_{\pi-y}^{\pi}\left(R_{1} \sin \lambda\right)^{2} \mathrm{~d} \lambda=\frac{R_{1}^{2}}{2} \times\left(\gamma-\frac{\sin 2 \gamma}{2}\right) ;
\end{aligned}
$$

- $r m s$ switch $(Q 1, Q 3)$ current $\quad I_{Q \mid R M S}=\left(\frac{R B+R C}{\omega_{0} T_{S}}\right)^{\frac{1}{2}}$;

- $r m s$ switch $(Q 2, Q 4)$ current $\quad I_{Q 2 R M S}=\left(\frac{R C}{\omega_{0} T_{S}}\right)^{\frac{1}{2}}$; 
- average diode $(D 1, D 3)$ current $I_{D 1 A V}=\frac{D A}{\omega_{0} T_{S}}$

- average diode $(D 2, D 4)$ current $I_{D 2 A V}=\frac{D A+D B}{\omega_{0} T_{S}}$;

- switch $(Q 1, Q 3)$ turn-off current $I_{Q 1 \circ f}=0$

- switch $(Q 2, Q 4)$ turn-off current $I_{Q 2 o f f}=0$

- switch $(Q 1, Q 3)$ turn-on current $I_{Q 10 n}=R_{2} \sin \zeta$

- switch $(Q 2, Q 4)$ turn-on current $I_{Q 2 o n}=R_{2} \sin \delta$.

The expressions for calculating salient features under other operating modes are tabulated in Appendix B.5.

Output characteristics: Employing the above-derived expressions, various circuit characteristics can be generated using the flowchart shown in Figure 2.19. Figure 2.20 shows the dc control-to-output characteristics for the CM-SRC at several frequencies below the resonant frequency. In the figure, the average inductor current (output current) is plotted as a function of the pulse-width, $\beta_{S}$, of $v_{S}$, and the output-to-input voltage ratio, $V_{\text {ON. }}$ A $\beta_{S}$ angle of $180^{\circ}$ corresponds to a full pulse width of $v_{S}$ (duty ratio $=1$ ). A dotted line is used in each graph to indicate the boundary of natural commutation. Above the dotted line, all the transistors are naturally commutated. Below the dotted line, at least two of the transistors, $Q 1$ and $Q 3$, are force-commutated. It can be seen that the converter is able to regulate the output from no load to a full load. A minimum load, however, has to be maintained to achieve natural commutation of all the transistors. The converter usually operates in the mixed-commutation region under light load where Q2,Q4 are naturally commutated and Q1,Q3 are force-commutated. 


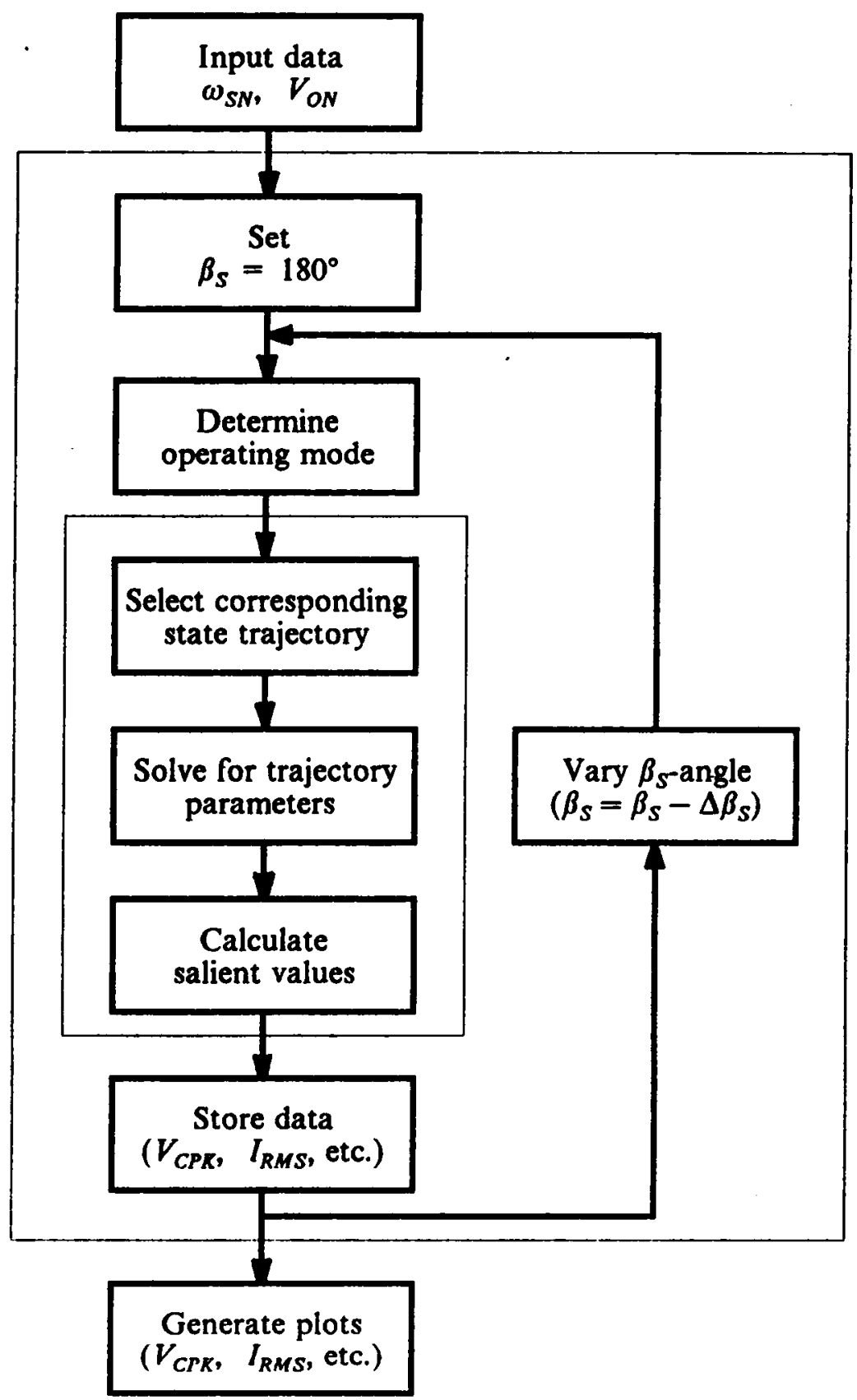

Figure 2.19 A Flowchart Used to Generate Plots of DC Characteristics 


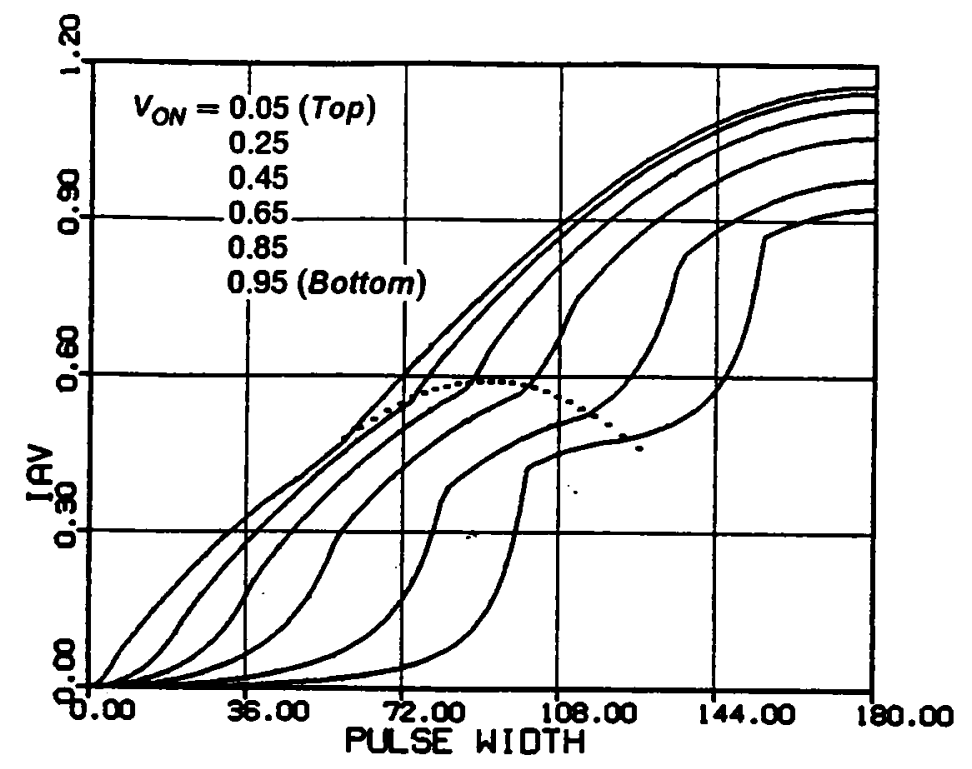

(a) $\omega_{S N}=0.7$

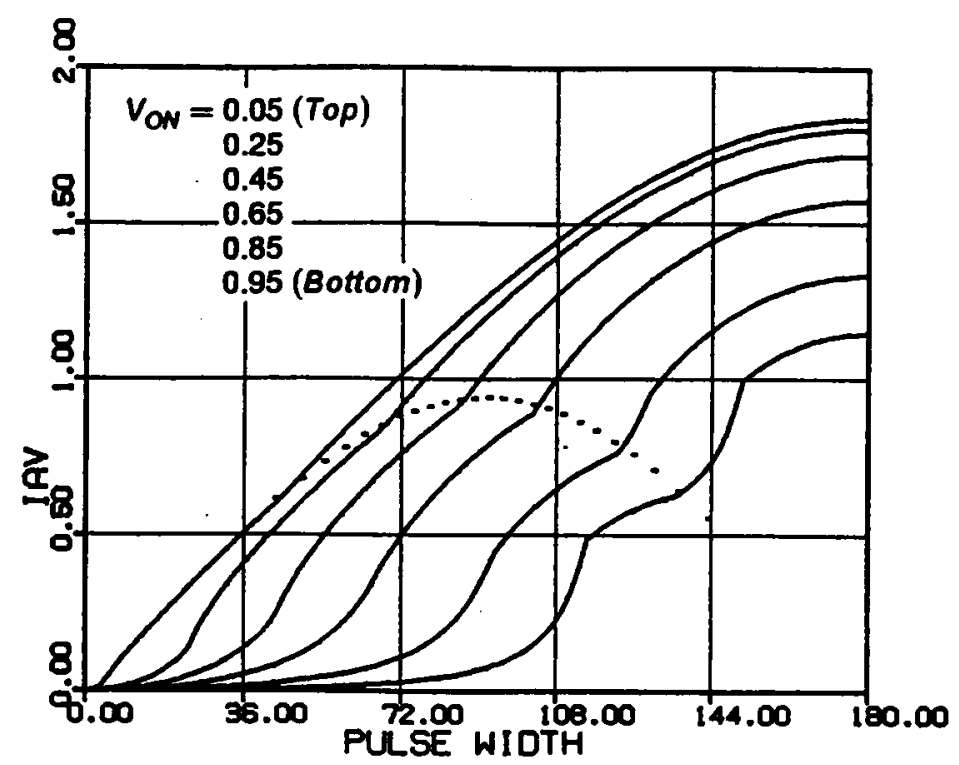

(b) $\omega_{S N}=0.8$

Figure 2.20 DC Control-to-Output Characteristics Below Resonant Frequency 


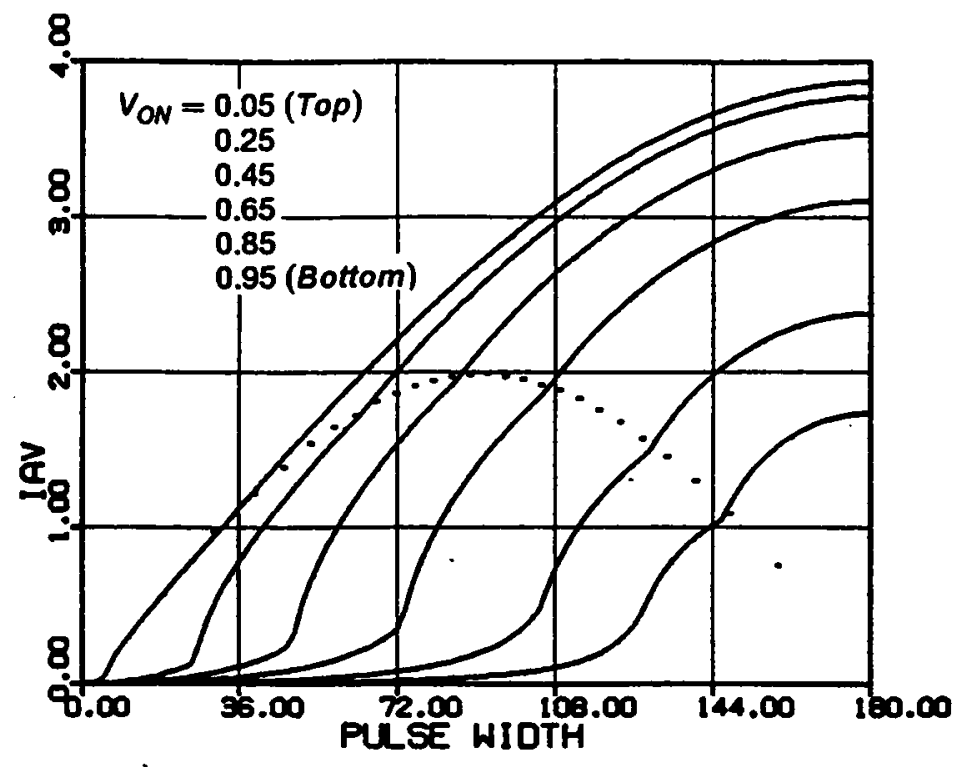

(c) $\omega_{S N}=0.9$

Figure 2.20 Continued 
Figure 2.21 shows other important dc characteristics for the CM-SRC at frequency $\omega_{S N}=0.8$. Notice that the currents of Q1,Q3 and the currents of Q2,Q4 are not balanced, as can be seen from Figures 2.21(c) and 2.21(d). Same phenomenon also exits between the currents of D1,D3 and D2,D4. This is due to the phase displacement in the triggering of the transistors. In general, transistors Q1,Q3 and diodes D2,D4 carry higher currents when the converter operates below the resonant frequency.

Similar dc characteristics for other frequencies can be found in Appendix B.6. 


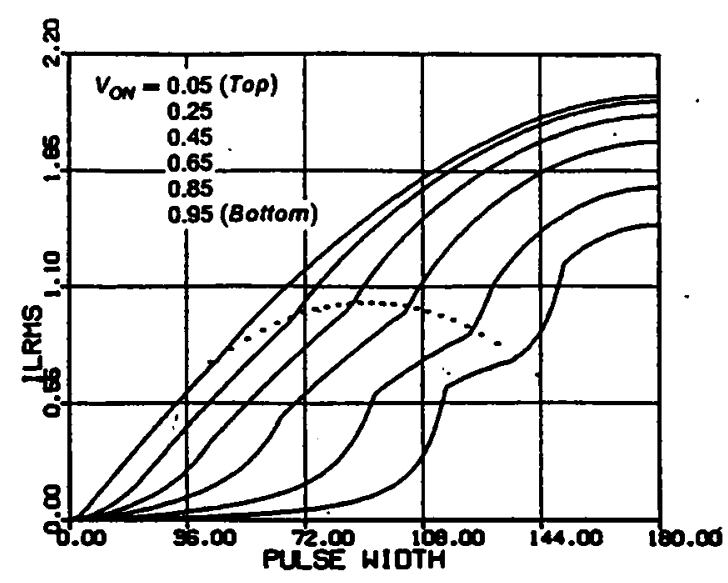

(a) RMS inductor current

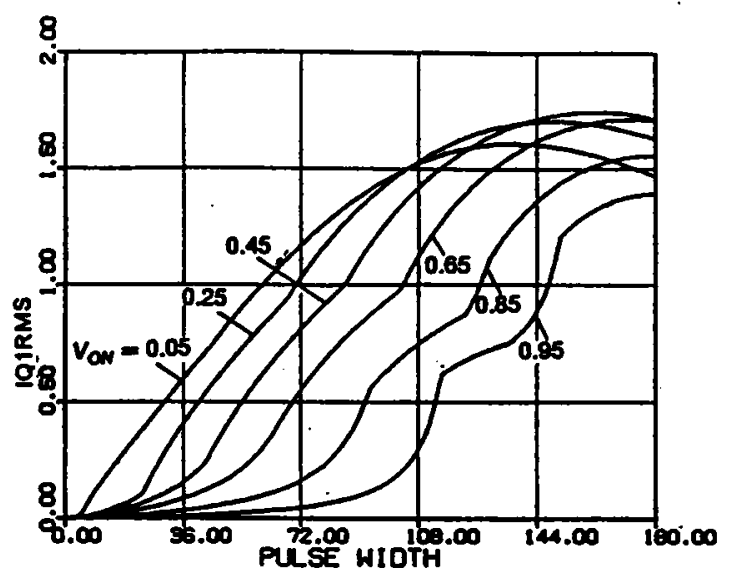

(c) RMS switch current (Q1,Q3)

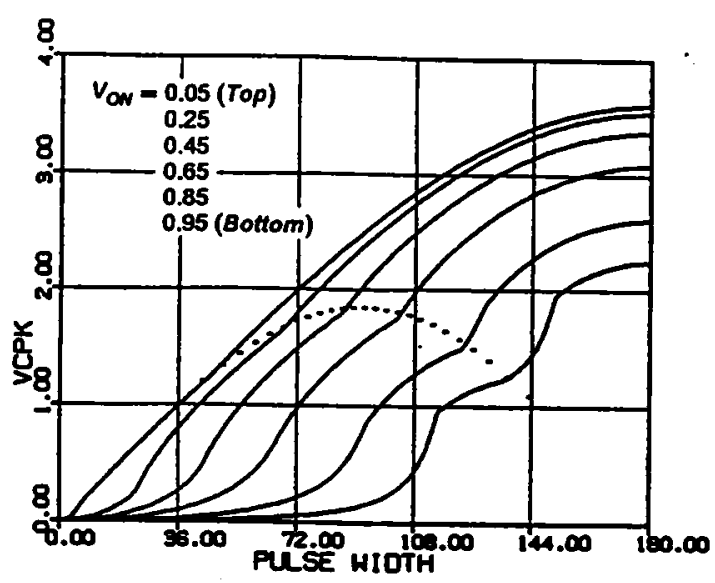

(b) Peak capacitor voltage

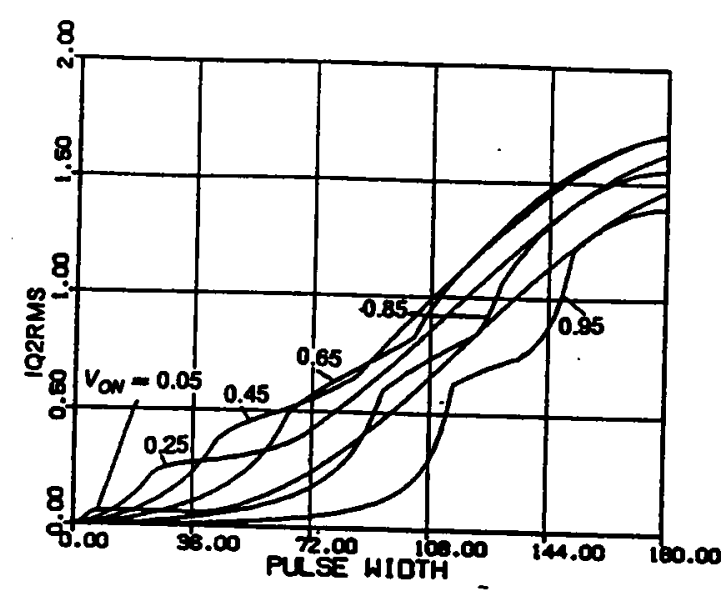

(d) RMS switch current (Q2,Q4)

Figure 2.21 Characteristics for Various Circuit Salient Features at $\omega_{S N}=0.8$ 


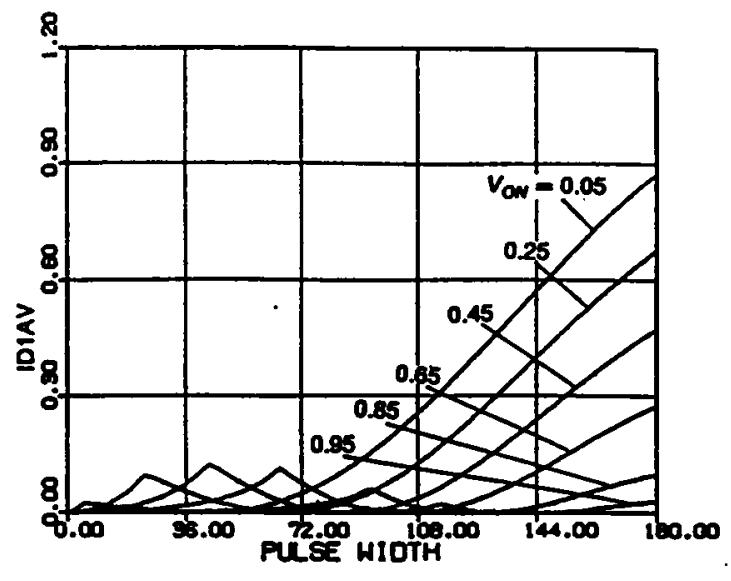

(e) Average diode current $(D I, D 3)$

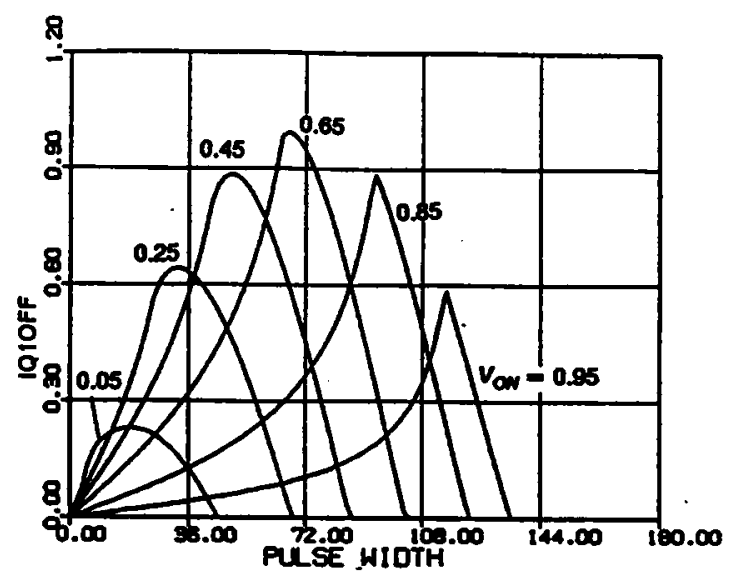

(g) Switch turn-off current (QI.Q3)

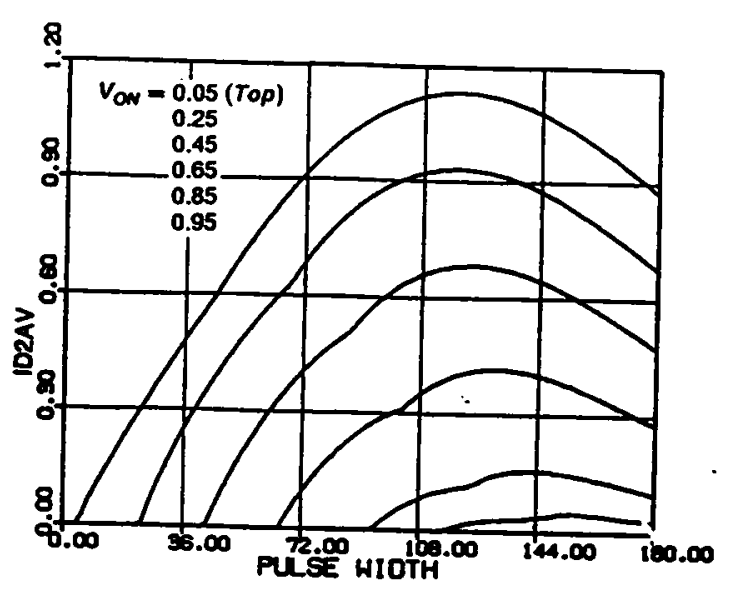

(f) Average diode current $(D 2, D 4)$

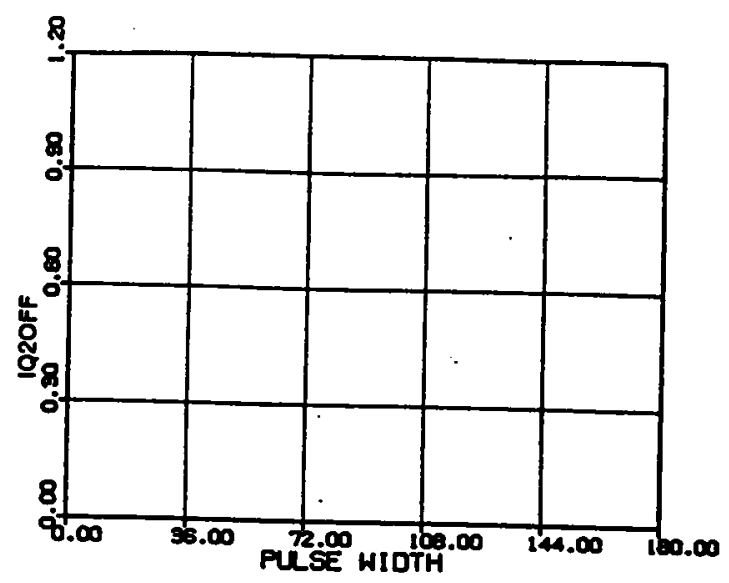

(h) Switch turn-off current (Q2,Q4)

Figure 2.21 Continued 


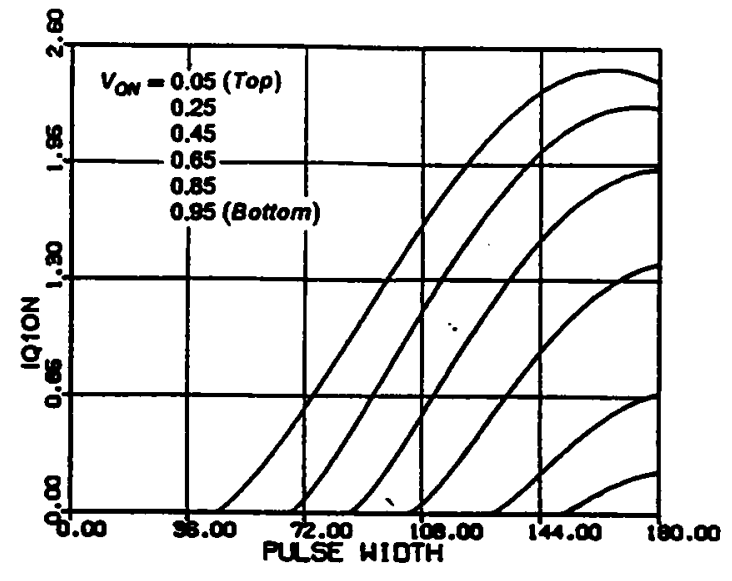

(i) Switch turn-on current $(Q 1, Q 3)$

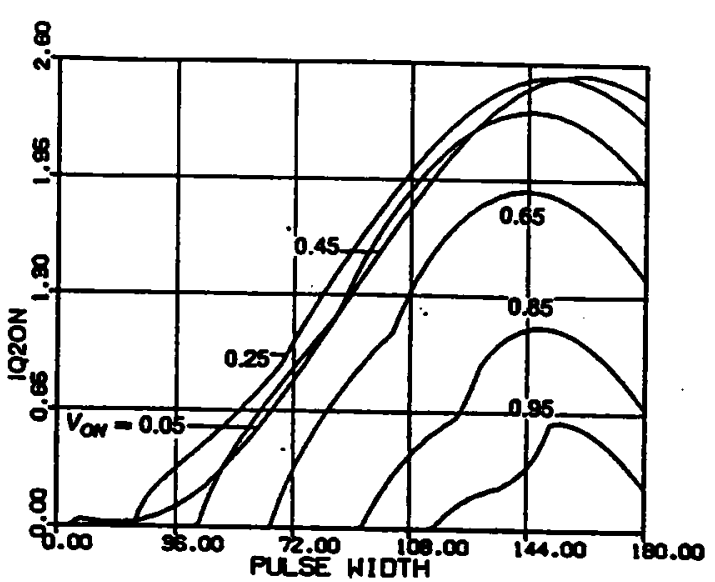

(j) Switch turn-on current (Q2,Q4)

Figure 2.21 Continued 


\subsubsection{DC characteristics above resonant frequency}

Trajectory parameters: The parameters for the equilibrium trajectories above resonant frequency are defined in Appendix B.7. The equations and the constraints for calculating these parameters are also included in the same Appendix.

Circuit salient features: The expressions for calculating various circuit salient features under different operating modes above resonant frequency are tabulated in Appendix B.8.

Output characteristics: Figure 2.22 shows the dc control-to-output characteristics at frequencies $\omega_{S N}=1.1,1.2,1.3$, and 1.4. The characteristics are similar to those obtained below resonant frequency. A dotted line is used in each figure to indicate the forcecommutation boundary. $\Lambda$ bove the dotted line, all the transistors are forcecommutated. Below the dotted line, transistors Q1 and Q3 are force-commutated while transistors Q2 and Q4 are naturally commutated. Again, the converter is able to regulate its output from no load to a full load. A minimum load, however, has to be maintained to achieve zero-voltage turn-on (force commutation) of all the transistors. The converter usually operate in the mixed-commutation region under light load.

Figure 2.23 shows other important dc characteristics at frequency $\omega_{S N}=1.2$. Again, the currents in the transistors and the currents in the diodes are unbalanced, as shown in Figures 2.23(c) and 2.23(d) and Figures 2.23(e) and 2.23(f), respectively. However, in this frequency range, transistors Q2,Q4 and diodes D1,D3 carry higher currents.

Similar dc characteristics for other frequencies can be found in Appendix B.9. 


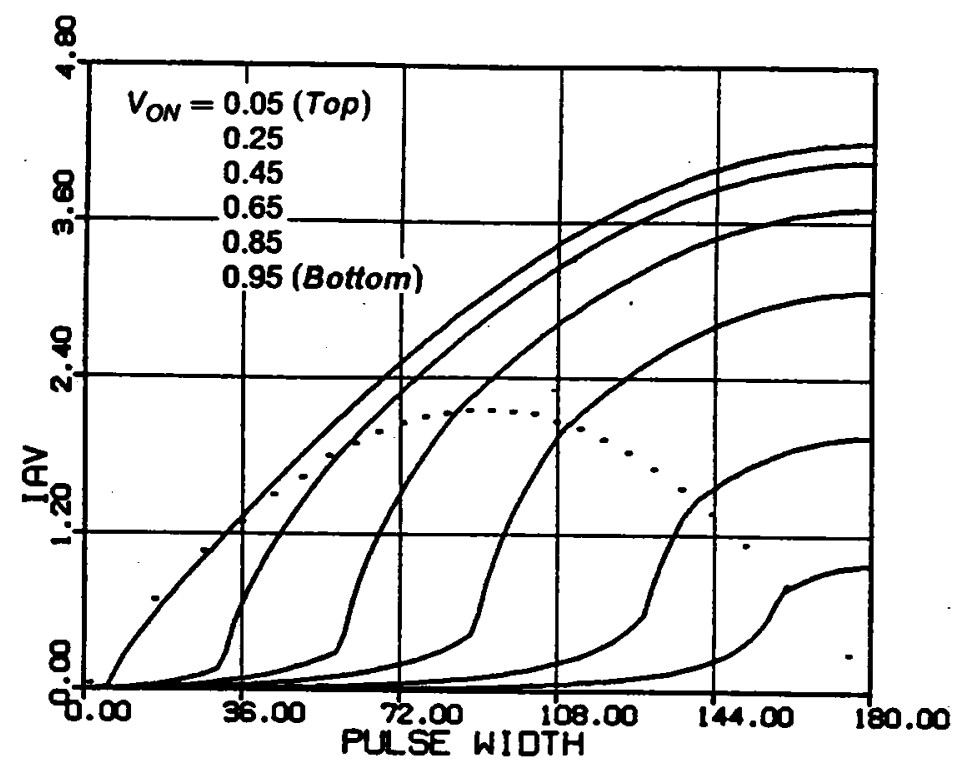

(a) $\omega_{S N}=1.1$

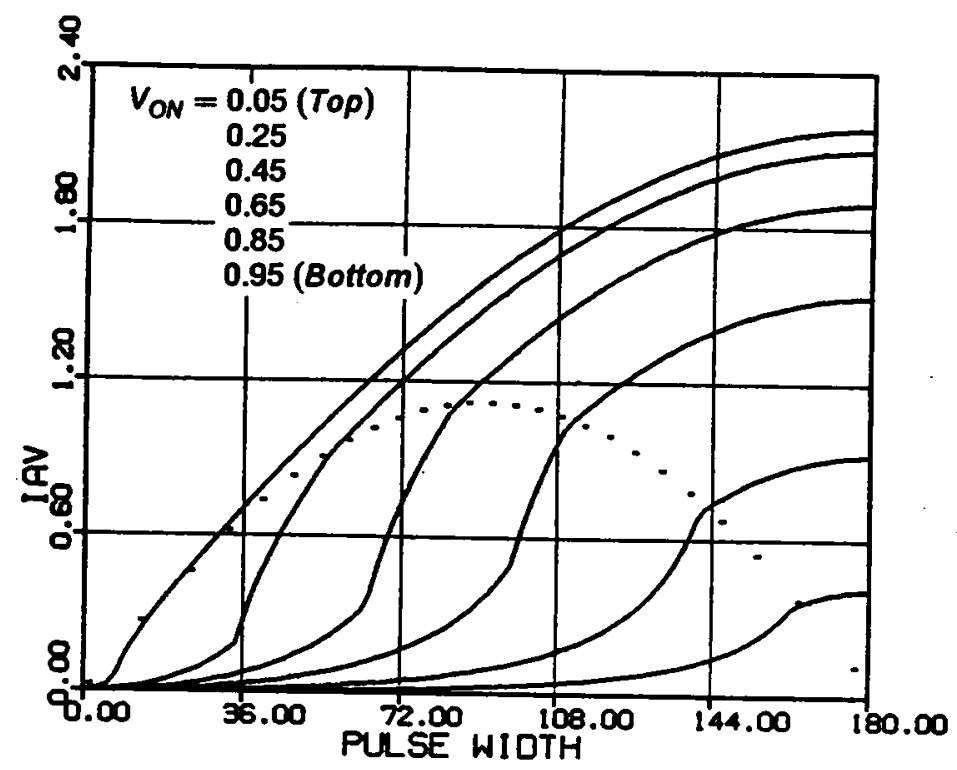

(b) $\omega_{S N}=1.2$

Figure 2.22 DC Control-to-Output Characteristics Above Resonant Frequency 


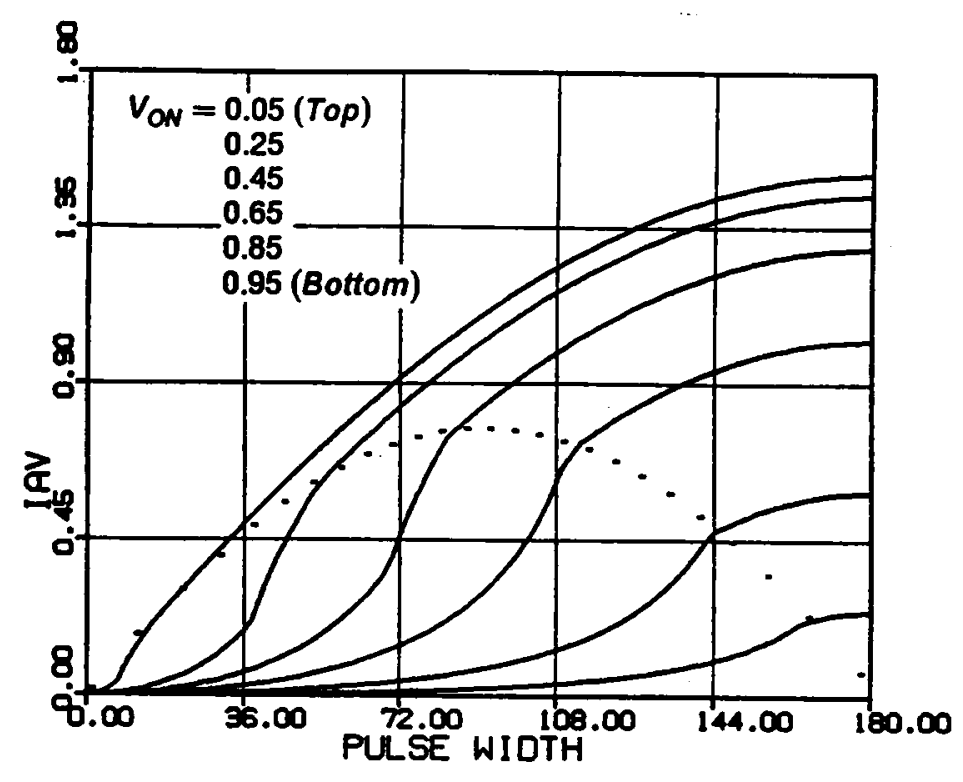

(c) $\omega_{S N}=1.3$

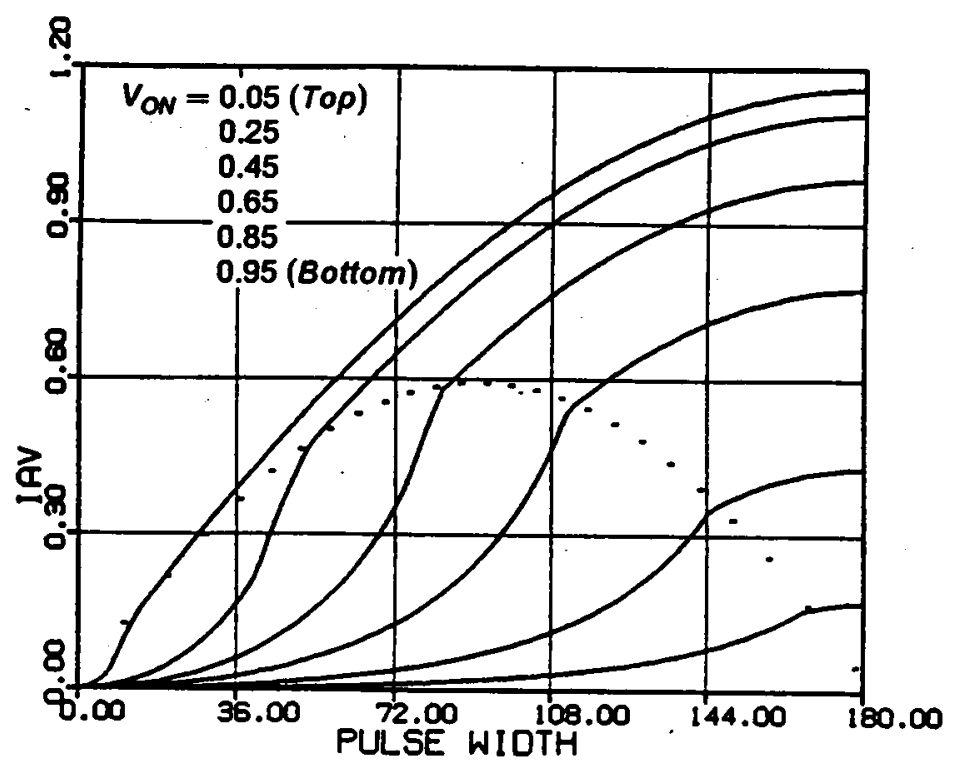

(d) $\omega_{S N}=1.4$

Figure 2.22 Continued 


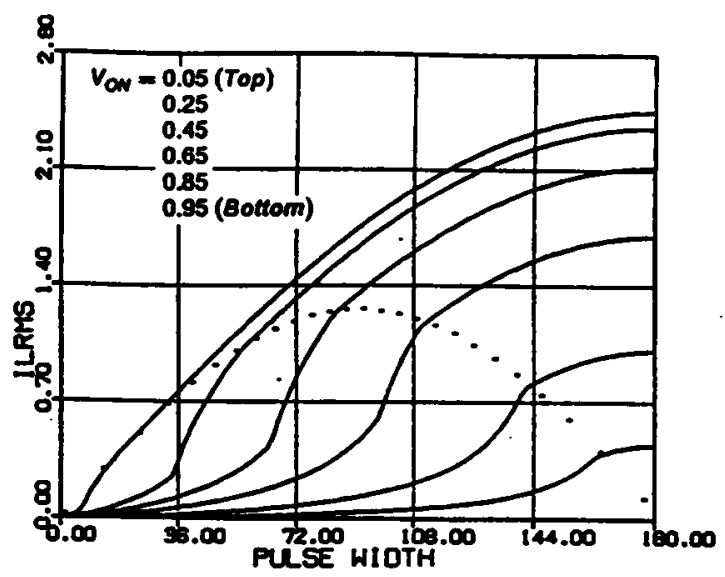

(a) RMS inductor current

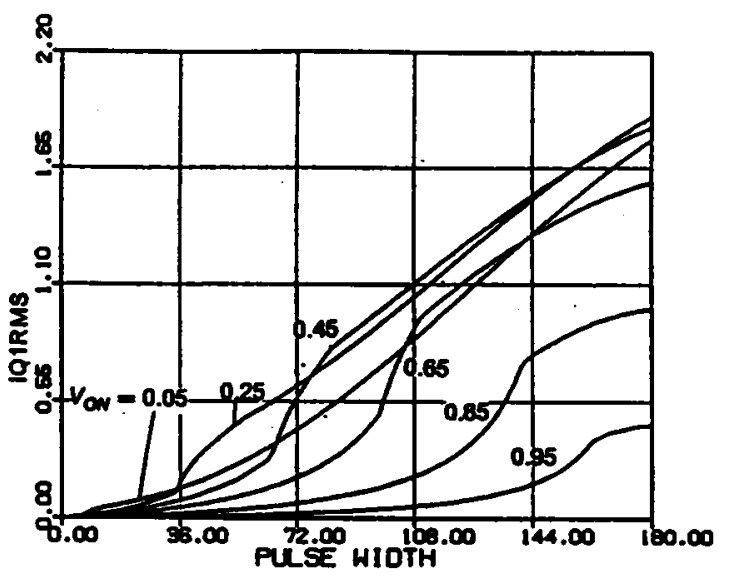

(c) RMS switch current (Q1,Q3)

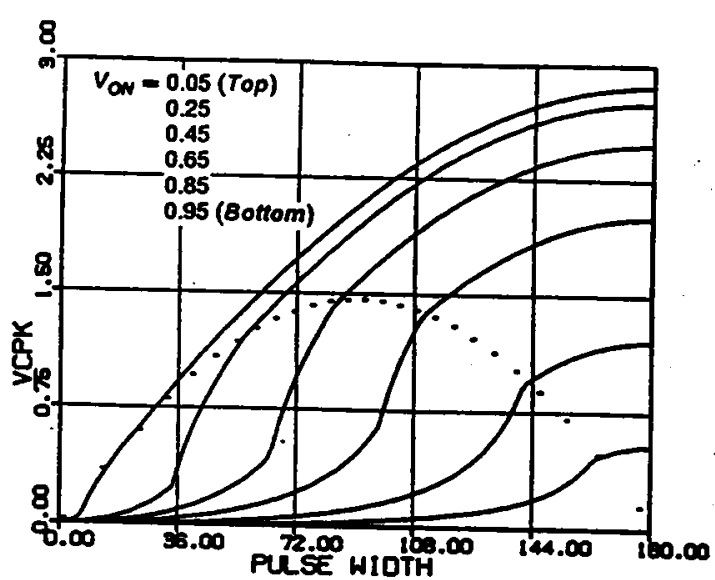

(b) Peak capacitor voltage

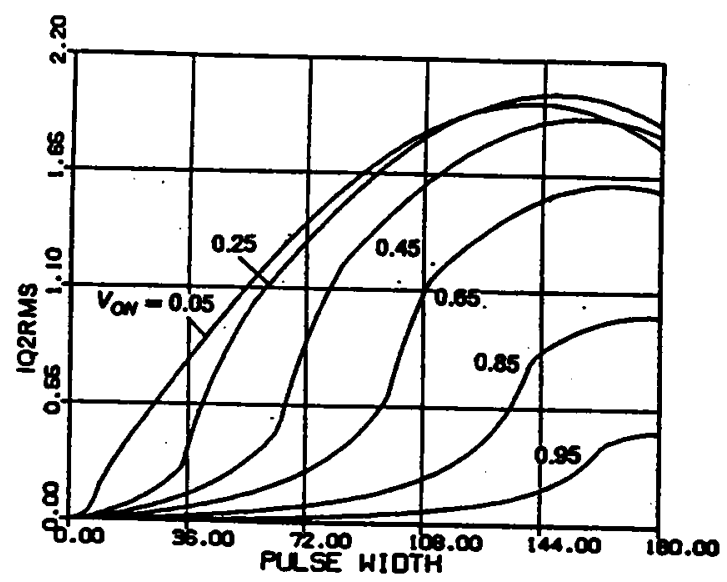

(d) RMS switch current (Q2,Q4)

Figure 2.23 Characteristics for Various Circuit Salient Features at $\omega_{S N}=1.2$ 


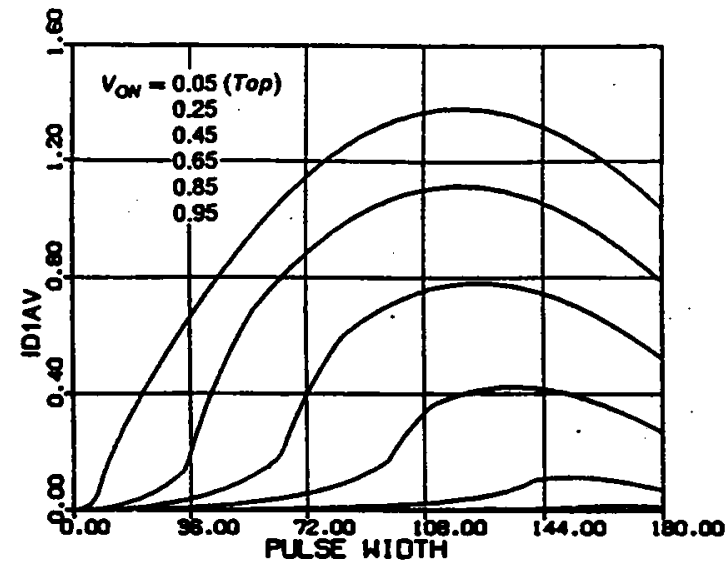

(e) Average diode current (DI,D3)

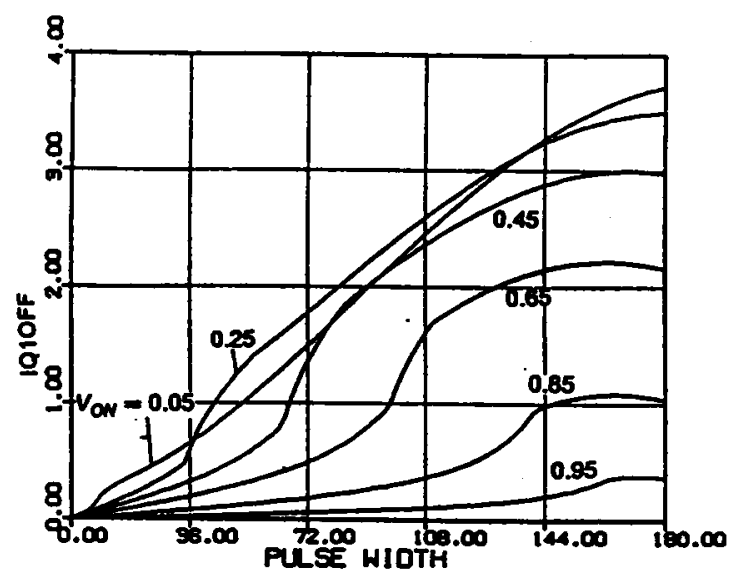

(g) Switch turn-off current (Q1,Q3)

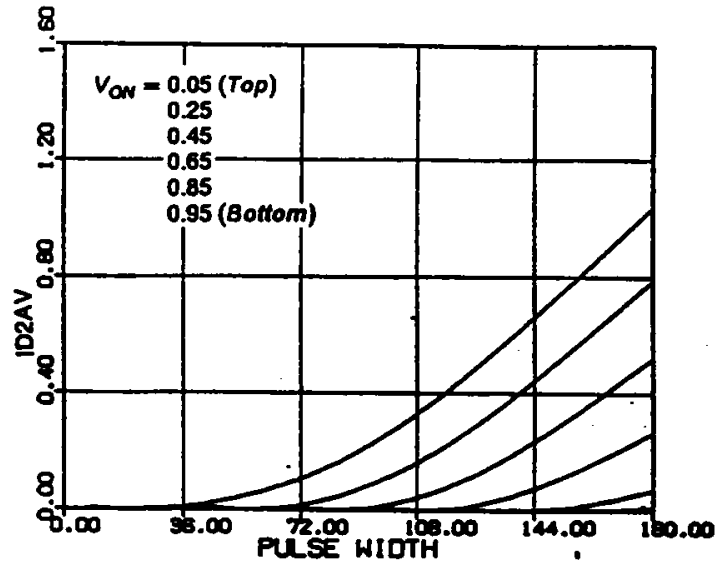

(f) Average diode current (D2,D4)

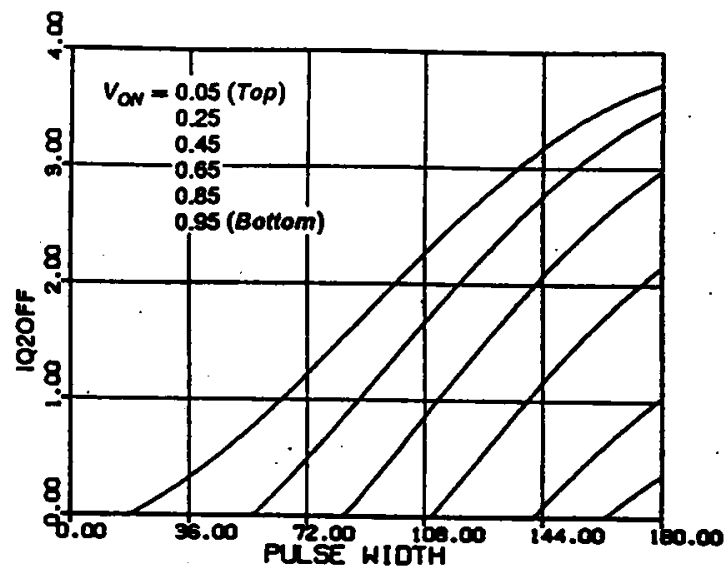

(h) Switch turn-off current (Q2,Q4)

Figure 2.23 Continued 


\subsection{DESIGN EXAMPLES}

The dc characteristics derived in the previous section are employed to facilitate design of a CM-SRC in this section. Three design examples are given. The first example designs the converter to operate in a natural-commutation region $\left(\omega_{S N}<1\right)$ such that all the transistors' turn-off losses are eliminated. The second example designs the converter to operate in a mixed-commutation region $\left(\omega_{S N}<1\right)$ such that the turn-on losses of Q1,Q3 and the turn-off losses of Q2,Q4 are eliminated. Lossless capacitor snubbers can be used across Q1 and Q3 to reduce their turn-off losses. The third example designs the converter to operate in a force-commutation region $\left(\omega_{S N}>1\right)$ such that all the transistors turn-on losses are eliminated. Lossless capacitor snubbers can be used across all the transistors to reduce their turn-ofr losses.

The converter is designed to satisfy the following requirements.

$$
\begin{aligned}
& \text { Input voltage }=40 \mathrm{~V} \sim 60 \mathrm{~V} \\
& \text { Output voltage }=5 \mathrm{~V} . \\
& \text { Output power }=40 \mathrm{~W} \sim 50 \mathrm{~W} .
\end{aligned}
$$

Assume an output transformer with turn ratio $n: l$ is used between the resonant tank and the load circuit. Then,

$$
\begin{aligned}
& V_{O N \max }=\frac{5 n}{40}, \quad V_{O N \min }=\frac{5 n}{60}, \\
& I_{A V \max }=\frac{10}{n \times 40 / Z_{0}}, \quad I_{A V \min }=\frac{8}{n \times 60 / Z_{0}} .
\end{aligned}
$$

(The load current range is from $8 \Lambda$ to $10 \Lambda$ ) 


\subsubsection{Example 1 - Design in Natural Commutation Region}

To achieve natural commutation of all the transistors, an operating frequency below resonant frequency must be chosen. Choose $\omega_{S N}=0.8$. From Figure 2.24(a), to ensure output regulation, choose

$$
I_{A V \max }=\frac{10}{n \times 40 / Z_{0}}=1.7
$$

The minimum $I_{A V}$ is then

$$
I_{A V \min }=\frac{8}{n \times 60 / Z_{0}}=0.9
$$

In order to maintain natural commutation of transistors at $I_{A V \min }, V_{\text {ONmin }}$ should be chosen less than or equal to 0.28 . Choose $V_{\text {ONmin }}=0.25$. This leads to

$$
n=V_{\text {ONmin }} \times \frac{60}{5}=3 \text {, }
$$

and

$$
V_{\text {ONmax }}=\frac{5 n}{40}=0.375
$$

The characteristic impedance, $Z_{0}$, is calculated as

$$
Z_{0}=I_{A V \max } \times n \times 40 \times \frac{1}{10}=18 \Omega .
$$

This value together with the resonant frequency, $\omega_{0}=1 / \sqrt{L C}$, can be used to determine the values of resonant capacitance $\mathrm{C}$ and resonant inductance $\mathrm{L}$. 
The normalized load range, as can be seen from Figure $2.24(\mathrm{a})$, is from $8 /\left(n \times 40 / Z_{0}\right)=1.36$ to $I_{A V \max }=1.7$ at $V_{O N \max }=0.375$ and from $I_{A V_{\min }}=0.9$ to $10 /\left(n \times 60 / Z_{0}\right)=1.125$ at $V_{\text {ONmin }}=0.25$.

The normalization factor for current, $I_{P . U}$, is equal to $40 / Z_{0}=2.22 \mathrm{~A}$ at $V_{\text {ONmax }}=0.375$, and equal to $60 / Z_{0}=3.33 A$ at $V_{O N \min }=0.25$.

The ranges of $\beta_{S}$ in this design are

$$
\begin{array}{ll}
\beta_{S}=113^{\circ} \sim 152^{\circ} & \text { at } V_{\text {ONmax }}, \\
\beta_{S}=71^{\circ} \sim 85^{\circ} & \text { at } V_{\text {ONmin }} .
\end{array}
$$

The converter operates in Mode I, as can be seen from Figure 2.10(n).

Using the above $\beta_{S}$ ranges, various important circuit features can be derived from Figure 2.24. In Figure 2.24, the ranges of various circuit features at $V_{O N_{\max }}$ and $V_{\text {ONmin }}$ are highlighted with dotted line segments. $A n{ }^{\prime} X^{\prime}$ is used to indicate the particular operating point where the maximum circuit feature occurs.

Since the characteristics are normalized, the obtained data should be denormalized to reflect the real values. For example, the maximum normalized rms switch current, $I_{Q 1 R M S \max }$ is 1.27 , as can be seen from Figure $2.24(\mathrm{~d})$. This value should be multiplied by the current normalization factor $I_{P . U .}=3.33 \Lambda$ at $V_{O N}=0.25$. Thus, the real maximum rms switch currents of $Q 1$ and $Q 3$ are

$$
I_{Q 1 R M S \max }=1.27 \times 3.33=4.23 \mathrm{~A} .
$$

The various circuit salient features for this design are summarized in the following:

$$
V_{C P K \max }=132 \mathrm{~V}, \quad I_{\text {LRMSmax }}=4.3 \mathrm{~A},
$$




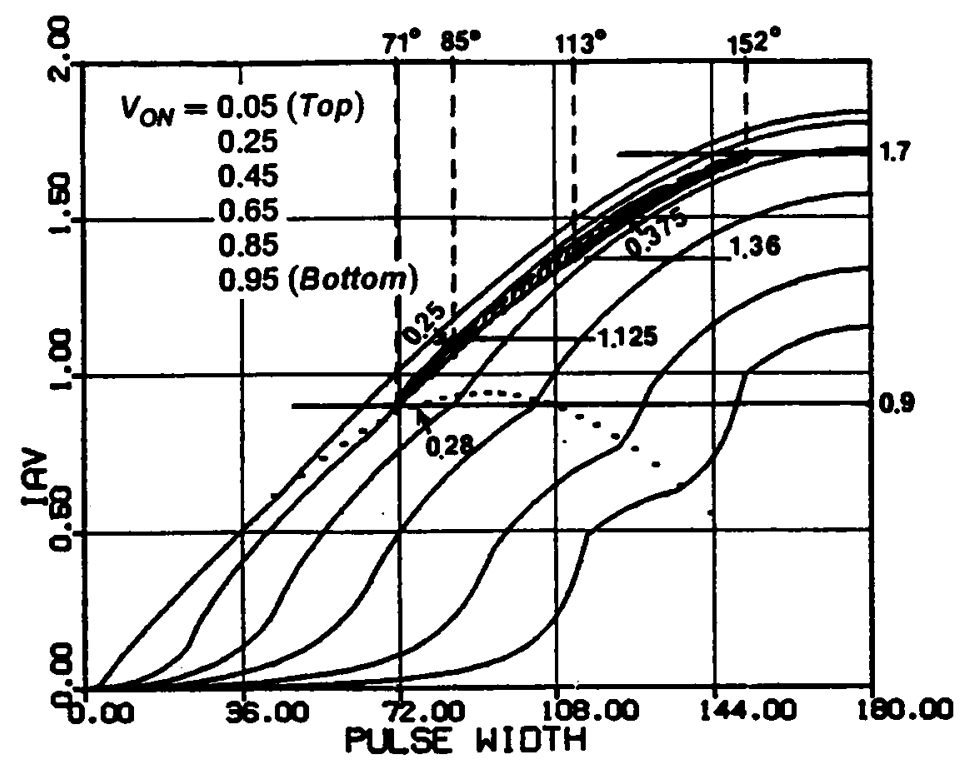

(a) Average inductor current (Load current)

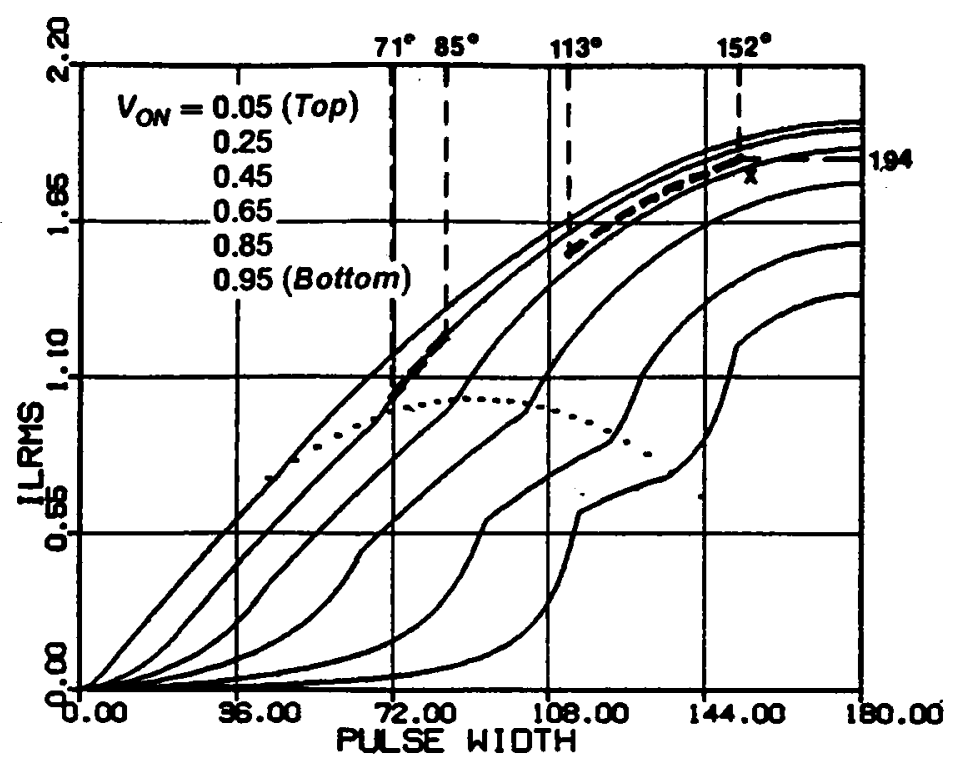

(b) RMS inductor current

Figure 2.24 Design Example in Natural Commutation Region 


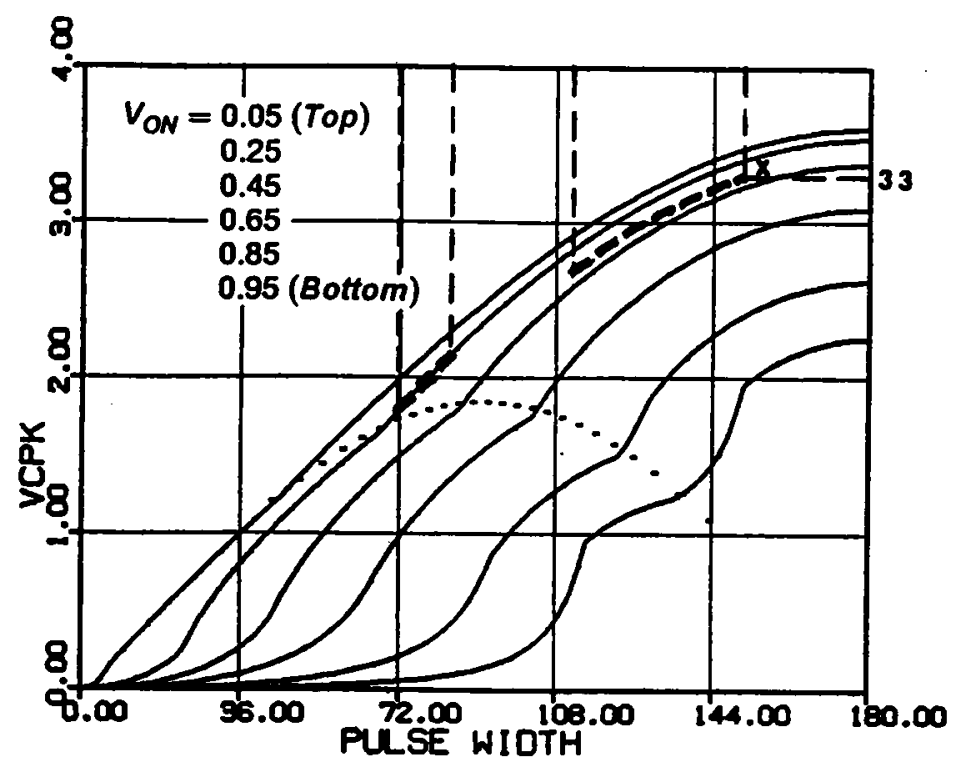

(c) Peak capacitor voltage

Figure 2.24 Continued 


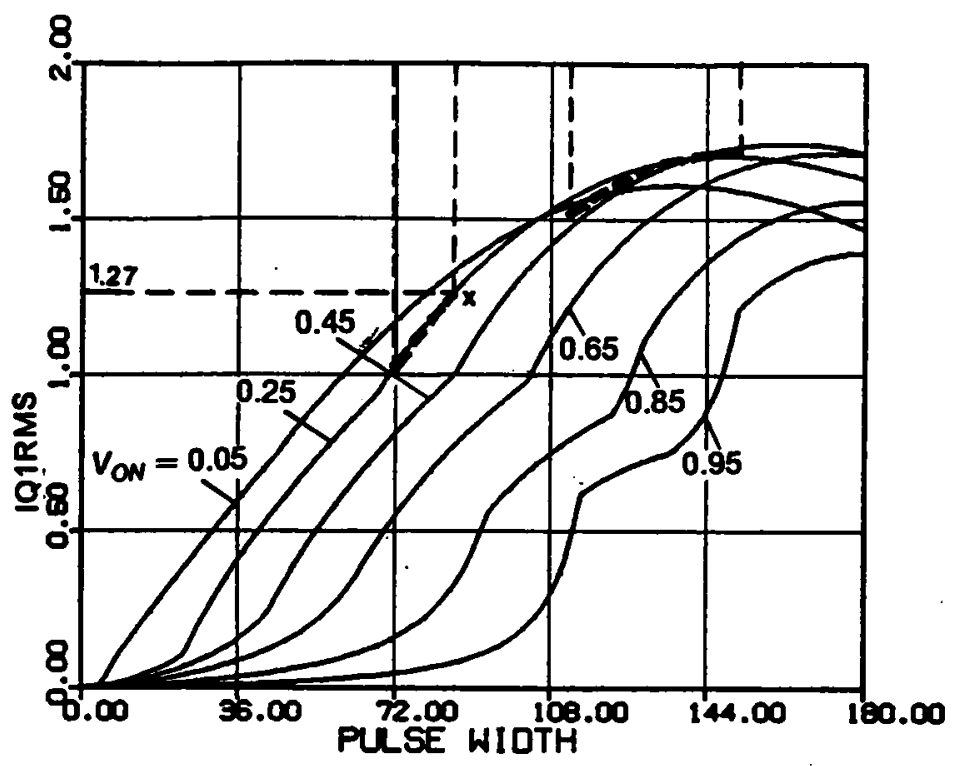

(d) RMS switch current (Q1,Q3)

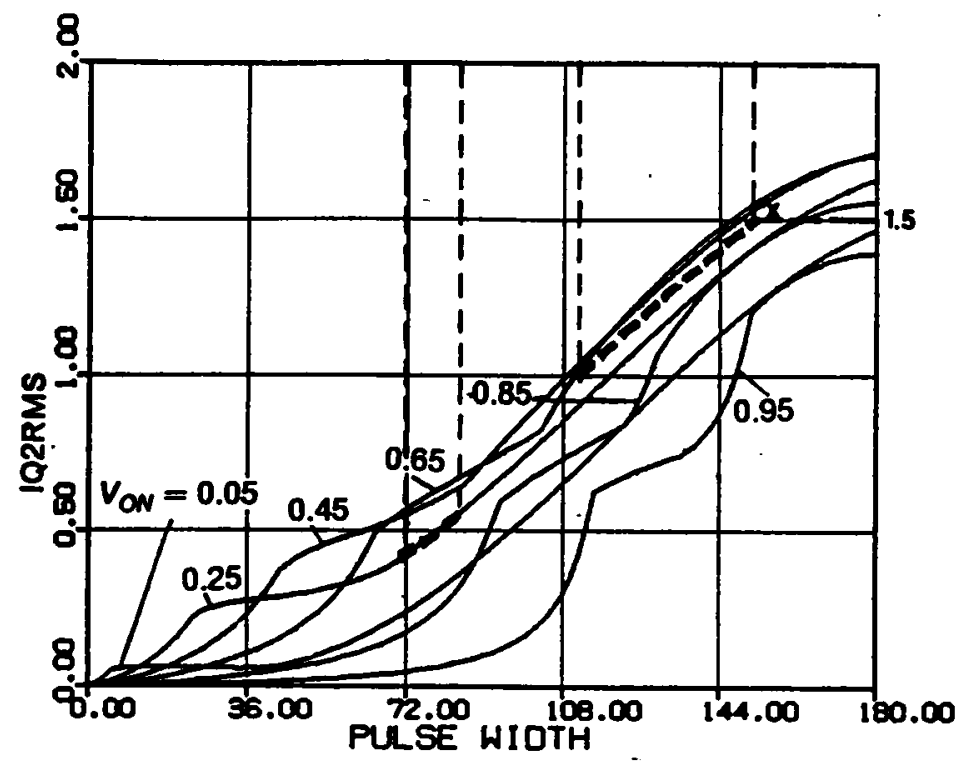

(e) RMS switch current (Q2,Q4)

Figure 2.24 Continued 


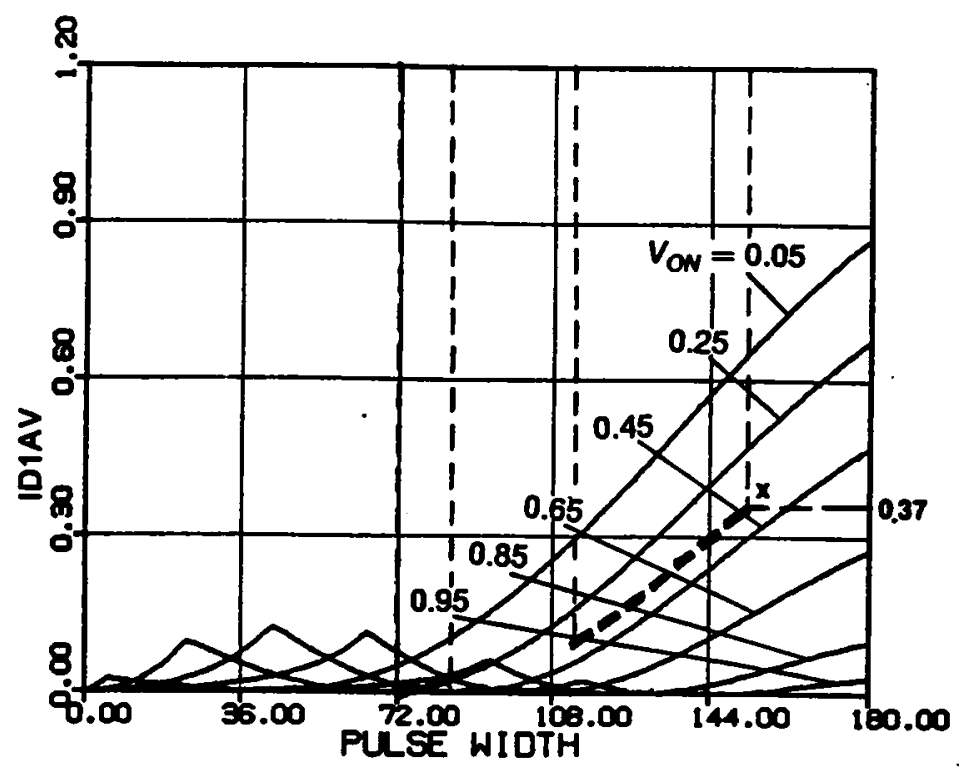

(f) Average diode current $(D I, D 3)$

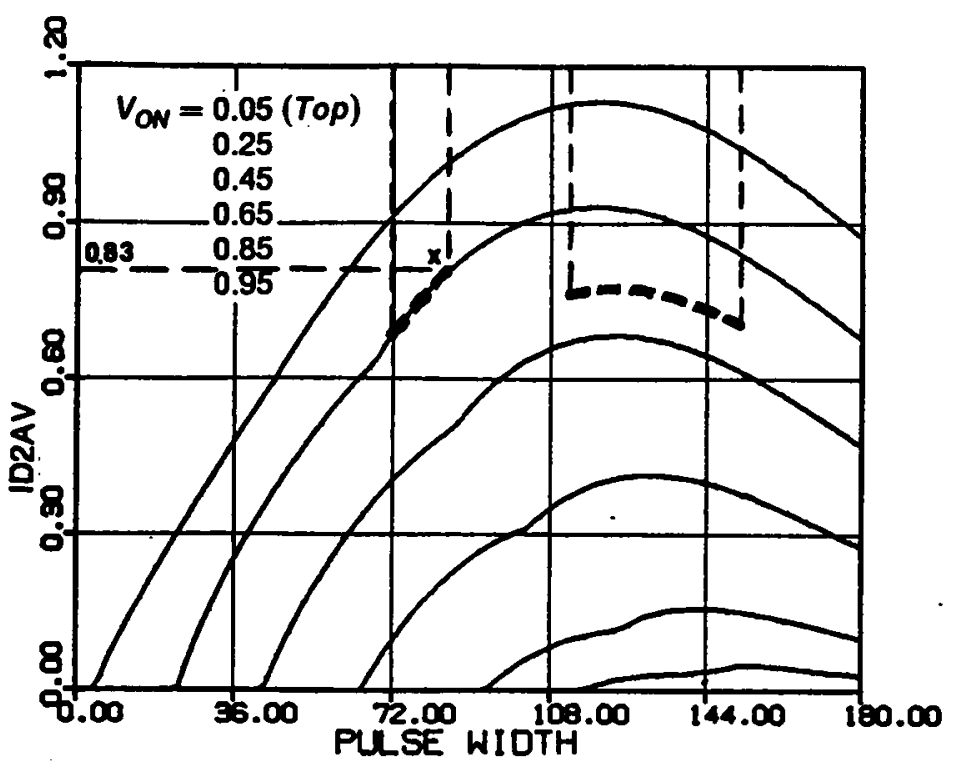

(g) Average diode current (D2,D4)

Figure 2.24 Continued 


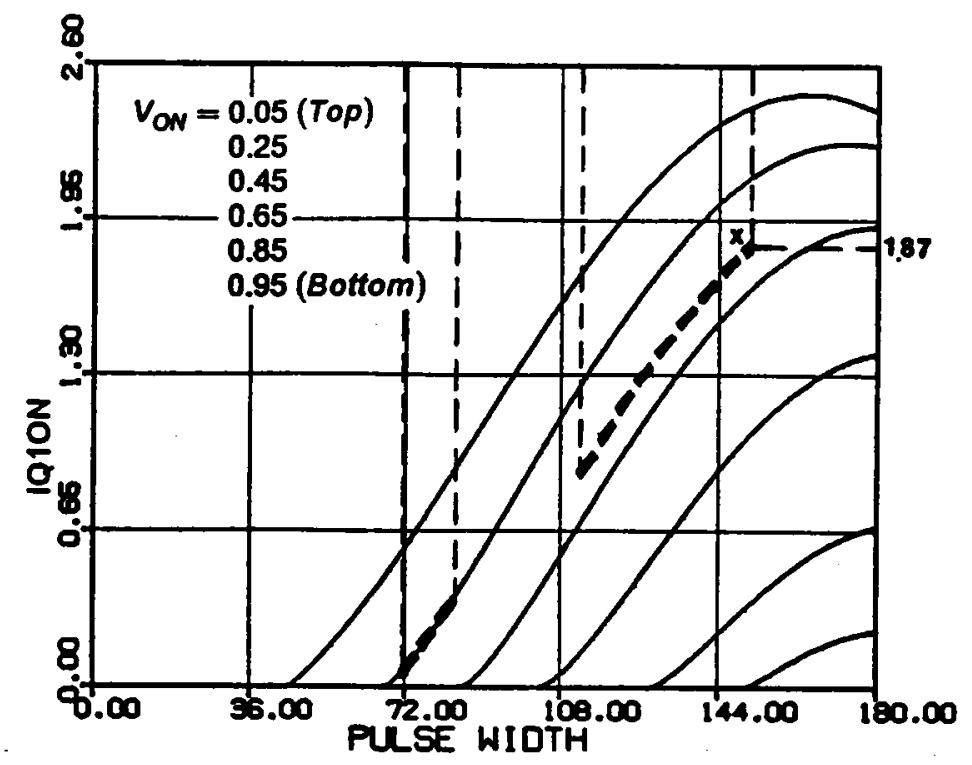

(h) Transistor turn-on Current (Q1,Q3)

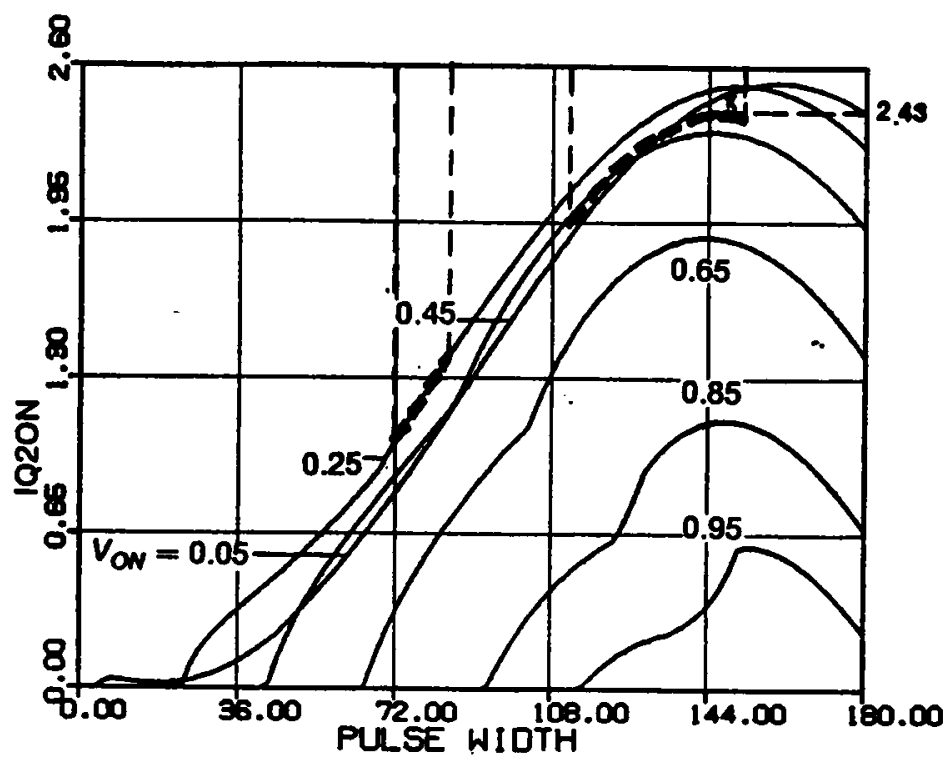

(i) Transistor turn-on Current $(Q 2, Q 4)$

Figure 2.24 Continued 


$$
\begin{array}{ll}
I_{Q 1 \text { RMSmax }}=4.23 \mathrm{~A}, & I_{Q 2 R M S \max }=3.3 \mathrm{~A}, \\
I_{D 1 A V \max }=0.83 \mathrm{~A},- & I_{D 2 A V \max }=2.75 \mathrm{~A}, \\
I_{Q 1 \text { offmax }}=0 . \mathrm{A} & I_{Q 20 f f_{\max }}=0 . \mathrm{A}, \\
I_{Q 1 \text { onmax }}=4.16 \mathrm{~A}, & I_{Q 2 \text { manax }}=5.39 \mathrm{~A} .
\end{array}
$$

\subsubsection{Example 2 - Design in Mixed-Commutation Region}

To achieve zero-voltage turn-on (force commutation) of Q1,Q3 and zero-current turn-off (natural commutation) of Q2,Q4, the operating frequency can be chosen either below or above resonant frequency. Choose $\omega_{S N}=0.8$. From Figure $2.25(\mathrm{a})$, to ensure force-commutation of $\mathrm{Q} 1$ and $\mathrm{Q} 3$, choose $I_{A V_{\max }}=10 /\left(n \times 40 / Z_{0}\right)=0.75$. This leads to

$$
I_{A V \min }=\frac{8}{n \times 60 / Z_{0}}=0.4
$$

Although $V_{\text {ONmax }}$ can be chosen anywhere between 0.15 and 0.85 , as shown in Figure 2.25(a), choose $V_{\text {ONmax }}=0.65$ to avoid possible Mode-IV operation (refer to Figure 2.11(f)). This leads to

$$
n=V_{\text {ONmax }} \times \frac{40}{5}=5.2 \text {, }
$$

and

$$
V_{\text {ONmin }}=\frac{5 n}{60}=0.43
$$

The characteristic impedance, $Z_{0}$, is calculated as 


$$
Z_{0}=I_{A V \max } \times n \times 40 \times \frac{1}{10}=15.6 \Omega
$$

The normalized load range, as can be seen from Figure 2.25(a), is from $8 /\left(n \times 40 / Z_{o}\right)=0.6$ to $I_{A V \max }=0.75$ at $V_{O N \max }=0.65$ and from $I_{A V \min }=0.4$ to $10 /\left(n \times 60 / Z_{o}\right)=0.5$ at $V_{O N \min }=0.43$.

The current normalization factor, $I_{P . U}$, is equal to $40 / Z_{0}=2.56 \mathrm{~A}$ at $V_{\text {ONmax }}=0.65$, and equal to $60 / Z_{0}=3.85 \mathrm{~A}$ at $V_{\text {ONmin }}=0.43$.

The ranges of $\beta_{s}$ in this design are

$$
\begin{array}{ll}
\beta_{S}=77^{\circ} \sim 87^{\circ} & \text { at } V_{\text {oNmax }}, \\
\beta_{S}=47^{\circ} \sim 52^{\circ} & \text { at } V_{\text {ONmin }} .
\end{array}
$$

The converter operates in Mode III, as can be seen from Figure 2.11( $)$.

Again, using the above $\beta_{S}$ ranges, the following salient features are obtained for this design,

$$
\begin{array}{ll}
V_{C P K \max }=57.2 \mathrm{~V}, & I_{\text {LRMSmax }}=2.14 \mathrm{~A}, \\
I_{Q 1 \text { RMSmax }}=2.0 \mathrm{~A}, & I_{Q 2 R M S \max }=1.76 \mathrm{~A}, \\
I_{D 1 A V \max }=0.3 \Lambda, & I_{D 2 A V \max }=0.8 \mathrm{~A}, \\
I_{Q 1 \text { offmax }}=3.3 \mathrm{~A} & I_{\text {Q2offmax }}=0 . \mathrm{A}, \\
I_{\text {Q1 } \operatorname{mmax}}=0 . \Lambda, & I_{\text {Q2onmax }}=1.95 \mathrm{~A} .
\end{array}
$$


The ranges of various salient features at $V_{O N \max }$ and $V_{\text {ONmin }}$ are highlighted with dotted line segments in Figure 2.25. 


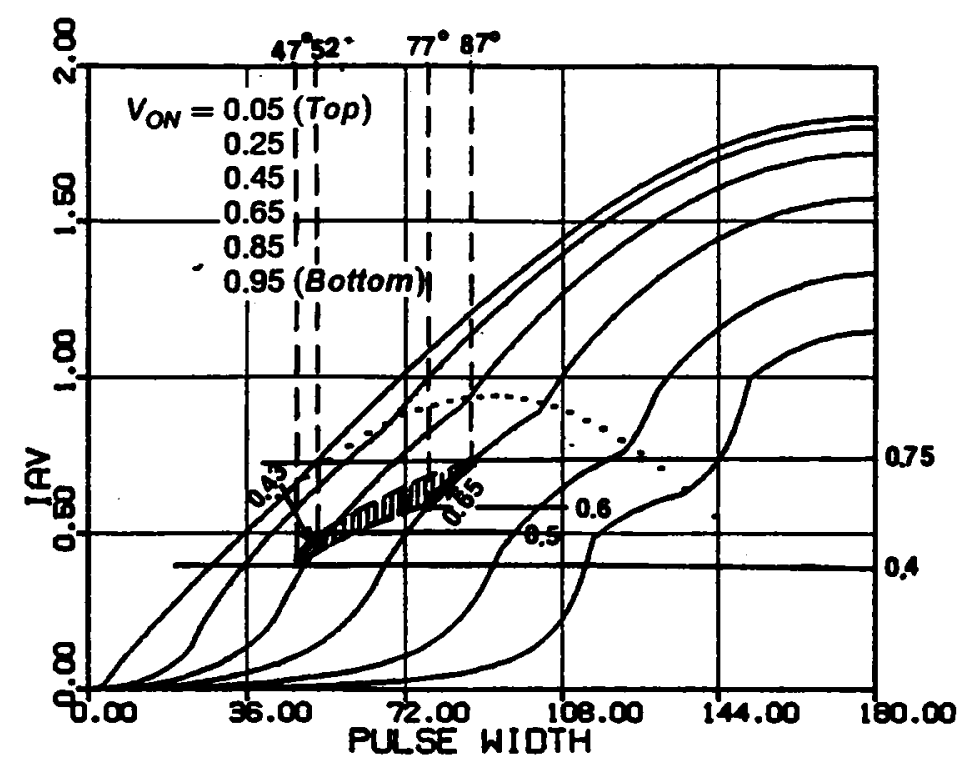

(a) Average inductor current (Load current)

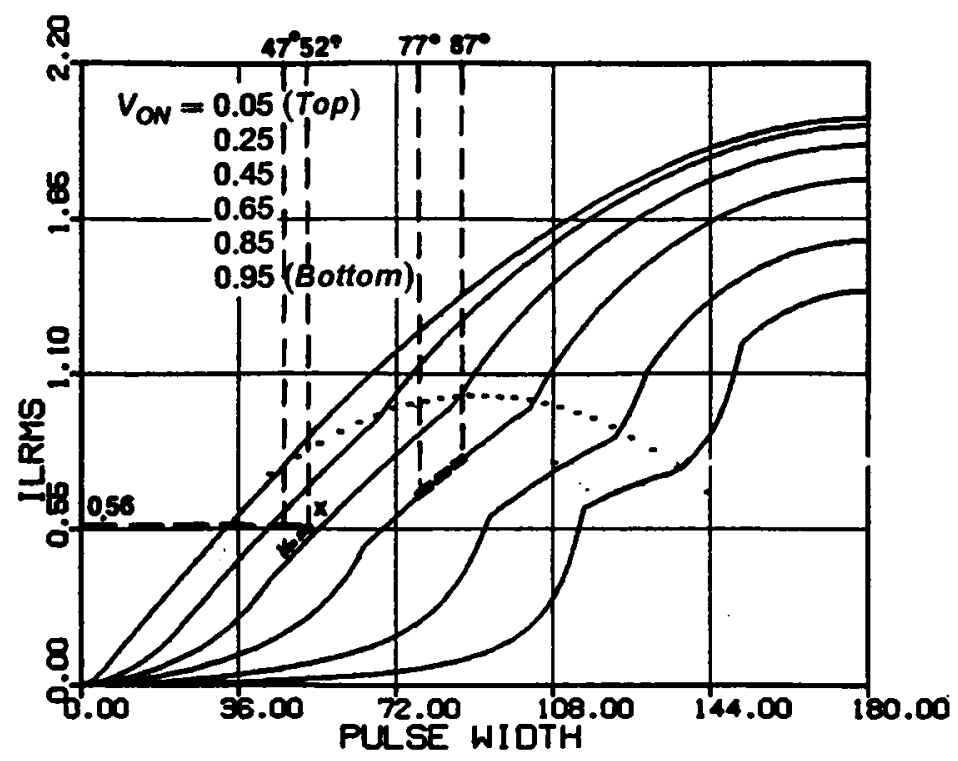

(b) RMS inductor current

Figure 2.25 Design Example in Mixed-Commutation Region 


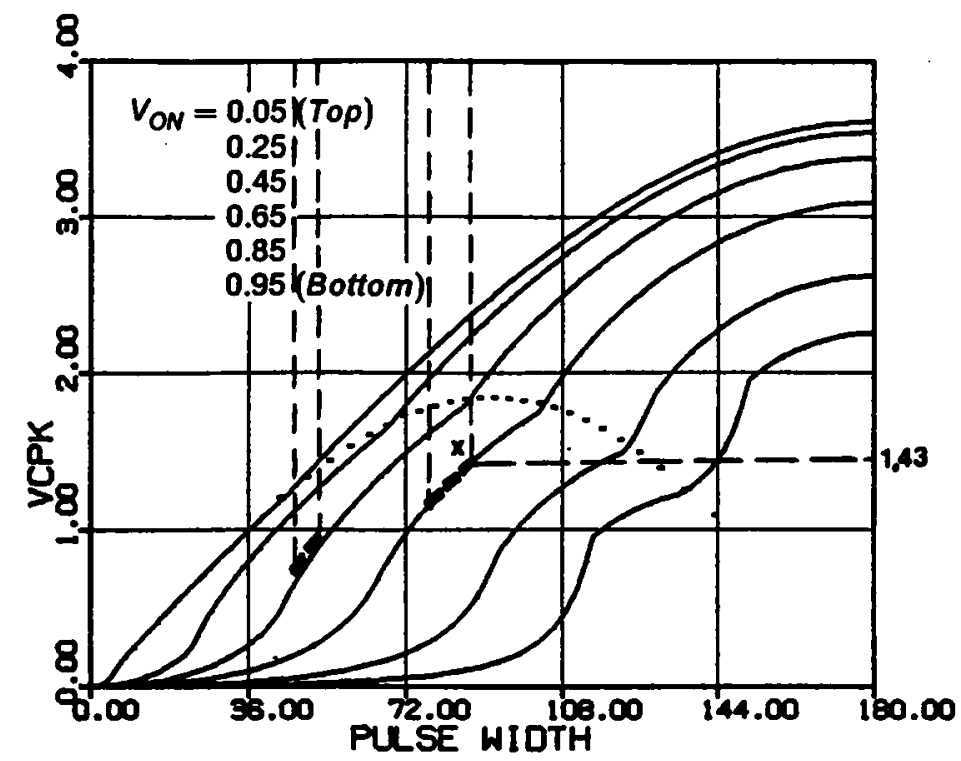

(c) Peak capacitor voltage

Figure 2.25 Continued 


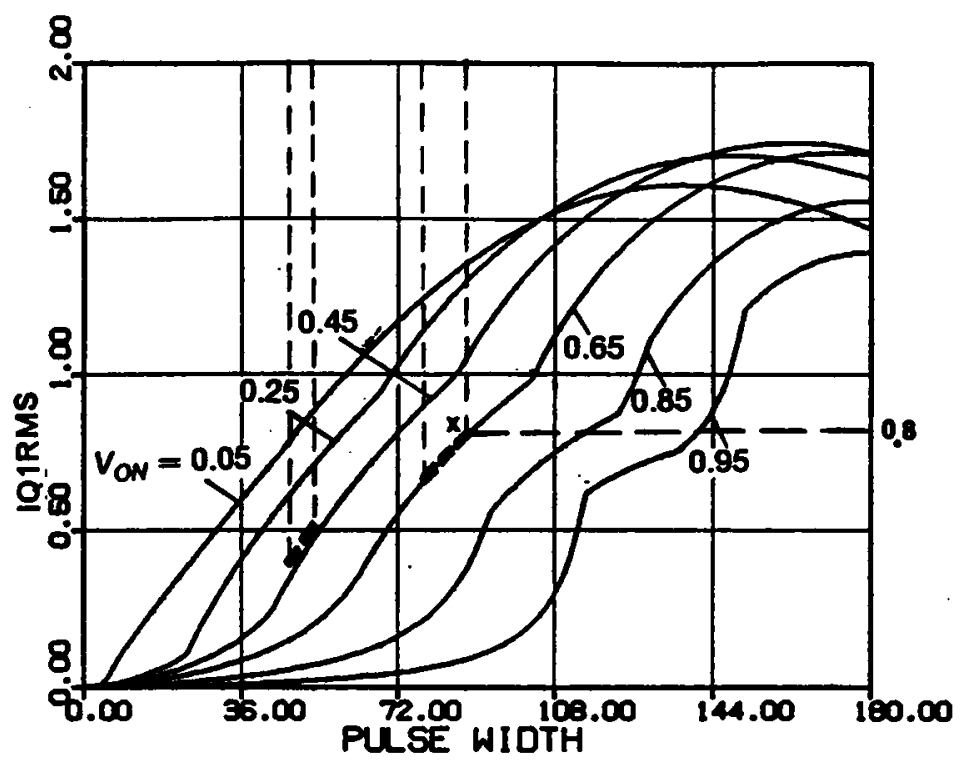

(d) RMS switch current $(Q 1, Q 3)$

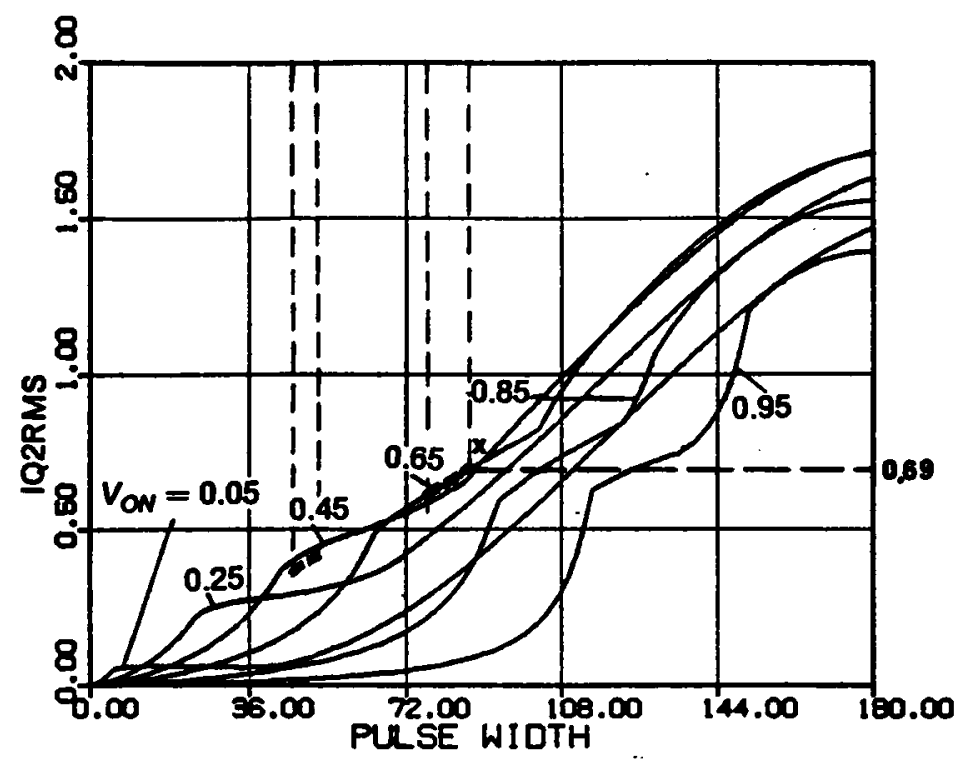

(e) RMS switch current (Q2,Q4)

Figure 2.25 Continued 


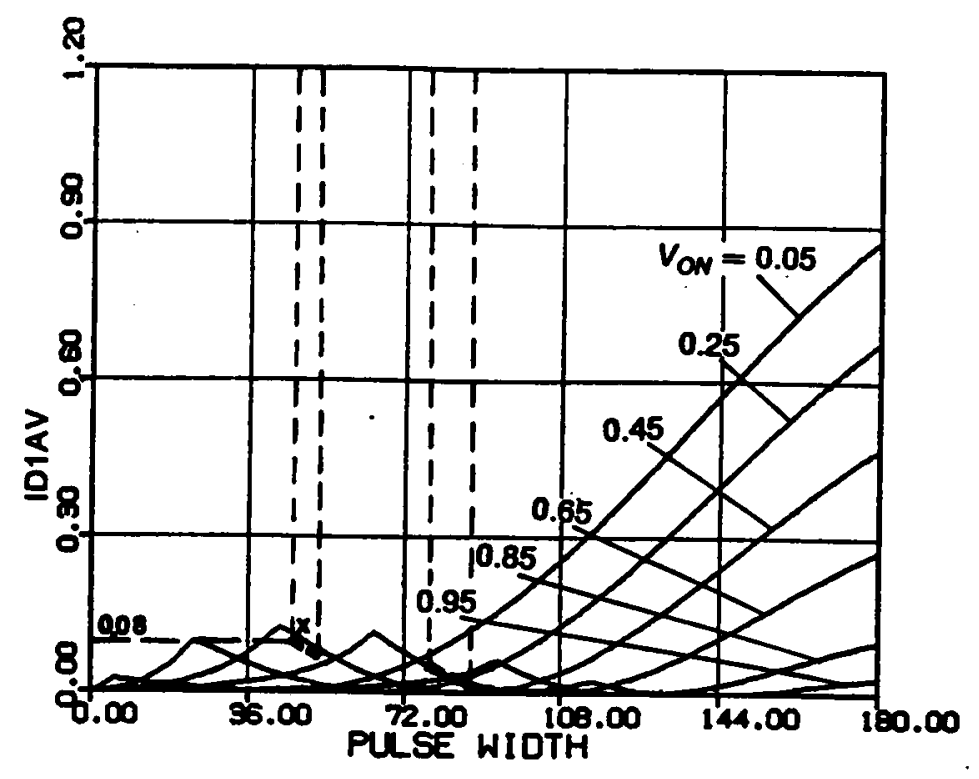

(f) Average diode current (D1,D3)

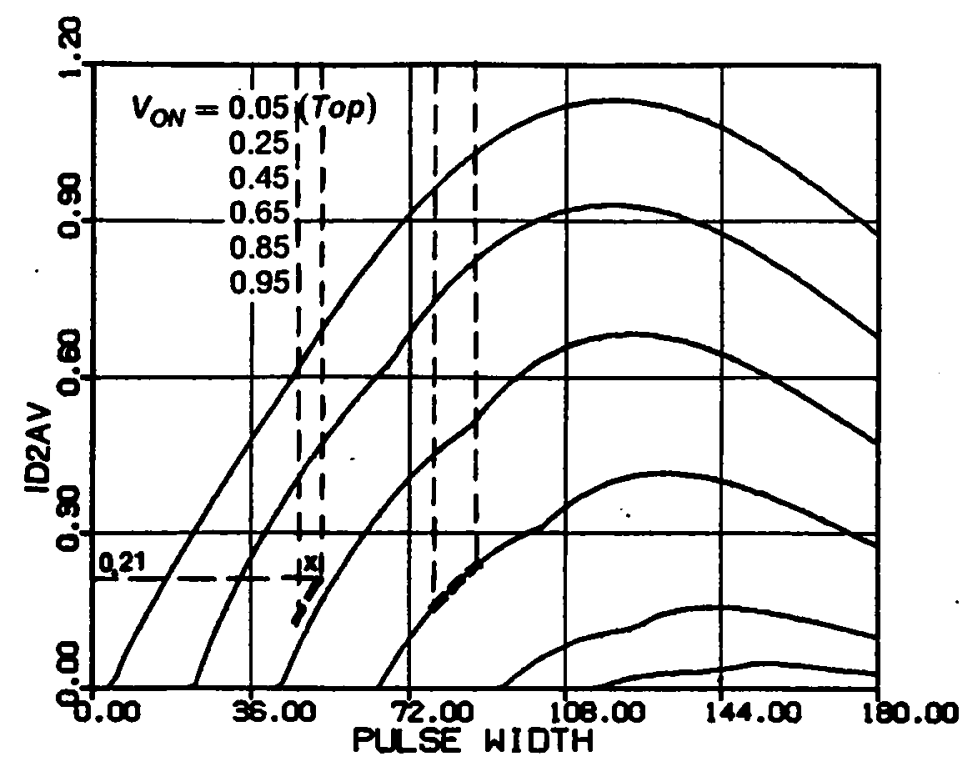

(g) Average diode current (D2,D4)

Figure 2.25 Continued 


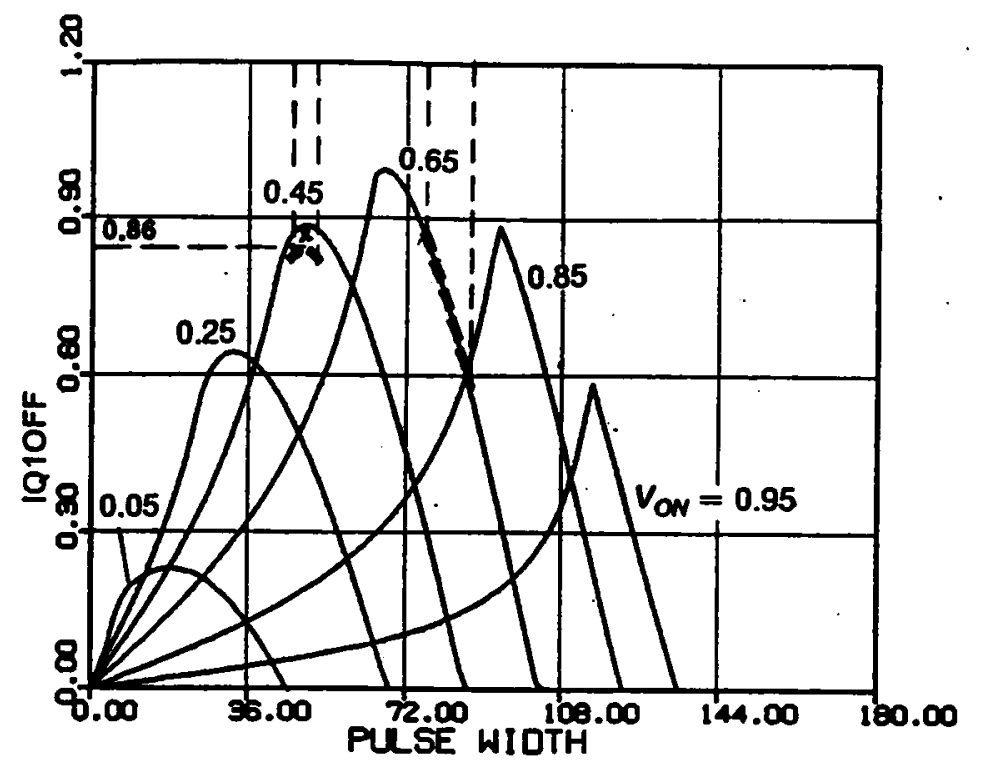

(h) Transistor turn-off Current $(Q 1, Q 3)$

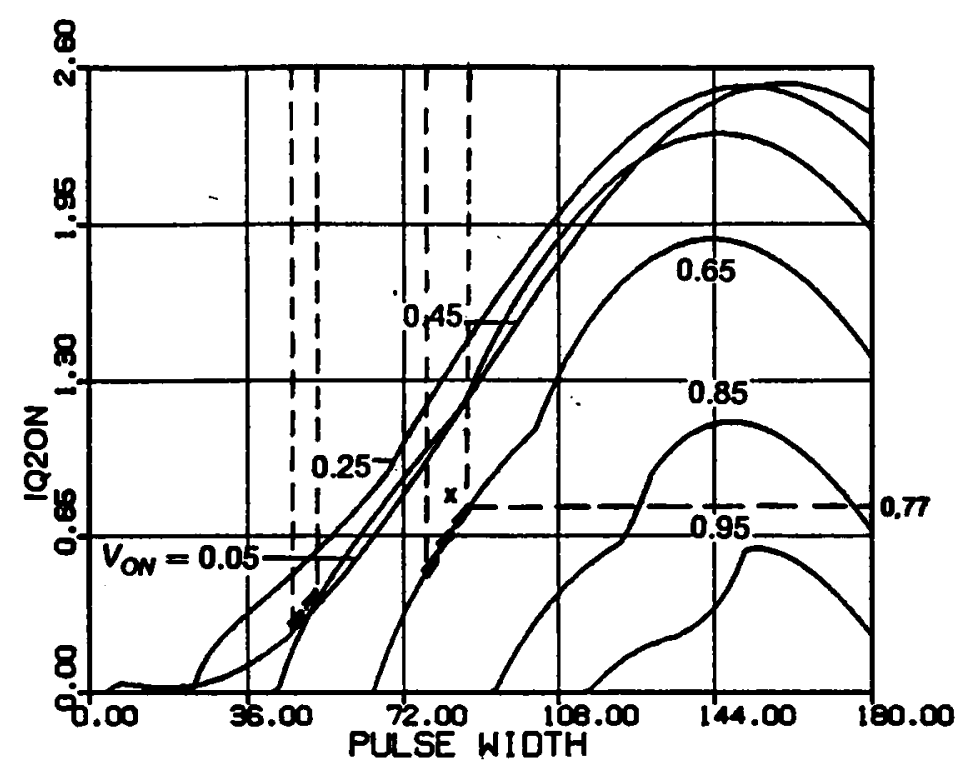

(i) Transistor turn-on Current (Q2,Q4)

Figure 2.25 Continued 


\subsubsection{Example 3 - Design in Force-Commutation Region}

To achieve zero-voltage turn-on (force commutation) of all the transistors, the operating frequency must be chosen above resonant frequency. Choose $\omega_{S N}=1.2$. From Figure 2.26(a), to ensure output regulation, choose

$$
I_{A V \max }=\frac{10}{n \times 40 / Z_{0}}=1.8
$$

The minimum $I_{A V}$ can then be determined as

$$
I_{A V \min }=\frac{8}{n \times 60 / Z_{0}}=0.96
$$

In order to maintain force commutation of transistors at $I_{A V \min }, V_{O N m m n}$ should be chosen less than or equal to 0.28 , as can be seen from Figure 2.26(a). Choose $V_{\text {ONmin }}=0.25$. This leads to

$$
n=V_{\text {oNmin }} \times \frac{60}{5}=3
$$

and

$$
V_{\text {ONmax }}=\frac{5 n}{40}=0.375
$$

The characteristic impedance, $Z_{0}$, is calculated as 


$$
Z_{0}=I_{A V \max } \times n \times 40 \times \frac{1}{10}=21.6 \Omega
$$

The normalized load range, as can be seen from Figure $2.26(\mathrm{a})$, is from $8 /\left(n \times 40 / Z_{0}\right)=1.44$ to $I_{A V \max }=1.8$. at $V_{o N \max }=0.375$ and from $I_{A V \min }=0.96$ to $10 /\left(n \times 60 / Z_{0}\right)=1.2$ at $V_{\text {oNmin }}=0.25$.

The normalization factor for current, $I_{P . U}$, is equal to $40 / Z_{0}=1.85 \mathrm{~A}$ at $V_{\text {ONmax }}=0.375$, and equal to $60 / Z_{0}=2.78 \mathrm{~A}$ at $V_{\text {oNmin }}=0.25$.

The ranges of $\beta_{S}$ in this design are

$$
\begin{array}{ll}
\beta_{S}=101^{\circ} \sim 139^{\circ} & \text { at } V_{\text {ONmax }} \\
\beta_{S}=59^{\circ} \sim 72^{\circ} & \text { at } V_{\text {ONmin }}
\end{array}
$$

The converter operates in Mode $A$, as can be seen from Figure 2.16(b).

Using the above $\beta_{S}$ ranges, the following salient features are obtained,

$$
\begin{array}{ll}
V_{\text {CPKmax }}=96 \mathrm{~V}, & I_{\text {LRMSmax }}=3.74 \mathrm{~A}, \\
I_{Q 1 \text { RMSmax }}=2.7 \mathrm{~A}, & I_{Q 2 R M S \max }=3.64 \mathrm{~A}, \\
I_{D 1 A V \max }=2.45 \mathrm{~A}, & I_{D 2 A V \max }=0.52 \mathrm{~A}, \\
I_{Q 1 \text { offmax }}=5.46 \Lambda & I_{Q 2 \text { offmax }}=4.1 \mathrm{~A}, \\
I_{Q 1 \text { maxmax }}=0 \mathrm{~A}, & I_{Q 2 \text { Ionmax }}=0 \mathrm{~A} .
\end{array}
$$

The ranges of various salient features at $V_{\text {oNmax }}$ and $V_{\text {oNmin }}$ are highlighted with dotted line segments in Figure 2.26. 


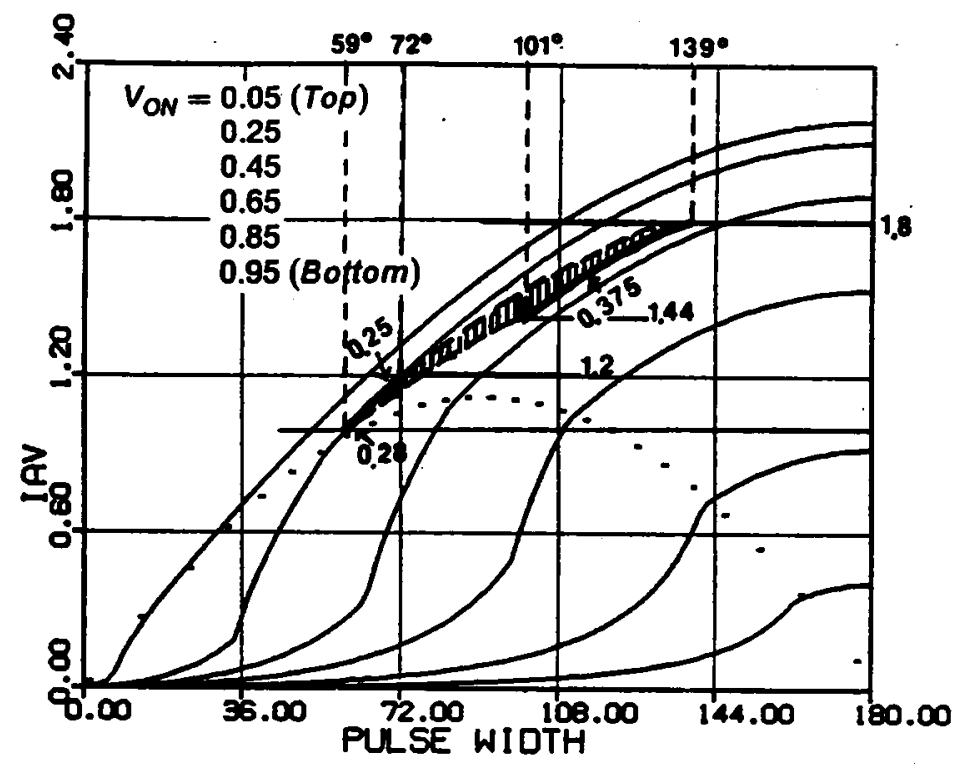

(a) Average inductor current (Load current)

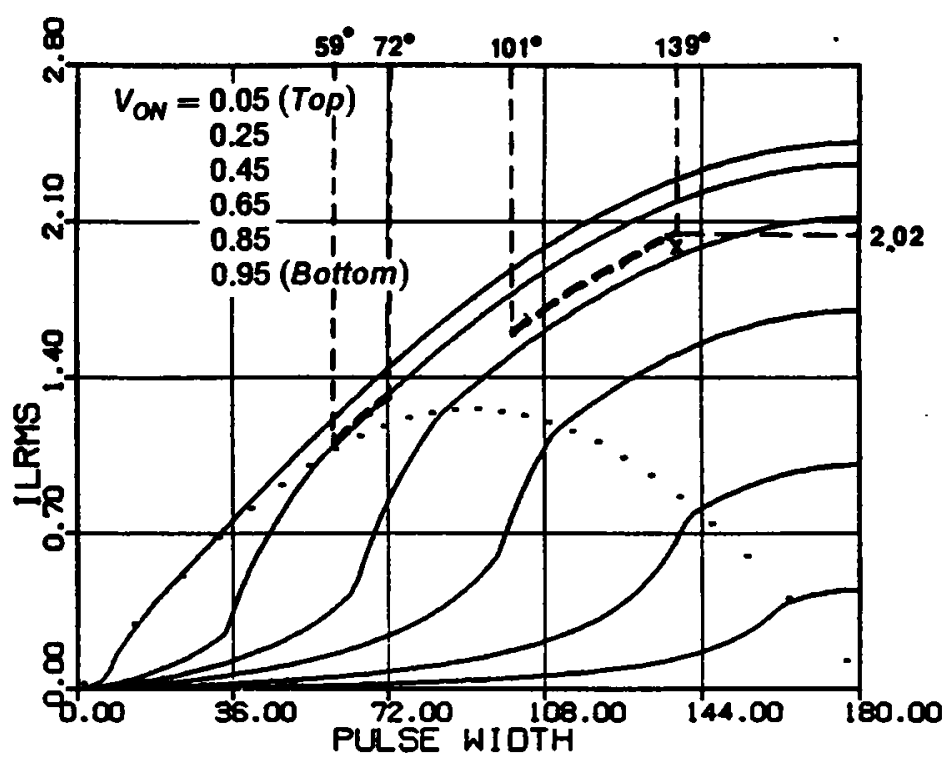

(b) RMS inductor current

Figure 2.26 Design Example in Force-Commutation Region 


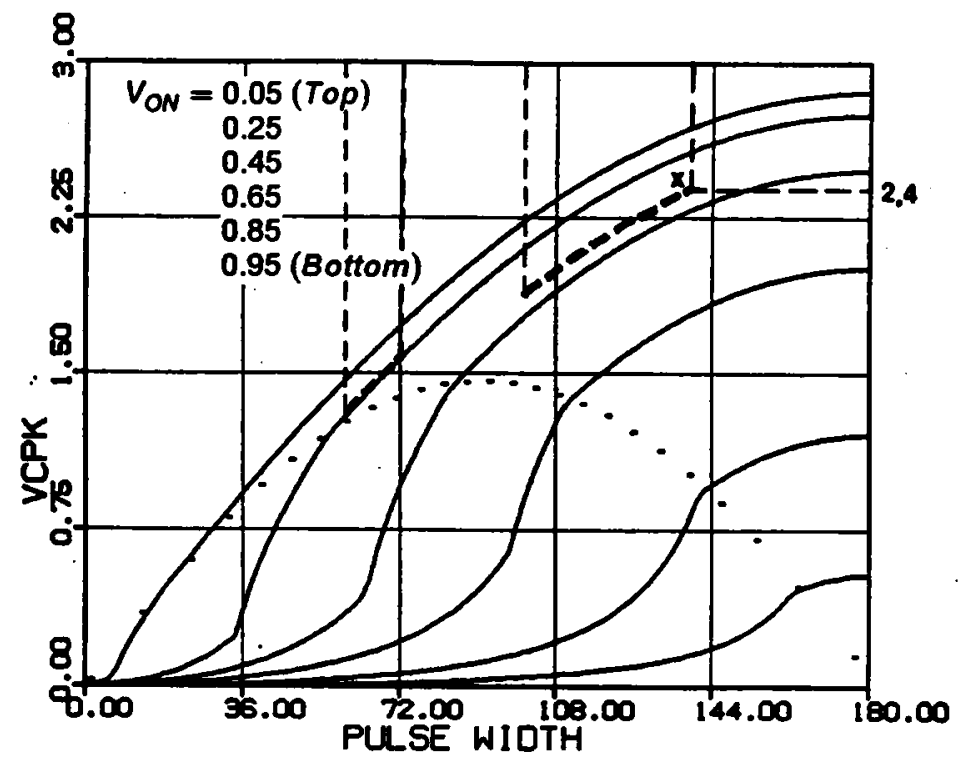

(c) Peak capacitor voltage

Figure 2.26 Continued 


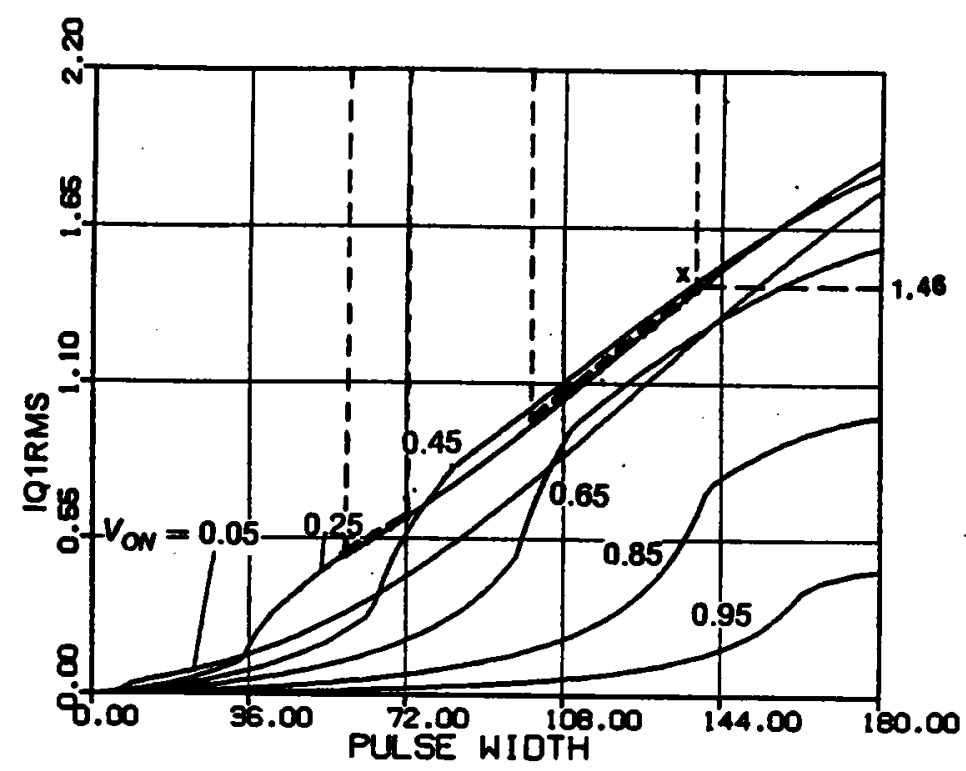

(d) RMS switch current $(Q 1, Q 3)$

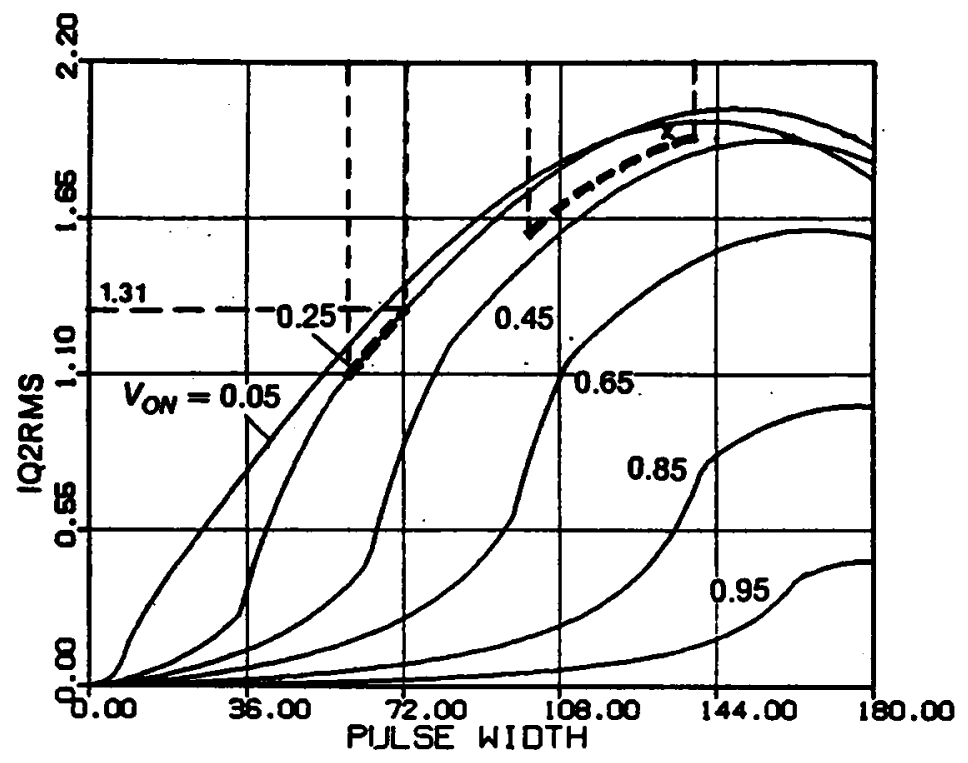

(e) RMS switch current $(Q 2, Q 4)$

Figure 2.26 Continued 


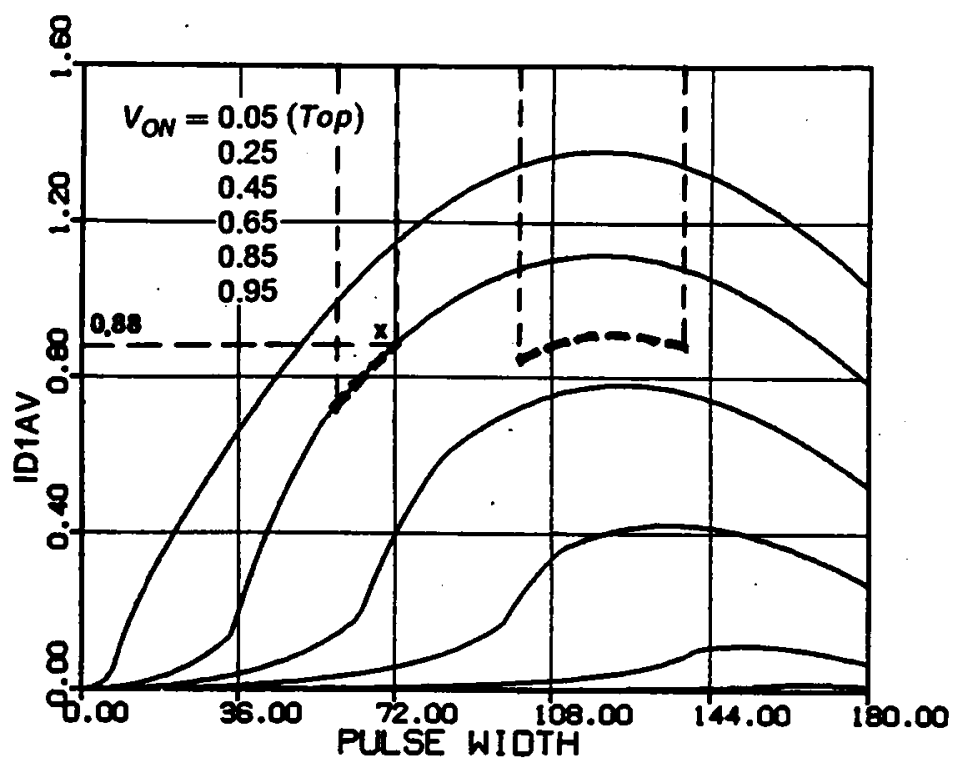

(f) Average diode current (DI,D3)

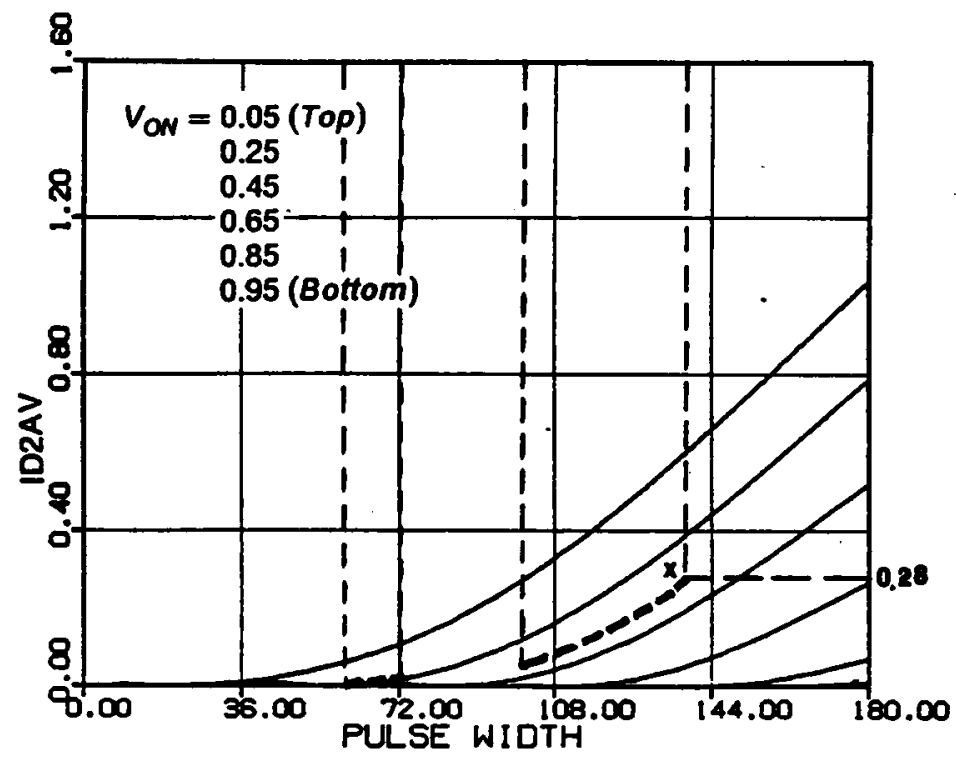

(g) Average diode current (D2,D4)

Figure 2.26 Continued 


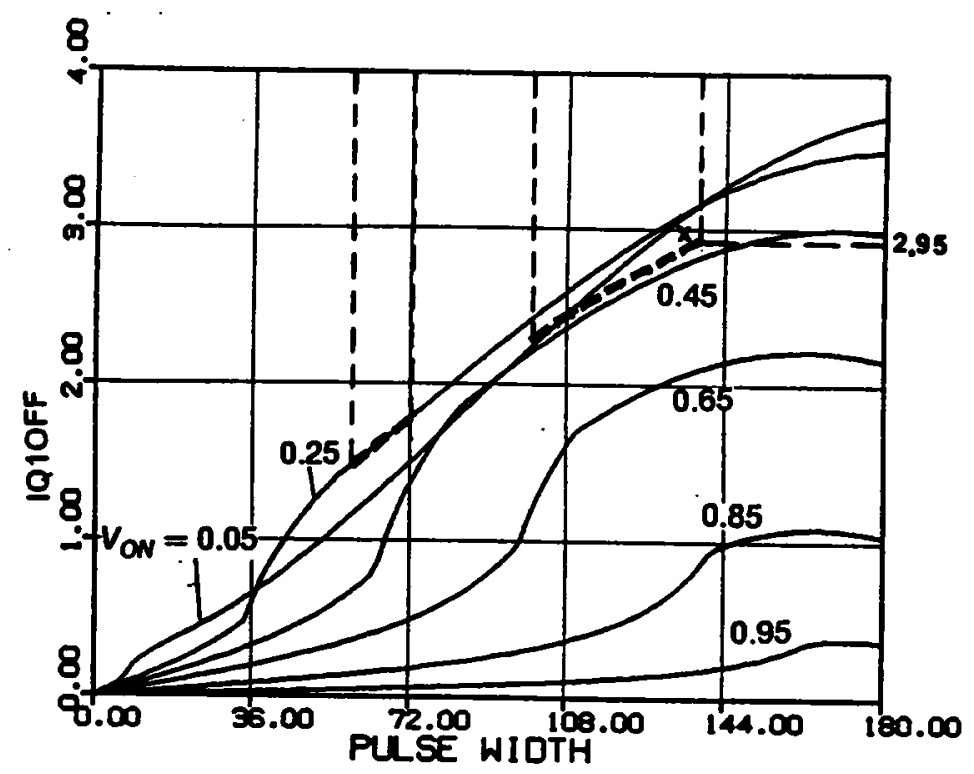

(h) Transistor turn-off Current (Q1,Q3)

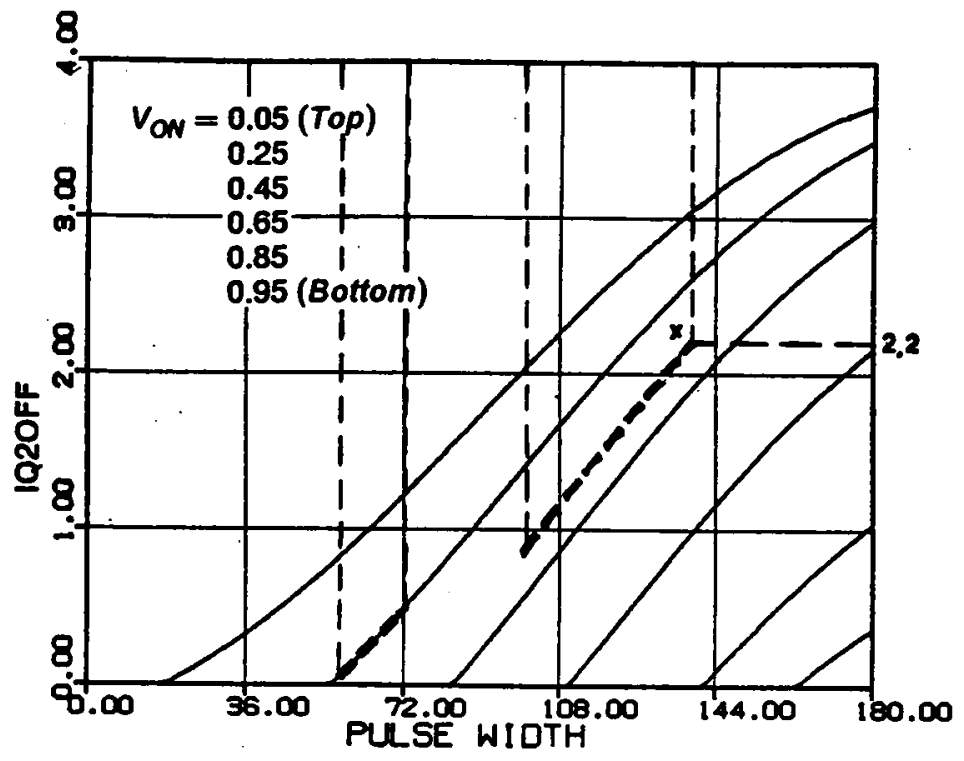

(i) Transistor turn-off Current (Q2,Q4)

Figure 2.26 Continued 
From the above examples, it can be seen that wider $\beta_{S}$ range and higher component ratings are usually required to design a CM-SRC in the natural- or in the forcecommutation region. 


\subsection{HARDWARE VERIFICATIONS}

Two prototype circuits have been built to verify the circuit operation of a CM-SRC. The first circuit is designed at $24 \mathrm{kIIz}$, with a resonant frequency of $42 \mathrm{kHz}$ ( $\left.\omega_{S N}=0.57 \simeq 0.6\right)$ to verify the operating modes below resonant frequency. The second circuit is designed at $100 \mathrm{kl} \mathrm{Iz}$ with a resonant frequency of $83.3 \mathrm{kHz}\left(\omega_{S N}=1.2\right)$ to verify the operating modes above resonant frequency. The experimented circuits are shown in Figure 2.27 and Figure 2.28 , respectively.

\subsubsection{Circuit Operation Below Resonant Frequency}

A series of experimental waveforms obtained from the circuit in Figure 2.27 are shown in Figure 2.29. These waveforms are obtained by fixing $V_{O N}$ at 0.2 while reducing angle $\beta_{S}$ from $180^{\circ}$ to $10^{\circ}$. The operating points are indicated by $x^{\prime} s$ in Figure 2.30. It can be seen that the converter sweeps through all the six operating modes. The tank energy decreases as $\beta_{S}$ is decreased. Circulating current in the resonant tank is small under light load since the pulse-width of the exciting voltage $v_{S}$ is reduced. The circuit is able to provide a load range from no load to full load which is not feasible for a conventional SRC. Figure $2.29(\mathrm{~h})$ shows an operating condition clearly illustrating Mode-IV operation. The waveforms are obtained at the same $\beta_{S}$ as in Figure 2.29(e) but with a $V_{O N}=0.1$.

Another serics of waveforms obtained analytically at the same operating points indicated in Figure 2.30 is shown in Figure 2.31. $\Lambda$ close resemblance can be observed between the waveforms in Figure 2.29 and Figure 2.31. The slight difference is caused by the fact that the normalized frequency of the experimented circuit is not exactly equal to 0.6. The losses in the converter have negligible effects on the waveforms at the chosen switching frequency. 


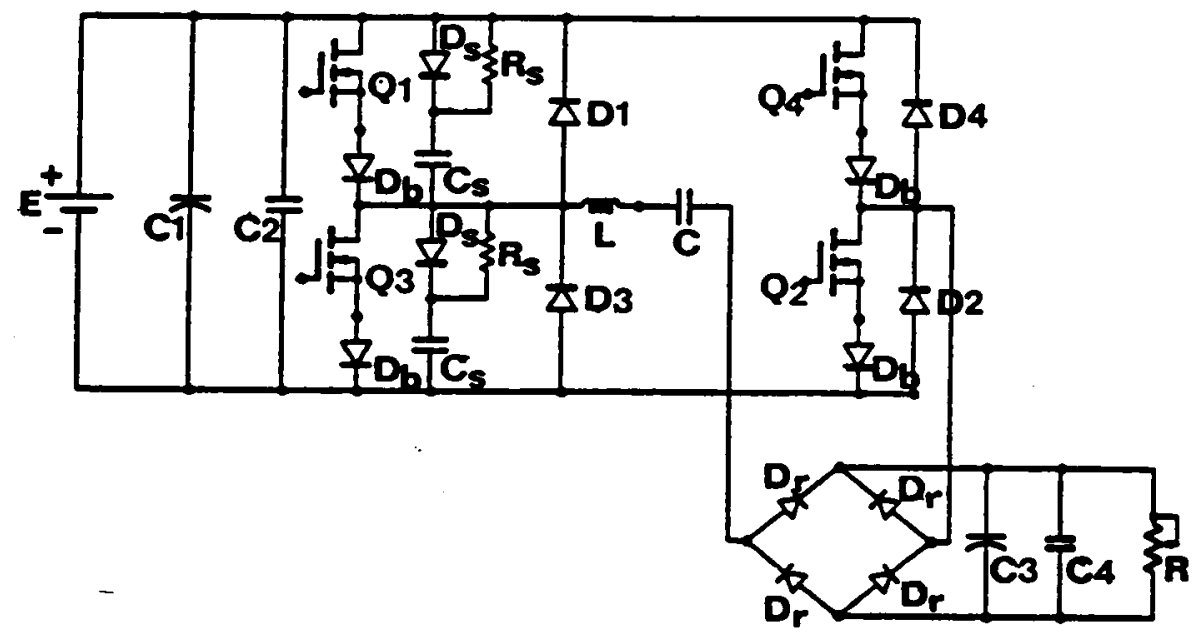

Q1,Q2,Q3,Q4: IRF520; D1,D2,D3,D4,Dr,Db: UES1303;

$C 1, C 3=2200 \mu F, C 2=100 n F, C 4=56 n F, R_{S}=56 \Omega$,

$C s=18 n F, L=105.2 \mu \mathrm{H}, \mathrm{C}=136.3 n F, E=30 \mathrm{~V}$.

Figure 2.27 A Breadboard Circuit Used to Verify Circuit Operations Below Resonant Frequency 


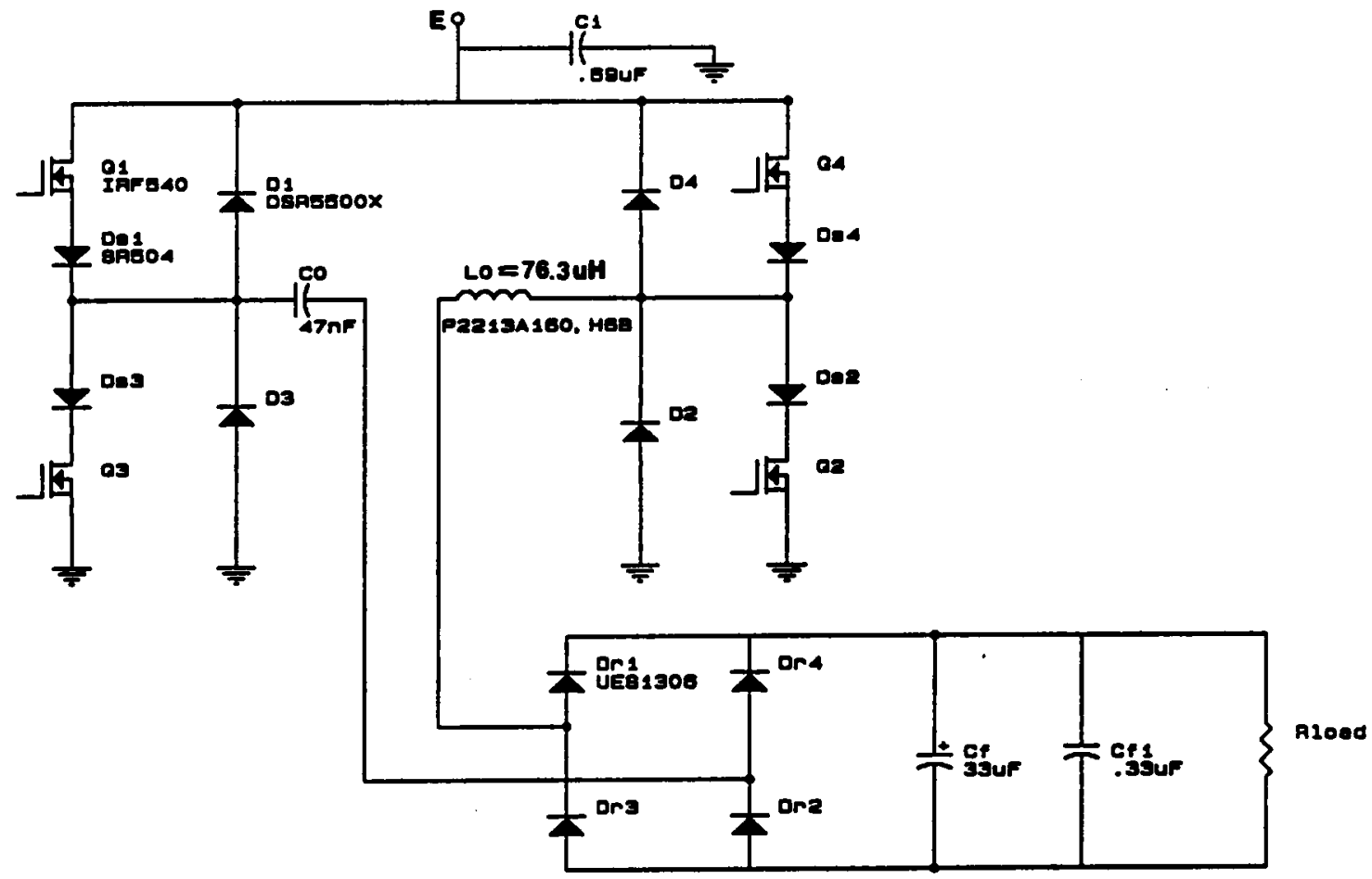

Q1,Q2,Q3,Q4: IRF540; D1,D2,D3,D4: DSR5500X;

Ds1,Ds1,Ds3,Ds4: SR504; Dr1,Dr2,Dr3,Dr4: UES1306;

$E=50 \mathrm{~V}, V_{0}=12.5 \mathrm{~V}$.

Figure 2.28 A Breadboard Circuit Used to Verify Circuit Operations Above Resonant Frequency 

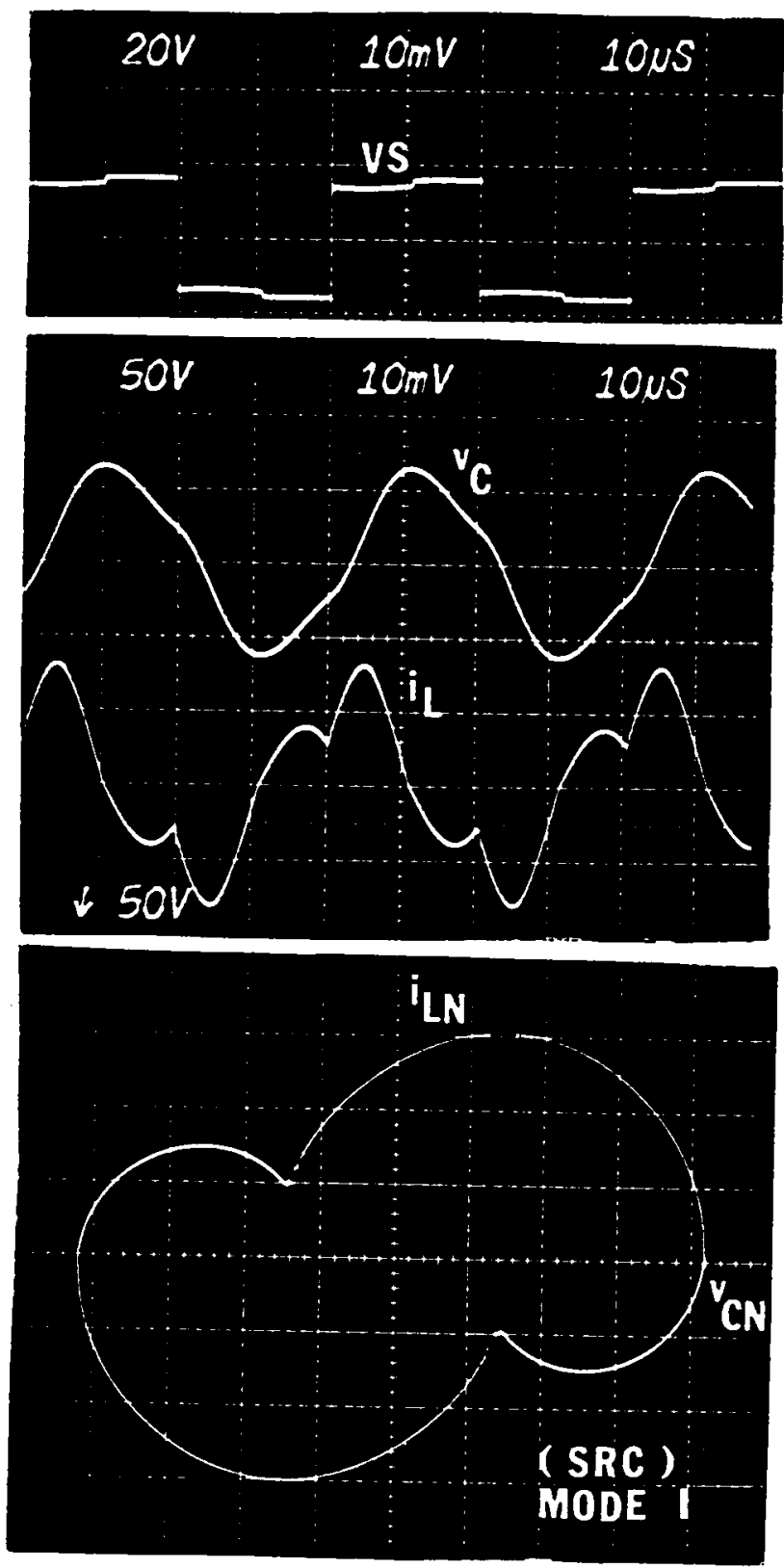

(a) SRC operation (operating point A)

Figure 2.29 Experimental Results from the Circuit in Figure 2.27 

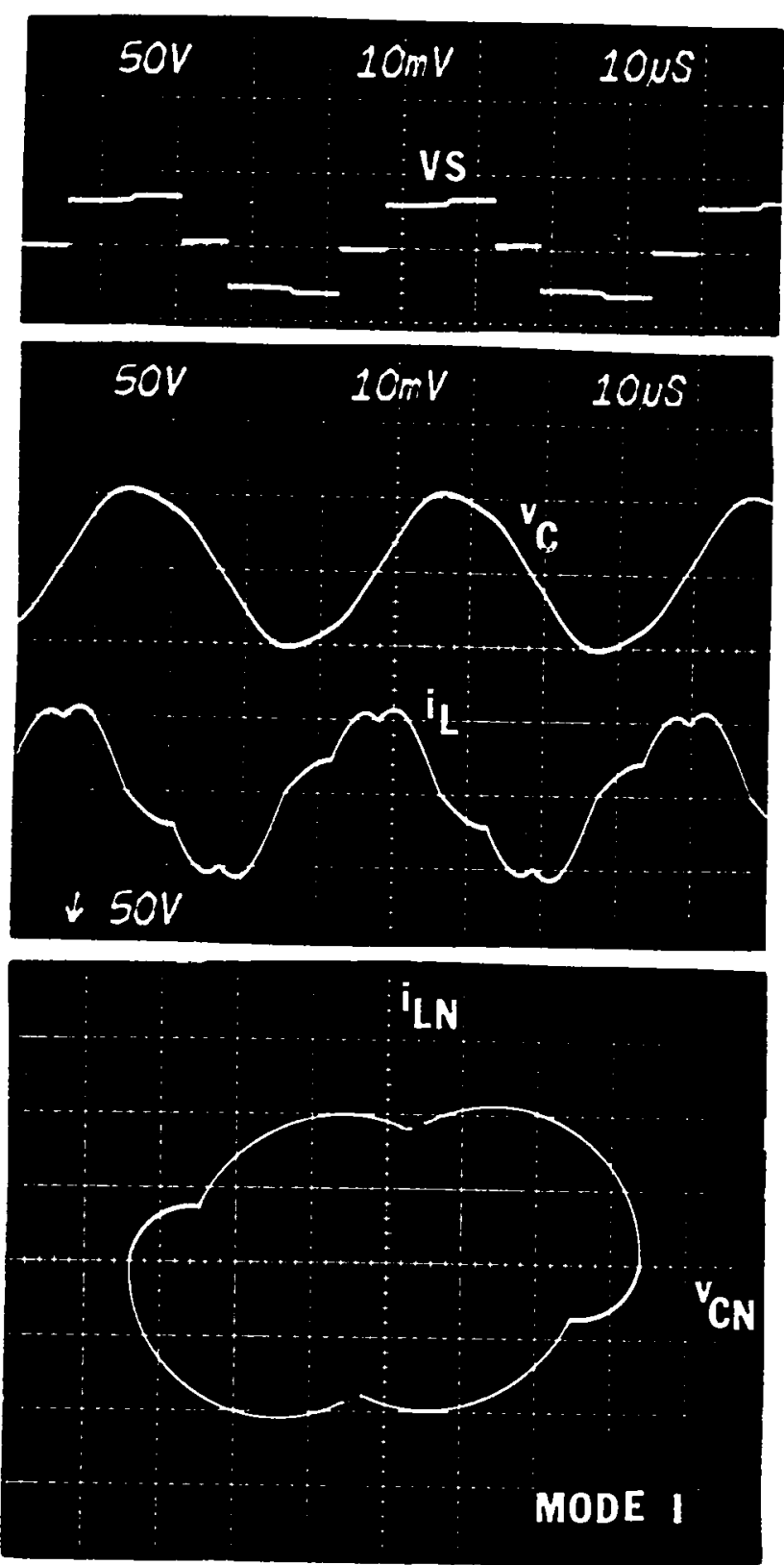

(b) Mode-I operation (operating point B)

Figure 2.29 Continued 

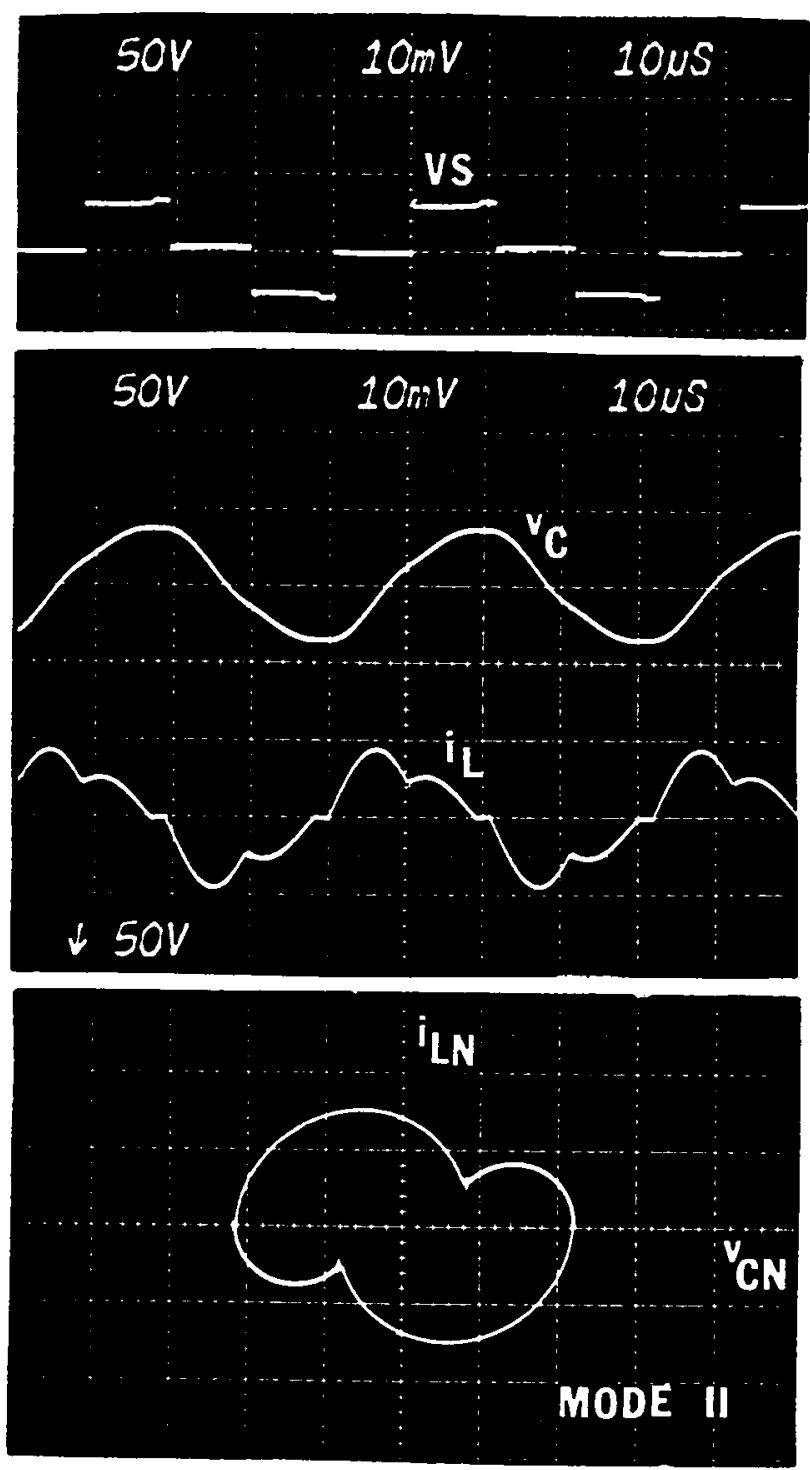

(c) Mode-II operation (operating point C)

Figure 2.29 Continued 

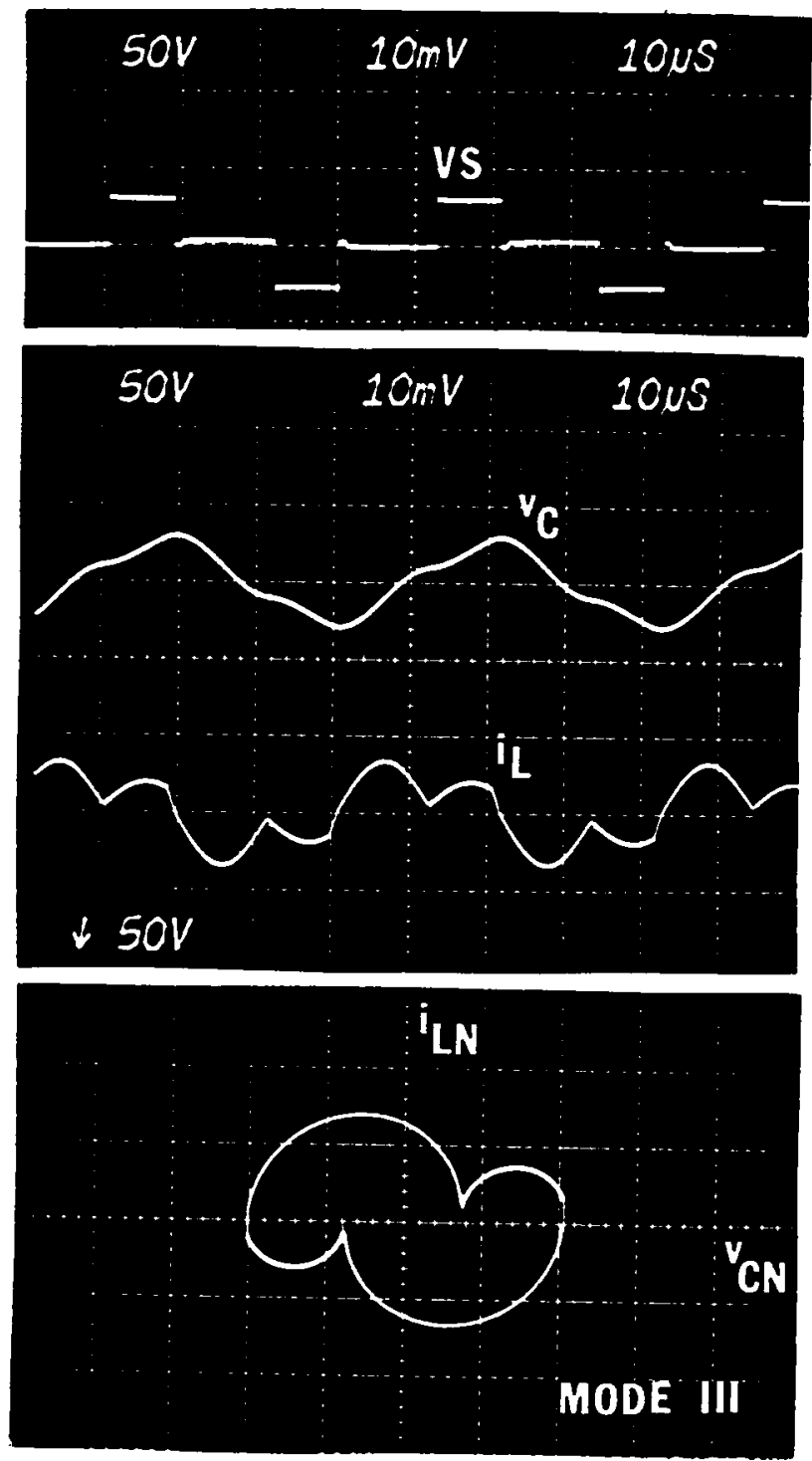

(d) Mode-lII operation (operating point D)

Figure 2.29 Continued 

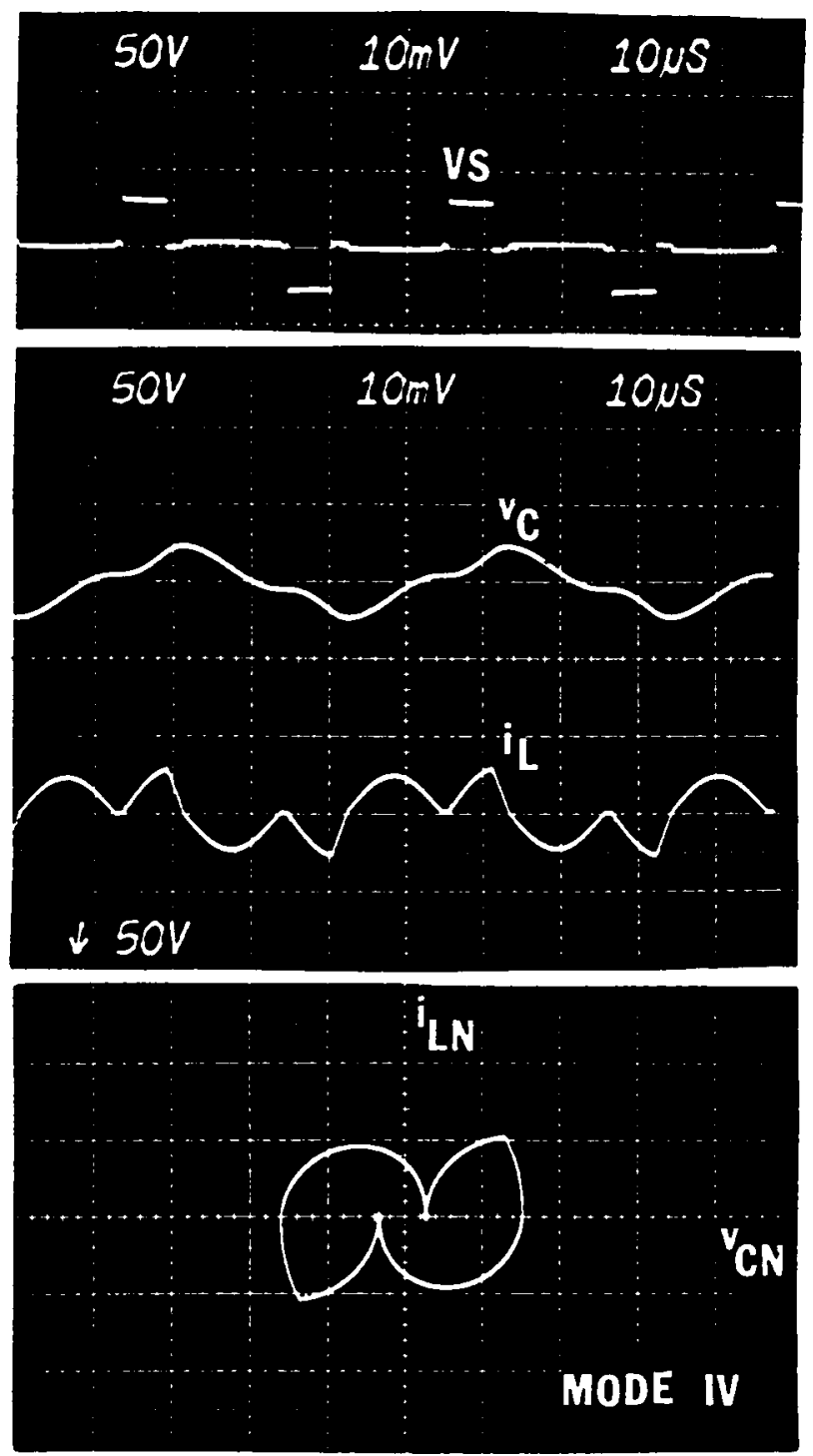

(e) Mode-IV operation (operating point E)

Figure 2.29 Continued 

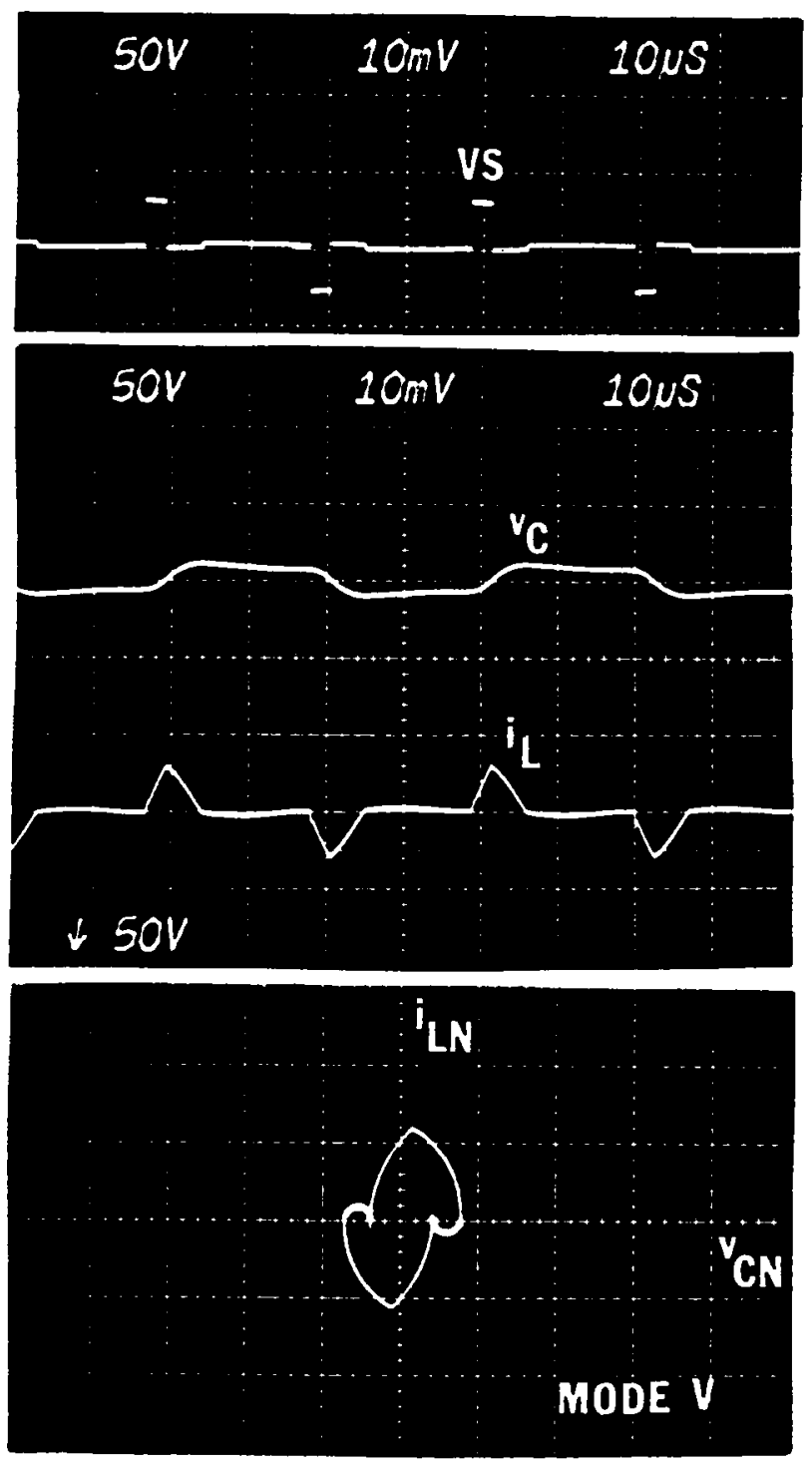

(f) Mode- $V$ operation (operating point F)

Figure 2.29 Continued 

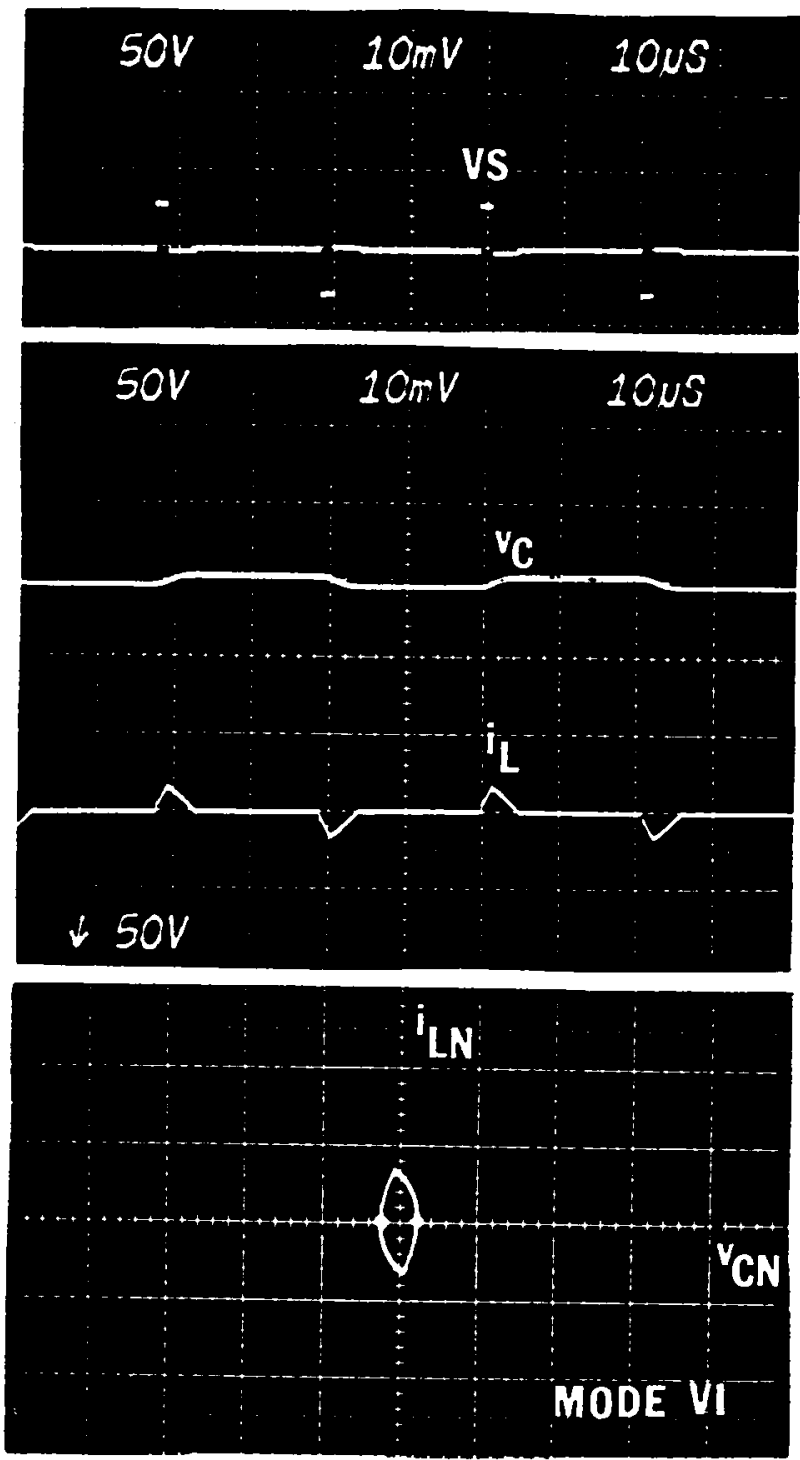

(g) Mode-VI operation (operating point G)

Figure 2.29 Continued 

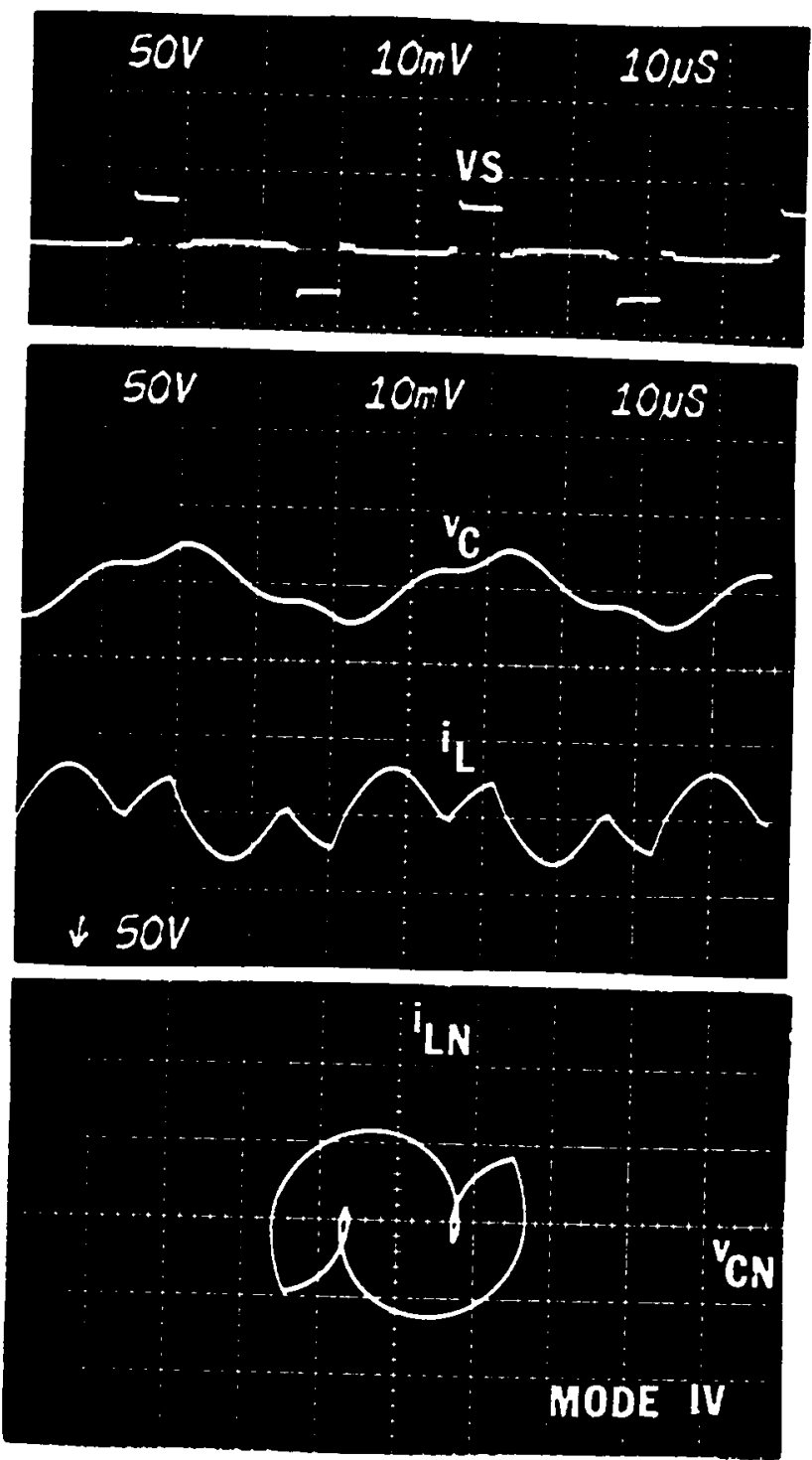

(h) Mode-IV Operation $\left(\beta_{S}=52^{\circ}, V_{O N}=0.1\right)$

Figure 2.29 Continued 


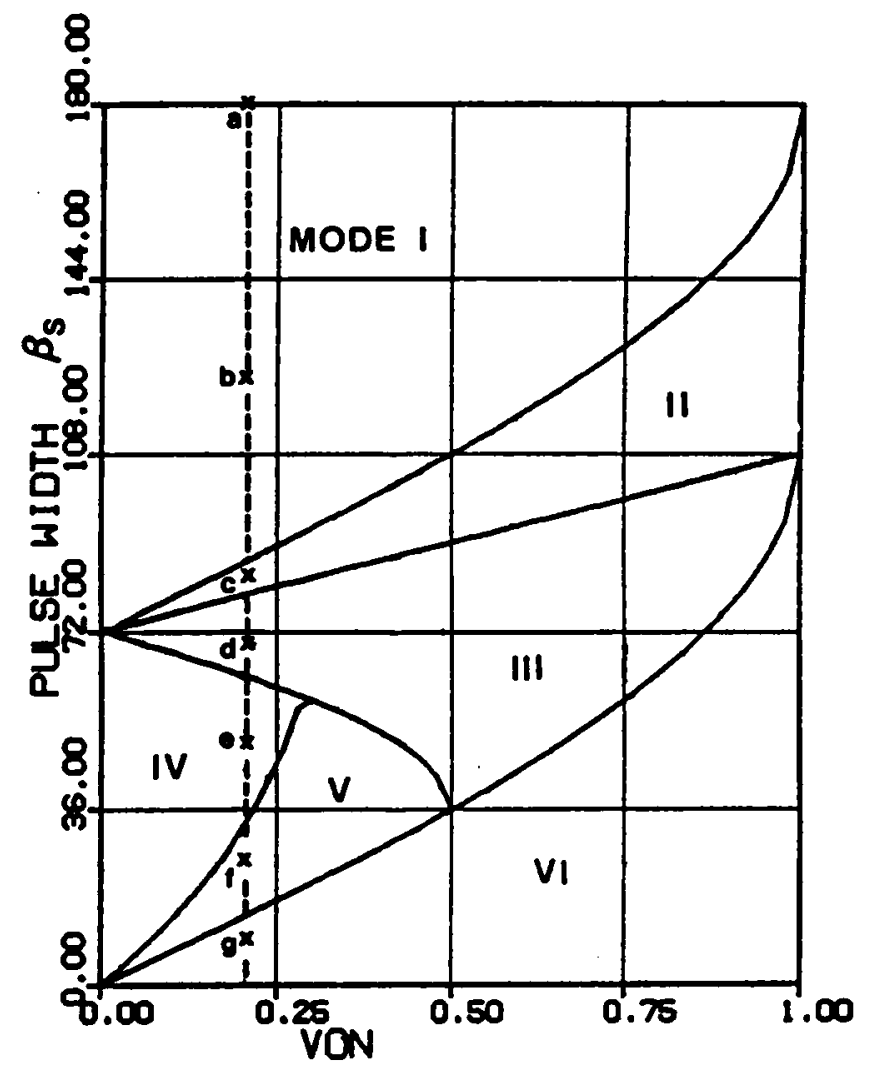

Figure 2.30 Operating Points for the Waveforms in Figure $2.29\left(\omega_{S N}=0.6\right)$ 


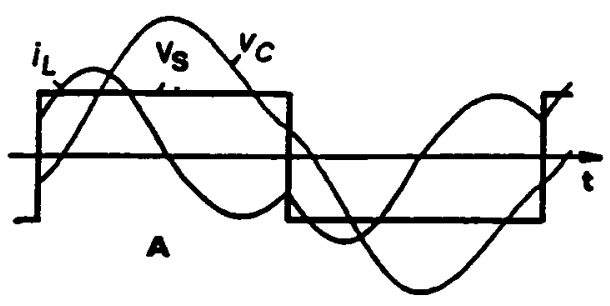

(a) SRC operation (operating point A)

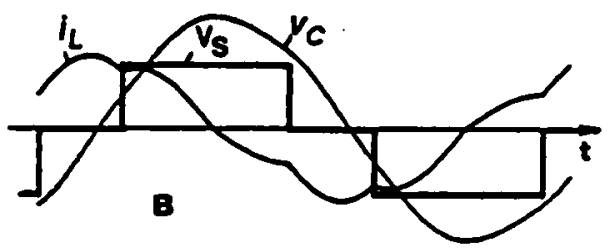

(b) Mode-I operation (operating point B)

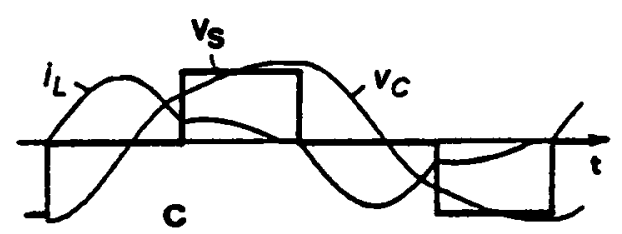

(c) Mode-II operation (operating point C)

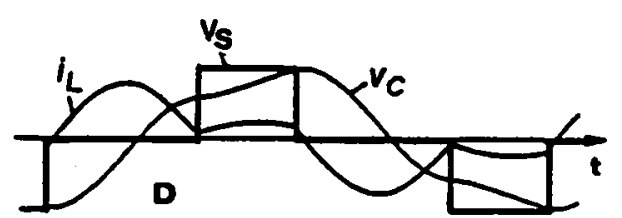

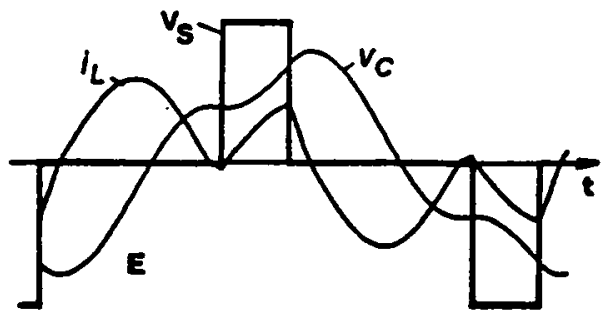

(e) Mode-IV operation (operating point E)

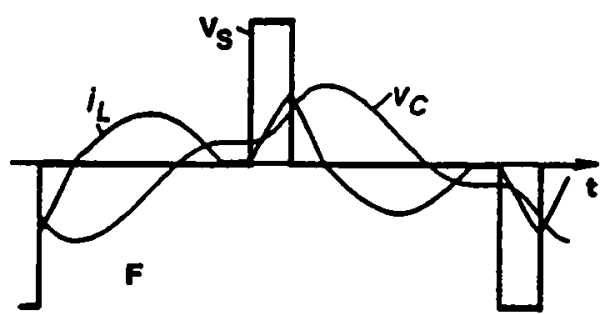

(f) Mode-V operation (operating point F)

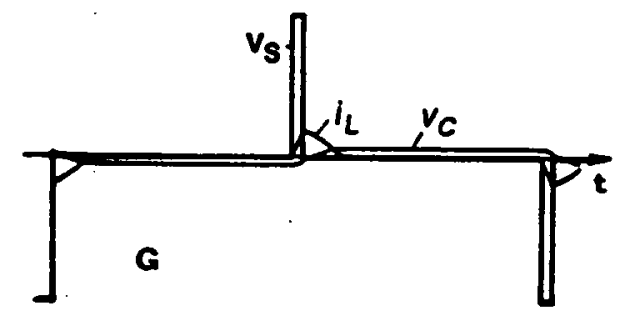

(g) Mode-VI operation (operating point G)

(d) Mode-III operation (operating point D)

Figure 2.31 Analytically Predicted Waveforms at the Operating Points Indicated in Figure 2.30 

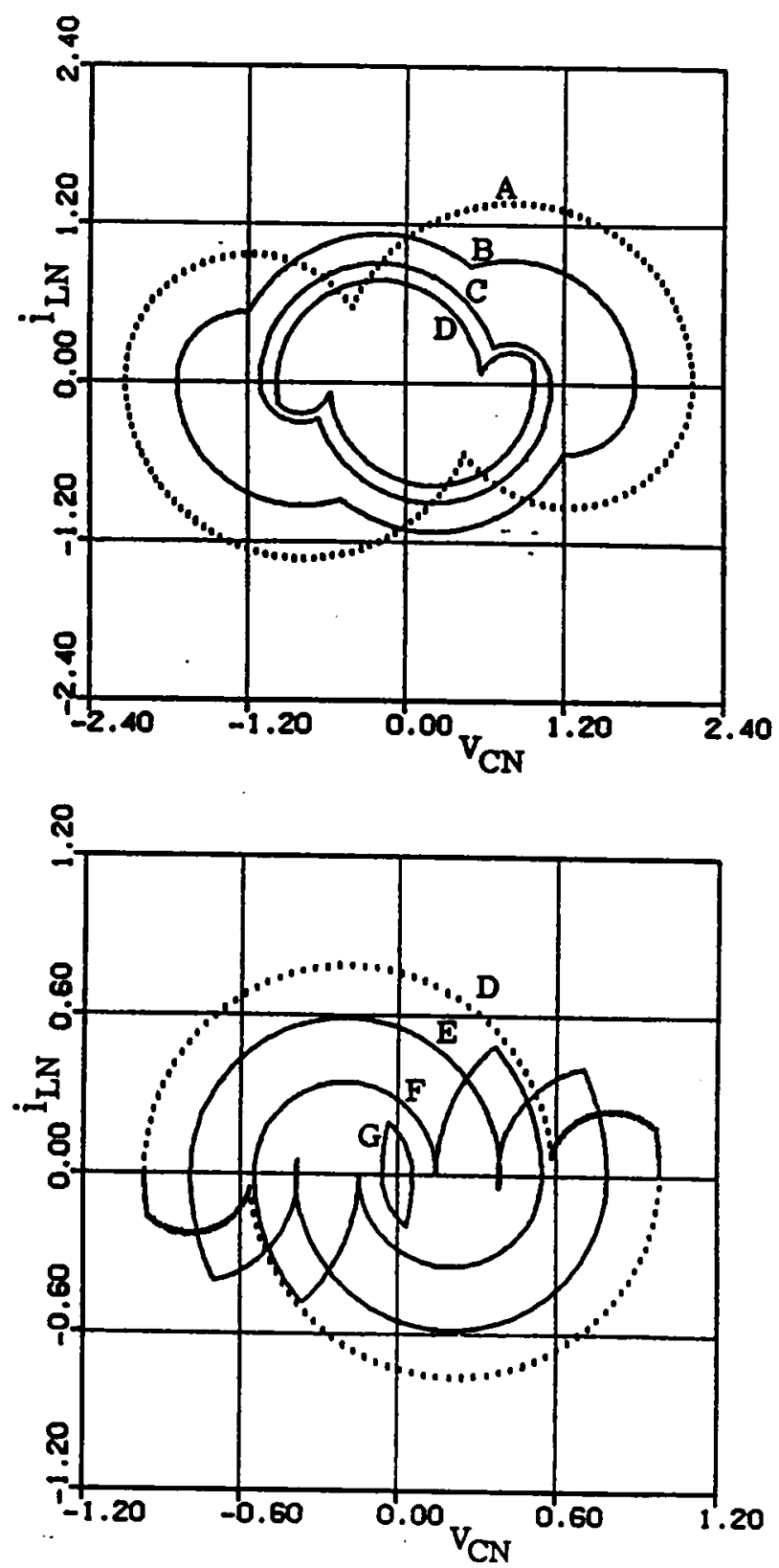

(h) Corresponding state trajectories

Figure 2.31 Continued 


\subsubsection{Circuit Operation Above Resonant Frequency}

Figure 2.32 shows a series of experimental waveforms obtained from the circuit in Figure 2.28. The waveforms are obtained by fixing $V_{O N}$ at 0.2 while reducing angle $\beta_{S}$ from $180^{\circ}$ to $29^{\circ}$. The operating points are indicated by asterisks in Figure 2.33. It can be seen that the converter sweeps through all the three operating modes above resonant frequency.

Another series of waveforms obtained analytically at the operating points $\mathbf{A}, \mathbf{B}, \mathbf{C}, \mathbf{D}$ in Figure 2.33 is shown in Figure 2.34. The waveforms resemble those shown in Figure 2.32 and little difference can be observed. $\Lambda$ gain, the tank energy decreases as $\beta_{S}$ is decreased. Circulating current in the resonant tank is small under light load. The converter is able to provide a load range from no load to a full load. 

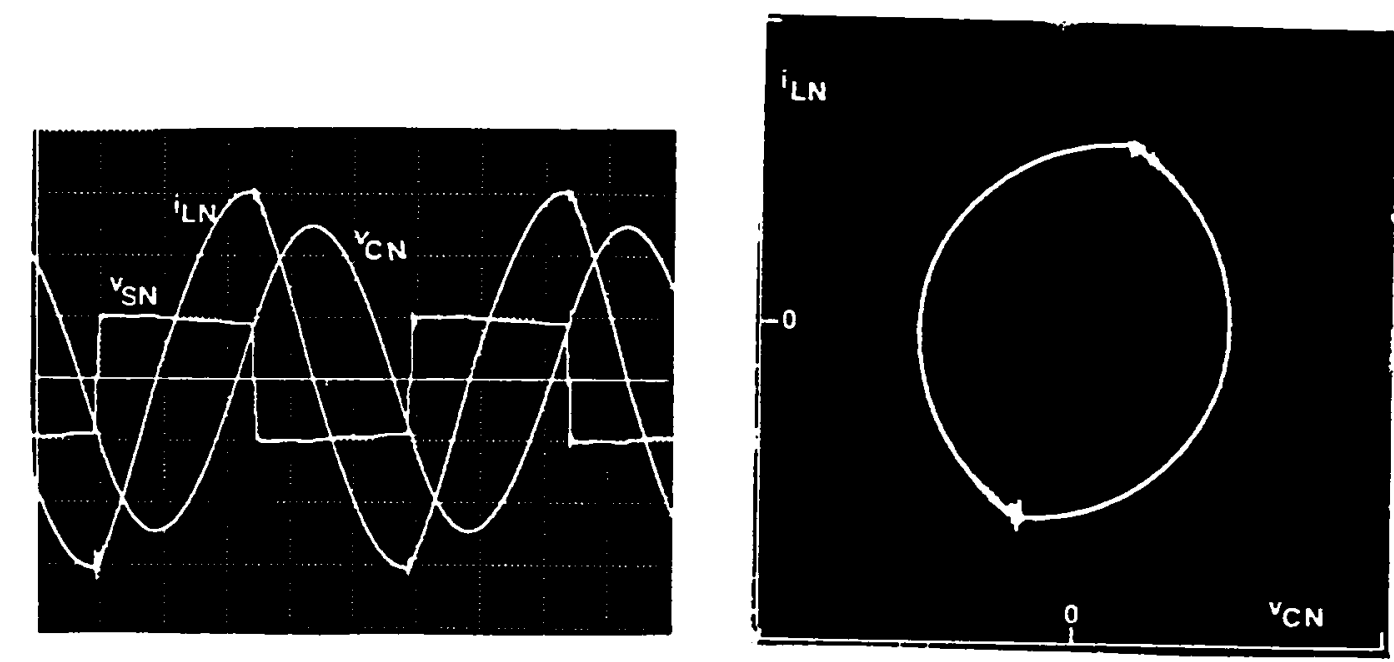

(a) SRC operation (operating point A)
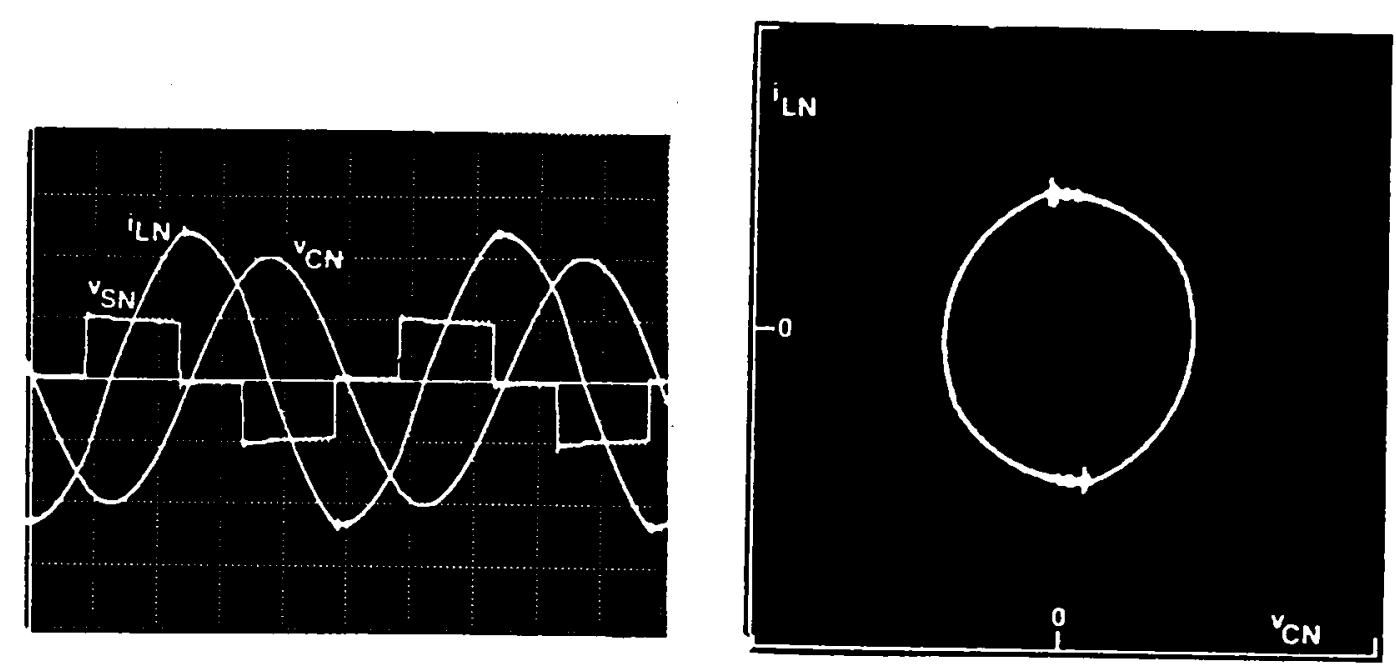

(b) Mode-A operation (operating point B)

Figure 2.32 Experimental Results from the Circuit in Figure 2.28 

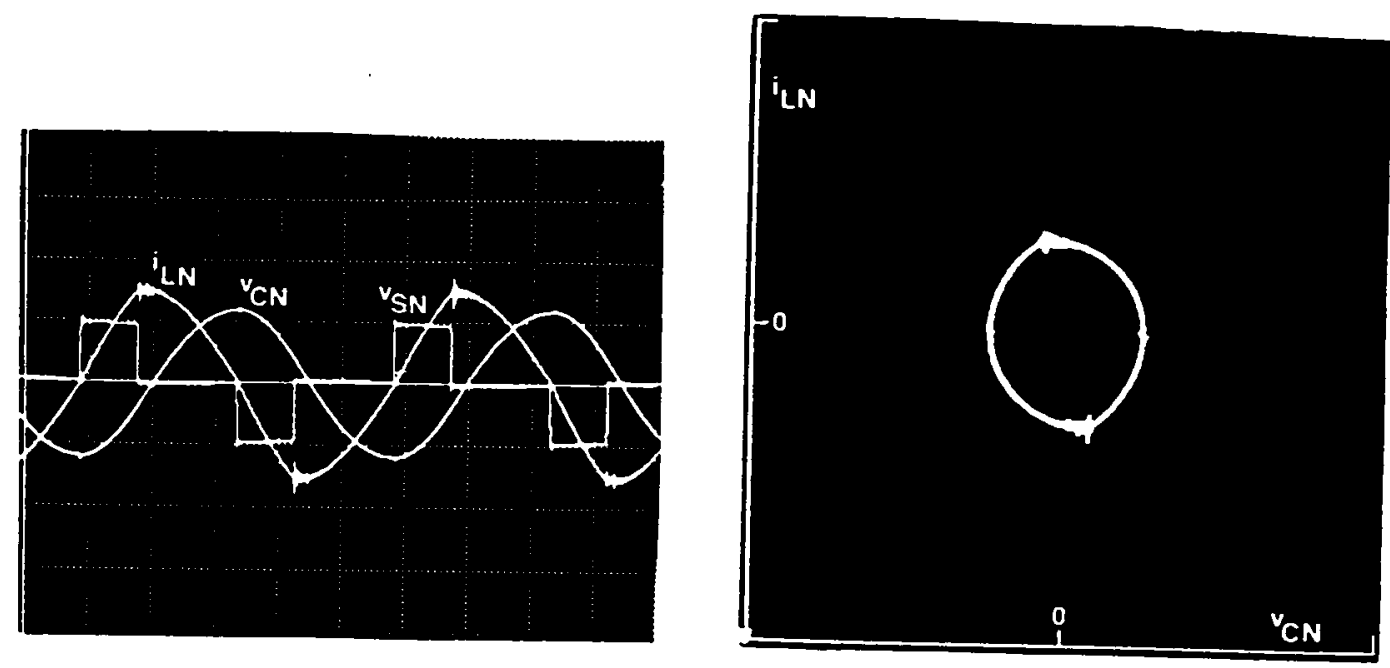

(c) Boundary Between Mode A and Mode B (operating point B)

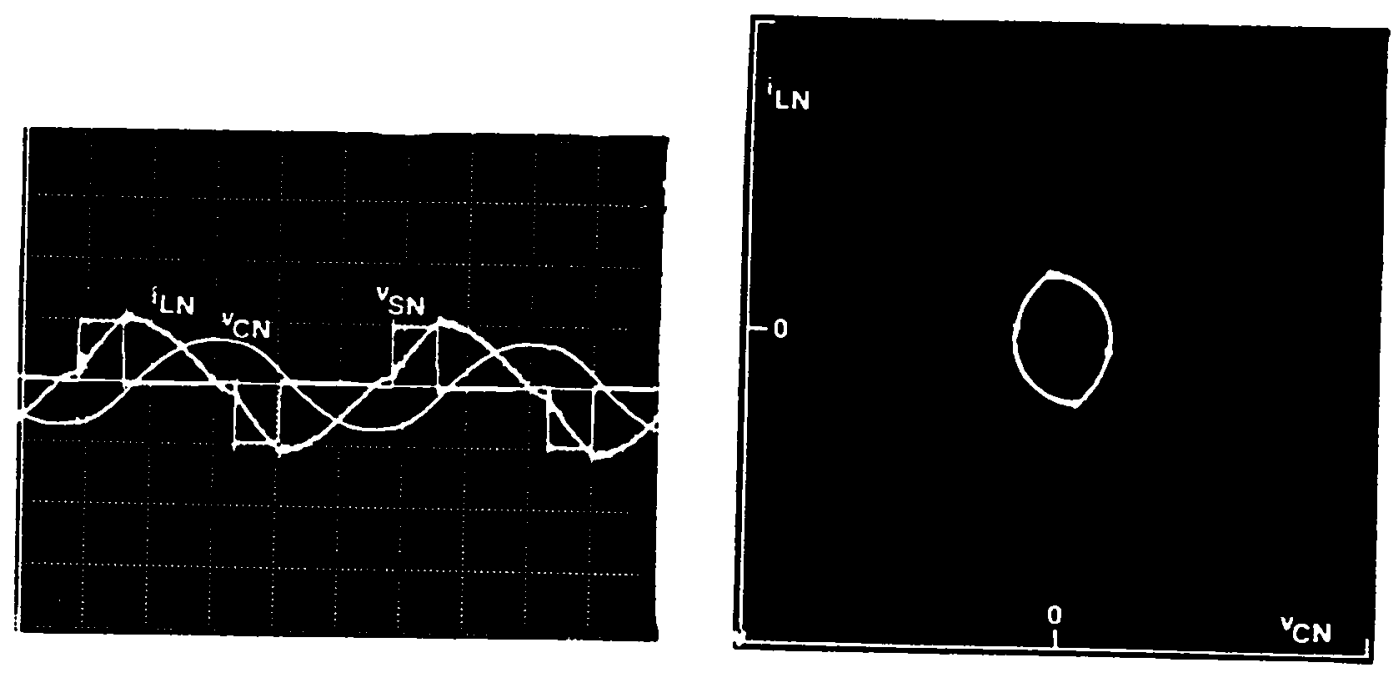

(d) Mode-B operation (operating point C)

Figure 2.32 Continued 

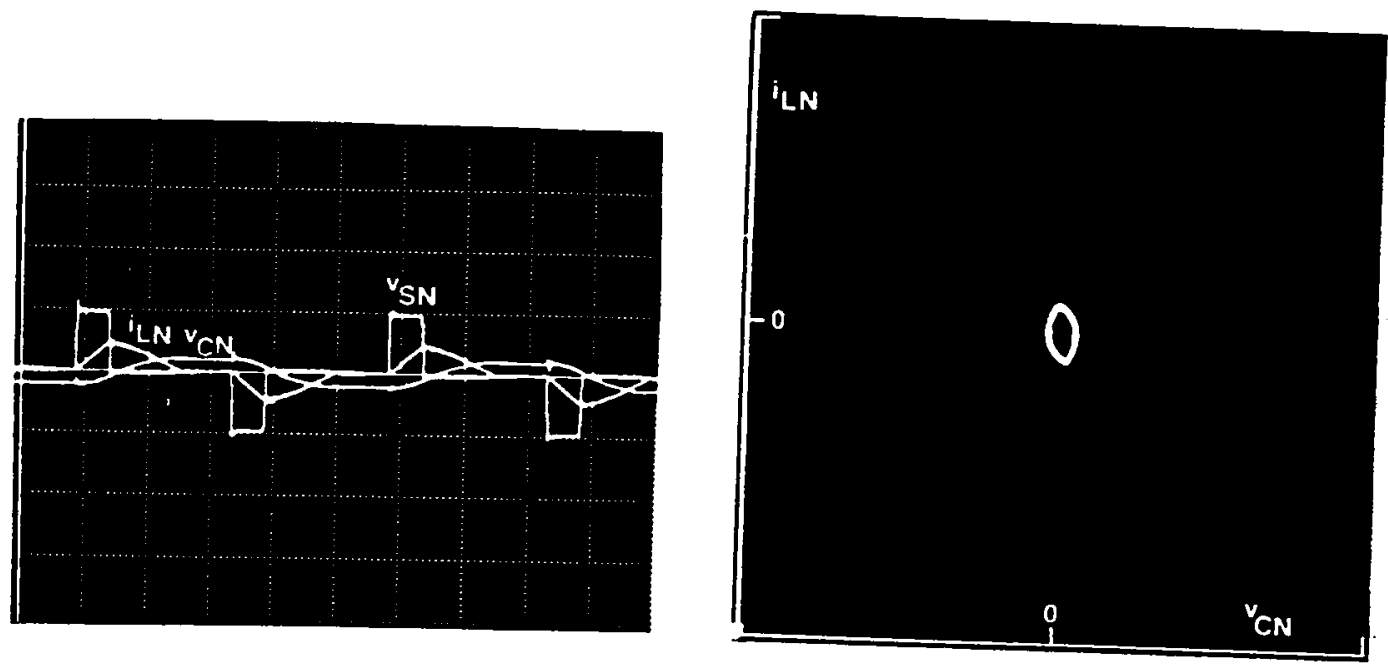

(e) Boundary Between Mode B and Mode C (operating point C')
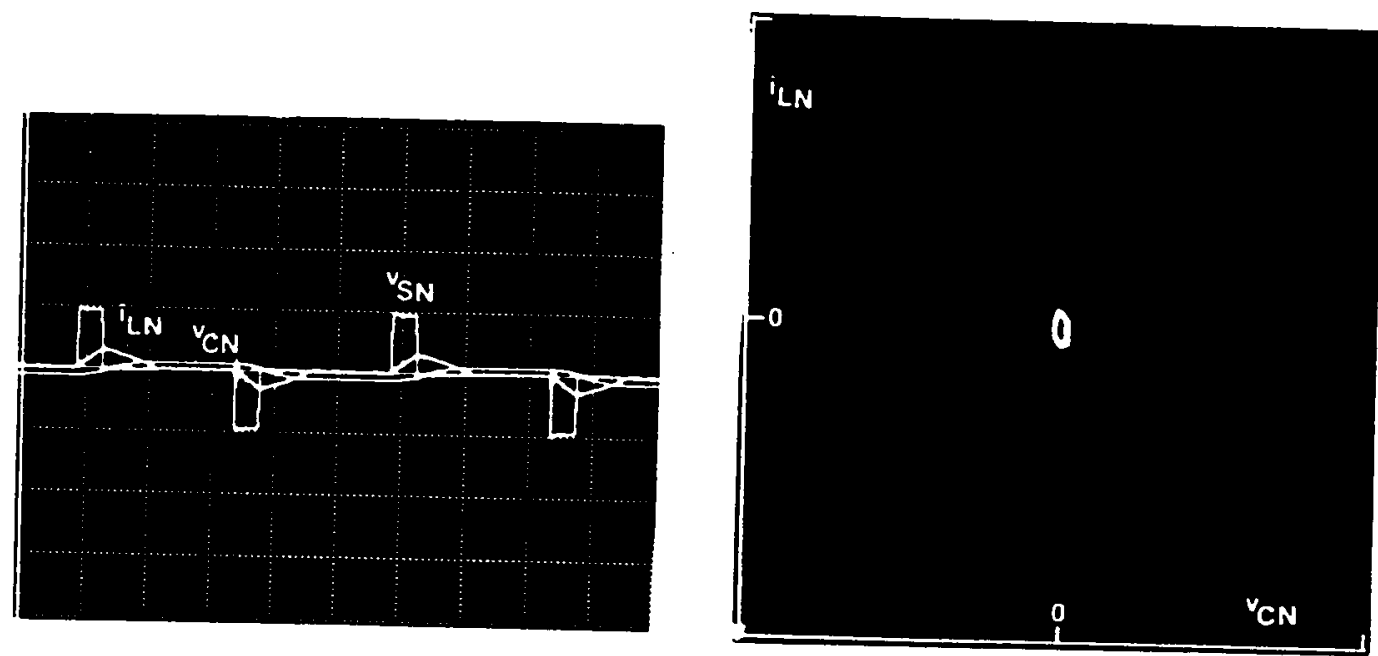

(f) Mode-C operation (operating point D)

Figure 2.32 Continued 


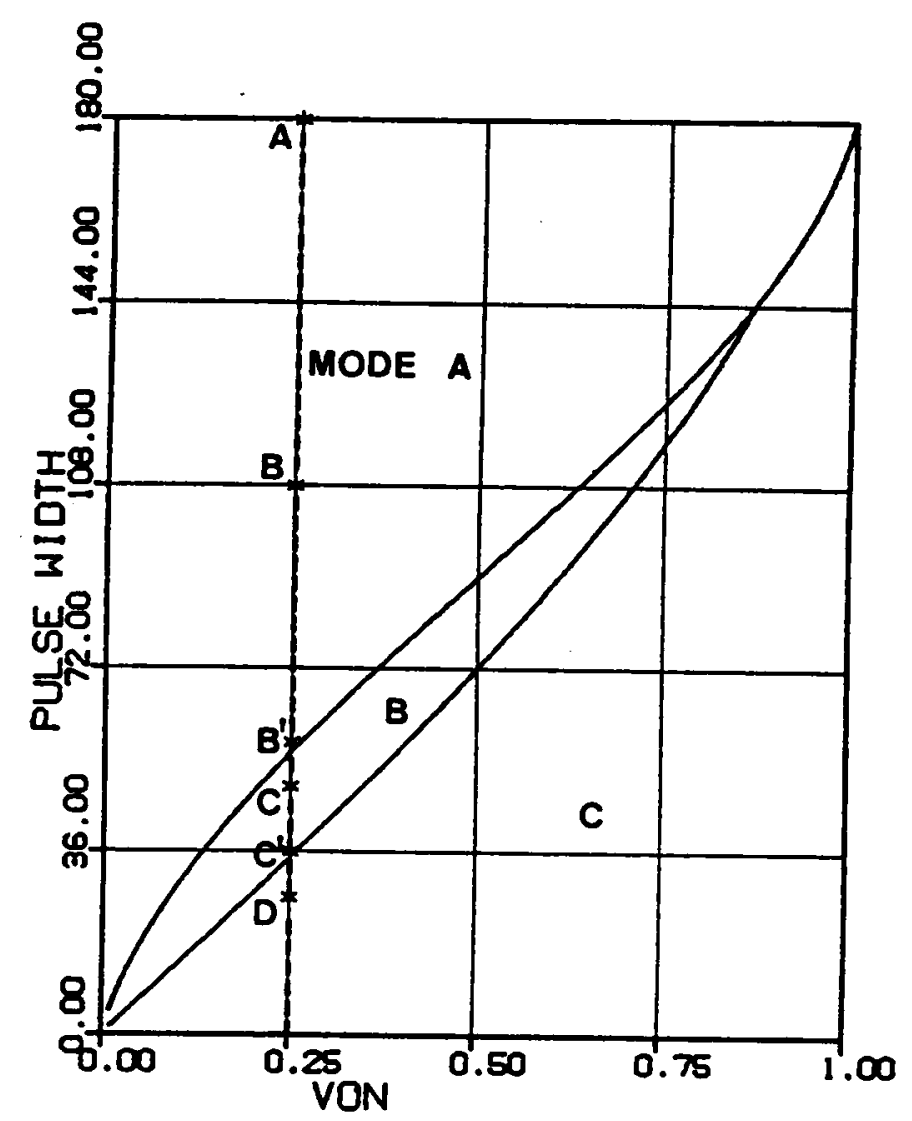

Figure 2.33 Operating Points for the Waveforms in Figure $2.31\left(\omega_{S N}=1.2\right)$ 


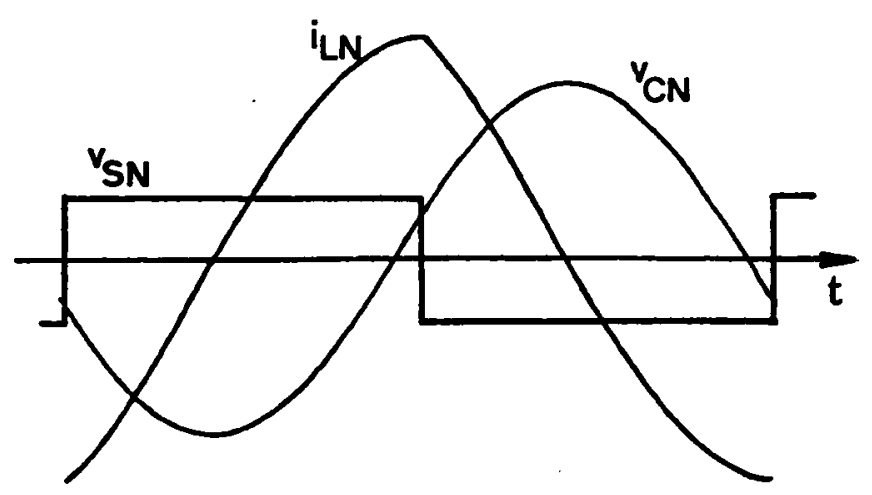

(a) SRC operation (operating point A)

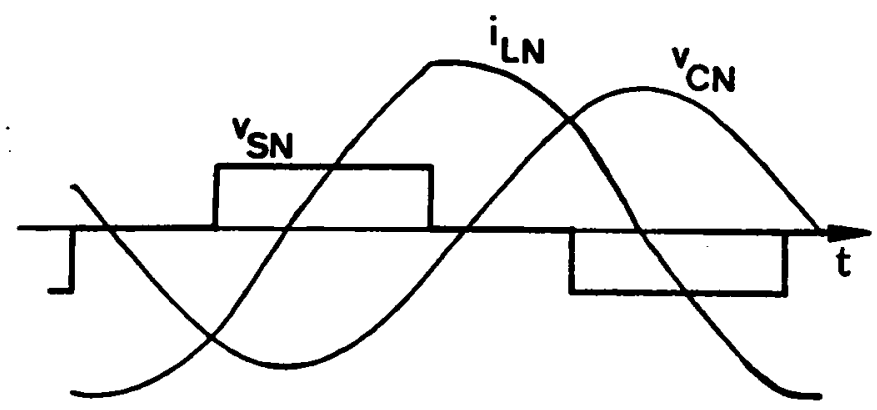

(b) Mode-A operation (operating point B)

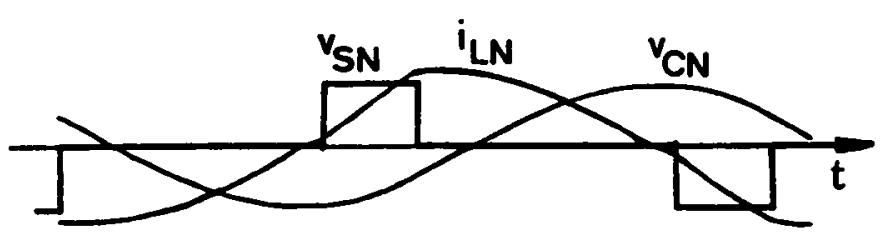

(c) Mode-B operation (operating point C)

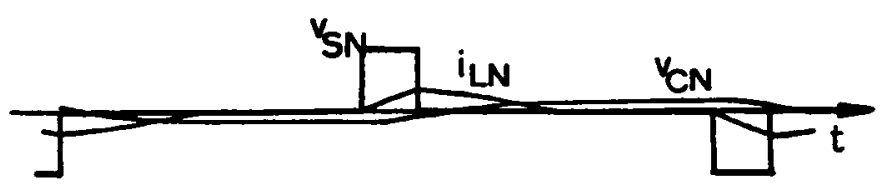

(d) Mode-C operation (operating point D)

Figure 2.34 Analytically Predicted Waveforms at Operating Points A,B,C,D in Figure 2.33 


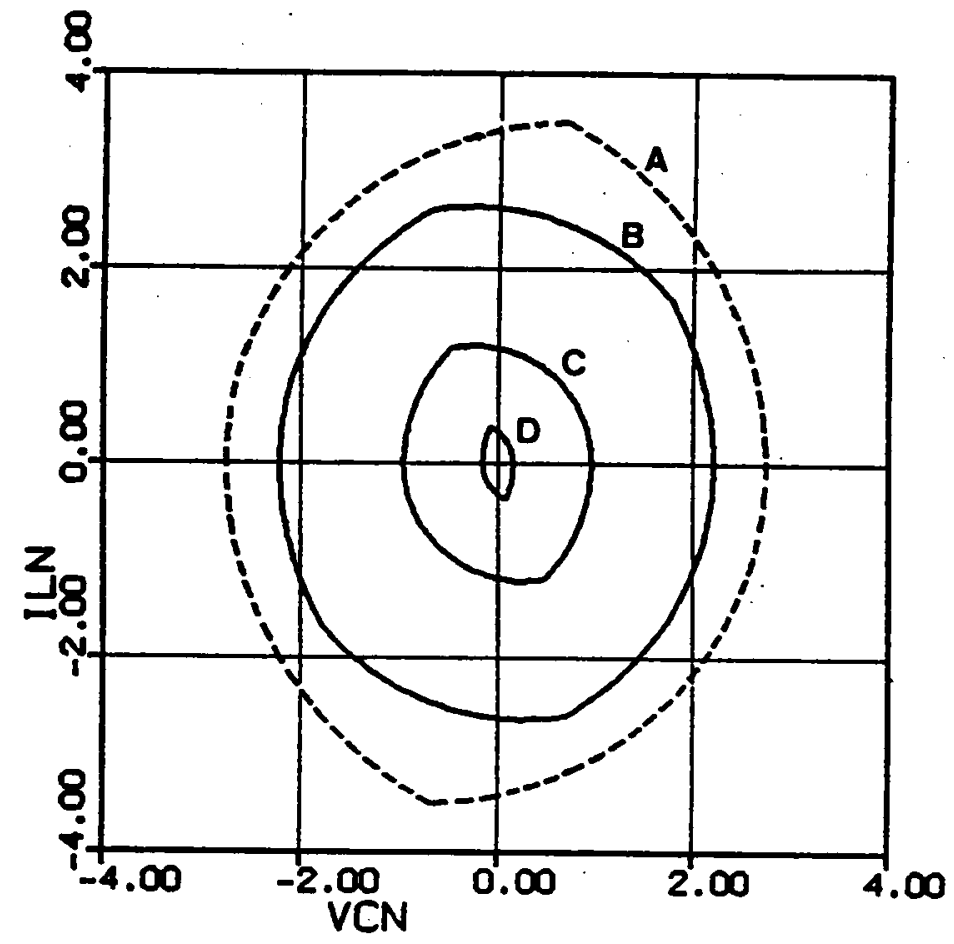

(h) Corresponding state trajectories

Figure 2.34 Continued 


\subsection{CONCLUSIONS}

A comprehensive analysis of the constant-frequency, clamped-mode, series-resonant converter has been performed in this chapter. Employing state-plane techniques, six operating modes below resonant frequency and three operating modes above resonant frequency are identified for the first time. These operating modes represent different device conduction sequences, resulting in different device commutation requirements.

To predict the converter's operating mode at a given condition, an algorithm is developed to define the regions of operation of the converter. The regions of operation can be used to determine the operating modes of the converter for a specific design.

There are mainly three different commutation regions for the converter. Each region has its advantages. In the natural commutation region, the turn-off losses of all the transistors are eliminated. In the mixed-commutation region, the turn-on losses of transistors Q1,Q3 and the turn-off losses of transistors Q2,Q4 are eliminated. Lossless capacitor snubbers can be used across Q1 and Q3. In the force-commutation region, the turn-on losses of all the transistors are eliminated. Lossless snubbers can be used across all the transistors.

State trajectories are conveniently used to calculate various circuit salient features. The parameters defining the trajectorics are solved using sets of nonlinear equations. Expressions for the output current, rms inductor current, peak capacitor voltage, switch currents and diode currents are then derived from these parameters.

Various dc characteristics have been derived. The characteristics are then employed to facilitate the converter design. Three design examples are given. The examples illustrate the procedures to design a CM-SRC operating in different commutation regions.

Finally, two breadboard circuits are used to verify the converter's operation. The presences of all the circuit operating modes, both below and above the resonant fre- 
quency, are verified. The experimental results are in good agreement with the analytical predictions. 


\section{CHAPTER 3.}

\section{ANALYSIS OF CLAMPED-MODE PARALLEL RESONANT CONVERTER}

\subsection{INTRODUCTION}

As discussed in Chapter 2, a clamped-mode series-resonant converter (CM-SRC) can be designed in three different regions to ohtain ccrtain desirable commutation features of the power switches. When a CM-SRC is designed to operate in the naturalcommutation region (below resonant frequency) or in the force-commutation region (above resonant frequency), a minimum load current has to be maintained. To achieve output regulation from no load to full load, the converter must be designed in the mixed commutation region where two of the power switches are naturally commutated while the other two are force-commutated.

Similar to a CM-SRC, a clamped-mode parallel-resonant converter (CM-PRC) can also be designed in three different regions to obtain cither natural commutation or mixed commutation or force commutation of its power switches. However, unlike the CM-SRC, it is possible to design a CM-PRC to regulate its output from no load to full load entirely in the natural or the force commutation regions. $\Lambda$ minimum load current 
has to be maintained if a CM-PRC is designed to operate in the mixed commutation region.

In this chapter, a complete $\mathrm{dc}$ characterization of the CM-PRC is presented. The analysis provides insights to the converter's operation and derives guidelines for the converter design. $\Lambda$ gain, graphical state-plane techniques are employed to identify all the possible circuit operating modes. The topological sequence and the device commutation requirements under each operating mode are detailed. The mode boundaries are determined and the regions for different commutations are defined. The dc control-tooutput characteristics are derived. Finally, three design examples are illustrated and the predicted operating modes are verified experimentally. 


\subsection{CIRCUIT OPERATION}

Figure 3.1(a) shows a clamped-mode, parallel-resonant converter with the controlled switches $\mathbf{S 1 , S 2 , S 3 , S 4 ~ i n ~ F i g u r e ~ \Lambda . 1 ( d ) ~ i m p l e m e n t e d ~ b y ~ t r a n s i s t o r s ~ Q 1 , Q 2 , Q 3 , Q 4 ~ a n d ~}$ their antiparallel diodes D1,D2,D3,D4, respectively. The transistors are triggered in a timing sequence as illustrated in Figure 3.1(b). All the transistors are driven with $50 \%$ duty-cycle gating signals. Transistors Q1,Q3 are triggered according to a clock signal whose frequency determines the operating frequency of the converter. Transistors $\mathrm{Q} 2, \mathrm{Q} 4$ are triggered with a controllable time delay, $\frac{\phi_{S}}{\omega_{S}}$, with respect to the triggering of Q1,Q3, respectively. The time delay is the interval during which the resonant tank is clamped as a short circuit. By controlling the time delay, the pulse-width, $\beta_{S}$, of the quasi-square-wave voltage, $v_{s}$, is controlled and the converter's output voltage, $V_{O}$, is regulated.

Depending upon the operating conditions, a CM-PRC may result in different modes of operation. Each mode of operation represents a unique conduction sequence of the switching devices.

A typical circuit operation of a CM-PRC: operating below resonant frequency is illustrated in Figure 3.2. $\Lambda \mathrm{t} t=0$, transistor $\mathrm{Q} 1$ turns on while diodes D3,D4 are conducting. Diode D3 is commutated due to the conduction of $\mathrm{Q} 1$ and the inductor current, $i_{l}$, resonates through $\mathrm{Q} 1$ and D4. $\Lambda \mathrm{t} t=\mathrm{tl}$, transistor $\mathrm{Q} 2$ is triggered and diode $\mathrm{D} 4$ is commutated. The inductor current resonates through $Q 1$ and $Q 2$. $\Lambda t t=t 2$, inductor current $i_{l}$, decreases to 7ero due to resonance. Transistors Q1.Q2 are naturally commutated and diodes D1,D2 conduct, subsequently. The inductor current resonates through $\mathrm{D} 1$ and D2. $\Lambda \mathrm{t}$ the end of the first half switching cycle, $\mathrm{t}=\frac{T_{S}}{2}$, transistor Q3 turns on, commutating diode D1. $\wedge$ similar process takes place in the second half switching cycle with the roles of Q1,Q2,D1,I)2 and Q3,Q4,D3,D4 interchanged, respectively. The renected load current, $i_{R}$, changes polarity whenever capacitor voltage $v_{C}$ crosses the 


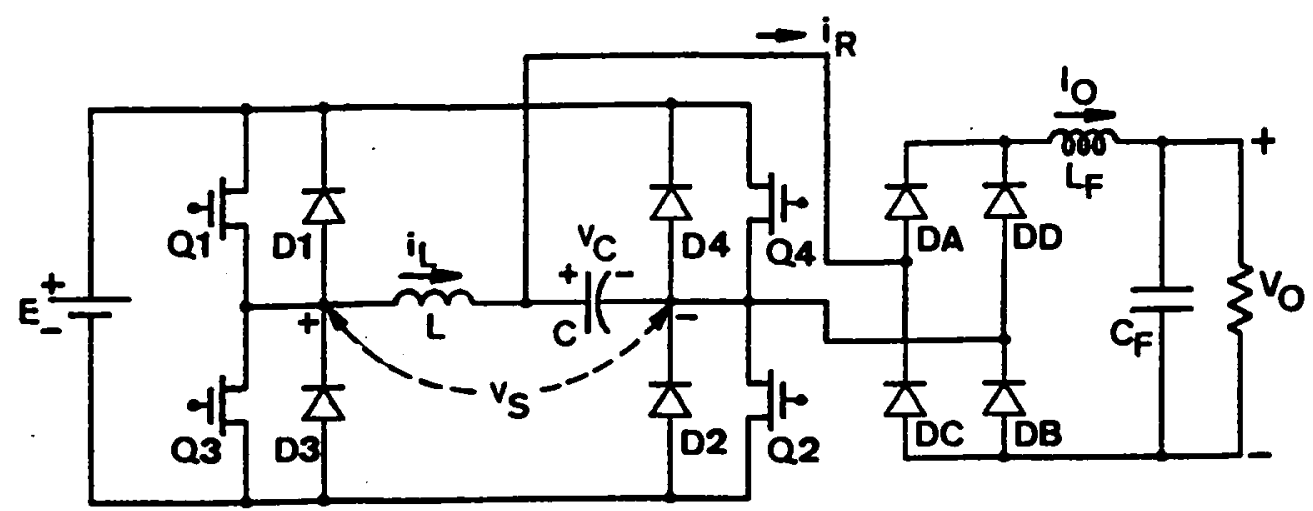

(a) Circuit diagram

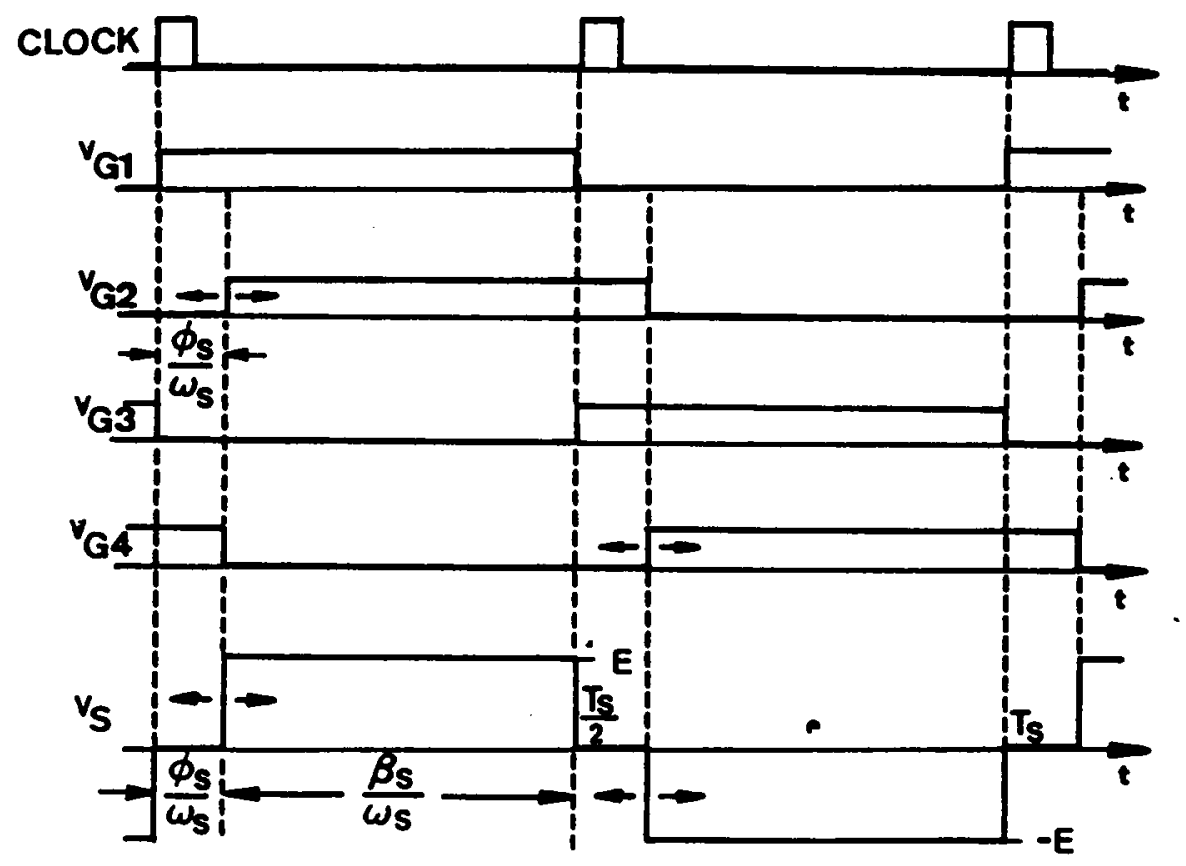

(b) Gating Signals

Figure 3.1 A Clamped-Mode Parallel-Resonant Converter 


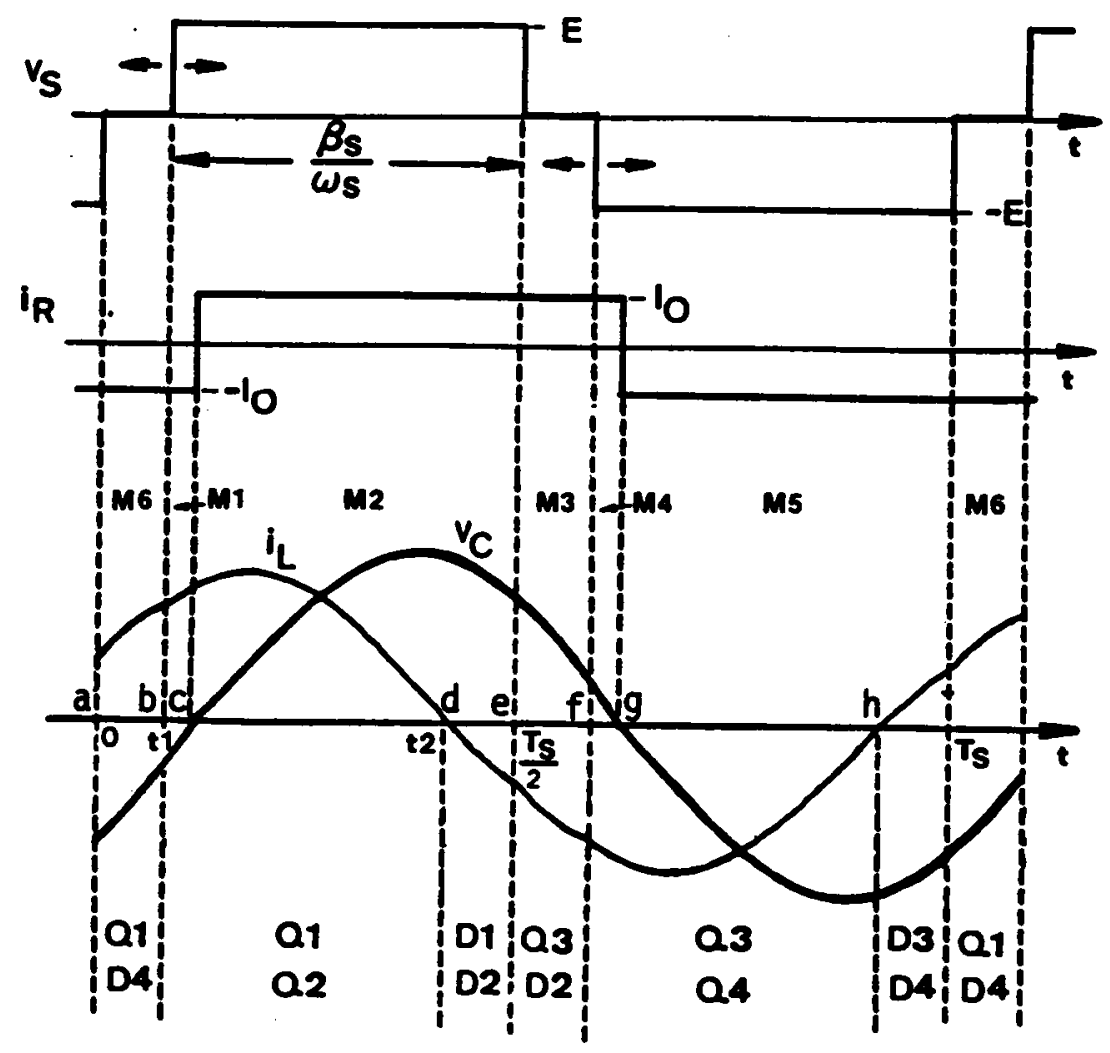

Figure 3.2 A Typical Circuit Operation Below Resonant Frequency 
zero-axis. In other words, the output bridge diodes switch from DA,DB to DC,DD or vice versa.

A typical circuit operation $n \Upsilon$ a CM-PRC operating above resonant frequency is illustrated in Figure 3.3. At $t=0$, transistor $Q 3$ is forced off and $Q 1$ is triggered with a gate voltage. Since the inductor current $i_{L}$ is negative, $Q 1$ can not conduct. Instead, diode D1 conducts. The inductor current resonates through $Q 4$ and D1. $\Lambda t t=t 1$, transistor Q4 is forced off. and Q2 is triggered. Transistor Q2 can not conduct since $i_{L}$ is still negative. Instead. diode D2 conducts. The inductor current resonates through D1 and D2. At $t=t 2$, inductor current $i_{t}$ increases to 7ero. Diodes D1,D2 turn off naturally and transistors Q1,Q2 conduct, subsequently. The inductor current resonates through Q1 and $\mathrm{Q}$. At the end of the first half switching cycle, $\mathrm{t}=\frac{T_{S}}{2}$, transistor $\mathrm{Q} 1$ is forced off and Q3 is triggered. A similar process occurs with the roles of Q1,Q2,D1,D2 and Q3,Q4,D3,D4 interchanged, respectively. The reflected load current, $i_{R}$, changes polarity whenever capacitor voltage $v_{r}$ crosses the zero-axis.

In the examples discussed above, the commutation features of the transistors, $\mathrm{Q} 1, \mathrm{Q} 2, \mathrm{Q} 3, \mathrm{Q} 4$, and the diodes, D1,D2,D3,D4, are the same as those in a conventional PRC. The commutation features for these devices, however, may change when the converter's operating conditions (such as frequency, load current, or pulse width of $v_{s}$ ) vary, as shall be seen in later sections. 


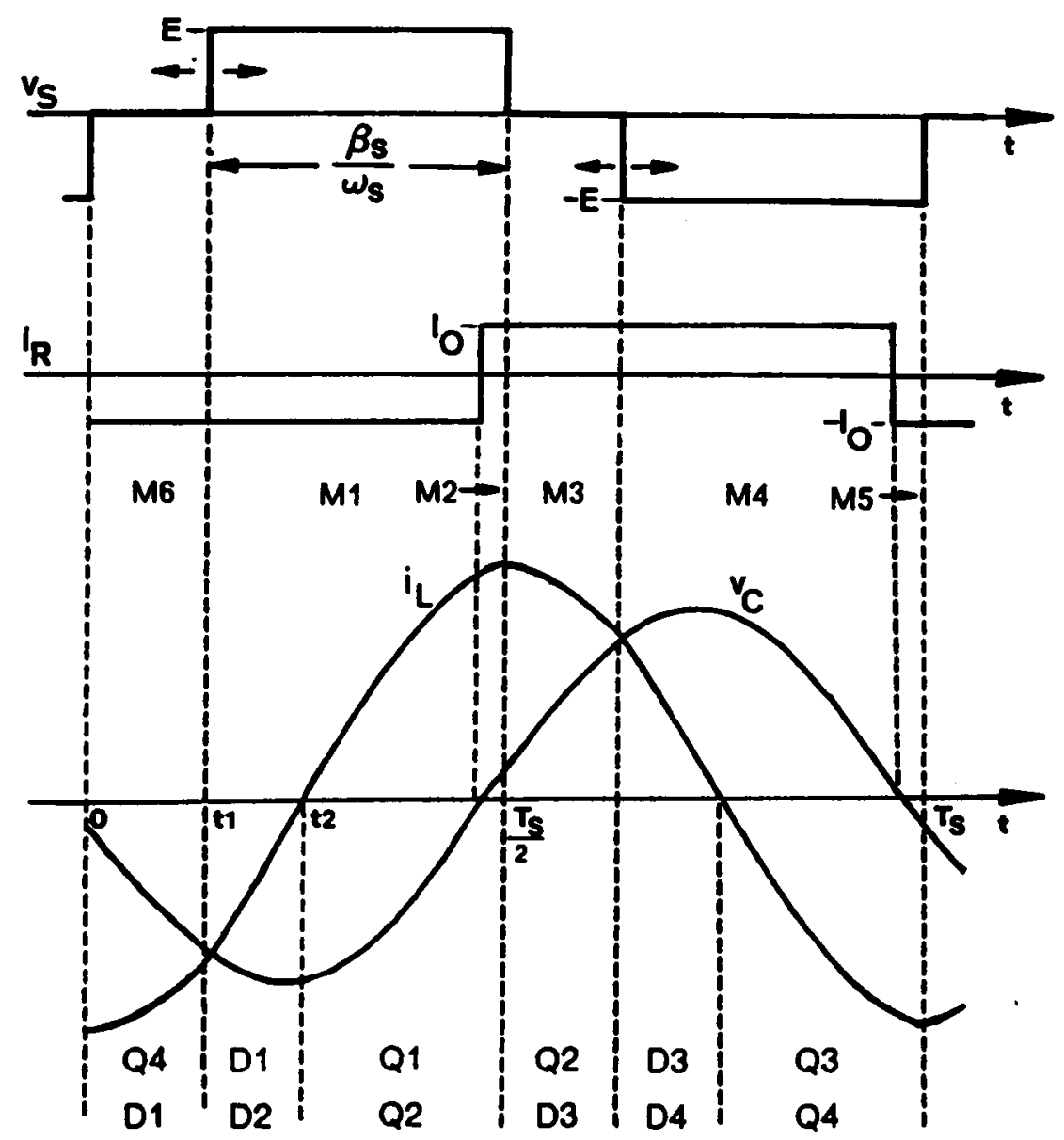

Figure 3.3 A Typical Circuit Operation Above Resonant Frequency 


\subsection{STATE-PLANE ANALYSIS}

In this section, graphical state-plane techniques are employed to analyze the CM-PRC. Various circuit operating modes are identified and regions of operation are defined. Important dc characteristics such as control-to-output transfer ratio, rms inductor current, peak capacitor voltage, rms switch currents, etc. are also derived.

\subsubsection{Assumptions}

The following assumptions are made during the analysis:

1. all the transistors are ideal, with zero switching time and no conduction drop;

2. the quality factor of the resonant tank is infinite; in other words, there is no loss in the tank circuit;

3. the output filter is large enough such that the output current, $I_{O}$, can be assumed constant during several switching cycles;

4. the transistors are driven with ideal $50 \%$-duty-cycle gating signals;

5. the switching frequency of the converter is greater than $50 \%$ of the resonant frequency.

\subsubsection{Circuit Topological Modes}

As illustrated in Figures 3.2 and 3.3, the one-cycle operation of a CM-PRC is composed of a sequence of linear circuits, cach corresponding to a particular switching in- 
terval. There are eight linear circuit topologies for a CM-PRC, as shown in Figure 3.4. These circuit topologies are referred to as circuit topological modes of a CM-PRC. Modes M1,M2,M3,M4,M5, and M6 are called "resonant modes". LC resonant actions occur in these modes. Mode ML is called "linear-charging mode". The resonant inductor is linearly charged or discharged by the source voltage in this mode. Mode MO is called "free-wheeling mode". The current in the resonant inductor freewheels through a transistor-diode pair of the input bridge in this mode. The circuit behavior of a CM-PRC under each topological mode can be described using the following equations.

For resonant modes M1,M2,M3,M4,M5, and M6,

$$
\begin{aligned}
& L \frac{d i_{L}}{d t}+v_{C}=v_{E}, \\
& C \frac{d v_{C}}{d t}-i_{L}=i_{E},
\end{aligned}
$$

where,

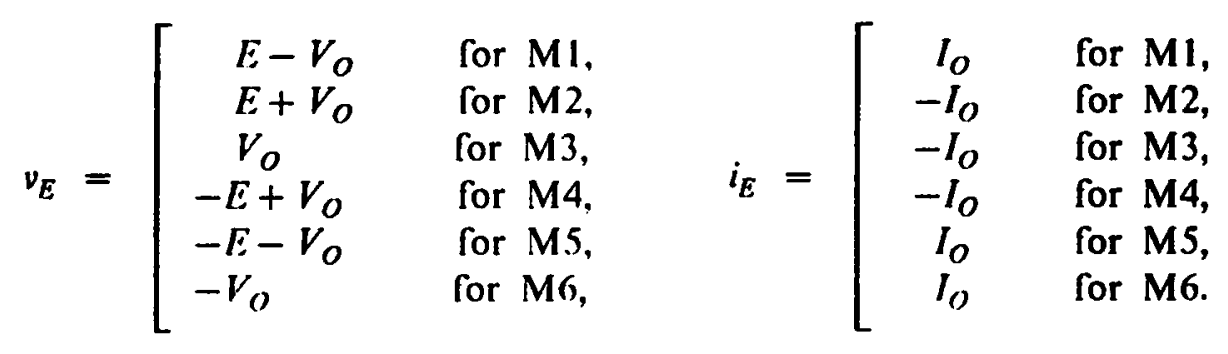

For linear-charging mode $\mathrm{Ml}$,

$$
\begin{aligned}
& \text { I. } \frac{d i_{L}}{d t}= \pm E \\
& { }_{C}=0 .
\end{aligned}
$$

For free-wheeling mode MO, 


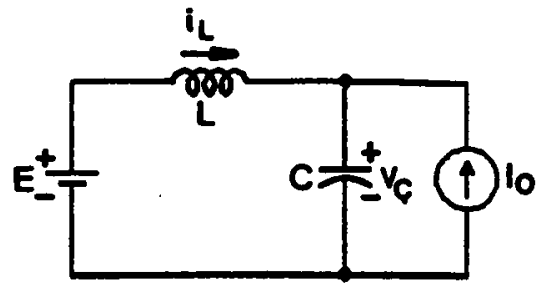

$$
\begin{array}{ll} 
& \left(i_{L N}+I_{O N}\right)^{2}+\left(V_{C N}-1\right)^{2}=R^{2} \\
\text { - } & R^{2}=\left(1-v_{C O N}\right)^{2}+\left(I_{L O N}+I_{O N}\right)^{2}
\end{array}
$$

(a) M1 : $v_{c}<0, Q 1, Q 2$ or $\mathrm{D} 1, \mathrm{D} 2$ on

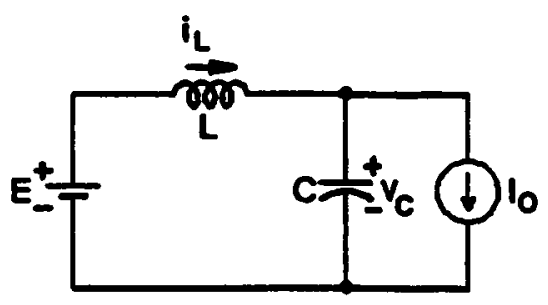

$$
\begin{aligned}
& \left(I_{L N}-I_{O N}\right)^{2}+\left(V_{C N}-1\right)^{2}=R^{2} \\
& \text { - } R^{2}=\left(1-V_{C O N}\right)^{2}+\left(I_{L O N}-I_{O N}\right)^{2}
\end{aligned}
$$

(b) $M 2: v_{c}>0, Q 1, Q 2$ or $D 1, D 2$ on

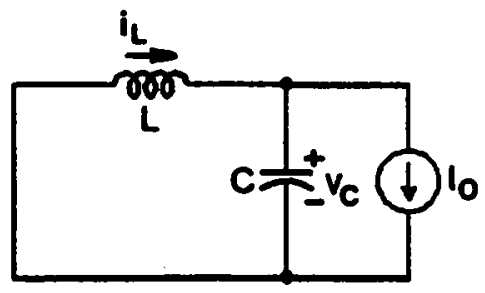

$$
\begin{aligned}
& \text { - }\left(i_{L N}-I_{O N}\right)^{2}+v_{C N}^{2}=R^{2} \\
& \text { - } R^{2}=V_{C O N}^{2}+\left(I_{L O N}-I_{O N}\right)^{2}
\end{aligned}
$$

(c) M3 : $v_{c}>0, \mathrm{Q1,D4}$ or Q4,D1 or Q2,D3 or Q3,D2 on

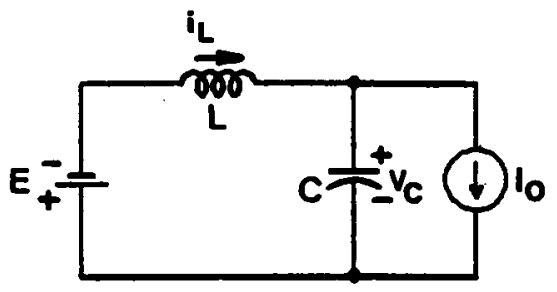

$$
\begin{aligned}
& \text { - }\left(i_{L N}-I_{O N}\right)^{2}+\left(v_{C N}+1\right)^{2}=R^{2} \\
& \text { - } R^{2}=\left(1+v_{C O N}\right)^{2}+\left(I_{L O N}-I_{O N}\right)^{2}
\end{aligned}
$$

(d) M4: $v_{C}>0, Q 3, Q 4$ or D3,D4 on

Figure 3.4 Circuit Topological Modes of a CM-PRC 


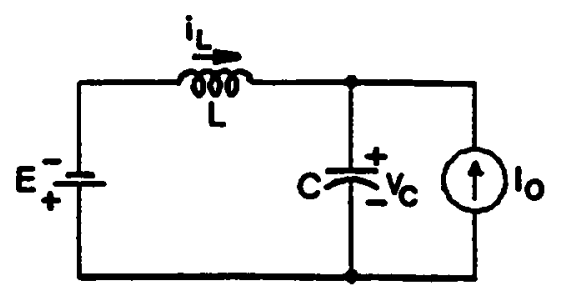

$\begin{array}{ll} & \left(i_{L N}+I_{O N}\right)^{2}+\left(v_{C N}+1\right)^{2}=R^{2} \\ - & R^{2}=\left(1+v_{C O N}\right)^{2}+\left(I_{L O N}+I_{O N}\right)^{2}\end{array}$

(e) M5 : $v_{c}<0, Q 3, Q 4$ or D3,D4 on

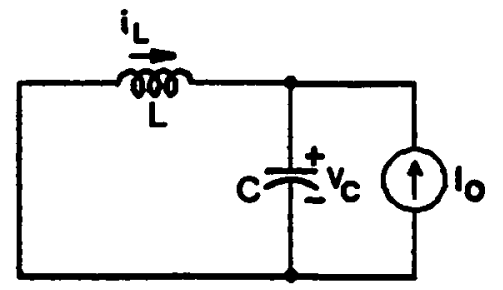

$$
\begin{aligned}
& \text { - }\left(I_{L N}+I_{O N}\right)^{2}+v_{C N}^{2}=R^{2} \\
& \text { - } R^{2}=V_{C O N}^{2}+\left(I_{L O N}+I_{O N}\right)^{2}
\end{aligned}
$$

(f) M6 : $v_{C}<0, Q 1, D 4$ or Q4,D1 or Q2,D3 or Q3,D2 on
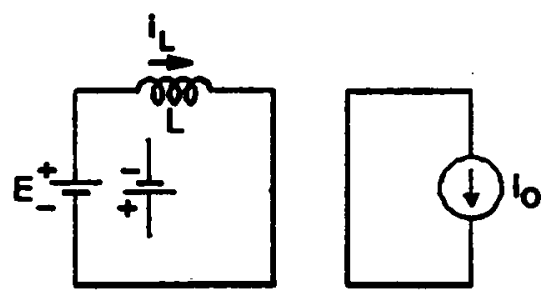

- $i_{L N}=I_{L O N} \pm \omega_{0} t, \quad v_{C N}=0$

(Linear-Charging Mode)

(g) $M L: v_{c}=0, Q 1, Q 2$ or D1,D2 or Q3,Q4 or D3,D4 on
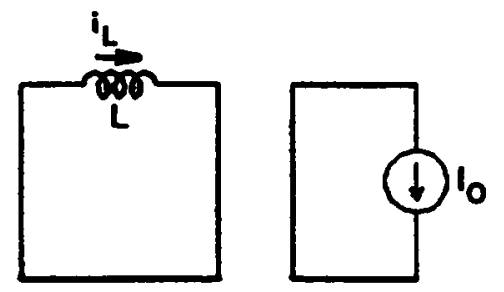

- $i_{L N}=I_{\text {LON }}, v_{C N}=0$

(Free-Wheeling Mode)

(h) MO: $v_{C}=0, Q 1, D 4$ or Q4,D1 or Q2,D3 or Q3,D2 on

Figure 3.4 Continued 


$$
\begin{aligned}
& L \frac{d i_{L}}{d t}=0, \\
& v_{C}=0 .
\end{aligned}
$$

By solving these equations, the expressions for the inductor current, $i_{L}$, and the capacitor voltage, $v_{C}$, in each topological mode can be derived. These expressions are normalized and shown in the following.

For resonant modes M1,M2,M3,M4,M5, and M6,

$$
\begin{aligned}
& i_{L N}=-\left(V_{C O N}-v_{E N}\right) \sin \omega_{0}\left(t-t_{0}\right)+\left(I_{L O N}+i_{E N}\right) \cos \omega_{0}\left(t-t_{0}\right)-i_{E N} \\
& v_{C N}=\left(V_{C O N}-v_{E N}\right) \cos \omega_{0}\left(t-t_{0}\right)+\left(I_{L O N}+i_{E N}\right) \sin \omega_{0}\left(t-t_{0}\right)+v_{E N}
\end{aligned}
$$

where, $t_{0}$ is the initial time and

$$
\begin{array}{lll}
\omega_{0} & =\frac{1}{\sqrt{L C}} \quad \text { is the angular resonant frequency, } \\
Z_{0} & =\sqrt{\frac{L}{C}} \quad \text { is the characteristic impedance, } \\
v_{C N} & =\frac{v_{C}}{E} \quad \text { is the normalized capacitor voltage, } \\
i_{L N} & =\frac{i_{L}}{E / Z_{0}} \quad \text { is the normalized inductor current, } \\
V_{C N} & =\frac{V_{C O}}{E} & \text { is the normalized initial capacitor voltage, } \\
I_{L O N} & =\frac{I_{L O}}{E / Z_{0}} & \text { is the normalized initial inductor current, } \\
v_{E N} & =\frac{v_{E}}{E,} & \\
i_{E N} & =\frac{i_{E}}{E .}
\end{array}
$$


For linear-charging mode $\mathrm{ML}$,

$$
\begin{aligned}
& i_{L N}=I_{L O N} \pm \omega_{0}\left(t-t_{0}\right), \\
& v_{C N}=0 .
\end{aligned}
$$

For free-wheeling mode M0,

$$
\begin{aligned}
& i_{L N}=I_{L O N}, \\
& v_{C N}=0 .
\end{aligned}
$$

The normalizing factors for the voltages and currents are $E$ and $E / Z_{0}$, respectively. 


\subsubsection{State Trajectories for Circuit Topological Modes}

From the expressions for $i_{L N}$ and $v_{C N}$ (eq. 3.4), state representation for the resonant modes M1,M2,M3,M4,M5, and M6 can be portrayed on the state plane. It can be seen that the trajectories for these topological modes are circular arcs with centers located at $\left(-v_{E N}, i_{E N}\right)$ and radii $R=\sqrt{\left(v_{E N}-V_{C O N}\right)^{2}+\left(i_{E N}+I_{L O N}\right)^{2}}$. A family of the trajectories for each resonant mode is plotted in Figure 3.5, where the centers of M1-M6 are indicated by ml-m6, respectively. The trajectories only exist in either the right-half or the left-half plane depending on the polarity of $v_{C}$. The solution trajectory corresponding to a specific switching interval is determined by the topological mode, the initial condition of resonant inductor current and resonant capacitor voltage, and the duration of the interval. Time is implicit in these trajectories. The elapsed time in a trajectory segment is measured by the angle subtended by the segment with respect to its center. For example, the amount of time elapsed from point $a$ to point $b$ in topological mode $M 1$ in Figure 3.5(a) is $\Delta t=y / \omega_{0}$. As time advances, the trajectories travel clockwise as indicated by the arrows.

The state trajectory for the linear-charging mode $M L$ is a line segment along the $i_{L N^{-}}$axis, as illustrated in Figure $3.5(\mathrm{~g})$. The amount of time elapsed in this mode is

proportional to the link, $\ell$, of the line segment, $\Delta t=\frac{\ell}{\omega_{0}}$. This mode occurs when voltage $v_{S}$ is equal to $\mathrm{E}$ or $-\mathrm{E}$ and the magnitude current of $i_{L N}$ is less than $I_{O N}$ when voltage $v_{C N}$ crosses the zero-axis. In this mode, the resonant capacitor $\mathrm{C}$ is shorted through the output bridge diodes.

The state trajectory for the free-wheeling mode MO is a stationary point on the $i_{L N}$-axis with coordinate $\left(0, I_{L, 0 N}\right)$ or $\left(0,-I_{I .0 N}\right)$, as indicated by a circle in Figure $3.5(\mathrm{~h})$. $\Lambda$ finite amount of time elapses at such a stationary point since both $i_{L N}$ and $v_{C N}$ are independent of time. This mode occurs at a similar condition as ML except that when $v_{C N}$ crosses the zero-axis, the voltage across the resonant tank is clamped at zero volt. 


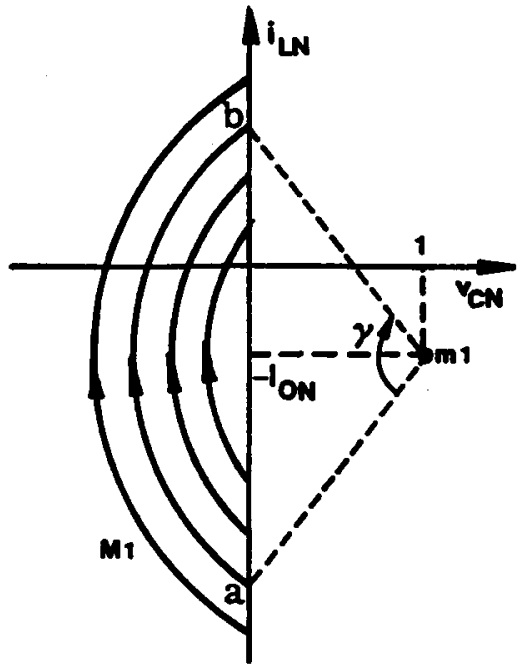

(a) Topological mode MI

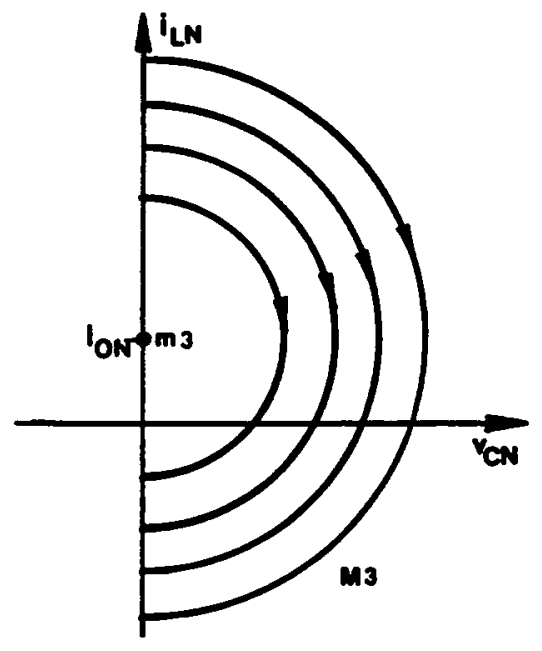

(c) Topological mode M3

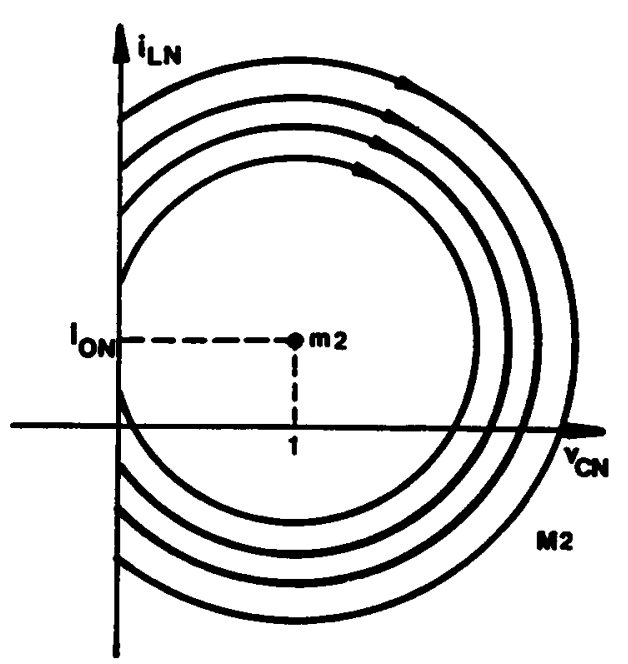

(b) Topological mode M2

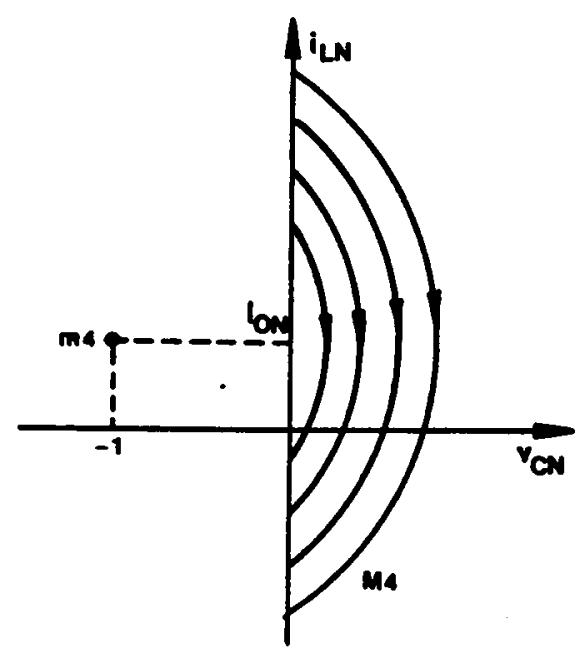

(d) Topological mode M4

Figure 3.5 Families of State Trajectory for Various Circuit Topological Modes 


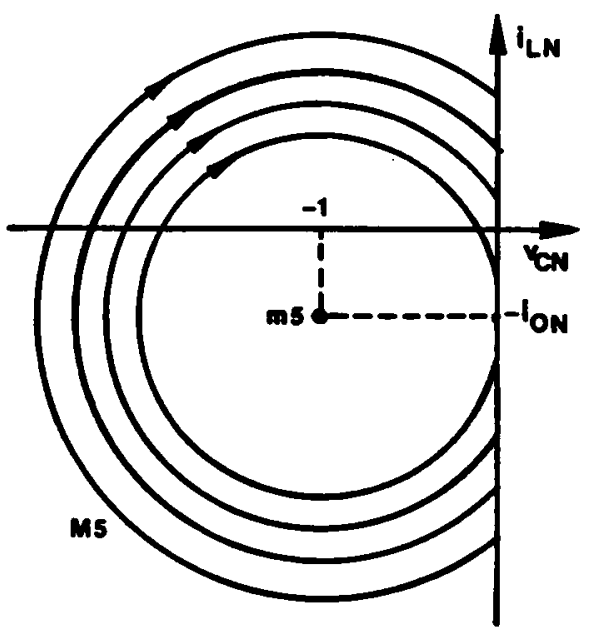

(e) Topological mode M5

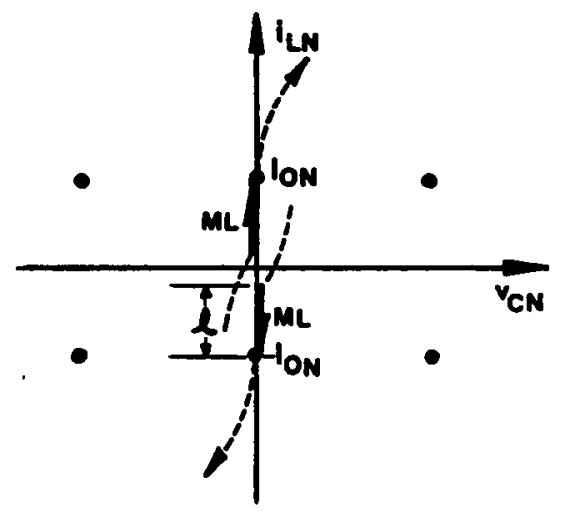

(g) Topological mode ML

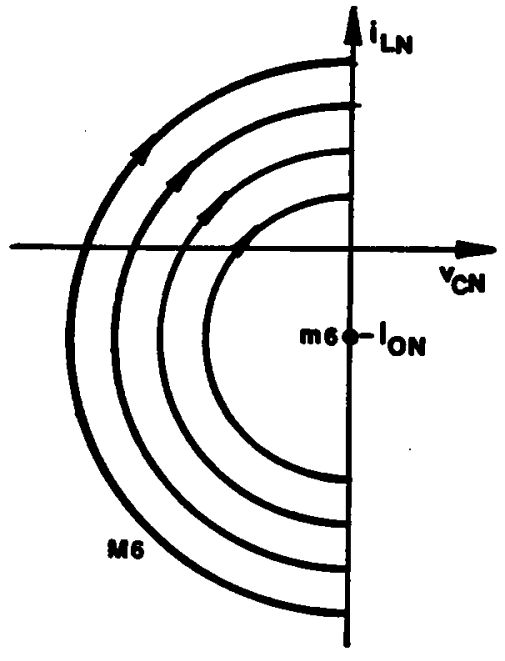

(f) Topological mode M6

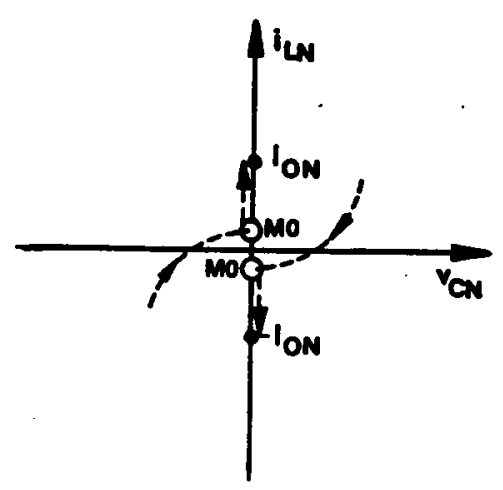

(h) Topological mode M0

Figure 3.5 Continued 


\subsubsection{Equilibrium Trajectory}

A steady-state operation of a CM-PRC can be represented by an equilibrium trajectory in the state plane [31]. An equilibrium trajectory is a closed contour composed of several trajectory segments. The solution trajectory is constructed via a particular sequence of circuit topological modes determined by the circuit operation.

An equilibrium state trajectory of a CM-PRC can be constructed using a composite diagram, as shown in Figure 3.6, which is generated by overlapping on the same $i_{\mathrm{LN}^{-}}$, $v_{C N}$ - axes the trajectories corresponding to each topological mode shown in Figure 3.5. The rules for constructing an equilibrium trajectory on the composite diagram are summarized in Appendix C.1. Figure 3.7 shows an example illustrating the construction of an equilibrium trajectory on the composite diagram. The constructed trajectory corresponds to the circuit operation described in Figure 3.2. In the trajectory, M6(M3) is initiated when Q1(Q3) is triggered at point a(e), M1(M4) is initiated when Q2(Q4) is triggered at point $b(f)$, and $M 2(M 5)$ is initiated when $v_{C}$ reverses polarity at point $c(g)$. 


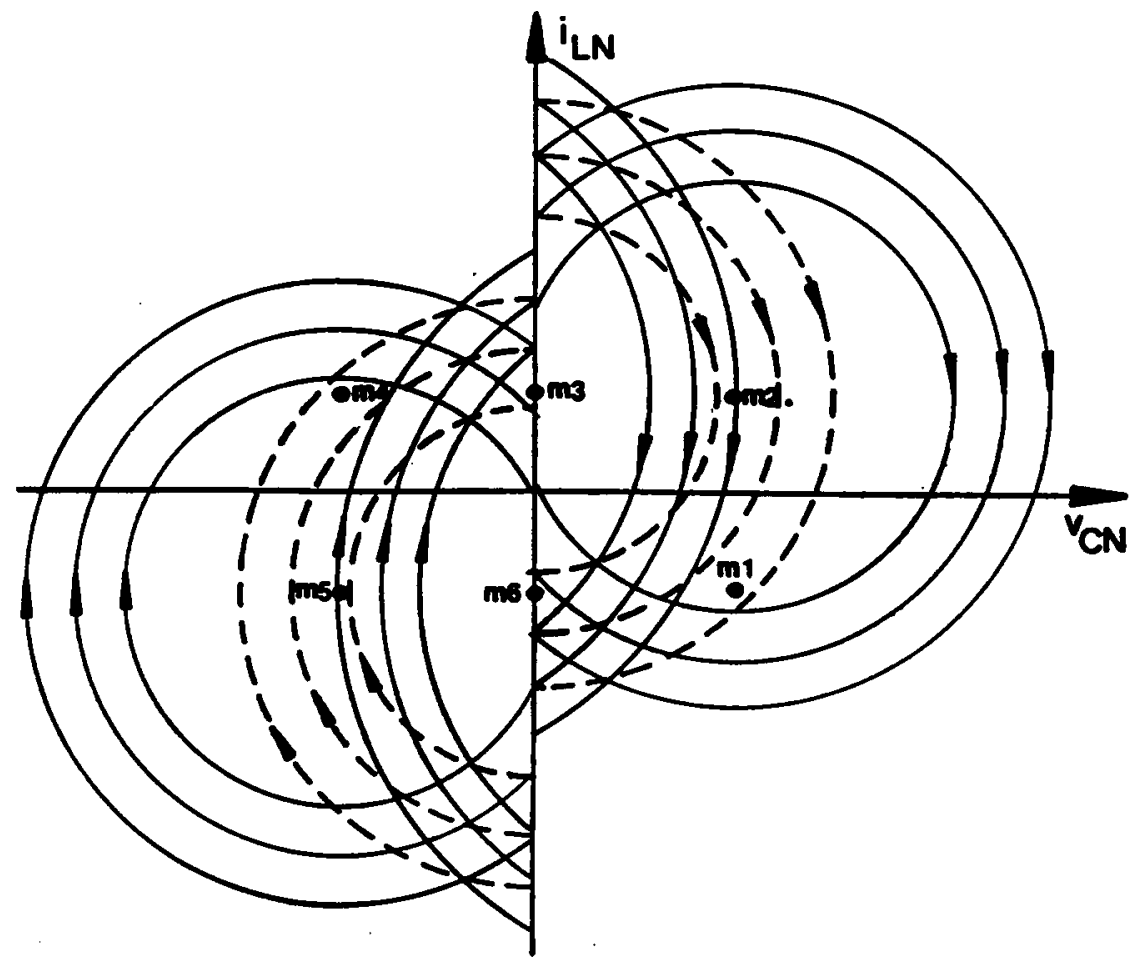

Figure 3.6 A Composite State Diagram for Constructing Equilibrium State Trajectories 


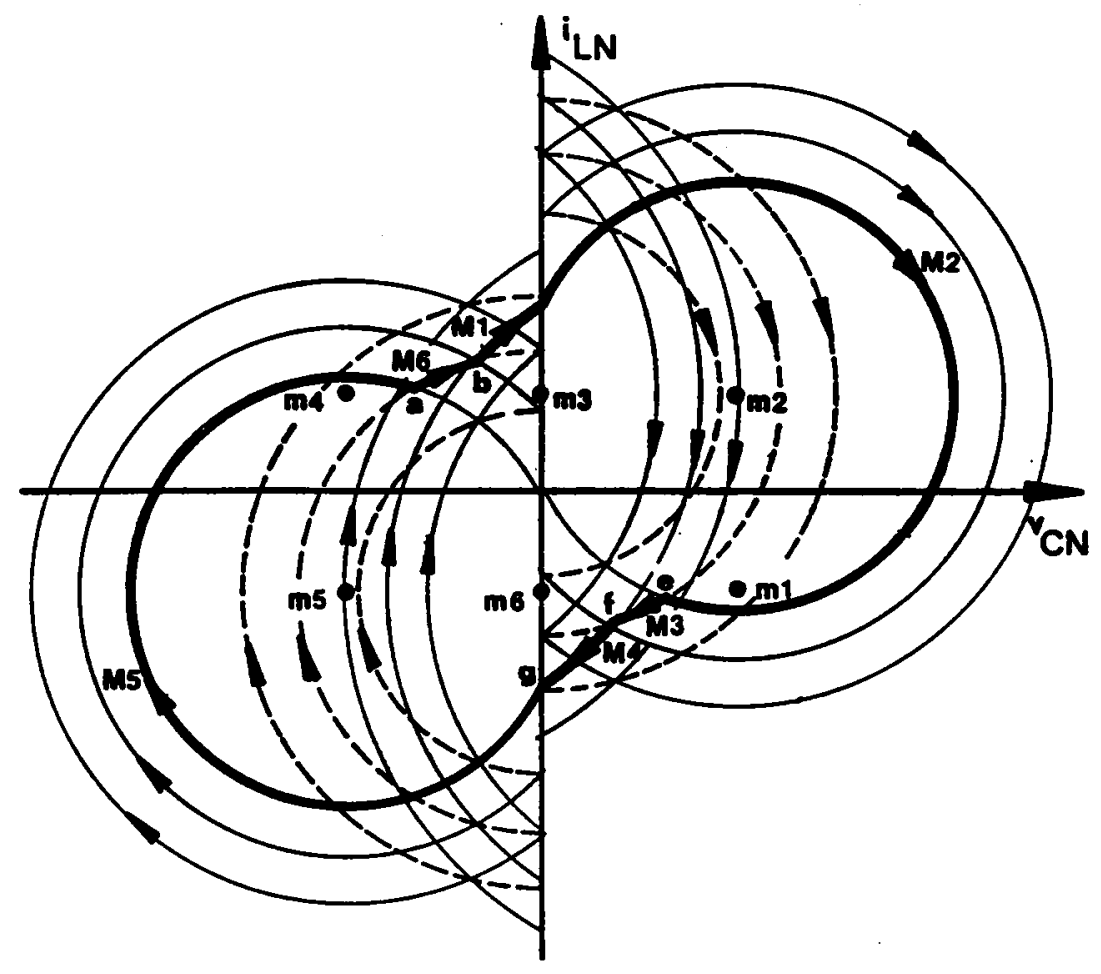

Figure 3.7 An Example Illustrating Construction of an Equilibrium State Trajectory on the Composite Diagram 


\subsubsection{Circuit Operating Modes}

Equilibrium trajectories representing various circuit operating modes of a CM-PRC have been constructed. These trajectories are discussed in the following under two categories : below resonant frequency and above resonant frequency.

\subsubsection{Operating modes below resonant frequency}

Figure 3.8 shows a series of equilibrium state trajectories together with their corresponding circuit waveforms which illustrate all the possible operating modes of a CM-PRC operating below resonant frequency.

\section{(a) Natural-commutation modes}

Trajectory $\mathbf{I}_{N}: \Lambda s$ shown in Ï̈gure $3.8(\mathrm{a})$, at $\mathrm{t}=\mathrm{a}$, transistor $\mathrm{Q} 1$ turns on commutating diode D3. The inductor current, $i_{L N}$, resonates through $\mathrm{Q} 1$ and D4 (M6). At $\mathrm{t}=\mathrm{b}$, transistor Q2 turns on commutating diode D4. The inductor current resonates through Q1 and Q2 (MI). At $t=c$, capacitor voltage $v_{C N}$ reverses polarity. The inductor current continues to resonate through Q1 and Q2 (M2). At $\mathrm{t}=\mathrm{d}$, inductor current $i_{L N}$ decreases to zero. Transistors Q1,Q2 turn off nalurally and diodes D1,D2 conducts subsequently. The inductor current resonant through D1 and D2 (M2). At $t=e$, transistor Q3 turns on commutating diode D1 and a similar process occurs with the roles of Q1,Q2,D1,D2 and Q3,Q4,D3,D4 interchanged, respectively. The topological mode sequence of this trajectory is M6-MI-M2-M3-M4-M.5, which is defined as "mode-I operation" of CM-PRC. Since the transistors are all naturally commutated, the circuit operation for this trajectory is referred to as "mode- $I_{N}$ opcration".

Trajectory $2_{N}: \Lambda s$ shown in Figure $3.8(\mathrm{~b})$, the circuit operation for this trajectory is similar to that for Trajectory $I_{N}$ except that $v_{C N}$ changes polarity before Q2(Q4) is triggered at $t=c(g)$. The topological mode sequence of this trajectory is 

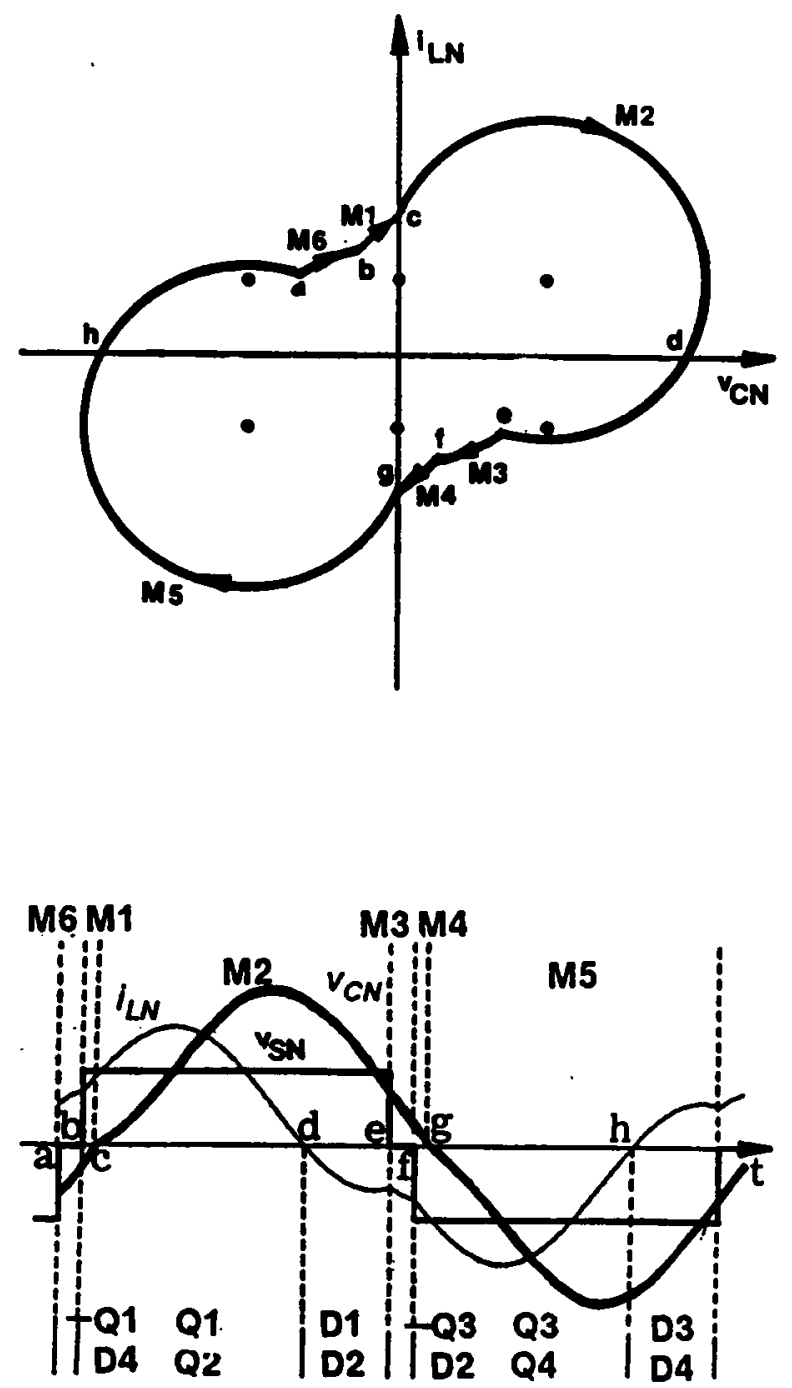

(a) Trajectory $1_{N}$ and its corresponding circuit waveforms(Mode- $I_{N}$ operation)

Figure 3.8 Equilibrium Trajectories of a CM-PRC Operating Below Resonant Frequency 

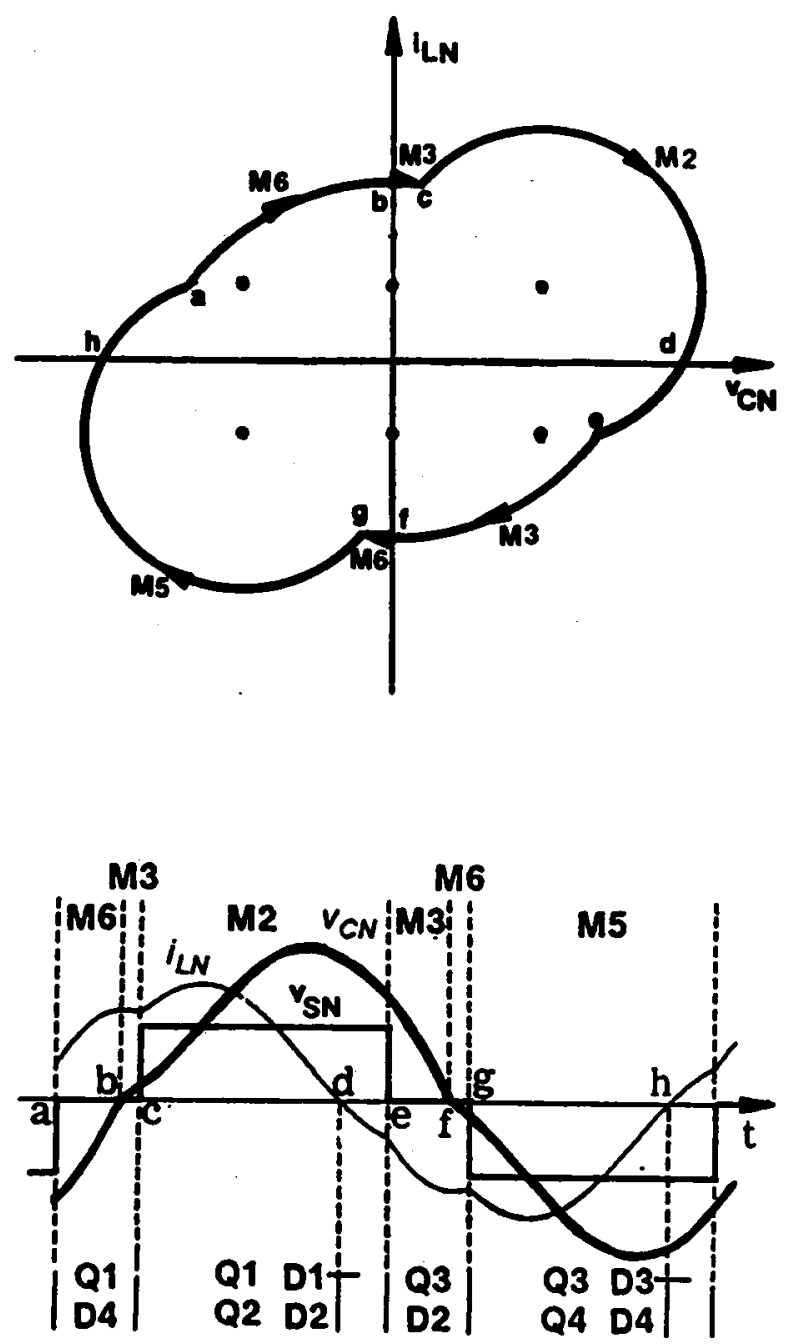

(b) Trajectory $2_{N}$ and its corresponding circuit waveforms(Mode- $I I_{N}$ operation)

Figure 3.8 Continued 
M6-M3-M2-M3-M6-M5, which is defined as "mode-II operation" of CM-PRC. The circuit operation for this trajectory is referred to as "mode- $I I_{N}$ operation".

Trajectory $3_{N}$ : As shown in Figure 3.8(c), the circuit operation for this trajectory is similar to that for Trajectory $1_{N}$ except that when $v_{C N}$ crosses the zero-axis, the magnitude of $i_{L N}$ is less than $I_{O N}$. As a result, an inductor linear-charging interval (ML) exists. The linear-charging interval ends when the magnitude of $i_{L N}$ increases beyond $I_{O N}$. The topological mode sequence of this trajectory is $M 6-M I-M L-M 2-M 3-M 4-M L-M 5$, which is defined as "mode-III operation" of CM-PRC. The circuit operation for this trajectory is referred to as "mode- $I I I_{N}$ operation".

Trajectory $4_{N}:$ As shown in Figure 3.8(d), the circuit operation for this trajectory is similar to that for Trajectory $3_{N}$ except that $v_{C N}$ crosses the zero-axis before Q2 or Q4 is triggered. Thus, in addition to the linear-charging interval, a free-wheeling interval (M0) also exists. The free-wheeling interval ends when $Q 2(Q 4)$ is triggered. The topological mode sequence of this trajectory is $M 6-M 0-M L-M 2-M 3-M 0-M L-M 5$, which is defined as "mode-IV operation" of CM-PRC. The circuit operation for this trajectory is referred to as "mode- $I V_{N}$ operation".

\section{(b) Mixed-commutation modes}

Trajectory $1_{M}$ : As shown in Figure 3.8(e), at $\mathrm{t}=\mathrm{a}$, transistor $\mathrm{Q} 3$ is forced off and $\mathrm{Q} 1$ is triggered. Transistor Q1 cannot conduct since $i_{L N}$ is negative. Instead, diode D1 conducts. The inductor current resonates through $Q 4$ and DI (M6). At $t=b$, inductor current $i_{I, N}$ increases to zero. Transistor $Q 4$ and diode DI turn off naturally and transistor Q1 and diode D4 conduct subsequently. The inductor current resonates through Q1 and D4 (M6). At $t=c$, transistor Q2 turns on commutating diode D4. The inductor current resonates through $\mathrm{Q} 1$ and $\mathrm{Q} 2(\mathrm{MI}) . \quad \Lambda \mathrm{t} t=\mathrm{d}$, capacitor voltage $v_{C N}$ changes polarity. The inductor current continues to resonate through $Q 1$ and Q2 (M2). At $t=e$, transistor Q1 is forced off and Q3 is triggered. A similar process occurs with the roles 

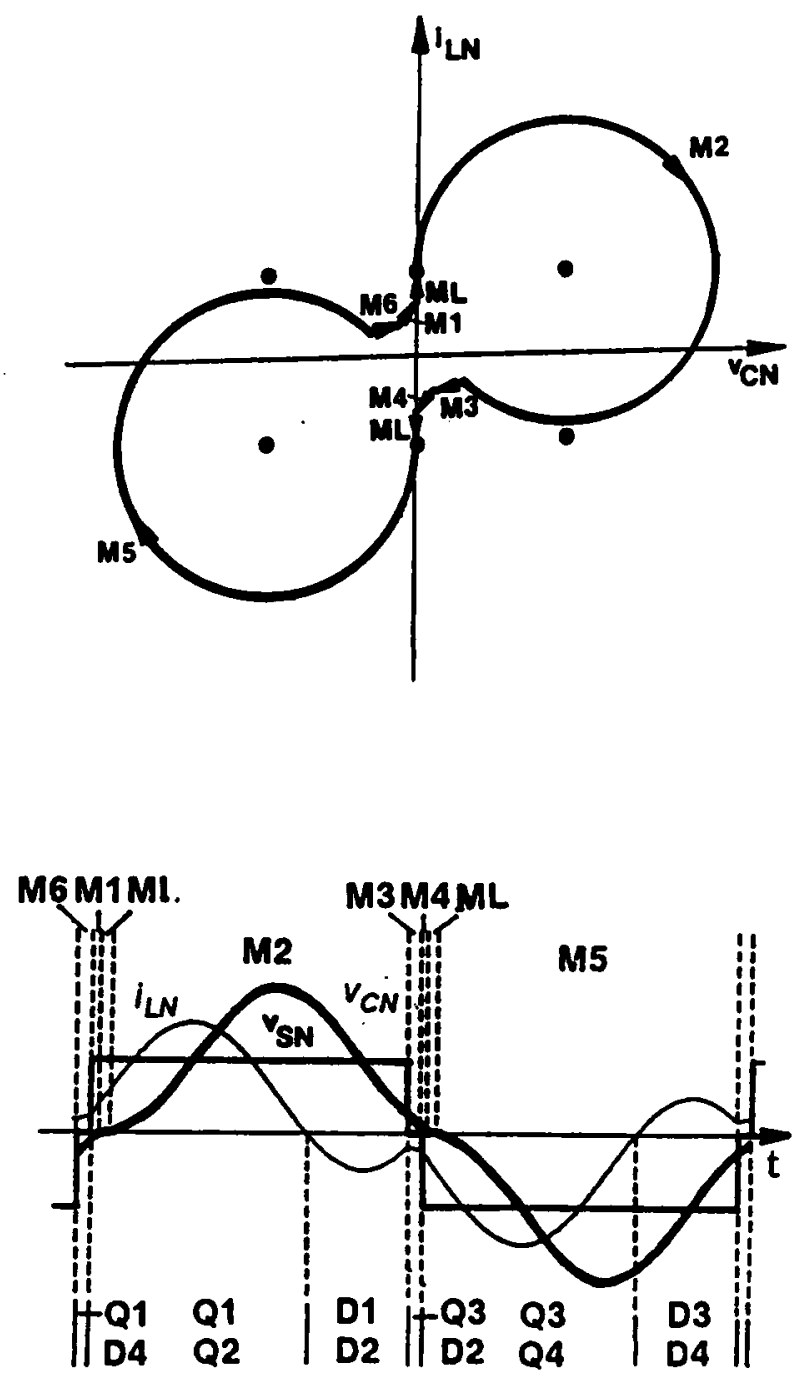

(c) Trajectory $3_{N}$ and its corresponding circuit waveforms(Mode- $I I I_{N}$ operation)

Figure 3.8 Continued 

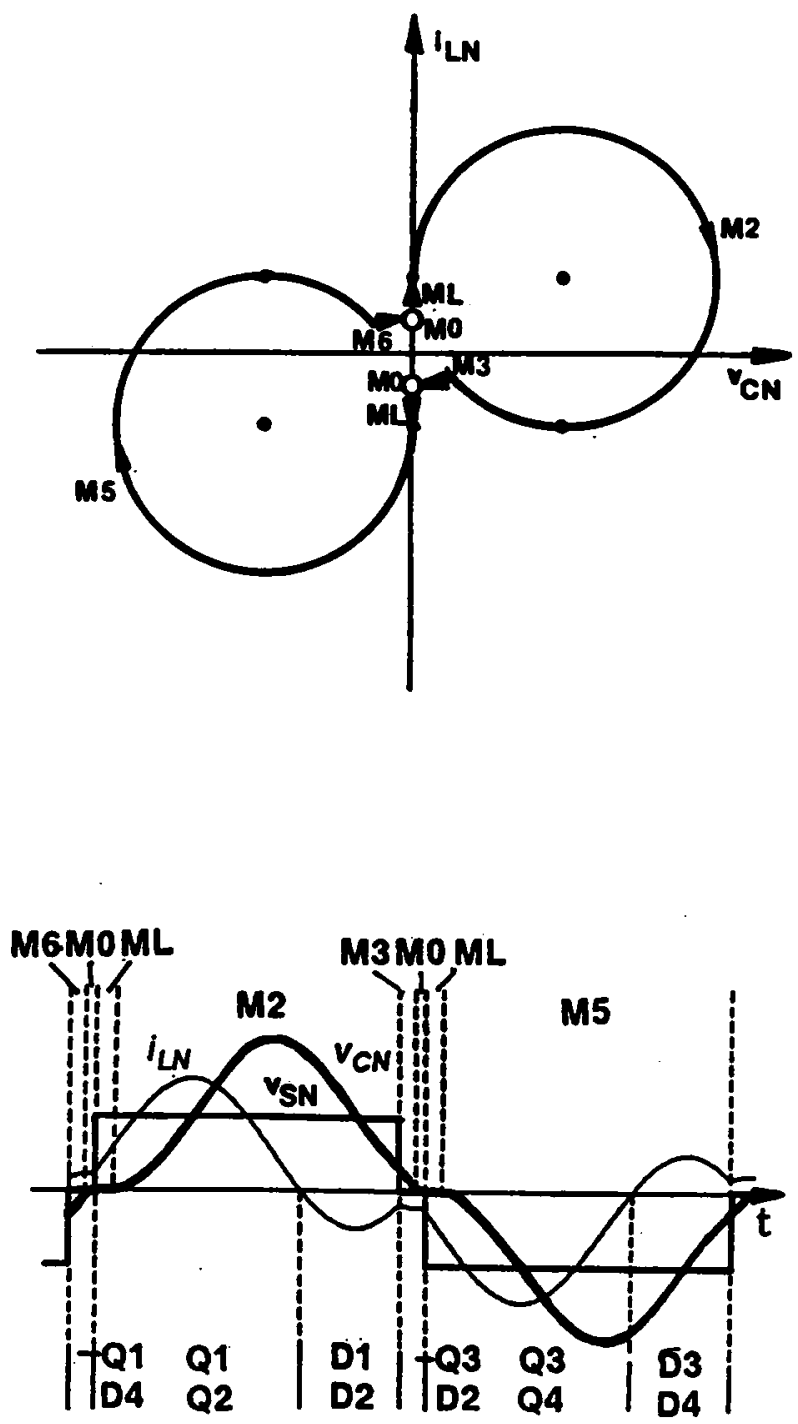

(d) Trajectory $4_{N}$ and its corresponding circuit waveforms(Mode- $I V_{N}$ operation)

Figure 3.8 Continued 

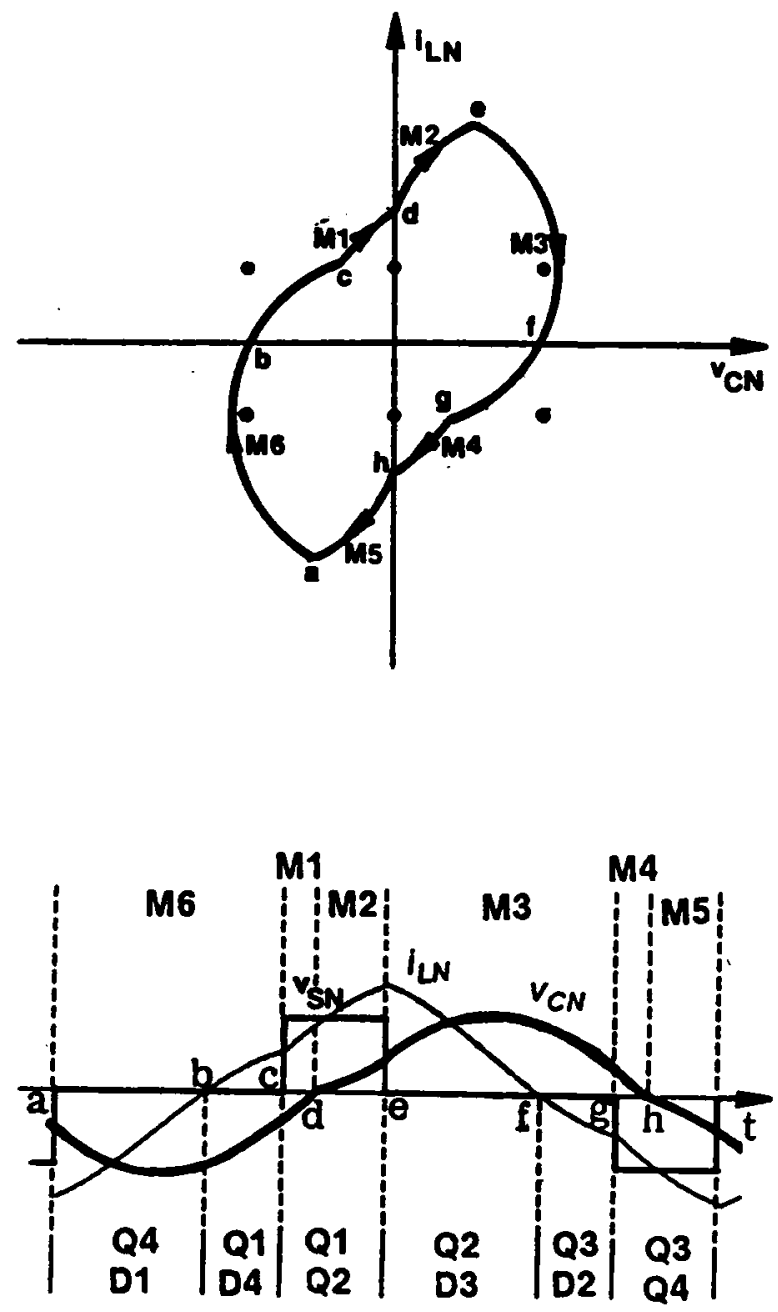

(e) Trajectory $I_{m}$ and its corresponding circuit waveforms(Mode- $I_{m}$ operation)

Figure 3.8 Continued 
of Q1,Q2,D1,Q2 and Q3,Q4,D3,D4 interchanged, respectively. The topological mode sequence of this trajectory is the same as that of Trajectory $1_{N}$. However, since transistors $\mathrm{Q1,Q3}$ are force-commutated while transistors $\mathrm{Q2,Q4}$ are naturally commutated, the circuit operation for this trajectory is referred to as "mode- $I_{M}$ operation".

Trajectory $2_{M}$ : As shown in Figure 3.8(f), the circuit operation for this trajectory is similar to that for Trajectory $1_{M}$ except that $v_{C N}$ changes polarity before Q2(Q4) is triggered at $t=d(h)$. The topological mode sequence of this trajectory is the same as that of Trajectory $2_{N}$. The circuit operation for this trajectory is referred to as "mode- $I I_{M}$ operation".

Trajectory $3_{M}$ : As shown in Figure 3.8(g), the circuit operation for this trajectory is similar to that for Trajectory $1_{M}$ except that when $v_{C N}$ crosses the zero-axis, the magnitude of $i_{L N}$ is less than $I_{O N}$. As a result, an inductor linear-charging interval (ML) exists. The linear-charging interval ends when the magnitude of $i_{L N}$ increases beyond $I_{O N}$. The topological mode sequence of this trajectory is the same as that of Trajectory $3_{N}$. The circuit operation for this trajectory is referred to as "mode- $I I I_{M}$ operation".

Trajectory $4_{M}:$ As shown in Figure 3.8(h), the circuit operation for this trajectory is similar to that for Trajectory $3_{M}$ except that $v_{C N}$ crosses the zero-axis before Q2 or Q4 is triggered. Thus, in addition to the linear-charging interval, a free-wheeling interval (M0) also exists. The free-wheeling interval ends when Q2(Q4) is triggered. The topological mode sequence of this trajectory is the same as that of Trajectory $4_{N}$. The circuit operation for this trajectory is referred to as "mode- $I V_{M}$ operation".

\section{(c) Force-commutation modes}

Trajectory $\mathrm{I}_{F}$ : As shown in Figure 3.8(i), at $\mathrm{t}=\mathrm{a}$, transistor $\mathrm{Q} 3$ is forced off and $\mathrm{Q} 1$ is triggered. Transistor Q1 cannot conduct since $i_{L N}$ is negative. Instead, diode D1 conducts. The inductor current resonates through Q4 and D1 (M6). At $t=b$, transistor Q4 is forced off and Q2 is triggered. Transistor Q2 cannot conduct since $i_{L N}$ is still neg- 

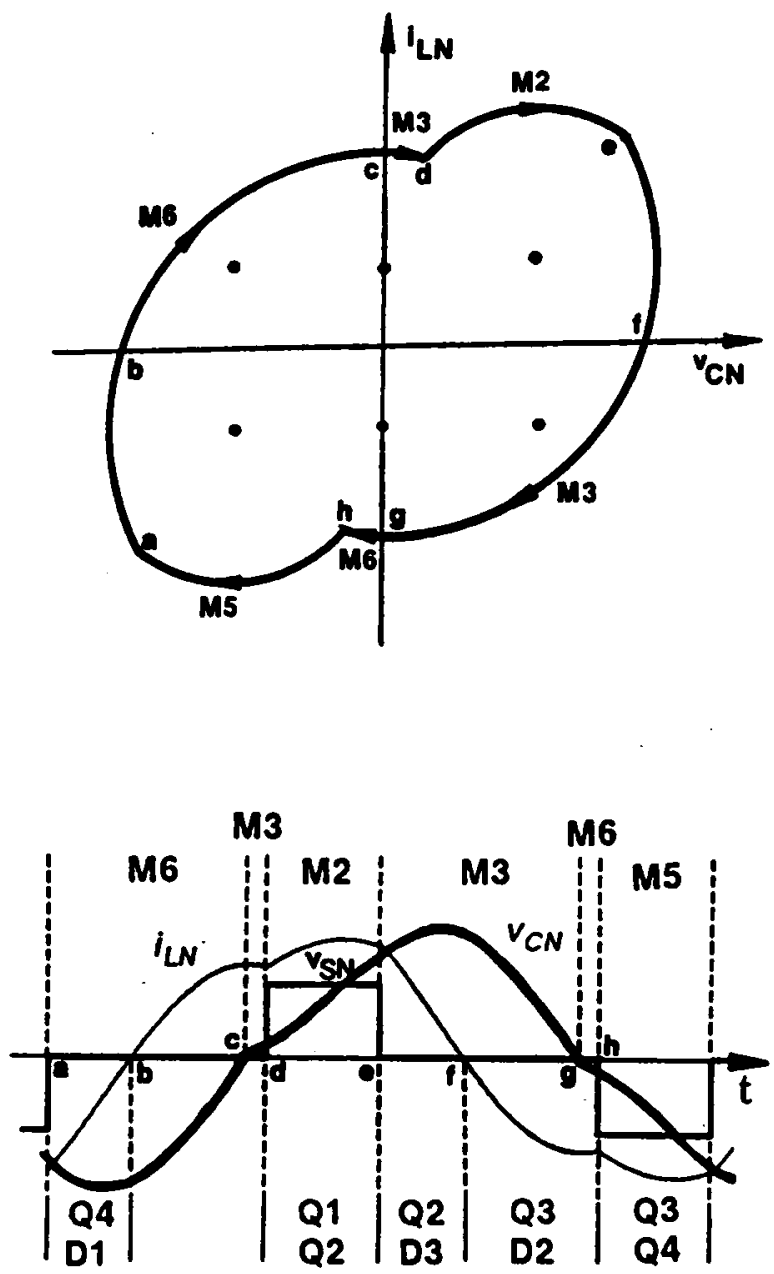

(f) Trajectory $2_{m}$ and its corresponding circuit waveforms(Mode- $I I_{m}$ operation)

Figure 3.8 Continued 

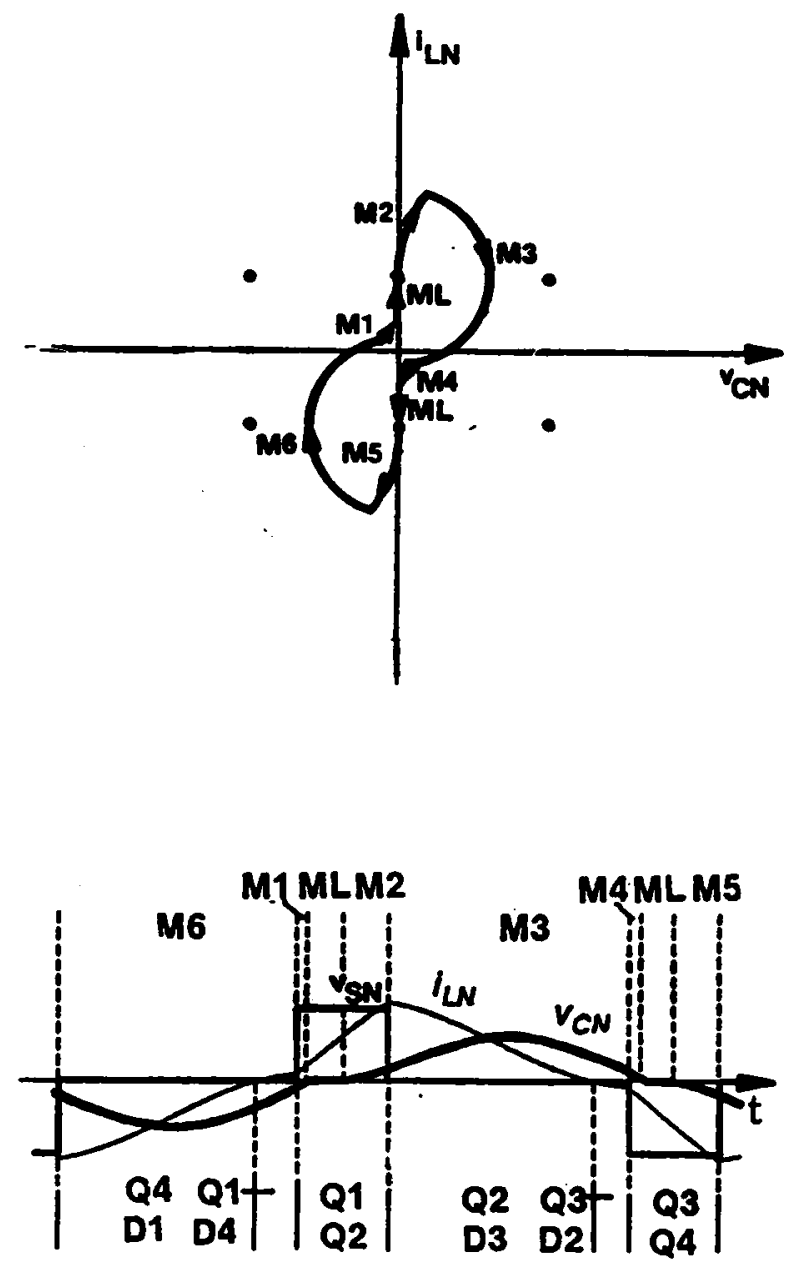

(g) Trajectory $3_{M}$ and its corresponding circuit waveforms(Mode- $I I I_{M}$ operation)

Figure 3.8 Continued 

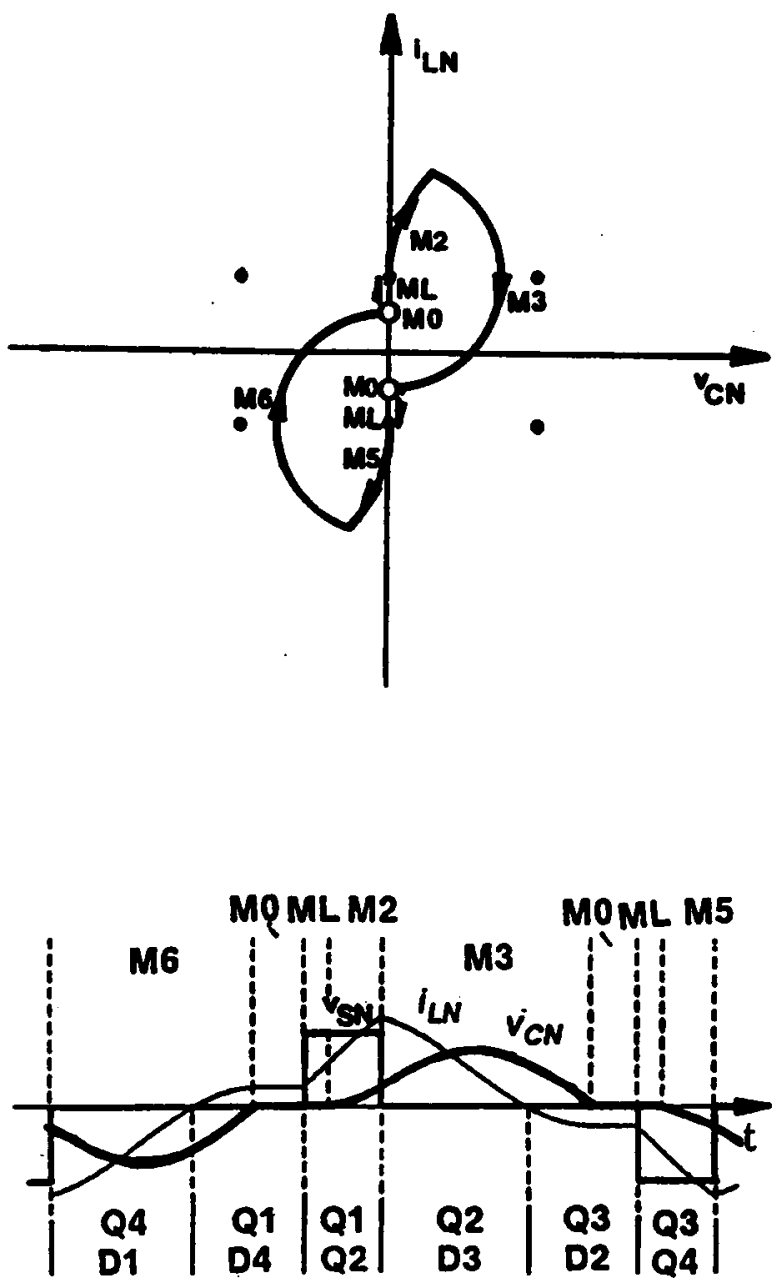

(h) Trajectory $4_{m}$ and its corresponding circuit waveforms(Mode- $I V_{m}$ operation)

Figure 3.8 Continued 

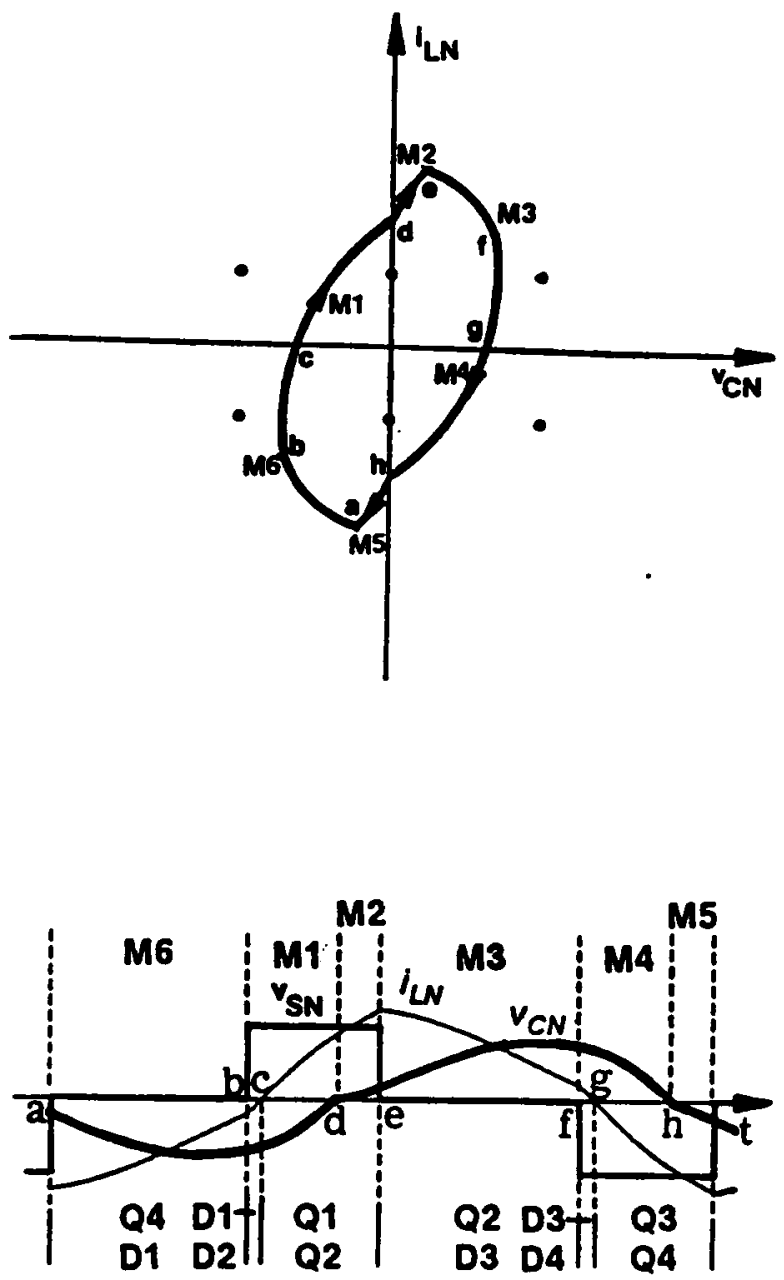

(i) Trajectory $I_{F}$ and its corresponding circuit waveforms(Mode- $I_{F}$ operation)

Figure 3.8 Continued 
ative. Instead, diode D2 conducts. The inductor current resonates through D1 and D2 (M1). At $\mathrm{t}=\mathrm{c}, i_{L N}$ increases to zero. Diodes D1,D2 turn off naturally and transistors Q1,Q2 conduct subsequently. The inductor current resonates through Q1 and Q2 (M1). At $\mathrm{t}=\mathrm{d}$, capacitor voltage $v_{C N}$ changes polarity. The inductor current continues to resonates through Q1 and Q2 (M2). At $\mathrm{t}=\mathrm{e}$, transistor $\mathrm{Q} 1$ is forced off and Q3 is triggered. A similar process occurs with the roles of Q1,Q2,D1,Q2 and Q3,Q4,D3,D4 interchanged, respectively. The topological mode sequence of this trajectory is the same as that of Trajectory $\mathbf{l}_{N}$. However, since all the transistors are force-commutated, the circuit operation for this trajectory is referred to as "mode- $I_{F}$ operation".

Trajectory $\mathbf{2}_{M L}$ : This trajectory is a special case of " mode-II operation". The transistors conduct twice during a switching period. $\Lambda s$ shown in Figure $3.8(\mathrm{j})$, at $\mathrm{t}=\mathrm{a}$, transistor Q3 is forced off and Q1 is triggered. Transistor Q1 cannot conduct since $i_{L N}$ is negative. Instead, diode D1 conducts. The inductor current resonates through Q4 and Dl (M6). At $\mathrm{t}=\mathrm{b}$, inductor current $i_{L N}$ increases to zero. Transistor Q4, diode D1 turn off naturally and transistor Q1, diode D4 conduct subsequently. The inductor current resonates through $\mathrm{Q} 1$ and $\mathrm{D} 4(\mathrm{M} 6) . \quad \Lambda \mathrm{t} t=\mathrm{b}^{\prime}$, capacitor voltage $v_{C N}$ changes polarity. The inductor current continues to resonates through Q1 and D4 (M3). At $t=c$, $i_{L N}$ changes polarity for the second time. Transistor Q1, diode D4 turn off naturally and transistor Q4, diode D1 conduct again. The inductor current resonates through Q4 and DI (M3). $\Lambda \mathrm{t} t=\mathrm{d}$, transistor $\mathrm{Q} 4$ is is forced off and Q2 is triggered. Transistor Q2 cannot conduct since $i_{I N}$ is negative. Instead, diode D2 conducts. The inductor current resonates through D1 and D2 (M2). $\Lambda \mathrm{t} t=\mathrm{e}, i_{L N}$ changes polarity for the third time. Diodes D1,D2 turn off naturally and transistors Q1,Q2 conduct subsequently. The inductor current resonates through $\mathrm{Q} 1$ and $\mathrm{Q} 2$ (M2). $\Lambda \mathrm{t} t=\mathrm{f}$, transistor $\mathrm{Q} 1$ is is forced off and Q3 is triggered. A similar process occurs with the roles of Q1,Q2,D1,D2 and Q3,Q4,D3,D4 interchanged, respectively. The topological mode sequence of this trajec- 

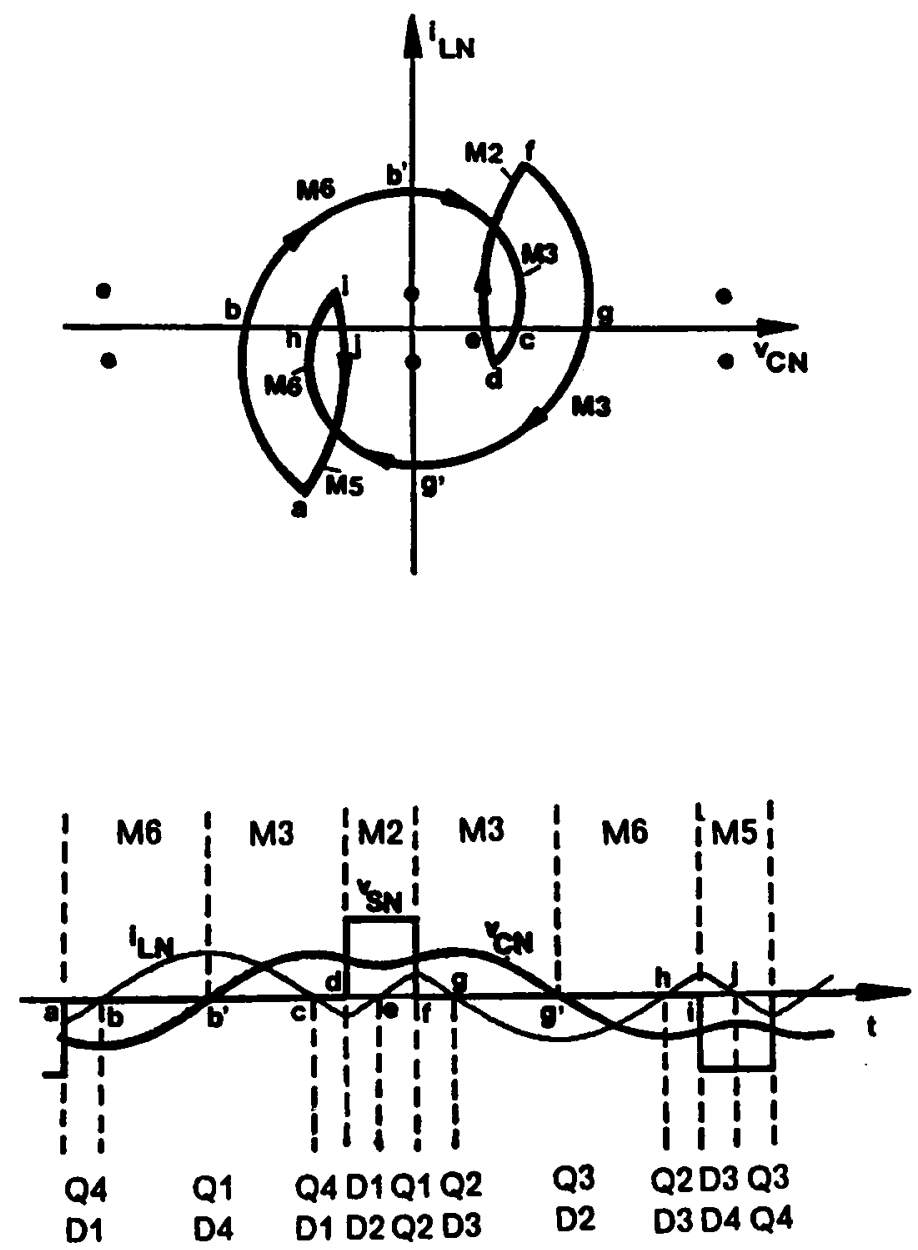

(j) Trajectory $2_{M L}$ and its corresponding circuit waveforms(Mode- $I I_{M}$ operation)

Figure 3.8 Continued 
tory is the same as that of Trajectory $2_{N}$. However, due to the multiple-conduction feature, the circuit operation for this trajectory is referred to as "mode- $I I_{M L}$ operaticin".

Trajectory $3_{F}$ : As shown in Figure 3.8(k), the circuit operation for this trajectory is similar to that for Trajectory $1_{F}$ except that when $v_{C N}$ crosses the zero-axis, the magnitude of $i_{L N}$ is less than $I_{O N}$. As a result, an inductor linear-charging interval (ML) exists. The linear-charging interval ends when the magnitude of $i_{L N}$ increases beyond $I_{O N}$. The topological mode sequence of this trajectory is the same as that of Trajectory $3_{N}$. The circuit operation for this trajectory is referred to as "mode- $I I I_{F}$ operation".

Trajectory 4F: As shown in Figure 3.8(1), the circuit operation for this trajectory is similar to that for Trajectory $3_{F}$ except that $v_{C N}$ crosses the zero-axis before Q2 or Q4 is triggered. Thus, in addition to the linear-charging interval, a free-wheeling interval (M0) also exists. The free-wheeling interval ends when Q2(Q4) is triggered. The topological mode sequence of this trajectory is the same as that of Trajectory $4_{N}$. The circuit operation for this trajectory is referred to as "mode- $I V_{F}$ operation".

Trajectory $5_{F}$ : As shown in Figure $3.8(\mathrm{~m})$, this trajectory is degenerated either from Trajectory $3_{F}$ or from Trajectory $4_{F}$. The capacitor voltage, $v_{C N}$, is always zero. In other words, the resonant capacitor, $\mathrm{C}$, is always shorted through the output bridge diodes. The resonant inductor, $\mathrm{L}$, is either linearly charged or discharged by the source voltage or shorted through two parallel switch branches in the input bridge. No resonant action occurs and no output voltage is generated. The topological mode sequence of this trajectory is MO-ML-M0-ML, which is defined as "mode-V operation" of CM-PRC. Since all the transistors are force-commutated, the circuit operation for this trajectory is referred to as "mode- $V_{F}$ operation". This operating mode can only exist momentarily when the energy in the output (filter) inductor is discharging into the load through the rectifier diodes. It cannot exist under stcady state. 

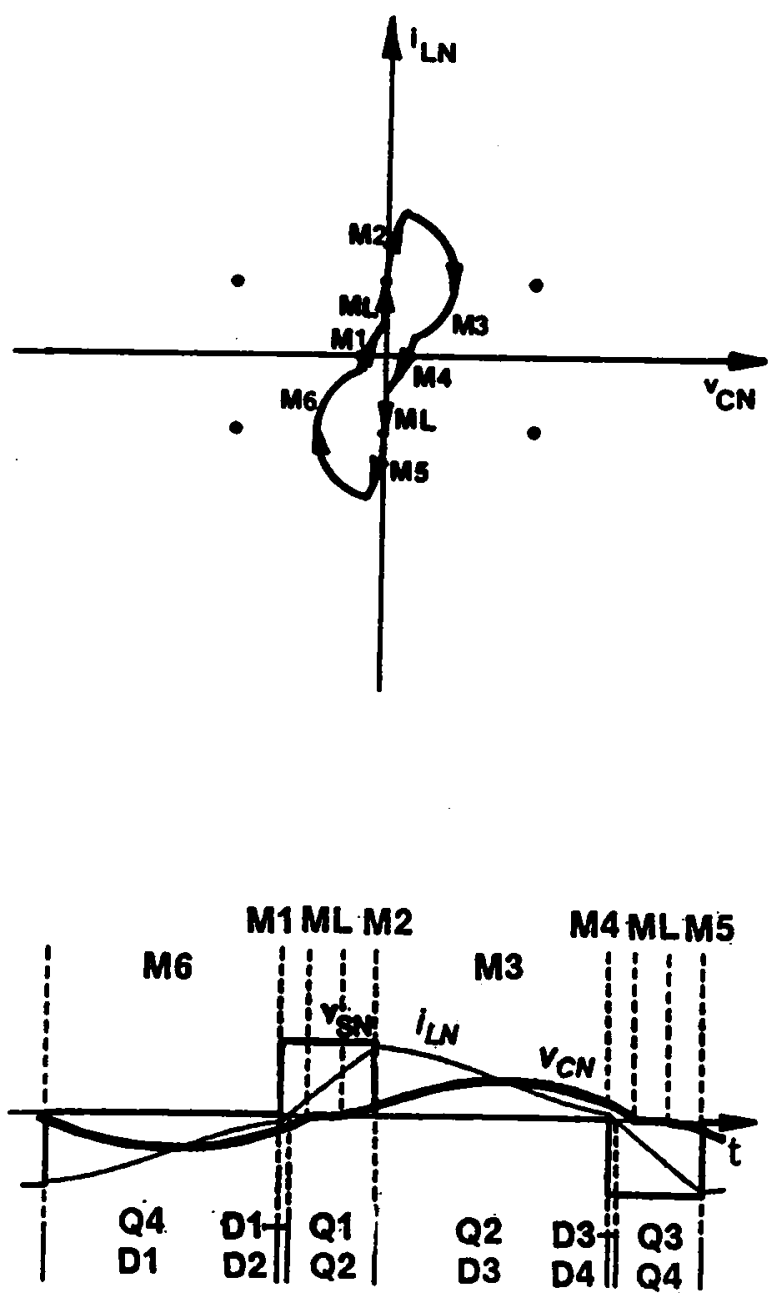

(k) Trajectory $3_{F}$ and its corresponding circuit waveforms(Mode- $I I I_{F}$ operation)

Figure 3.8 Continued 

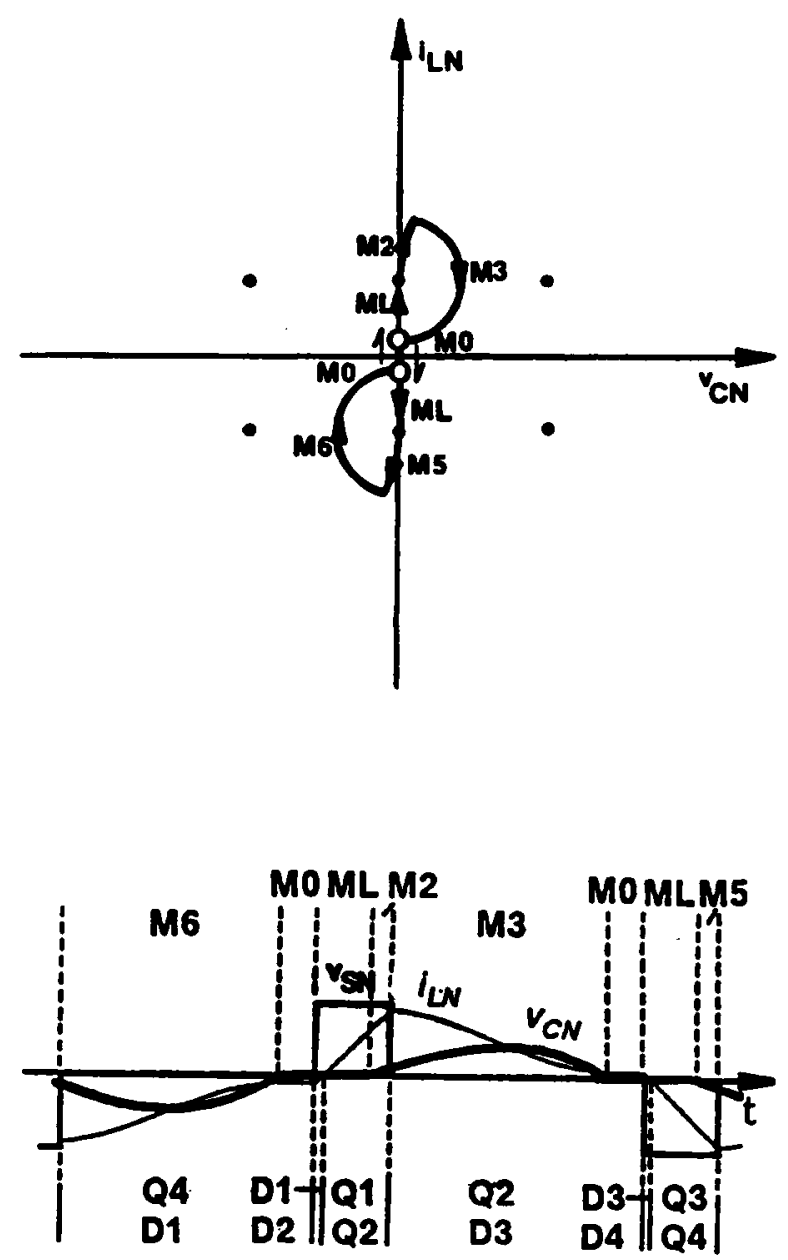

(I) Trajectory $4_{F}$ and its corresponding circuit waveforms(Mode- $I V_{F}$ operation)

Figure 3.8 Continued 

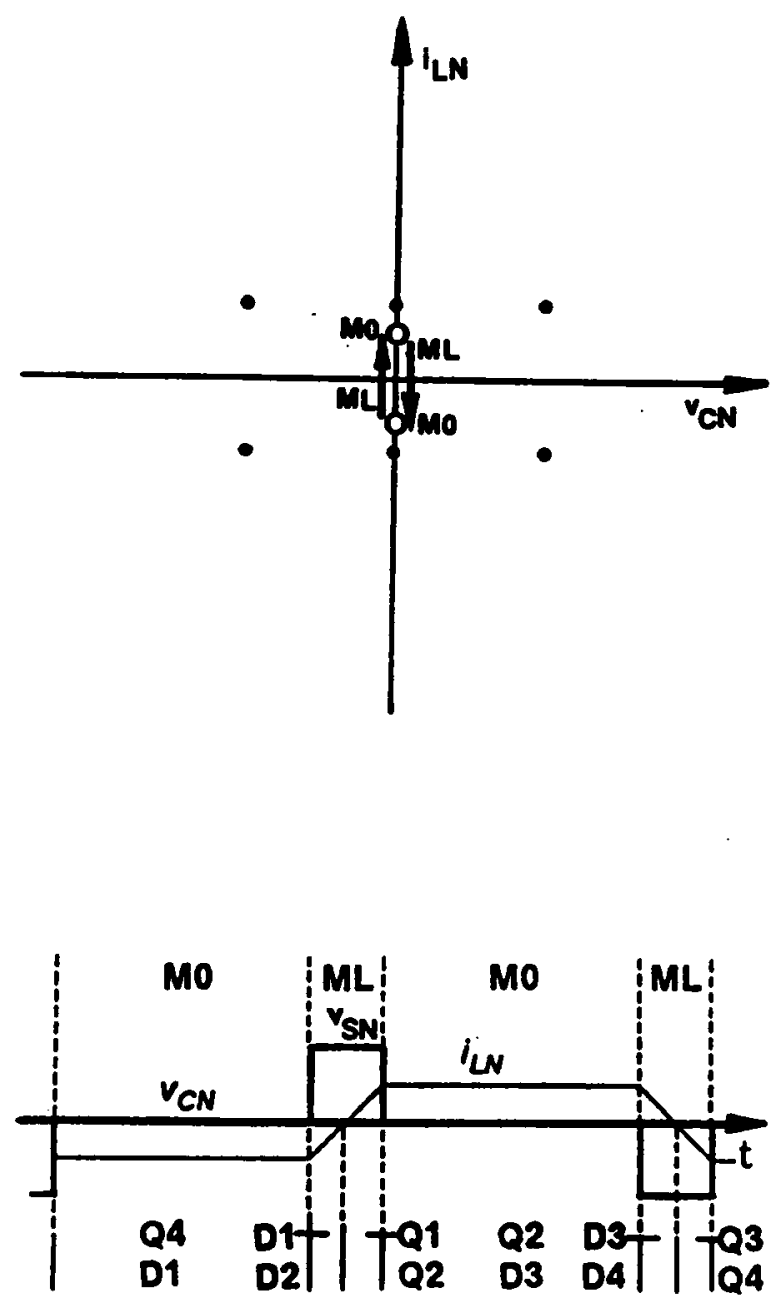

(m) Trajectory $S_{F}$ and its corresponding circuit waveforms(Mode- $V_{F}$ operation)

Figure 3.8 Continued 
Table 3.1 summarizes the operating modes below resonant frequency and their corresponding topological mode sequences. The subscript in each operating mode indicates the commutation features for the transistors, where " $\mathrm{N}$ " stands for natural commutation, " $M$ " stands for mixed commutation, "ML" stands for multiple conduction, and " $F "$ stands for force commutation.

\subsubsection{Operating modes above the resonant frequency}

Figure 3.9 shows a series of equilibrium state trajectories which illustrate all possible operating modes of a CM-PRC operating above resonant frequency. The trajectories shown in Figs. 3.9(a), (c), (d), (e) have already been discussed in the previous section. The trajectories shown in Figs. 3.9(b) and 3.9(f) are discussed in the following.

\section{(a) Force-commutation modes}

Trajectory 2A $A_{F}$ As shown in Figure 3.9(b), at $\mathrm{t}=\mathrm{a}$, transistor $\mathrm{Q} 3$ is forced off and Q1 is triggered. Transistor Q1 cannot conduct since $i_{L N}$ is negative. Instead, diode D1 conducts. The inductor current resonates through $\mathrm{Q}_{4}$ and D1 (M3). At $\mathrm{t}=\mathrm{a}^{\prime}$, capacitor voltage $v_{C N}$ changes polarity. The inductor current continues to resonate through Q4 and D1 (M6). At $\mathrm{t}=\mathrm{b}$, transistor Q4 is forced off and Q2 is triggered. Transistor Q2 cannot conduct since $i_{L N}$ is still negative. Instead, diode D2 conducts. The inductor current resonates through D1 and D2 (M1). $\Lambda \mathrm{t} t=\mathrm{c}$, inductor current $i_{L N}$ increases to zero. Diodes D1,D2 turn off naturally and transistors Q1,Q2 conduct subsequently. The inductor current resonates through $\mathrm{Q1}$ and $\mathrm{Q} 2$ (M1). At $\mathrm{t}=\mathrm{d}$, transistor $\mathrm{Q1}$ is forced off and Q3 is triggered. $\Lambda$ similar process occurs with the roles of Q1,Q2,D1,Q2 and Q3,Q4,D3,D4 interchanged, respectively. The topological mode sequence of this trajectory is M3-M6-MI-M6-M3-M4, which is defined as "mode-IIA operation" of CM-PRC. Since all the transistors are force-commutated, the circuit operation for this trajectory is referred to as "mode- $I I A_{F}$ operation". 
Table 3.1 Topological Mode Sequences for Circuit Operating Modes Below Resonant Frequency

\begin{tabular}{|c|c|}
\hline $\begin{array}{c}\text { Operating } \\
\text { Modes }\end{array}$ & Topological Mode Sequences \\
\hline$I_{N}, I_{M}, I_{F}$ & M6-M1-M2-M3-M4-M5 \\
\hline$I I_{N}, I I_{M}, I I_{M L}$ & M6-M3-M2-M3-M6-M5 \\
\hline$I I I_{N}, I I I_{M}, I I I_{F}$ & M6-M1-ML-M2-M3-M4-ML-M5 \\
\hline$I V_{N}, I V_{M}, I V_{F}$ & M6-M0-ML-M2-M3-M0-ML-M5 \\
\hline$V_{F}$ & M0-ML-MO-ML \\
\hline
\end{tabular}



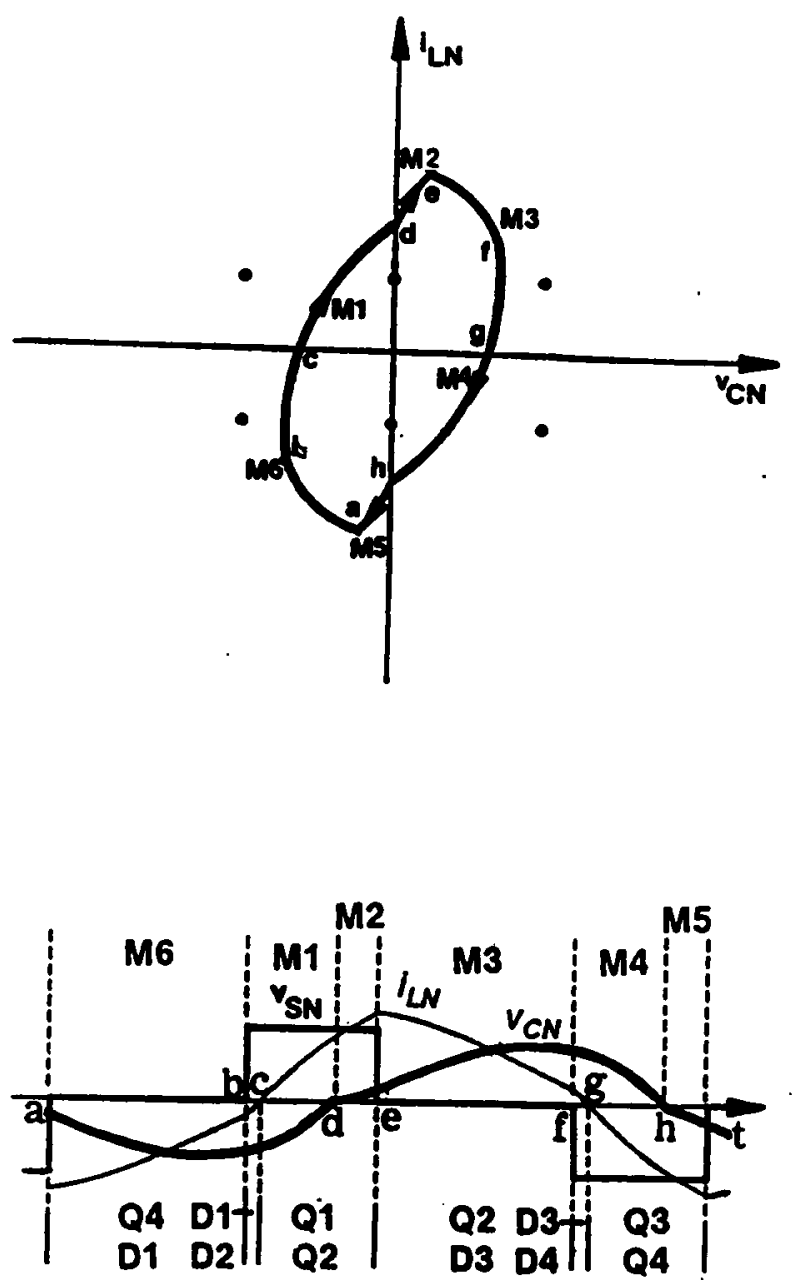

(a) Trajectory $I_{F}$ and its corresponding circuit waveforms(Mode- $I_{F}$ operation) 

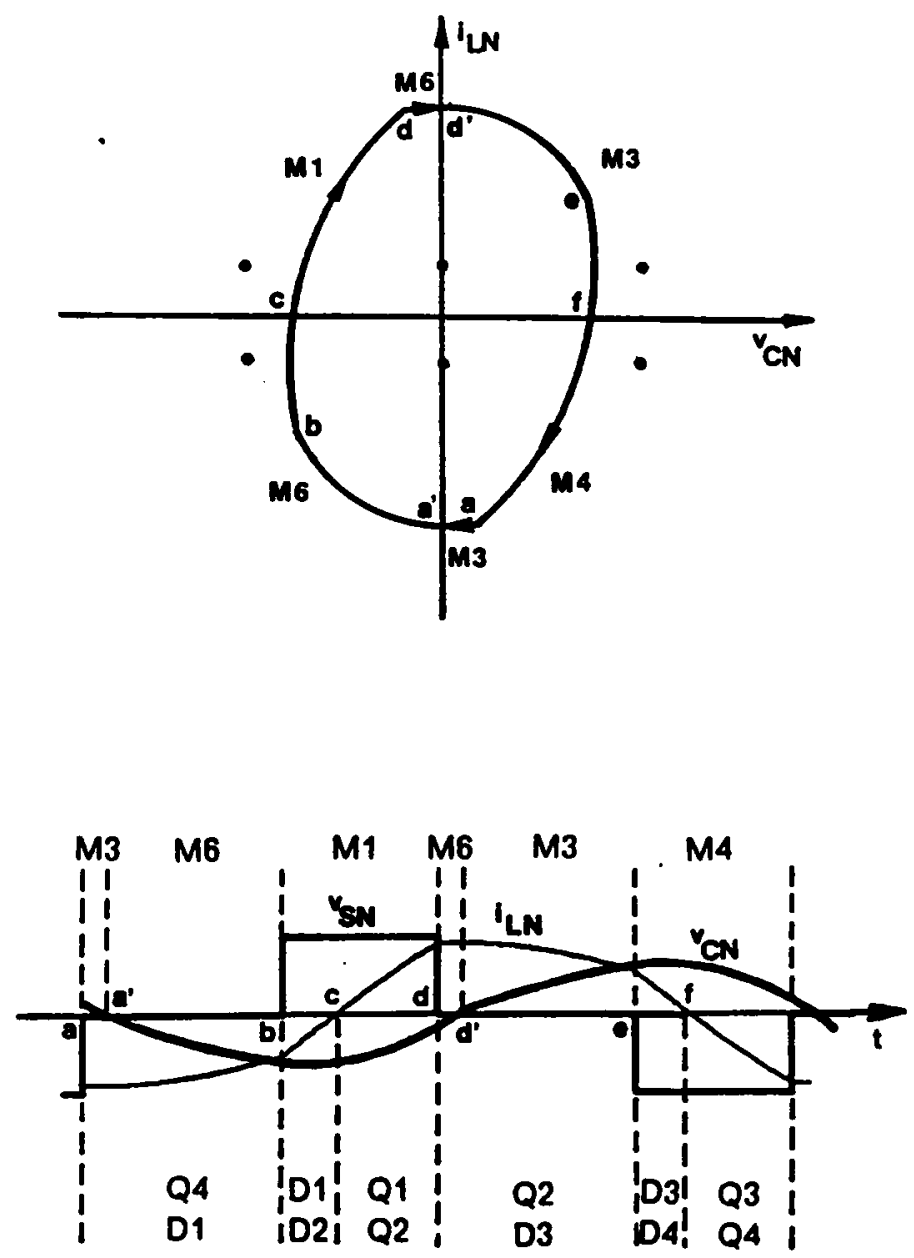

(b) Trajectory $2 A_{F}$ and its corresponding circuit waveforms(Mode- $I I A_{F}$ operation)

Figure 3.9 Continued 

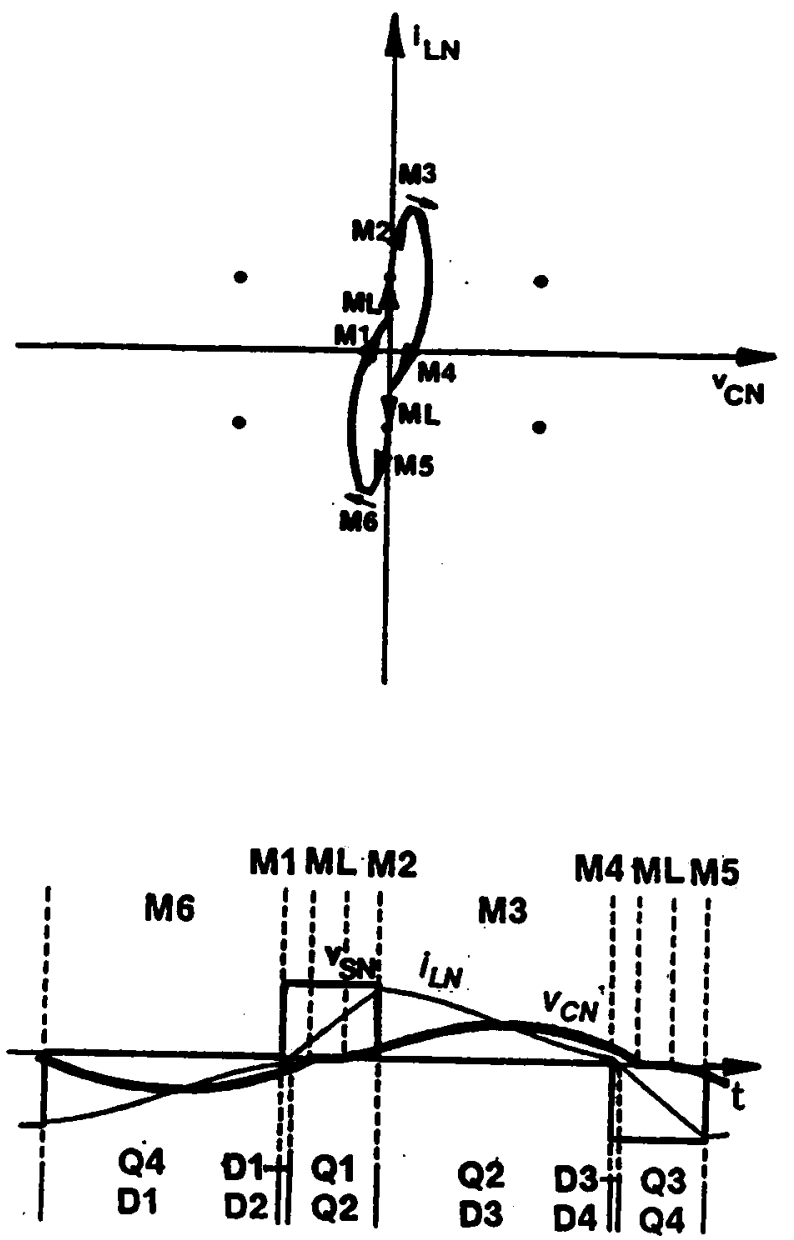

(c) Trajectory $3_{F}$ and its corresponding circuit waveforms(Mode- $I I I_{F}$ operation)

Figure 3.9 Continued 

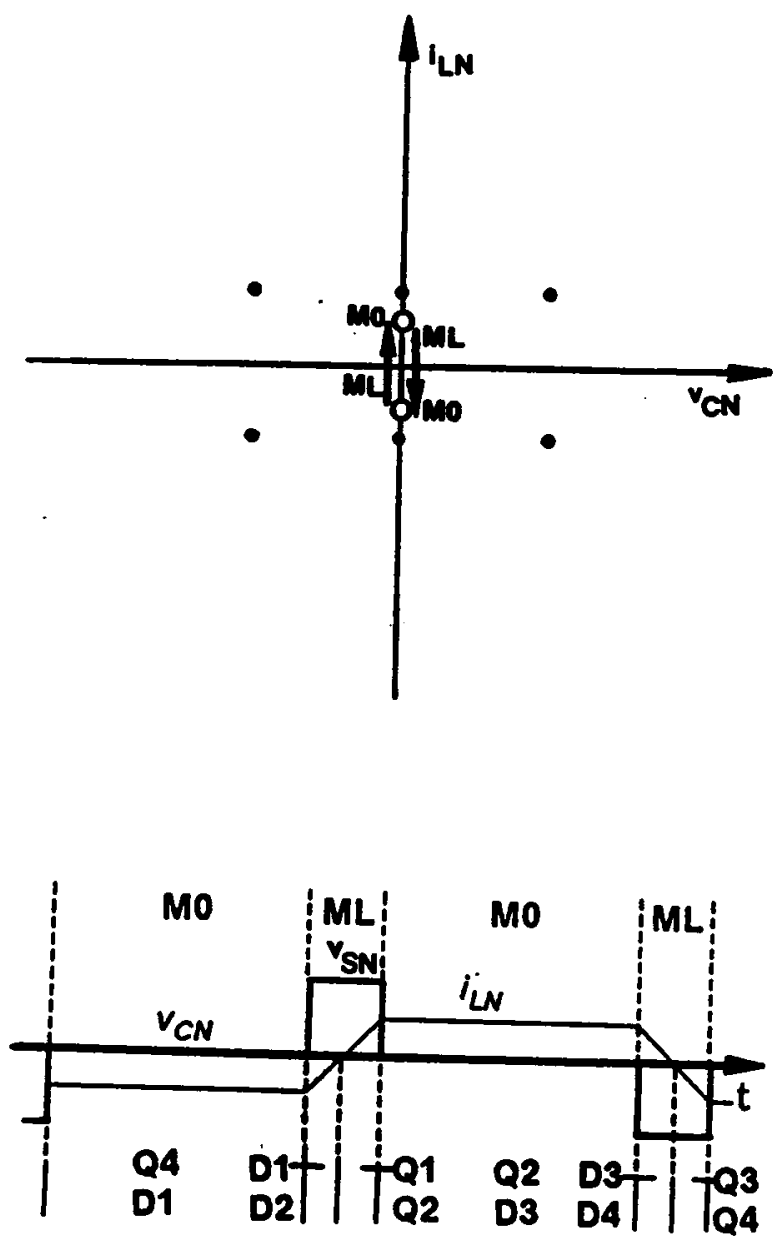

(d) Trajectory $S_{F}$ and its corresponding circuit waveforms(Mode- $V_{F}$ operation)

Figure 3.9 Continued 
(b) Mixed-commutation modes

Trajectory $2 A_{M}$ : As shown in Figure 3.9(f), at $\mathrm{t}=\mathrm{a}$, transistor $\mathrm{Q} 3$ is forced off and Q1 is triggered. Transistor Q1 cannot conduct since $i_{L N}$ is negative. Instead, diode D1 conducts. The inductor current resonates through Q4 and D1 (M3). At $t=a^{\circ}$, capacitor voltage $v_{C N}$ changes polarity. The inductor current continues to resonate through Q4 and D1 (M6). At $\mathrm{t}=\mathrm{b}$, inductor current $i_{L N}$ increases to zero. Transistor Q4, diode D1 turn off naturally and transistors Q1, diode D4 conduct subsequently. The inductor current resonates through $\mathrm{Q} 1$ and D4 (M6). At $\mathrm{t}=\mathrm{c}$, transistor $\mathrm{Q} 2$ is triggered commutating diode D4. The inductor current resonates through $Q 1$ and $Q 2(M 1) . A t t=d$, transistor $\mathrm{Q} 1$ is forced off and Q3 is triggered. A similar process occurs with the roles of Q1,Q2,D1,Q2 and Q3,Q4,D3,D4 interchanged, respectively. The topological mode sequence of this trajectory is the same as that of Trajectory $2 A_{F}$. However, since transistors Q1,Q3 are force-commutated while Q2,Q4 are naturally commutated, the circuit operation for this trajectory is referred to as "mode- $I I A_{M}$ operation".

Table 3.2 summarizes the operating modes above resonant frequency and their corresponding topological mode sequences.

It should be noted that the trajectories discussed above are used only to qualitatively illustrate the existence of various operating modes of a CM-PRC. The frequencies corresponding to the trajectories may not be the same. Some trajectories exist only for a certain range of frequency and load current, as shall be seen in the following section. 

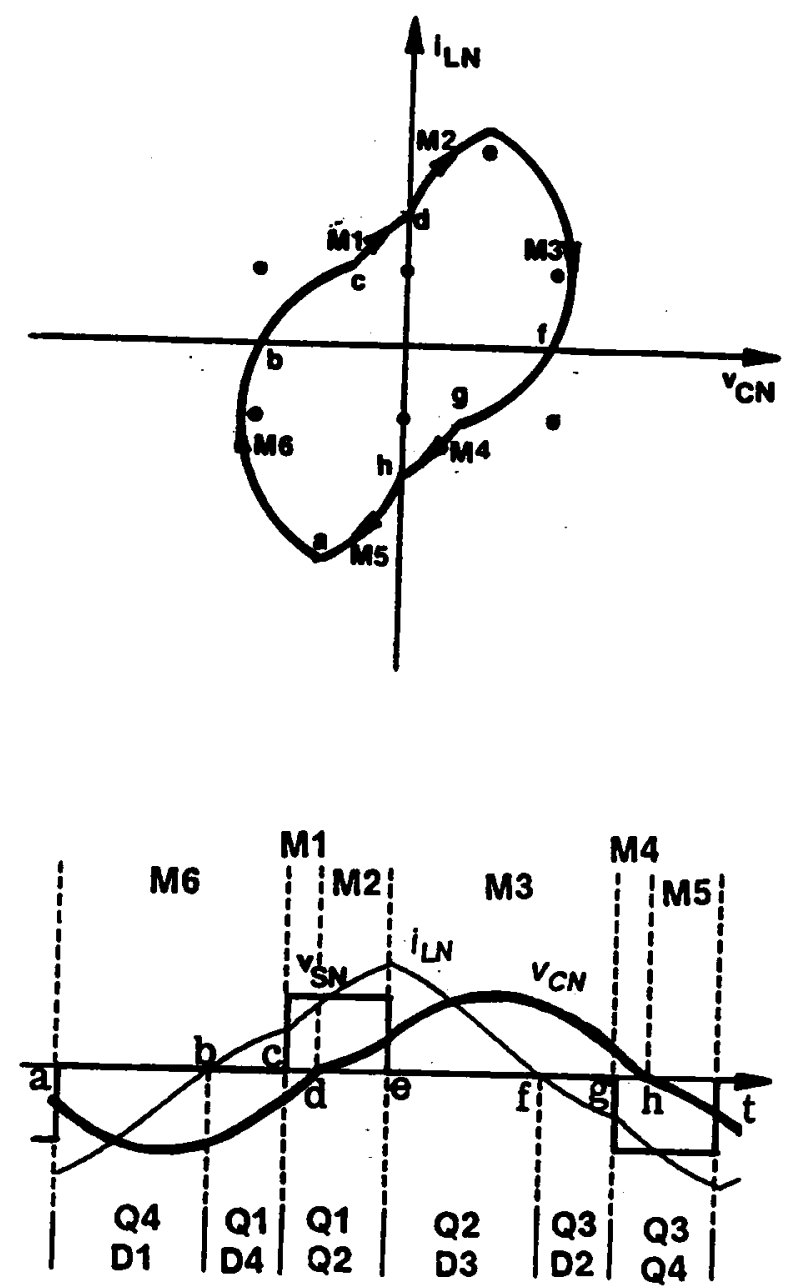

(e) Trajectory $I_{m}$ and its corresponding circuit waveforms(Mode- $I_{m}$ operation)

Figure 3.9 Continued 

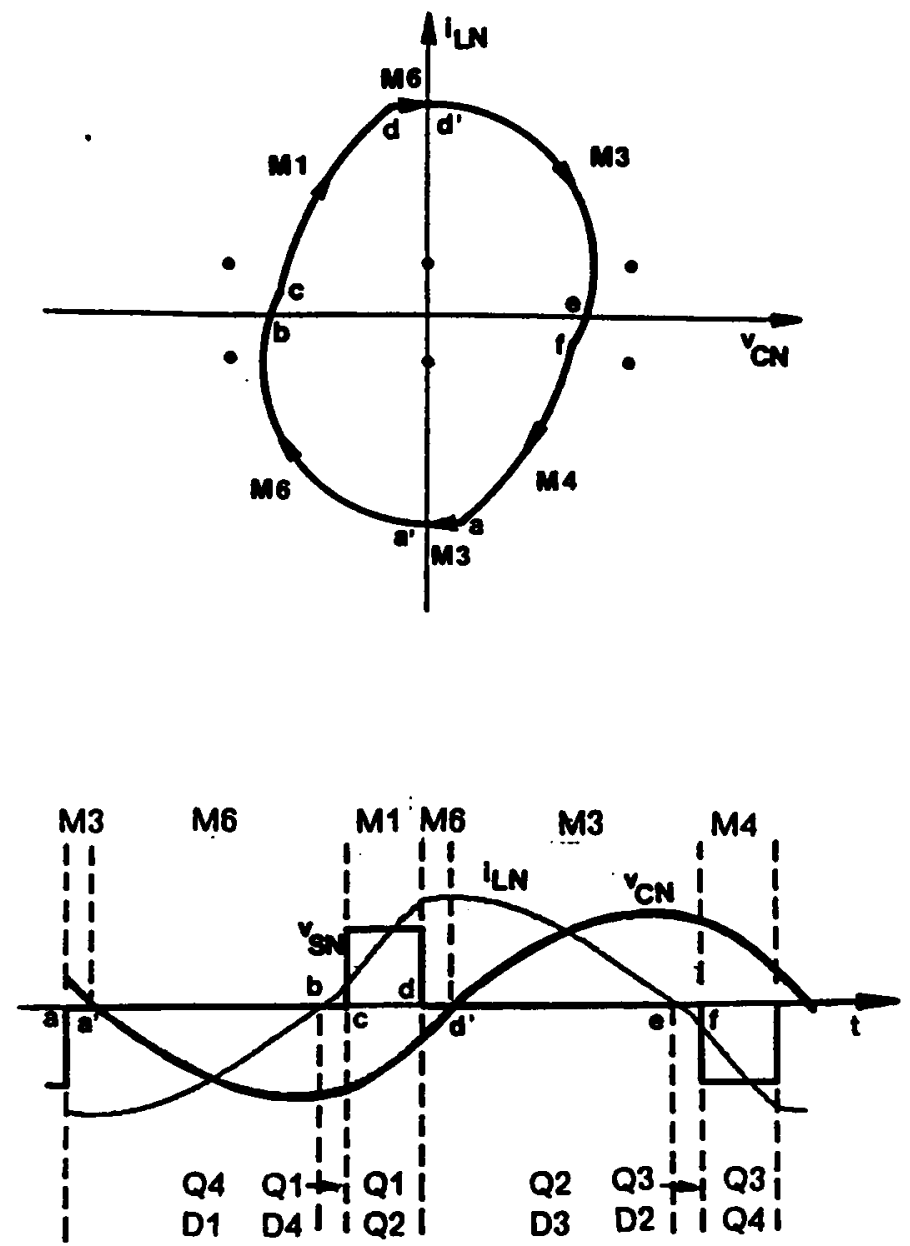

(f) Trajectory $2 A_{M}$ and its corresponding circuit waveforms(Mode-II $A_{M}$ operation)

Figure 3.9 Continued 
Table 3.2 Topological Mode Sequences for Circuit Operating Modes Above Resonant Frequency

\begin{tabular}{|c|c|}
\hline $\begin{array}{c}\text { Operating } \\
\text { Modes }\end{array}$ & Topological Mode Sequences \\
\hline$I_{M_{1}} I_{F}$ & $M 6-M 1-M 2-M 3-M 4-M 5$ \\
\hline$I I A_{M}, I I A_{F}$ & $M 3-M 6-M 1-M 6-M 3-M 4$ \\
\hline$I I I_{F}$ & $M 6-M 1-M L-M 2-M 3-M 4-M L-M 5$ \\
\hline$V_{F}$ & $M 0-M L-M 0-M L$ \\
\hline
\end{tabular}




\subsubsection{Regions of Operation}

As mentioned in Chapter 2, it is important to know the converter's mode of operation for a specific design such that circuit devices such as diodes and snubbers can be optimally selected for the converter. In this section, regions of operation for a CM-PRC are defined to determine the converter's mode of operation for given normalized switching frequency, $\omega_{S N}=\frac{\omega_{S}}{\omega_{0}}$, pulse width, $\beta_{S}$, of the quasi-square-wave voltage $v_{S}$, and the normalized load current, $I_{O N}=\frac{Z_{0} \times I_{O}}{E}$.

\subsubsection{Operating regions below resonant frequency}

Figure 3.10 shows the operating regions of a CM-PRC operating below resonant frequency. In the figure, the mode of operation is represented as a function of the pulse width, $\beta_{S}$, of the quasi-square-wave voltage, $v_{S}$, and the load current, $I_{O_{N}}$, at several frequencies $\omega_{S N}=0.6,0.7,0.8$, and 0.9. $\wedge \beta_{S}$ angle of $180^{\circ}$ is equivalent to a full pulse-width of $v_{S}$ (duty ratio $=1$ ). Three dotted lines are shown in each figure to indicate boundaries for different device commutation conditions. $\Lambda$ bove the dotted line ${ }^{N} \mathrm{~N}$ is the natural-commutation region in which all the transistors are naturally commutated. Between the dotted lines " $\mathrm{N}$ " and " $\mathrm{F}$ " is the mixed-commutation region in which transistors Q1,Q3 are force-commutated and Q2,Q4 are naturally commutated. Below the dotted line " $F$ " is the force-commutation region in which all the transistors are forcecommutated. The dotted line "MI." defines a region corresponding to mode- $I I_{M L}$ operation, where the transistors conduct twicc during a switching period. This multiple-conduction region is undesirable and should be avoided. Given the range of $I_{O N}, \beta_{S}$, and a specific operating frequency, the converter's modes of operation can be easily determined. For example, if $\omega_{S N}$ equals $0.8, I_{O N}$ ranges from 0.25 to 0.5 , and $\beta_{S}$ ranges from $144^{\circ}$ to $108^{\circ}$, the converter will operates either in mode $I_{N}$ or in mode $I I_{N}$, as indicated by the shaded area in rigurc 3.10(c). 


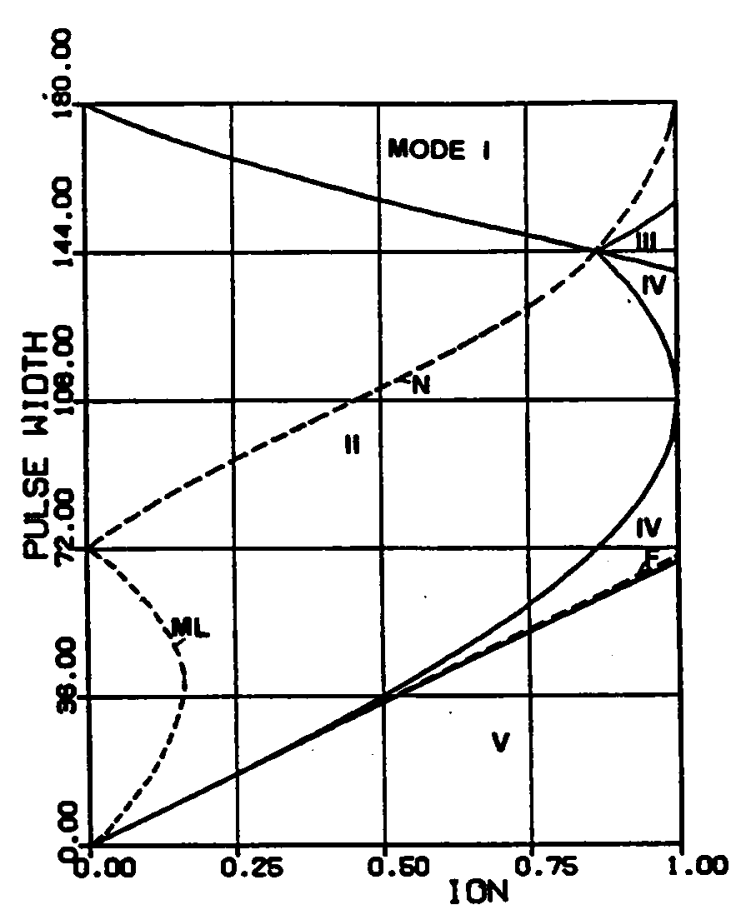

(a) $\omega_{S N}=0.6$

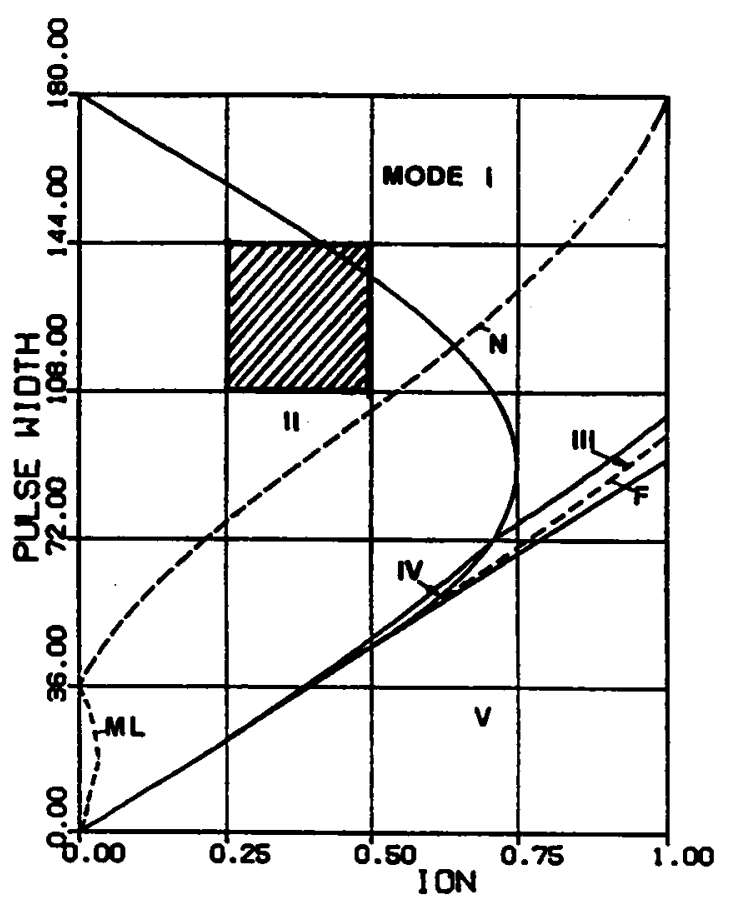

(c) $\omega_{S N}=0.8$

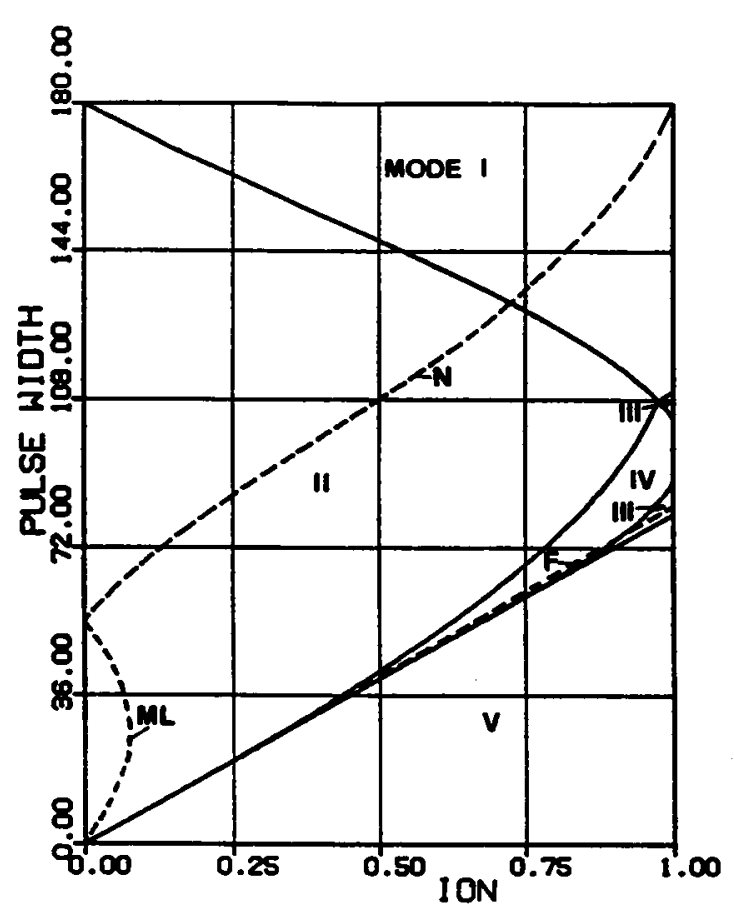

(b) $\omega_{S N}=0.7$

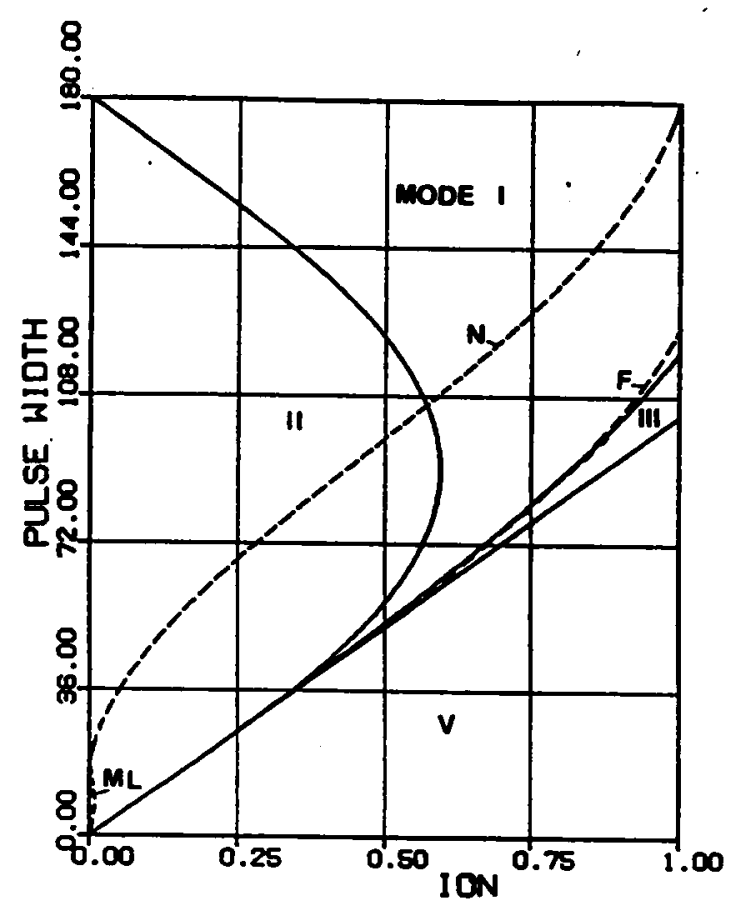

(d) $\omega_{S N}=0.9$

Figure 3.10 Regions of Operation Below Rcsonant Frequency 
For other frequencies not shown in Figure 3.10, an algorithm is developed in $\Lambda p$ pendix C.2 which can be used to plot similar graphs to those in Figure 3.10.

\subsubsection{Operating regions above resonant frequency}

Figure 3.11 shows sevcral operating regions above resonant frequency. These regions are derived from the algorithm developed in Appendix C.3. A dotted line is used in the figures for $\omega_{S N}=1.1$ and 1.2 to define the mixed-commutation region. Inside the dotted line, transistors $\mathrm{Q} 1, \mathrm{Q} 3$ are force-commutated and $\mathrm{Q} 2, \mathrm{Q} 4$ are naturally commutated. Outside the dotted line, all the transistors are force-commutated. The mixedcommutation region disappears when the normalized switching frequency increases beyond 1.2 , as can be seen from the figure. 


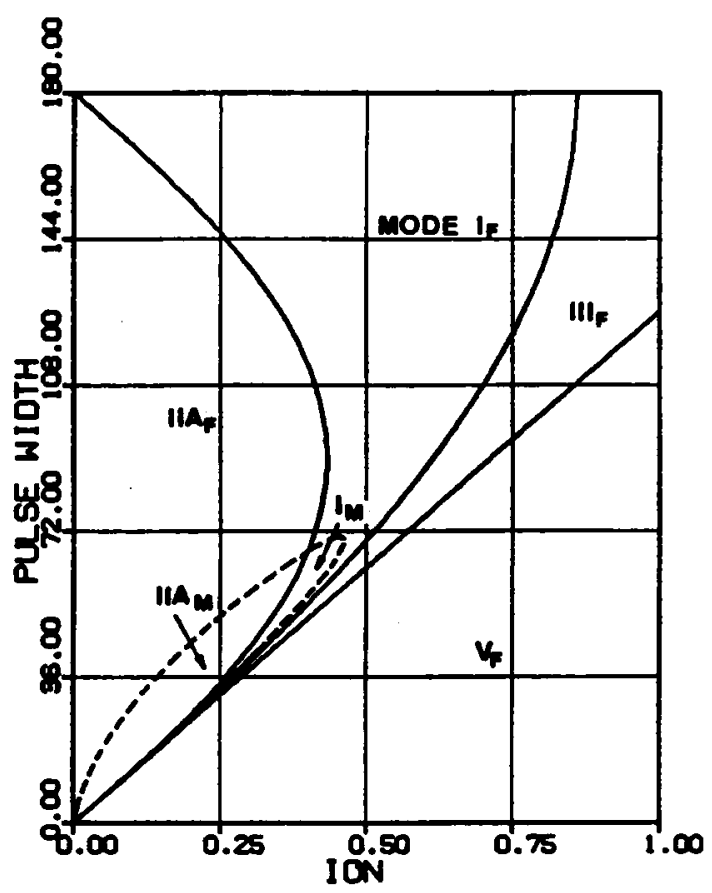

(a) $\omega_{S N}=1.1$

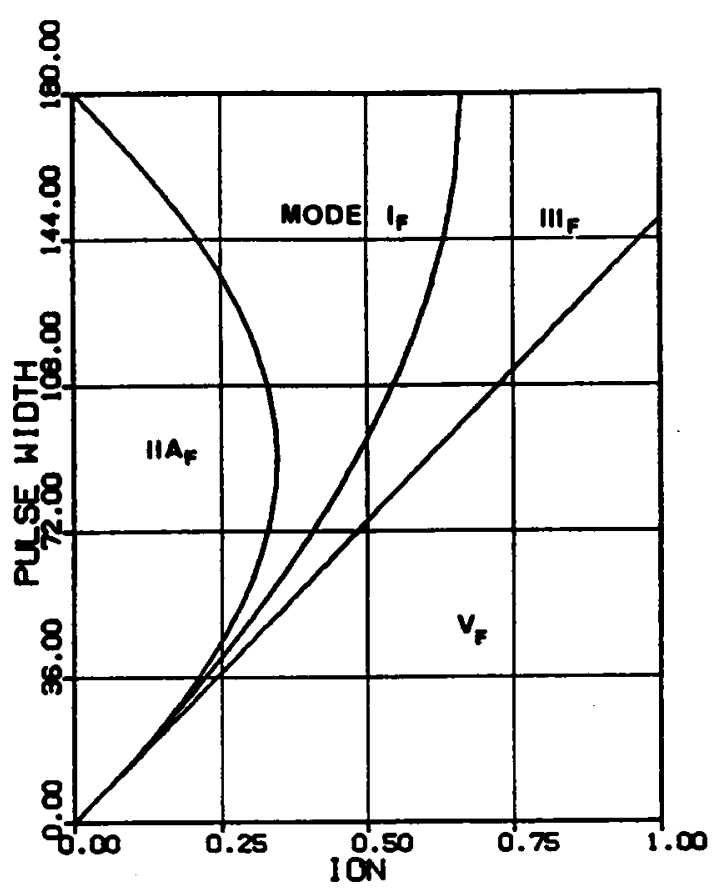

(c) $\omega_{S N}=1.3$

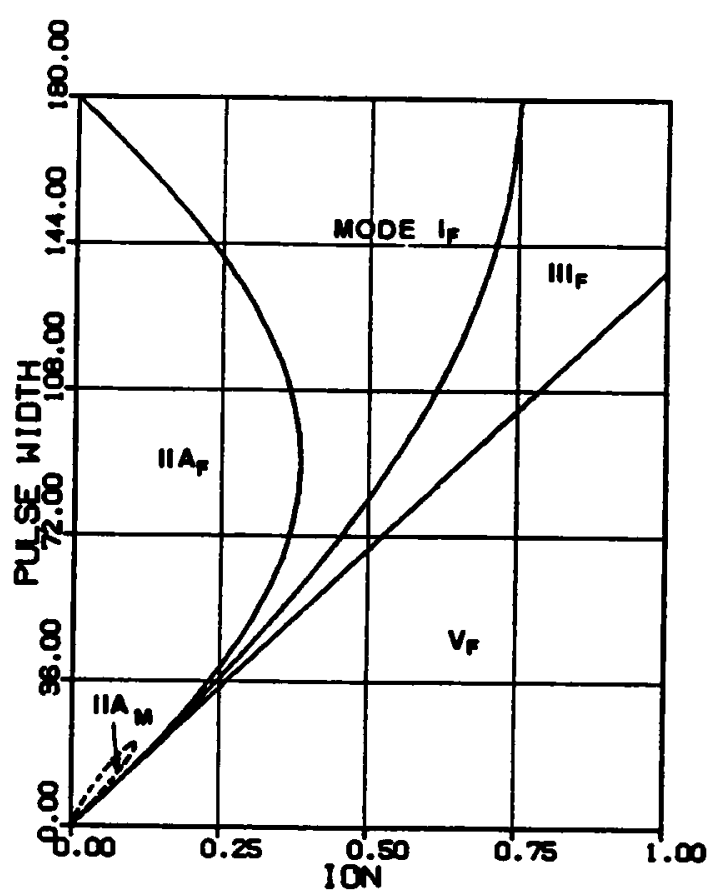

(b) $\omega_{S N}=1.2$

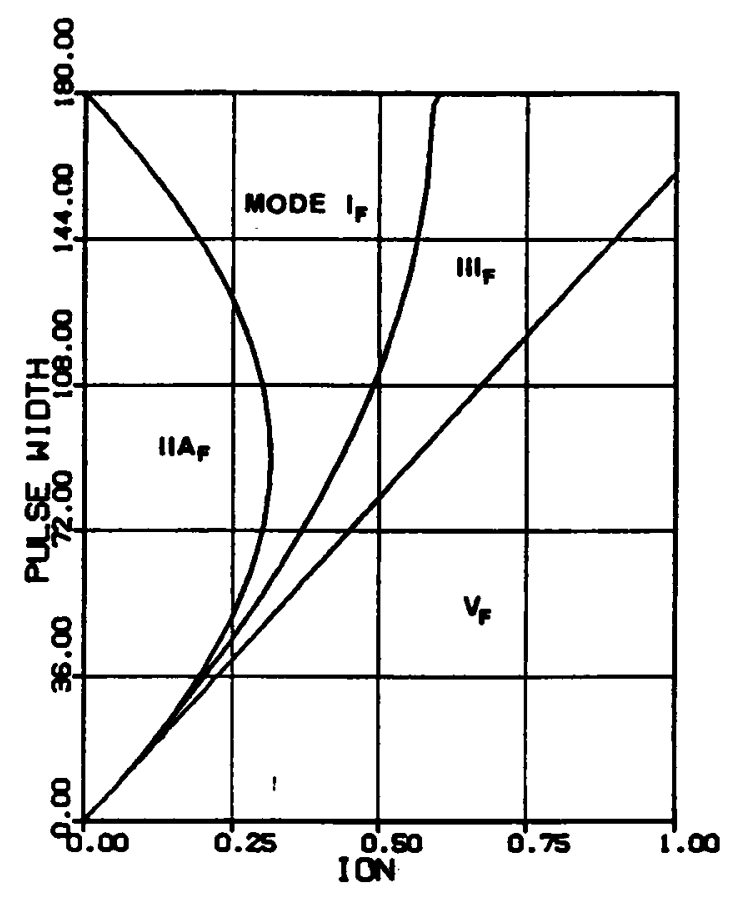

(d) $\omega_{S N}=1.4$

Figure 3.11 Regions of Operation Above Resonant Frequency 


\subsubsection{Characteristics}

Once the regions of operation is defined, the dc characteristics for a CM-PRC can be derived following a similar approach as in Section 2.3.7. The equations for solving trajectory parameters for various operating modes below and above resonant frequency are summarized in Appendix C.4. A Fortran program for calculating salient circuit features such as control-to-output transfer ratio, rms inductor current, peak capacitor voltage, rms switch currents, etc. is included in Appendix C.5.

\subsubsection{DC characteristics below resonant frequency}

Figure 3.12 shows the dc control-to-output characteristics for several frequencies below the resonant frequency. In these figures, the output voltage (average capacitor voltage), $V_{o N}$, is plotted as a function of the pulse-width, $\beta_{S}$, of $v_{S}$, and the output current, $I_{O N}$. Two dotted lines are used in each graph to indicate the boundaries of natural commutation and force commutation. $\Lambda$ bove the dotted line " $\mathrm{N}$, all the transistors are naturally commutated. Between the dotted lines " $N$ " and " $F$ ", transistors $Q 1, Q 3$ are force-commutated and $\mathrm{Q} 2, \mathrm{Q} 4$ are naturally commutated. Below the dotted line " $\mathrm{F}^{\prime \prime}$, all the transistors are force-commutated. It can be seen that the converter is able to regulate the output from no load to a full load in the natural-commutation region. The region, however, is limited, especially when the switching frequency is below $80 \%$ of the resonant frequency. It is thus dificult to design a $P M-P R C$ in the natural-commutation region when the output-to-input voltage ratio, $V_{O N}$, varies over a wide range. For example, if $\omega_{S N}=0.8$ and $V_{O N}$ ranges from 1.2 to $1.8, I_{O N}$ has to be limited less than 0.15 , as can be seen from Figure 3.12(c). This can only be achieved either by using a small characteristic impedance or by limiting the load current to a small valuc. In either case, the circulating current in the tank circuit is high and the components are subjected to excessive stresses. 


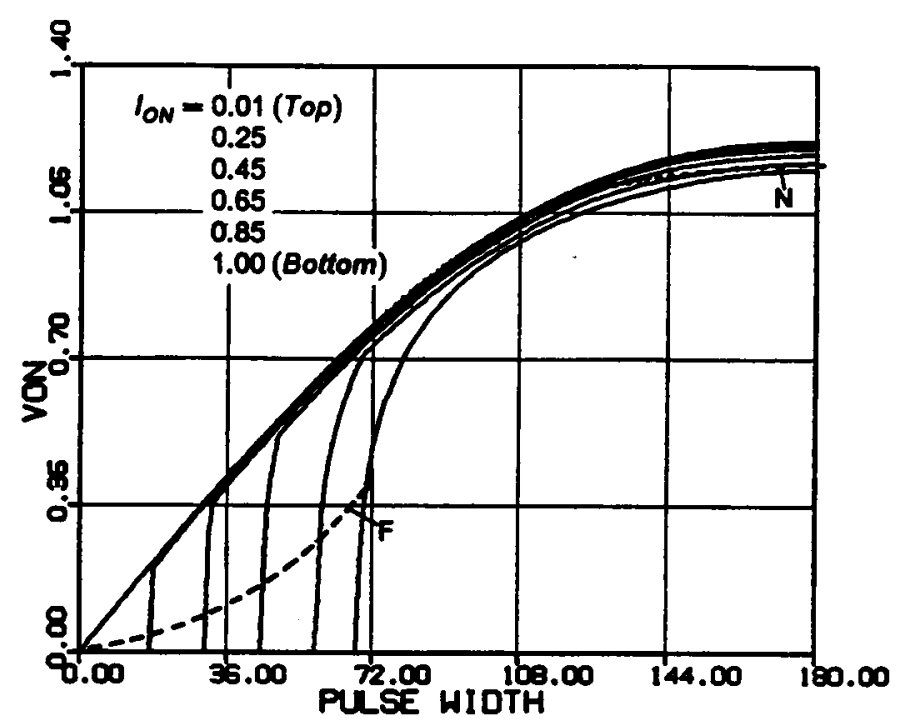

(a) $\omega_{S N}=0.6$

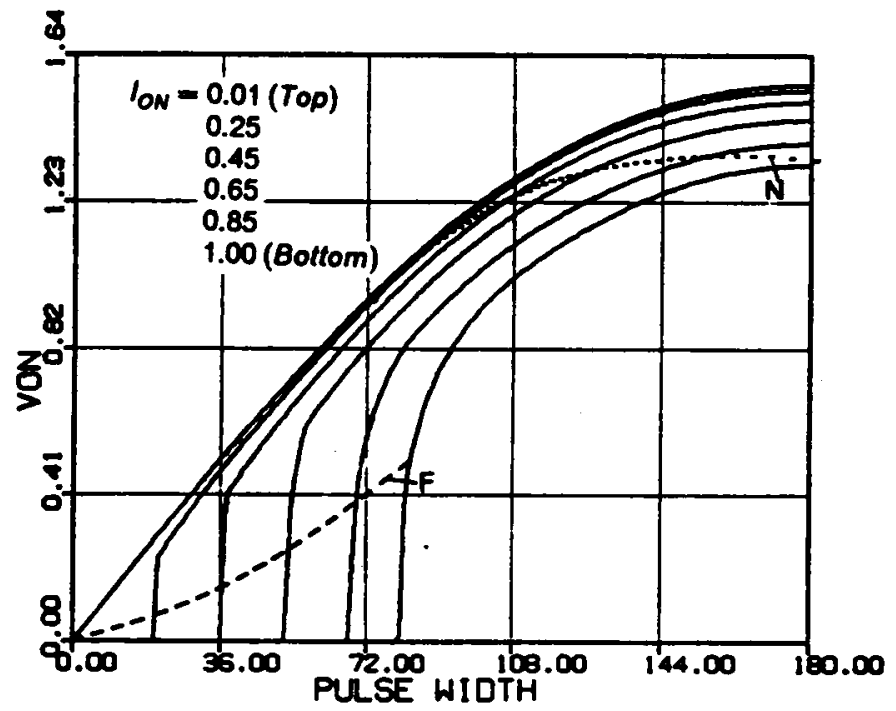

(b) $\omega_{S N}=0.7$

Figure 3.12 DC Control-to-Output Characteristics Below Resonant Frequency 


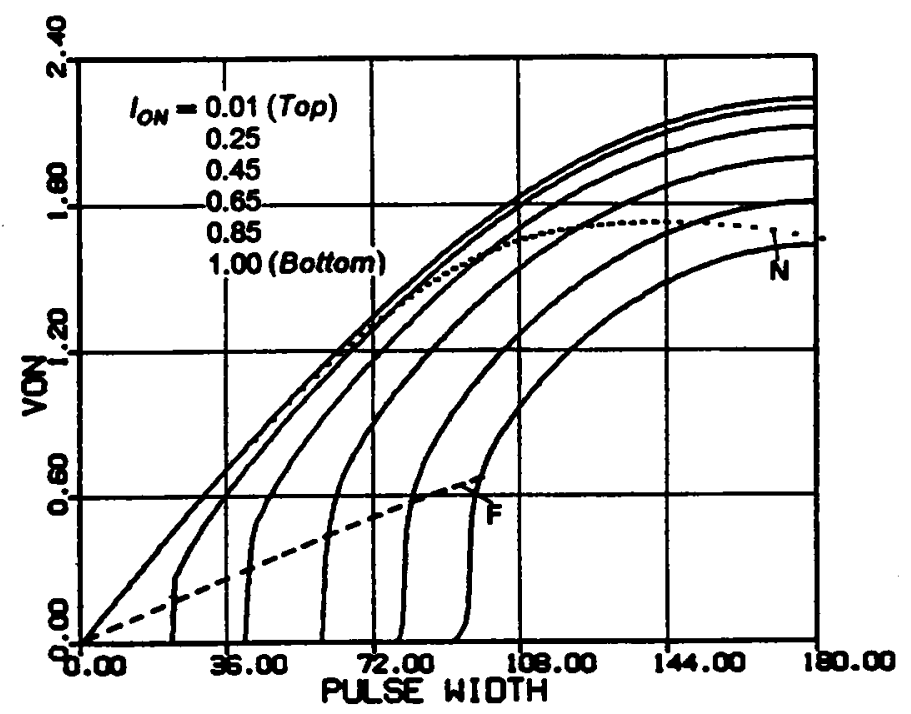

(c) $\omega_{S N}=0.8$

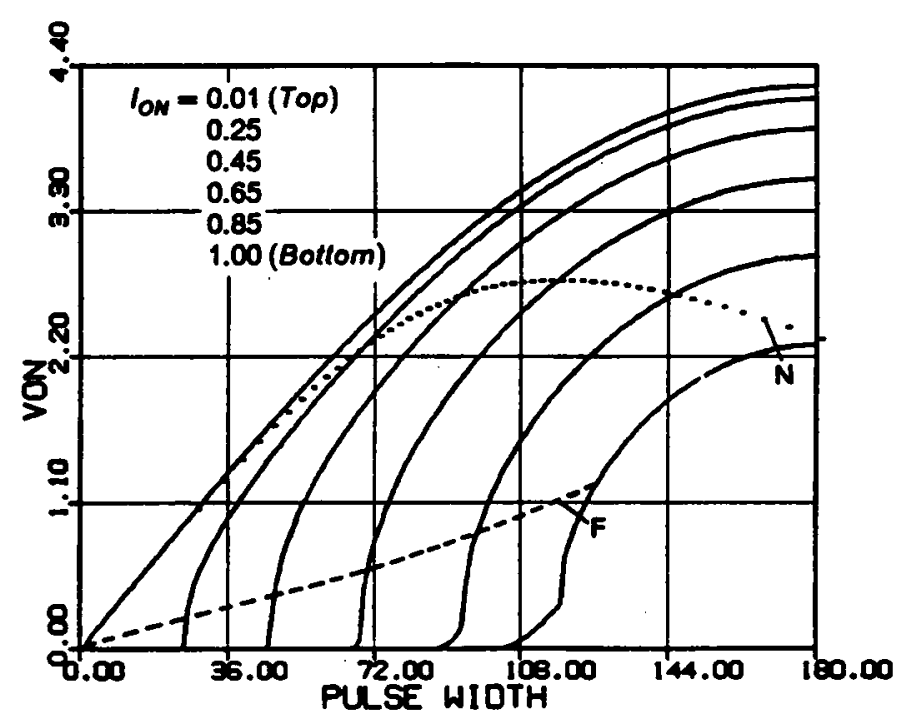

(d) $\omega_{S N}=0.9$

Figure 3.12 Continued 


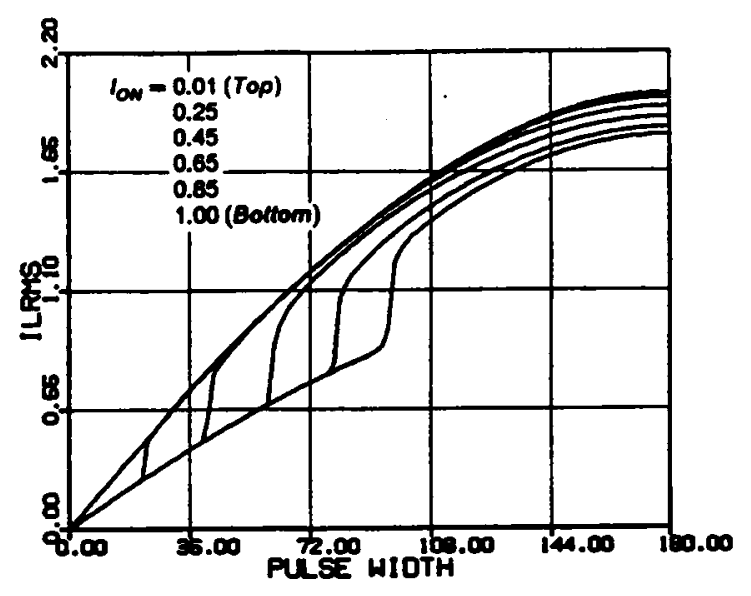

(a) RMS inductor current

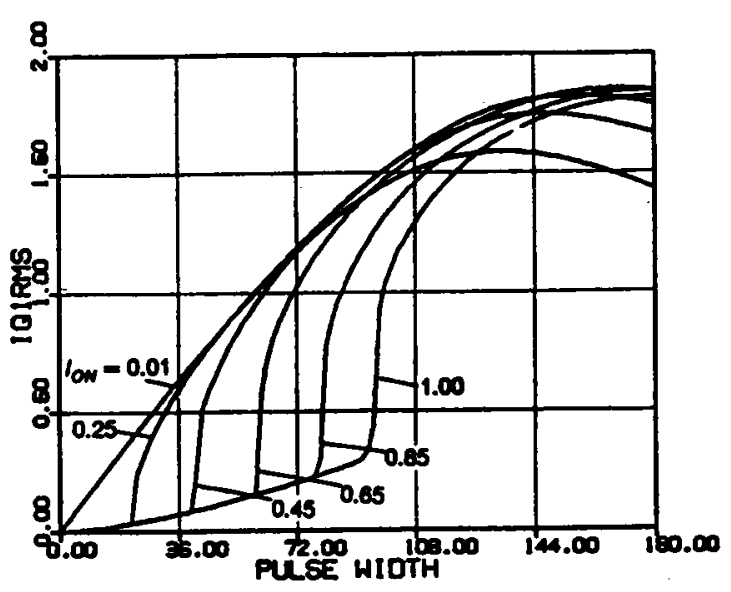

(c) RMS switch current (Q1.Q3)

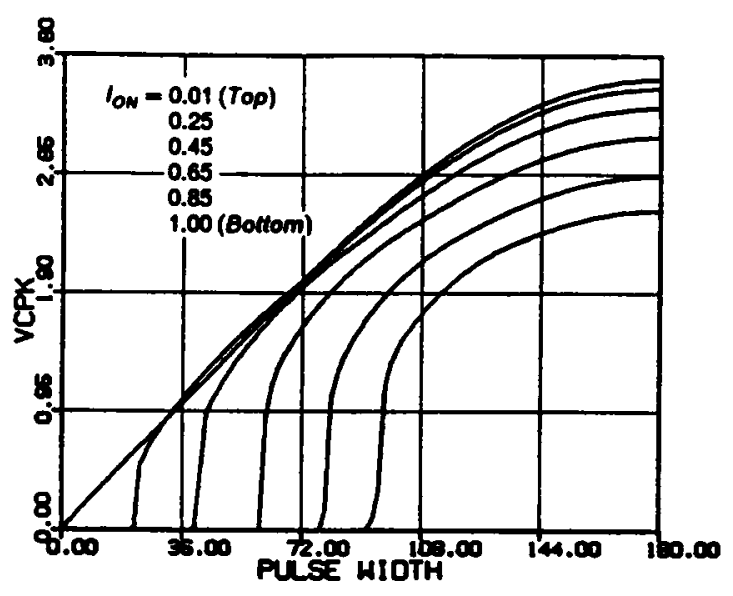

(b) Peak capacitor voltage

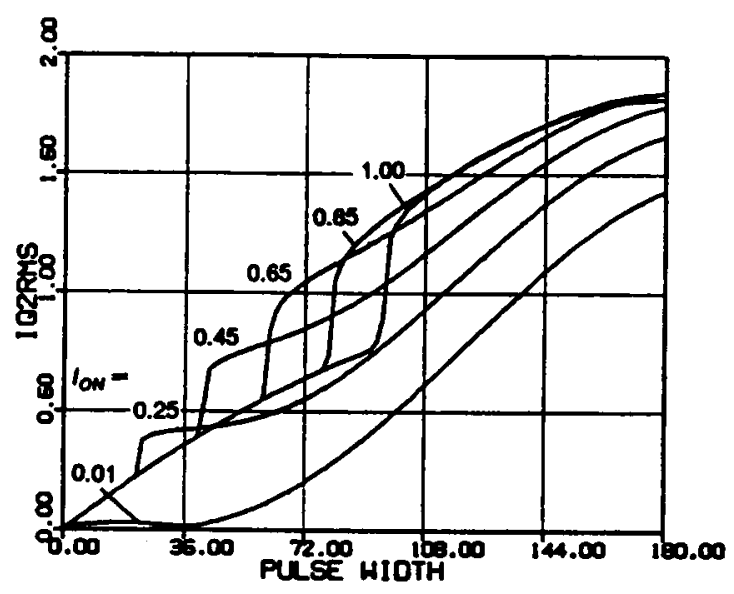

(d) RMS switch current $(Q 2, Q 4)$

Figure 3.13 Characteristics for Various Circuit Salient Features at $\omega_{S N}=0.8$ 


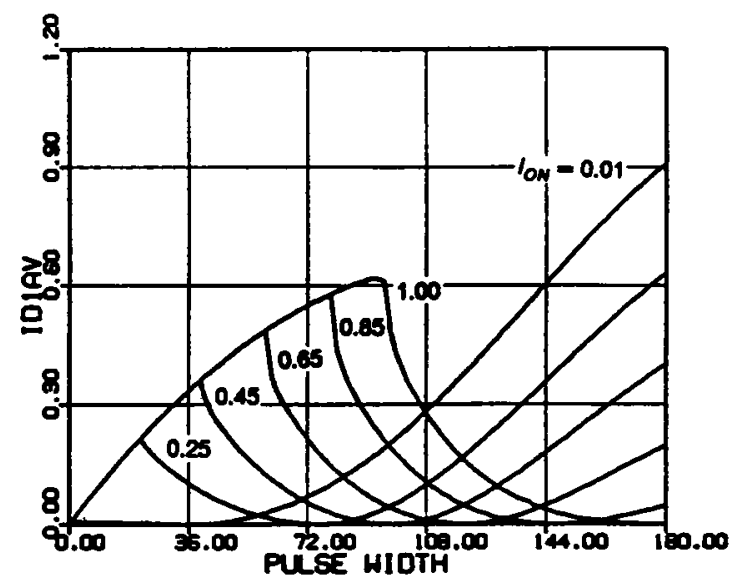

(e) Average diode current (DI,D3)

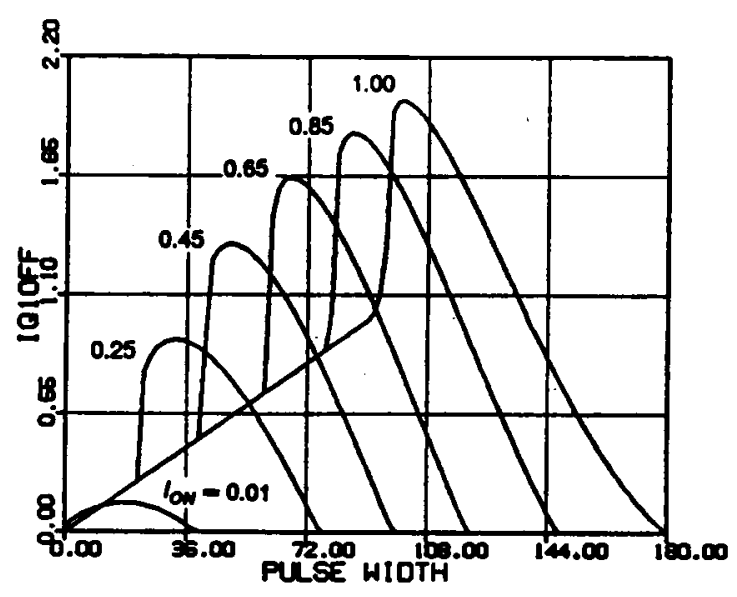

(g) Switch turn-off current (Q1,Q3)

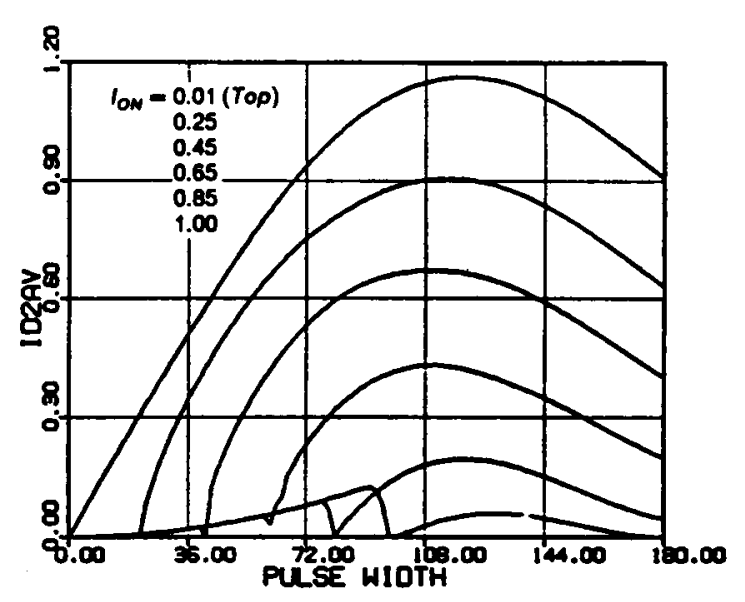

(f) Average diode current (D2,D4)

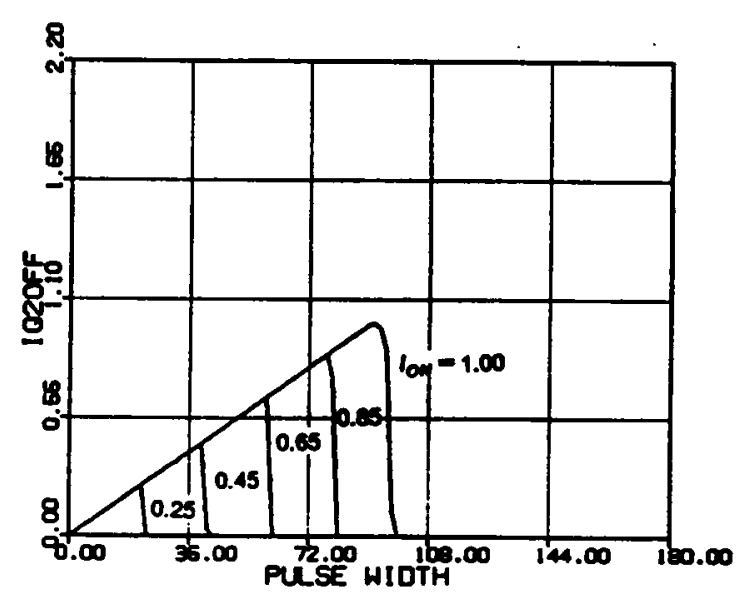

(h) Switch turn-off current (Q2,Q4)

Figure 3.13 Continued 


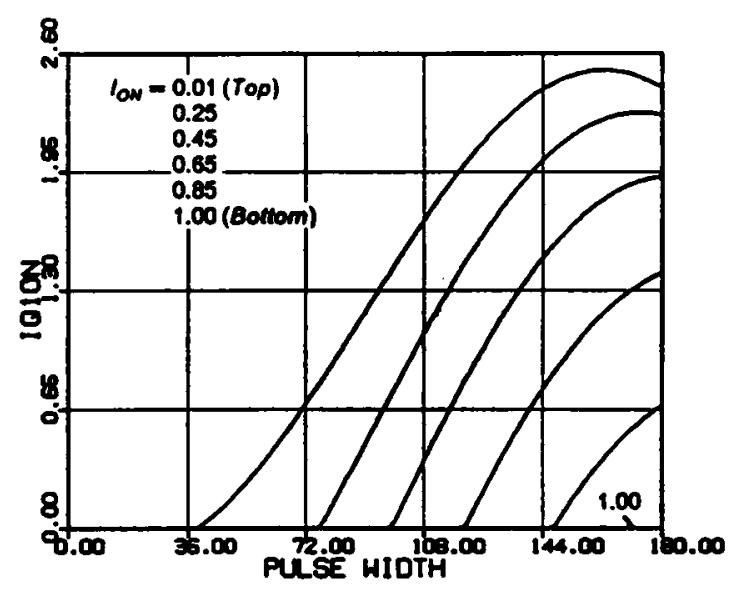

(i) Switch turn-on currént $\left(Q 1, Q^{3}\right)$

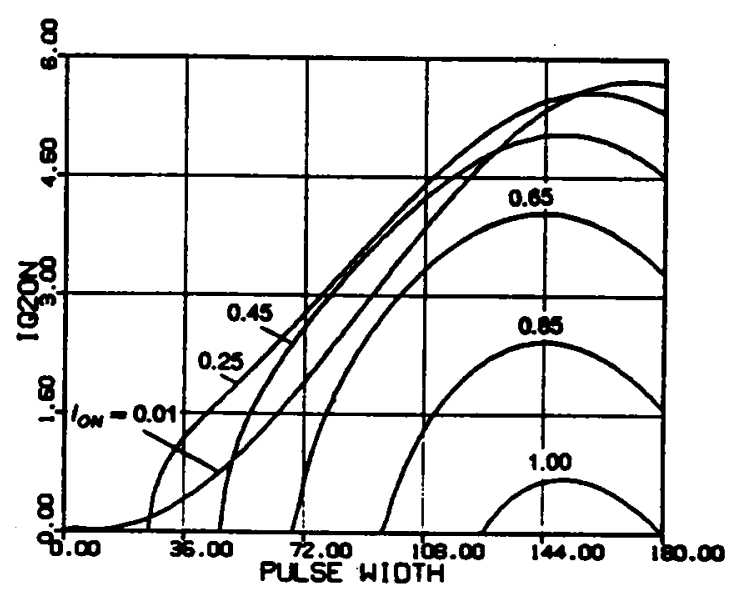

(j) Switch turn-on current (Q2,Q4)

Figure 3.13 Continued 
An alternative is to design the converter in the mixed-commutation region. From Figure 3.12, it can be seen that the mixed-commutation mode can be operated over a wide range of load current and output-to-input voltage ratio. However, a minimum load has to be maintained if the converter is to be designed in this region.

The force-commutation region in this frequency range is very limited and is impractical to use. essentially useless. It allows only a small variation in the output-to-input voltage ratio. It requires a minimum load current. Most of all, since the slopes of the characteristics in this region are very steep, the converter is very difficult to control.

Figure 3.13 shows other important dc characteristics at frequency $\omega_{S N}=0.8$. Notice that the currents of Q1,Q3 and the currents of Q2,Q4 are not balanced, as can be seen from Figures 3.13(c) and (d). Same phenomenon exits between the currents of D1,D3 and D2,D4. This is due to the phase displacement in the triggering of the transistors. In general, transistors Q1,Q3 and diodes D2,D4 carry higher currents when CM-PRC operates below the resonant frequency.

Similar dc characteristics for the frequency, $\omega_{S N}=0.9$, can be found in Appendix C.6.

\subsubsection{DC characteristics above resonant frequency}

Figure $3.14(\mathrm{a}) \sim(\mathrm{d})$ shows the dc control-to-output characteristics for $\omega_{S N}=1.1,1.2,1.3$, and 1.4 , respectively. $\Lambda$ dotted line is used in the figures for $\omega_{S N}$ $=1.1$ and 1.2 to indicate the mixed-commutation boundary. Inside the dotted line, transistors Q1,Q3 are force-commutated and Q2.Q4 are naturally commutated. Outside the dotted line, all the transistors are forcc-commutated. It can be seen that in this frequency range, the converter is able to regulate its output from no load to full load over a wide range of output-to-input voltage ratio.

Figure 3.15 shows other important dc characteristics at frequency $\omega_{S N}=1.2$. Again, the currents in the transistors and the currents in the diodes are unbalanced, as shown 
in Figures 3.15(c) and (d) and Figures 3.15(e) and (f), respectively. However, in this frequency range, transistors Q2,Q4 and diodes D1,D3 carry higher currents.

Similar dc characteristics for other frequencies can be found in $\Lambda$ ppendix C.7. 


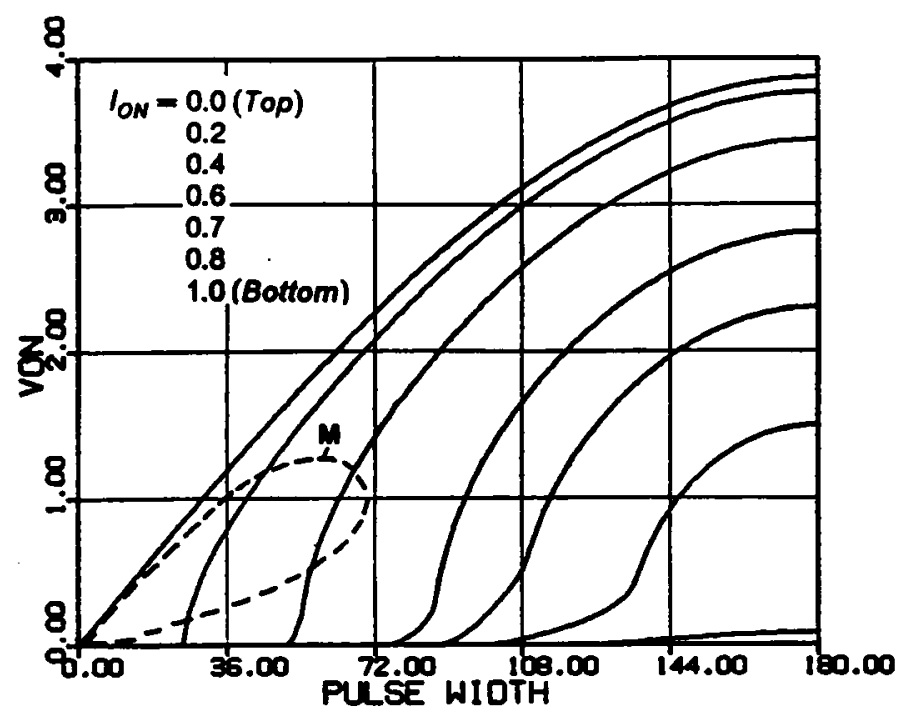

(a) $\omega_{S N}=1.1$

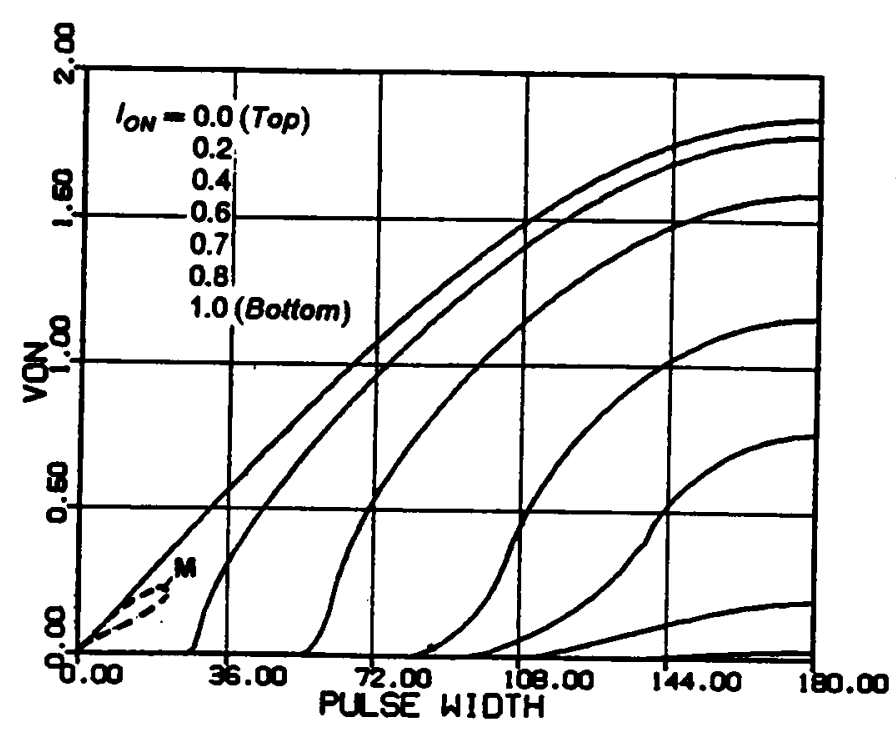

(b) $\omega_{S N}=1.2$

Figure 3.14 DC Control-to-Output Characteristics Above Resonant Frequency 


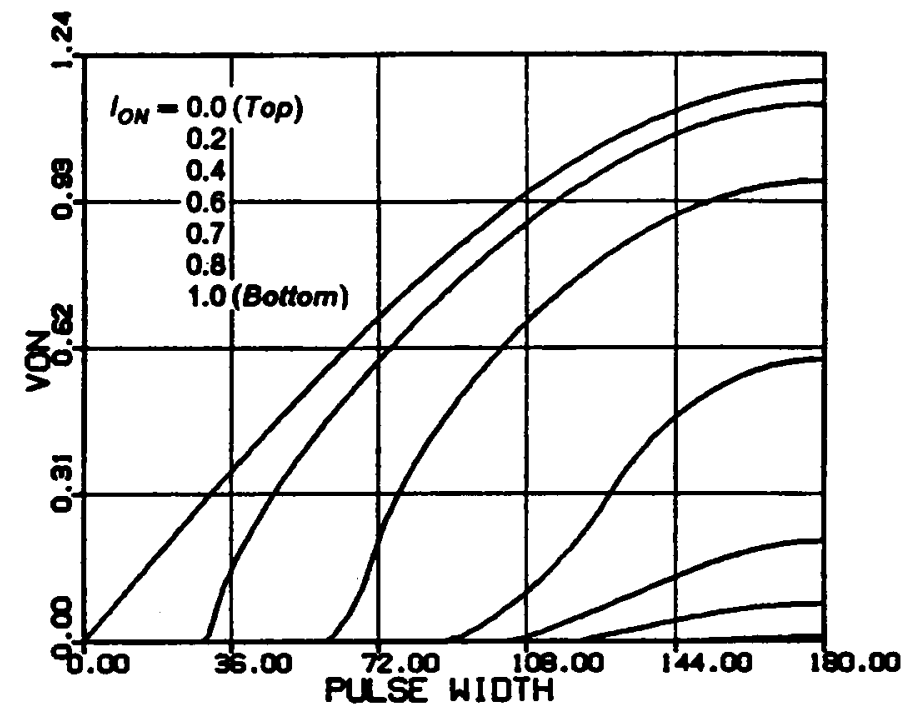

(c) $\omega_{S N}=1.3$

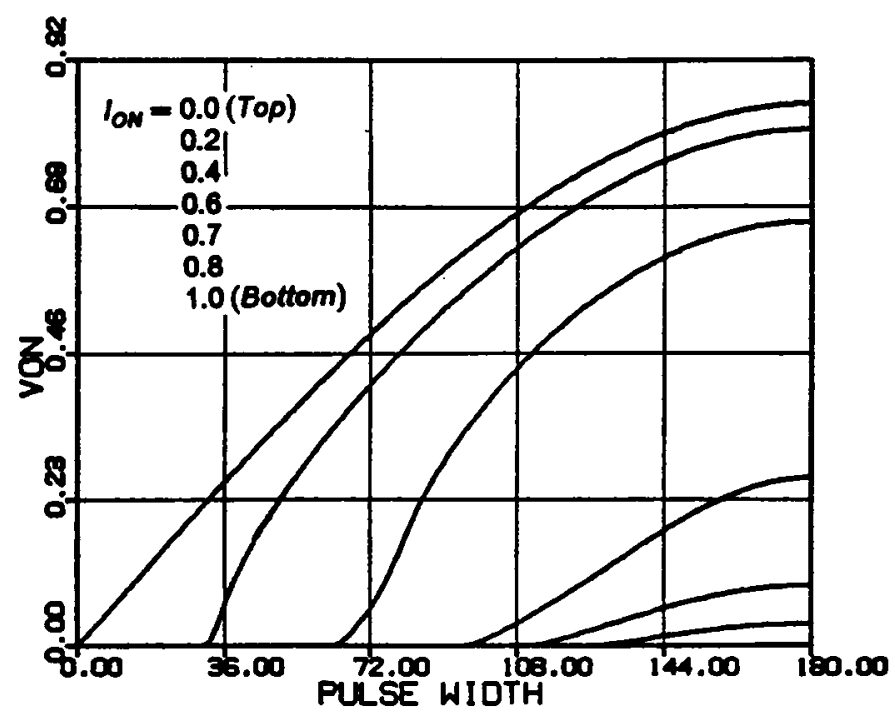

(d) $\omega_{S N}=1.4$

Figure 3.14 Continued 


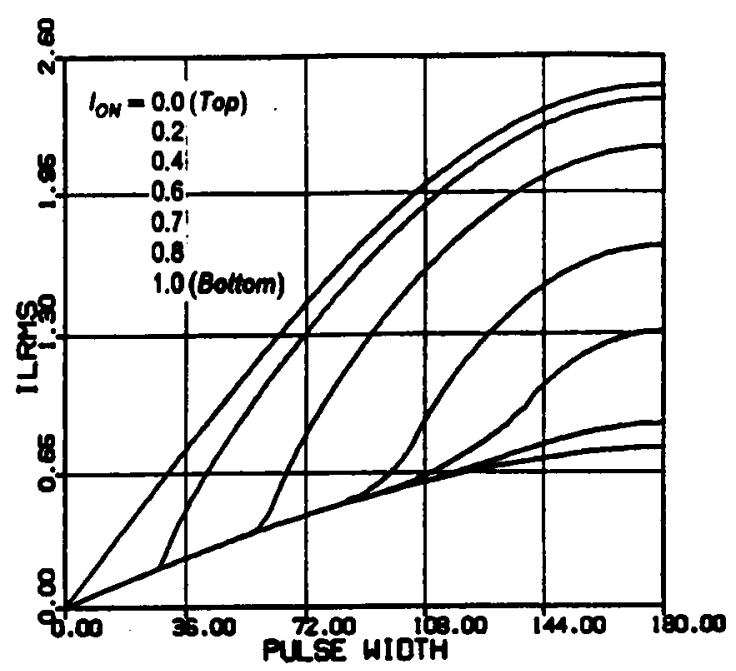

(a) RMS inductor current

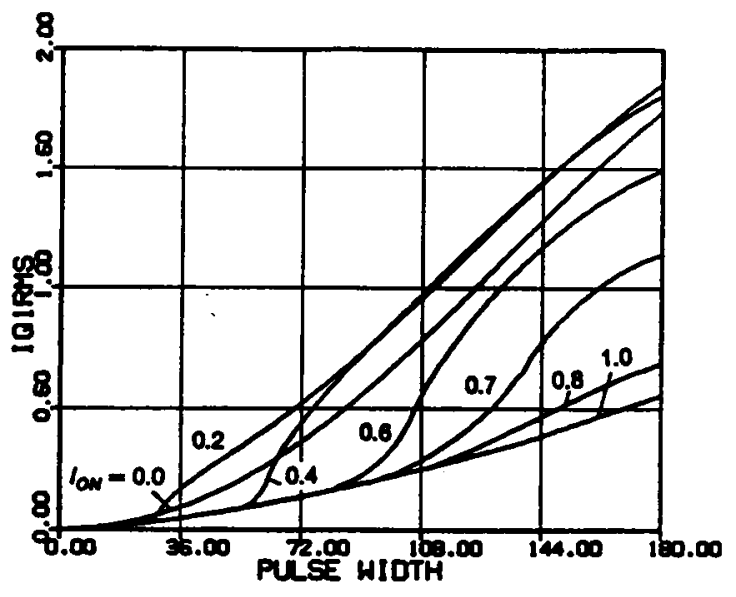

(c) RMS switch current $(Q 1, Q 3)$

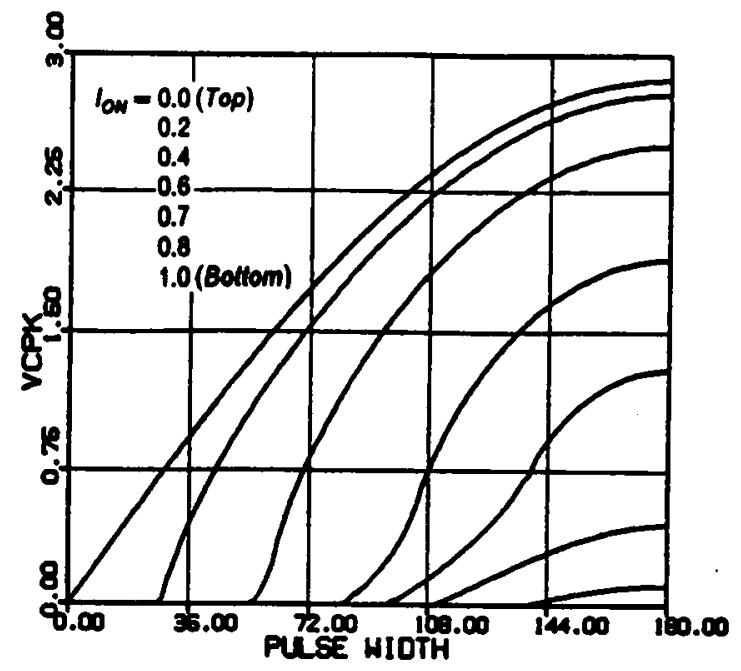

(b) Peak capacitor voltage

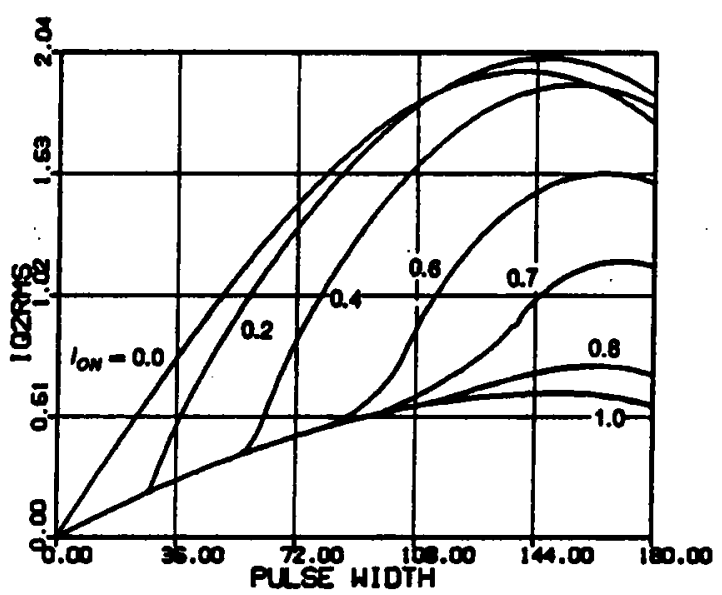

(d) RMS switch current $(Q 2, Q 4)$

Figure 3.15 Characteristics for Various Circuit Salient Features at $\omega_{S N}=1.2$ 


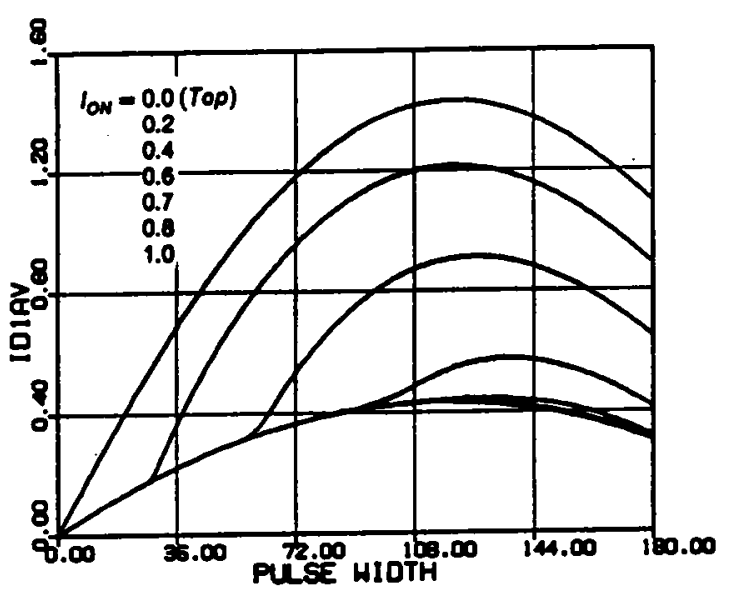

(e) Average diode current (DI,D3)

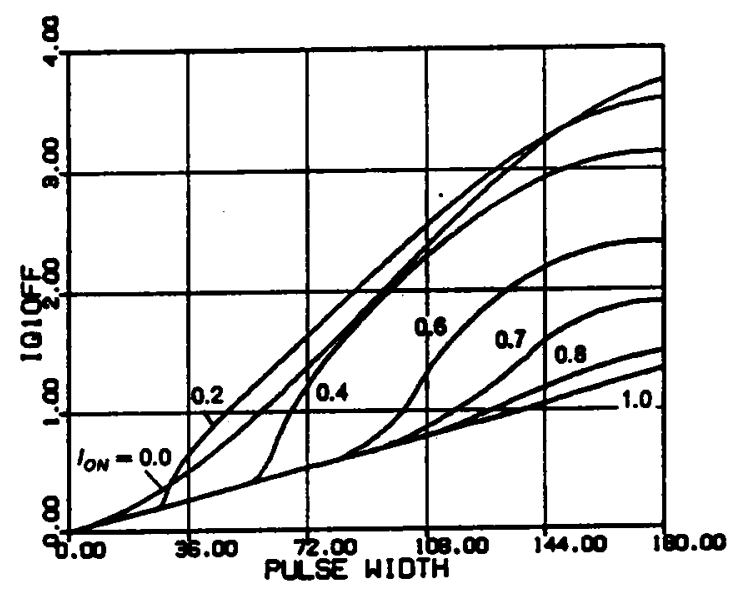

(g) Switch turn-off current $(Q 1, Q 3)$

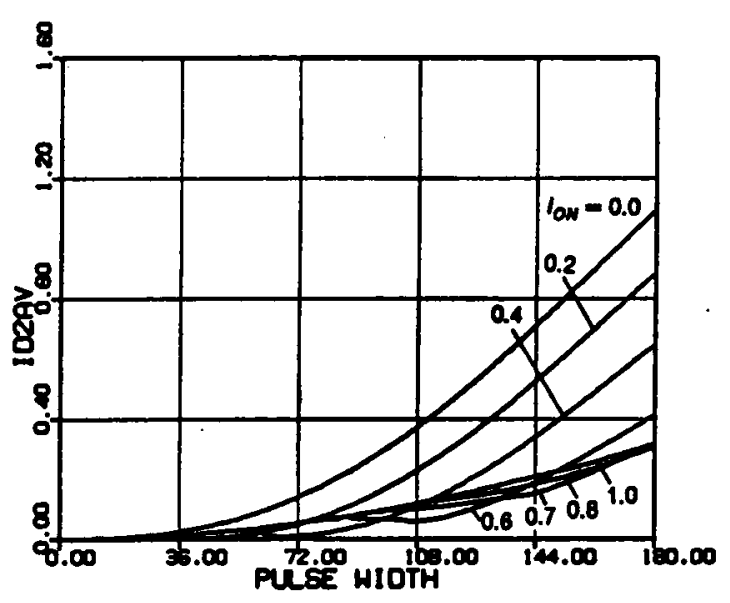

(f) Average diode current (D2,D4)

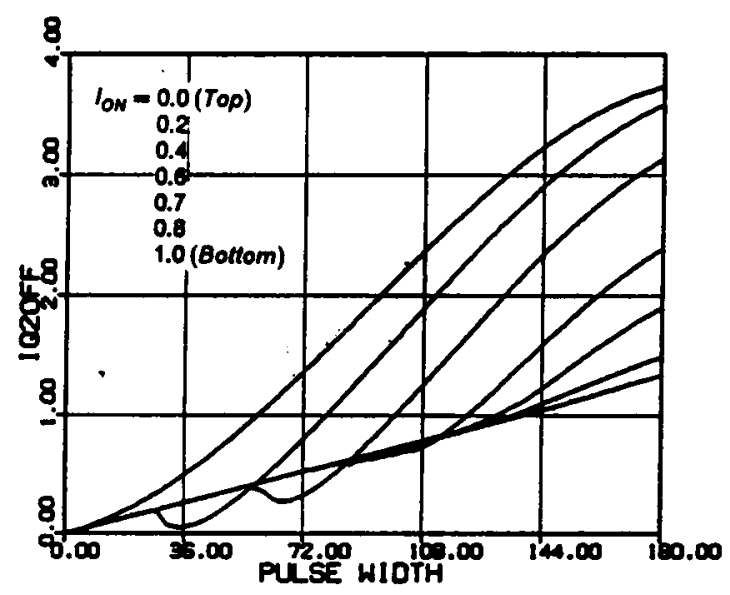

(h) Switch turn-off current (Q2,Q4)

Figure 3.15 Continued 


\subsection{DESIGN EXAMPLES}

The dc characteristics derived in the previous section are employed to facilitate design of a CM-PRC in this section. Three design examples are given. The first example designs the converter to operate in a natural commutation region $\left(\omega_{S N}<1\right)$ such that all the transistors' turn-off losses are eliminated. The second example designs the converter to operate in a mixed-commutation region $\left(\omega_{S N}<1\right)$ such that the turn-on losses of Q1,Q3 and the turn-off losses of Q2,Q4 are eliminated. Lossless snubbers can be used across Q1 and Q3 to reduce their turn-off losses. The third example designs the converter to operate in a force-commutation region $\left(\omega_{S N}>1\right)$ such that all the transistors' turn-on losses are eliminated. Lossless snubbers can be used across all the transistors to reduce their turn-off losses.

The converter is designed to satisfy the following requirements.

$$
\begin{aligned}
& \text { Input voltage }=40 \mathrm{~V} \sim 60 \mathrm{~V} . \\
& \text { Output voltage }=5 \mathrm{~V} . \\
& \text { Output poiver }=40 \mathrm{~W} \sim 50 \mathrm{~W} .
\end{aligned}
$$

Assume an output transformer with turn ratio $n: l$ is used between the resonant tank and the load circuit. Then,

$$
\begin{aligned}
& V_{\text {ONmax }}=\frac{5 n}{40}, \quad V_{O N \min }=\frac{5 n}{60}, \\
& I_{\text {ONmax }}=\frac{10}{\left.n \times 40 / 7_{10}\right)}, \quad I_{\text {ONmin }}=\frac{8}{n \times 60 / Z_{0}} .
\end{aligned}
$$

(The load current range is from $8 \Lambda$ to $10 \Lambda$. .) 


\subsubsection{Example 1 - Design in Natural-Commutation Region}

To achieve natural commutation of all the transistors, an operating frequency below the resonant frequency must be chosen. Choose $\omega_{S N}=0.8$.

Case I. From Figure 3.16, to obtain maximum achievable load current magnitude, choose

$$
V_{\text {oNmin }}=\frac{5 \times n}{60}=1.7 .
$$

The transformer turn ratio $n$ can then be calculated as

$$
n=\frac{60 \times 1.7}{5}=21 \text {, }
$$

and the maximum $V_{O N}$ is

$$
V_{\text {ONmax }}=\frac{5 \times 21}{40}=2.62 .
$$

From Figure 3.16, this voltage level can not be achieved. Thus, the design is impossible.

Case II. From Figure 3.16, to ensure output regulation for the entire input voltage range, choose

$$
V_{\text {ONmax }}=\frac{5 \times n}{40}=2.13
$$

The transformer turn ratio $n$ is calculated as

$$
n=\frac{40 \times 2.13}{5}=17 \text {, }
$$




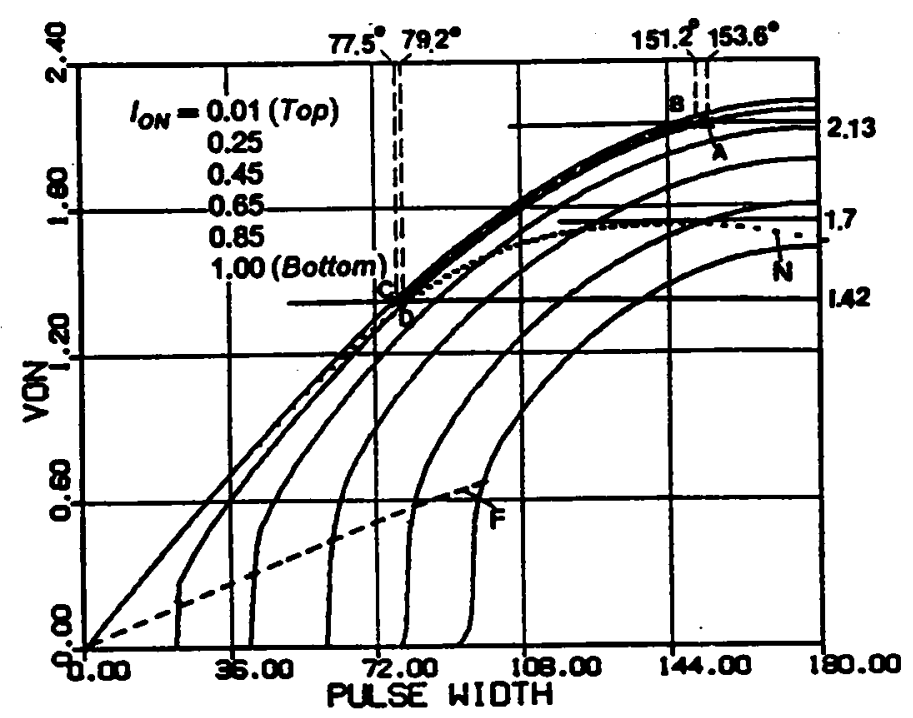

Figure 3.16 Design Example in Natural Commutation Region 
and the minimum $V_{O N}$ is

$$
V_{\text {oNmin }}=\frac{5 \times 17}{60}=1.42
$$

From Figure 3.16, it can be seen that the load current at $V_{\text {ONmin }}$ can not exceed 0.26 if natural commutation is to be maintained. To ensure this, choose

$$
I_{\text {ONmax }}=\frac{10}{n \times 40 / Z_{0}}=0.25
$$

The characteristic impedance is calculated as

$$
Z_{0}=I_{\text {ONmax }} \times n \times 40 \times \frac{1}{10}=17 \Omega
$$

The normalized load range at $V_{O N \max }$ is from $8 /\left(n \times 40 / Z_{0}\right)=0.2$ to $I_{\text {ONmax }}=0.25$, which are indicated by points $B$ and $A$ in Figure 3.16, respectively. The normalized load range at $V_{\text {ONmin }}$ is from $8 /\left(n \times 60 / Z_{0}\right)=0.13$ to $10 /\left(n \times 60 / Z_{0}\right)=0.17$, which are indicated by points $C$ and $D$ in Figure 3.16, respectively.

The normalization factor for the current, $I_{P . U}$, is equal to $40 / Z_{0}=2.35 \mathrm{~A}$ at $V_{\text {ONmax }}$ (ponits $A, B$ ) and equal to $60 / Z_{0}=3.531$ at $V_{\text {ONmin }}$ (points D).

The four boundary points, A,B,C,D, define the operating region for the converter in this design, which is indicated by the shaded area in Figure 3.16. The $\beta_{S}$ angles corresponding to these points are A: $153.6^{\circ}, \mathrm{B}: 151.2^{\circ}, \mathrm{C}: 77.5^{\circ}, \mathrm{D}: 79.2^{\circ}$. By mapping these boundary points (using their corresponding $\beta_{O N}$ and $I_{O N}$ ) into other characteristics as illustrated in Figure 3.17, various important circuit features can be obtained. The circuit's salient feature usually occurs at one of the four boundary points.

The following salient features are obtained for this design, 


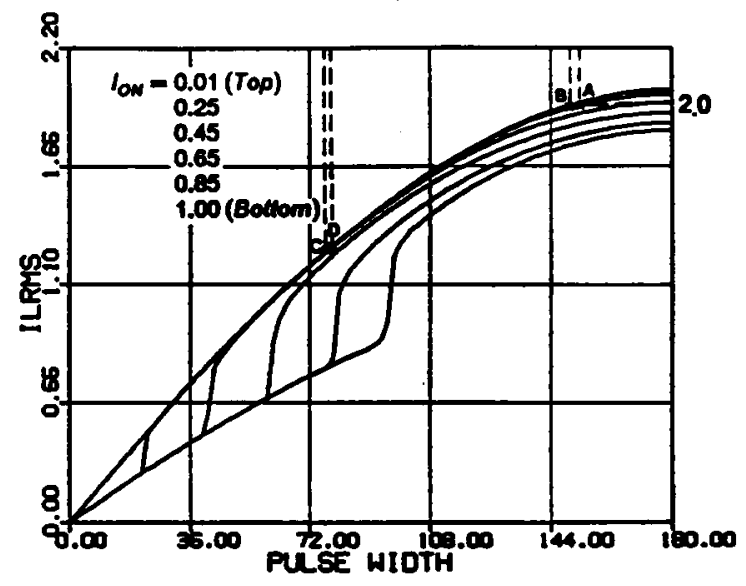

(a) RMS inductor current

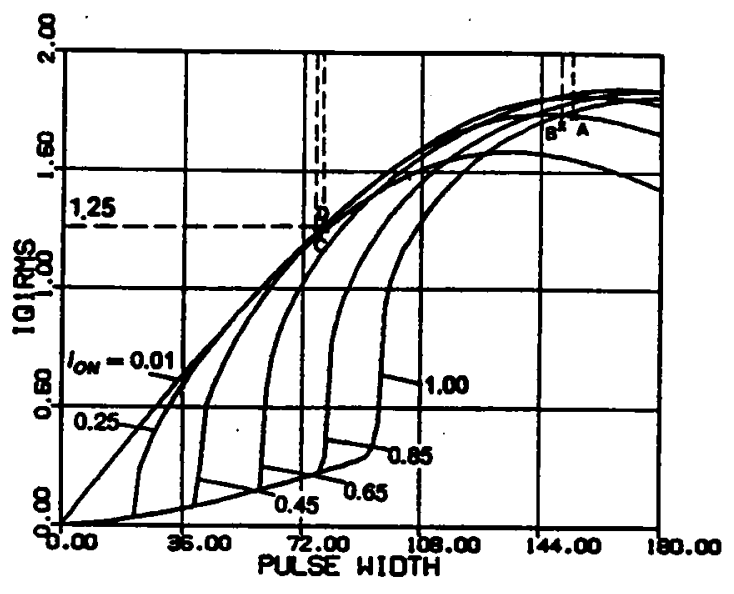

(c) RMS switch current $(Q I, Q 3)$

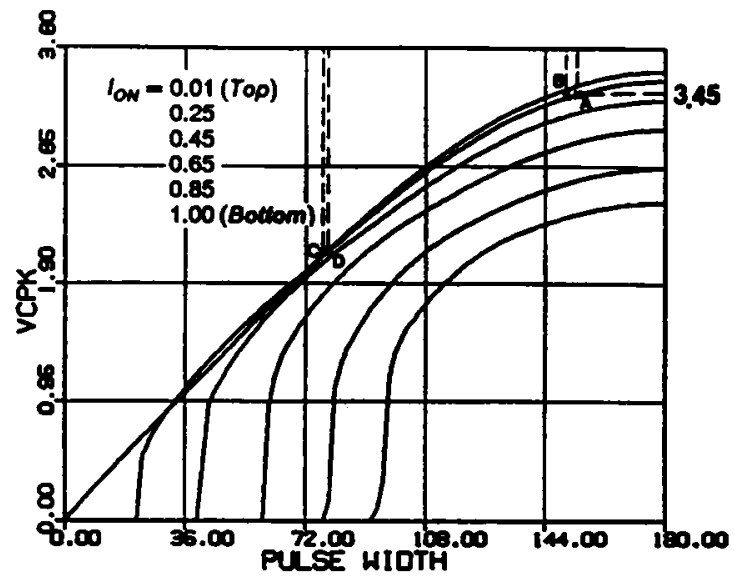

(b) Peak capacitor voltage

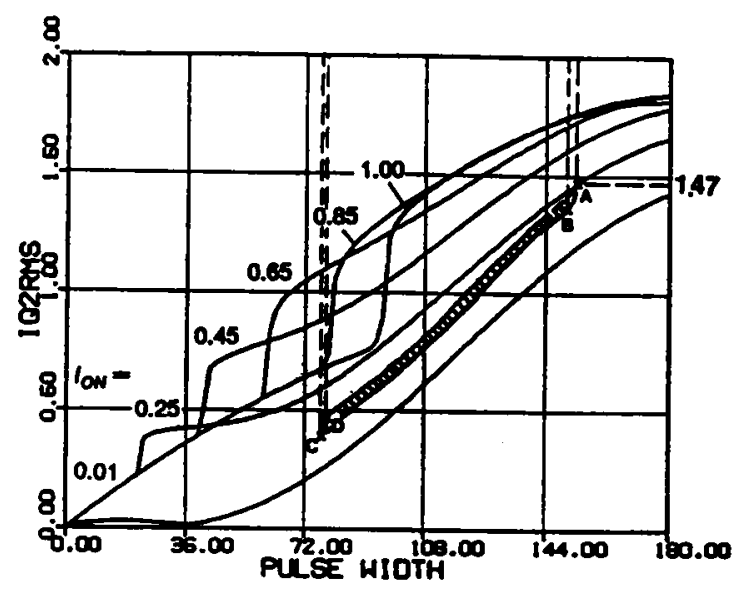

(d) RMS switch current $(Q 2, Q 4)$

Figure 3.17 Circuit Salient Features for the Design in Figure 3.16 


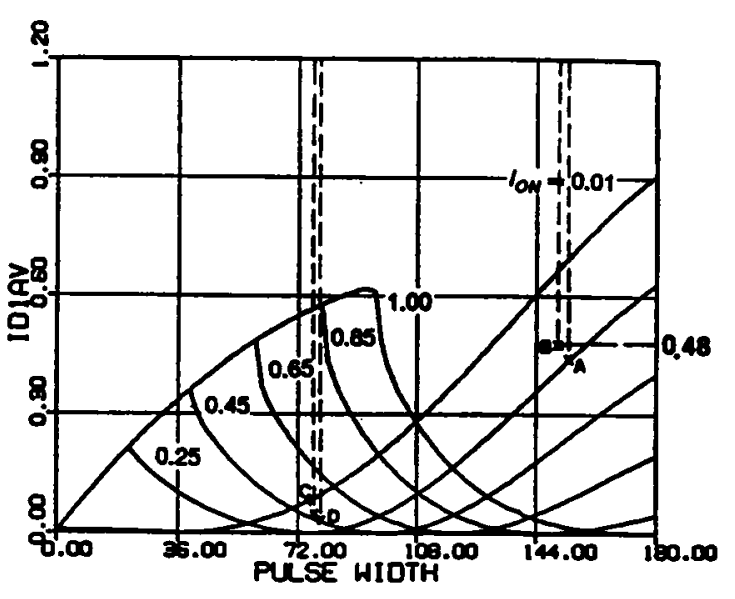

(e) Average diode current (DI,D3,

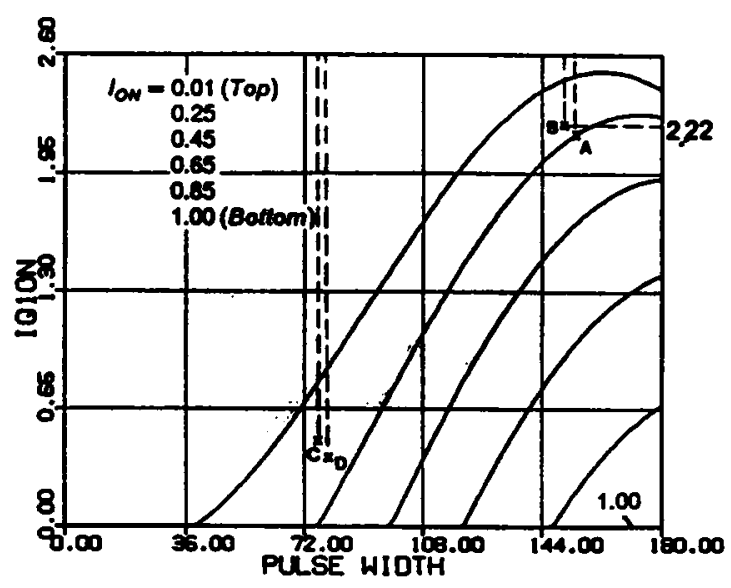

(g) Switch turn-on current (Q1,Q3)

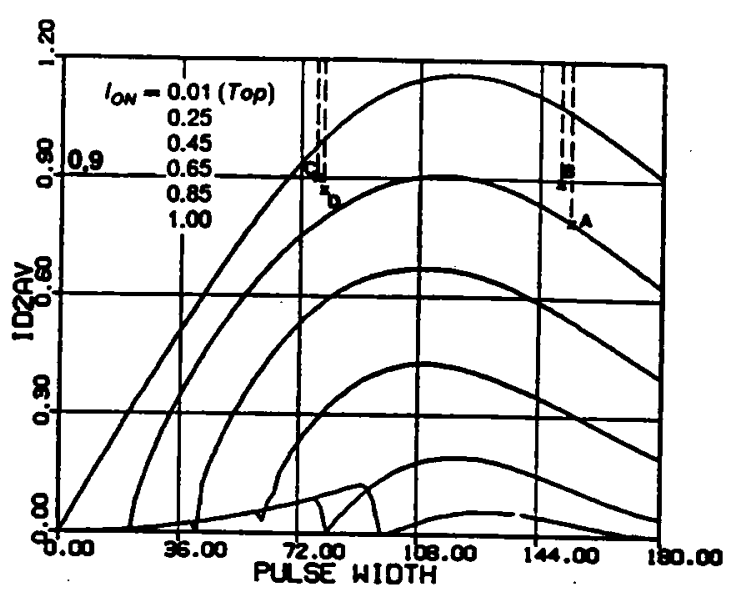

(f) Average diode current (D2,D4)

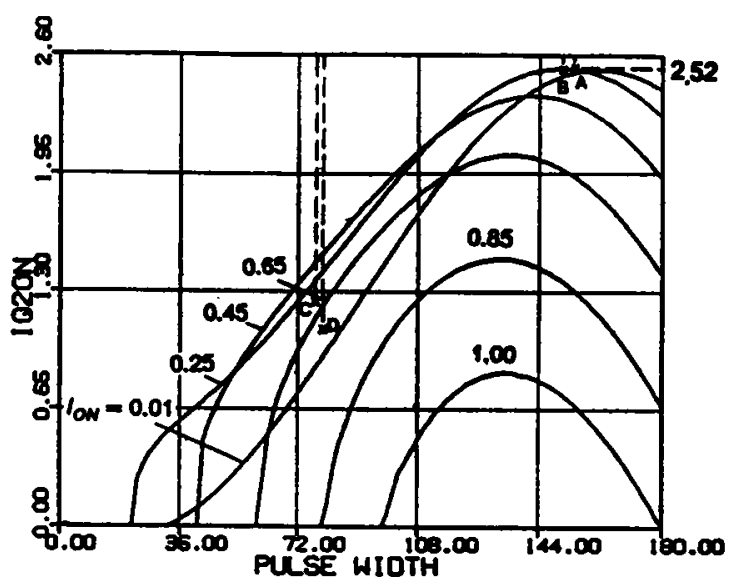

(h) Switch turn-on current $(Q 2 ; Q 4)$

Figure 3.17 Continued 


$$
\begin{array}{ll}
V_{C P K \max }=138 . \mathrm{V}, & I_{L R M S \max }=4.7 \mathrm{~A}, \\
I_{Q 1 \text { RMSmax }}=4.4 \mathrm{~A}, & I_{Q 2 R M S \max }=3.45 \mathrm{~A}, \\
I_{D 1 A V \max }=1.13 \mathrm{~A}, & I_{D 2 A V \max }=3.18 \mathrm{~A}, \\
I_{Q 1 \text { offmax }}=0 . \mathrm{A}, & I_{Q 20 J I_{\max }}=0 . \mathrm{A}, \\
I_{Q 1 \text { m max }}=5.23 \mathrm{~A}, & I_{Q 2 \text { onmax }}=6.39 \mathrm{~A} .
\end{array}
$$

\subsubsection{Example 2 - Design in Mixed-Commutation Region}

To achieve zero-voltage turn-on (force commutation) of $\mathrm{Q} 1, \mathrm{Q} 3$ and zero-current turn-off (natural commutation) of Q2,Q4, the operating frequency should be chosen below the resonant frequency. Choose $\omega_{S N}=0.8$. From Figure 3.18, to ensure Q1 and Q3 are force-commutated, choose

$$
V_{\text {ONmax }}=\frac{5 \times n}{40}=1.5 .
$$

The transformer turn ratio $n$ is calculated as

$$
n=\frac{40 \times 1.5}{5}=12
$$

The minimum $V_{O N}$ is

$$
V_{\text {ONmin }}=\frac{5 \times 12}{60}=1.0
$$

To obtain a high characteristic impedance, $I_{O N}$ should be chosen as high as possible.

\section{Choose}

$$
I_{\text {ONmax }}=\frac{10}{n \times 40 / Z_{0}}=1.0 .
$$




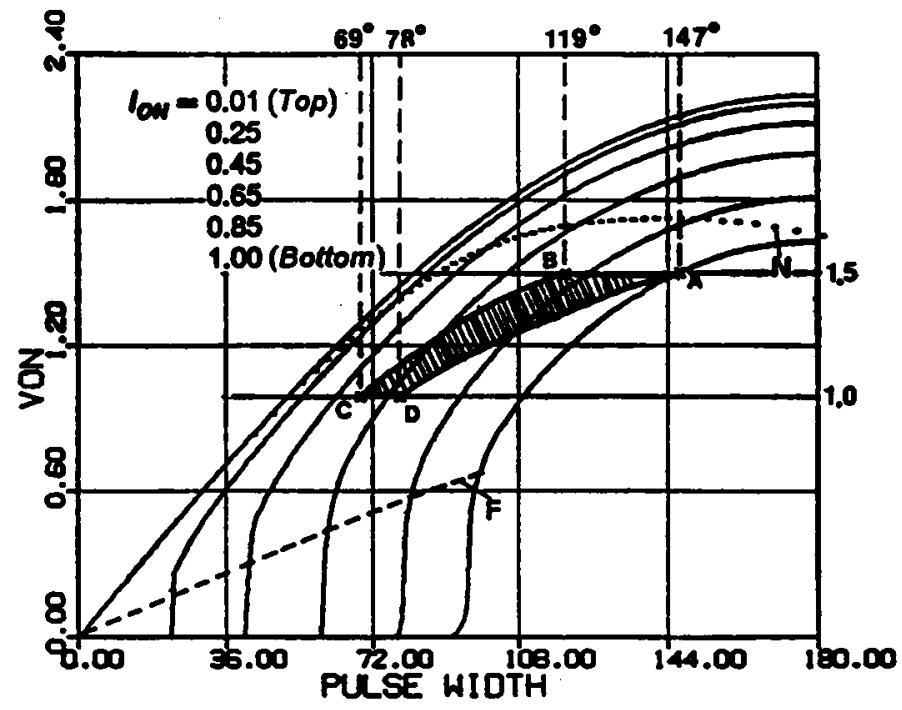

Figure 3.18 Design Example in Mixed-Commutation Region 
The characteristic impedance is calculated as

$$
Z_{0}=I_{\text {ONmax }} \times n \times 40 \times \frac{1}{10}=48 \Omega .
$$

The normalized load range at $V_{\text {ONmax }}$ is from $8 /\left(n \times 40 / Z_{0}\right)=0.8$ to $I_{\text {ONmax }}=1.0$, which are indicated by points $B$ and $A$ in Figure 3.18, respectively. The normalized load range at $V_{\text {ONmin }}$ is from $8 /\left(n \times 60 / Z_{0}\right)=0.53$ to $10 /\left(n \times 60 / Z_{0}\right)=0.67$, which are indicated by points $C$ and $D$ in Figure 3.18, respectively.

The normalization factor for current, $I_{P, U}$, is equal to $40 / Z_{0}=0.833 \mathrm{~A}$ at $V_{\text {ONmax }}$ (ponits $\mathrm{A}, \mathrm{B}$ ) and equal to $60 / Z_{0}=1.25 A$ at $V_{\text {ONmin }}$ (points $\mathrm{C}, \mathrm{D}$ ).

The four boundary points, A,B,C,D, define the operating region for the converter in this design, which is indicated by the shaded area in Figure 3.18. The $\beta_{S}$ angles corresponding to these points are A: $147^{\circ}$, B: $119^{\circ}$, C: $69^{\circ}$, D: $78^{\circ}$. By mapping these boundary points (using their corresponding $\beta_{O N}$ and $I_{O N}$ ) into other characteristics as illustrated in Figure 3.19, various important circuit features are obtained and shown in the following:

$$
\begin{aligned}
& V_{C P K \max }=107 . \mathrm{V}, \quad I_{\text {LRMSmax }}=1.5 \mathrm{~A}, \\
& I_{Q 1 R M S \max }=1.44 \Lambda, \quad I_{Q 2 R M S \max }=1.43 \mathrm{~A}, \\
& I_{D 1 A V \max }=0.25 \Lambda, \quad I_{D 2 \Lambda V \max }=0.5 \Lambda, \\
& I_{\text {QIffmax }}=1.92 \Lambda \quad I_{Q 2 n / \text { max }}=0 . \Lambda \text {, } \\
& I_{\text {Q1onmax }}=0 . \Lambda, \quad I_{\text {Q2onmax }}=1.4 \AA .
\end{aligned}
$$

\subsubsection{Example 3 - Design in Force-Commutation Region}




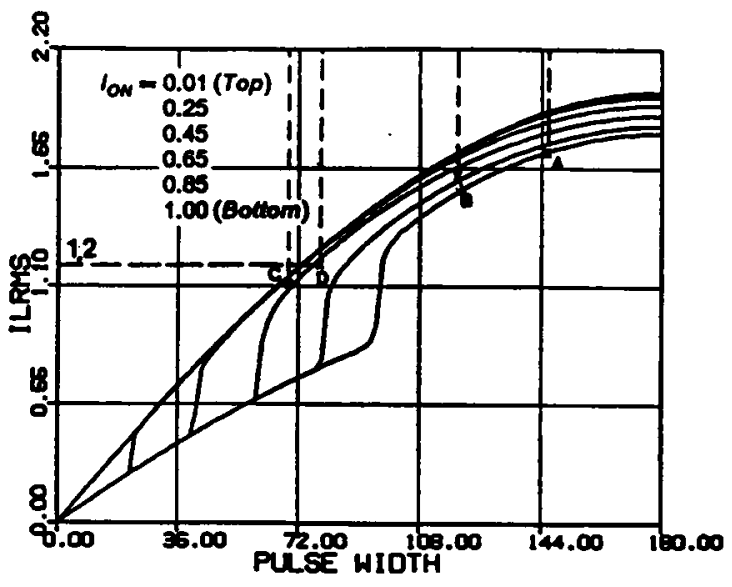

(a) RMS inductor current

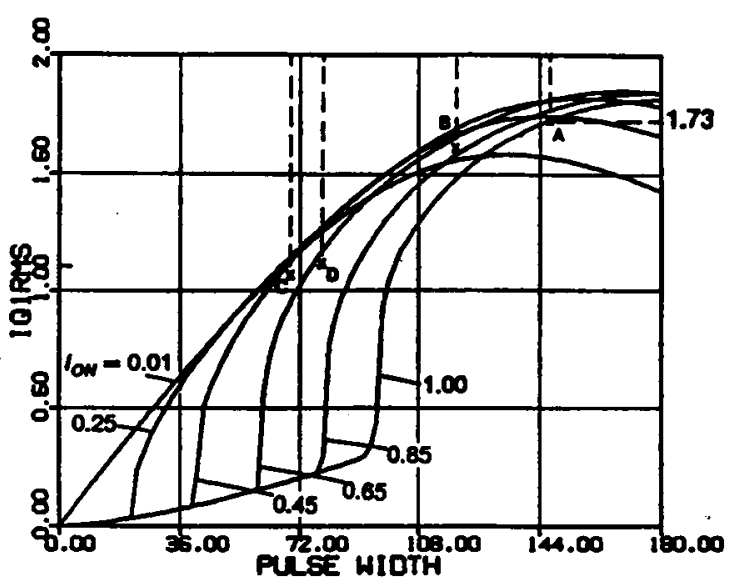

(c) RMS switch current (Q1,Q3)

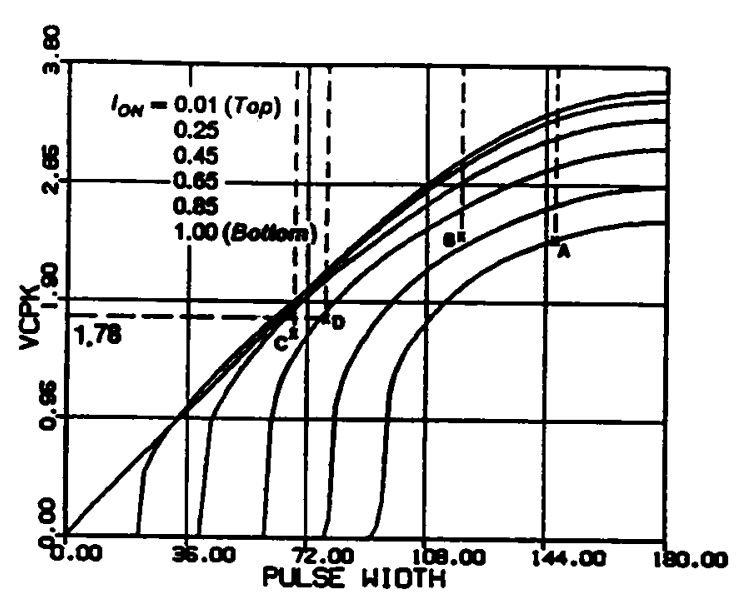

(b) Peak capacitor voltage

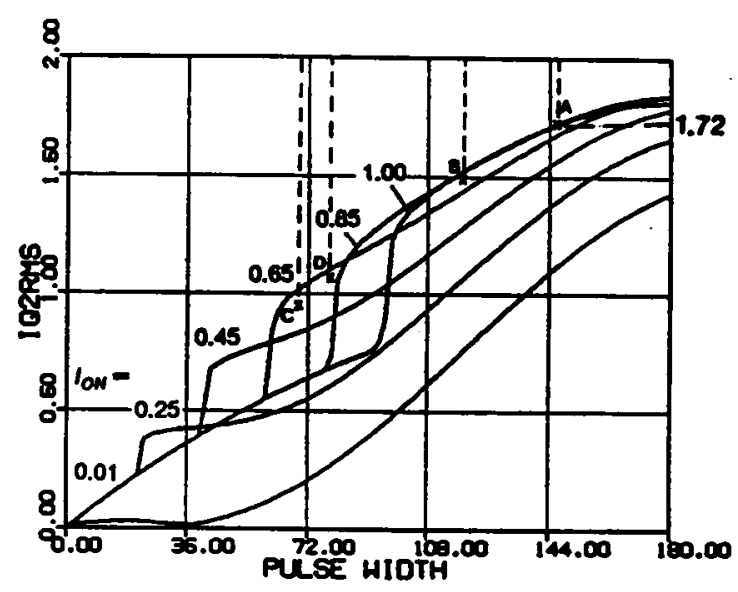

(d) RMS switch current $(Q 2, Q 4)$

Figure 3.19 Circuit Salient Features for the Design in Figure 3.18 


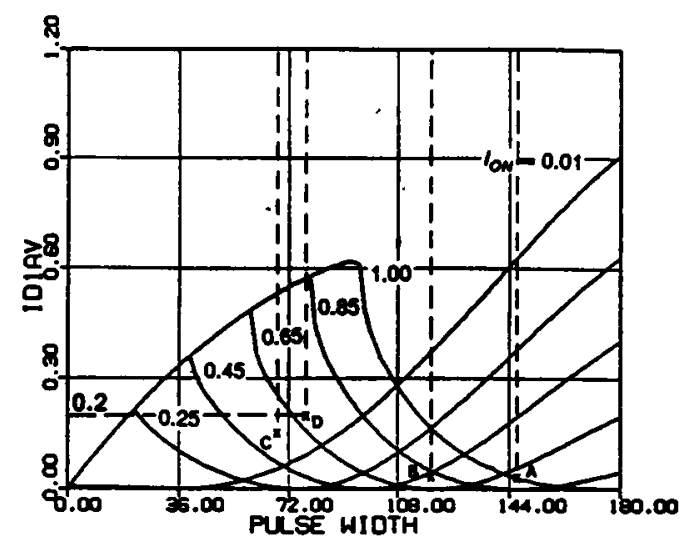

(e) Average diode current (DI,D3)

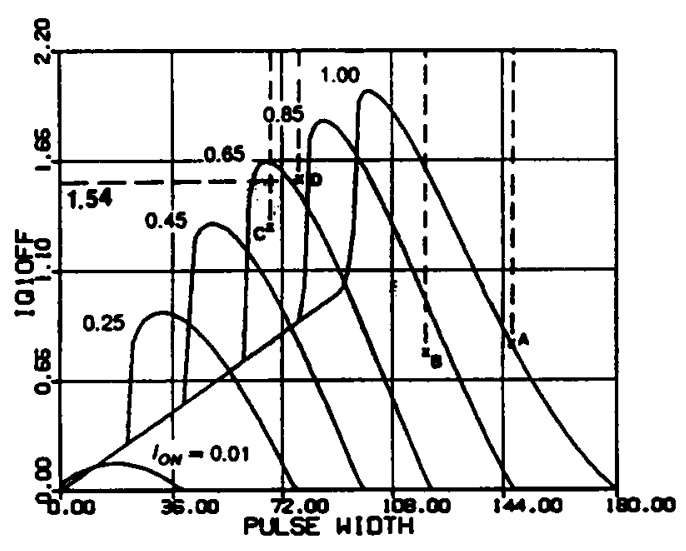

(g) Switch turn-off current (QI,Q3)

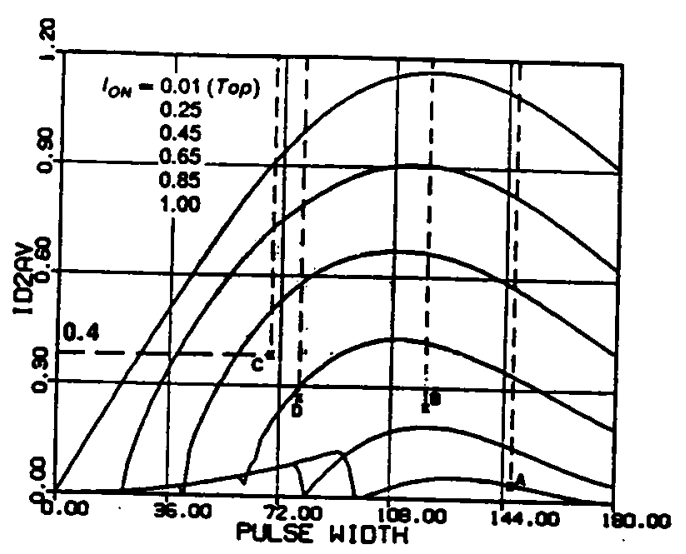

(f) Average diode current (D2,D4)

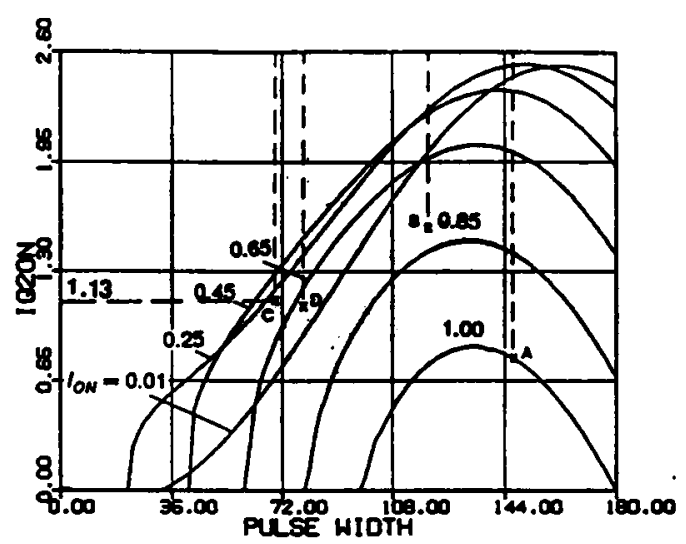

(h) Switch turn-on current (Q2,Q4)

Figure 3.19 Continued 
To achieve zero-voltage turn-on (force commutation) of all the transistors, the operating frequency must be chosen above the resonant frequency. Choose $\omega_{S N}=1.2$. From Figure 3.20, to ensure force commutation of all the transistors (avoiding the mixedcommutation region), choose

$$
V_{\text {ONmin }}=\frac{5 \times n}{60}=0.4
$$

The transformer turn ratio $n$ is calculated as

$$
n=\frac{60 \times 0.4}{5}=4.8
$$

The maximum $V_{O N}$ is

$$
V_{\text {oNmax }}=\frac{5 \times 4.8}{40}=0.6
$$

From Figure 3.20, $I_{\text {ONmax }}$ must not exceed 0.72 . Choose

$$
I_{O N \max }=\frac{10}{n \times 40 / Z_{0}}=0.7
$$

The characteristic impedance is calculated as

$$
Z_{n}=I_{\text {ONmax }} \times n \times 40 \times \frac{1}{10}=13.44 \Omega .
$$

The normalized load range at $V_{\text {oNmax }}$ is from $8 /\left(n \times 40 / 7_{0}\right)=0.56$ to $I_{\text {ONmax }}=0.7$, which are indicated by points $B$ and $A$ in Figure 3.20, respectively. The normalized load range at $V_{\text {ONmin }}$ is from $8 /\left(n \times 60 / Z_{n}\right)=0.37$ to $10 /\left(n \times 60 / Z_{0}\right)=0.47$, which are indicated by points $\mathbf{C}$ and $\mathbf{D}$ in Figure 3.20, respectively.

The normalization factor for current, $I_{P . U}$, is equal to $40 / Z_{0}=2.98 \mathrm{~A}$ at $V_{\text {ONmax }}$ (ponits $A, B$ ) and equal to $60 / Z_{0}=4.46 .1$ at $V_{\text {oNmin }}$ (points $C, D$ ). 


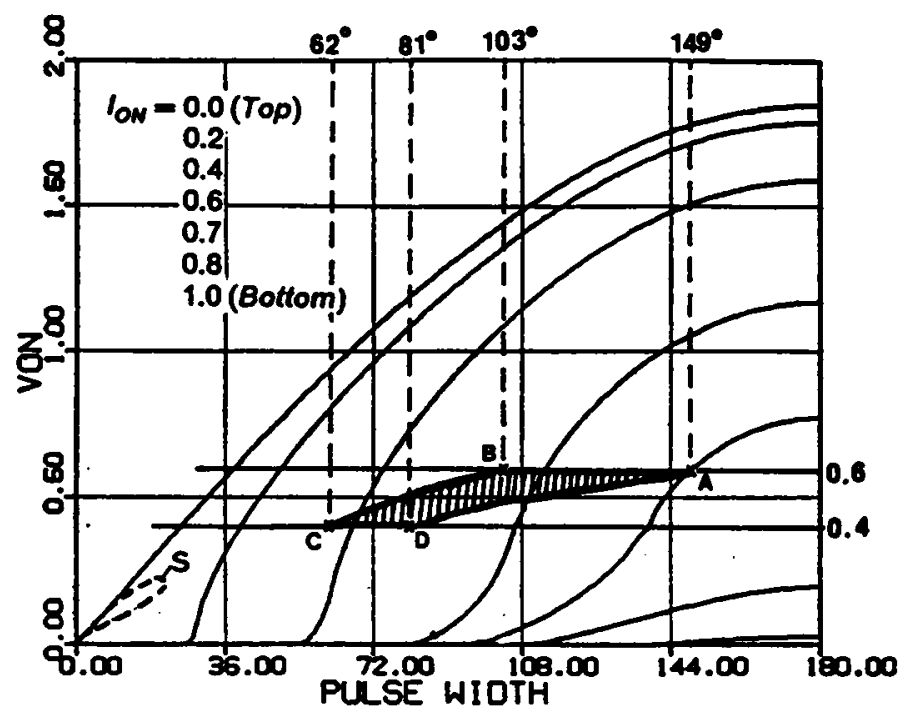

Figure 3.20 Design Example in Force-Commutation Region 


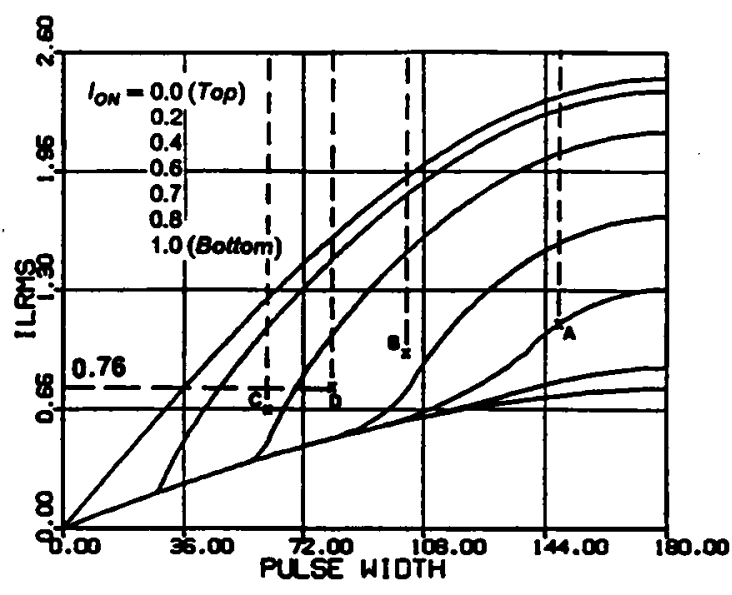

(a) RMS inductor current

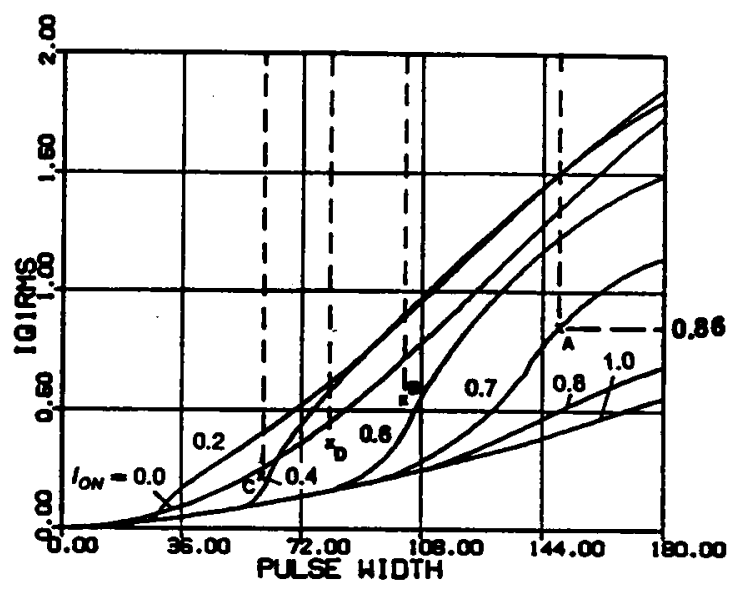

(c) RMS switch current (QI,Q3)

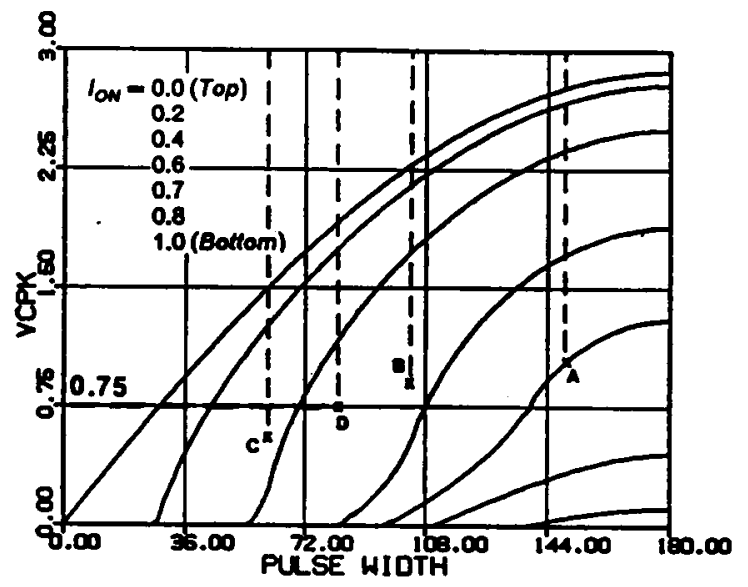

(b) Peak capacitor voltage

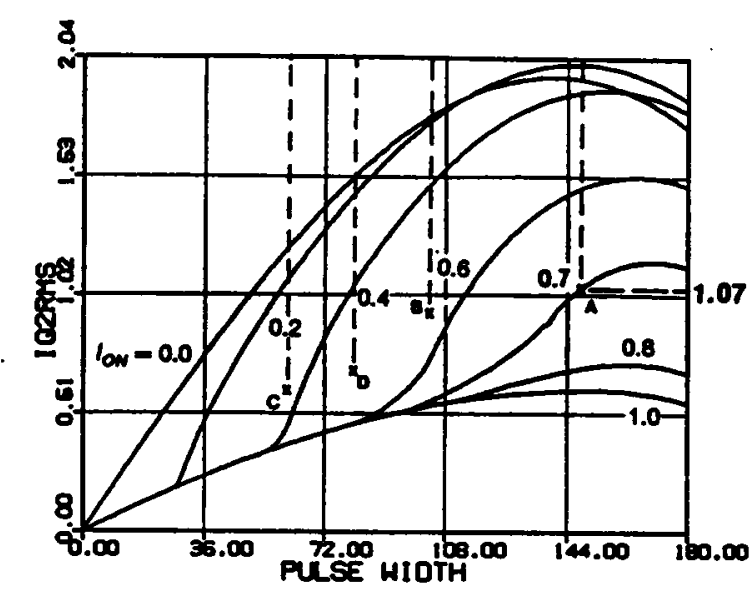

(d) RMS switch current (Q2,Q4)

Figure 3.21 Circuit Salient Features for the Design in Figure 3.20 


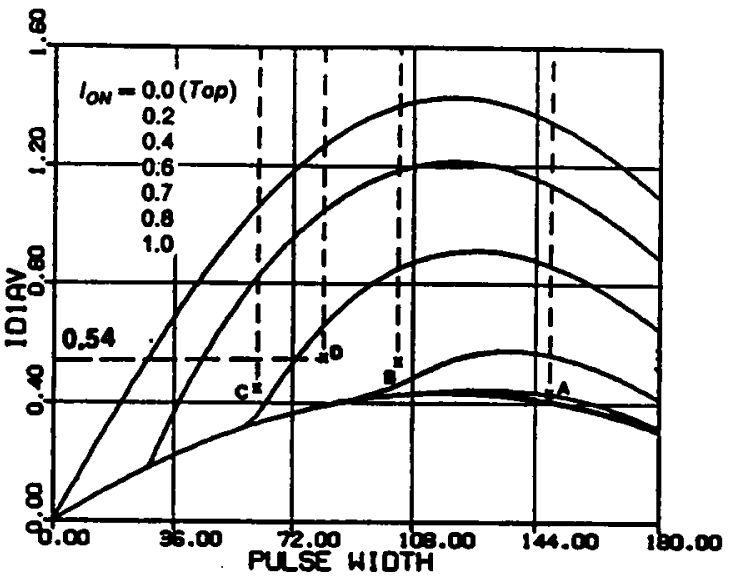

(e) Average diode current (DI,D3)

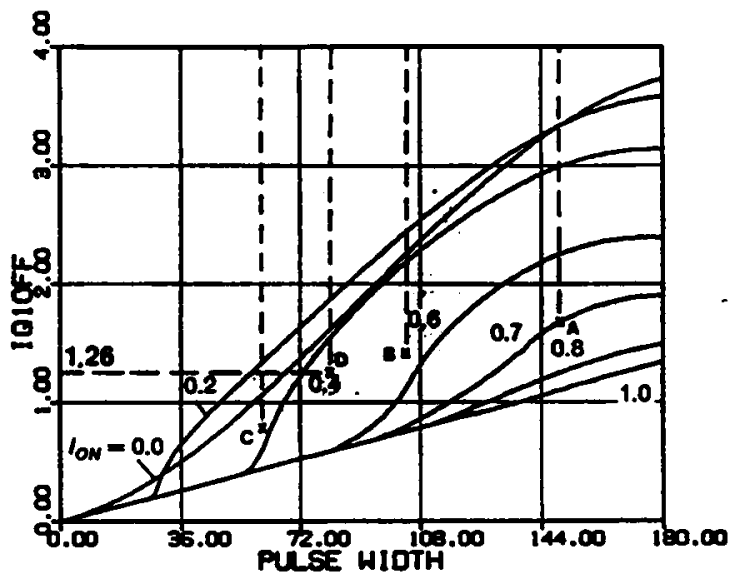

(g) Switch turn-off current (Q1,Q3)

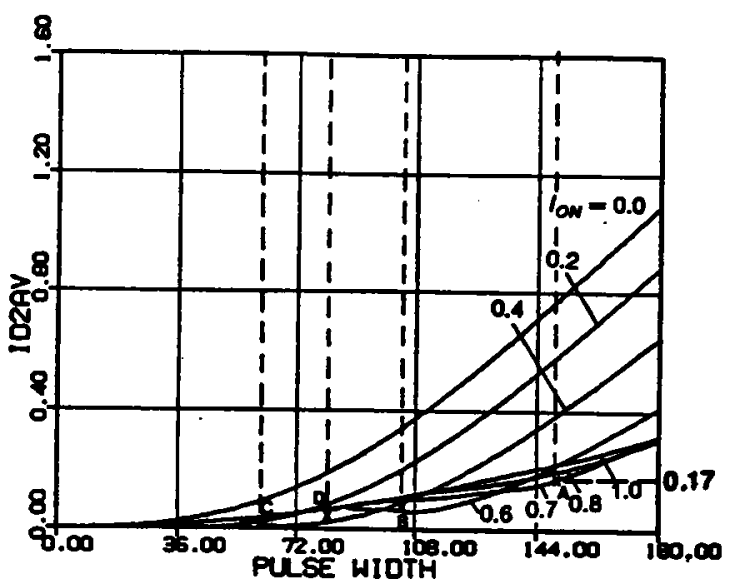

(f) Average diode current (D2,D4)

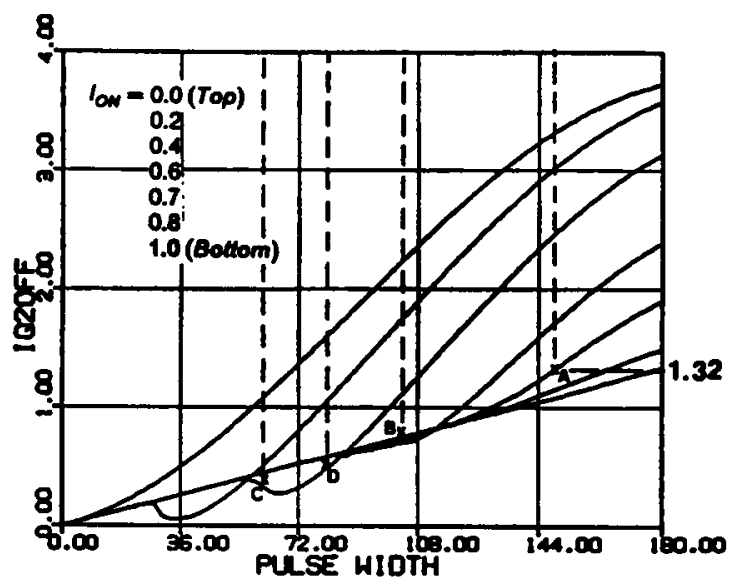

(h) Switch turn-off current (Q2,Q4)

Figure 3.21 Continued 
The four boundary points, $\mathbf{A}, \mathbf{B}, \mathbf{C}, \mathbf{D}$, define the operating region for the converter in this design, which is indicated by the shaded area in Figure 3.20. The $\beta_{S}$ angles corresponding to these points are A: $149^{\circ}, \mathrm{B}: 103^{\circ}, \mathrm{C}: 62^{\circ}, \mathrm{D}: 81^{\circ}$. By mapping these boundary points (using their corresponding $\beta_{O N}$ and $I_{O N}$ ) into other characteristics as illustrated in Figure 3.21, various important circuit features are obtained and shown in the following:

$$
\begin{aligned}
& V_{\text {CPKmax }}=45 . \mathrm{V} \quad I_{\text {LRMSmax }}=3.37 \mathrm{~A} \text {, } \\
& I_{Q 1 R M S \max }=2.35 \Lambda, \quad I_{\text {Q2RMSmax }}=3.2 \AA \text {, } \\
& I_{D 1 A V_{\max }}=2.4 \Lambda, \quad I_{D 2 \Lambda V \max }=0.51 \mathrm{~A}, \\
& I_{\text {Q1 offmax }}=5.61 \mathrm{~A} \quad I_{\text {Q20Ifmax }}=3.94 \mathrm{~A} \text {, } \\
& I_{\text {Q Ionmax }}=0 . \Lambda, \quad I_{\text {Q2onmax }}=0 . \AA \text {. }
\end{aligned}
$$

From the above examples, it can be seen that higher component ratings are usually required when a CM-PRC is to be designed either in the natural- or in the force commutation region. 


\subsection{HARDWARE EXPERIMENTS}

A prototype circuit, as shown in Figure 3.22, was built to verify the circuit operation of a CM-PRC. The circuit was designed at $105.4 \mathrm{kHz}$ with a resonant frequency of $145 \mathrm{kHz}\left(\omega_{S N}=0.72\right)$.

Figure 3.23 shows the experimental results. All the predicted operating modes below resonant frequency have been illustrated except Mode V operation. (Mode V operation does not exist under steady state.) The state trajectories are obtained by externally calibrating the inductor current so that the ratio of the y-axis scale to the $\mathrm{x}$-axis scale is the same as the ratio of $i_{L N}$ to $v_{C N}$.

Figure 3.23(g) shows a trajectory close to Mode V operation. This trajectory is obtained by shorting the output resistor $(R=0 . \Omega)$. Slight asymmetry in the trajectories exists due to the imbalance in the gating signals. 


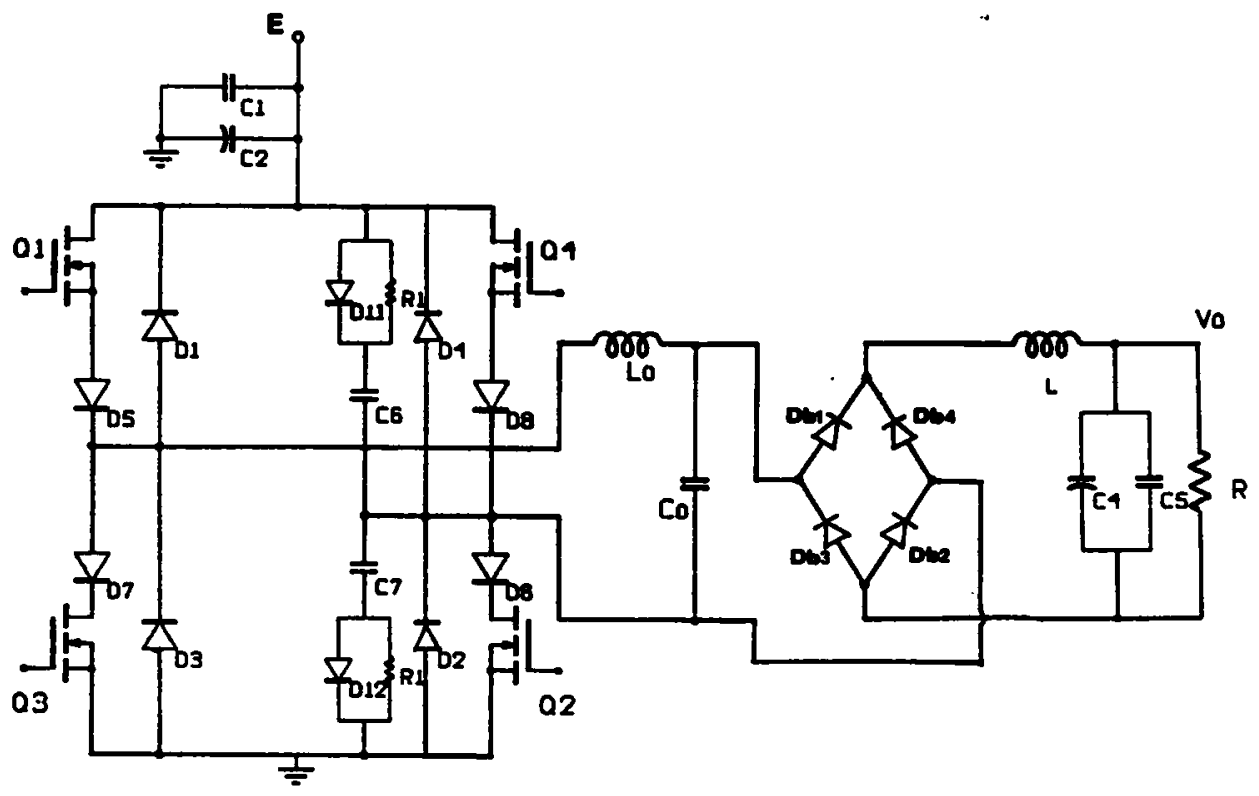

Q1,Q2,Q3,Q4: IRF450; D1,D2,D3,D4,Db1,Db2,Db3,Db4: UES1403;

$\mathrm{D} 11, \mathrm{D} 12, \mathrm{D} 5, \mathrm{D} 6, \mathrm{D} 7, \mathrm{D} 8:$ IR31DQ06; $\mathrm{C} 2=40 \mu \mathrm{F}, \mathrm{C1}, \mathrm{C} 5=0.01 \mu \mathrm{F}$;

$C 4=10 \mu F, C 6, C 7=1000 p F, R 1=150 \Omega, L=15.6 \mathrm{mH}$;

$L O=55.32 \mu H, C=21.87 n F, E=50 \mathrm{~V}$.

Figure 3.22 A Breadboard Circuit Used to Verify Circuit Operations Below Resonant Frequency 

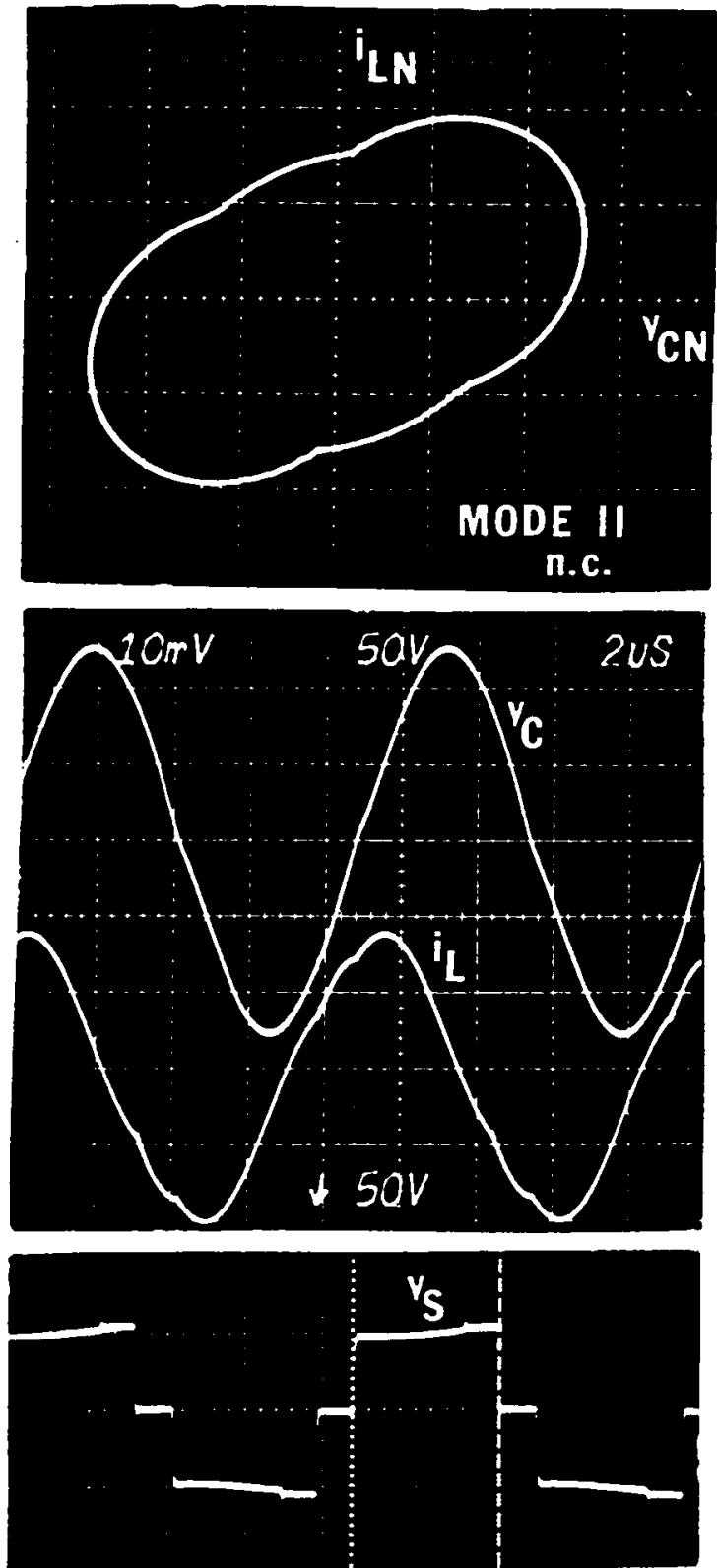

(a) Mode- $I I_{N}$ operation

Figure 3.23 Experimental Results from the Circuit in Figure 3.22 


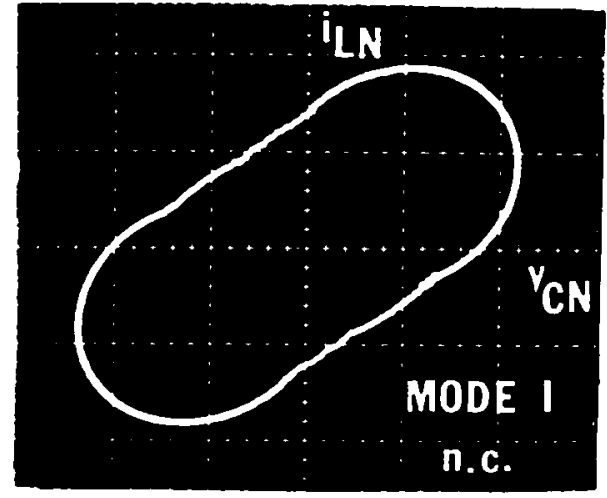

(b) Mode- $I_{N}$ operation

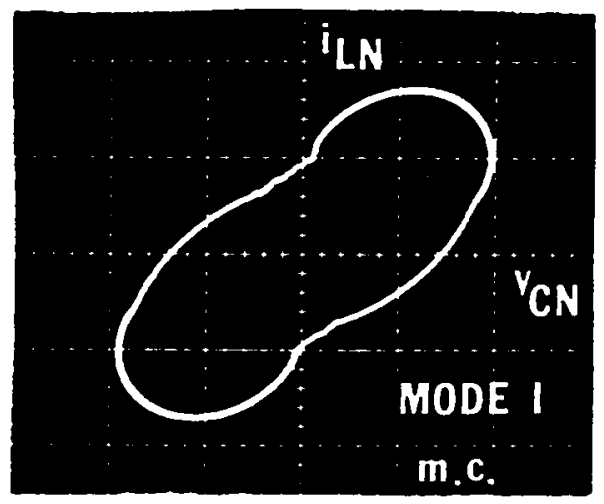

(c) Mode-I operation

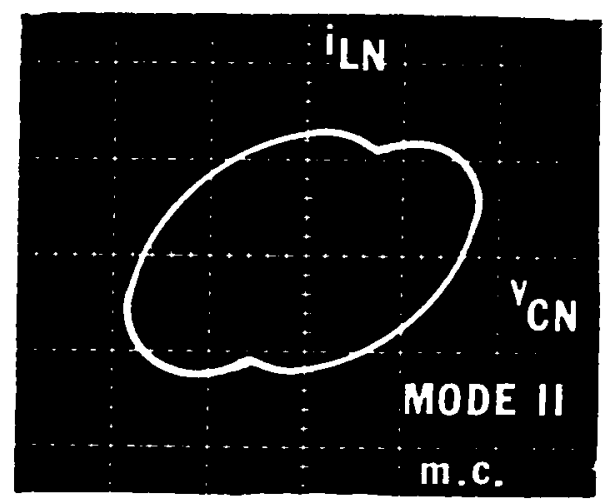

(d) Mode- $I I_{M}$ operation

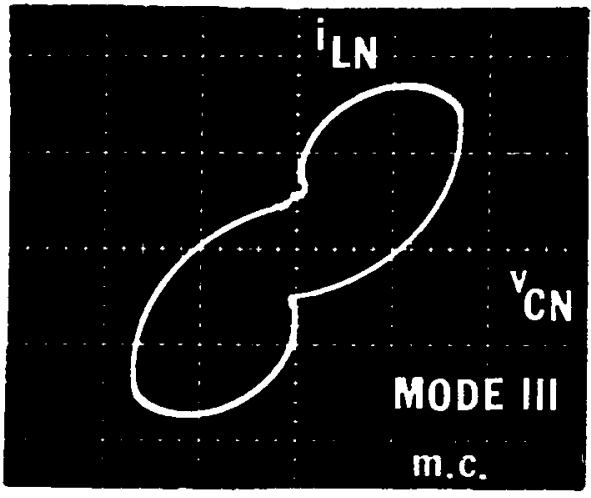

(e) Mode- $I I I_{m}$ operation

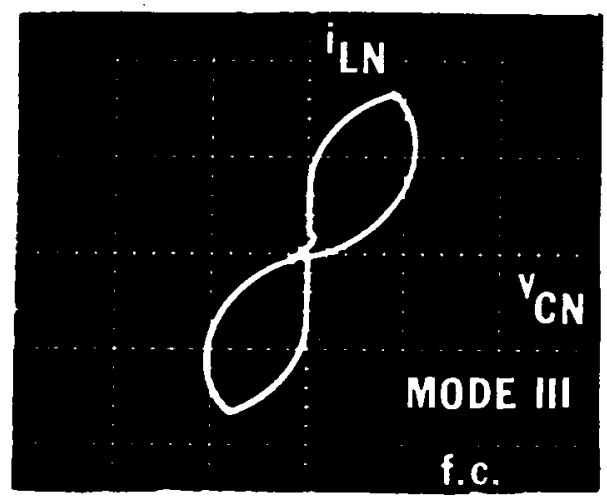

(f) Mode- $I I I_{F}$ operation

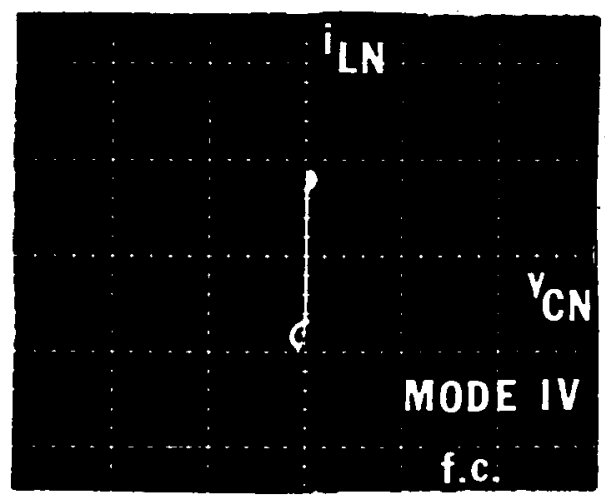

(g) Mode- $I V_{F}$ operation

Figure 3.23 Continued 


\subsection{CONCLUSIONS}

A complete analysis of the constant-frequency, clamped-mode, parallel-resonant converter has been performed in this chapter. Employing state-plane analysis techniques, all the circuit operating modes are identified for the frequency range above $50 \%$ of the resonant frequency. The operating modes are categorized into six different circuit topological mode sequences, each representing a unique device conduction sequence.

Three different commutation modes for the transistors exist: natural commutation, mixed commutation, and force commutation. To predict the converter's mode of operation and determine the transistors' commutation conditions, an algorithm is developed to define the regions of operation.

Unlike the CM-SRC, a CM-PRC, can be designed to operate in the natural or the force commutation region from no load to full load. Design in natural commutation region, however, is difficult when the output-to-input voltage ratio varies over a wide range since the natural commutation region is very limited, especially at frequencies below $80 \%$ of the resonant frequency. High circulating current usually exists in the converter since a small charactcristic impedance must be used.

The converter can be easily designed in the force-commutation region for a wide range of input voltage and output current since a very large force commutation region exists for the converter when the operating frequency is above the resonant frequency.

The converter can also be designed in the mixed-commutation region at frequencies below the resonant frequency. $\Lambda$ minimum load current, however, has to be maintained.

Three design examples are given. The examples illustrate how to employ the dc characteristics to design a CM-PRC in different commutation regions. The results show that higher component ratings are usually required to design the converter in either the natural or the force commutation region. 
Finally, a $105 \mathrm{kHz}$ prototype circuit is breadboarded to verify the operation of a CM-PRC. All the predicted operating modes below resonant frequency are experimentally substantiated. 


\section{CHAPTER 4.}

\section{CONCLUSIONS}

The distinct advantages of resonant converters, such as zero-current turn-off, zerovoltage turn-on, low EMI, and fast responses, have attracted much attention in power electronics industry. Numerous efforts have been made to extend the soft switching resonant techniques into other types of converters such that the performances of these converters can be improved. For example, recently a new family of quasi-resonant converters and multi-resonant converters were developed which can be operated above one megahertz and can achieve very high power density [47-56]. These converters are obtained by properly adding LC resonant components into conventional PWM converters such that zero-current and/or 7ero-voltage switching features can be obtained. These converters are, however, controlled via frequency modulation, as in the conventional resonant converters.

Variable-frequency operation often results in poor utilization of magnetic components and capacitor filters since these components has to be designed according to the lowest operating frequency. The bandwidth of the control loop is also limited by the lowest operating frequency. Variable-frequency opcration can also results in undesirable low beat frequencies which are difficult to filter. 
In view of this, efforts have been made to search for circuit topologies and control techniques which enable resonant converters to operate at a constant frequency.

Recently, two fixed frequency clamped-mode resonant (series and parallel) converters were proposed which show show very promising features, such as simple control (similar to PWM), no load to full load operation, low circulating current under light load, and zero-current or zero-voltage switching.

In the dissertation, the behaviors of both the clamped-mode series-resonant converter and the clamped-mode parallel-resonant converter were completely characterized to provide insights to the complex circuit opcrations and to derive design guidelines. State-plane analysis techniques were employed to identify for the first time various circuit operating modes and to define mode boundaries and operating regions. Based on the state plane analysis, important dc characteristics, such as control-to-output transfer ratio, inductor rms current, peak capacitor voltage, switch rms currents, average diode currents, turn-on current and turn-off current of the switches were derived to facilitate design of the converters.

Three different regions, natural commutation region, forced commutation region, and mixed mode of natural and forced commutation region, exist for both the CM-SRC and the CM-PRC. In the natural commutation region, all the controlled switches turn off at zero current. Fast antiparallel diodes are required for all the switches. In the mixed commutation region, two of the switches turn off at zero current while the other two turn on at zero voltage. last antiparallel diodes are required for the two switches which are naturally commutated, while slow antiparallel diodes and simple lossless capacitor snubbers can be used for the other two stwitches which are turned on at zero voltage. In the force commutation region, all the switches turn on at zero voltage. In this case, slow antiparallel diodes and simple lossless capacitor snubbers can be used for all the switches. To optimize the design and the utilization of available components, it is sug- 
gested that the converter be designed to opcrate strictly within a specific commutation region, although it is not necessary.

For a CM-SRC, design in both natural and force commutation requires a minimum load to be maintained. To achieve output regulation from no load to full load, the converter has to be designed in the mixed commutation region.

For a CM-PRC, design in the mixed-commutation mode requires a minimum load. The converter can be designed to regulate the output from no load to full load in both the natural and force commutation regions. However, design in the natural commutation region is not practical since the region for natural commutation is very limited.

In applications where MOSFETS are used as switching devices, it is most desirable to design the converters in the force commutation regions where all the switches are turned on at zero voltage. The slow recovery internal diodes can be used and the output capacitors of the MOSFETS can be utilized as the lossless snubbers. The CM-PRC operating above resonant frequency is found to be most attractive since zero-voltage turn-on can be implemented for all the switches in an operation from no load to full load.

The clamped-mode SRC and PRC are emerging as the mainstay in high performance and high power conversion applications. The clamped-mode concept can be further extended to applications in dc-ac potver inversions, where a high frequency ac output or an ac distributed bus is required. 


\section{REFERENCES}

1. N. Marpham, " $\Lambda$ S SCR Inverter with Good Regulation and Sinewave Output", IEEE Transactions on Industrial and General Applications, IGA-3, No.2, Mar/Apr 1967, pp. 176 187.

2. V.T. Ranganathan, P.D. Ziogas, and V.R. Stefanovic, "A Regulated DC-DC Voltage Source Converter Using a Iligh-Frequency Link", IEEE Transactions on Industrial Applications, Vol. IA-18, No.3, pp. 279 287, May/June 1982.

3. R.L. Steigerwald, "High-Frequency Resonant Transistor DC-DC Converters", IEEE Transactions on Industrial Tilectronics, Vol. IE-31, No.2, pp. 181 191, May 1984.

4. $\quad F . C$. Schwarz, " $\Lambda$ Method of Resonant Current Pulse Modulation for Power Converters", IEEE Transactions of Industrial Electronics and Control Instrumentation, IECI-17, No.3, May 1970, pp. 209 221.

5. F.C. Schwarz, " $\wedge$ n Improved Method of Resonant Current Pulse Modulation for Power Converters", IFEE Power Flectronics Specialists Conference, Record, 1975, pp. 194 204.

6. J.G. Kassakian, " $\wedge$ new Current Mode Sinc Wave Inverter", IEEE Transactions on Industrial $\Lambda$ pplications, I $\Lambda-18$, No.3, May/June 1982, pp. 273 278.

7. J.G. Kassakian, $\Lambda . F$. (ioldberg, and I.R. Moretti, " $\Lambda$ Comparative Evaluation of Series and Parallel Structure for Iligh Frequency Transistor Inverters", IEEE Power Electronics Specialists Conference, Record, 1982, pp. 20 26. pp. 273 278.

8. J.B. Klassens, "ICC to $\Lambda C$ Series Resonant Converter System with High Internal Frequency Gencrating Synthesized Waveforms for Multikilowatt Power I.evels", IFEE Power Electronics Specialists Conference, Record. 1984, pp. $99 \sim 110$. 
9. S. Muroyama and K. Sakakibara, "Characteristics of a New Series Resonant Converter", IEEE INTELEC Conference Record, 1982, pp. 111 116.

10. R.J. King and T.A. Stuart, " $\Lambda$ Normalized Model for the Half-Bridge Series Resonant Converter", IEEE Transactions on Aerospace and Electronics Systems, AES-17, No.2, Mar 1981, pp 190 198.

11. R.J. King and T.A. Stuart, "Modeling the Full Bridge Series Resonant Converter", IEEE Transactions on Nerospace and Electronics Systems, AES-18, No.4, Mar 1982, pp 449 459.

12. V. Vorperian and S. Cuk, "A Complete DC Analysis of the Series Resonant Converter", IEEE Power Electronics Specialists Conference, Record, 1982, pp. 85 100.

13. V. Vorperian and S. Cuk, "Small Signal Analysis of Resonant Converters", IEEE Power Electronics Specialists Conference, Record, 1983, pp. 269 282.

14. A. F. Witulski and R.W. Erickson, "Steady State Analysis of the Series Resonant Converter", IEEE Transactions on Aerospace and Electronic Systems, AES-21, No.6, November 198.

15. S.D. Jolnnson and R.W. Erickson, "Steady State Analysis and Design of the Parallel Resonant Converter", ITI: E Power Electronics Specialists Conference, Record 1986.

16. R.J. King and T.A. Stuart, "Inherent Overload Protection for the Series Resonant Converters", IEEE Transactions on Aerospace and Electronic Systems, AES-19, No. 6, November 1983, pp. 829 830.

17. R.R. Robson and D.J. Ilancock, " $\Lambda$ 10KW Series Resonant Converter Design, Transistor Characterization, and Base Drive Optimization", IEEE Power Electronics Specialists Conference, Record, 1982, pp. 33 44.

18. R. Oruganti and F.C. Lee, "Resonant Power Processors: Part I - State Plane Analysis", IEEE IAS Annual Meeting, Conference Record, 1984, pp. 860 867.

19. . R. Oruganti and F.C. L.ee, "Resonant Power Processors: Part II - Methods of Control", IEEE I $\Lambda$ S $\Lambda$ nnual Mceting, Conference Record, 1984, pp. 868 878.

20. V.T. Ranganathan, P.D. Ziogas, and V.R. Stefonovic, " $\Lambda$ DC- $\Lambda$ C Power Conversion Technique using Twin Resonant Iligh Frequency Links", IEEE Transactions on Industrial $\Lambda$ pplications, I $\Lambda-19$, No. 3, May/June 1983, pp. 393 400.

21. V. Vorperian, "High-Q Approximations in the Small-Signal Analysis of Resonant Converters", IEEE PESC Record, 1985, pp. 707 715.

22. R.J. King and T. $\Lambda$. Stuart, " $\Lambda$ Large Signal Dynamic Simulation for the Series Resonant Converter," IEEF: Transactions on $\Lambda$ erospace and Electronic Systems, AES-19, No.6, November 1983, pp. 859 870. 
23. R.J. King and T. $\Lambda$. Stuart, "Transformer Induced Instability of the Series Resonant Converter," IEEE Transactions on Aerospace and Flectronic Systems, AES-19, No.3, May 1983, pp. 474 482.

24. D.L. Cronin, "2800 Watt Series Inverter DC Power Supply", IEEE PESC Conference Record, 1971, pp. 117 123.

25. J. Biess, L. Inouye, and J.II. Shank, "High Voltage Series Resonant Inverter Ion Engine Screen Supply", IEEE PESC Conference Record, 1974, pp. 97 105.

26. D. Champers, "Designing High Power SCR Resonant Converters for Very High Frequency Operation", Proceedings of Powercon-9, 1982, Session F, Paper 2.

27. R.R. Robson, "Designing a 25 Kilowatt High Frequency Series Resonant DC/DC Converter", Proceedings of Powercon-11, 1984, Session H1, Paper 3.

28. E.E. Buchanan, Jr. and E.J. Miller, "Resonant Switching Power Conversion Technique", IEEE PESC Conference Record, 1975, pp. 183 193.

29. E.J. Miller, "Resonant Switching Power Conversions", IEEE PESC Conference Record, 1976, pp. 206 211.

30. R. Redl, B. Molnar, and N.O. Sokal, "Class-E Resonant Regulated DC/DC Power Converters: $\Lambda$ nalysis of Operation, and Experimental Results at 1.5MII", IEEE PESC Conference Record, 1983, pp. 50 60.

31. R. Oruganti and F.C. Lee, "State-Plane Analysis of Parallel Resonant Converters", IEEE PESC Conference Record, 1985, pp. 56 73.

32. K. Karube, ' $\Gamma$. Nomura, and T. Nakano, "Iligh-Frequency Resonant MOS-FET DC-DC Converter", IIEEE PESC Conference Record, 1988, pp. 26 34.

33. V.T. Ranganathan, P.D. Tiogas, V. Stefanovic, "Performance Characteristics of High Frequency Links under Forward and Regenerative Power Flow Conditions", IEEE I $\Lambda$ S $\Lambda$ nnual Meeting, Conference Record, 1983, pp. 831 839.

34. F.S. Tsai, R. Oruganti, and F.C. I.ee, " $\Lambda$ Novel Control for Bidirectional Power Flow of a Parallel Resonant Converter", IFE:E I $\Lambda$ S $\Lambda$ nnual Meeting, Conference Record, 198.5, pp. 1124 1129.

35. I.J. Pitcl, "Phase-Modulated, Resonant Power Conversion Techniques for High Iirequency I.ink Inverters", II:EE I $\Lambda$ S Annual Meeting , Conference Record, 1985, pp. $1163 \sim 1172$.

36. F.S. Tsai and F.C. Lee, "Constant-Frequency, Phase-Controlled Resonant Power Processor", IEEE I $\Lambda$ S $\Lambda$ nnual Meeting, Conference Record, 1986, pp. 617 622.

37. F.S. Tsai, "Constant-Frequency Resonant Power Processors", MS Thesis, Electrical Engineering Department, Virginia Polytechnic Institute \& State University, June, 198.5. 
38. O.D. Patterson, D.M. Divan, "Pseudo-Resonant Full Bridge DC-DC Converter", IEEE PESC Conference Record, 1987, pp. 424 430.

39. K. Harada and W. Gu, "Controlled Resonant -Converters with Switching Frequency Fixed", IEEE PESC Conference Record, 1987, pp. 431 438.

40. K.D.T. Ngo, "Analysis of a Series Resonant Converter Pulse-Width-Modulated or Current Controlled for Low Switching Losses", IEEE PESC Conference Record, 1987, pp. 527 536.

41. S.G. Trabert and R.W. Erickson, "Steady State Analysis of the Duty-Cycle Controlled Series Resonant Converter", IEEE PESC Conference Record, 1987, pp. 545 556.

42. F.S. Tsai, P. Materu, and F.C. Lee, "Constant-Frequency, Clamped-Mode Resonant Converters", IEEE PESC Conference Record, 1987, pp. 557 566.

43. F.S. Tsai, Y. Chin, and F.C. Lee, "State-Plane Analysis of Clamped-Mode Parallel Resonant Converter", IEEE INTELEC Conference Record, 1987, pp. 220 227.

44. J. Sabate, "Clamped-Mode Fixed-Frequency Series-Resonant Converter: OffLine $\Lambda$ pplications, $\Lambda$ nalysis, and Implementation", MS Thesis, Electrical Engineering Department, Virginia Polytechnic Institute \& State University, November, 1988.

45. K. Kuwabara, J. Chida, and E. Miyachika, "A Constant-Frequency, SeriesResonant DC-DC Converter with PWM Controlled Output", IEEE PESC Conference Record, 1988, pp. 563 .566.

46. B. Ray and T. $\Lambda$. Stuart, "A Cascaded Schwarz Converter for High Frequency Power Converter", IEEE PESC Conference Record, 1988, pp. 1199 1206.

47. K.H. Liu and F.C. Lee, "Resonant Switches - A Unified Approach to Improve Performances of Switching Converters", IEEE INTELEC Conference Record, 1984, pp. 344 351.

48. K.H. Liu, R. Oruganti, and F.C. I.ee, "Resonant Switches - Topologies and Characteristics", IEIEE PISSC. Conference Record, 1985, pp. 860 867.

49. K.II. Liu and F.C. Lce, "Zero-Voltage Switching Techniques in DC/DC Converters", IEEE PESC. Conference Record, 1986, pp. 58 70.

50. R. Oruganti and F.C. Lee, "Effects of Parasitic Losses on the Performance of Series Resonant Converters", IEEF: I $\Lambda \mathrm{S} \wedge$ nnual Meeting, Conference Record, 1985, pp. 1233 1243.

51. M.M. Jovanovic, K.II. Liu, and F.C. I.ee, "State-Plane Analysis of QuasiResonant Converters", IFEE Transactions on Power Electronics, PE-2, No. 5, January 1987. 
52. V. Vorperian, R. Tymerski, K.H. Liu, and F.C. Lee, "Generalized Resonant Switches Part 1: Topologies", Proceedings, Power Electronics Seminar, Virginia Power Electronics Center, Virginia Polytechnic Institute \& State University, November 1986.

53. V. Vorperian, R. 'Tymerski, K.HI. Liu, and F.C. Lee, "Generalized Resonant Switches Part 2: Analysis and Circuit Models", Proceedings, Power Electronics Seminar, Virginia Power Electronics Center, Virginia Polytechnic Institute \& State University, November 1986.

54. W. Tabisz, P. Gradzki, and F.C. Lee, "Zero-Voltage-Switched Quasi-Resonant Buck and Flyback Converters - Experimental Results at $10 \mathrm{MHz}$, IEEE PESC Conference Record, 1987, pp. 404 413.

55. A.W. Lotfi, V. Vorperian, and F.C. Lee, "Comparison of Stresses in QuasiResonant and Pulse-Width-Modulated Converters", IEEE PESC Conference Record, 1988, pp. 591 598.

56. W. Tabisz and F.C. Lee, "Zero-Voltage-Switching Multi-Resonant Technique - A Novel Approach to Improve Performance of High-Frequency QuasiResonant Converters", IEEE PESC Conference Record, 1988, pp. 9 17. 


\section{APPENDIX A.1 \\ DERIVATION OF CLAMPED-MODE, PARALLEL- \\ RESONANT CONVERTER}

Figure A.1 illustrates the combination of the two resonant inverters in a phasecontrolled, parallel-resonant ennverter(PC-PRC). The outputs of the inverters are connected in series. Viewing tank voltages $v_{1}$ and $v_{2}$ as new voltage sources, a simplified circuit for the PC-PRC is shown in Figure $\Lambda .1(b)$. The circuit equations for the PC-PRC are

$$
\begin{aligned}
& v_{1}=v_{C 1}+l \cdot \frac{d i_{L I}}{d t}, \\
& i_{t, 1}=C \cdot \frac{d v_{C 1}}{d t}+i_{O}, \\
& v_{2}=v_{C 2}+I \cdot \frac{d i_{L 2}}{d t}, \\
& i_{t .2}=\left(\cdot \frac{d v_{C 2}}{d t}+i_{o)},\right.
\end{aligned}
$$

and

$$
v_{o}=\left(v_{c 1}+v_{c_{2}}\right) .
$$

By adding Equ. $(\Lambda .1)$ to $(\Lambda .3)$, and $(\Lambda .2)$ and $(\Lambda .4)$, we obtain 


$$
\begin{gathered}
\left(v_{1}+v_{2}\right)=\left(v_{C 1}+v_{C 2}\right)+L\left(\frac{d i_{L 1}}{d t}+\frac{d i_{L 2}}{d t}\right), \\
\left(i_{L 1}+i_{l .2}\right)=C\left(\frac{d v_{C 1}}{d t}+\frac{d v_{C 2}}{d t}\right)+2 i_{O} .
\end{gathered}
$$

Rewriting Eqs. $(\boldsymbol{\Lambda . 6 )}$ and $(\boldsymbol{\Lambda} .7)$,

$$
\begin{gathered}
\left(v_{1}+v_{2}\right)=v_{O}+(2 L) \frac{d}{d t}\left(\frac{i_{L 1}+i_{L 2}}{2}\right), \\
\frac{\left(i_{L 1}+i_{L, 2}\right)}{2}=i_{O}+\left(\frac{C}{2}\right) \frac{d}{d t} v_{O} .
\end{gathered}
$$

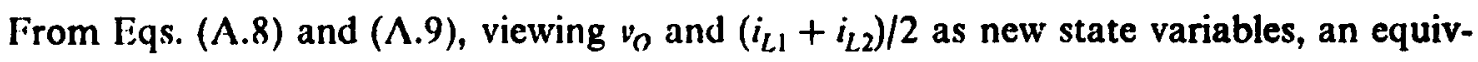
alent circuit for the PC-PRC can be derived, as shown in Figure A.1(c), where

$$
\begin{gathered}
v_{C}=v_{O}, \\
i_{L}=\frac{\left(i_{L 1}+i_{L 2}\right)}{2},
\end{gathered}
$$

and

$$
v_{s}=v_{1}+v_{2}
$$

The quasi-square-wave voltage $v_{s}$, as shown in Figure $\Lambda .1(e)$, can be realized using the full-bridge circuit shown in Figure $\Lambda . I(d)$, which is a clamped-mode, parallel-resonant converter(PC-PRC). 

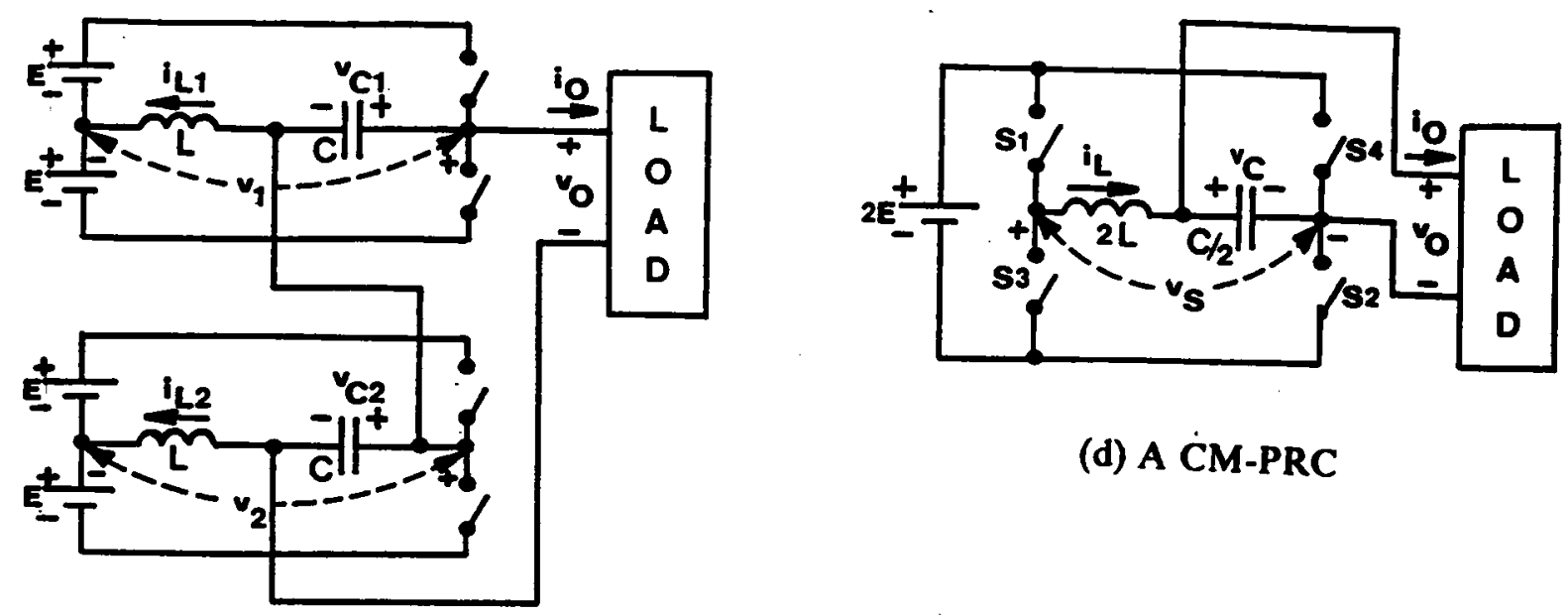

(a) A PC-PRC
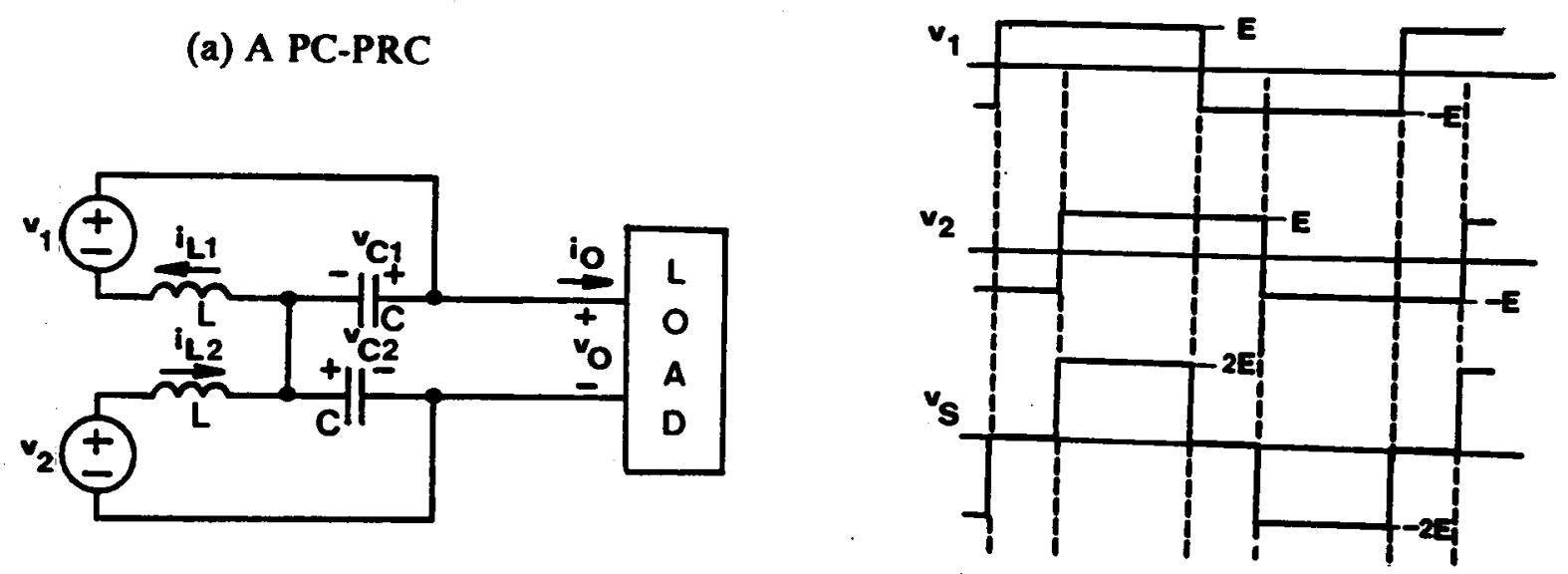

(b) Simplified circuit

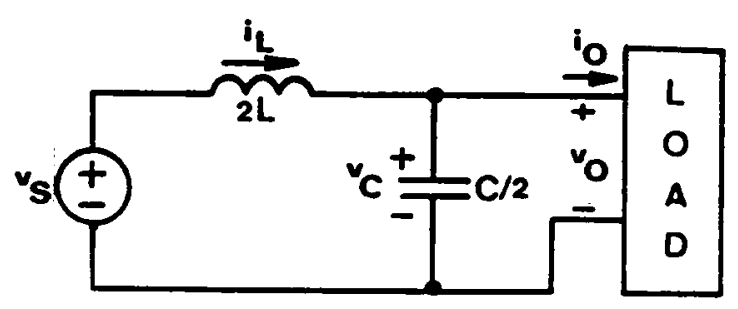

(c) Equivalent circuit

(d) A CंM-PRC

(e) Equivalent source voltage

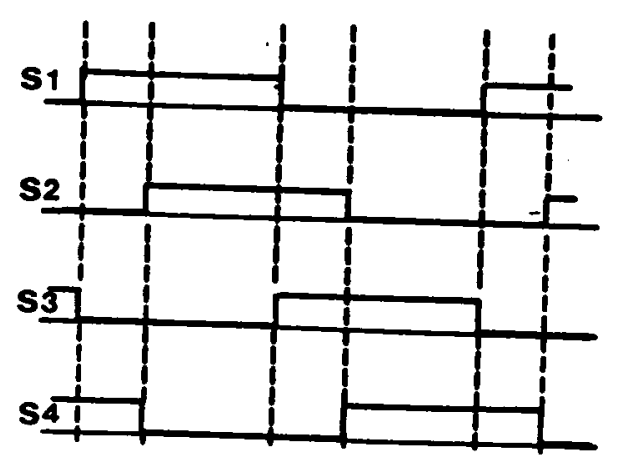

(f) Switch conduction sequence

Figure A.1 Derivation of Clamped-Mode Parallel-Resonant Converter 


\section{APPENDIX B.1 \\ RULES FOR CONSTRUCTING EQUILIBRIUM STATE TRAJECTORIES OF A CM-SRC}

An equilibrium state trajectory constructed in the composite diagram discussed in Section 2.3.4 must satisfy the following rules. These rules are obtained from the converter's operation.

- On the upper half plane $\left(i_{I, N}>0\right)$

- Triggering Q1 or forcing Q1 of initiates M6.

- Triggering Q2 initiates MI.

- Forcing Q2 off initiates M5.

- On the lower half plane $\left(i_{L N}<0\right)$

- $\quad$ rriggering $\mathrm{Q3}$ or forcing $\mathrm{Q3}$ ofr initiates $\mathrm{M} 3$.

- $\quad$ Triggering Q4 initiates M4.

- Forcing Q4 off initiates M2.

- On the $v_{C N}$-axis $\left(i_{L N}=0\right)$

- If the previous topological mode is M2, 
- M1 is initiated if $v_{C N}<1-V_{O N}$ (trajectory crosses $v_{C N}$-axis on the left-hand side of $\mathrm{ml}$ );

- M6 is initiated if $v_{C N}<-V_{O N}$ (trajectory crosses $v_{C N}$-axis on the left-hand side of $\mathrm{m6}$ ) and $\mathrm{Ql}$ is forced off the instant of crossing.

- If the previous topological mode is M3,

$\Delta \quad$ M6 is initiated if $v_{C N}<-V_{O N}$ (trajectory crosses $v_{C N}$-axis on the left-hand side of $\mathrm{m6}$ );

$\triangle \mathrm{M} 1$ is initiated if $v_{C N}<1-V_{O N}$ (trajectory crosses $v_{C N}$-axis on the left-hand side of $\mathrm{ml}$ ) and Q2 is triggered at the instant of crossing;

$\Delta \quad M 5$ is initiated if $v_{C N}<-1-V_{O N}$ (trajectory crosses $v_{C N}$-axis on the lefthand side of $\mathrm{m} .5$ ) and $\mathrm{Q} 2$ is forced off at the instant of crossing;

- MO is initiated if $v_{C N}>-V_{O N}$ (trajectory crosses $v_{C N}$-axis on the right-hand side of $\mathrm{m6}$ ). If the crossing point of the trajectory is to the left of $\mathrm{ml}$ ( $\left.v_{C N}<1-V_{O N}\right)$, MO will he terminated when $Q 2$ is triggered which initiates M1.

- If the previous topological mode is M4,

$\Delta \quad$ M5 is initiated if $v_{C N}<-1-V_{O N}$ (trajectory crosses $v_{C N}$-axis on the lefthand side o( $\mathrm{m} .5)$;

$\triangle \quad M 6$ is initiated if $v_{C N}<-V_{O N}$ (trajectory crosses $v_{C N}$-axis on the left-hand side of m6) and $Q 1$ is triggered at the instant of crossing;

$\Delta \quad \mathrm{M} 0$ is initiated if $v_{C N}>-1-V_{O N}$ (trajectory crosses $v_{C N}$-axis on the righthand side of $\mathrm{m} 5$ ). If the crossing point of the trajectory is to the left of $\mathrm{m} 6$ $\left(v_{C N}<-V_{O N}\right)$, MO will be terminated when $\mathrm{Ql}$ is triggered which initiates M6. 
- If the previous topological mode is M5,

- M1 is initiated if $v_{C N}>-1+V_{O N}$ (trajectory crosses $v_{C N}$-axis on the righthand side of $\mathrm{m} 4$ );

- M3 is initiated if $v_{C N}>V_{O N}$ (trajectory crosses $v_{C N}$-axis on the right-hand side of $\mathrm{m} 3$ ) and $\mathrm{Q} 3$ is forced off the instant of crossing.

- If the previous topological mode is M6,

- M3 is initiated if $v_{C N}>V_{O N}$ (trajectory crosses $v_{C N}$-axis on the right-hand side of $\mathrm{m} 3$ );

- M4 is initiated if $v_{C N}>-1+V_{O N}$ (trajectory crosses $v_{C N}$-axis on the righthand side of $\mathrm{m} 4$ ) and $\mathrm{Q} 4$ is triggered at the instant of crossing;

- M2 is initiated if $v_{C N} t l+V_{O N}$ (trajectory crosses $v_{C N}$-axis on the right-hand side of $\mathrm{m} 2$ ) and $\mathrm{Q} 4$ is forced off at the instant of crossing;

$\triangle M 0$ is initiated if $v_{C N}<V_{O N}$ (trajectory crosses $v_{C N}$-axis on the left-hand side of $\mathrm{m} 3$ ). If the crossing point of the trajectory is to the right of $\mathrm{m} 4$ ( $\left.v_{C N}>-1+V_{O N}\right)$, M0 will be terminated when Q4 is triggered which initiates M4.

- If the previous topological mode is MI,

$\triangle \mathrm{M} 2$ is initiated if $v_{C N}>1+V_{O N}$ (trajectory crosses $v_{C N}$-axis on the righthand side of $\mathrm{m} 2$ );

- M3 is initiated if $v_{C N}>V_{O N}$ (trajectory crosses $v_{C N}$-axis on the right-hand side of $\mathrm{m} 3$ ) and $\mathrm{Q} 4$ is triggered at the instant of crossing; 
$\Delta \quad \mathrm{M} 0$ is initiated if $v_{C N}<1+V_{O N}$ (trajectory crosses $v_{C N}$-axis on the left-hand side of $\mathrm{m} 2$ ). If the crossing point of the trajectory is to the right of $\mathrm{m} 3$ ( $\left.v_{C N}>V_{O N}\right)$, M0 will be terminated when Q4 is triggered which initiates M3. 


\section{APPENDIX B.2}

\section{PREDICTION OF MODE TRANSITIONS OF A CM-SRC OPERATING BELOW RESONANT FREQUENCY}

Consider the boundary trajectories, $T_{34}$, between Mode III and Mode IV, and $T_{35}$, between Mode III and Mode V, as shown in Figure B.2.1 and Figure B.2.2, respectively. These two trajectories are constructed via same topological mode sequence and can be represented by the same equations,

$$
\begin{aligned}
& \cos \zeta=\frac{1+\left(1-R+2 V_{O N}\right)^{2}-R^{2}}{2\left(1-R+2 V_{O N}\right)}, \\
& \cos \delta=\frac{1+R^{2}-\left(1-R+2 V_{O N}\right)^{2}}{2 R}, \\
& \omega_{S N}=\frac{\pi}{\pi+\delta+\zeta}
\end{aligned}
$$

The parameter $R$ can be used to determine whether a trajectory defined by equations (B.2.1) is a $T_{34}$, or $T_{45}$. When $R \leq 1-2 V_{O N}$, equations (B.2.1) represent a $T_{34}$. When $R>1-2 V_{O N}$, equations (B.2.1) represent a $T_{35}$.

Such a boundary trajectory can only be constructed with $R$ in between $V_{O N}$ and 1 , as illustrated in Figure B.2.3. The frequency, $\omega_{T N}$, of the trajectory is related to $R$ in a way as shown in Figure B.2.4, where

$$
\begin{aligned}
& \omega_{3 N}=\frac{\pi}{\pi+2 \cos ^{-1}\left(\frac{1}{1+2 V_{O N}}\right)} \\
& \omega_{4 N}=\frac{\pi}{\pi+\cos ^{-1} V_{O N}+\cos ^{-1}\left(1-2 V_{O N}^{2}\right)}
\end{aligned}
$$

This relationship can be used to determine the transitions of operating modes as follows. 
(a). $V_{O N} \leq 0.5$

(a.1) If $\omega_{S N}>\omega_{3 N}$, such a boundary trajectory does not exist, implying both mode-IV and mode-V operations do not exist. The converter's operation transits from Mode III directly to Mode VI, as indicated by S3 in Figure 2.10(c).

The boundary $\beta_{S}$ angle, $\beta_{36}$, separating mode III and Mode VI, can be calculated from trajectory $T_{36}$, as shown in Figure B.2.5,

$$
\beta_{36}=\omega_{S N} \times \delta, \quad \delta=\cos ^{-1}\left(1-2 V_{O N}^{2}\right)
$$

(a.2) If $\omega_{S N}=\omega_{3 N}$, one such boundary trajectory exists. The converter's operation transits from mode III to the boundary trajectory back to Mode III then to Mode VI, as indicated by $\mathbf{S} 4$ in Figure 2.10(c). If the boundary trajectory is viewed as a special case of Mode-III operation, the converter's mode transition sequence is the same as (a.1).

(a.3) If $\omega_{4 N} \leq \omega_{S N}<\omega_{3 N}$, two such boundary trajectories exist. Equations (B.2.1) have two sets of solutions, $\left(R_{1}, \zeta_{1}, \delta_{1}\right)$ and $\left(R_{2}, \zeta_{2}, \delta_{2}\right)$.

- (a.3.1) If $R_{1} \leq 1-2 V_{O N}$ and $R_{2}>1-2 V_{O N}$, a boundary trajectory, $T_{34}$, between Mode III and Mode IV and another boundary trajectory, $T_{35}$, between Mode III and Mode V exist, implying both Mode-IV and Mode-V operations exist. The converter's operation transits from Mode III to Mode IV to Mode V back to Mode III to Mode VI, as indicated by S5 in Figure 2.10(d).

The boundary $\beta_{S}$ angle, $\beta_{34}$, between Mode III and Mode IV is equal to $\omega_{S N} \times \delta_{1}$

The boundary $\beta_{S}$ angle, $\beta_{45}$, between Mode IV and Mode $\mathrm{V}$ can be calculated from trajectory $T_{45}$, as shown in Figure B.2.6, 


$$
\beta_{45}=\omega_{S N} \times \delta, \quad \delta=\cos ^{-1}\left(1-\frac{6 V_{O N}^{2}}{1-2 V_{O N}}\right)
$$

The boundary $\beta_{S}$ angle, $\beta_{53}$, between Mode $V$ and Mode III is equal to $\omega_{S N} \times \delta_{2}$

The boundary $\beta_{S}$ angle, $\beta_{36}$, between Mode III and Mode VI is calculated as in (a.1).

- (a.3.2) If $R_{1} \leq 1-2 V_{O N}$ and $R_{2} \leq 1-2 V_{O N}$, two boundary trajectories exist between Mode III and Mode IV, implying Mode-V operation does not exist. The converter's operation transits from Mode III to Mode IV back to Mode III to Mode VI, as indicated by $\mathbf{S 6}$ in Figure 2.10(e).

The boundary $\beta_{S}$ angle, $\beta_{34}$, between Mode III and Mode IV is equal to $\omega_{S N} \times \delta_{1}$

The boundary $\beta_{S}$ angle, $\beta_{43}$, between Mode IV and Mode III is equal to $\omega_{S N} \times \delta_{2}$.

The boundary $\beta_{s}$ angle, $\beta_{36}$, between Mode III and Mode VI is calculated as in (a.1).

- (a.3.3) If $R_{1}>1-2 V_{O N}$ and $R_{2}>1-2 V_{O N}$, two boundary trajectories exist between Mode III and Mode V, implying Mode-IV operation does not exist. The converter's operation transits from Mode III to Mode V back to Mode III to Mode VI, as indicated by $\mathbf{S 7}$ in Figure 2.10(c).

The boundary $\beta_{s}$ angle, $\beta_{35}$, between Mode III and Mode $V$ is equal to $\omega_{S N} \times \delta_{1}$.

The boundary $\beta_{S}$ angle, $\beta_{53}$, between Mode $V$ and Mode III is equal to $\omega_{S N} \times \delta_{2}$ 
The boundary $\beta_{S}$ angle, $\beta_{36}$, between Mode III and Mode VI is calculated as in (a.1).

(a.4) If $0.5 \leq \omega_{S N}<\omega_{4 N}$, one such boundary trajectory exists. Equations (B.2.1) have one solution, $\left(R_{1}, \zeta_{1}, \delta_{1}\right)$.

- (a.4.1) If $R_{1} \leq 1-2 V_{O N}$, a boundary trajectory exists between Mode III and Mode IV. Since a Mode-IV trajectory can not transit directly into a Mode-VI trajectory, Mode- $\mathrm{V}$ operation must also exist. The converter's operation transits from Mode III to Mode IV to Mode V to Mode VI, as indicated by S8 in Figure 2.10(b).

The boundary $\beta_{S}$ angle, $\beta_{34}$, between Mode III and Mode IV is equal to $\omega_{S N} \times \delta_{1}$.

The boundary $\beta_{S}$ angle, $\beta_{45}$, between Mode IV and Mode $V$ is calculated as in (a.3.1).

The boundary $\beta_{S}$ angle, $\beta_{56}$, between Mode V and Mode VI can be calculated from trajectory $T_{56}$, as shown in Figure B.2.7,

$$
\beta_{56}=\omega_{S N} \times \delta, \quad \delta=\cos ^{-1}\left(1-2 V_{O N}^{2}\right) .
$$

- (a.4.2) If $R_{1}>1-2 V_{O N}$, a boundary trajectory exists between Mode III and Mode $\mathrm{V}$, implying Mode-IV operation does not exist. The converter's operation transits from Mode III to Mode V to Mode VI, as indicated by S9 in Figure 2.10(d).

The boundary $\beta_{S}$ angle, $\beta_{35}$, hetween Mode III and Mode $V$ is equal to $\omega_{S N} \times \delta_{1}$.

The boundary $\beta_{S}$ angle, $\beta_{56}$, between Mode $V$ and Mode VI is calculated as in (a.4.1). 
(b). $V_{\text {ON }}>0.5$

(b.1) If $\omega_{S N}>\omega_{4 N}$, such a boundary trajectory does not exist, implying both Mode-IV and Mode-V operations do not exist. The converter's operation transits from Mode III directly to Mode VI, as in (a.1).

(b.2) If $\omega_{S N} \leq \omega_{4 N}$, one such boundary trajectory exists. Since $R>V_{O N}>1-2 V_{O N}$, the trajectory is between Mode III and Mode V, implying Mode-IV operation does not exist. The converter's operation transits from Mode III to Mode V to Mode VI, as in (a.4.2). 


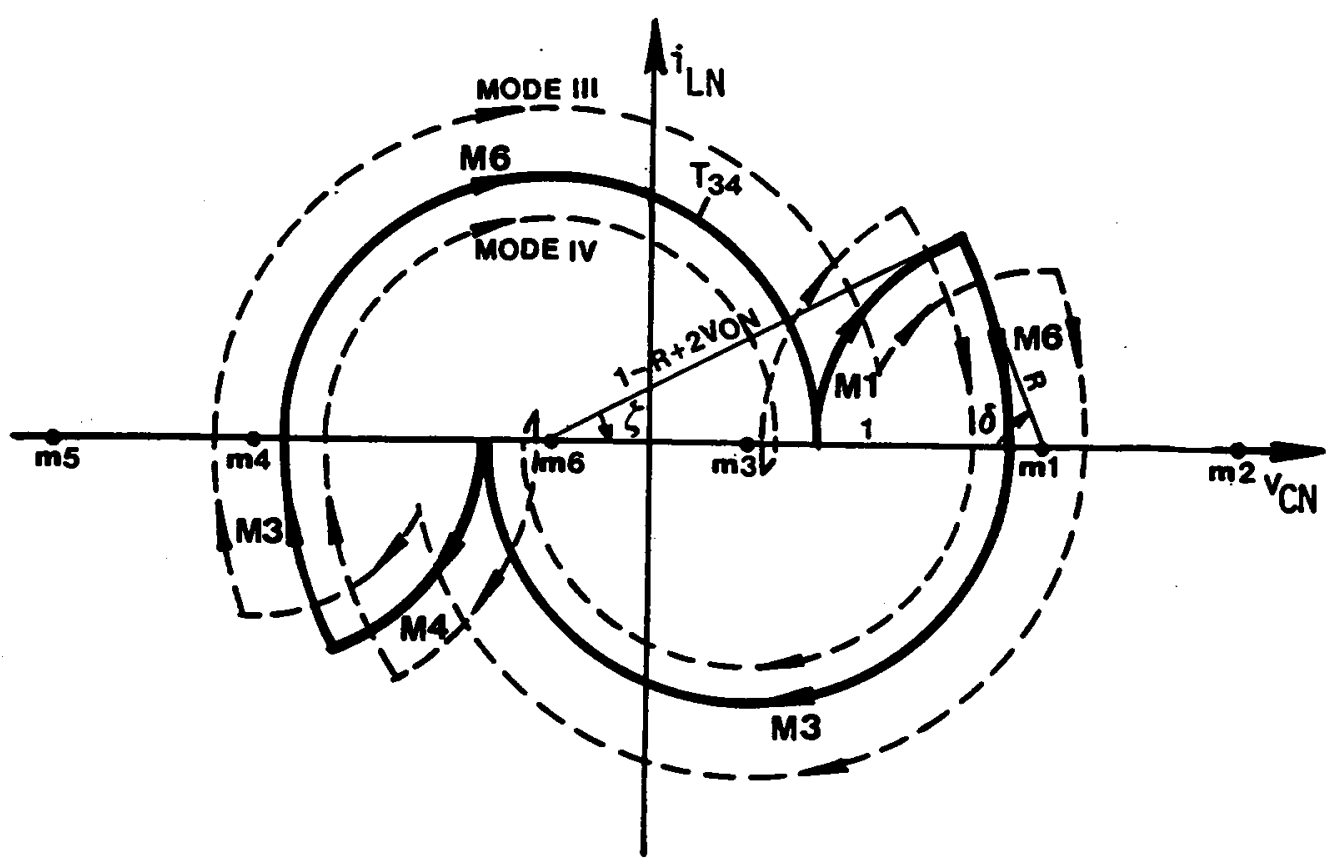

Figure B.2.1 Boundary Trajectory Between Mode III and Mode IV 


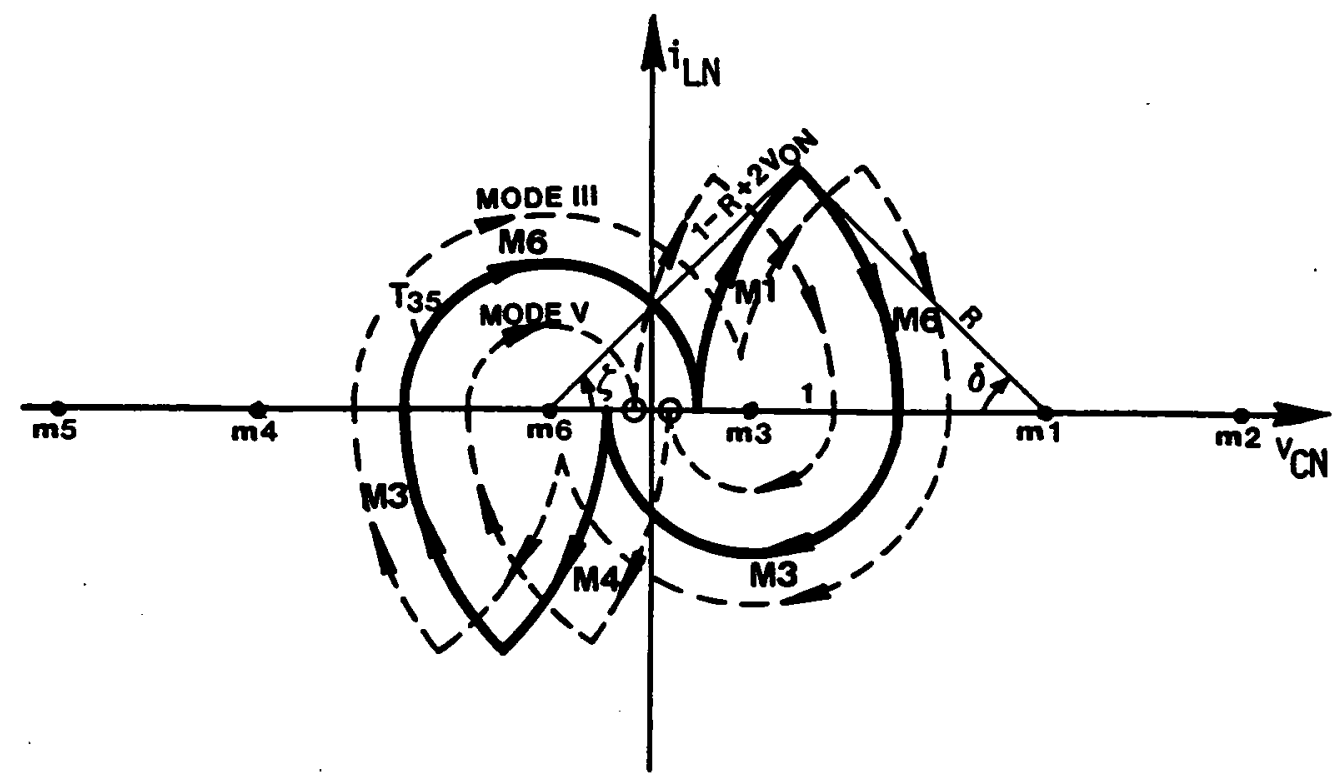

Figure B.2.2 Boundary Trajectory Between Mode III and Mode V 


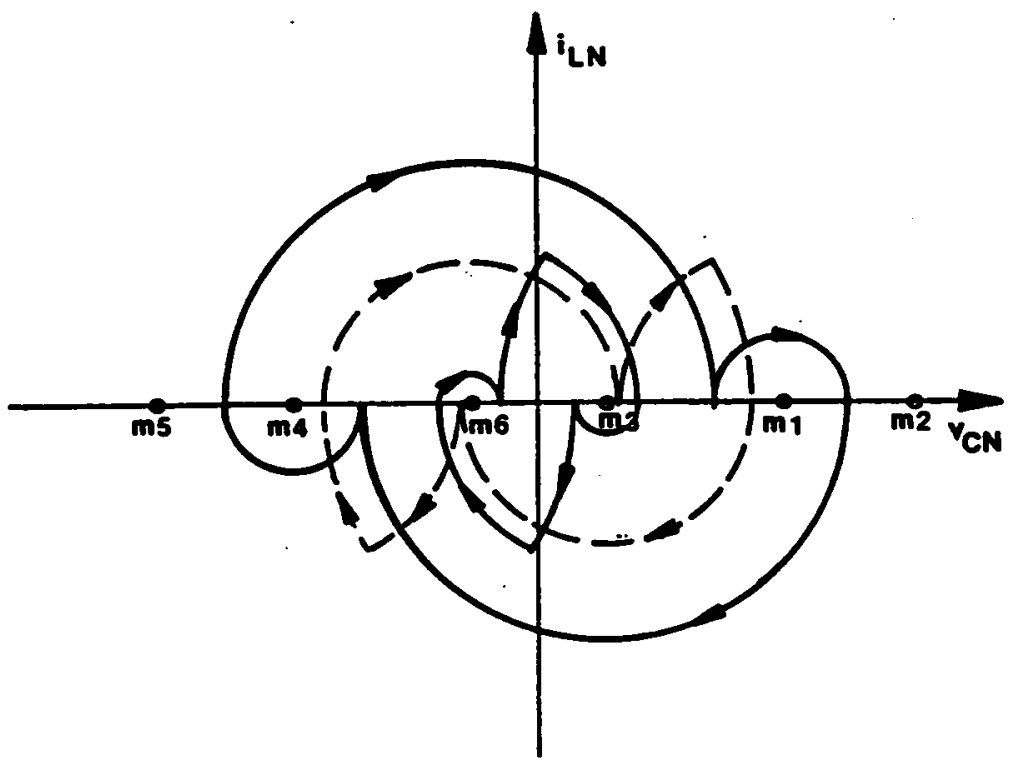

Figure B.2.3 Extreme Trajectory Described by Equation (B.2.1) 


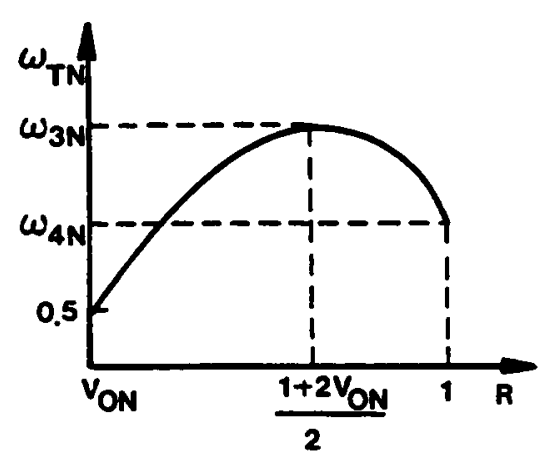

$v_{\text {ON }} \leq 0.5$

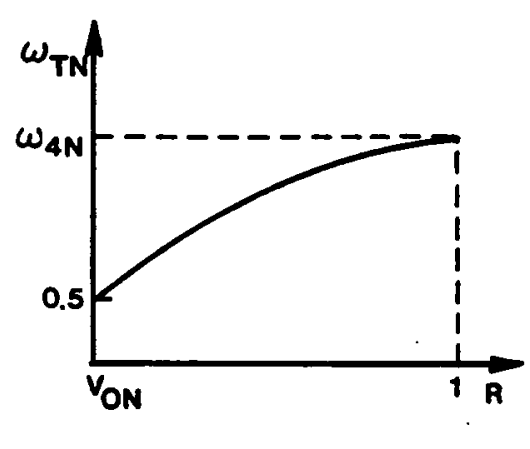

$v_{\text {ON }}>0.5$

Figure B.2.4 Relationship Between Frequency of the Trajectory Described by Equation (B.2.1) and Radius $R$ 


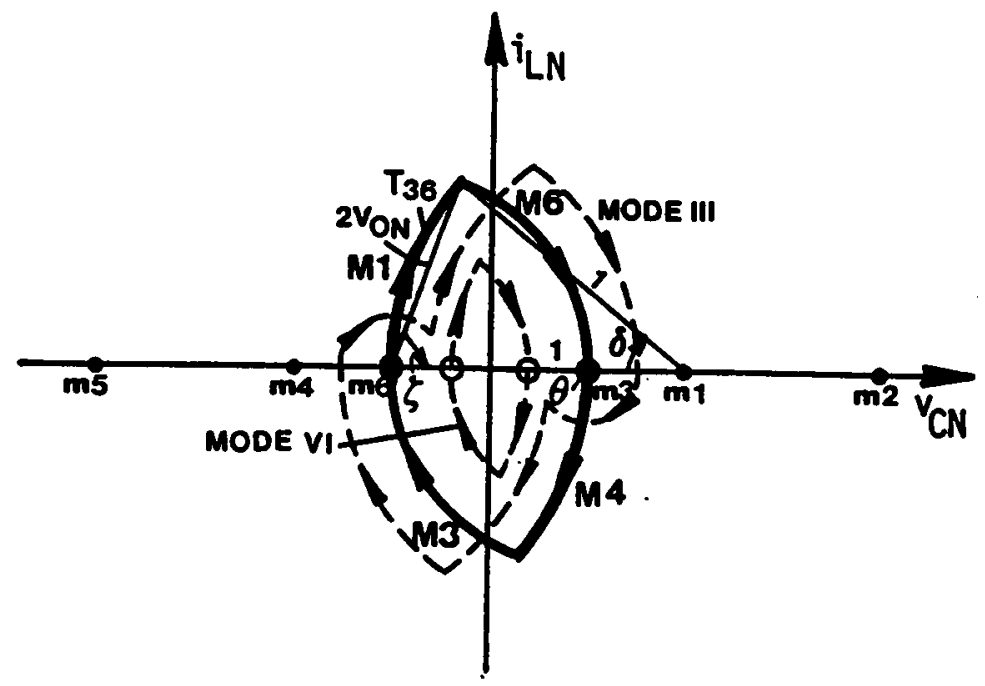

Figure B.2.5 Boundary Trajectory Between Mode III and Mode VI 


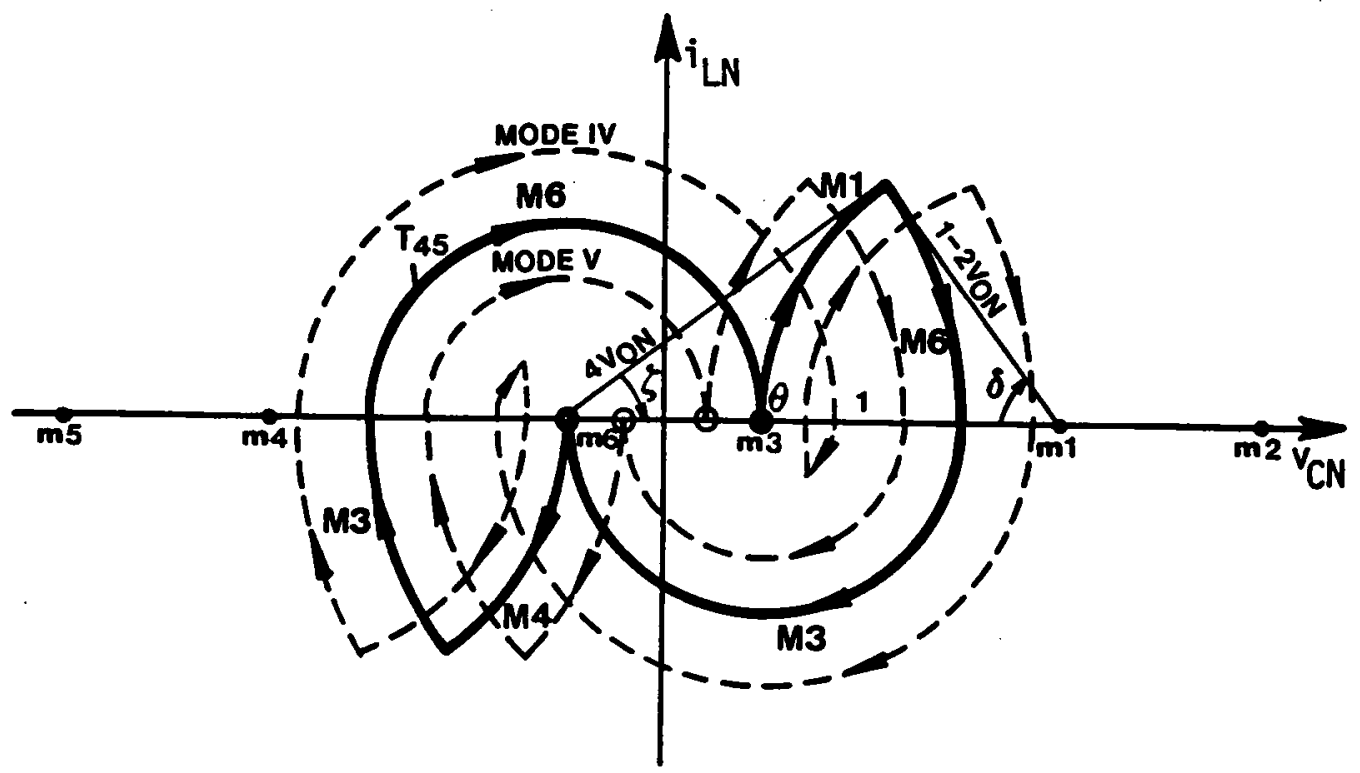

Figure B.2.6 Boundary Trajectory Between Mode IV and Mode V 


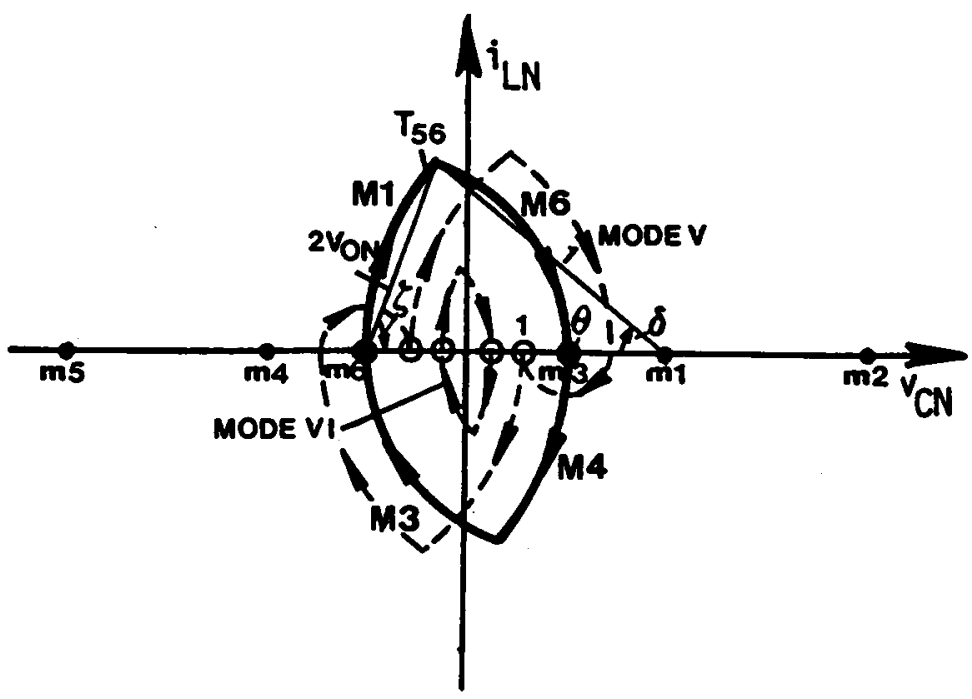

Figure B.2.7 Boundary Trajectory Between Mode V and Mode VI 
FORTRAN Program for Determining Mode Transitions and Mode Boundaries Below Resonant Frequency

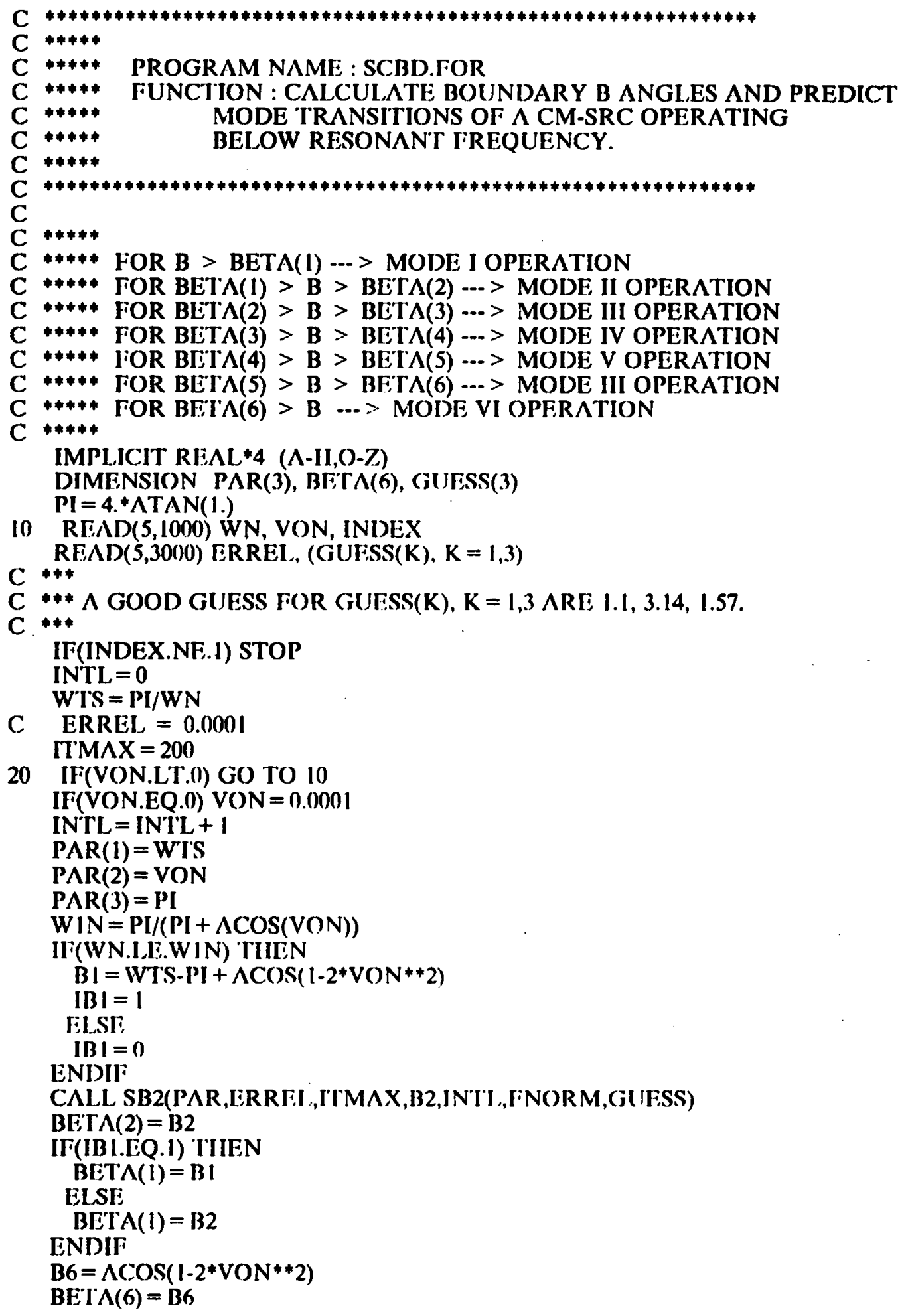




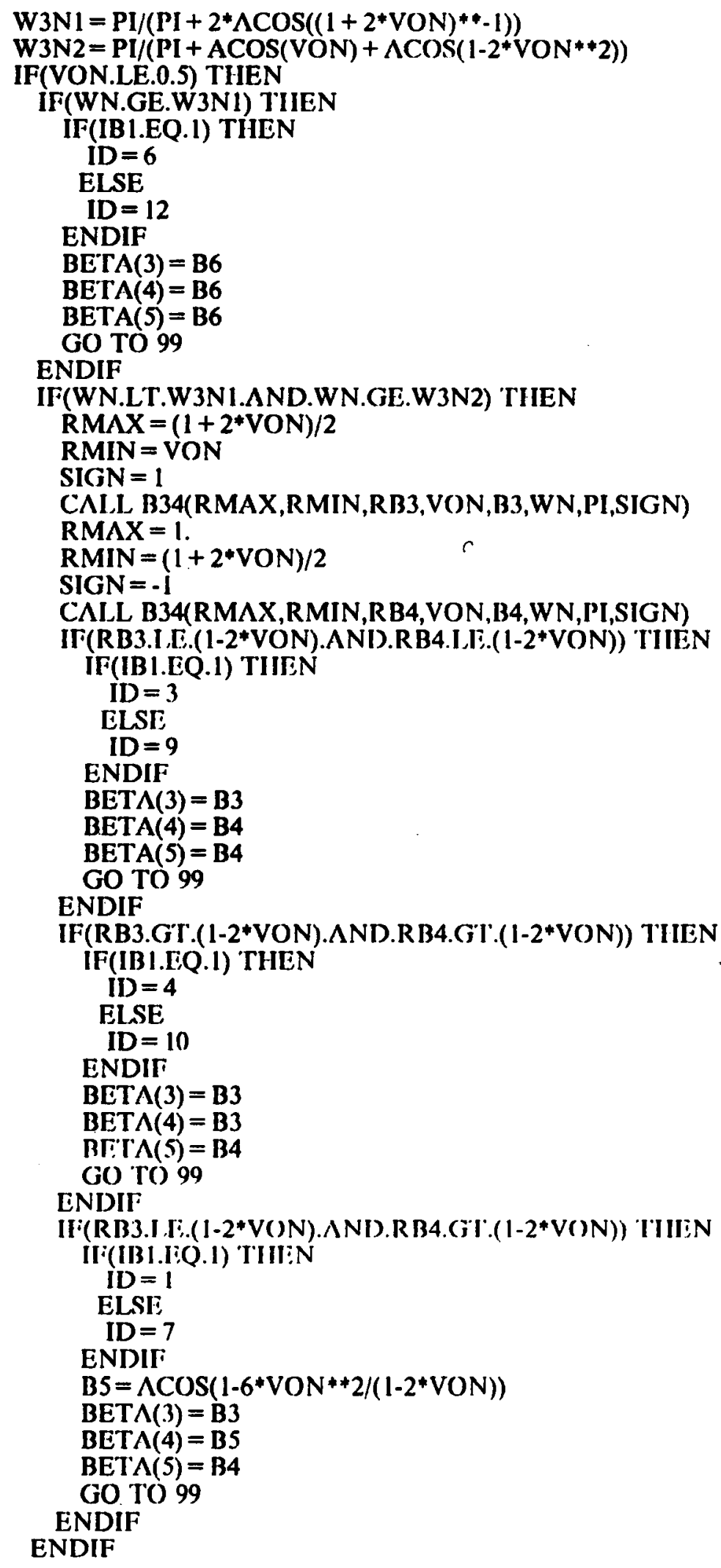




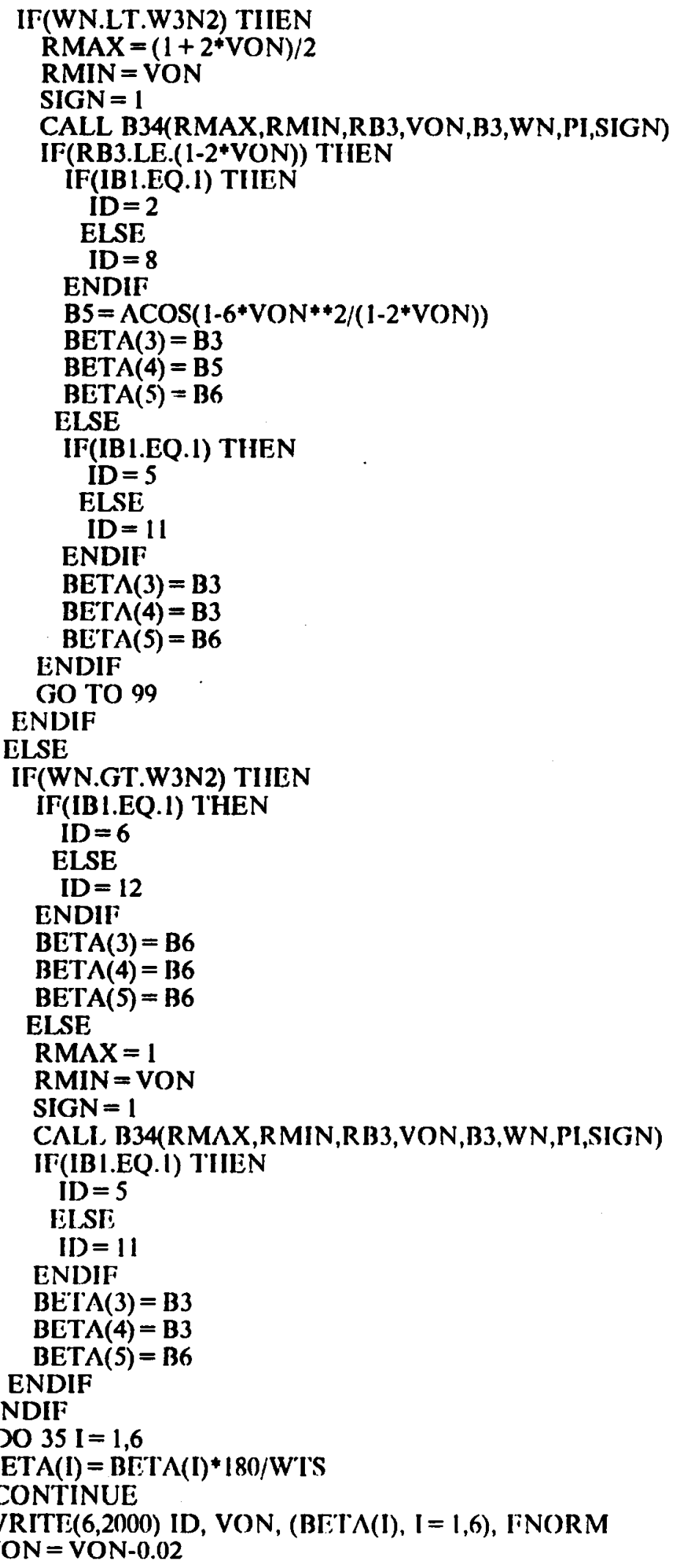




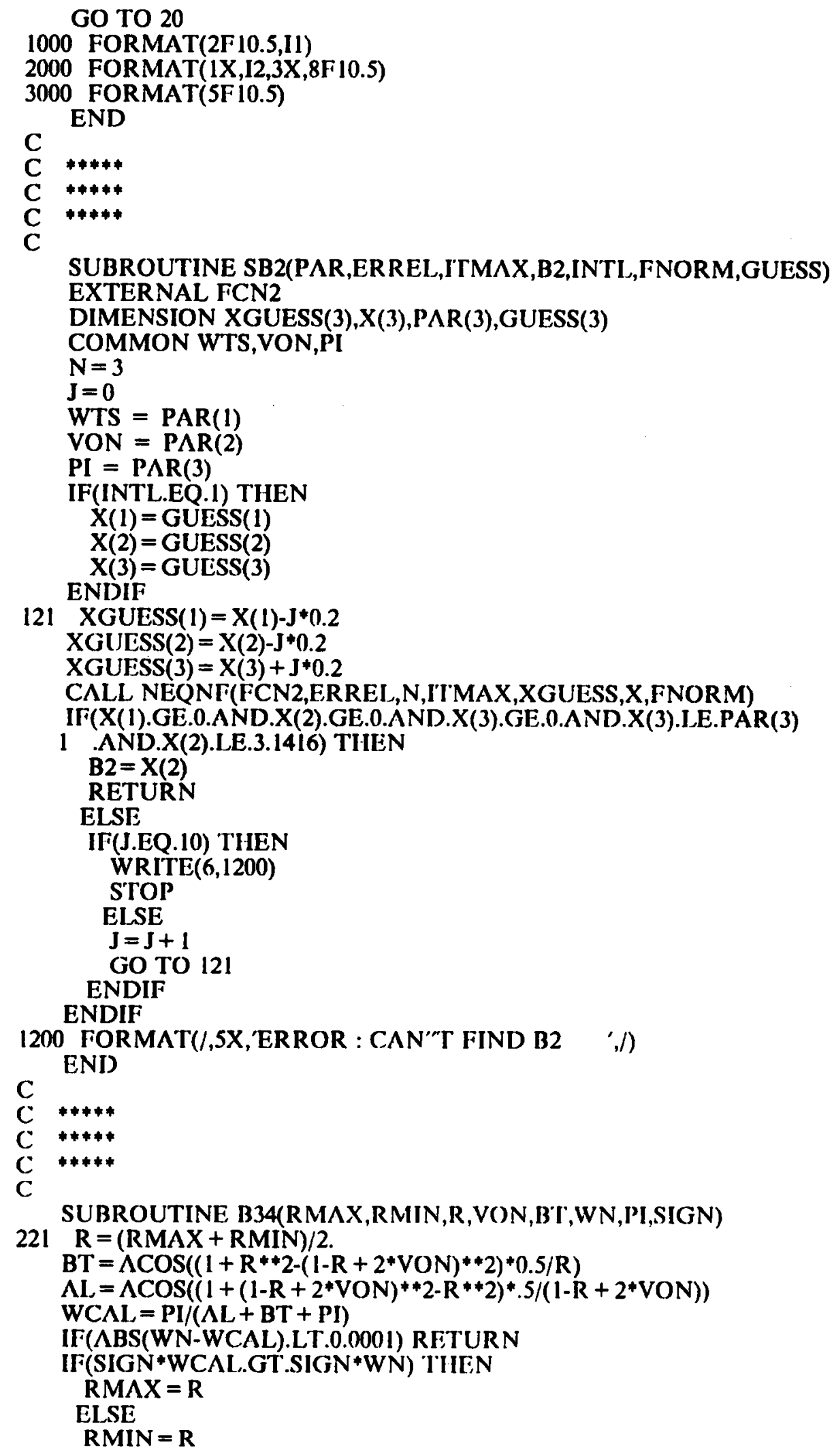




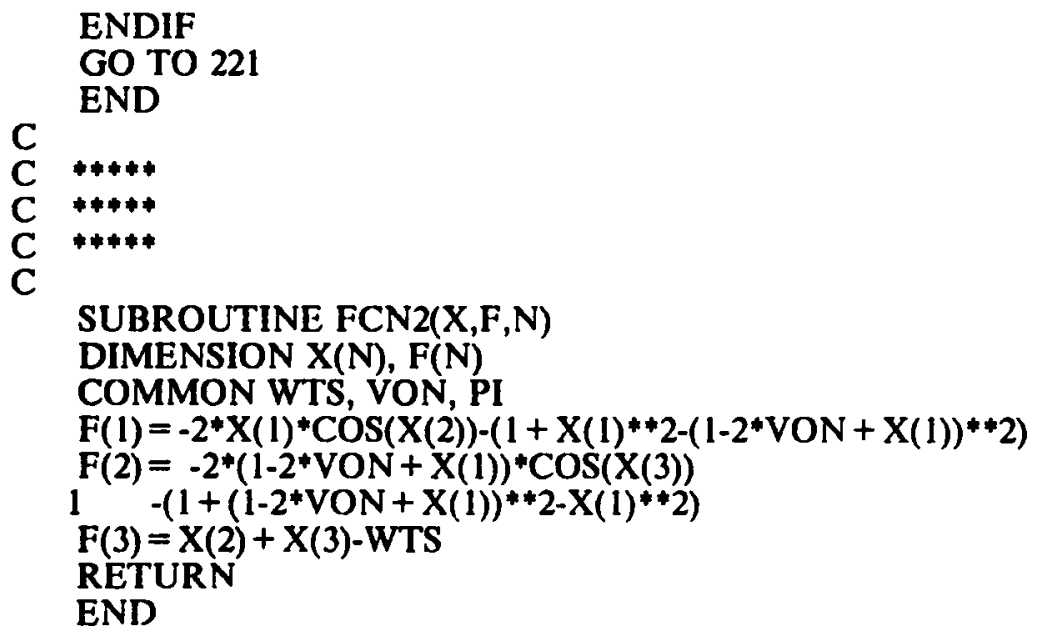




\section{APPENDIX B.3}

\section{PREDICTION OF MODE TRANSITIONS OF A CM-SRC OPERATING ABOVE RESONANT FREQUENCY}

Consider the boundary trajectories, $T_{A B}$, between Mode $\Lambda$ and Mode $B$, and $T_{A C}$, between Mode $\mathrm{A}$ and Mode $\mathrm{C}$, as shown in Figure B.3.1 and Figure B.3.2, respectively. These two trajectories are constructed via same topological mode sequence and can be represented by the same equations,

$$
\begin{aligned}
& \cos \delta=\frac{1+\left(R_{1}-1+2 V_{O N}\right)^{2}-R_{1}^{2}}{2\left(R_{1}-1+2 V_{O N}\right)}, \\
& \cos \beta=\frac{1+R_{1}^{2}-\left(R_{1}-1+2 V_{O N}\right)^{2}}{2 R_{1}}, \\
& \omega_{S N}=\frac{\pi}{\delta+\beta} .
\end{aligned}
$$

The parameter $R_{1}$ can be used to distinguish between trajectories $T_{A B}$ and $T_{A C}$. When $R_{1}>1$, equations (B.3.1) represent a $T_{A B}$. When $R_{1}<1$, equations (B.2.1) represent a $T_{A C} \cdot$

As illustrated in Figure B.3.3, the frequency of a trajectory represented by equation (B.3.1) increases as $R_{1}$ decreases. Thus, the frequency, $\omega_{1 N}$, separating trajectory $T_{A B}$ from trajectory $T_{A C}$ occurs at $R_{\mathrm{I}}=1$ and is calculated as

$$
\omega_{1 N}=\frac{\pi}{\delta+\beta}
$$

where, $\delta=\cos ^{-1}\left(V_{O N}\right)$ and $\beta=\cos ^{-1}\left(1-2 V_{O N}^{2}\right)$. 
If the converter's operating frequency, $\omega_{S N}$, is greater than $\omega_{I N}$, boundary trajectory $T_{A C}$ exists. This implies that Mode B operation does not exist. As $\beta_{S}$ decreases, the converter's operation transits from Mode $\Lambda$ to Mode $C$, as indicated by SA in Figure 2.13(a). The boundary $\beta_{S}$ angle, $\beta_{A C}$, can be calculated by solving equation (B.3.1). Angle $\beta_{A C}$ is equal to $\beta \times \omega_{S N}$.

If $\omega_{S N}<\omega_{1 N}$, boundary trajectory $T_{A B}$ exists. Thus, as $\beta_{S}$ decreases, the converter's operation transits from Mode A to Mode B to Mode C, as indicated by SB in Figure 2.13(a). The boundary $\beta_{S}$ angle, $\beta_{A B}$, is calculated by solving equation (B.3.1). Angle $\beta_{A B}$ is equal to $\beta \times \omega_{S N}$.

The boundary $\beta_{S}$ angle, $\beta_{B C}$, between Mode $B$ and Mode $C$ can be calculated from trajectory $T_{B C}$, as shown in Figure B.3.4. Angle $\beta_{B C}$ is equal to $\beta \times \omega_{S N}$, where $\beta=\cos ^{-1}\left(1-2 V_{O N}^{2}\right)$. 


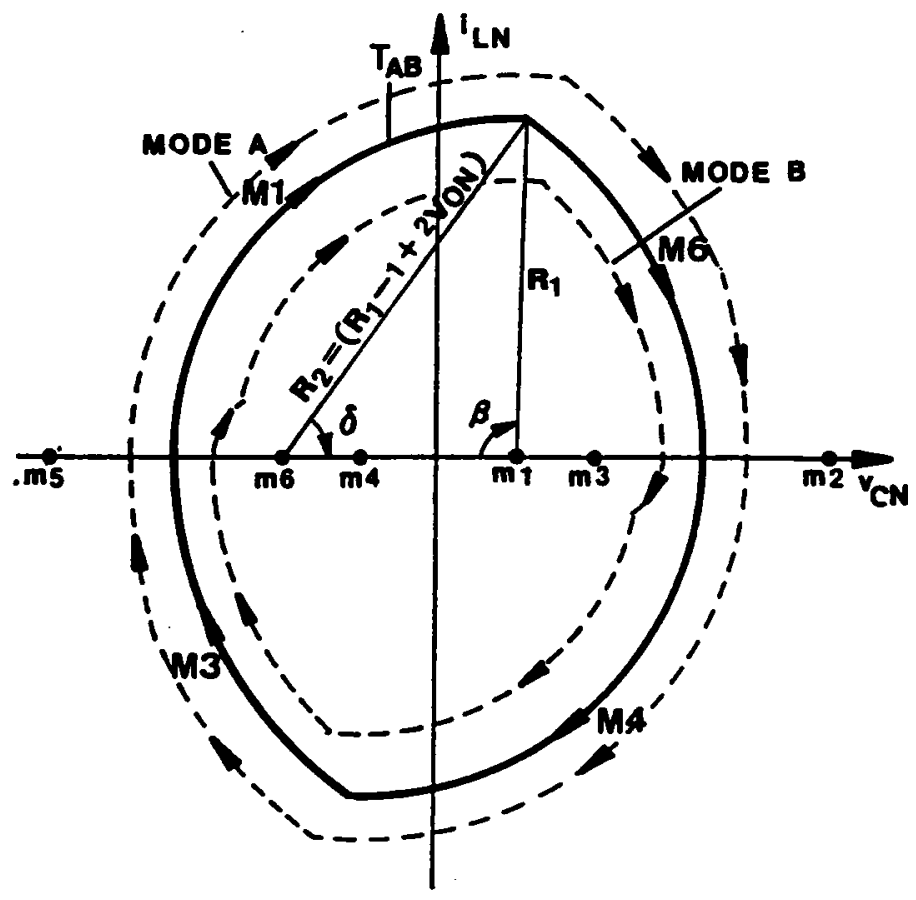

Figure B.3.1 Boundary Trajectory Between Mode A and Mode B 


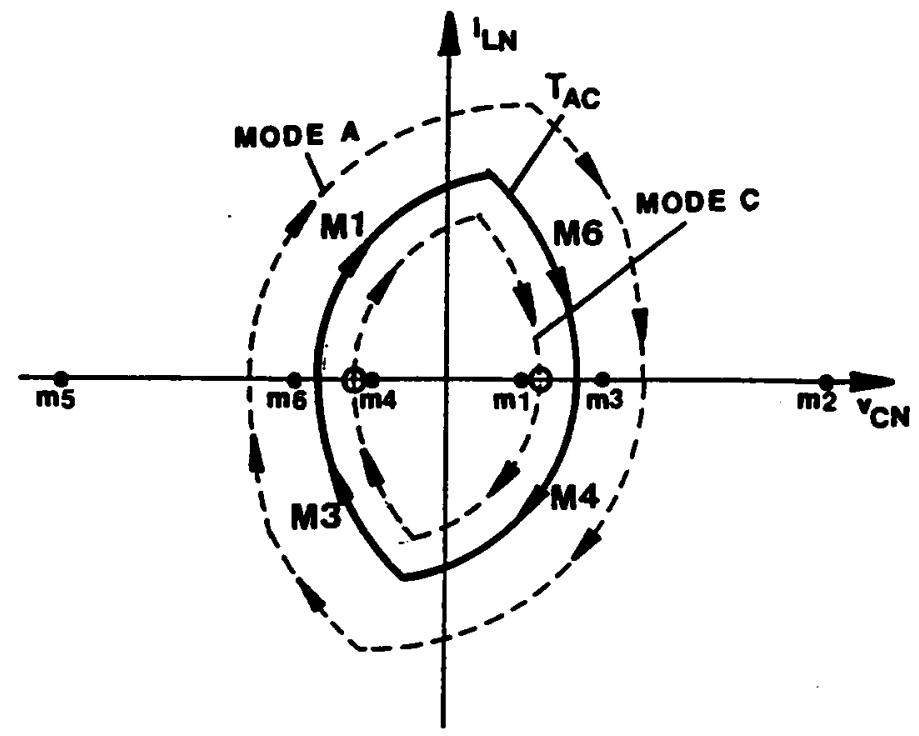

Figure B.3.2 Boundary Trajectory Between Mode A and Mode C 


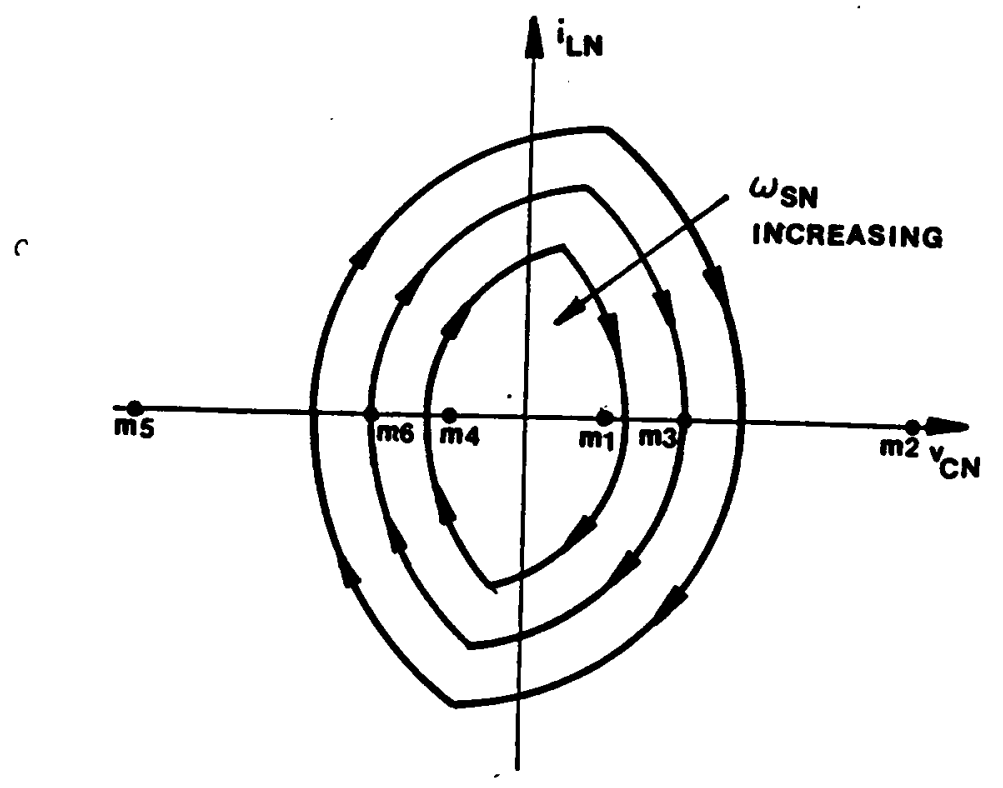

Figure B.3.3 Relationship Between Frequency of the Trajectory Described by Equation (B.3.1) and Radius $R_{1}$ 


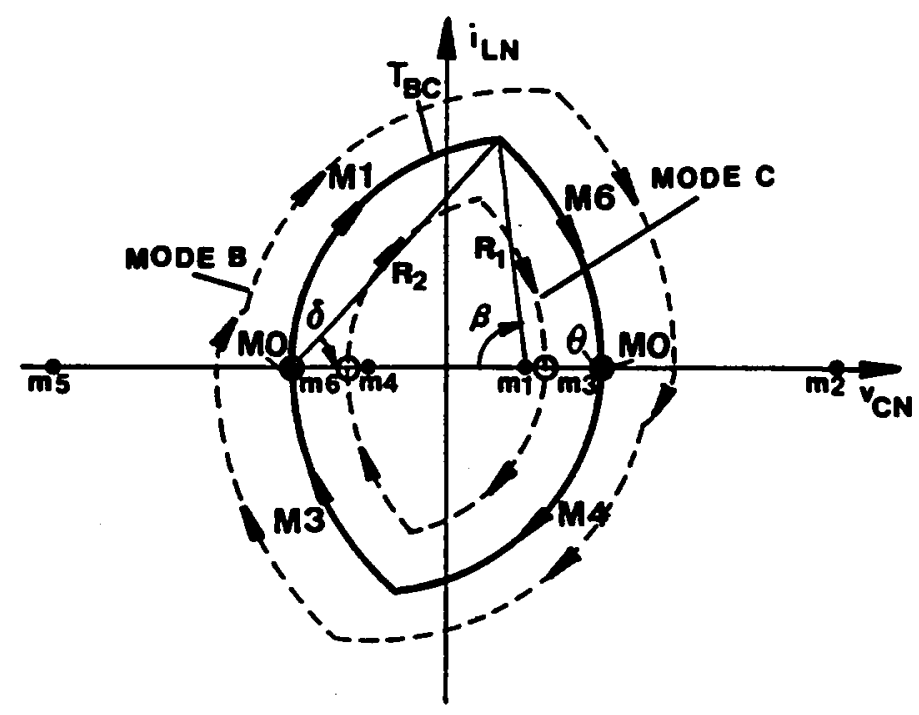

Figure B.3.4 Boundary Trajectory Between Mode B and Mode C 
FORTRAN Program for Determining Mode Transitions and Mode Boundaries Above Resonant Frequency

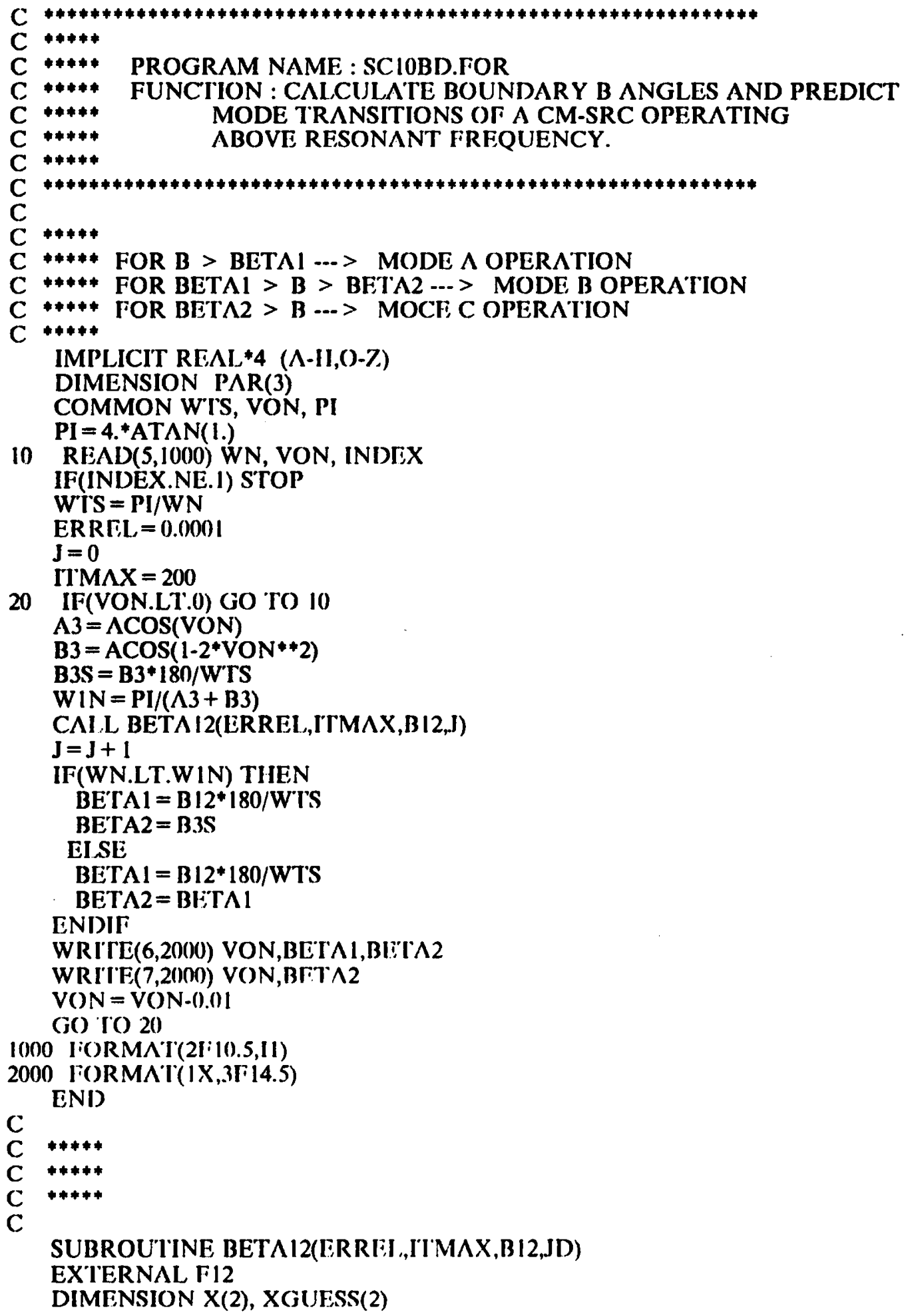

10 READ $(5,1000)$ WN, VON, INDEXX

IF(INDEX.NE.1) STOP

WTS $=$ PI/WN

ERRF,L $=0.0001$

$J=0$

IIM $\Lambda X=200$

20 IF(VON.LT.0) GO TO 10

$\mathrm{A} 3=\Lambda \operatorname{COS}(\mathrm{VON})$

$\mathrm{B} 3=\mathrm{ACOS}(1-2 * \mathrm{VON} * 2)$

$\mathrm{B} 3 \mathrm{~S}=\mathrm{B} 3 * 180 / \mathrm{W}$ TS

$\mathrm{W} I N=\operatorname{PI} /(\Lambda 3+B 3)$

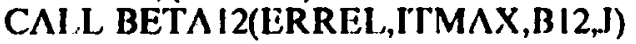

$\mathbf{J}=\mathbf{J}+\mathbf{1}$

IF(WN.LT.WIN) THEN

BETA1 $=B 12 * 180 /$ WTS

BETA2 $=$ B.3S

EISE

BETA $1=B 12 * 180 /$ WTS BETA2 = BETA

ENIDIF

WRITE $(6,2000)$ VON,BET $\wedge 1$, BLIT $\wedge 2$

WRI'IE $(7,2000)$ VON,BFT $\wedge 2$

$\mathrm{VON}=\mathrm{VON}-(0.01$

GO TO 20

I000 IFORMAT $(2 \mathrm{I}: 10.5,11)$

2000 FORMAT $(1 X, 3 F 14.5)$

ENI

C

C $* * * *$

C $* * * * *$

C $* * * * *$

C

SUBROUTINE BET $\Lambda 12$ (ERRII,ITM $\Lambda X, B 12, J D)$

EXI'ERNAL F12

DIMENSION X(2), XGUESS(2) 


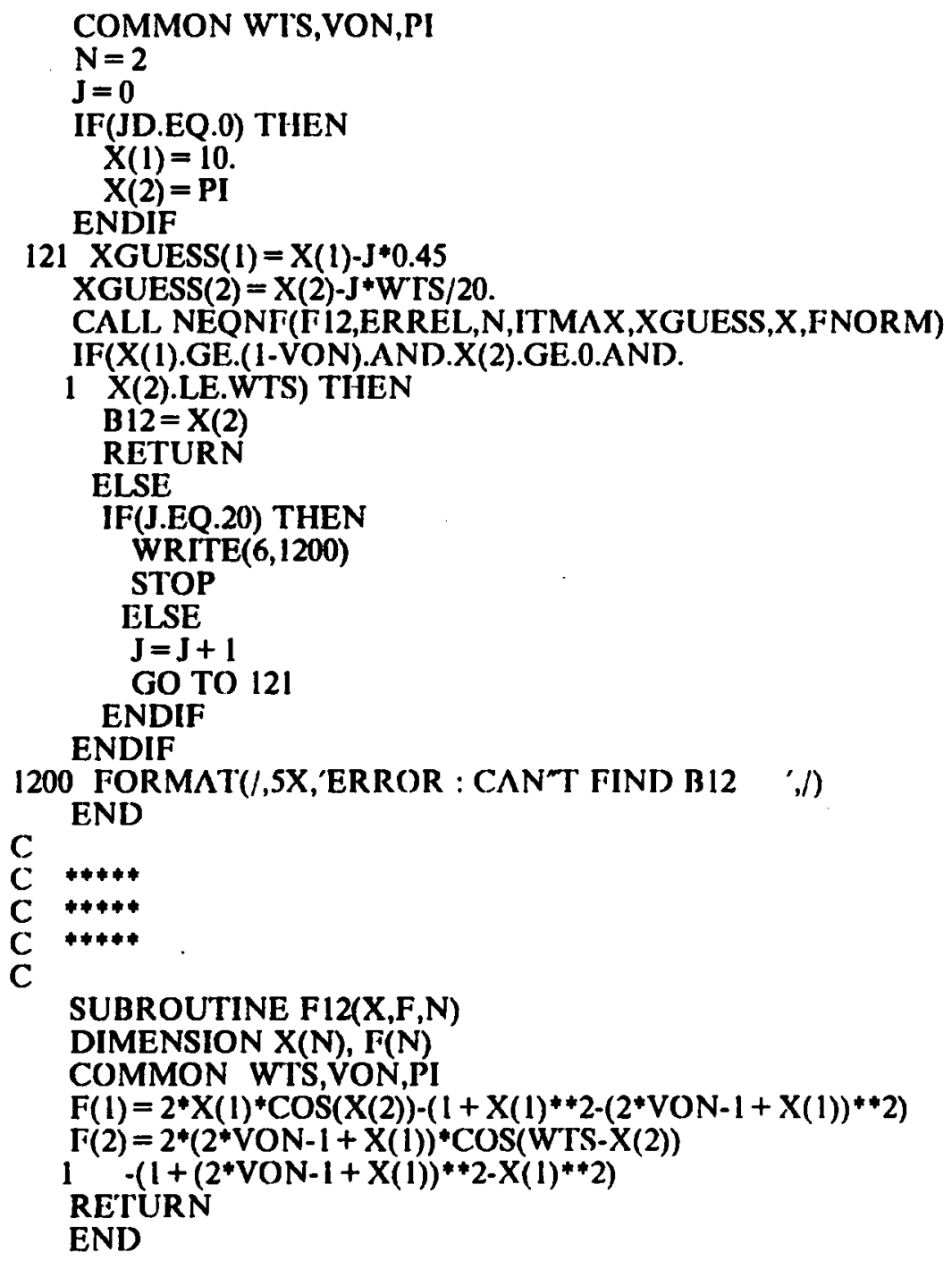




\section{APPENDIX B.4 \\ CALCULATION OF TRAJECTORY PARAMETERS BELOW RESONANT FREQUENCY}

The parameters for the equilibrium trajectories of a CM-SRC can be obtained by solving sets of nonlinear equations. The equations are derived from the geometrical relationship among the parameters. Notice that the distance between any two centers (ml-m6) of topological modes are known.

The equations for solving the parameters of Mode-I Trajectory have already be shown in Section 2.3.7.1.

The equations for solving the parameters of Mode-II Trajectory are shown in Figure B.4.1.

The equations for solving the parameters of Mode-III Trajectory are shown in Figure B.4.2.

The equations for solving the parameters of Mode-IV Trajectory are shown in Figure B.4.3.

The equations for solving the parameters of Mode-V Trajectory are shown in Figure B.4.4.

The equations for solving the parameters of Mode-VI Trajectory are shown in Figure B.4.5. 


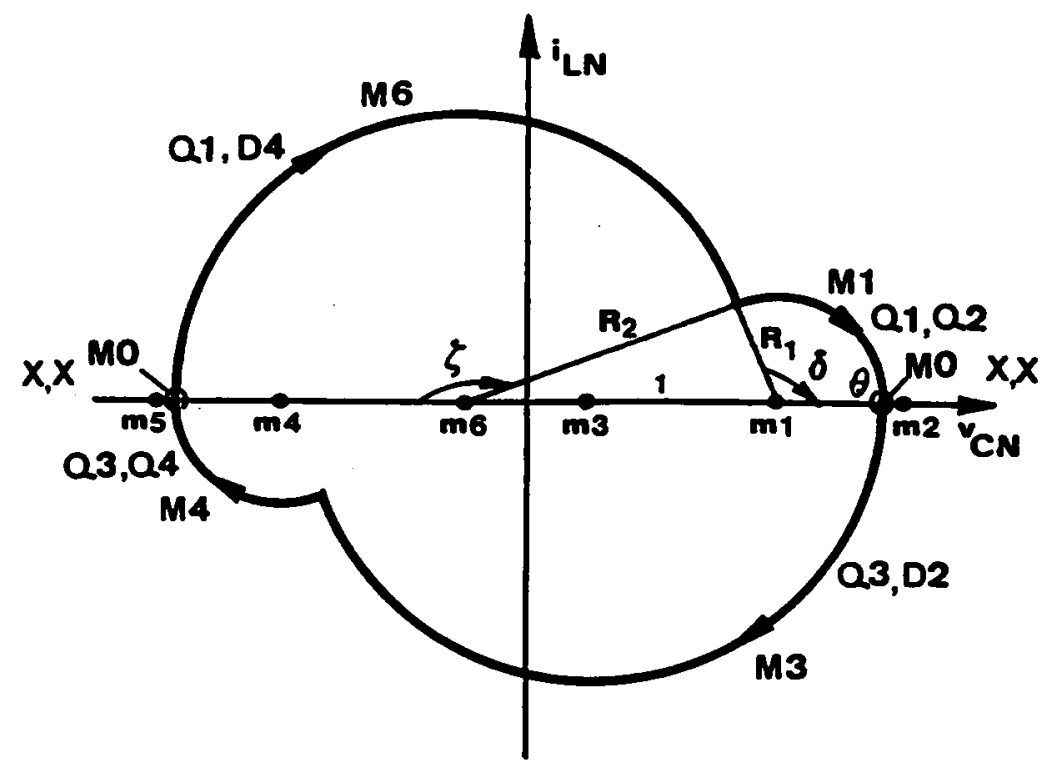

$$
\begin{gathered}
\cos (\pi-\zeta)=\frac{1+R_{2}^{2}-R_{1}^{2}}{2 R_{2}}, \\
\cos (\pi-\delta)=\frac{1+R_{1}^{2}-R_{2}^{2}}{2 R_{1}}, \\
\frac{\beta_{S}}{\omega_{S N}}=\delta+\theta, \\
\frac{\pi}{\omega_{S N}}=\delta+\theta+\zeta, \\
R_{2}=R_{1}+1-2 V_{O N .} \\
0 \leq R_{1} \leq 2 V_{O N}, 0 \leq R_{2} \leq 1, \quad 0 \leq \zeta, \delta, \theta \leq \pi .
\end{gathered}
$$

Figure B.4.1 Mode-II Trajectory Below Resonant Frequency 


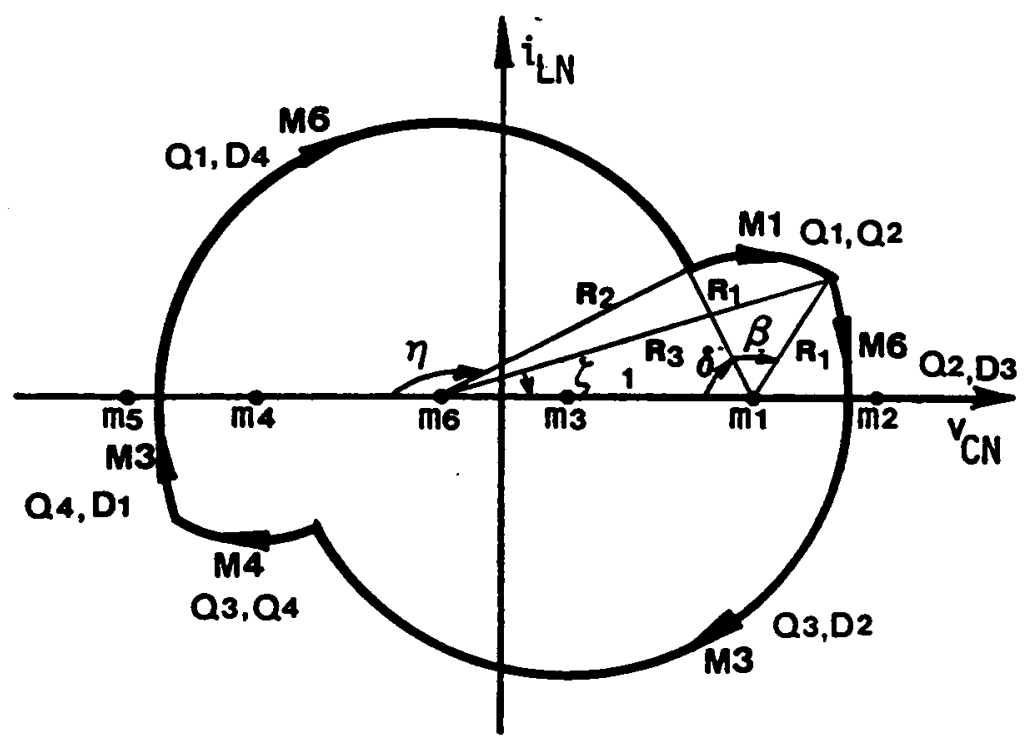

$$
\begin{aligned}
& \cos (\pi-\eta)=\frac{1+R_{2}^{2}-R_{1}^{2}}{2 R_{2}}, \\
& \cos \delta=\frac{1+R_{1}^{2}-R_{2}^{2}}{2 R_{1}}, \\
& \cos \zeta=\frac{1+R_{3}^{2}-R_{1}^{2}}{2 R_{3}}, \\
& \cos \left(\delta+\frac{\beta_{S}}{\omega_{S N}}\right)=\frac{1+R_{1}^{2}-R_{3}^{2}}{2 R_{1}}, \\
& \frac{\pi}{\omega_{S N}}=\zeta+\eta+\frac{\beta_{S}}{\omega_{S N}}, \\
& R_{3}=R_{2}+2 V_{O N} . \\
& 0 \leq R_{1}, R_{2}, R_{3}, \quad 0 \leq \eta, \zeta . \delta \leq \pi
\end{aligned}
$$

Figure B.4.2 Mode-III Trajectory Below Resonant Frequency 


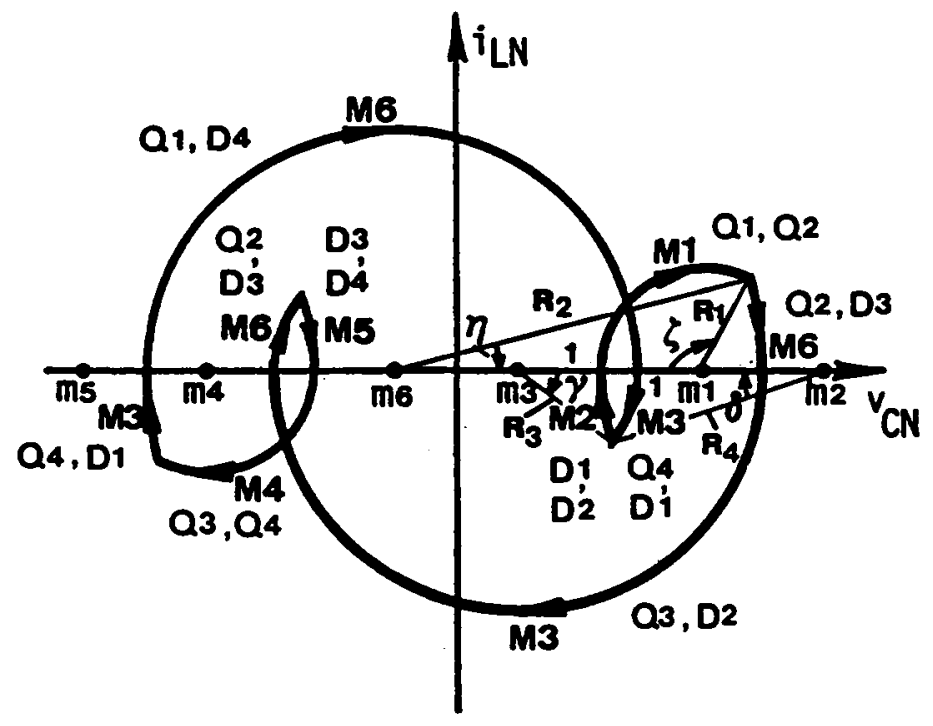

$$
\begin{aligned}
& \cos \eta=\frac{1+R_{2}^{2}-R_{1}^{2}}{2 R_{2}}, \quad \cos \zeta=\frac{1+R_{1}^{2}-R_{2}^{2}}{2 R_{1}}, \\
& \cos \gamma=\frac{1+R_{3}^{2}-R_{4}^{2}}{2 R_{3}}, \quad \cos \delta=\frac{1+R_{4}^{2}-R_{3}^{2}}{2 R_{4}}, \\
& \frac{\beta_{S}}{\omega_{S N}}=\delta+\zeta, \\
& \frac{\pi}{\omega_{S N}}=\eta+\pi+\gamma+\frac{\beta_{S}}{\omega_{S N}}, \\
& R_{4}=R_{1}+2 V_{O N}, \\
& R_{2}=R_{3}+4 V_{O N} . \\
& 0 \leq R_{1}, R_{2}, R_{3}, R_{4}, \quad 0 \leq \eta, \zeta . \gamma, \delta \leq \pi .
\end{aligned}
$$

Figure B.4.3 Mode-IV Trajectory Below Resonant Frequency 


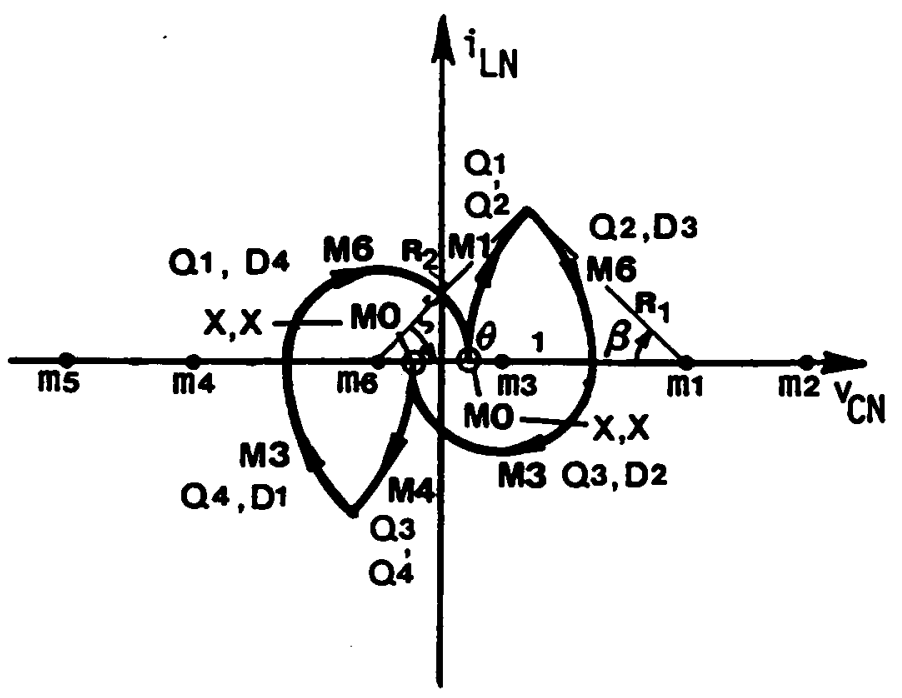

$$
\begin{gathered}
\cos \zeta=\frac{1+R_{2}^{2}-R_{1}^{2}}{2 R_{2}}, \\
\cos \left(\frac{\beta_{S}}{\omega_{S N}}\right)=\frac{1+R_{1}^{2}-R_{2}^{2}}{2 R_{1}}, \\
\frac{\pi}{\omega_{S N}}=\pi+\theta+\zeta+\frac{\beta_{S}}{\omega_{S N}}, \\
R_{2}=2 V_{O N}+1-R_{1} . \\
0 \leq R_{1} \leq 1,2 V_{O N} \leq R_{2}, \quad 0 \leq \zeta, \theta \leq \pi .
\end{gathered}
$$

Figure B.4.4 Mode-V Trajectory Below Resonant Frequency 


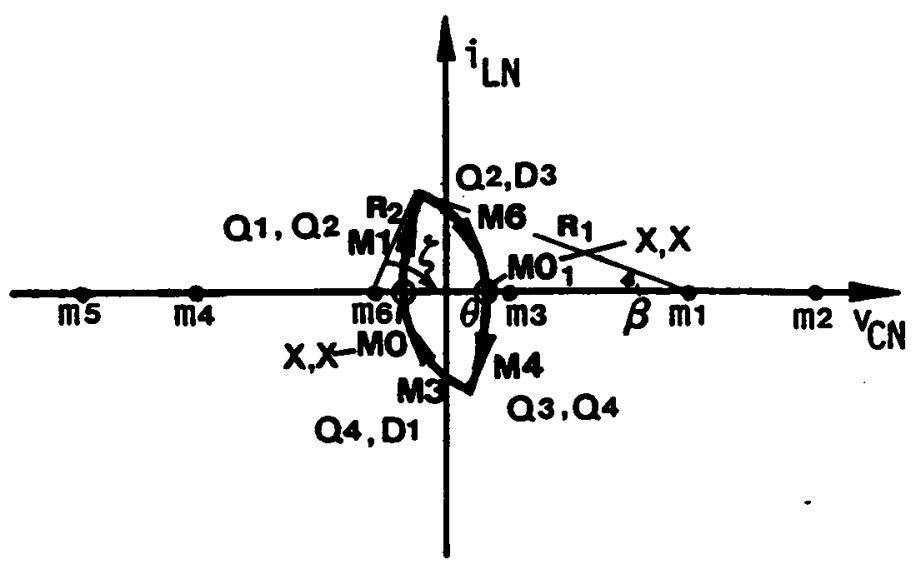

$$
\begin{gathered}
\cos \zeta=\frac{1+R_{2}^{2}-R_{1}^{2}}{2 R_{2}}, \\
\cos \left(\frac{\beta_{S}}{\omega_{S N}}\right)=\frac{1+R_{1}^{2}-R_{2}^{2}}{2 R_{1}}, \\
\frac{\pi}{\omega_{S N}}=\theta+\zeta+\frac{\beta_{S}}{\omega_{S N}}, \\
R_{2}=R_{1}-1+2 V_{O N .} \\
0 \leq R_{1} \leq 1,0 \leq R_{2} \leq 2 V_{O N}, \quad 0 \leq \zeta \leq \pi, 0 \leq \theta \leq 2 \pi .
\end{gathered}
$$

Figure B.4.5 Mode-VI Trajectory Below Resonant Frequency 
FORTRAN Program for Calculating Trajectory Parameters Below Resonant Frequency

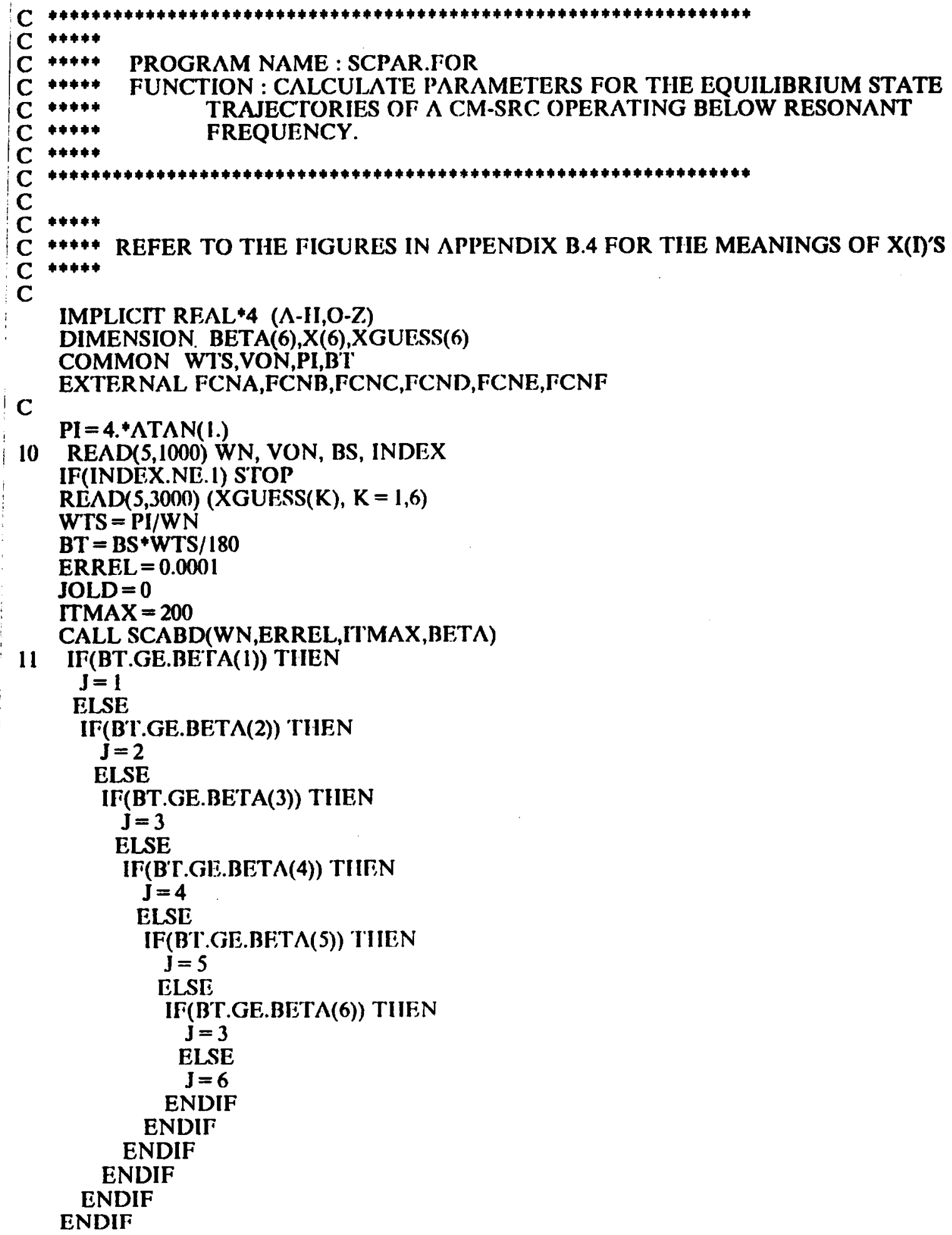

10 READ $(5,1000)$ WN, VON, BS, INDEX

IF(INDEX.NL. I) STOP

$\operatorname{RE} \Lambda D(5,3000)$ (XGUESS(K), $K=1,6$ )

WTS $=$ PI $/$ WN

$\mathrm{BT}=\mathrm{BS} * \mathrm{WTS} / 180$

ERREL $=0.0001$

JOLD $=0$

ITMAX $=200$

CALL SCABD(WN,ERREL,ITMAX,BETA)

11 IF(BT.GE.BETA(1)) THEN

$\mathrm{J}=1$

ESSE

IF(B'I.GE.BET $\Lambda(2))$ THEN

$\mathrm{J}=\mathbf{2}$

ELSE

IF(BT.GE.BE'TA(3)) THEN

$\mathrm{J}=3$

ELSE

IF(B'T.GIE.BETA(4)) TIIFN

$\mathrm{J}=4$

ELSE

IF(BT.GE.BFT $\Lambda(5))$ THEN

$\mathrm{J}=5$

ESLSE

IF(B'T.GE.BETA(6)) TIIEN

$\mathrm{J}=\mathbf{3}$

ELSE

$J=6$

ENDIF

ENDIF

ENDIF

ENDIF

ENDIF

ENDIF 
$I=0$

GO TO $(100,200,300,400,500,6(1) 0), J$

$100 \mathrm{~N}=6$

101 IF(J.NE.JOLD) THEN

$X(1)=X G U E S S(1)$

$X(2)=X G U E S S(2)$

$X(3)=X$ XUESS $(3)$

$X(4)=X$ XUESS $(4)$

$X(5)=X$ XUESS $(5)$

$X(6)=X G U E S S(6)$

ENDIF

XGUESS $(1)=X(1)-1 * 0.1$

XGUESS $(2)=X(2) \cdot I^{*} 0.05$

XGUESS $(3)=X(3)-I * 0.1$

XGUESS $(4)=X(4)-I * 0.1$

XGUESS $(5)=X(5)+I^{*} 0.1$

XGUESS $(6)=X(6)-I * 0.1$

CALL NEQNF(FCNA,ISRREL,N,ITMAX,XGUESS,X,FNORM)

IF(X(1).GT.0.AND.X(2).GT.0.AND.X(3).GE.0.AND.X(4).GE.0

1 .AND.X(5).GE.O.AND.X(6).GE.0.AND.X(3).LE.PI.AND.X(4)

1 .LE.PI.AND.X(5).LE.PI.AND.X(6).LF.PI) GO TO 999

IF(I.GE.20) STOP

$I=I+1$

GO TO 101

$200 \mathrm{~N}=4$

201 IF(J.NE.JOL.D) THEN

$$
X(1)=2 \text {. }
$$

$X(2)=P I$

$X(3)=0$.

$X(4)=P I$

ENDIF

XGUESS $(1)=\mathrm{X}(1)-\mathrm{I} * 0.2$

XGUESS $(2)=X(2)-I * 0.2$

XGUESS $(3)=X(3)+1 * 0.1$

$X G U E S S(4)=X(4)-I * 0.2$

CALL NEQNF(ICNB,ERREL,N,ITM XX,XGUESS,X,FNORM)

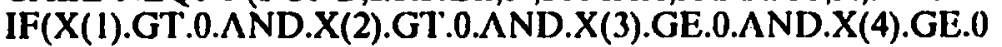

1 AND.X(2).LE.PI.AND.X(3).LE.PI.AND.X(4).LE.PI) GO TO 999

IF(I.GE.20) STOP

$I=I+I$

GO TO 201

$300 \mathrm{~N}=5$

$30 I$ IF(J.NE.JOI.I) TIIEN

$$
\begin{aligned}
& X(1)=1.5 \\
& X(2)=2 . \\
& X(3)=P I / 2 \\
& X(4)=P I \\
& X(5)=P I / 2 .
\end{aligned}
$$

ENDIF

XGUESS $(1)=X(1)-I * 0.15$

XGUESS $(2)=X(2)-I * 0.2$

XGUESS $(3)=X(3)$

XGUESS $(4)=X(4)-1 * 0.2$

XGUESS $(5)=X(5)-I * 0.2$

CALL NEQNF(FCNC,ERREL, N,ITM $\Lambda X, X G U L S S, X, F N O R M)$

IF(X(1).GT.0.AND.X(2).GT.0.AND.X(3).GE.0.AND.X(4).GE.0

1 .AND.X(5).GE.O.ANID.X(3).I.E.PI.AND.X(4)

1 .LE.PI.AND.X(5).LE.PI) GO TO 999 
IF(I.GE.20) STOP

$I=I+I$

GO TO 301

$400 \mathrm{~N}=6$

401 IF(J.NE.JOLD) THEN

$\mathrm{X}(1)=1 .-2 * \mathrm{VON}$

$\mathrm{X}(2)=2 * \mathrm{VON}$

$\mathrm{X}(3)=\mathrm{PI} / 4$

$X(4)=3 * P I / 4$

$\mathrm{X}(5)=\mathrm{PI} / 4$

$\mathrm{X}(6)=\mathrm{PI} / 4$

ENDIF

XGUESS $(1)=X(1)-1 * 0.1$

XGUESS $(2)=X(2)+1 * 0.15$

XGUESS $(3)=X(3)-I^{*} 0.1$

XGUESS $(4)=X(4)-I * 0.15$

XGUESS $(5)=X(5)-I * 0.1$

XGUESS(6) $=\mathrm{X}(6)-I^{*} 0.1$

CALL NEQNF(FCND,ERREL,N,ITMAX,XGUESS,X,FNORM)

IF(X(1).GT.0.AND.X(2).GT.0.AND.X(3).GE.0.AND.X(4).GE.0

1 AND.X(5).GE.O.AND.X(6).GE.O.AND.X(3).LE.PI.AND.X(4)

1 .LE.PI.AND.X(5).LE.PI.AND.X(6).LE.PI) GO TO 999

IF(I.GE.20) STOP

$I=I+1$

GO TO 401

$500 \mathrm{~N}=3$

501 IF(J.NE.JOLD) THEN

$\mathrm{X}(1)=1-2 * \mathrm{VON}$

$\mathrm{X}(3)=\mathrm{PI} / 2$

ENDIF

XGUESS $(1)=X(1)+I * 0.1$

XGUESS $(3)=X(3)-I^{*} 0.1$

XGUESS $(2)=$ WTS-BT-XGUESS $(3)$

CALL NEQNF(FCNE,ERREL,N,ITMAX,XGUISSS,X,FNORM)

IF(X(1).GT.0.AND.X(1).LE.1.AND.X(2).GE.0.AND.X(3).GE..0

1 .AND.X(2).LE.PI.AND.X(3).LE.PI) GO) TO 999

IF(I.GE.20) STOP

$I=I+1$

GO TO 501

$600 \mathrm{~N}=3$

601 IF(J.NE.JOLD) THEN

$$
X(1)=1 \text {. }
$$

$X(3)=P I / 2$.

ENDIF

XGUESS $(1)=X(1)-1 * 0.1$

XGUESS $(3)=X(3)-I^{*} 0.1$

XGUIESS(2) = WIS-BT-XGUESS(3)

CALL NEQNF(FCNF,ERREL,N,ITMAX,XGUESS,X,FNORM)

IF(X(1).GT.0.AND.X(2).GE.0.AND.X(3).GE.0.AND.X(1).LE.I

1 .AND.X(2).LE.(2*PI).AND.X(3).LE.PI) GO TO 999

IF(I.GE.20) STOP

$I=I+1$

GO TO 601

999 WRITE $(6,2000) \mathrm{J}, \mathrm{BT}, \mathrm{VON},(\mathrm{X}(\mathrm{K}), \mathrm{K}=1, \mathrm{~N})$

JOLD $=\mathbf{J}$

IF(BT.EQ.0.001) GO TO 10

$\mathrm{BT}=\mathrm{BT}-\mathrm{WTS} / 100$

IF(BT.LE.0) B'T $=0.001$ 
GO TO 11

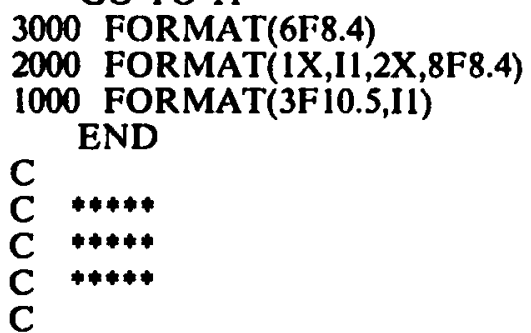

SUBROUTINE SCABD(WN,ERREL,ITMAX,BETA)

DIMENSION X(3),BET $\Lambda$ (6),XGUESS(3)

C

COMMON WTS, VON,PI,B'T

INTL $=1$

$\mathrm{W} I \mathrm{~N}=\mathrm{PI} /(\mathrm{PI}+\mathrm{ACOS}(\mathrm{VON}))$

CALL SB2(ERREL,ITMAX,B2,INTL)

IF(WN.LE.WIN) THEN

$\mathrm{Bl}=\mathrm{WTS}-\mathrm{PI}+\mathrm{ACOS}\left(1-2^{*} \mathrm{VON}^{* * 2}\right)$

ELSE

$\mathrm{BI}=\mathrm{B2}$

ENDIF

$\mathrm{B} 6=\mathrm{ACOS}(1-2 * \mathrm{VON} * * 2)$

$\operatorname{BETA}(1)=\mathrm{B1}$

BETA (2) = B2

BET $\Lambda(6)=$ B6

IF(VON.LE.0.5) THIEN

VONR $=((\operatorname{COS}(\mathrm{PI} *(1-\mathrm{WN}) * .5 / \mathrm{WN})) * *-1-1) * .5$

IF(VON.GT.VONR) TIIEN

ID $=6$

$\operatorname{BETA}(3)=B 6$

BETA(4) $=$ B6

BETA $(5)=$ B6

GO TO 99

ENDIF

$\mathrm{WIN}=\mathrm{PI} /(\mathrm{PI}+2 * \Lambda \operatorname{COS}((1+2 * \mathrm{VON}) * *-1))$

$\mathrm{W} 2 \mathrm{~N}=\mathrm{PI} /(\mathrm{PI}+\mathrm{ACOS}(\mathrm{VON})+\mathrm{ACOS}(1-2 * \mathrm{VON} * * 2))$

IF(WN.GE.WIN) THEN

ID $=6$

BETA $(3)=B 6$

$\operatorname{BET\Lambda }(4)=B 6$

BETA(5) $=$ B6

GO TO 99

ENDIF

IF(WN.LT.W1N.AND.WN.GIE.W2N) TIIEN

$\mathrm{RM} \Lambda \mathrm{X}=(1+2 * \mathrm{VON}) / 2$

RMIN $=$ VON

SIGN $=1$

CALL B34(RMAX,RMIN,RUP,VON,BUP,WN,PI,SIGN)

$\operatorname{RMAX}=1$.

RMIN $=(1+2 *$ VON $) / 2$

SIGN $=-1$

CALL B34(RMAX,RMIN,RDN,VON,BDN,WN,PI,SIGN)

IF(RUP.LE.(1-2*VON).AND.RDN.LE.(1-2*VON)) THEN

$\mathrm{ID}=3$

$\mathrm{BETA}(3)=\mathrm{BUP}$

BETA (4) $=$ BDN

$\operatorname{BETA}(5)=$ BDN 


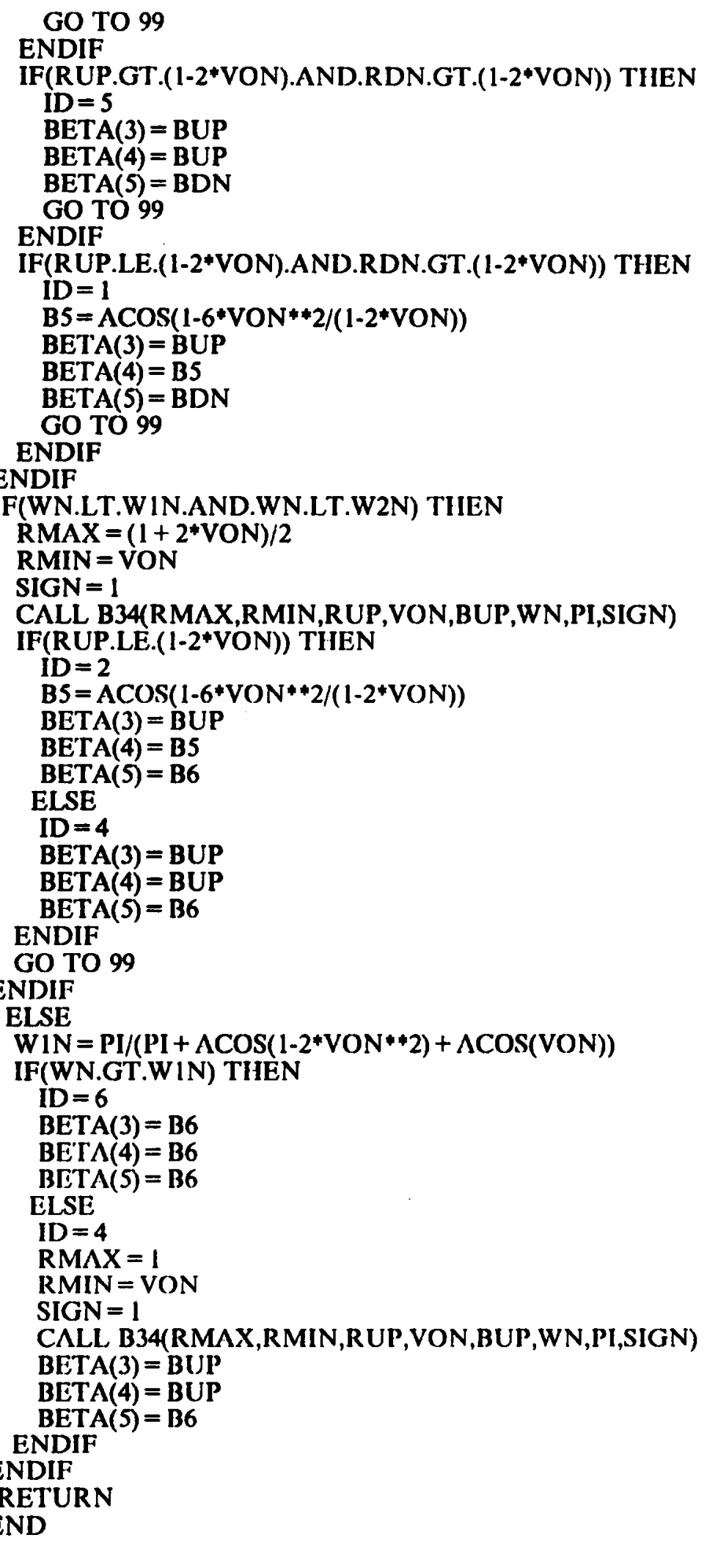


C

SUBROUTINE SB2(ERREL,ITMAX,B2,INTL)

EXTERNAL FCN2

DIMENSION X(3),XGUESS(3)

COMMON WTS,VON,PI,BT

$\mathrm{N}=3$

$\mathbf{J}=\mathbf{0}$

IF(INTL.EQ.1) THEN

$$
\begin{aligned}
& X(1)=1.1 \\
& X(2)=3.14 \\
& X(3)=1.57
\end{aligned}
$$

ENDIF

$121 \mathrm{XGUESS}(1)=\mathrm{X}(1)-\mathrm{J} * 0.1$

XGUESS $(2)=X(2) \cdot J * 0.15$

XGUESS $(3)=X(3)$

CALL NEQNF(FCN2,ERREL,N,ITMAX,XGUESS,X,FNORM)

IF(X(1).GE.0.AND.X(2).GE.0.AND.X(3).GE.0.AND.X(3).LE.PI

1 .AND.X(2).LE.3.1416) THEN

$\mathrm{B} 2=\mathrm{X}(2)$

RETURN

ELSE

IF(J.EQ.10) THEN

WRITE $(6,1200)$

STOP

ELSE

$\mathrm{J}=\mathrm{J}+\mathbf{1}$

GO TO 121

ENDIF

ENDIF

1200 FORMAT(/,5X,'ERROR : CAN'"T FIND B2 , , )

C END

C $* * * * *$

C $\$ * * * *$

C $* * * *$

C

SUBROUTINE B34(RMAX,RMIN,R,VON,BT,WN,PI,SIGN)

$221 \mathrm{R}=(\mathrm{RMAX}+\mathrm{RMIN}) / 2$.

$\mathrm{BT}=\Lambda \mathrm{COS}((1+\mathrm{R} * * 2-(1-\mathrm{R}+2 * \mathrm{VON}) * * 2) * 0.5 / \mathrm{R})$

$A L=\Lambda \operatorname{COS}((1+(1-R+2 * V O N) * * 2-R * * 2) * .5 /(1-R+2 * V O N))$

$\mathrm{WCAL}=\mathrm{PI} /(\Lambda \mathrm{L}+\mathrm{BT}+\mathrm{PI})$

IF( $\Lambda B S(W N-W C \Lambda L) . L T .0 .0001)$ RE:TURN

IF(SIGN*WCAL.GT.SIGN*WN) TIIIEN

$\mathrm{RM} \Lambda \mathrm{X}=\mathrm{R}$

EISSE

RMIN $=\mathbf{R}$

ENDIF

GO TO 221

$\mathrm{C}$

END

C

C

C

SUBROUTINE FCN2(X,F,N) 


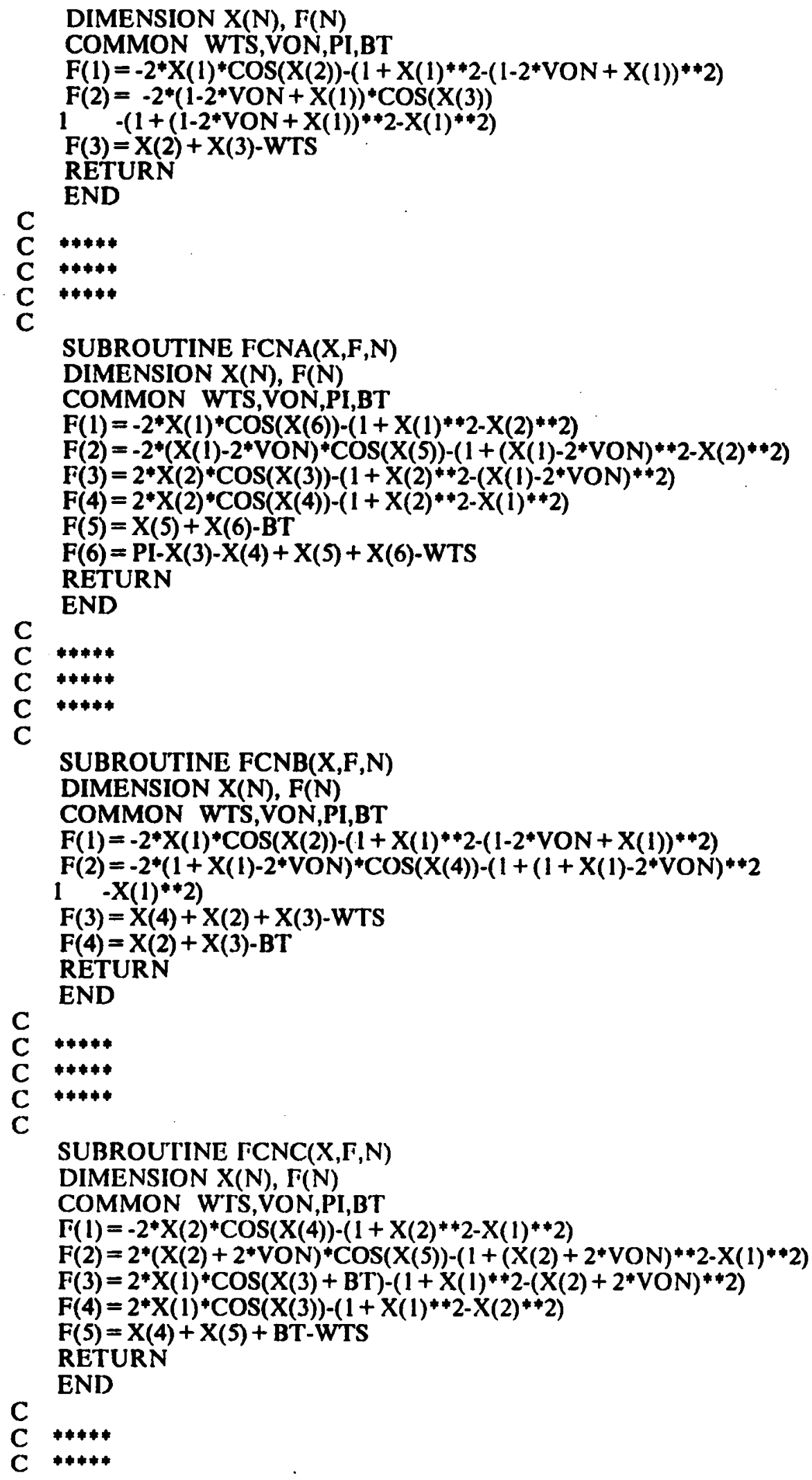


C

SUBROUTINE FCND(X,F,N)

DIMENSION $X(N), F(N)$

COMMON WTS, VON,PI,BT

$\mathrm{F}(1)=2 * \mathrm{X}(1)^{*} \mathrm{COS}(\mathrm{X}(4))-\left(1+\mathrm{X}(1) * * 2-\mathrm{X}(2)^{* * 2}\right)$

$F(2)=2 *(X(1)+2 * V O N) * \operatorname{COS}(X(3))-(1+(X(1)+2 * V O N) * * 2$

$1-(\mathrm{X}(2)-4 * \mathrm{VON}) * * 2)$

$F(3)=2^{*} X(2)^{*} \operatorname{COS}(X(6))-(1+X(2) *+2-X(1) *+2)$

$\mathrm{F}(4)=2 *(\mathrm{X}(2)-4 * \mathrm{VON}) * \mathrm{COS}(\mathrm{X}(5))-(1+(\mathrm{X}(2)-4 * \mathrm{VON}) * 2$

$1-(\mathrm{X}(1)+2 * \mathrm{VON}) *+2)$

$F(5)=X(3)+X(4)-B T$

$F(6)=X(5)+X(6)+B T+P I-W T S$

RETURN

C

END

C $+* * * *$

C $\$ * * * *$

C $* * * * *$

C

SUBROUTINE FCNE(X,F,N)

DIMENSION X(N), $F(N)$

COMMON WTS,VON,PI,BT

$\mathrm{F}(1)=2^{*} \mathrm{X}(1) * \mathrm{COS}(\mathrm{BT})-\left(1+\mathrm{X}(1) * * 2-\left(1-\mathrm{X}(1)+2^{*} \mathrm{VON}\right) * * 2\right)$

$F(2)=2 *(1-X(1)+2 * V O N) * \operatorname{COS}(X(3))-(1+(1-X(1)+2 * V O N) * 2$

$1 \quad-X(1)^{* * 2)}$

$F(3)=X(3)+B^{\prime} \Gamma+X(2)+P I-W r S$

RETURN

END

C

C $* * * *$

C $\$ * * * *$

C $* * * * *$

C

SUBROUTINE FCNF $(\mathrm{X}, \mathrm{F}, \mathrm{N})$

DIMENSION $X(N), F(N)$

COMMON WTS,VON,PI,BT

$\mathrm{F}(1)=2 * \mathrm{X}(1) * \mathrm{COS}(\mathrm{BT})-(1+\mathrm{X}(1) * * 2-(\mathrm{X}(1)-1+2 * \mathrm{VON}) * * 2)$

$F(2)=2 *(X(1)-1+2 * \operatorname{VON}) * \operatorname{COS}(X(3))-(1+(X(1)-1+2 * \mathrm{VON}) * 2$

$1-X(1) * * 2)$

$F(3)=X(3)+B T+X(2)-W T S$

RETURN

END 


\begin{tabular}{|c|c|c|c|c|c|c|c|c|c|c|c|c|}
\hline \multirow{6}{*}{ 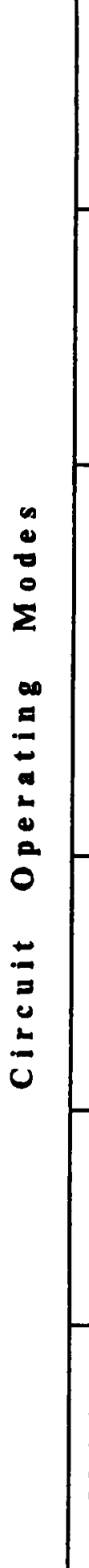 } & $\begin{array}{l}5 \\
\frac{8}{8} \\
\Sigma\end{array}$ & $\begin{array}{l}3 \\
\vdots \\
1 \\
2\end{array}$ & \begin{tabular}{l|l}
0 & \\
+ & \multirow{2}{0}{} \\
8 & 3
\end{tabular} & 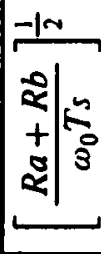 & $\frac{-10}{2 \mid \frac{\pi}{3}}$ & 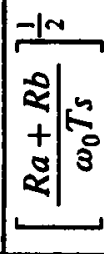 & $0 \mid \overbrace{3}^{n}$ & 0 & $\begin{array}{l}\infty \\
.5 \\
-\overline{6} \\
\alpha\end{array}$ & 0 & 0 & 0 \\
\hline & $\frac{7}{8}$ & $\begin{array}{c}z \\
0 \\
1 \\
2\end{array}$ & \begin{tabular}{l|l}
0 & \\
+ & \\
0 & $n$ \\
0 & 0 \\
+ & \\
0 &
\end{tabular} & 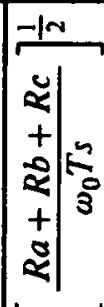 & 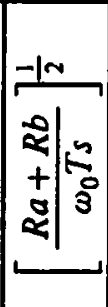 & 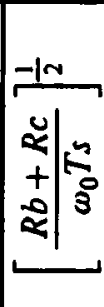 & $\checkmark) \mid \frac{n}{s}$ & $5 \mid \frac{5}{8}$ & 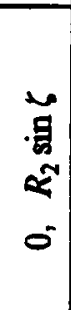 & 0 & $\begin{array}{l}0 \\
0\end{array}$ & 0 \\
\hline & $\begin{array}{l}z \\
\frac{0}{8} \\
\Sigma\end{array}$ & $\begin{array}{c}3 \\
\vdots \\
1 \\
2\end{array}$ & \begin{tabular}{c|c}
0 & \\
+ & \\
0 & \\
0 & \\
+ & \\
$u$ & 5 \\
0 & 0 \\
+ & \\
0 & \\
0 & \\
0 &
\end{tabular} & 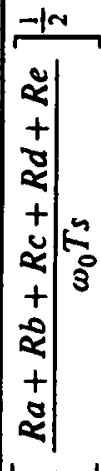 & 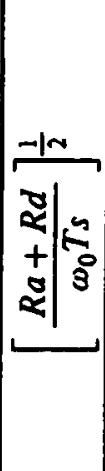 & 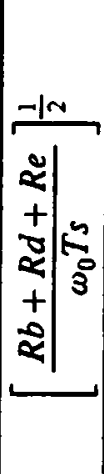 & \begin{tabular}{l|l}
0 & \\
+ & \\
$u$ & \multirow{2}{*}{} \\
0 & 3 \\
+ & 3 \\
0 &
\end{tabular} & \begin{tabular}{l|l}
0 & \\
+ & $\frac{1}{0}$ \\
0 & 3 \\
0 &
\end{tabular} & 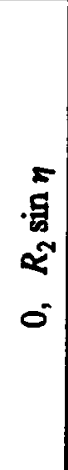 & $\begin{array}{l}\infty \\
5 \\
5 \\
2 \\
0\end{array}$ & $\begin{array}{l}0 \\
0\end{array}$ & $\begin{array}{l}0 \\
0\end{array}$ \\
\hline & $\begin{array}{l}\text { 를 } \\
\frac{ \pm}{8} \\
\Sigma\end{array}$ & $\begin{array}{l}3 \\
\vdots \\
+ \\
x^{2}\end{array}$ & \begin{tabular}{l|l}
0 & \\
+ & \\
0 & $n$ \\
0 & \\
+ & 3 \\
0 &
\end{tabular} & 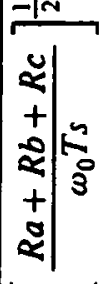 & 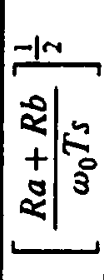 & 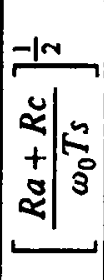 & ดे| & $\left.0\right|_{3} ^{4}$ & $\begin{array}{l}\text { है } \\
\text { ₹ี }\end{array}$ & 0 & 0 & $\begin{array}{l}\infty \\
.9 \\
\alpha \\
\alpha\end{array}$ \\
\hline & $\frac{2}{8}$ & $\begin{array}{c}a^{2} \\
1 \\
7 \\
2\end{array}$ & \begin{tabular}{l|l}
0 & \\
+ & $n$ \\
0 & 3 \\
0 &
\end{tabular} & 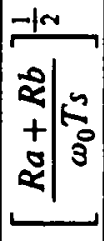 & 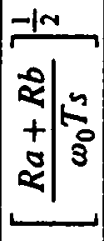 & $\frac{-10}{20}$ & 0 & $\left.\Xi\right|_{3} ^{n}$ & 0 & 0 & 0 & $\begin{array}{l}\infty \\
.5 \\
2 \\
2\end{array}$ \\
\hline & $\frac{9}{8}$ & $\begin{array}{c}\vdots \\
\vdots \\
\frac{5}{7} \\
2\end{array}$ & \begin{tabular}{c|c}
0 & \\
+ & \\
0 & 4 \\
0 & 0 \\
+ & 3 \\
0 &
\end{tabular} & 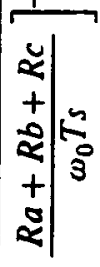 & 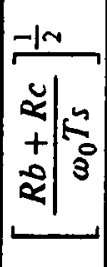 & $\frac{-10}{2 \mid \frac{5}{0}}$ & $5 \mid \frac{5}{3}$ & \begin{tabular}{l|l}
0 & $n$ \\
+ & 0 \\
0 & 3
\end{tabular} & 0 & 0 & . & $\begin{array}{l}\infty \\
.9 \\
2\end{array}$ \\
\hline 莺 莺 & $\begin{array}{l}8 \\
8 \\
8 \\
8\end{array}$ & $2^{\frac{4}{3}}$ & $\exists$ & 产 & $\underset{\mathbf{a}}{\mathbf{a}}$ & 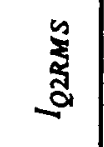 & $\vec{\Xi}$ & $\vec{\Xi}$ & 8 & ఫ్వ & $\frac{5}{9}$ & ఫ్ \\
\hline
\end{tabular}




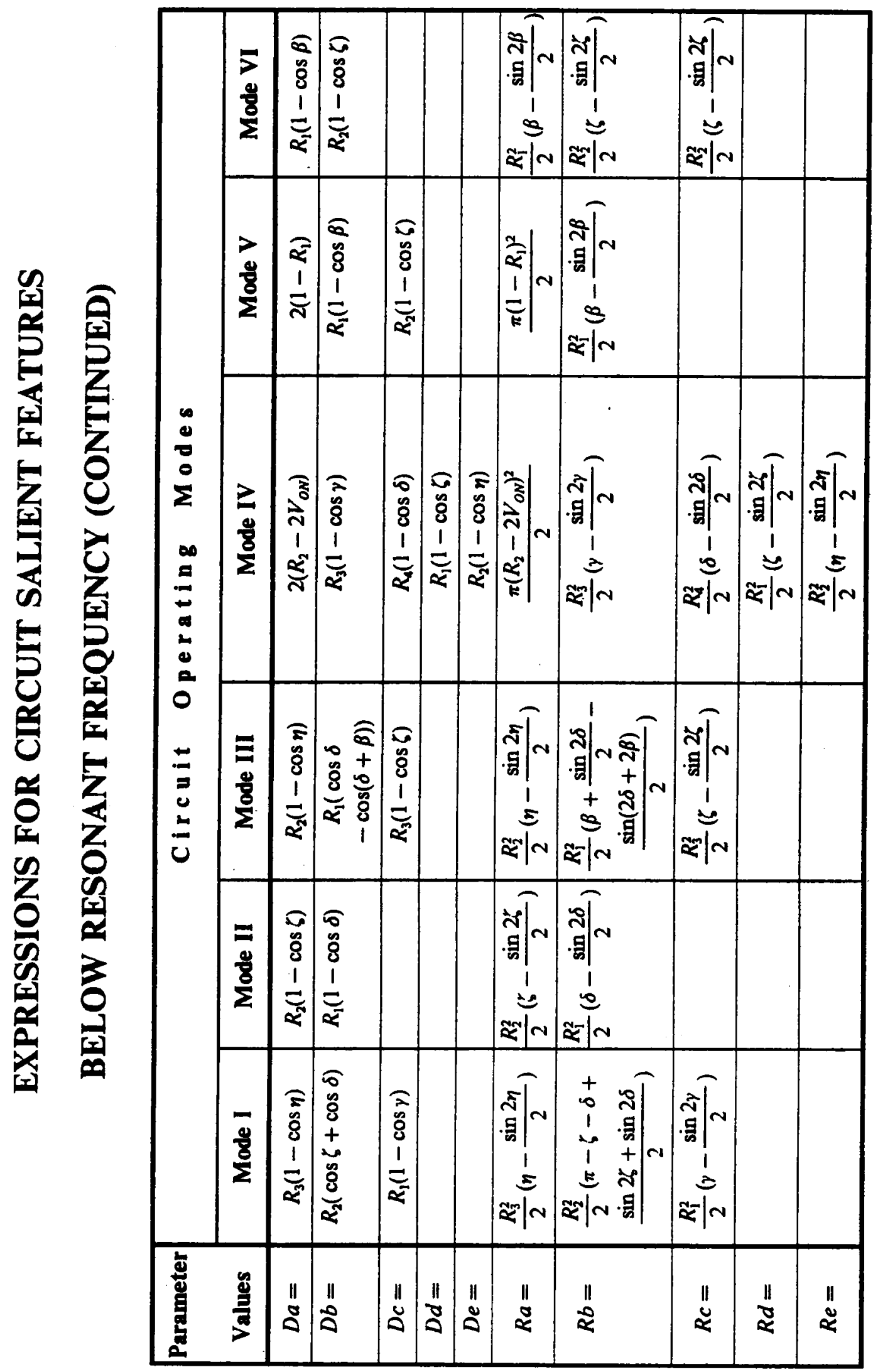


FORTRAN Programs for Calculating Circuit Salient Features of a CM-SRC Operating Below Resonant Frequency

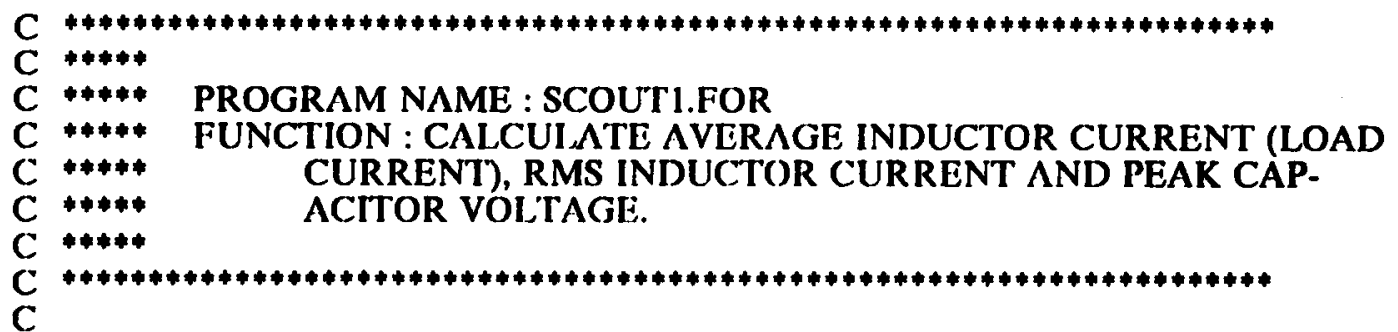

REAL IAV, ILRMS, VPK

DIMENSION X(6)

$P I=4{ }^{*} \Lambda T A N(1$.

$10 \operatorname{READ}(5,1050)$ WN

WTS $=$ PI $/$ WN

$11 \operatorname{READ}(5,1000) \mathrm{J}, \mathrm{BT}, \mathrm{VON},(\mathrm{X}(\mathrm{K}), \mathrm{K}=1,6)$

C

C ***** TIIE INPU'IS ARE OBTAINED FROM PROGRAM SCPAR.FOR

C

IF(J.EQ.0) STOP

GO TO $(100,200,300,400,500,600)$, J

$100 \mathrm{R}=\mathrm{X}(1)$

$\mathrm{R} 1=\mathrm{X}(2)$

$\mathrm{B} 1=\mathrm{X}(3)$

$\mathrm{B} 2=\mathrm{X}(4)$

$\Lambda \mathrm{L}=\mathrm{X}(5)$

$\mathrm{GA}=\mathrm{X}(6)$

$\mathrm{IAV}=\left(\left(\mathrm{R}-2^{*} \mathrm{VON}\right) *(1-\operatorname{COS}(\mathrm{AL}))+\mathrm{R} I^{*}(\mathrm{COS}(\mathrm{BI})+\mathrm{COS}(\mathrm{B} 2))+\mathrm{R}^{*}(1-\operatorname{COS}(\mathrm{GA}))\right)$

1 /WTS

ILRMS $=\left(\left(0.5 *(R-2 * \mathrm{VON}) * * 2 *(\mathrm{AL}-0.5 * \operatorname{SIN}(2 * \Lambda L))+0.5 * \mathrm{R} I^{* * 2 *}(\mathrm{PI}-\right.\right.$

$1 B 1-B 2+0.5 * \operatorname{SIN}(2 * B 1)+0.5 * \operatorname{SIN}(2 * B 2))+0.5 * R * * 2 *(G \Lambda$ -

$10.5 * \operatorname{SIN}(2 * \mathrm{GA}))) / \mathrm{WTS}) * * 0.5$

$\mathrm{VPK}=1+\mathrm{R}-\mathrm{VON}$

GO TO 999

$200 \mathrm{R}=\mathrm{X}(1)$

$B 1=X(2)$

$\mathbf{F I}=\mathbf{X}(\mathbf{3})$

$\mathbf{A L}=\mathbf{X}(4)$

$I A V=\left(\left(1-2^{*} \mathrm{VON}+\mathrm{R}\right) *(1-\operatorname{COS}(\mathrm{AL}))+\mathrm{R}^{*}(1-\operatorname{COS}(\mathrm{BI}))\right) / \mathrm{WTS}$

ILRMS $=\left(\left(0.5^{*}\left(1-2^{*} \mathrm{VON}+\mathrm{R}\right)^{* *} 2^{*}\left(\Lambda \mathrm{L}-.^{*} \operatorname{SIN}\left(2^{*} \Lambda \mathrm{I}\right)\right)\right)\right.$

$\left.\left.1+.5 * R^{* * 2 *}(\mathrm{~B} 1-.5 * \mathrm{SIN}(2 * \mathrm{~B} 1))\right) / \mathrm{WTS}\right)^{* *} 0.5$

VPK $=1+R-V O N$

GO TO 999

$300 \mathrm{R}=\mathrm{X}(1)$

$\mathrm{R} I=\mathrm{X}(2)$

$\mathrm{BI}=\mathrm{X}(\mathbf{3 )}$

$\Lambda \mathrm{L}=\mathrm{X}(4)$

$\mathbf{G A}=\mathbf{X}(5)$

$I A V=\left(R I^{*}(1-\operatorname{COS}(A L))+R^{*}\left(\operatorname{COS}(B I)-\operatorname{COS}\left(B I+B^{\prime} T\right)\right)+\left(R 1+2^{*} \mathrm{VON}\right) *\right.$

$1 \quad(1-\operatorname{COS}(\mathrm{GA}))) /$ WTS

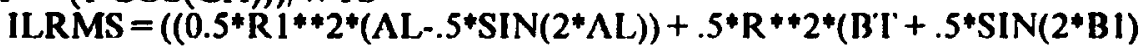

$\left.\left.1 \quad-.5 * \operatorname{SIN}\left(2^{*}(\mathrm{~B} 1+\mathrm{BT})\right)\right)+.5^{*}\left(\mathrm{R} 1+2^{*} \mathrm{VON}\right) * 2^{*}\left(\mathrm{G} \Lambda-.5 * \mathrm{SIN}\left(2^{*} \mathrm{G} \Lambda\right)\right)\right)$

1 (WTS)**0.5

$\mathrm{VPK}=\mathrm{R} 1+\mathrm{VON}$

GO TO 999

400) $\mathrm{R}=\mathrm{X}(1)$

$\mathbf{R} I=\mathbf{X}(2)$

$\mathbf{B} 1=\mathbf{X}(3)$ 


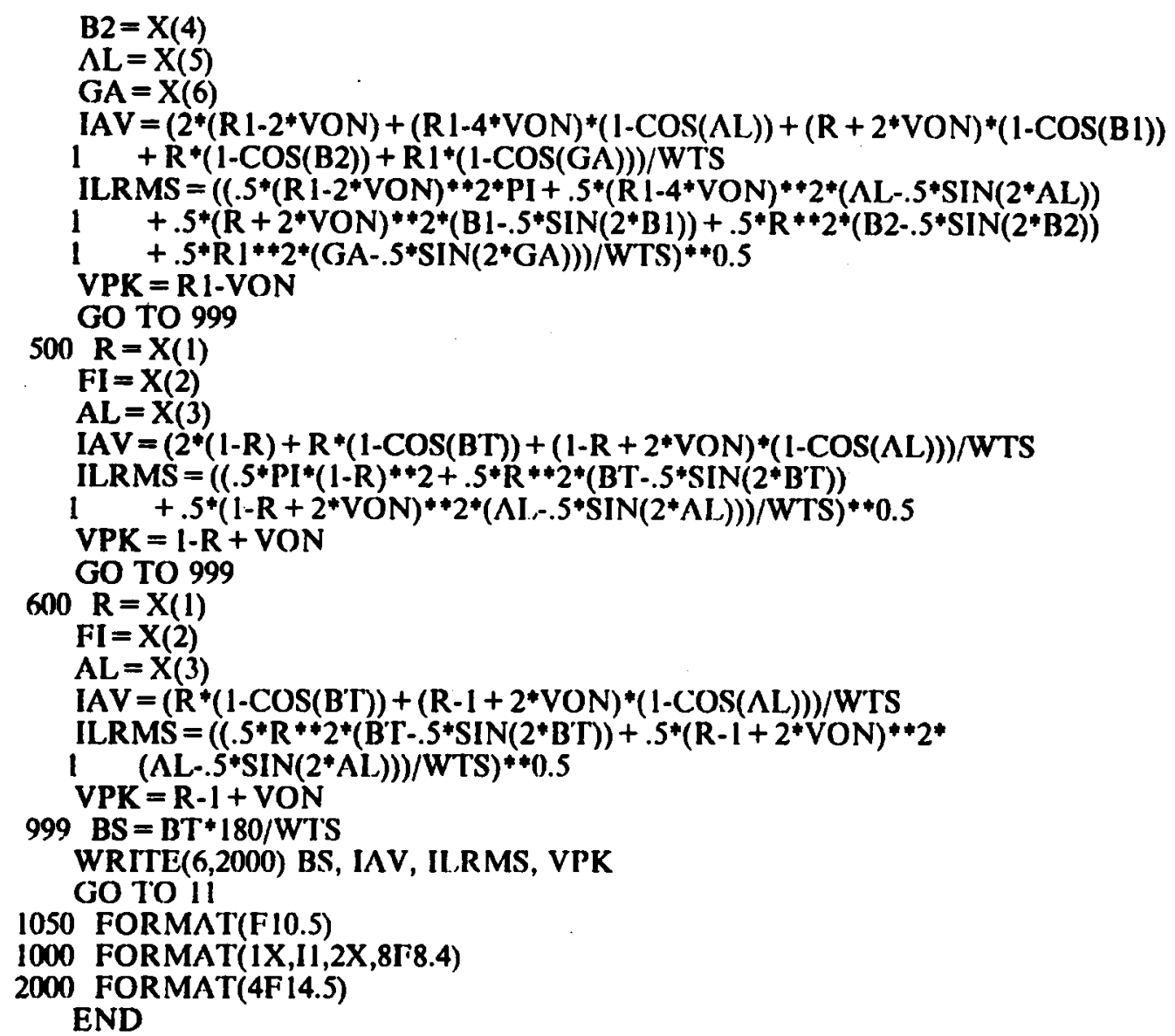




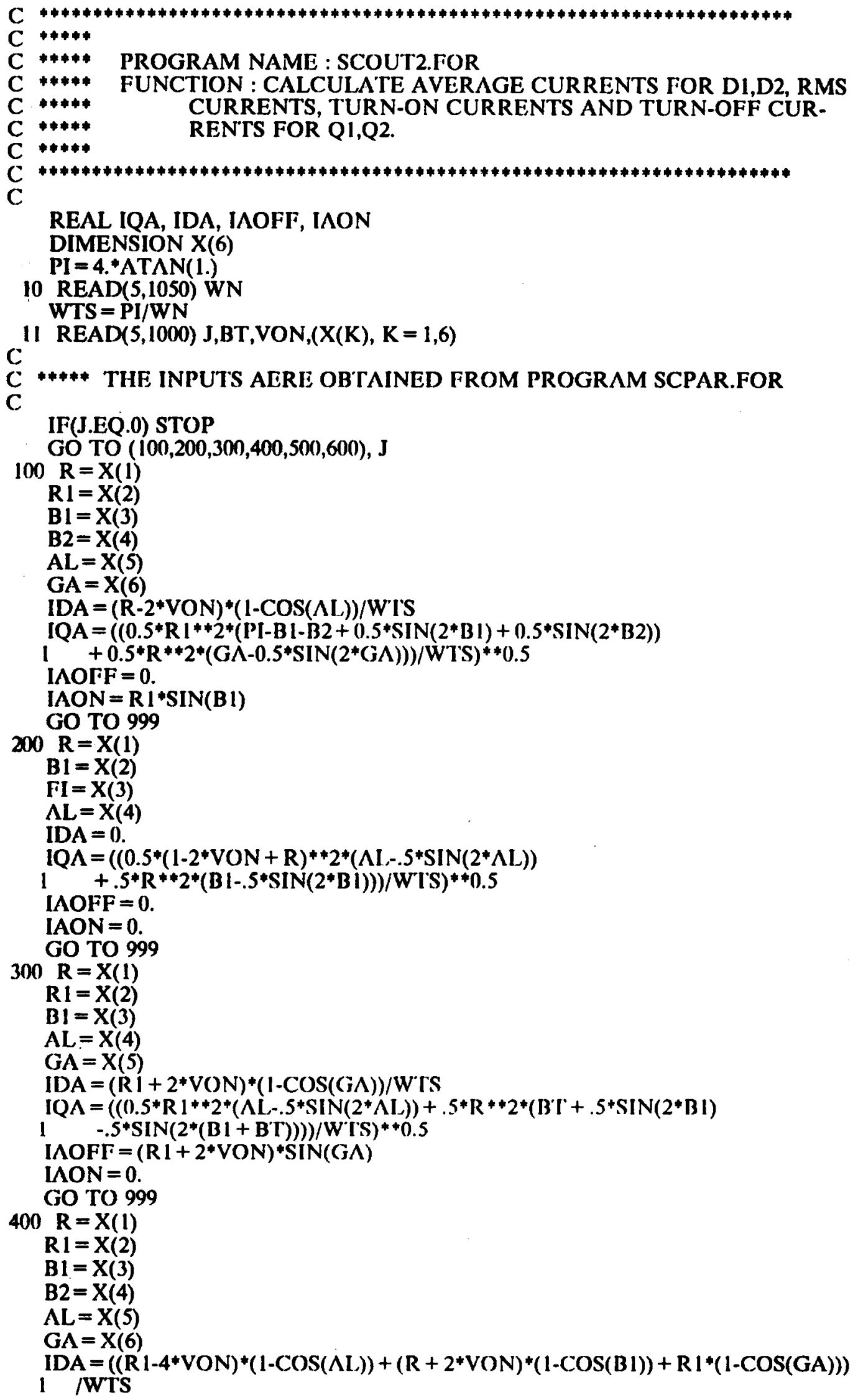




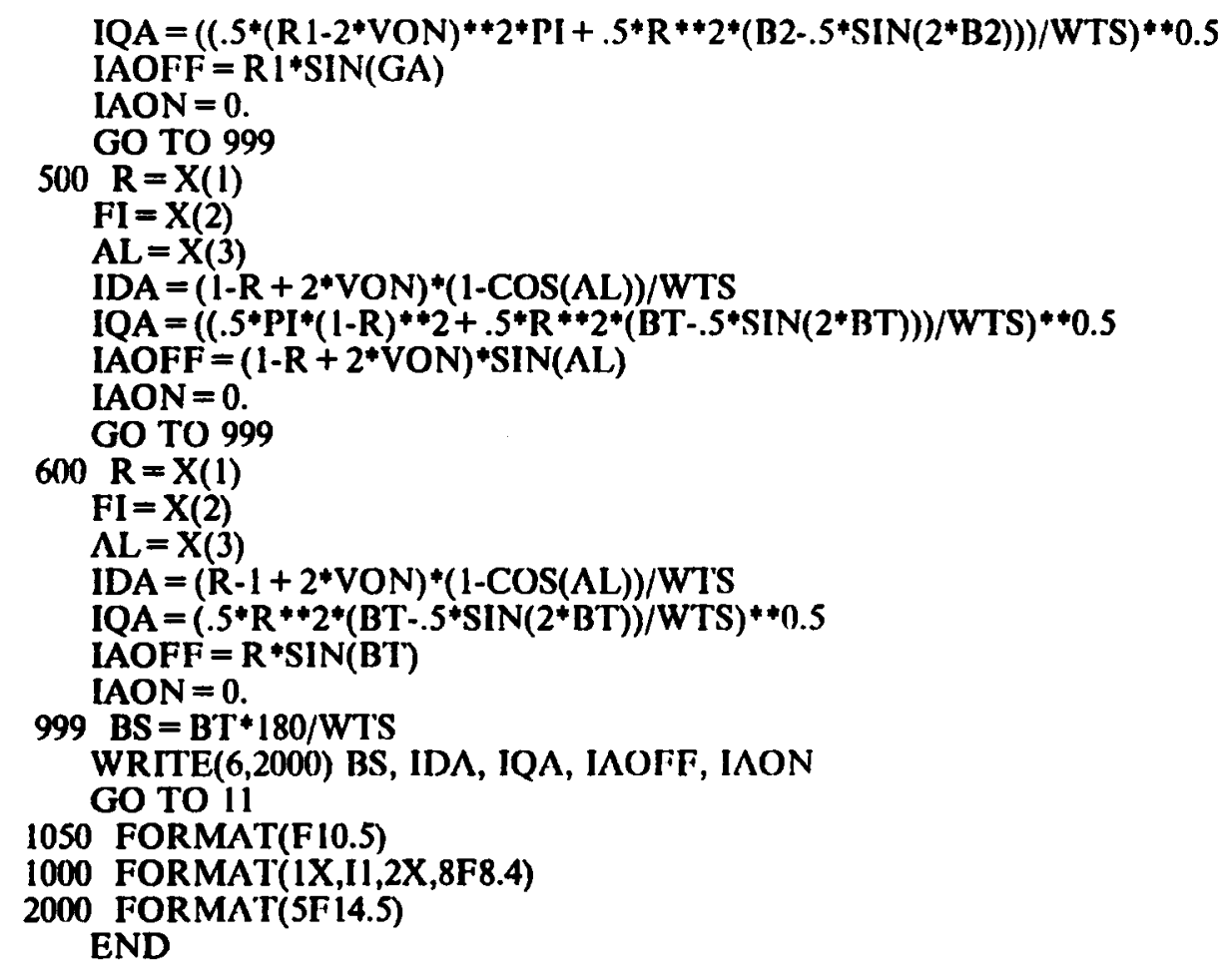




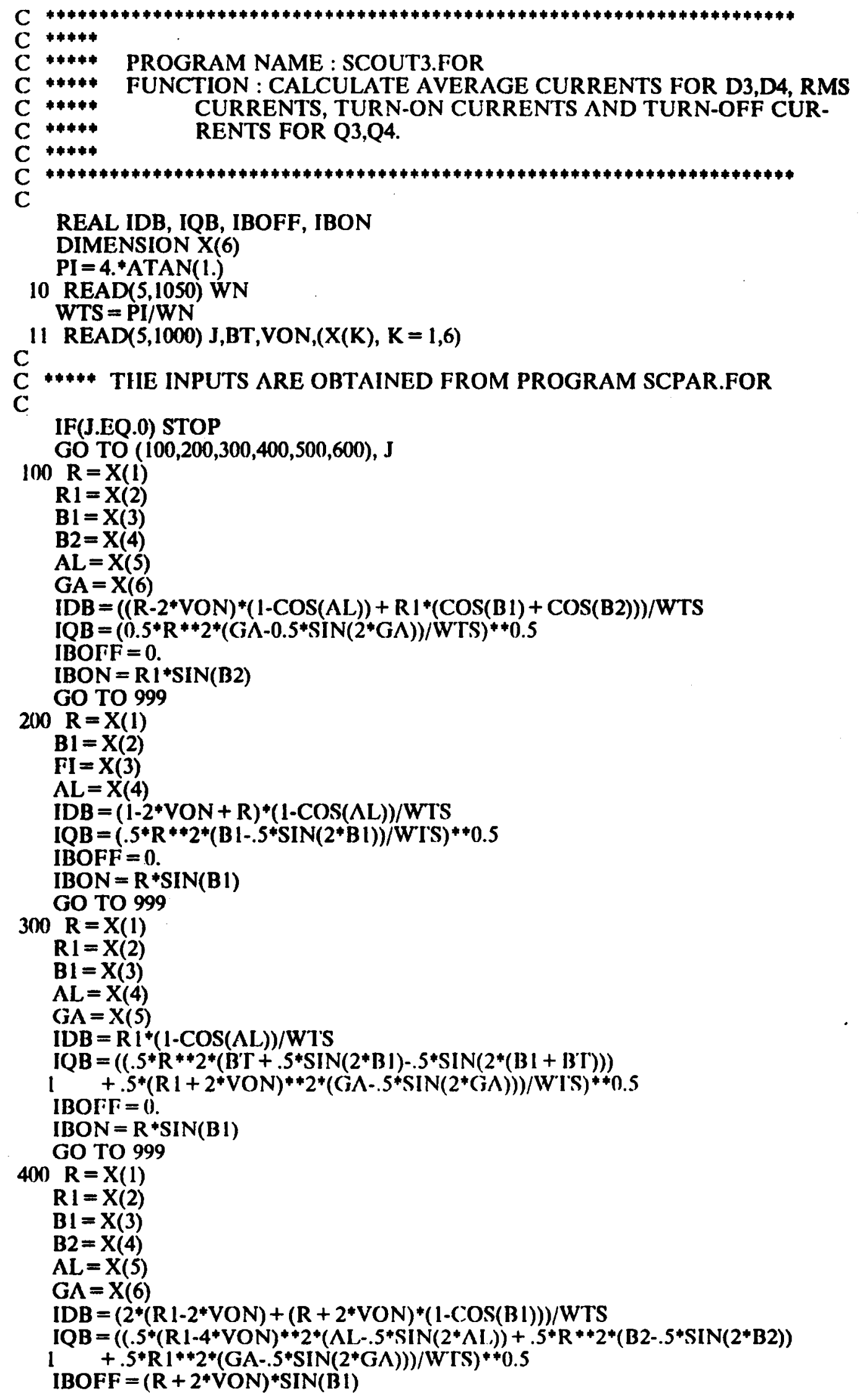


IBON $=0$.

GO TO 999

$500 \mathrm{R}=\mathrm{X}(1)$

$\mathrm{FI}=\mathrm{X}(2)$

$A L=X(3)$

IDB $=2 *(1-R) / W T S$

$\mathrm{IQB}=((.5 * \mathrm{R} * 2 *(\mathrm{BT}-.5 * \operatorname{SIN}(2 * \mathrm{BT}))+.5 *(1-\mathrm{R}+2 * \mathrm{VON}) * 2 *(\mathrm{AL}-$

$1 \quad .5 * \operatorname{SIN}(2 * A L))) / \mathrm{WTS}) * 0.5$

IBOFF $=0$.

IBON $=0$.

GO TO 999

$600 \mathrm{R}=\mathrm{X}(1)$

$\mathbf{F I}=\mathbf{X}(2)$

$\mathrm{AL}=\mathrm{X}(3)$

$\mathrm{IDB}=0$.

$\mathrm{IQB}=((.5 * \mathrm{R} * 2 *(\mathrm{BT}-.5 * \operatorname{SIN}(2 * \mathrm{BT}))+.5 *(\mathrm{R}-1+2 * \mathrm{VON}) * * 2 *$

$1 \quad(\mathrm{AL}-.5 * \operatorname{SIN}(2 * \mathrm{AL}))) / \mathrm{WTS}) * 0.5$

IBOFF $=0$.

IBON $=0$.

$999 \mathrm{BS}=\mathrm{BT} * 180 / \mathrm{WTS}$

WRITE $(6,2000)$ BS, IDB, IQB, IBOFF, IBON

GO TO 11

1050 FORMAT(F10.5)

1000 FORMAT(1X,11,2X,8F8.4)

2000 FORMAT(SF 14.5)

END 


\section{APPENDIX B.6 \\ DC CHARACTERISTICS BELOW \\ RESONANT FREQUENCY}

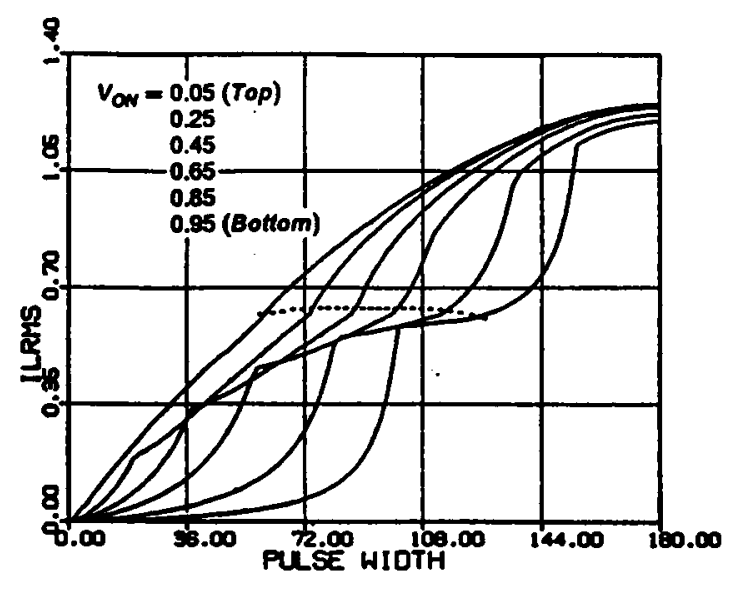

- RMS switch current (Q2.Q4)

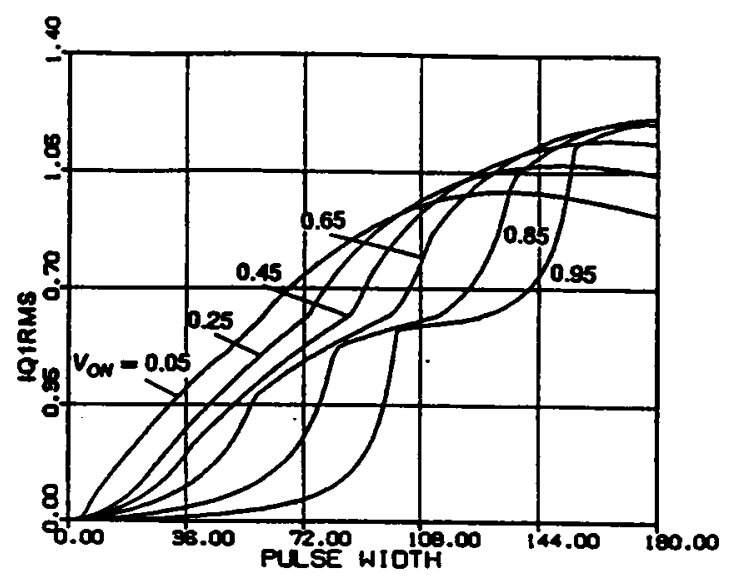

- RMS switch current $(Q 1, Q 3)$

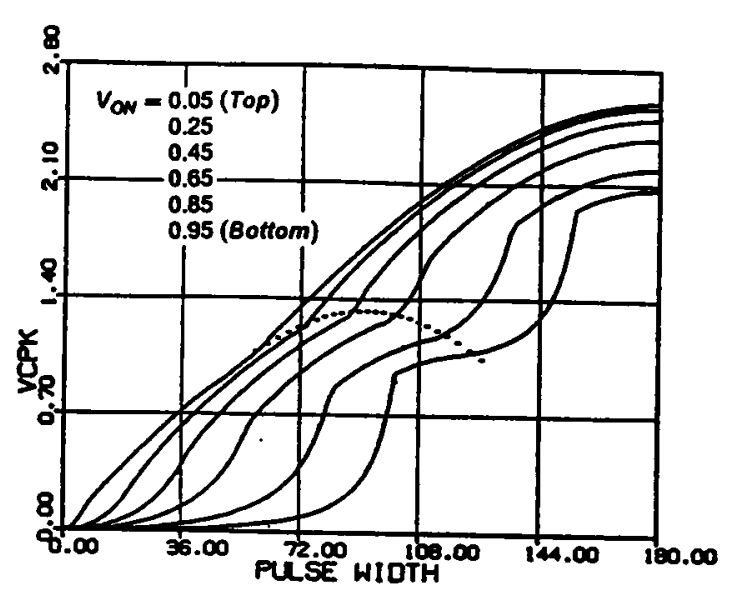

- Peak capacitor voltage

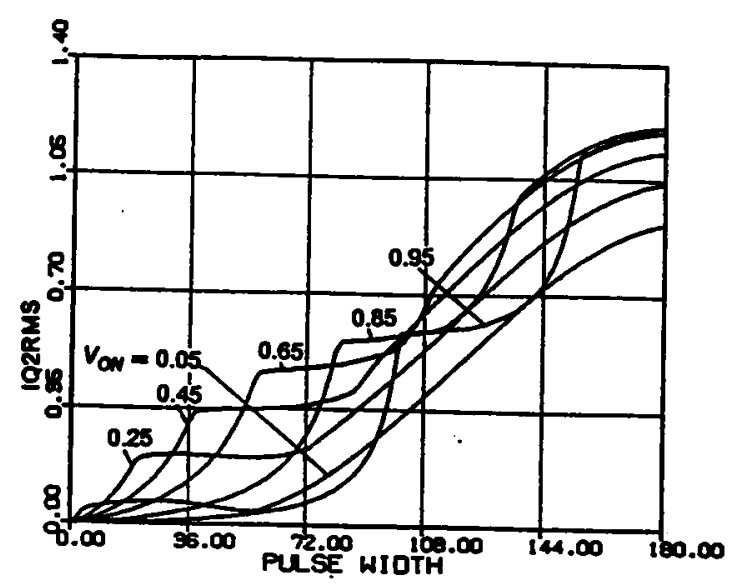

- RMS switch current (Q2,Q4)

Figure B.6.1 DC Characteristics for $\omega_{S N}=0.7$. 


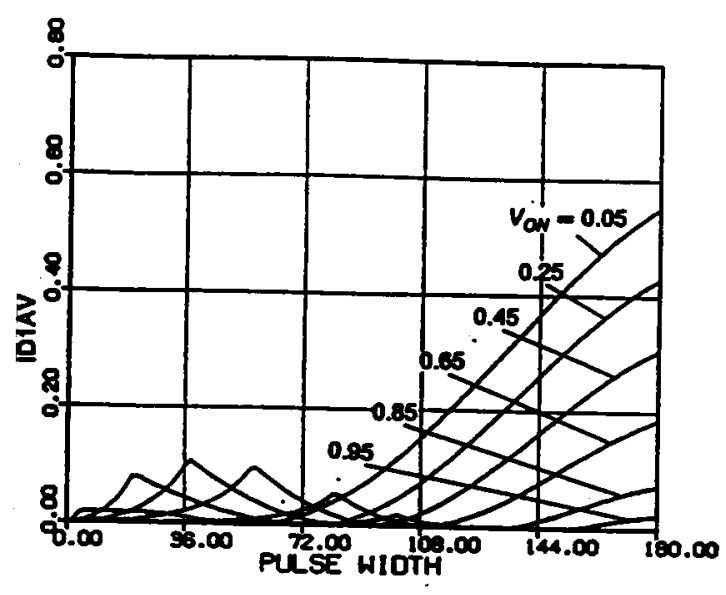

- Average diode current (DI,D3)

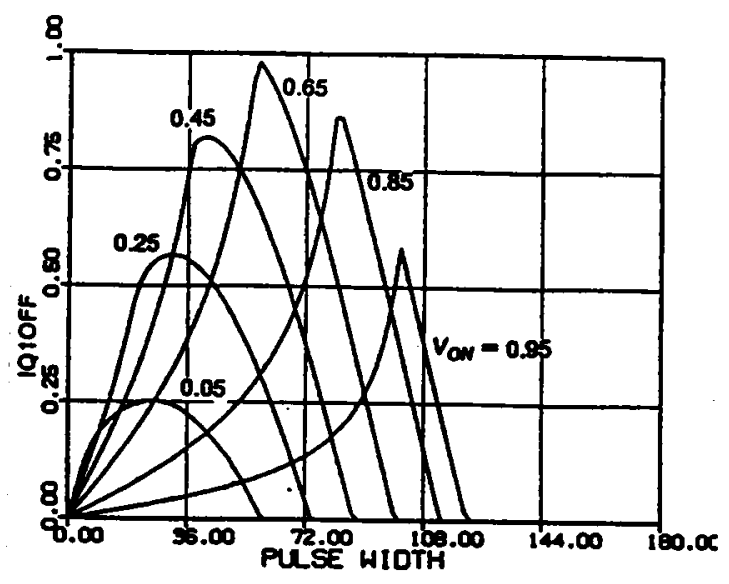

- Switch turn-off current (QI,Q3)

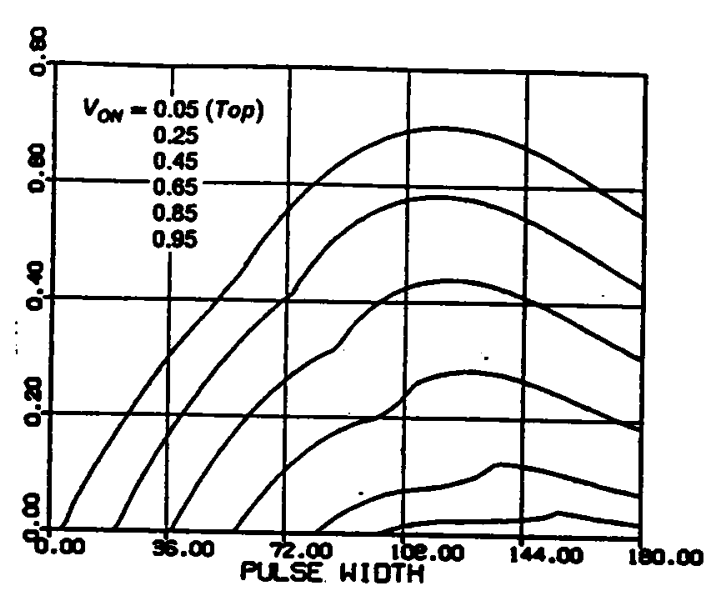

- Average diode current (D2,D4)

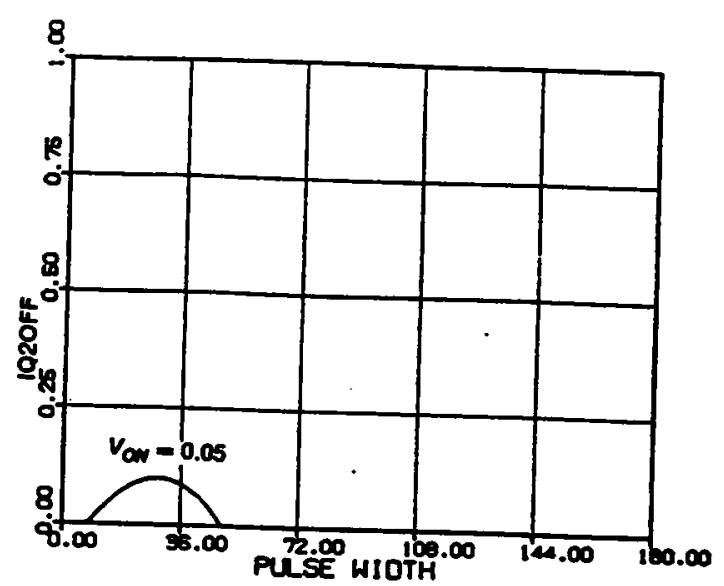

- Switch turn-off current $(Q 2, Q 4)$

Figure B.6.1 Continued 


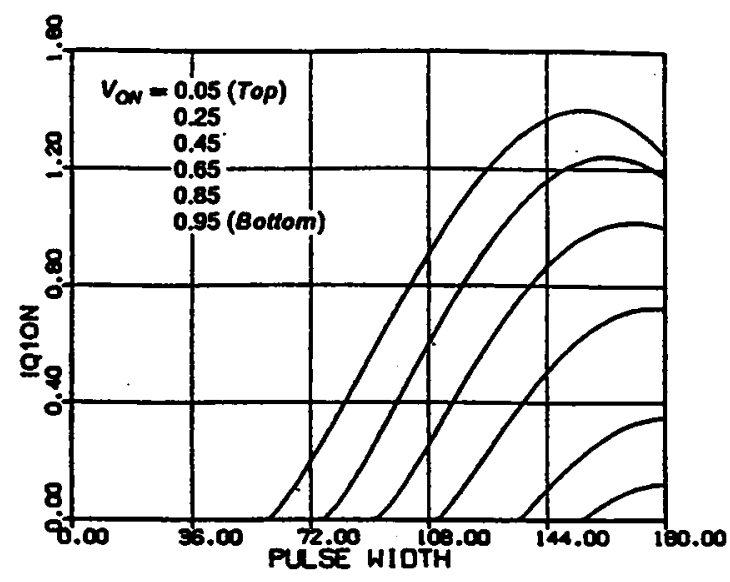

- Switch turn-on current $(Q 1, Q 3)$

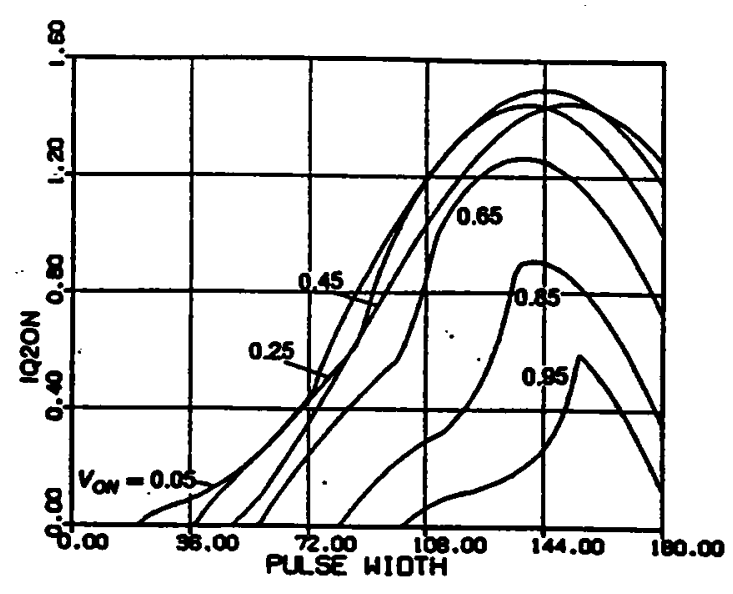

- Switch turn-on current (Q2.Q4)

Figure B.6.1 Continued 


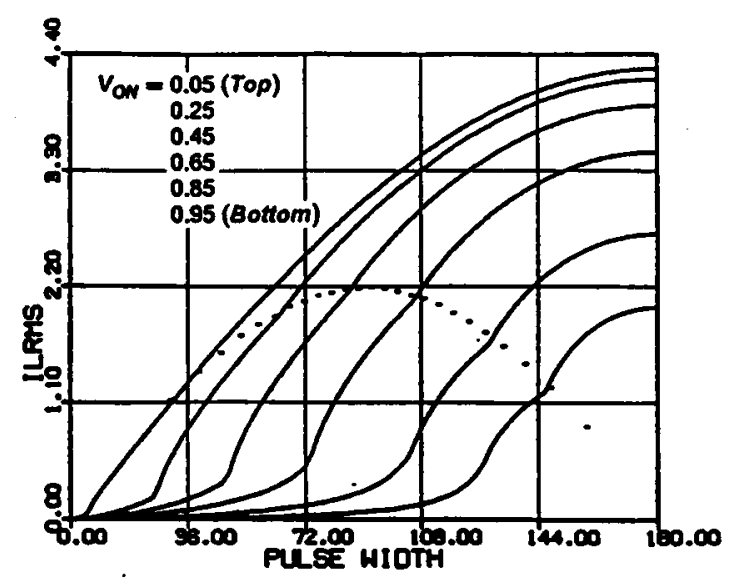

- RMS inductor current

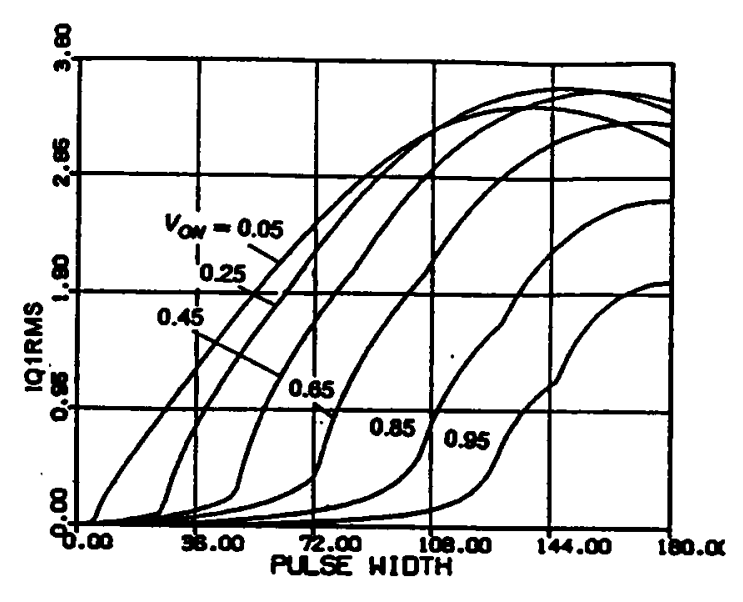

- RMS switch current (QI,Q3)

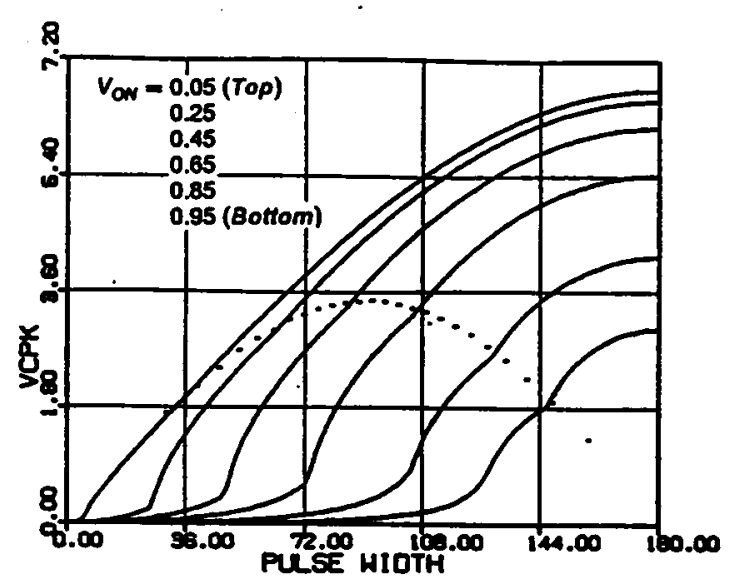

- Peak capacitor voltage

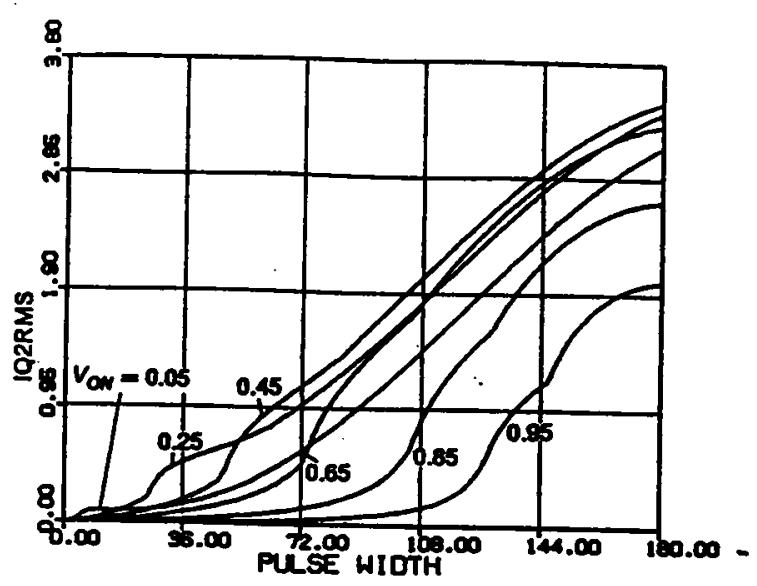

- RMS switch current (Q2,Q4)

Figure B.6.2 DC Characteristics for $\omega_{S N}=0.9$. 


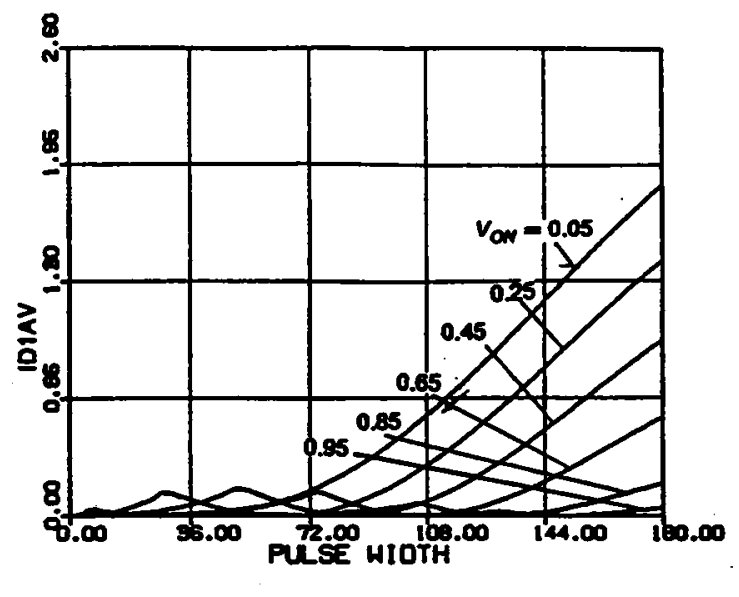

- Average diode current (DI,D3)

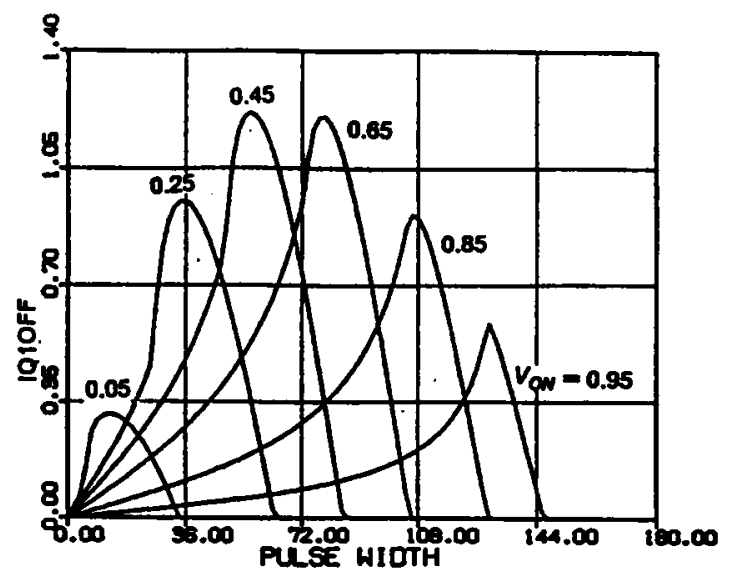

- Switch turn-off current (QI,Q3)

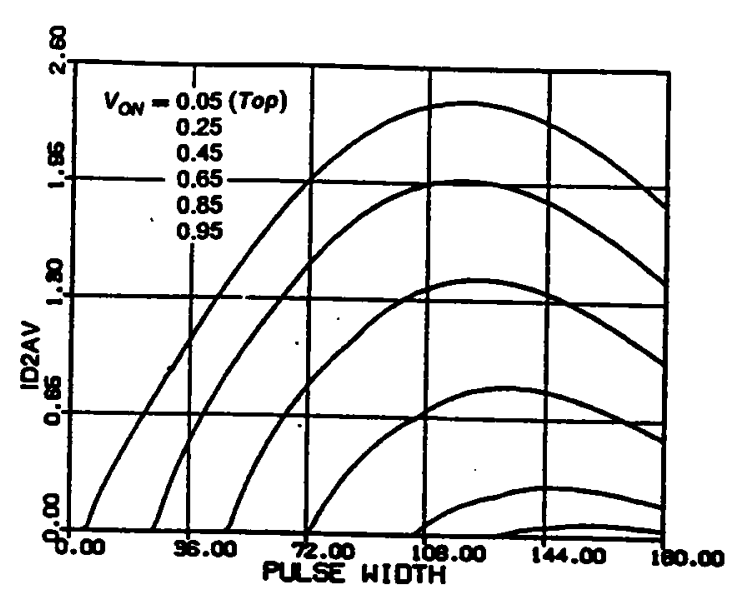

- Average diode current (D2,D4)

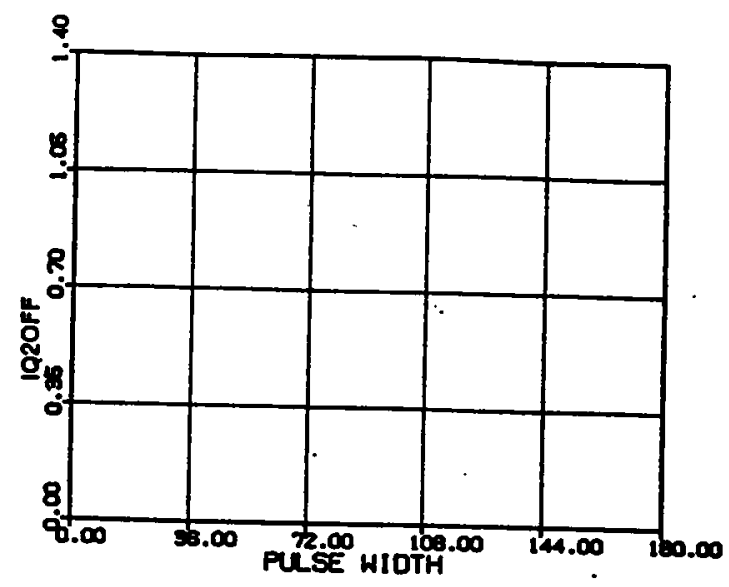

- Switch turn-off current $(Q 2, Q 4)$

Figure B.6.2 Continued 


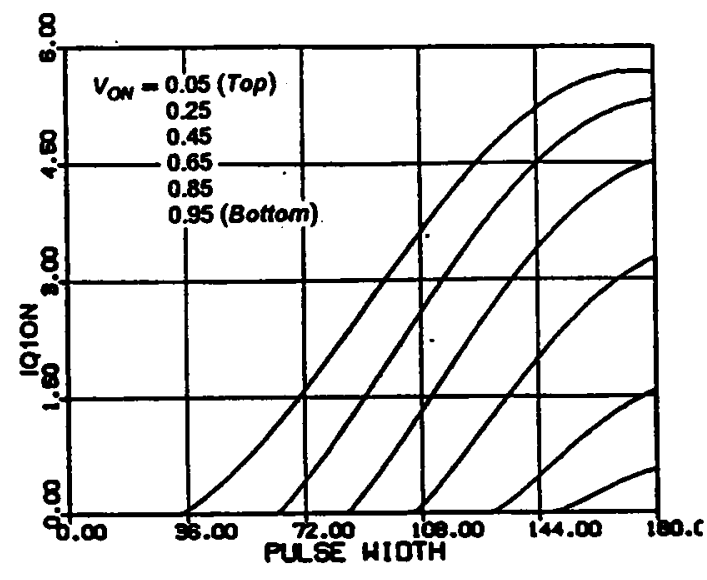

- Switch turn-on current $(Q 1, Q 3)$

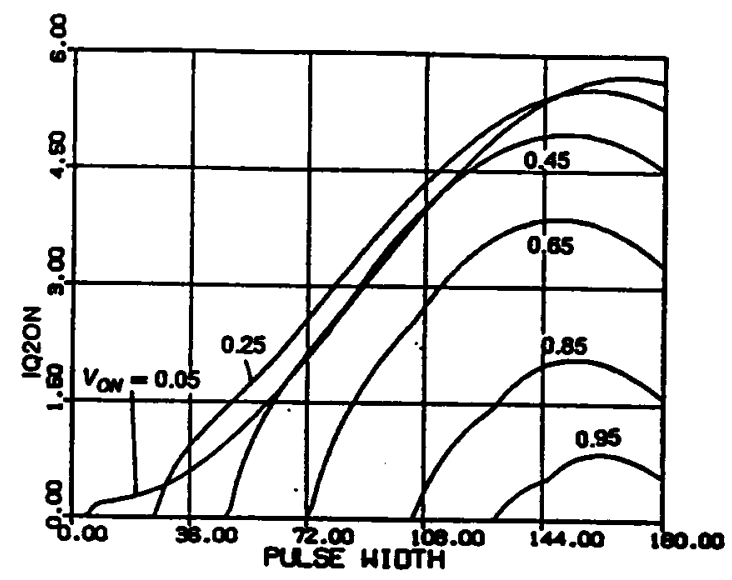

- Switch turn-on current $(Q 2, Q 4)$

Figure B.6.2 Continued 


\section{APPENDIX B.7 \\ CALCULATION OF TRAJECTORY PARAMETERS \\ ABOVE RESONANT FREQUENCY}

The equations for solving the parameters of the equilibrium trajectories of a CM-SRC operating above resonant frequency are included in this section.

The equations for solving the parameters of Mode- $\Lambda$ Trajectory are shown in Figure B.7.1.

The equations for solving the parameters of Mode-B Trajectory are shown in Figure B.7.2.

The equations for solving the parameters of Mode-C Trajectory are shown in Figure B.7.3. 


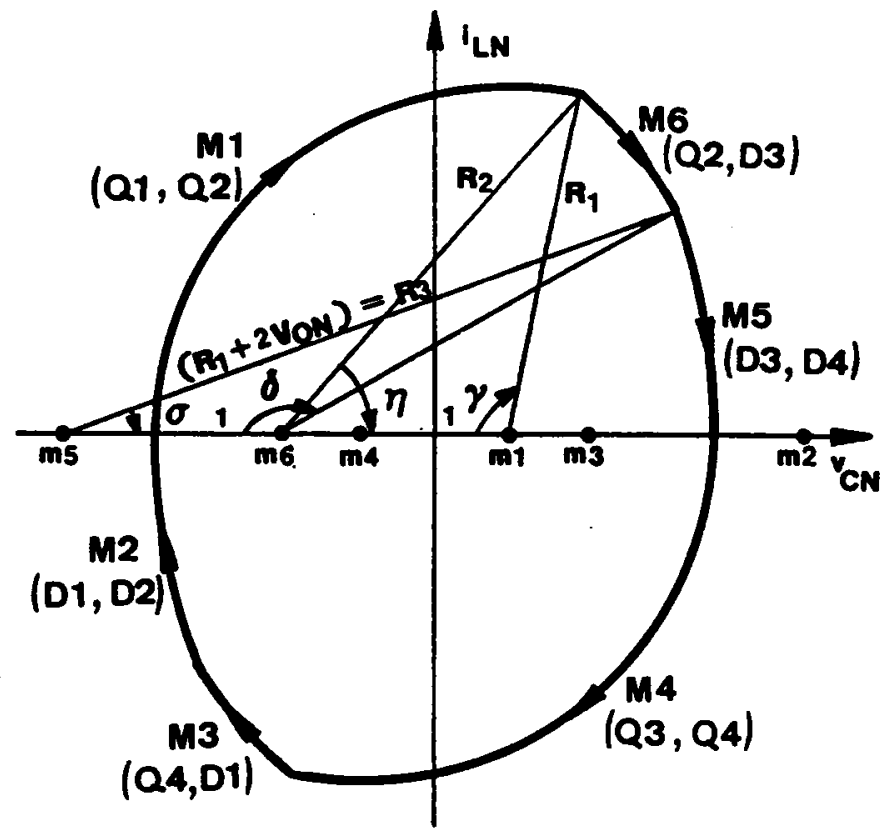

$$
\begin{aligned}
& \cos \gamma=\frac{1+R_{1}^{2}-R_{2}^{2}}{2 R_{1}}, \\
& \cos \eta=\frac{1+R_{2}^{2}-R_{1}^{2}}{2 R_{2}}, \\
& \cos \delta=\frac{1+R_{1}^{2}-\left(R_{1}+2 V_{O N}\right)^{2}}{2 R_{1}}, \\
& \cos \left(\frac{\beta_{S}}{\omega_{S N}}-\gamma\right)=\frac{1+\left(R_{1}+2 V_{O N}\right)^{2}-R_{2}^{2}}{2\left(R_{1}+2 V_{O N}\right)} \\
& \frac{\pi}{\omega_{S N}}=\delta+\eta-\pi+\frac{\beta_{S}}{\omega_{S N}} . \\
& \left(1-V_{O N}\right) \leq R_{1}, 0 \leq R_{2}, \quad 0 \leq \eta . \delta \leq \pi, 0 \leq \gamma \leq \frac{\pi}{\omega_{S N}}
\end{aligned}
$$

Figure B.7.1 Mode-A Trajectory Above Resonant Frequency 


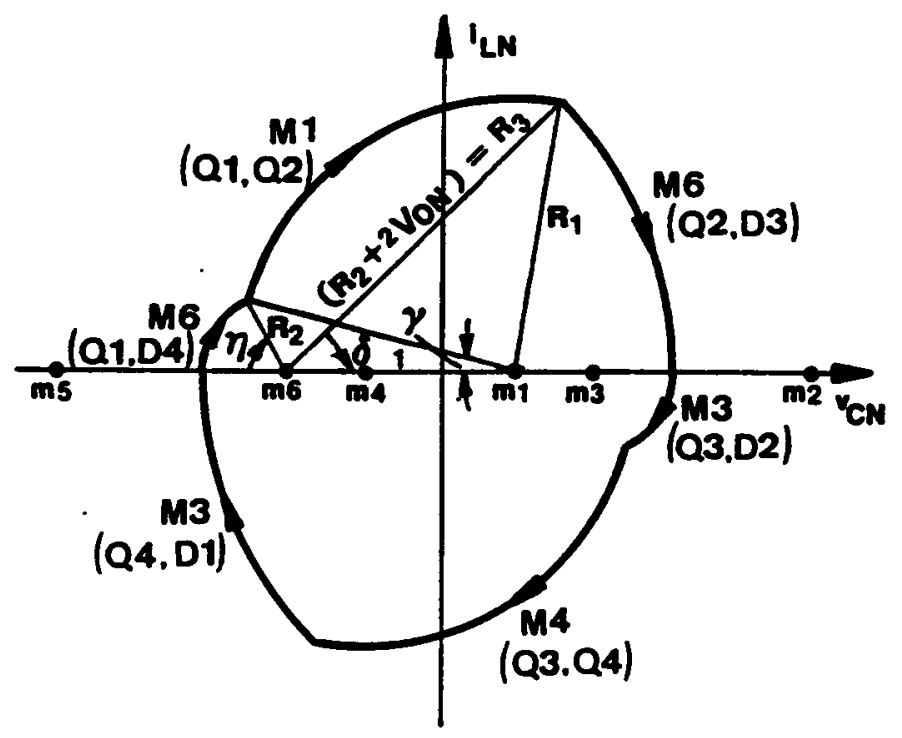

$$
\begin{aligned}
& \cos \gamma=\frac{1+R_{1}^{2}-R_{2}^{2}}{2 R_{1}}, \\
& \cos (\pi-\eta)=\frac{1+R_{2}^{2}-R_{1}^{2}}{2 R_{2}}, \\
& \cos \left(\gamma+\frac{\beta_{S}}{\omega_{S N}}\right)=\frac{1+R_{1}^{2}-\left(R_{2}+2 V_{O N}\right)^{2}}{2 R_{1}} \\
& \cos \delta=\frac{1+\left(R_{2}+2 V_{O N}\right)^{2}-R_{1}^{2}}{2\left(R_{2}+2 V_{O N}\right)}, \\
& \frac{\pi}{\omega_{S N}}=\delta+\eta+\frac{\beta_{S}}{\omega_{S N}} . \\
& 0 \leq R_{1}, \quad R_{2}, \quad 0 \leq \gamma \leq \pi, 0 \leq \eta, \delta \leq \frac{\pi}{\omega_{S N}}
\end{aligned}
$$

Figure B.7.2 Mode-B Trajectory Above Resonant Frequency 


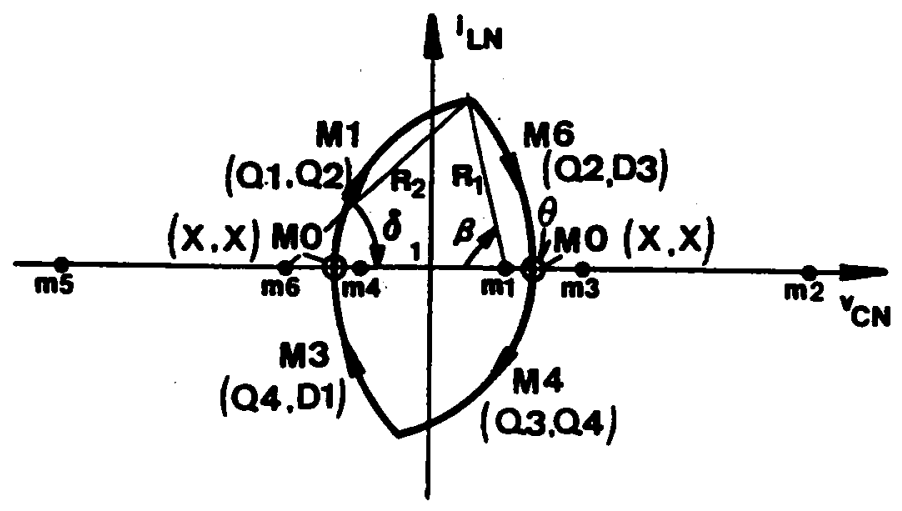

$$
\begin{gathered}
\cos \left(\frac{\beta_{S}}{\omega_{S N}}\right)=\frac{1+R_{1}^{2}-R_{2}^{2}}{2 R_{1}}, \\
\cos \delta=\frac{1+R_{2}^{2}-R_{1}^{2}}{2 R_{2}}, \\
\frac{\pi}{\omega_{S N}}=\delta+\theta+\frac{\beta_{S}}{\omega_{S N}}, \\
R_{2}=R_{1}-1+2 V_{O N} . \\
\left(1-V_{O N}\right) \leq R_{1} \leq 1, V_{O N} \leq R_{2} \leq 2 V_{O N}, \quad 0 \leq \theta, \delta \leq \frac{\pi}{\omega_{S N}}
\end{gathered}
$$

Figure B.7.3 Mode-C Trajectory Above Resonant Frequency 
FORTRAN Program for Calculating Trajectory Parameters Above Resonant Frequency

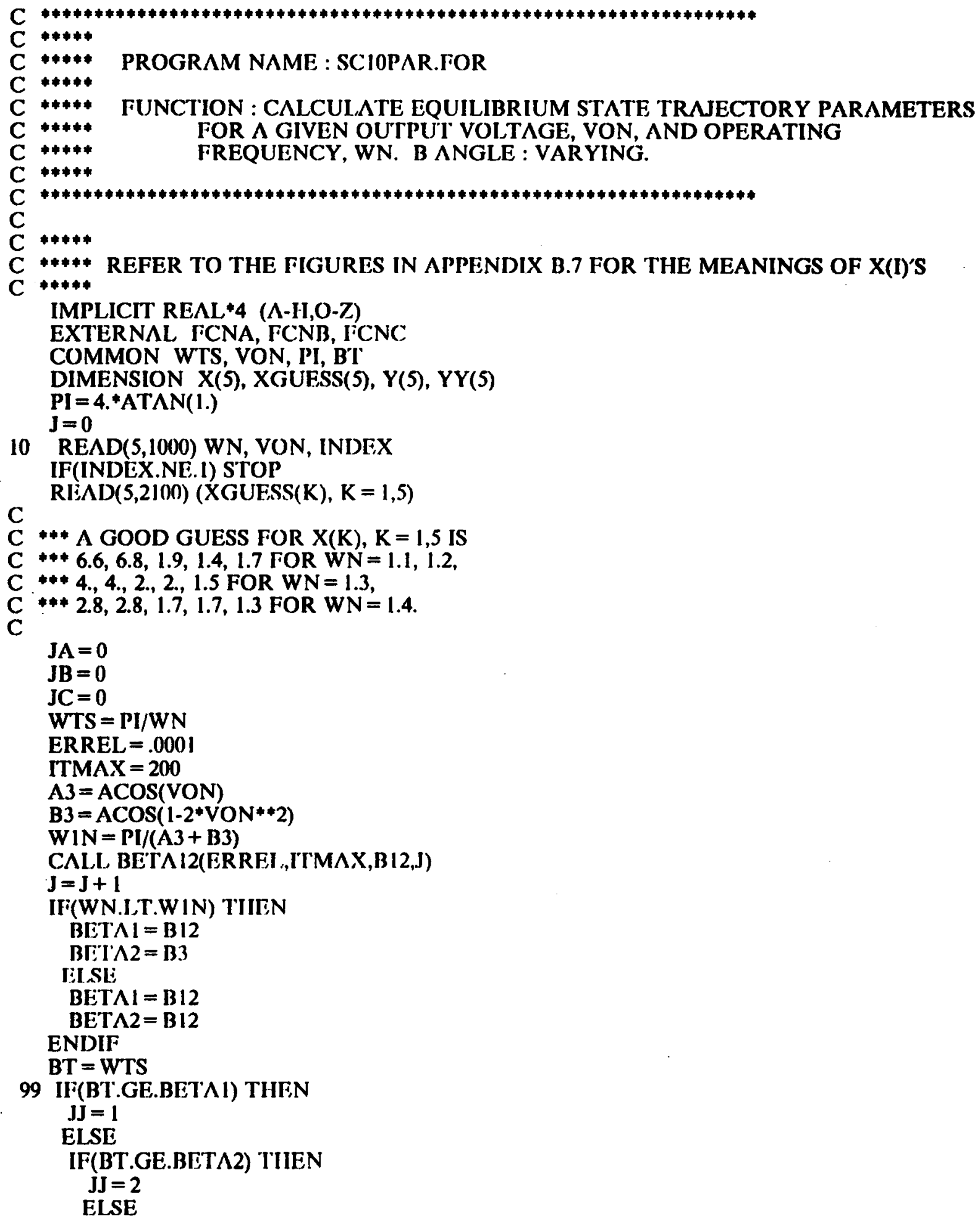




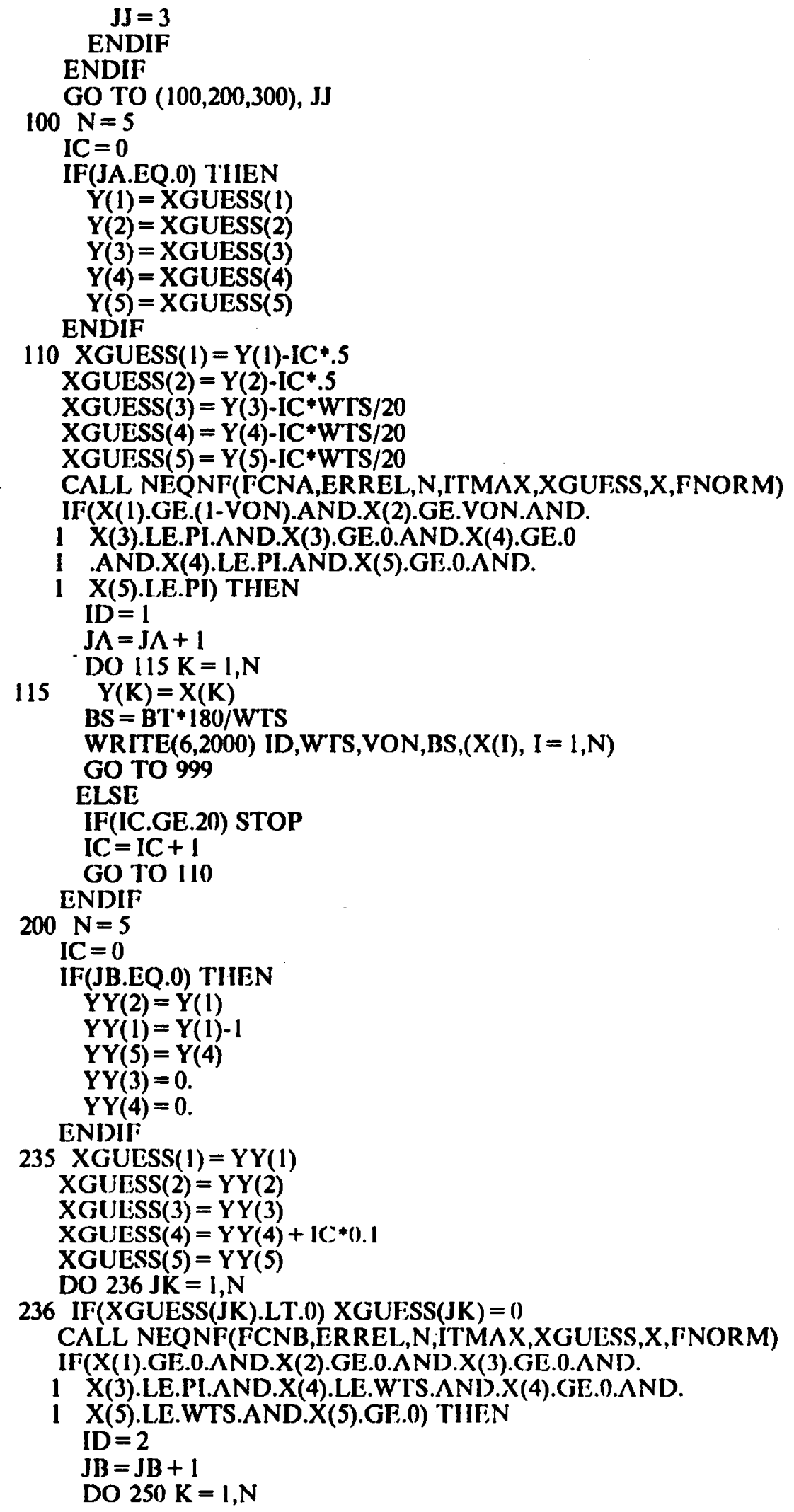




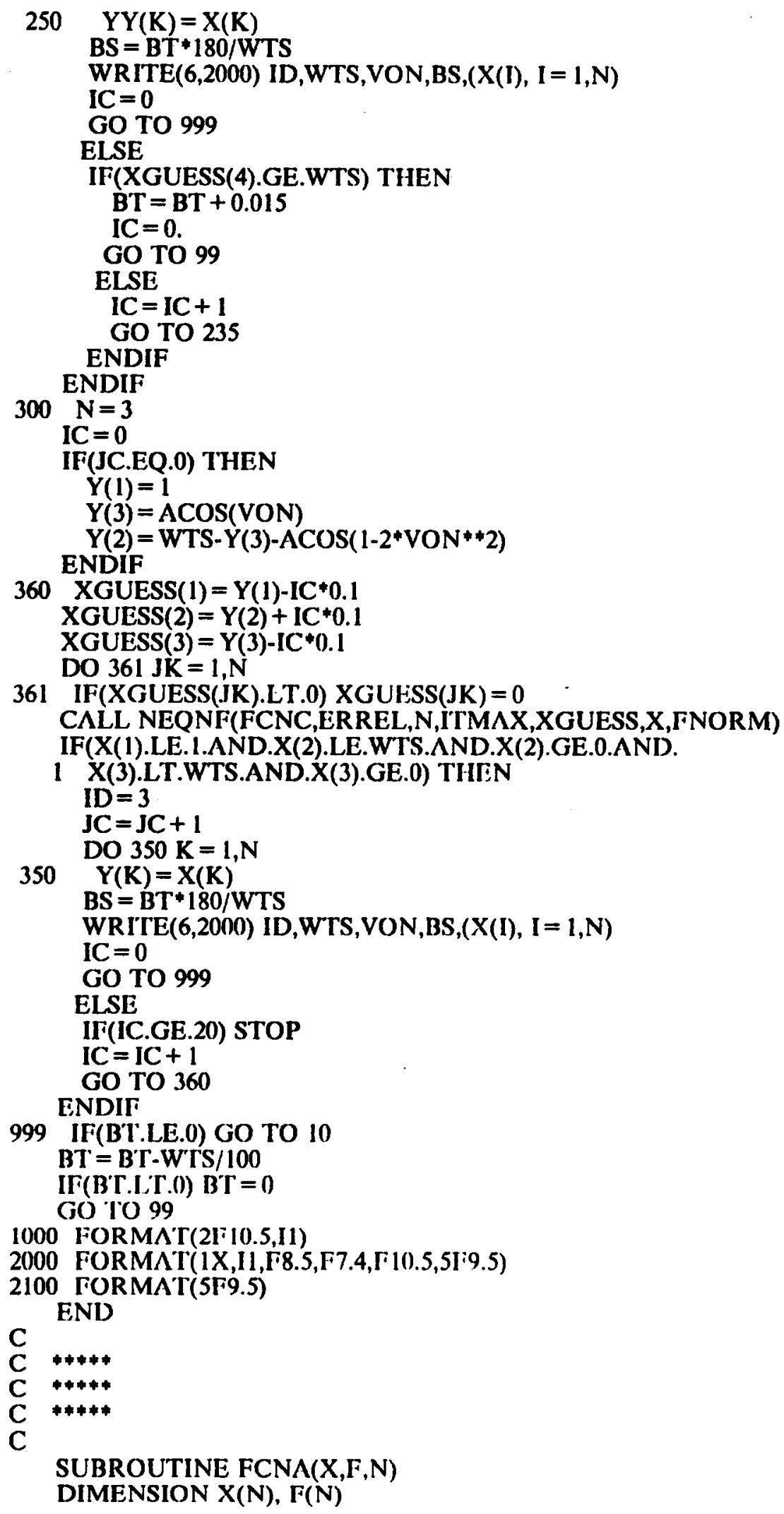


COMMON WIS, VON, PI, BT

$\mathrm{F}(1)=2 *(\mathrm{X}(1)+2 * \mathrm{VON}) * \mathrm{COS}(\mathrm{BT}-\mathrm{X}(5))-(1+(\mathrm{X}(1)+$ $12 * \mathrm{VON}) * * 2-\mathrm{X}(2) * * 2)$

$F(2)=2 * X(2) * \operatorname{COS}(X(3))-(1+X(2) * * 2-(X(1)+2 * \operatorname{VON}) * * 2)$

$\mathrm{F}(3)=2 * \mathrm{X}(2) * \operatorname{Cos}(\mathrm{X}(4))-(1+\mathrm{X}(2) * * 2-\mathrm{X}(1) * * 2)$

$F(4)=2 * X(1)^{*} \operatorname{Cos}(X(5))-\left(1+X(1)^{* * 2-X(2) * * 2)}\right.$

$F(5)=$ WTS-BT-X(3)-X(4) + PI

RETURN

C

END

C
C
C

SUBROUTINE FCNB(X,F,N)

DIMENSION $X(N), F(N)$

COMMON WTS, VON, PI, BT

$F(1)=-2 * X(1) * \operatorname{COS}(X(5))-\left(1+X(1)^{* * 2-X(2) * * 2)}\right.$

$F(2)=2 *(X(1)+2 * V O N) * \operatorname{COS}(X(4))$

$1-\left(1+(\mathrm{X}(1)+2 * \mathrm{VON}) *+2-\mathrm{X}(2)^{* * 2}\right)$

$\mathrm{F}(3)=2^{*} \mathrm{X}(2) * \operatorname{COS}(\mathrm{X}(3))-\left(1+\mathrm{X}(2)^{* *}-\mathrm{X}(1)^{* * 2)}\right.$

$\mathrm{F}(4)=2 * \mathrm{X}(2) * \operatorname{COS}(\mathrm{X}(3)+\mathrm{BT})-(1+\mathrm{X}(2) * 2-(\mathrm{X}(1)+2 * \mathrm{VON}) * * 2)$

$F(5)=X(5)+X(4)-W T S+B T$

RETURN

$\mathrm{C}$

END

C

C

C

SUBROUTINE FCNC $(X, F, N)$

DIMENSION $X(N), F(N)$

COMMON WTS, VON, PI, BT

$\mathrm{F}(1)=2 * \mathrm{X}(1)^{*} \mathrm{COS}(\mathrm{BT})-\left(1+\mathrm{X}(1)^{* *} 2-(\mathrm{X}(1)-1+2 * \mathrm{VON}) * * 2\right)$

$F(2)=2 *(X(1)-1+2 * \operatorname{VON}) * \operatorname{COS}(X(2))$

$1-(1+(\mathrm{X}(1)-1+2 * \mathrm{VON}) * 2-\mathrm{X}(1) * 2)$

$F(3)=X(2)+X(3)+B T-W T S$

RETURN

C

END

C $* * * * * *$

C $* * * *$

C

SUBROUTINE BETA12(ERREI,ITM XX,B12,JD)

EX'IERNAI, F12

COMMON W'IS, VON, Pl, B'I

DIMENSION X(2), XGUESS(2)

$\mathrm{N}=\mathbf{2}$

$\mathrm{J}=0$

IF(JD.EQ.0) THEN

$\mathrm{X}(1)=10$.

$X(2)=$ WTS

ENDIF

$121 \mathrm{XGUESS}(1)=\mathrm{X}(1)-\mathrm{J} * 0.45$

XGUESS $(2)=X(2)-J * W T S / 20$.

CALL NEQNF( F 12,ERREL,N,ITM $\Lambda X, X G U E S S, X, F N O R M)$

IF(X(1).GE.(1-VON).AND.X(2).GE.0.ANI). 
1 X(2).LE.WTS) THEN

$\mathrm{B} 12=\mathrm{X}(2)$

RETURN

ELSE

IF(J.EQ.20) THEN

WRITE $(6,1200)$

STOP

\section{ELSE}

$\mathrm{J}=\mathrm{J}+1$

GO TO 121

ENDIF

ENDIF

1200 FORMAT(/,5X,'ERROR : CAN"T FIND B 12 ',/)

C

END

C $* * * * *$

C $* * * * *$

C $* * * * *$

C

SUBROUTINE F12(X,F,N)

DIMENSION $X(N), F(N)$

COMMON WTS, VON, PI, BT

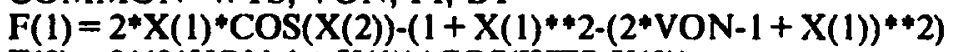

$\mathrm{F}(2)=2 *(2 * \mathrm{VON}-1+\mathrm{X}(1)) * \operatorname{COS}(\mathrm{WTS}-\mathrm{X}(2))$

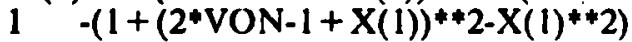

RETURN

END 


\section{APPENDIX B.8}

\section{EXPRESSIONS FOR CIRCUIT SALIENT FEATURES ABOVE RESONANT FREQUENCY}

\begin{tabular}{|c|c|c|c|}
\hline \multirow{2}{*}{$\begin{array}{l}\text { Circuit } \\
\text { Salient } \\
\text { Features }\end{array}$} & \multicolumn{2}{|r|}{ Operating } & \multirow{2}{*}{$\frac{\text { Modes }}{\text { Mode C }}$} \\
\hline & Mode A & Mode B & \\
\hline$V_{C P K}$ & $R_{\mathrm{I}}-1+V_{O N}$ & $R_{2}+V_{O N}$ & $R_{1}-1+V_{O N}$ \\
\hline$I_{A V}$ & $\frac{D a+D b+D c}{\omega_{0} T s}$ & $\frac{D a+D b+D c}{\omega_{0} T s}$ & $\frac{D a+D b}{\omega_{0} T s}$ \\
\hline$I_{R M S}$ & {$\left[\frac{R a+R b+R c}{\omega_{0} T_{s}}\right]^{\frac{1}{2}}$} & {$\left[\frac{R a+R b+R c}{\omega_{0} T s}\right]^{\frac{1}{2}}$} & $\left.\frac{R a+R b}{\omega_{0} T s}\right]^{\frac{1}{2}}$ \\
\hline$I_{Q \mid R M S}$ & {$\left[\frac{R a}{\omega_{0} T s}\right]^{\frac{1}{2}}$} & {$\left[\frac{R a+R b}{\omega_{0} T s}\right]^{\frac{1}{2}}$} & {$\left[\frac{R a}{\omega_{0} T_{s}}\right]^{\frac{1}{2}}$} \\
\hline$I_{Q 2 R M S}$ & {$\left[\frac{R a+R b}{\omega_{0} T s}\right]^{\frac{1}{2}}$} & {$\left[\frac{R b+R c}{\omega_{0} T s}\right]^{\frac{1}{2}}$} & {$\left[\frac{R a+R b}{\omega_{0} T s}\right]^{\frac{1}{2}}$} \\
\hline$I_{D 1 A V}$ & $\frac{D b+D c}{\omega_{0} T s}$ & $\frac{D c}{\omega_{0} 7 s}$ & $\frac{D b}{\omega_{0} T s}$ \\
\hline$I_{D 2 A V}$ & $\frac{D c}{\omega_{0} T s}$ & $\frac{D a}{\omega_{0} T s}$ & 0 \\
\hline$I_{Q \mid \circ J}$ & $R_{2} \sin \eta$ & $R_{3} \sin \delta$ & $R_{2} \sin \delta$ \\
\hline$I_{Q 200}$ & $R_{3} \sin \sigma$ & 0 & $\mathbf{0}$ \\
\hline$I_{\text {Qlon }}$ & 0 & 0 & $\mathbf{0}$ \\
\hline$I_{Q 20 n}$ & $\mathbf{0}$ & $R_{1} \sin \gamma$ & $\mathbf{0}$ \\
\hline$D a=$ & $R_{1}(1-\cos \gamma)$ & $R_{2}(1-\cos \eta)$ & $R_{1}(1-\cos \beta)$ \\
\hline$D b=$ & $-R_{2}(\cos \eta+\cos \delta)$ & $R_{1}(\cos y-\cos (\gamma+\beta))$ & $R_{2}(1-\cos \delta)$ \\
\hline$D C=$ & $R_{3}(1-\cos \sigma)$ & $R_{3}(1-\cos \delta)$ & \\
\hline$R a=$ & $\frac{R_{1}^{2}}{2}\left(\gamma-\frac{\sin 2 \gamma}{2}\right)$ & $\frac{R_{2}^{2}}{2}\left(\eta-\frac{\sin 2 \eta}{2}\right)$ & $\frac{R_{1}^{2}}{2}\left(\beta-\frac{\sin 2 \beta}{2}\right)$ \\
\hline$R b=$ & $\begin{array}{l}\frac{R_{2}^{2}}{2}(\delta+\eta-\pi- \\
\left.\left(\frac{\sin 2 \delta}{2}+\frac{\sin 2 \eta}{2}\right)\right)\end{array}$ & $\begin{array}{c}\frac{R_{1}^{2}}{2}\left(\beta+\frac{\sin 2 \gamma}{2}-\right. \\
\left.\frac{\sin (2 \gamma+2 \beta)}{2}\right)\end{array}$ & $\frac{R_{2}^{2}}{2}\left(\delta-\frac{\sin 2 \delta}{2}\right)$ \\
\hline$R c=$ & $\frac{R_{3}^{2}}{2}\left(\sigma-\frac{\sin 2 \sigma}{2}\right)$ & $\frac{R_{3}^{2}}{2}\left(\delta-\frac{\sin 2 \delta}{2}\right)$ & \\
\hline
\end{tabular}


FORTRAN Programs for Calculating Circuit Salient Features of a CM-SRC Operating Above Resonant Frequency

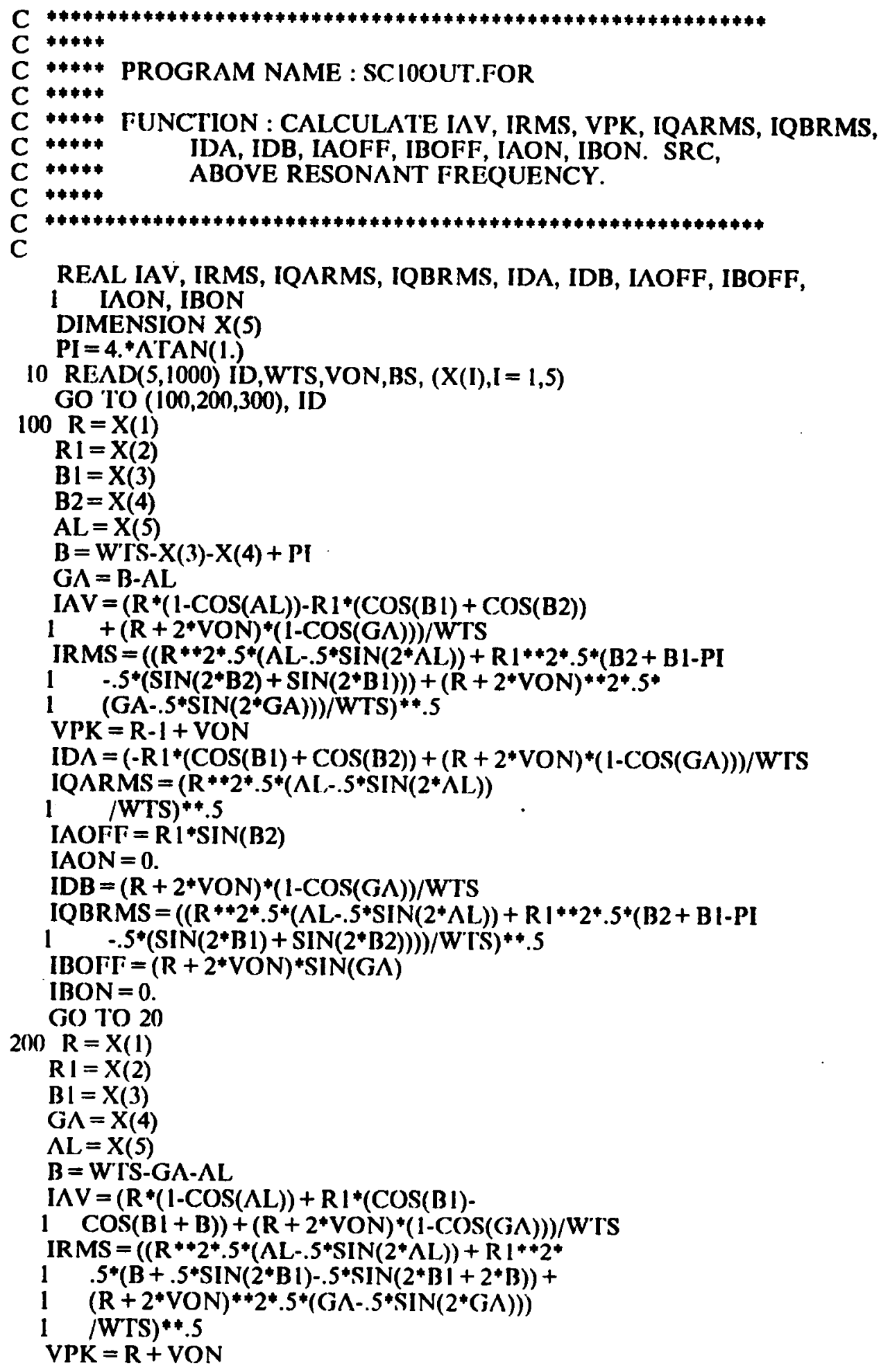




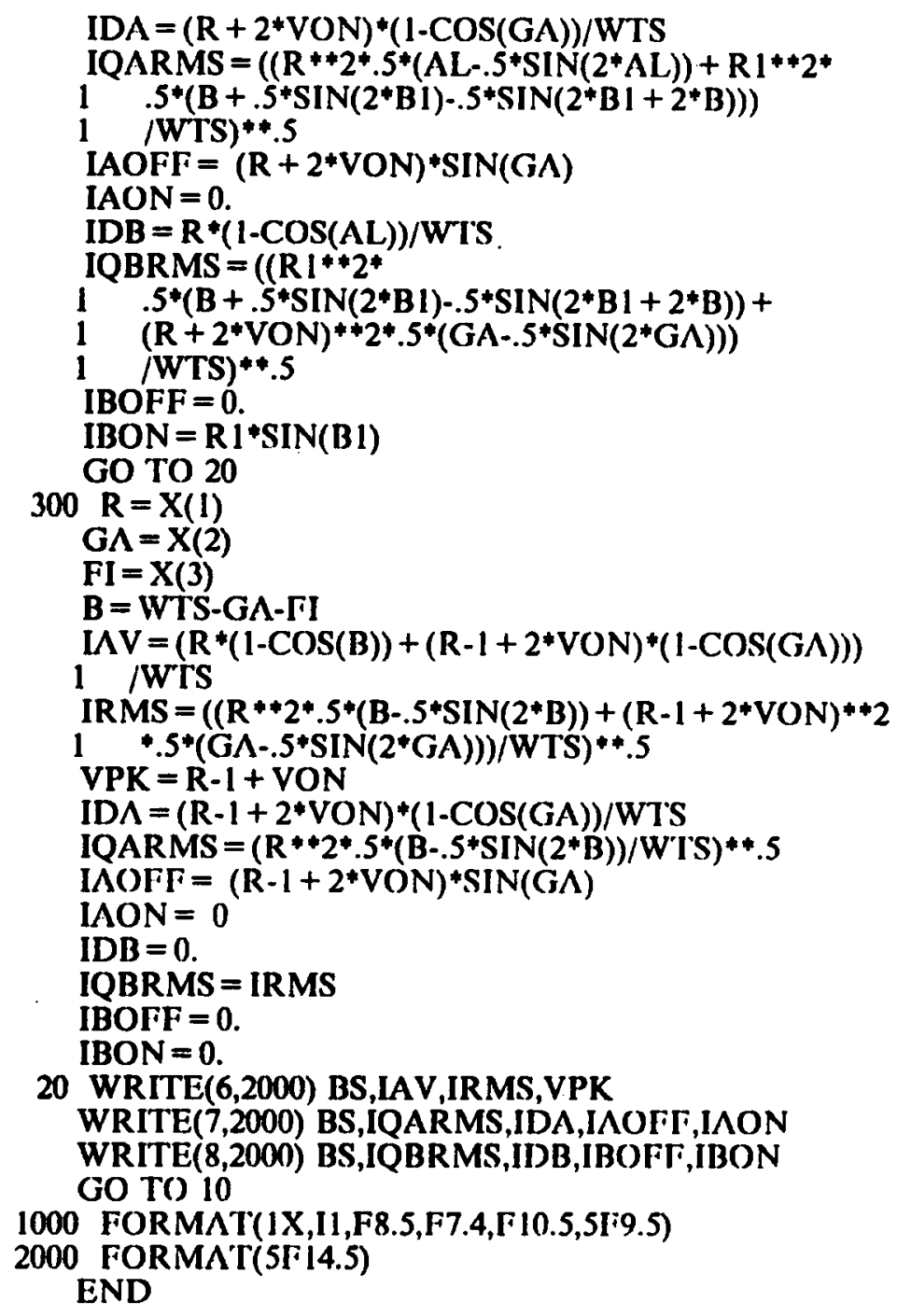




\section{APPENDIX B.9 \\ DC CHARACTERISTICS ABOVE \\ RESONANT FREQUENCY}

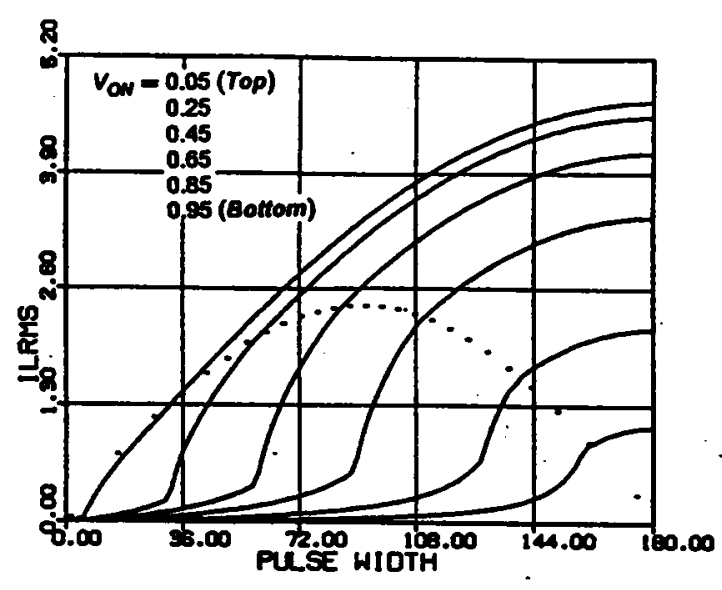

- RMS inductor current

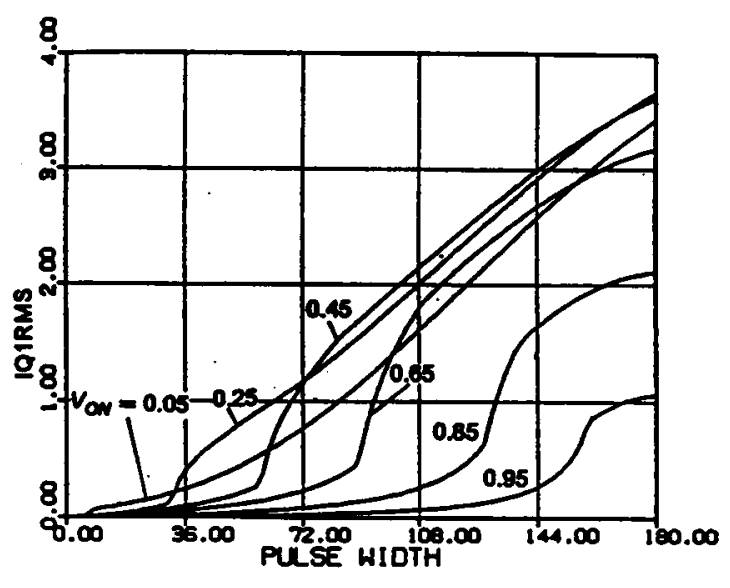

- RMS switch current (Q1,Q3)

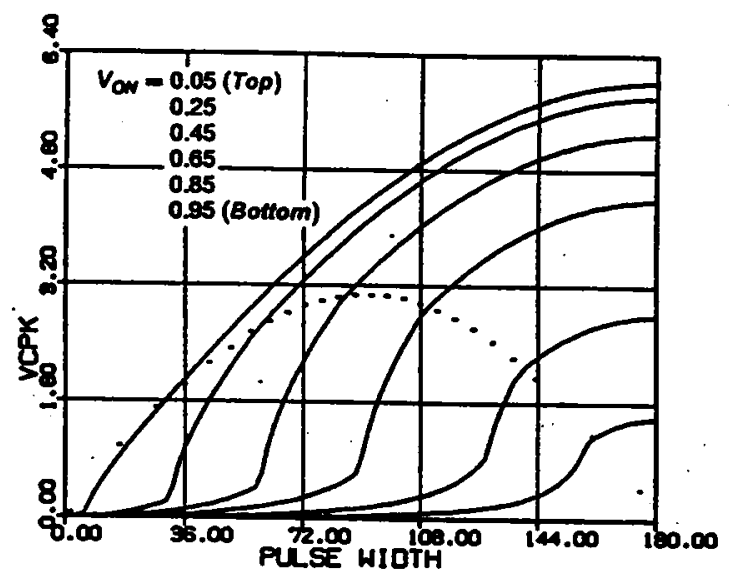

- Peak capacitor voltage

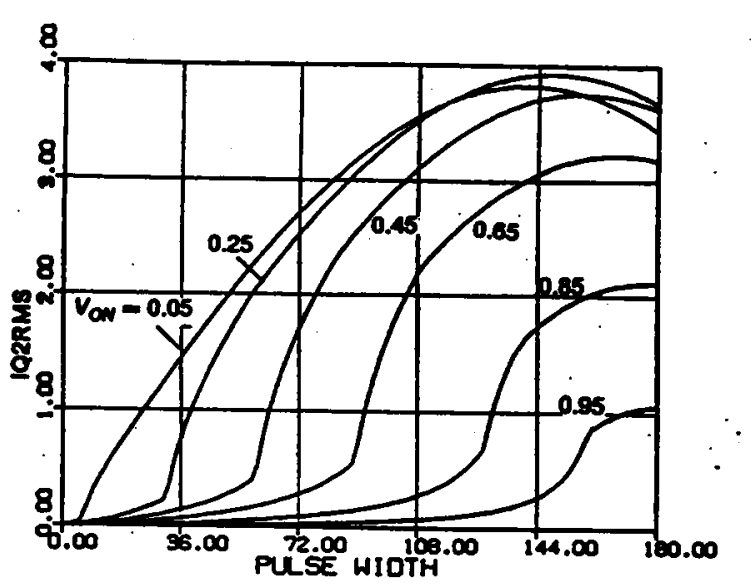

- RMS switch current (Q2.Q4)

Figure B.9.1 DC Characteristics for $\omega_{S N}=1.1$ 


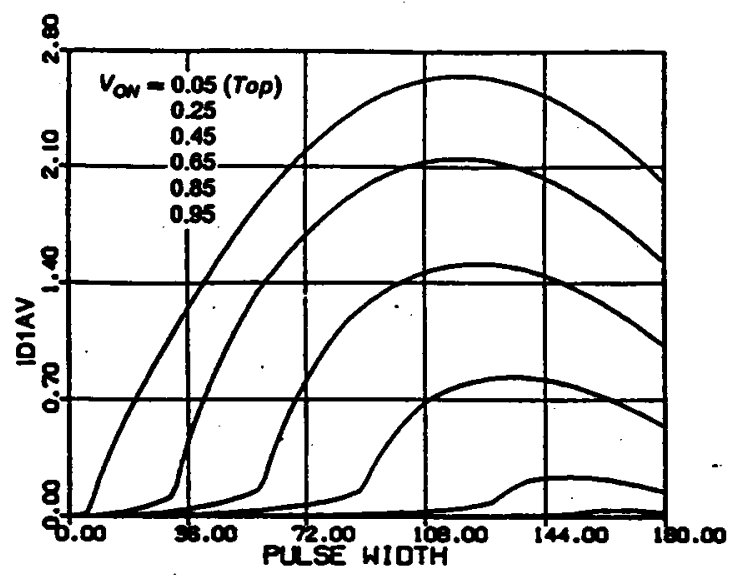

- Average diode current (DI,D3)

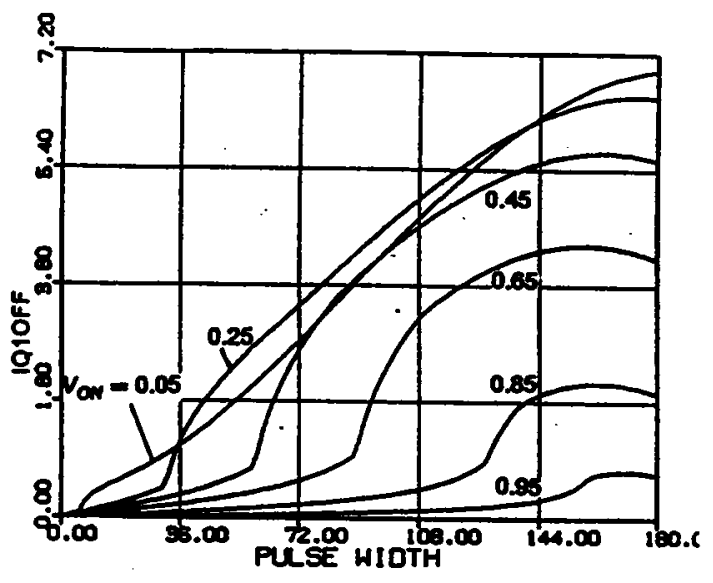

- Switch turn-off current $\left(Q 1, Q^{3}\right)$

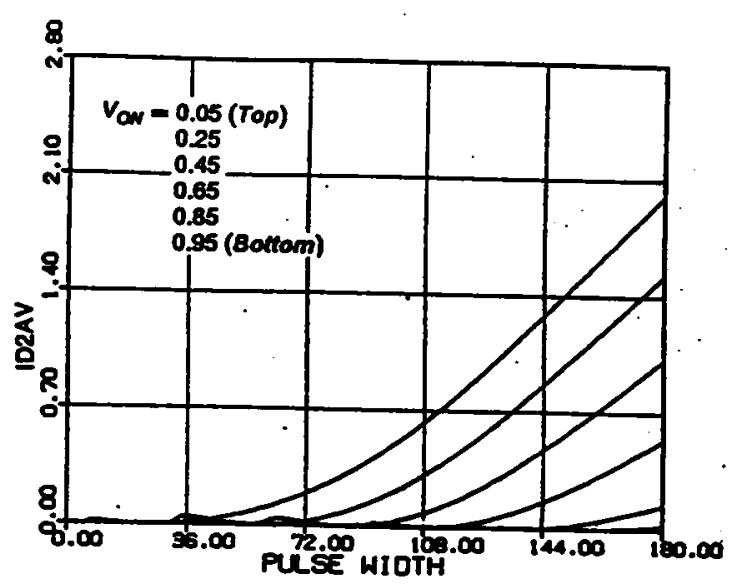

- Average diode current $(D 2, D 4)$

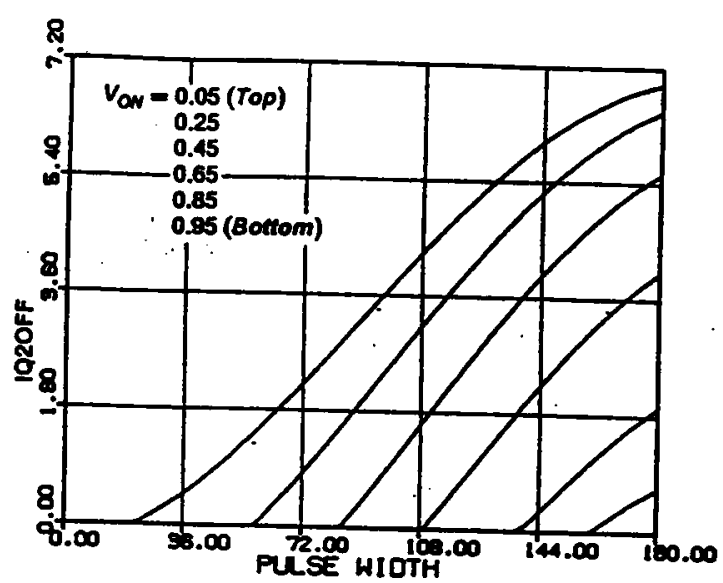

- Switch turn-off current (Q2.Q4)

Figure B.9.1 Continued 


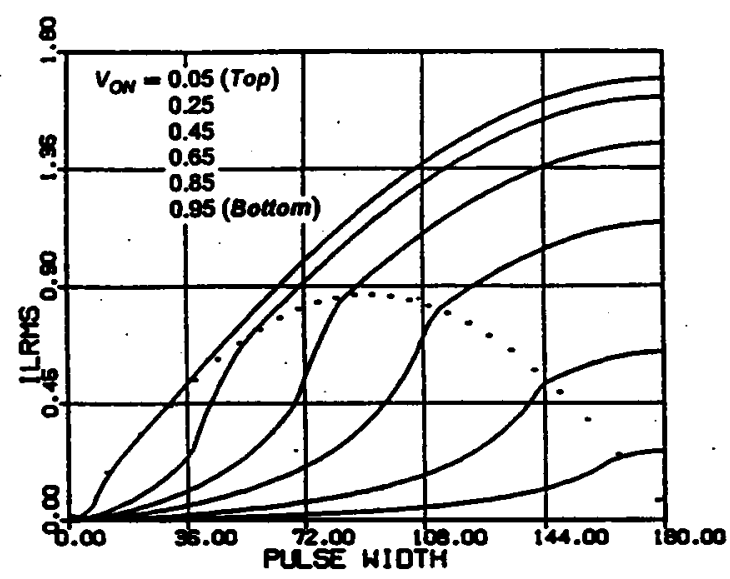

- RMS inductor current

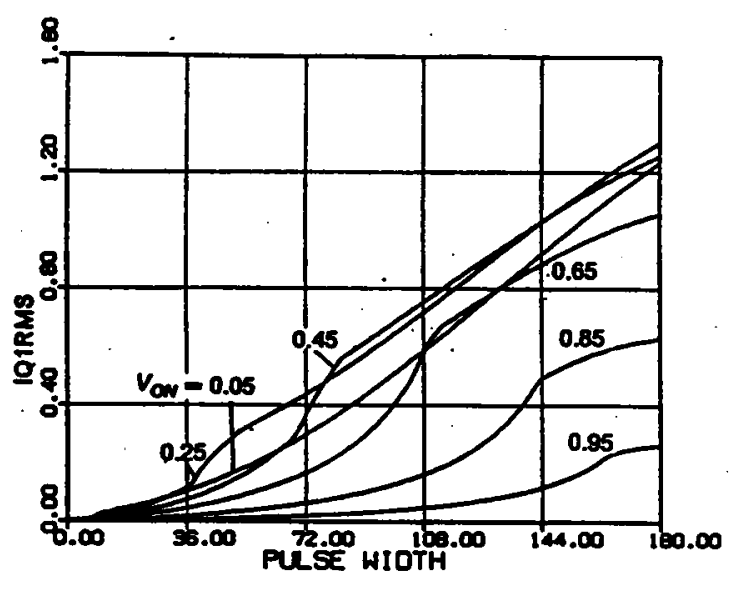

- RMS switch current (QI,Q3)

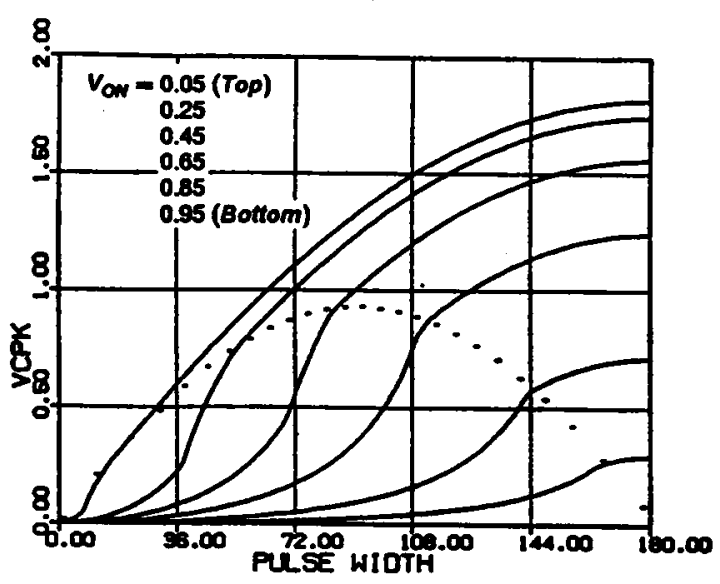

- Peak capacitor voltage

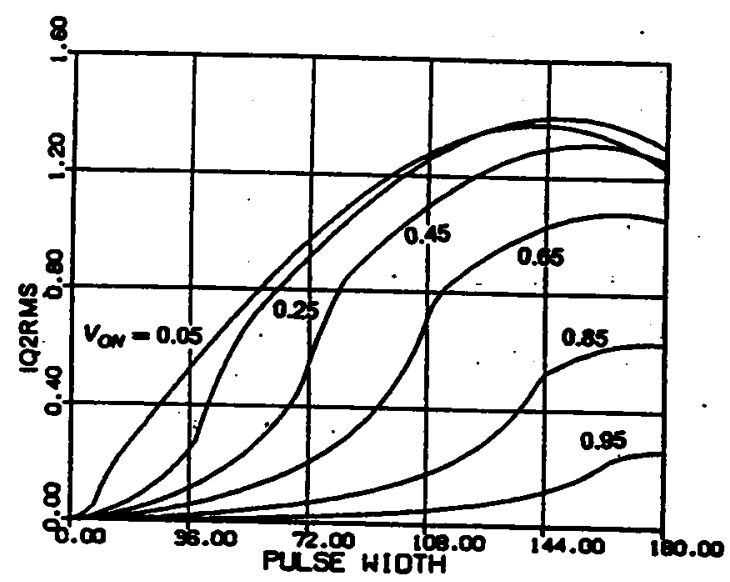

- RMS switch current (Q2,Q4)

Figure B.9.2 DC Characteristics for $\omega_{S N}=1.3$ 


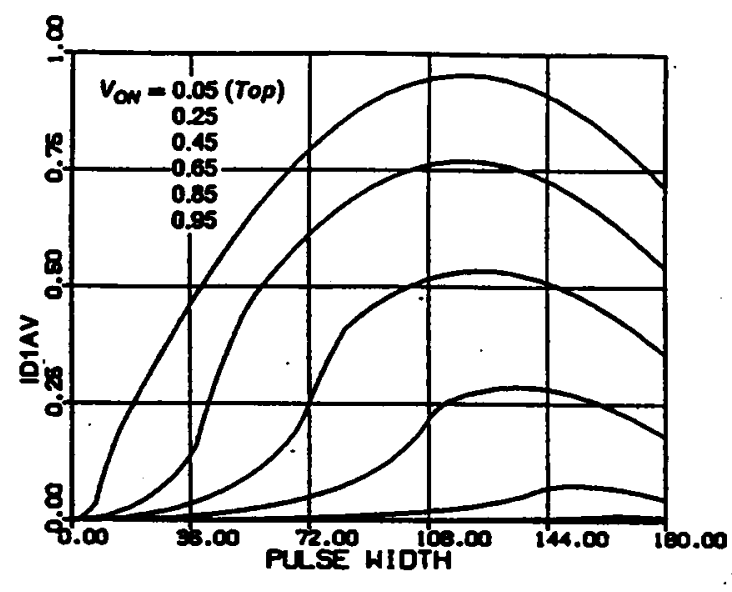

- Average diode current $(D I, D 3)$

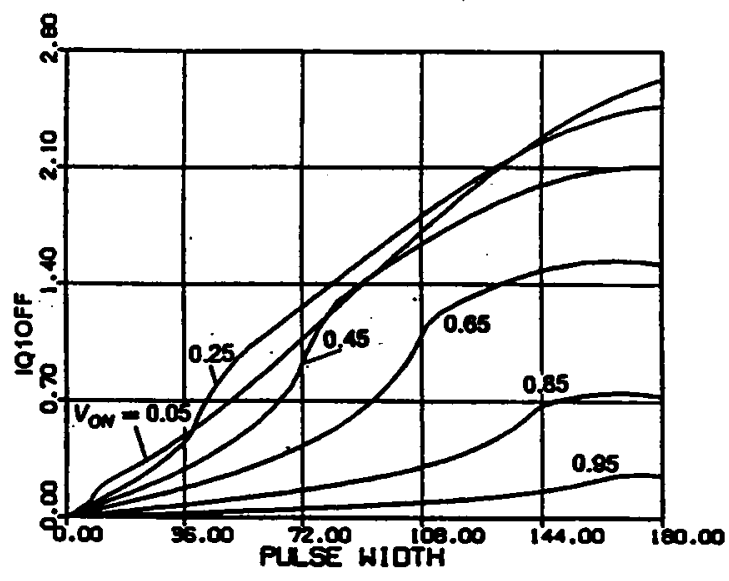

- Switch turn-off current (Q1,Q3)

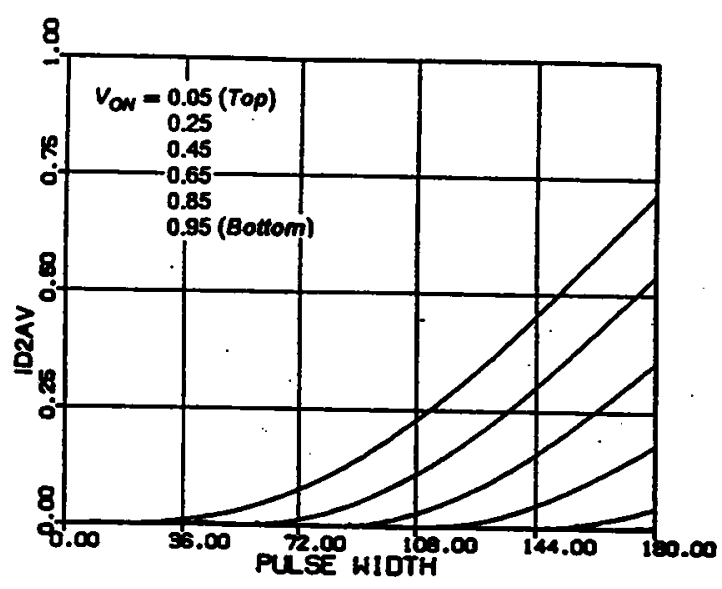

- Average diode current (D2,D4)

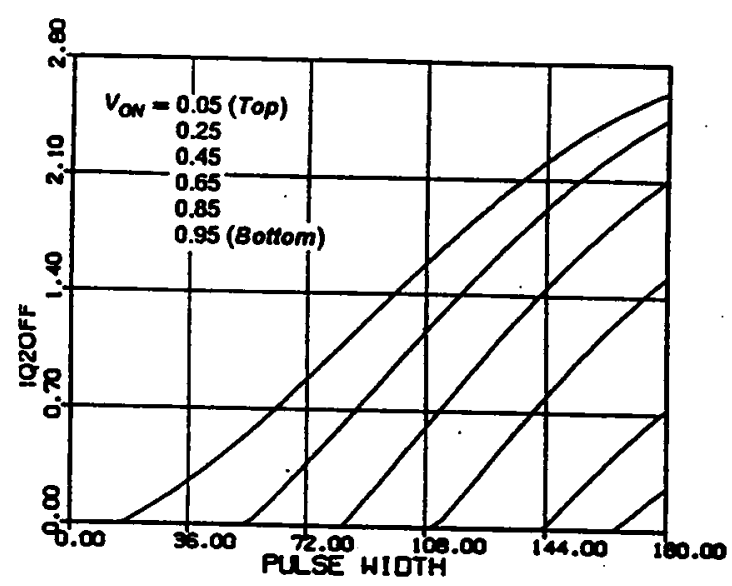

- Switch turn-off current (Q2,Q4)

Figure B.9.2 Continued 


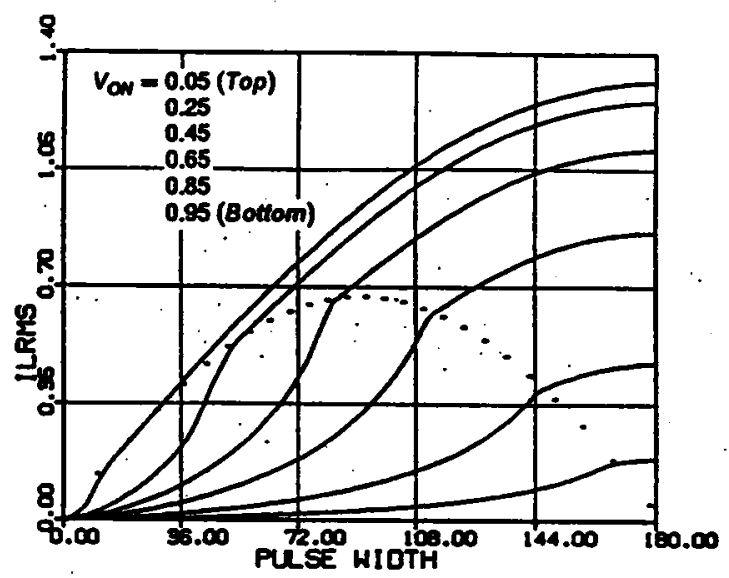

- $R M S$ inductor current

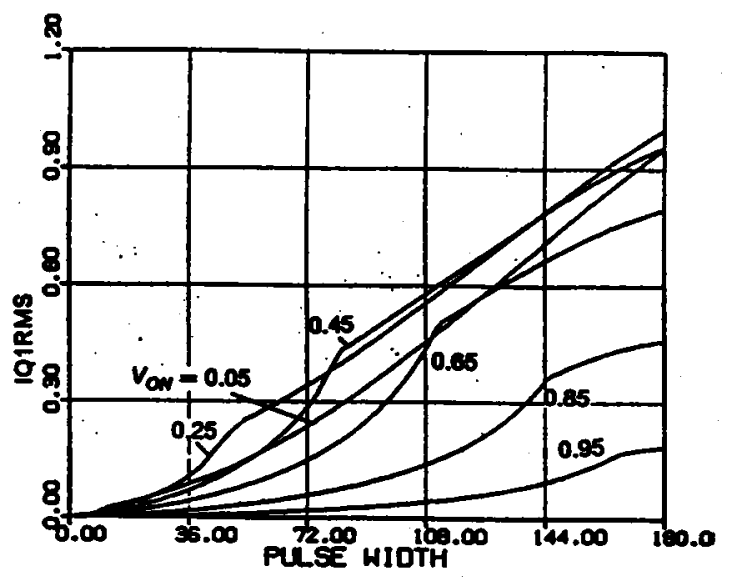

- RMS switch current (Q1,Q3)

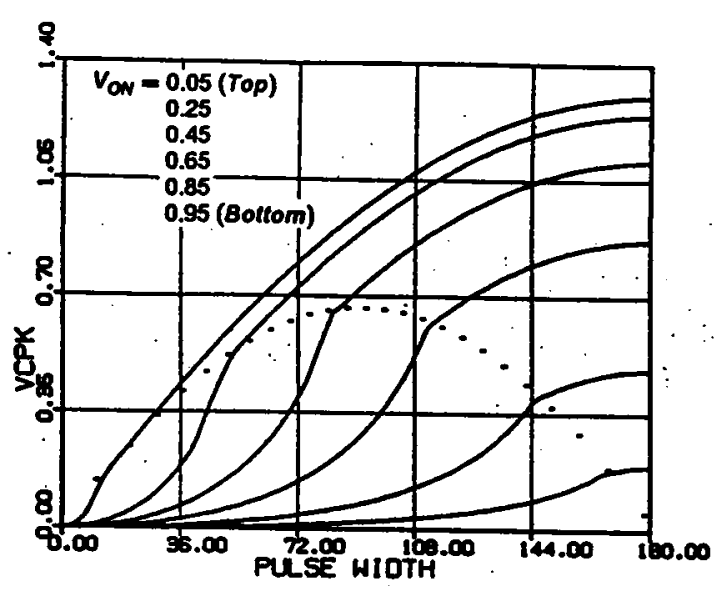

- Peak capacitor voltage

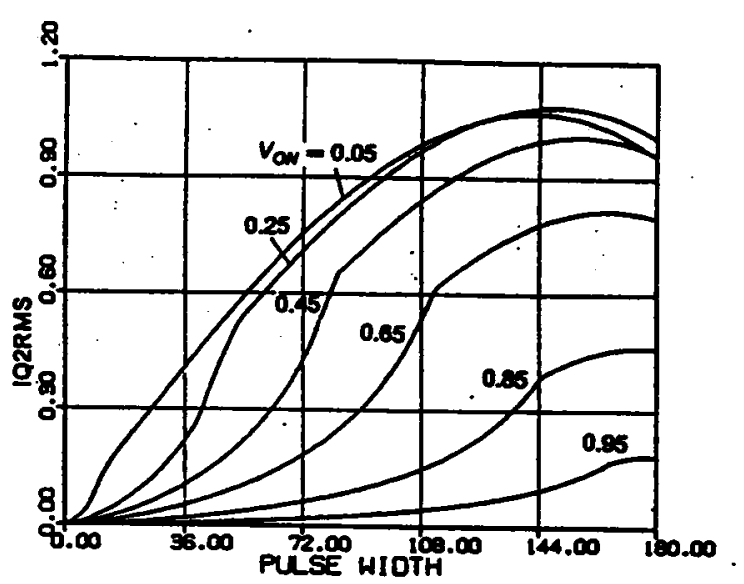

- RMS switch current (Q2,Q4)

Figure B.9.3 DC Characteristics for $\omega_{S N}=1.4$ 


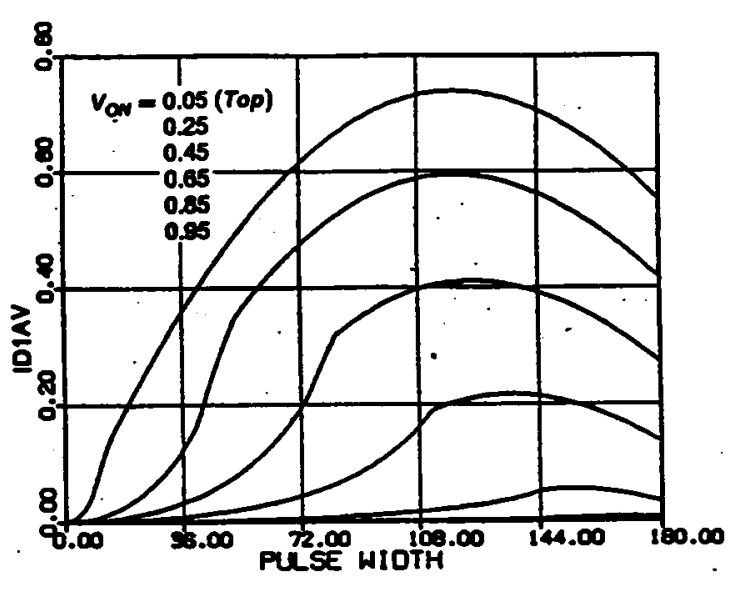

- Average diode current (DI,D3)

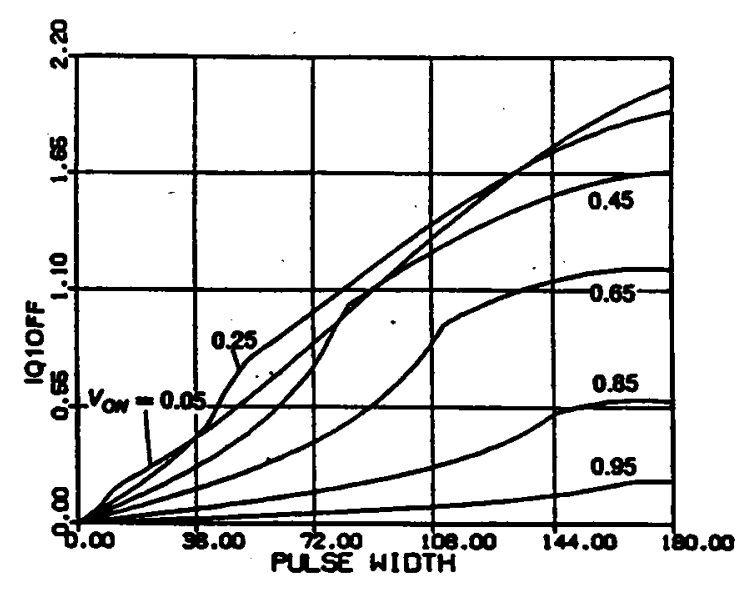

- Switch turn-off current (Q2,Q4)

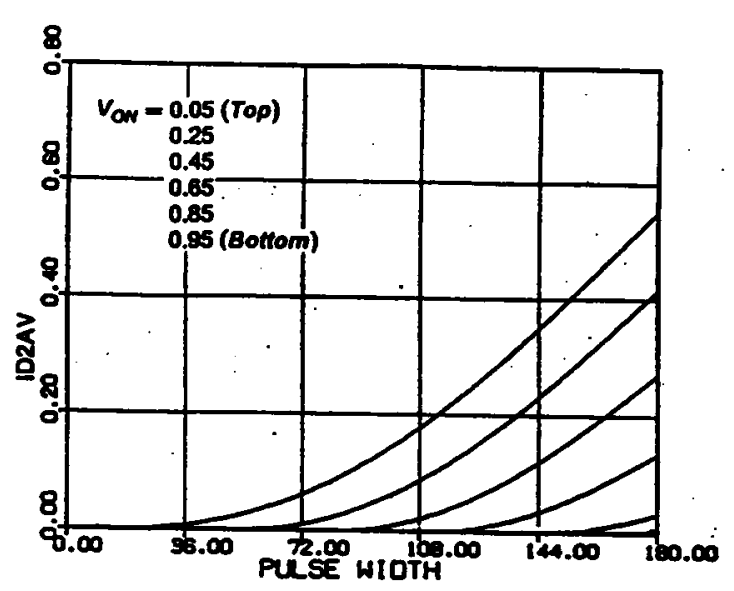

- Average diode current $(D 2, D 4)$

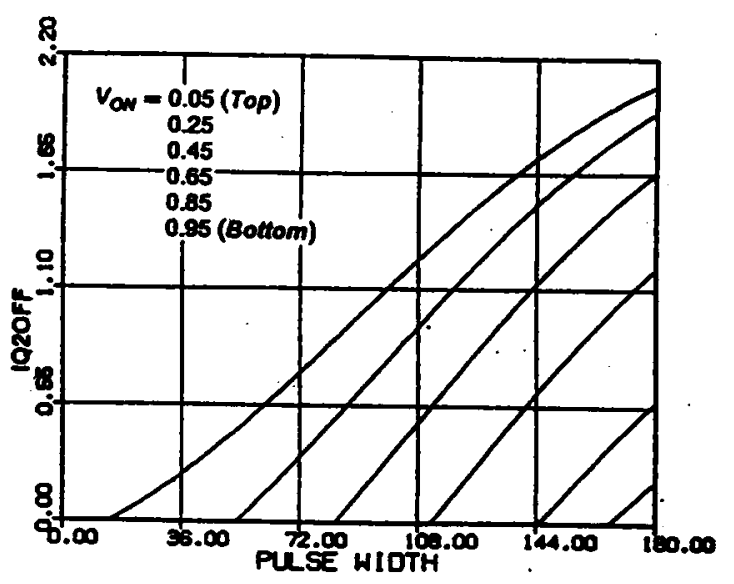

- Switch turn-off current (QI,Q3)

Figure B.9.3 Continued 


\section{APPENDIX B.10 \\ GENERATION OF CIRCUIT WAVEFORMS OF A CM-SRC}

Fortran Program for Generating Capacitor Voltage and Inductor Current
Waveforms (Below Resonant Frequency)

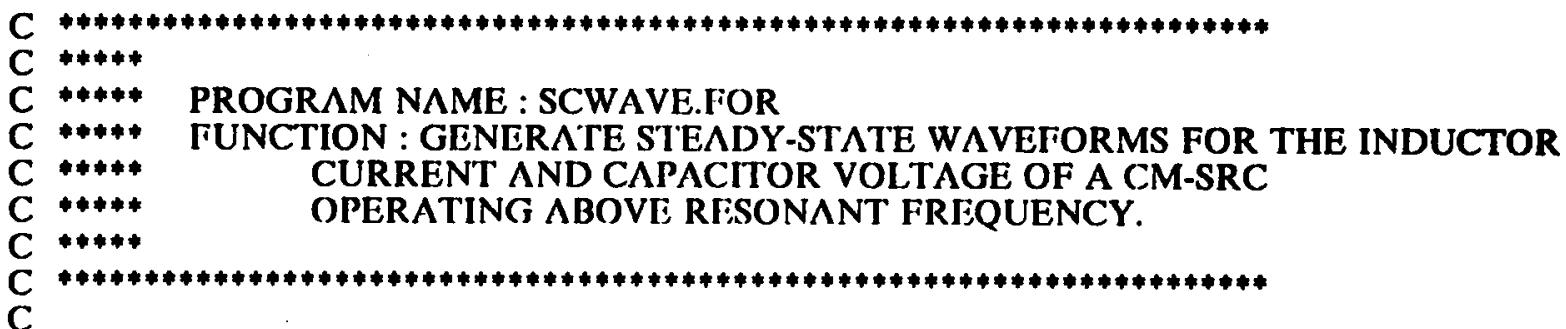

REAL ILN, VCN

DIMENSION X(6)

$\mathrm{PI}=4 . * \Lambda T A N(1$.

$10 \operatorname{RE} \Lambda \mathrm{D}(5,1050) \mathrm{WN}$

11 READ $(5,1000)$ J,BT,VON,(X(K), $K=1,6)$

$\mathrm{C}$

C **** THE INPUTS ARE OB'TAINED I'ROM PROGRAM SCPAR.FOR

C

IF(J.EQ.0) STOP

$\mathrm{WT}=0$.

$Y T=0$.

GO TO $(100,200,300,400,500,600)$, J

$100 \mathrm{R}=\mathrm{X}(1)$

$R I=X(2)$

$\mathrm{BI}=\mathrm{X}(3)$

$B 2=X(4)$

$\mathrm{AL}=\mathrm{X}(5)$

$G \Lambda=X(6)$

$\mathbf{T}=\mathbf{P I}-\mathrm{B} \mathbf{I}$

101 IF(T.LT.B2) GO TO 102

ILN $=$ RI*SIN('T)

$\mathrm{VCN}=\mathrm{R} 1^{*} \mathrm{COS}(\mathrm{T})-\mathrm{VON}$

WRITE $(7,2000)$ WT, IIN

WRITE(6,2000) WT,VCN,II,N

$\mathrm{T}=\mathrm{T}-(\mathrm{PI}-\mathrm{B} 2-\mathrm{BI}) / 30$.

$W^{\prime} \mathrm{I}=\mathrm{WT}+(\mathrm{PI}-\mathrm{B} 2-\mathrm{BI}) * 180 * \mathrm{WN} / \mathrm{PI} / 30$.

GO TO 101

$102 \mathrm{~T}=\mathrm{GA}$

$\mathrm{YT}=\mathrm{YT}+(\mathrm{PI}-\mathrm{B} 2-\mathrm{B} 1)$

$\mathrm{WT}=\mathrm{YT} * 180 * \mathrm{WN} / \mathrm{PI}$

103 IF(T.LT.0) GO TO 104

ILN $=\mathbf{R} * \operatorname{SIN}(\mathrm{T})$

$\mathrm{VCN}=\mathrm{R} * \operatorname{COS}(\mathrm{T})+1-\mathrm{VON}$

WRITE(7,2000) WT, ILN

WRITE $(6,2000)$ WT,VCN,IL,N

$\mathrm{T}=\mathrm{T} \cdot \mathrm{GA} / 50$.

$W T=W T+G \Lambda * 180 * W N / P I / 50$. 


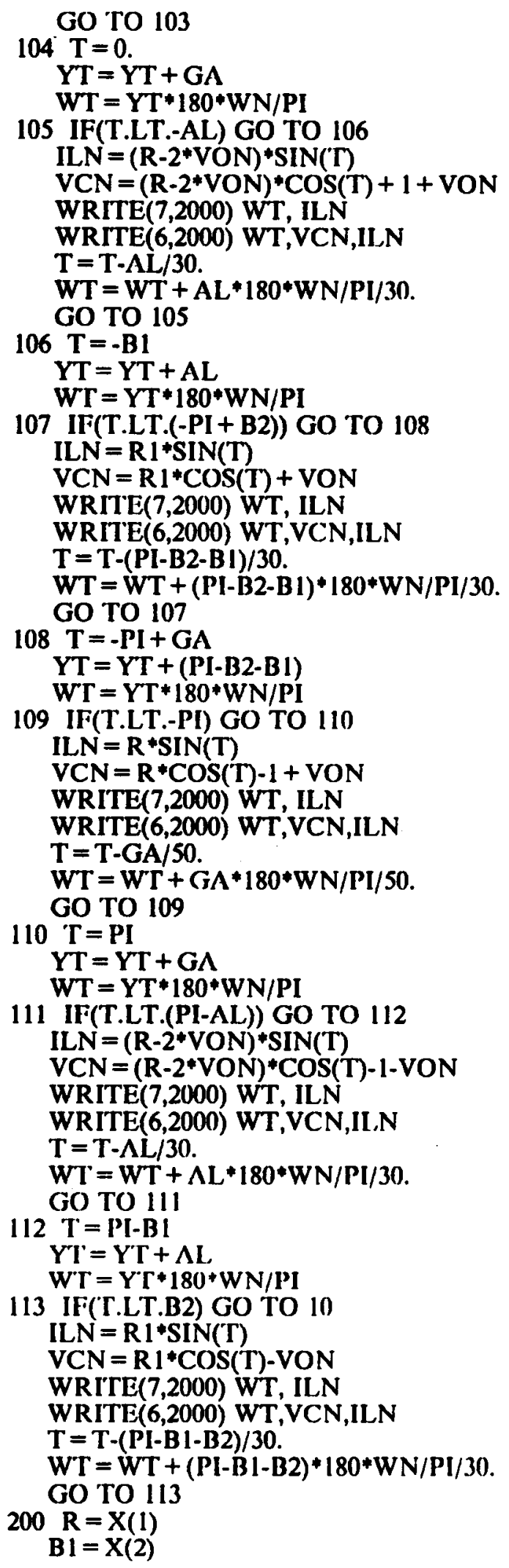


$F I=X(3)$

$A L=X(4)$

$\mathbf{T}=\mathbf{P I}$

201 IF(T.LT.(PI-AL)) GO TO 202

ILN $=(\mathrm{R}+1-2 * \mathrm{VON}) * \operatorname{SIN}(\mathrm{T})$

$V C N=(R+1-2 * V O N) * C O S(T)-V O N$

WRITE $(7,2000)$ WT, ILN

WRITE $(6,2000)$ WT, VCN,ILN

$\mathrm{T}=\mathrm{T}-\mathrm{AL} / 50$.

$\mathrm{WT}=\mathrm{WT}+\Lambda \mathrm{L} * 180^{*} \mathrm{WN} / \mathrm{PI} / 50$.

GO TO 201

$202 \mathrm{~T}=\mathrm{B} 1$

$\mathbf{Y T}=\mathbf{Y T}+\mathbf{A L}$

$\mathrm{WT}=\mathrm{YT} * 180 * \mathrm{WN} / \mathrm{PI}$

203 IF(T.LT.0) GO TO 204

IL $N=R * \operatorname{SIN}(T)$

$\mathrm{VCN}=\mathrm{R} * \mathrm{COS}(\mathrm{T})+1-\mathrm{VON}$

WRITE $(7,2000)$ WT, ILN

WRITE $(6,2000)$ WT,VCN,ILN

$\mathrm{T}=\mathrm{T}-\mathrm{B} 1 / 30$.

$W T=W T+B I * 180 * W N / P I / 30$.

GO TO 203

$204 \mathrm{~T}=\mathrm{FI}$

$Y T=Y T+B 1$

$\mathrm{WT}=\mathrm{YT} * 180 * \mathrm{WN} / \mathrm{PI}$

205 IF(T.LT.0) GO TO 206

ILN $=0$.

$\mathrm{VCN}=\mathrm{R}+1-\mathrm{VON}$

WRITE $(7,2000)$ WT,ILN

WRITE $(6,2000)$ WT,VCN,ILN

$\mathrm{T}=\mathrm{T}-\mathrm{FI} / 30$.

$\mathrm{WT}=\mathrm{WT}+\mathrm{FI} * 180 * \mathrm{WN} / \mathrm{PI} / 30$.

GO TO 205

$206 \mathrm{~T}=0$.

$\mathrm{YT}=\mathrm{YT}+\mathrm{FI}$

$\mathrm{WT}=\mathrm{YT} * 180 * \mathrm{WN} / \mathrm{PI}$

207 IF(T.LT.-AL) GO TO 208

ILN $=(\mathrm{R}+1-2 * \mathrm{VON}) * \mathrm{SIN}(\mathrm{T})$

$\mathrm{VCN}=(\mathrm{R}+1-2 * \mathrm{VON}) * \mathrm{COS}(\mathrm{T})+\mathrm{VON}$

WRITE $(7,2000)$ WT, ILN

WRITE $(6,2000)$ WT,VCN,ILN

$\mathrm{T}=\mathrm{T} \cdot \mathrm{AL} / \mathrm{SO}$.

$\mathrm{WT}=\mathrm{WT}+\Lambda \mathrm{L} * 180 * \mathrm{WN} / \mathrm{PI} / 50$.

GO TO 207

$208 \mathrm{~T}=-\mathrm{PI}+\mathrm{B} 1$

$Y^{\prime} \mathrm{T}=\mathrm{Y}^{\prime} \mathrm{T}+\Lambda \mathrm{L}$,

$\mathrm{WT}=\mathrm{Y} T * 180 * \mathrm{WN} / \mathrm{PI}$

209 II'(I.LT.-PI) GO TO 210

ILN $=R * \operatorname{SIN}(T)$

$\mathrm{VCN}=\mathrm{R} * \operatorname{COS}(\mathrm{T})-1+\mathrm{VON}$

WRITE $(7,2000)$ WT, ILN

WRITE $(6,2000)$ WT,VCN,ILN

$\mathbf{T}=\mathrm{T} \cdot \mathrm{B} \mathbf{1} / 30$.

$\mathrm{WT}=\mathrm{WT}+\mathrm{BI} * 180^{*} \mathrm{WN} / \mathrm{PI} / 30$.

GO TO 209

$210 \mathrm{~T}=\mathrm{FI}$

$\mathrm{YT}=\mathrm{YT}+\mathrm{B} 1$

$W T=Y T * 180 * W N / P I$ 
211 IF(T.LT.0.) GO TO 212

ILN $=0$.

$\mathrm{VCN}=-\mathrm{R}-1+\mathrm{VON}$

WRITE $(7,2000)$ WT, ILN

.. WRITE $(6,2000)$ WT,VCN,ILN

$\mathrm{T}=\mathrm{T}-\mathrm{FI} / 30$.

$\mathrm{WT}=\mathrm{WT}+\mathrm{FI} * 180 * \mathrm{WN} / \mathrm{PI} / 30$.

GO TO 211

$212 \mathrm{~T}=\mathrm{PI}$

$Y T=Y T+F I$

$W T=Y T * 180 * W N / P I$

213 IF(T.LT.(PI-AL)) GO TO 10

ILN $=(R+1-2 * V O N) * \operatorname{SIN}(T)$

$\mathrm{VCN}=(\mathrm{R}+1-2 * \mathrm{VON}) * \mathrm{COS}(\mathrm{T})-\mathrm{VON}$

WRITE $(7,2000)$ WT, ILN

WRITE $(6,2000)$ WT, VCN,ILN

$\mathrm{T}=\mathrm{T}-\mathrm{AL} / 50$.

$W T=W T+A L * 180 * W N / P I / 50$.

GO TO 213

$300 \mathrm{R}=\mathrm{X}(1)$

$\mathrm{R} 1=\mathbf{X}(2)$

$\mathrm{B} 1=\mathrm{X}(3)$

$A L=X(4)$

$\mathrm{GA}=\mathbf{X}(5)$

$T=-P I+G \Lambda$

301 IF(T.LT.-PI) GO TO 302

II N $=(\mathrm{R} 1+2 * \mathrm{VON}) * \operatorname{SIN}(\mathrm{T})$

$\mathrm{VCN}=(\mathrm{R} 1+2 * \mathrm{VON}) * \mathrm{COS}(\mathrm{T})+\mathrm{VON}$

WRITE $(7,2000)$ WT, ILN

WRITE $(6,2000)$ WT,VCN,IL,N

$\mathrm{T}=\mathrm{T}-\mathrm{GA} / \mathbf{3 0}$.

$W T=W T+G \Lambda * 180 * W N / P I / 30$.

GO TO 301

$302 \mathrm{~T}=\mathrm{PI}$

$\mathrm{YT}=\mathrm{YT}+\mathrm{G \Lambda}$

$\mathrm{WT}=\mathrm{YT} * 180 * \mathrm{WN} / \mathrm{PI}$

303 IF(T.LT.(PI- $\Lambda$ L)) GO TO 304

ILN $=$ R I*SIN(T)

$\mathrm{VCN}=\mathrm{R} 1 * \mathrm{COS}(\mathrm{T})-\mathrm{VON}$

WRITE $(7,2000)$ WT,ILN

WRITE $(6,2000)$ WT, VCN,IL,N

$\mathrm{T}=\mathrm{T}-\mathrm{AL} / 50$.

$\mathrm{WT}=\mathrm{WT}+\mathrm{AL}, * 180 * \mathrm{WN} / \mathrm{PI} / 50$.

GO TO 303

$304 \mathrm{~T}=\mathrm{PI}-\mathrm{B} 1$

$\mathrm{YT}=\mathrm{YT}+\Lambda \mathrm{L}$

$\mathrm{W}^{\prime} \mathrm{I}=\mathrm{Y}^{\prime} \mathrm{T}^{*} 180^{*} \mathrm{WN} / \mathrm{PI}$

305 IF('T.LT.(PI-BI-BT)) GO TO 306

ILN $=\mathrm{R} * \mathrm{SIN}(\mathrm{T})$

$\mathrm{VCN}=\mathrm{R}^{*} \mathrm{COS}(\mathrm{T})+1-\mathrm{VON}$

WRITE $(7,2000)$ WT, ILN

WRITE $(6,2000)$ WT,VCN,ILN

$\mathrm{T}=\mathbf{} \mathrm{\Gamma}-\mathrm{BT} / 30$.

$W T=W T+B T^{*} * 180 * W N / P I / 30$.

GO TO 305

$306 \mathrm{~T}=\mathrm{GA}$

$\mathrm{YT}=\mathrm{YT}+\mathrm{BT}$

$W T=Y T * 180 * W N / P I$ 
307 IF(T.LT.0) GO TO 308

ILN $=(\mathrm{R} 1+2 * \mathrm{VON}) * \mathrm{SIN}(\mathrm{T})$

$\mathrm{VCN}=(\mathrm{R} 1+2 * \mathrm{VON}) * \mathrm{COS}(\mathrm{T})-\mathrm{VON}$

WRITE $(7,2000)$ WT,ILN

WRITE $(6,2000)$ WT,VCN,ILN

$\mathrm{T}=\mathrm{T} \cdot \mathrm{GA} / 30$.

$W T=W T+G A * 180 * W N / P I / 30$.

GO TO 307

$308 \mathrm{~T}=0$.

$\mathbf{Y T}=\mathbf{Y T}+\mathbf{G A}$

$\mathrm{WT}=\mathrm{YT} * 180 * \mathrm{WN} / \mathrm{PI}$

309 IF(T.LT.-AL) GO TO 310

ILN $=$ R $1 * \operatorname{SIN}(\mathrm{T})$

$\mathrm{VCN}=\mathrm{R} 1+\operatorname{COS}(\mathrm{T})+\mathrm{VON}$

WRITE $(7,2000)$ WT, ILN

WRITE $(6,2000)$ WT,VCN,ILN

$\mathrm{T}=\mathrm{T}-\mathrm{AL} / 50$.

$\mathrm{WT}=\mathrm{WT}+\mathrm{AL} * 180 * \mathrm{WN} / \mathrm{PI} / 50$.

GO TO 309

$310 \mathrm{~T}=-\mathrm{B} 1$

$Y T=Y T+A L$

$W T=Y T * 180 * W N / P I$

311 IF(T.LT.(-B1-BT)) GO TO 312

ILN $=R * \operatorname{SIN}(\mathrm{T})$

$\mathrm{VCN}=\mathrm{R} * \operatorname{COS}(\mathrm{T})-1+\mathrm{VON}$

WRITE $(7,2000)$ WT, IL,N

WRITE $(6,2000)$ WT,VCN,ILN

$\mathrm{T}=\mathrm{T}-\mathrm{BT} / 30$.

$W T=W T+B T * 180 * W N / P I / 30$.

GO TO 311

$312 \mathrm{~T}=-\mathrm{PI}+\mathrm{GA}$

$Y \mathrm{~T}=\mathrm{YT}+\mathrm{BT}$

$W T=Y T * 180^{*} W N / P I$

313 IF(T.LT.-PI) GO TO 10

ILN $=(R 1+2 *$ VON $) * \operatorname{SIN}(T)$

$\mathrm{VCN}=(\mathrm{R} I+2 * \mathrm{VON}) * \operatorname{COS}(\mathrm{I})+\mathrm{VON}$

WRITE $(7,2000)$ WT, ILN

WRITE $(6,2000)$ WT, VCN,ILN

$\mathrm{T}=\mathrm{T}-\mathrm{GA} / \mathbf{3 0}$.

$W T=W T+G \Lambda * 180 * W N / P I / 30$.

GO TO 313

$400 \mathrm{R}=\mathrm{X}(1)$

$\mathrm{R} 1=\mathbf{X}(2)$

$\mathrm{B} 1=\mathrm{X}(3)$

$B 2=X(4)$

$\mathrm{AL}=\mathrm{X}(5)$

$\mathrm{G} \Lambda=\mathrm{X}(6)$

$\mathrm{T}=-\mathrm{PI}+\mathrm{G \Lambda}$

401 IF(T.LT.-PI) GO TO 402

ILN $=\mathrm{RI} * \mathrm{SIN}(\mathrm{T})$

$\mathrm{VCN}=\mathrm{R} I{ }^{*} \mathrm{COS}(\mathrm{T})+\mathrm{VON}$

WRITE $(7,2000)$ WT, ILN

WRITE $(6,2000)$ WT, VCN,ILN

$\mathrm{T}=\mathrm{T}-\mathrm{GA} / 30$.

$\mathrm{WT}=\mathrm{WT}+\mathrm{G} \Lambda * 180 * \mathrm{WN} / \mathrm{PI} / 30$.

GO TO 401

$402 \mathrm{~T}=\mathrm{PI}$

$\mathbf{Y T}=\mathbf{Y T}+\mathrm{GA}$ 


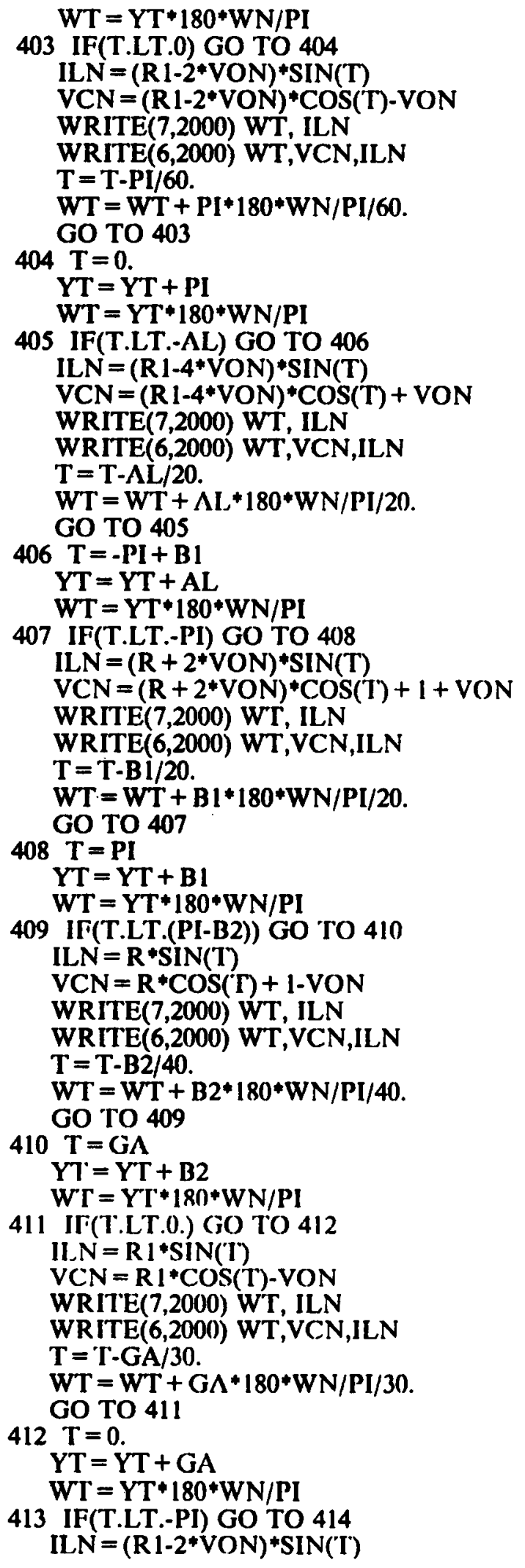


$\mathrm{VCN}=(\mathrm{R} 1-2 * \mathrm{VON}) * \mathrm{COS}(\mathrm{T})+\mathrm{VON}$

WRITE $(7,2000)$ WT, ILN

WRITE $(6,2000)$ WT,VCN,ILN

$\mathrm{T}=\mathrm{T} \cdot \mathrm{PI} / 60$.

$\mathrm{WT}=\mathrm{WT}+\mathrm{PI} * 180 * \mathrm{WN} / \mathrm{PI} / 60$.

GO TO 413

$414 \mathrm{~T}=\mathrm{PI}$

$\mathbf{Y T}=\mathbf{Y T}+\mathbf{P I}$

$\mathrm{WT}=\mathrm{YT} * 180 * \mathrm{WN} / \mathrm{PI}$

415 IF(T.LT.(PI- $\lambda$ L)) GO TO 416

ILN $=(R 1-4 * \mathrm{VON}) * \operatorname{SIN}(\mathrm{T})$

$\mathrm{VCN}=\left(\mathrm{R} 1-4^{*} \mathrm{VON}\right) * \mathrm{COS}(\mathrm{T})-\mathrm{VON}$

WRITE $(7,2000)$ WT, ILN

WRITE $(6,2000)$ WT,VCN,ILN

$\mathrm{T}=\mathrm{T}-\mathrm{AL} / 20$.

$W T=W T+A L * 180 * W N / P I / 20$.

GO TO 415

$416 \mathrm{~T}=\mathrm{B1}$

$Y T=Y T+A L$

$\mathrm{WT}=\mathrm{YT} * 180 * \mathrm{WN} / \mathrm{PI}$

417 IF('T.LT.0.) GO TO 418

ILN $=(\mathrm{R}+2 * \mathrm{VON}) * \mathrm{SIN}(\mathrm{T})$

$\mathrm{VCN}=\left(\mathrm{R}+2^{*} \mathrm{VON}\right) * \mathrm{COS}(\mathrm{T})-1-\mathrm{VON}$

WRITE $(7,2000)$ WT, ILN

WRITE $(6,2000)$ WT,VCN,ILN

$\mathrm{T}=\mathrm{T} \cdot \mathrm{B} \mathbf{1} / 20$.

$\mathrm{WT}=\mathrm{WT}+\mathrm{BI} * 180 * \mathrm{WN} / \mathrm{PI} / 20$.

GO TO 417

$418 \mathrm{~T}=0$.

$Y T=Y T+B 1$

$\mathrm{WT}=\mathrm{YT} * 180^{*} \mathrm{WN} / \mathrm{PI}$

419 IF(T.LT.-B2) GO TO 420

ILN $=R * \operatorname{SIN}(\mathrm{T})$

$\mathrm{VCN}=\mathrm{R} * \operatorname{COS}(\mathrm{T})-1+\mathrm{VON}$

WRITE $(7,2000)$ WT, ILN

WRITE $(6,2000)$ WT,VCN,II,N

$\mathrm{T}=\mathrm{T}-\mathrm{B} 2 / 40$.

$\mathrm{WT}=\mathrm{WT}+\mathrm{B2} * 180 * \mathrm{WN} / \mathrm{PI} / 40$.

GO TO 419

$420 \mathrm{~T}=-\mathrm{PI}+\mathrm{GA}$

$\mathrm{YT}=\mathrm{YT}+\mathrm{B} 2$

$\mathrm{WT}=\mathrm{YT} * 180 * \mathrm{WN} / \mathrm{PI}$

421 IF(T.LT.-PI) GO TO 10

ILN $=$ R $1 *$ SIN $(T)$

$\mathrm{VCN}=\mathrm{R} 1{ }^{*} \mathrm{COS}(\mathrm{T})+\mathrm{VON}$

WRITE(7,2000) WT, ILN

WRI'TE $(6,2000)$ WT, VCN,ILN

$\mathrm{T}=\mathrm{T}-\mathrm{G} \Lambda / 30$.

$W T=W T+G \Lambda * 180 * W N / P I / 30$. GO TO 421

$500 \mathrm{R}=\mathrm{X}(1)$

$\mathrm{FI}=\mathrm{X}(2)$

$\mathbf{A L}=\mathrm{X}(3)$

$\mathrm{T}=-\mathrm{PI}+\mathrm{AL}$

501 IF(T.LT.-PI) GO TO 502

$\mathrm{ILN}=(1-\mathrm{R}+2 * \mathrm{VON}) * \mathrm{SIN}(\mathrm{T})$

$\mathrm{VCN}=(1-\mathrm{R}+2 * \mathrm{VON}) * \mathrm{COS}(\mathrm{T})+\mathrm{VON}$

WRITE $(7,2000)$ WT, IL,N 
WRITE $(6,2000)$ WT,VCN,ILN

$\mathrm{T}=\mathrm{T}-\mathrm{AL} / 30$.

$W T=W T+A L * 180 * W N / P I / 30$.

GO TO 501

$502 \mathrm{~T}=\mathrm{PI}$

$\mathrm{YT}=\mathrm{YT}+\mathrm{AL}$

$\mathrm{WT}=\mathrm{YT} * 180 * \mathrm{WN} / \mathrm{PI}$

503 IF(T.LT.0) GO TO 504

ILN $=(1-R) * \operatorname{SIN}(T)$

$\mathrm{VCN}=(1-\mathrm{R}) * \mathrm{COS}(\mathrm{T})-\mathrm{VON}$

WRITE $(7,2000)$ WT, ILN

WRITE $(6,2000)$ WT,VCN,ILN

$\mathrm{T}=\mathrm{T}-\mathrm{PI} / 50$.

$\mathrm{WT}=\mathrm{WT}+\mathrm{PI} * 180 * \mathrm{WN} / \mathrm{PI} / 50$.

GO TO 503

$504 \mathrm{~T}=\mathrm{FI}$

$\mathrm{YT}=\mathrm{YT}+\mathrm{PI}$

$\mathrm{WT}=\mathrm{YT} * 180 * \mathrm{WN} / \mathrm{PI}$

505 IF(T.LT.0.) GO TO 506

ILN $=0$.

$\mathrm{VCN}=1-\mathrm{R}-\mathrm{VON}$

WRITE $(7,2000)$ WT, ILN

WRITE $(6,2000)$ WT,VCN,ILN

$\mathrm{T}=\mathrm{T} \cdot \mathrm{FI} / \mathbf{3 0}$.

$\mathrm{WT}=\mathrm{WT}+\mathrm{FI} I+180 * \mathrm{WN} / \mathrm{PI} / 30$.

GO TO 505

$506 \mathrm{~T}=\mathrm{PI}$

$Y T=Y T+F I$

$\mathrm{WT}=\mathrm{YT} * 180^{*} \mathrm{WN} / \mathrm{PI}$

507 IF(T.LT.(PI-BT)) GO TO 508

ILN $=R * \operatorname{SIN}(T)$

$\mathrm{VCN}=\mathrm{R} * \mathrm{COS}(\mathrm{T})+1-\mathrm{VON}$

WRITE(7,2000) WT, ILN

WRITE $(6,2000)$ WT, VCN,ILN

$\mathrm{T}=\mathrm{T} \cdot \mathrm{BT} / 30$.

$W \Gamma=W T+B^{\prime} \Gamma * 180 * W N / P I / 30$.

GO TO 507

$508 \mathrm{~T}=\mathrm{AL}$

$\mathbf{Y T}=\mathbf{Y T}+\mathbf{B T}$

$\mathrm{WT}=\mathrm{YT} * 180 * \mathrm{WN} / \mathrm{PI}$

509 IF(T.LT.0.) GO TO 510

ILN $=(1-R+2 *$ VON $) *$ SIN (T)

$\mathrm{VCN}=(1-\mathrm{R}+2 * \mathrm{VON}) * \mathrm{COS}(\mathrm{T})-\mathrm{VON}$

WRITE $(7,2000)$ WT, ILN

WRITE( $(6,2000)$ W'T,VCN,ILN

$\mathrm{T}=\mathrm{T}-\Lambda \mathrm{L} / 30$.

$\mathrm{WT}=\mathrm{WT}+\Lambda \mathrm{L} * 180 * \mathrm{WN} / \mathrm{PI} / 30$.

GO TO 509

$510 \mathrm{~T}=0$.

$\mathbf{Y T}=\mathbf{Y T}+\mathbf{A L}$

$\mathrm{WT}=\mathrm{YT} * 180 * \mathrm{WN} / \mathrm{PI}$

511 IF(T.LT.-PI) GO TO 512

ILN $=(1-R)^{*} \operatorname{SIN}(T)$

$\mathrm{VCN}=(1-\mathrm{R})+\operatorname{COS}(\mathrm{T})+\mathrm{VON}$

WRITE $(7,2000)$ WT, ILN

WRITE $(6,2000)$ WT,VCN,IL,N

$\mathrm{T}=\mathrm{T}-\mathrm{PI} / 50$.

$\mathrm{WT}=\mathrm{WT}+\mathrm{PI} * 180 * \mathrm{WN} / \mathrm{PI} / 50$. 


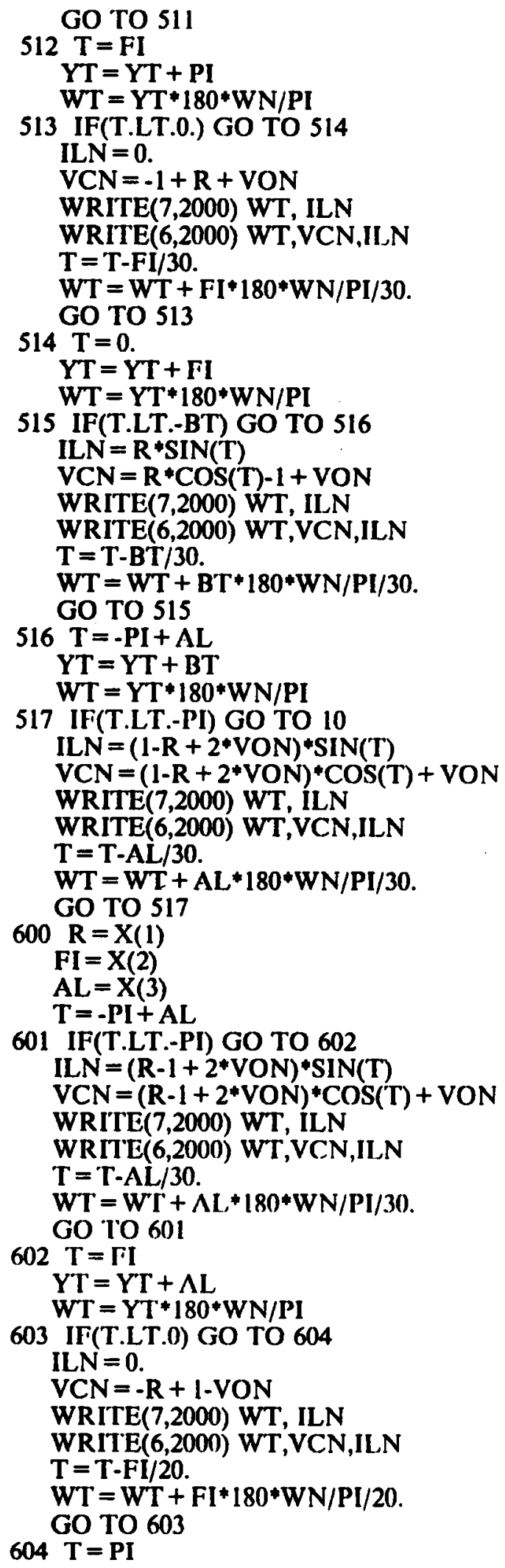




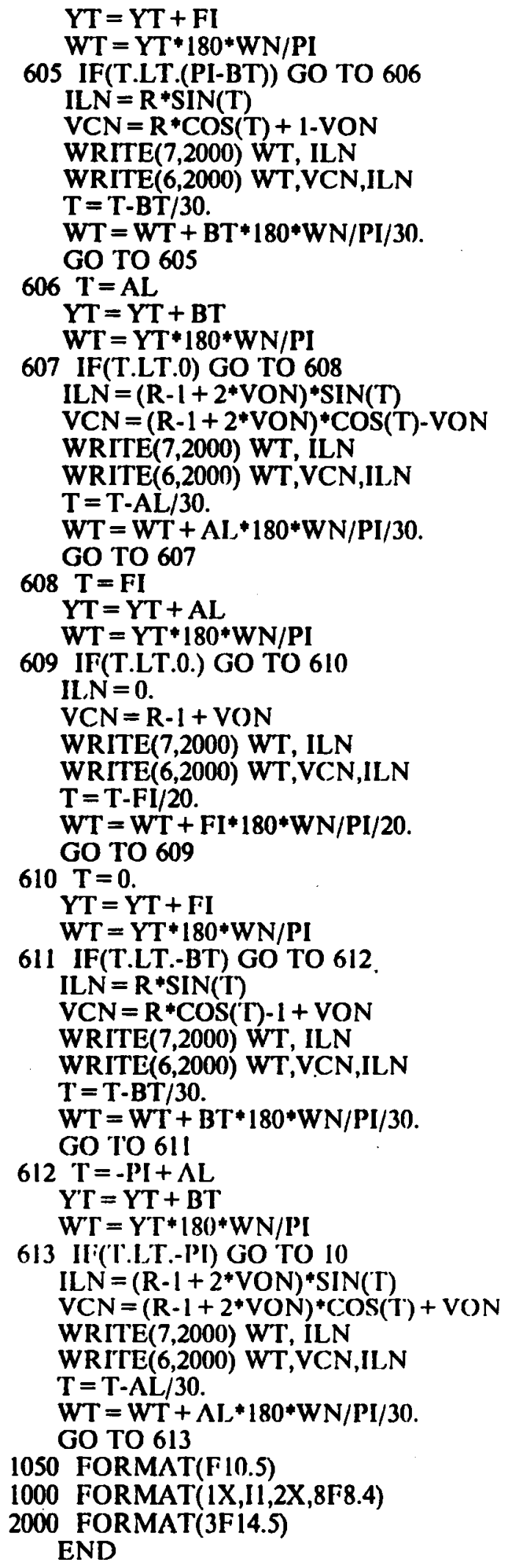


Fortran Program for Generating Capacitor Voltage and Inductor Current Waveforms (Above Resonant Frequency)

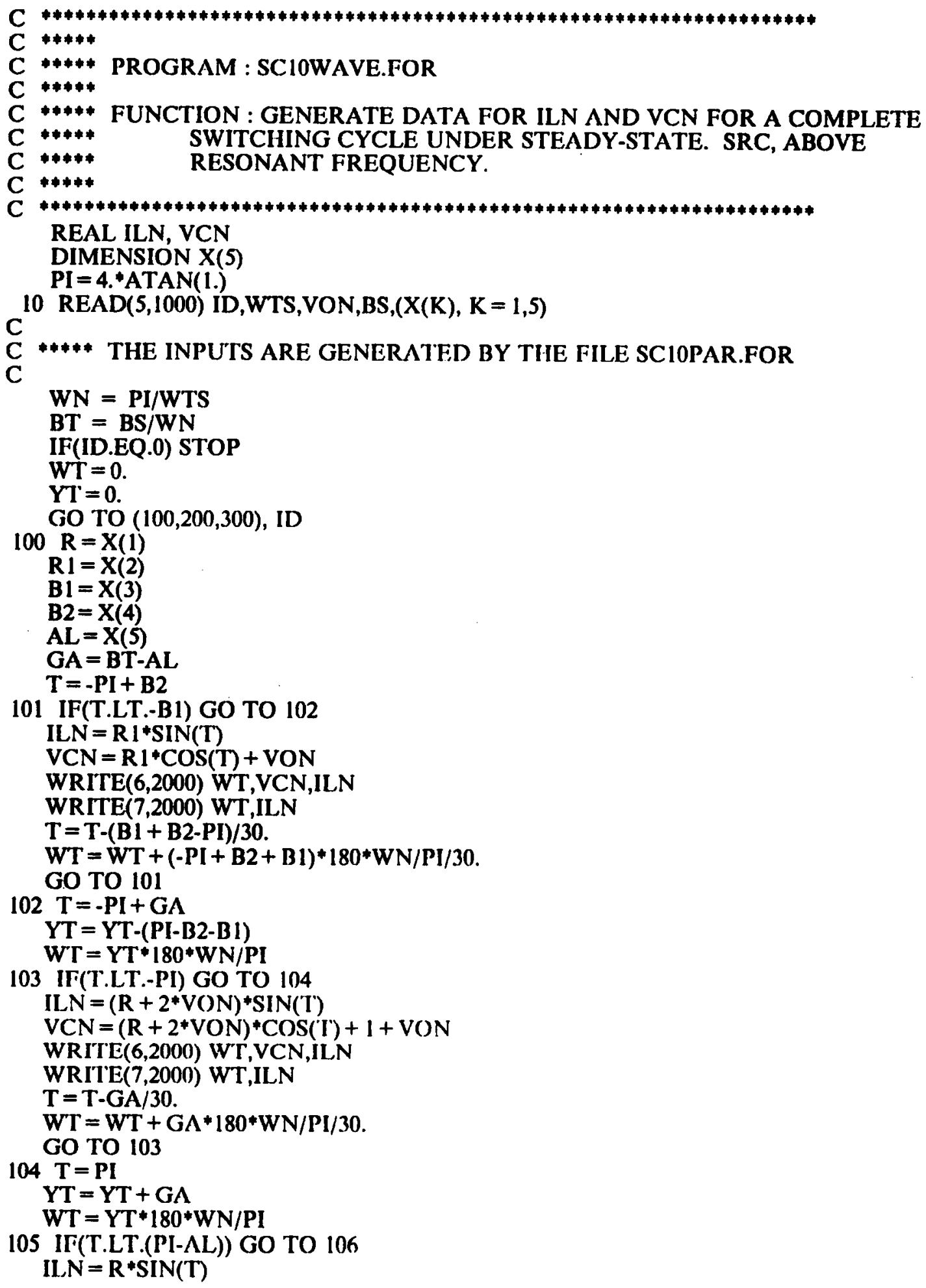




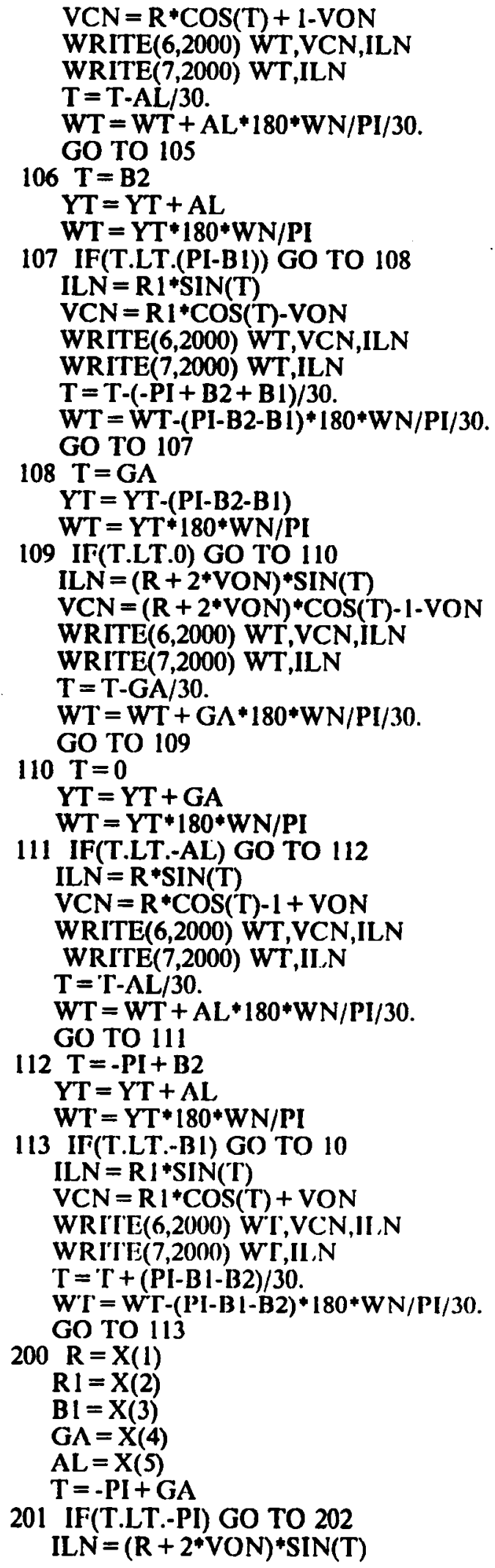


$\mathrm{VCN}=(\mathrm{R}+2 * \mathrm{VON}) * \mathrm{COS}(\mathrm{T})+\mathrm{VON}$

WRITE $(6,2000)$ WT,VCN,ILN

WRITE $(7,2000)$ WT,ILN

$\mathrm{T}=\mathrm{T} \cdot \mathrm{GA} / \mathbf{3 0}$.

$\mathrm{WT}=\mathrm{WT}+\mathrm{GA} * 180 * \mathrm{WN} / \mathrm{PI} / 30$.

GO TO 201

$202 \mathrm{~T}=\mathrm{PI}$

$Y T=Y T+G A$

$W T=Y T * 180 * W N / P I$

203 IF(T.LT.(PI-AL)) GO TO 204

ILN $=\mathbf{R} * \operatorname{SIN}(T)$

$\mathrm{VCN}=\mathrm{R} * \operatorname{COS}(\mathrm{T})-\mathrm{VON}$

WRITE $(6,2000)$ WT,VCN,ILN

WRITE $(7,2000)$ WT,ILN

$\mathrm{T}=\mathrm{T}-\mathrm{AL} / 30$.

$W T=W T+\Lambda L * 180 * W N / P I / 30$.

GO TO 203

$204 \mathrm{~T}=\mathrm{PI}-\mathrm{BI}$

$\mathrm{YT}=\mathrm{YT}+\mathrm{AL}$

$\mathrm{WT}=\mathrm{YT} * 180 * \mathrm{WN} / \mathrm{PI}$

205 IF(T.LT.(PI-B1-BT)) GO TO 206

ILN $=\mathrm{R} 1 *$ SIN $(\mathrm{T})$

$\mathrm{VCN}=\mathrm{R} 1 * \mathrm{COS}(\mathrm{T})+1-\mathrm{VON}$

WRITE $(6,2000)$ WT, VCN,ILN

WRITE $(7,2000)$ WT,ILN

$\mathrm{T}=\mathrm{T} \cdot \mathrm{BT} / 30$.

$\mathrm{WT}=\mathrm{WT}+\mathrm{BT} * 180 * \mathrm{WN} / \mathrm{PI} / 30$.

GO TO 205

$206 \mathrm{~T}=\mathrm{GA}$

$Y T=Y T+B T$

$W T=Y T * 180 * W N / P I$

207 IF(T.LT.0) GO TO 208

$\mathrm{ILN}=(\mathrm{R}+2 * \mathrm{VON}) * \mathrm{SIN}(\mathrm{T})$

$\mathrm{VCN}=(\mathrm{R}+2 * \mathrm{VON}) * \mathrm{COS}(\mathrm{I})-\mathrm{VON}$

WRITE $(6,2000)$ WT,VCN,ILN

WRITE(7,2000) WT,ILN

$\mathrm{T}=\mathrm{T} \cdot \mathrm{GA} / 30$.

$W T=W T+G \Lambda * 180 * W N / P I / 30$.

GO TO 207

$208 \mathrm{~T}=0$.

$\mathbf{Y T}=\mathbf{Y T}+\mathrm{G \Lambda}$

$\mathrm{WT}=\mathrm{YT} * 180 * \mathrm{WN} / \mathrm{PI}$

209 IF(T.LT.-AL) GO TO 210

ILN $=R * \operatorname{SIN}(T)$

$\mathrm{VCN}=\mathrm{R} * \mathrm{COS}(\mathrm{T})+\mathrm{VON}$

WRITE $(6,2000)$ WT,VCN,ILN

WRITE $(7,2000)$ WT,II.N

$\mathrm{T}=\mathrm{T} \cdot \mathbf{-} \mathbf{\Lambda} / 30$.

$\mathrm{WT}=\mathrm{WT}+\Lambda \mathrm{L} * 180 * \mathrm{WN} / \mathrm{PI} / 30$.

GO TO 209

$210 \mathrm{~T}=-\mathrm{Bl}$

$\mathbf{Y T}=\mathbf{Y T}+\Lambda \mathbf{L}$

$\mathrm{WT}=\mathrm{YT} * 180 * \mathrm{WN} / \mathrm{PI}$

211 IF(T.LT.(-B1-BT)) GO TO 212

ILN $=$ RI*SIN(T)

$\mathrm{VCN}=\mathrm{R} 1{ }^{*} \mathrm{COS}(\mathrm{T})-1+\mathrm{VON}$

WRITE $(6,2000)$ WT,VCN,II,N

WRITE $(7,2000)$ WT,ILN 


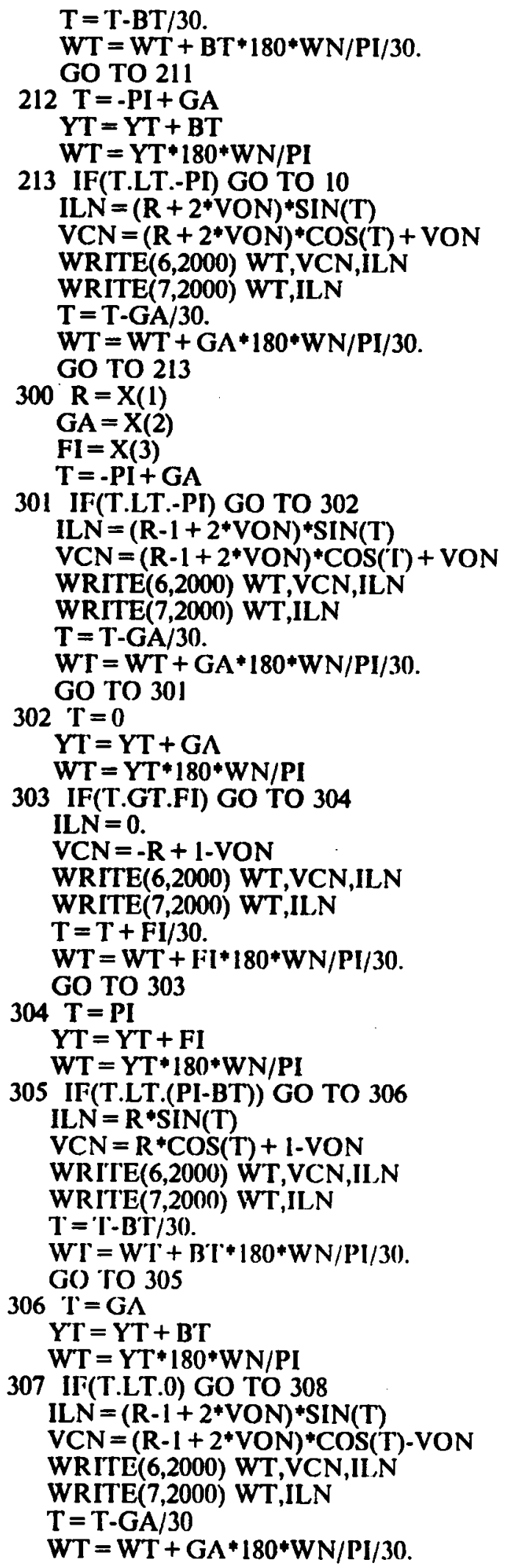




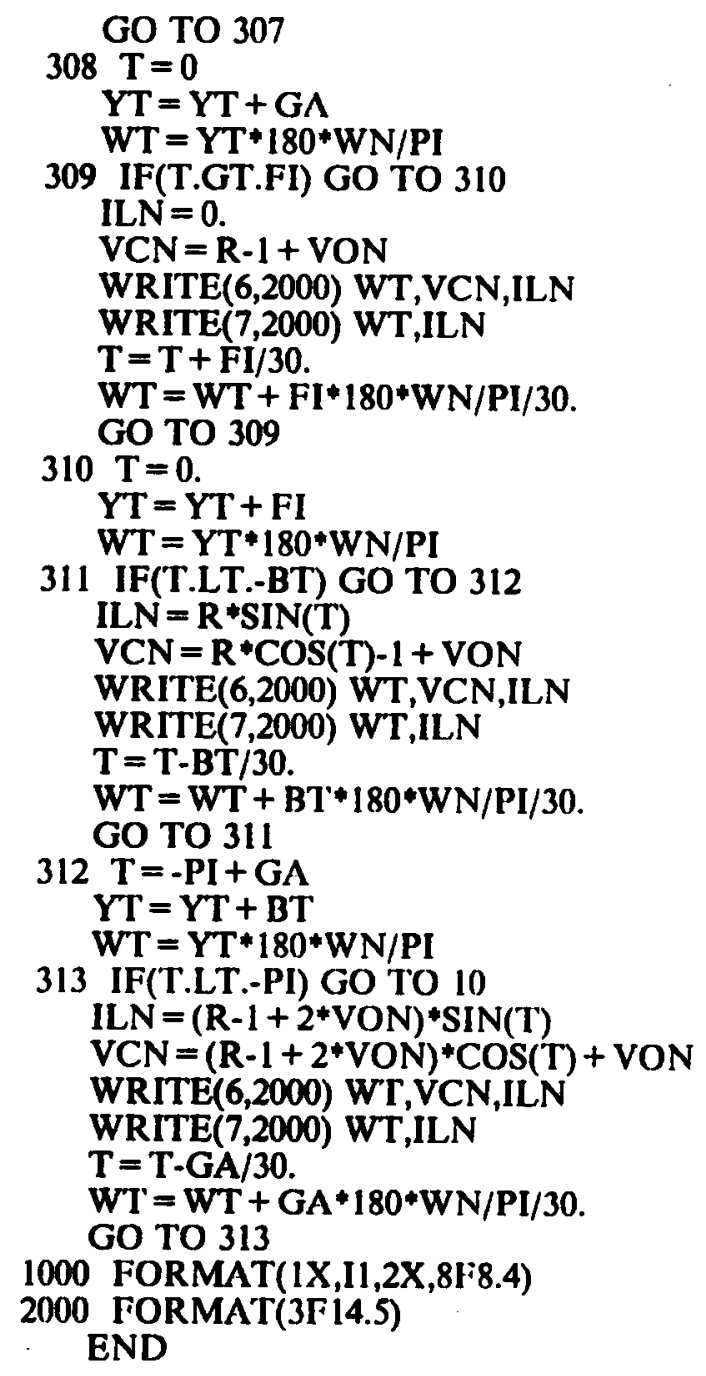




\section{APPENDIX C.1 \\ RULES FOR CONSTRUCTING EQUILIBRIUM STATE TRAJECTORIES OF A CM-PRC}

An equilibrium state trajectory constructed in the composite diagram discussed in Section 3.3.4 must satisfy the following rules. These rules are obtained from the converter's operation.

- On the left half plane $\left(v_{C N}<0\right)$,

- triggering of Q1 initiates M6;

- triggering of $\mathrm{Q} 2$ initiates $\mathrm{M1}$;

- $\quad$ triggering of Q4 initiates M5.

- On the right half plane $\left(v_{C N}>0\right)$,

- triggering of $\mathrm{Q}^{3}$ initiates $\mathrm{M} 3$;

- $\quad$ triggering of Q4 initiates $M 4$;

- $\quad$ triggering of Q2 initiates $\mathrm{M} 2$.

- On the $i_{L N^{-}}$axis $\left(v_{C N}=0\right)$,

- if the magnitude of $i_{L N}$ is greater than $l_{O N}$, 
- triggering of $\mathrm{Q} 2$ initiates $\mathrm{M} 2$;

- triggering of Q4 initiates M5;

- if the magnitude of $i_{L N}$ is greater than $I_{O N}$ and no triggering event occurs,

- M2 is initiated if the previous topological mode is M1;

- M3 is initiated if the previous topological mode is M6;

- M5 is initiated if the previous topological mode is M4;

- M6 is initiated if the previous topological mode is M3;

- if the magnitude of $i_{L N}$ is equal to $I_{O N}$,

- M0 is initiated if the previous topological mode is M3 or M6; M0 will be terminated when $\mathrm{Q} 2$ or $\mathrm{Q} 4$ is triggered which initiates $\mathrm{M} 2$ or $\mathrm{M} 5$, respectively;

- M2 is initiated if the previous topological mode is $M L$ and $i_{L N}>0$;

- M5 is initiated if the previous topological mode is $M L$ and $i_{L N}<0$;

- if the magnitude of $i_{L N}$ is less than $I_{O N}$,

- M0 is initiated if the previous topological mode is M3 or M6; M0 will be terminated when $\mathrm{Q} 2$ or $\mathrm{Q} 4$ is triggered which initiates $\mathrm{ML}$;

- ML is initiated if previous topological mode is M1 or M4. 
$\Lambda$ systematic way to construct equilibrium trajectories of a CM-PRC is illustrated in Figure C.1.1. Starting with an equilibrium trajectory $\left(a_{0} s c_{0} t a_{0}\right.$ in Figure C.1.1(a) or $a_{0} s s^{\prime} c_{0} t t^{\prime} a_{0}$ in Figure C.1.1(b)) corresponding to conventional PRC operation, the triggering instant of $\mathrm{Ql}(\mathrm{Q} 3), a_{0}, a_{1}, a_{2}, \ldots\left(c_{0}, c_{1}, c_{2}, \ldots\right)$, is gradually moved counterclockwise along the trajectory of M5(M2). By doing so, the effective duty ratio of the quasi-square-wave voltage is gradually reduced and various equilibrium trajectories can be obtained.

In Figure C.1.1(a), trajectory $a_{1} b_{1} s c_{1} d_{1} t a_{1}$ corresponds to a mode- $I_{N}$ operation. Trajectory $a_{2} b_{2} c_{2} d_{2} a_{2}$ corresponds to a mode-IIN operation. Trajectory $a_{4} b_{4} c_{4} d_{4} a_{4}$ corresponds to a mode- $I I_{s}$ operation. Trajectory $a_{5} b_{5} s c_{5} d_{5} t a_{5}$ corresponds to a mode$I_{S}$ operation. Trajectory $a_{7} b_{7} s c_{7} d_{7} t a_{7}$ corresponds to a mode- $I_{F}$ operation.

In Figure C.1.1(b), trajectory $a_{1} b_{1} s s^{\prime} c_{1} d_{1} t t^{\prime} a_{1}$ corresponds to a mode- $I I I_{N}$ operation. Trajectory $a_{2} b_{2} s^{\prime} c_{2} d_{2} t^{\prime} a_{2}$ corresponds to a mode- $I V_{N}$ operation. Trajectory $a_{6} b_{6} s^{\prime} c_{6} d_{6} t^{\prime} a_{6}$ corresponds to a mode- $I V_{s}$ operation. Trajectory $a_{7} b_{7} s s^{\prime} c_{7} d_{7} t t^{\prime} a_{7}$ corresponds to a mode-IIII $I_{S}$ operation. Trajectory $a_{9} b_{9} s s^{\prime} c_{9} d_{9} t t^{\prime} a_{9}$ corresponds to a mode$I I I_{F}$ operation. Trajectory $a_{10} b_{10} s^{\prime} c_{10} d_{10} t^{\prime} a_{10}$ corresponds to a mode- $I V_{F}$ operation.

Figure C.1.2 shows the construction of equilibrium trajectories above resonant frequency. In Figure C.1.2(a), trajectory $a_{1} b_{1} s c_{1} d_{1} t a_{1}$ represents a mode- $I_{F}$ operation. Trajectory $a_{1} b_{1}, s c_{1} d_{1}, t a_{1}$ represents a mode- $I_{S}$ operation. Trajectory $a_{2} b_{2} c_{2} d_{2} a_{2}$ represents a mode- $I I A_{F}$ operation. Trajectory $a_{2} b_{2} c_{2} d_{2} a_{2}$ represents a mode- $I I A_{S}$ operation.

In Figurc C.1.2(b), trajectory $a_{1} b_{1} s s^{\prime} c_{1} d_{1} t t^{\prime} a_{1}$ represents a mode- $I I I_{F}$ operation. Trajectory $a_{2} b_{2} s^{\prime} c_{2} d_{2} t^{\prime} a_{2}$ represents a mode- $I V_{F}$ operation. It can be shown, however, that mode-IV operation cannot exist at frequencies above resonant frequency. 

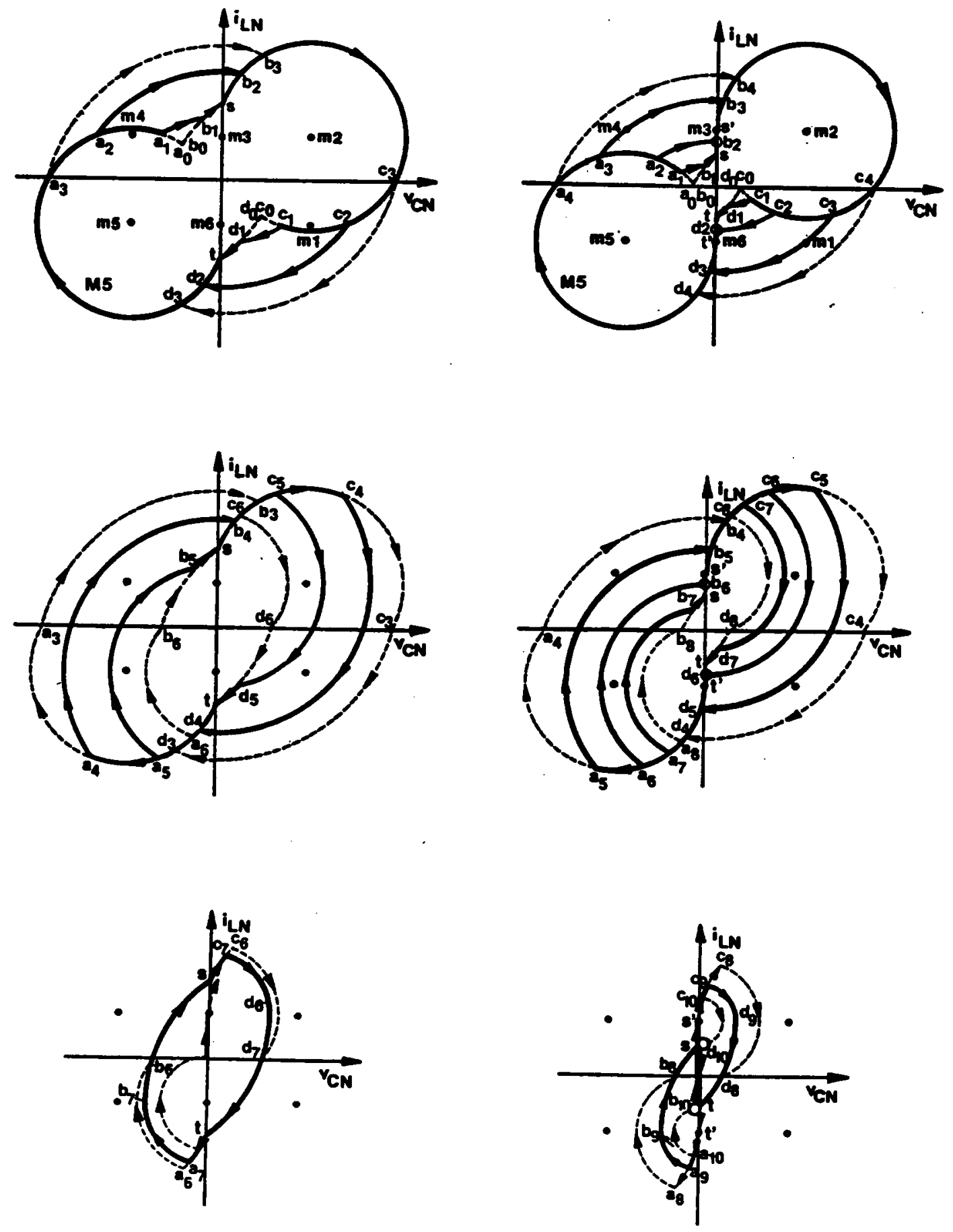

(a) Trajectories for continuous $v_{C N}$

(b) Trajectories for discontinuous $v_{C N}$

Figure C.1.1 Systematical Construction of Equilibrium Trajectories Below Resonant Frequency 


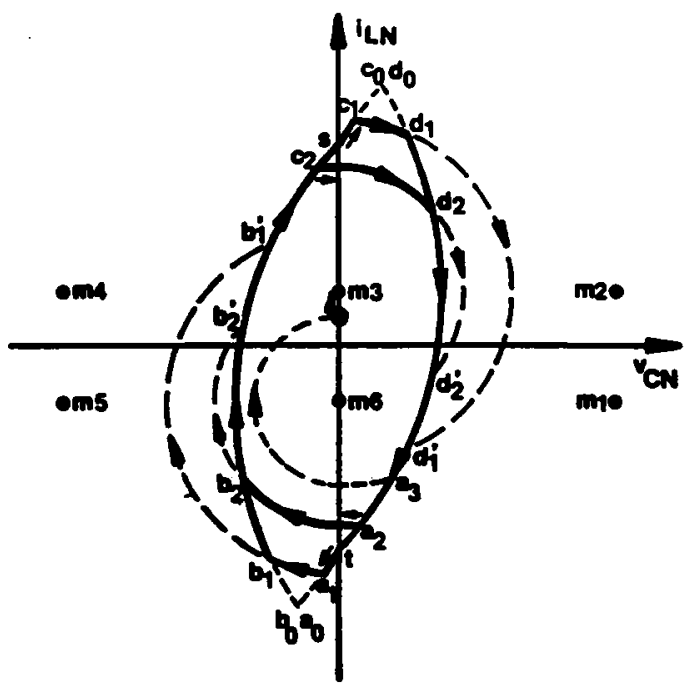

(a) Trajectories for continuous $v_{C N}$

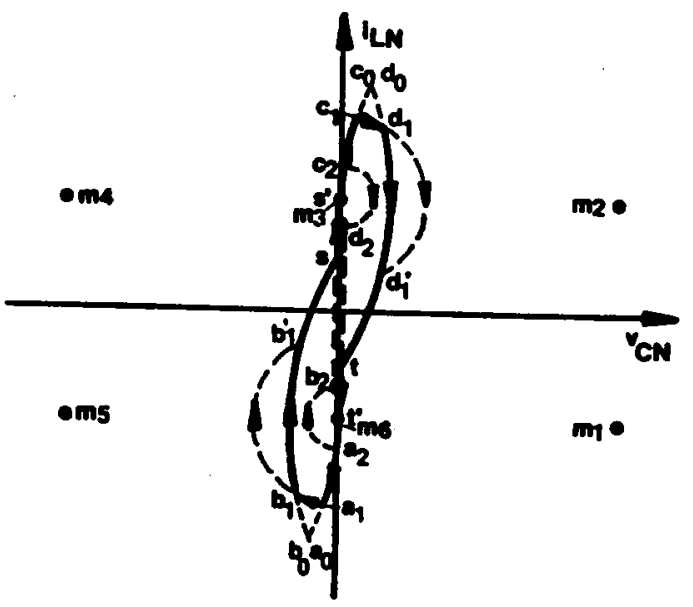

(b) Trajectories for discontinuous $v_{C N}$ Figure C.1.2 Systematical Construction of Equilibrium Trajectories
Above Resonant Frequency 


\section{APPENDIX C.2}

\section{PREDICTION OF MODE TRANSITIONS OF A CM-PRC OPERATING BELOW RESONANT FREQUENCY}

Assume the waveform for the capacitor voltage is continuous when $\beta_{S}$ is equal to $180^{\circ}$ (PRC operation). This requires $[\ldots]$

$$
I_{O N} \leq-0.5 \times \sin \left(\frac{\pi}{\omega_{S N}}\right) \times \sqrt{\frac{1+\left(1-2\left(\cos \left(\frac{\pi}{\omega_{S N}}\right)-1\right)\right)}{\sin ^{2}\left(\frac{\pi}{\omega_{S N}}\right)}}
$$

$\Lambda s \beta_{S}$ decreases, the first operating mode for the converter is mode $\mathbf{I}$.

Further decreasing $\beta_{S}$, the converter's operation may transit into mode II or mode III. Consider the boundary trajectories, $T_{A 12}$ and $T_{R 12}$, between mode I and mode II, as shown in Figure C.2.1. These trajectories can be represented by the equations

$$
\left[\begin{array}{l}
\cos \rho=\frac{1}{R}, \\
\cos \sigma=\frac{1+R^{2}-R_{1}^{2}}{2 R} \\
\cos \gamma=\frac{1+R_{1}^{2}-R^{2}}{2 R_{1}} \\
\frac{\pi}{\omega_{S N}}=\sigma-\rho+\frac{\pi}{2}-\gamma \\
R_{1}=2 I_{O . V}+\sqrt{R^{2}-1}
\end{array}\right.
$$

and can be constructed only when $R+1 \geq R_{1}$ which leads to

$$
2 I_{O N} \leq R_{1} \leq R_{1 \max }, \quad R_{1 \max }=\left[\begin{array}{ll}
\text { infinite } & \text { when } I_{O N} \leq 0.5 \\
\frac{2 I_{O N}^{2}}{2 I_{O N}-1} & \text { when } I_{O N}>0.5
\end{array}\right.
$$


The corresponding frequency to these trajectories are shown in Figure C.2.2 as a function of radius $R$, where

$$
\begin{array}{ll}
\omega_{1}=\frac{\pi}{\pi+\sigma / 2}, & \sigma=\cos ^{-1}\left(1-2 I_{O N}^{2}\right), \\
\omega_{2}=\frac{\pi}{\pi+\sigma / 2}, & \sigma=2 \pi-\cos ^{-1}\left(1-2 I_{O N}^{2}\right),
\end{array}
$$

$\omega_{3}$ is the frequency occurs at $R_{\max }=R_{1 \max }-1$, and $\omega_{H}$ is the highest possible frequency which can be numerically determined. If we define

$$
\omega_{12 \max }=\left[\begin{array}{cc}
1 & \text { for } I_{O N} \leq 0.5 \\
\omega_{H} & \text { for } I_{O N}>0.5
\end{array}\right.
$$

from Figure C.2.2, the transition of the operating mode can be determined as follows.

(a). If $\omega_{12 \max }<\omega_{S N}$, the converter's operation transits form mode I to mode III since there is no boundary trajectory exists between mode I and mode II. The boundary $\beta_{S}$ angle, $\beta_{13}$, between mode $I$ and mode III can be calculated by solving equation C.2.7, $\beta_{13}=\left(\alpha_{2}-\alpha_{1}+\sigma\right) \times \omega_{S N}$.

(b). If $\omega_{1} \leq \omega_{S N}<\omega_{12 \max }$, the converter's operation transits form mode I to mode II back to mode I to mode III since two houndary trajectories between mode I and mode II exist. Two sets of solutions, $\left(R, R_{1}, \sigma, \rho, \gamma\right)$ and $\left(R^{\prime}, R_{1}{ }^{\prime}, \sigma^{\prime}, \rho^{\prime}, \gamma^{\prime}\right)$, can be obtained when equation C.2.2 is solved. The boundary $\beta_{S}$ angles between mode I and mode II are $\beta_{121}=(\sigma-\rho) \times \omega_{S N}$ and $\beta_{12 R}=\left(\sigma^{\prime}-\rho^{\prime}\right) \times \omega_{S N}$. The boundary $\beta_{S N}$ angle between mode I and mode III is calculated as in (a).

(c). If $\omega_{2} \leq \omega_{S N}<\omega_{1}$, the converter's operation transits from mode I to mode II to mode IV, since there is one boundary trajectory between mode I and mode II exists. The transition from mode II to mode IV can be easily justified since, besides mode I, 
mode IV is the only possile operating mode for the converter to transit into from mode II. One solution, $\left(R, R_{1}, \sigma, \rho, \gamma\right)$, for equation C.2.2 exists. The boundary $\beta_{S}$ angle between mode I and mode II is $\beta_{12}=(\sigma-\rho) \times \omega_{S N}$. The boundary $\beta_{S}$ angle between mode II and mode IV is $\beta_{24}=\sigma \times \omega_{S N}, \sigma=\cos ^{-1}\left(1-2 I_{O_{N}}^{2}\right)$, which is calculated from Figure C.2.5(b).

(d). If $\omega_{S N}<\omega_{2}$, the converter's operation transits form mode I to mode III, since there is no boundary trajectory exists between mode I and mode II. The boundary $\beta_{S}$ angle, $\beta_{13}$, between mode I and mode III is calculated as in $(a)$.

Before proceeding to determine the mode transitions as $\beta_{S}$ is further decreased, consider the boundary trajectories, $T_{A 34}$ and $T_{B 34}$, between mode III and Mode IV, as shown in Figure C.2.3. These trajectories can be described by equations

$$
\left[\begin{array}{l}
\cos \sigma=\frac{2-R^{2}}{2}, \\
\ell=2 I_{O N}-R, \\
\frac{\pi}{\omega_{S N}}=\pi+\frac{\sigma}{2}+\ell .
\end{array}\right.
$$

The corresponding frequency to these trajectories are shown in Figure C.2.4 as a function of radius $R$, where

$$
\begin{gathered}
\omega_{L A}=\frac{\pi}{2 \pi+2 I_{O N}}, \\
\omega_{I B}=\frac{\pi}{\pi+2 I_{O N}}, \\
\omega_{M}=\left[\begin{array}{cc}
\omega_{1} & \text { for } I_{O N} \leq \frac{\sqrt{3}}{2}, \\
\frac{\pi}{2 I_{O N}+2.4567} & \text { for } I_{O N}>\frac{\sqrt{3}}{2},
\end{array}\right.
\end{gathered}
$$

and $\omega_{1}, \omega_{2}$ are the same as in equation (C.2.3). 
Figure C.2.5 shows the boundary trajectories mode I and mode III, and mode II and mode IV. The houndary trajectory between mode I and mode III can be described by equations

$$
\left[\begin{array}{l}
\cos \sigma=\frac{2-R_{1}^{2}}{2}, \\
\cos \eta=\frac{1+R_{1}^{2}-R_{2}^{2}}{2 R_{1}}, \\
\cos \alpha_{1}=\frac{1+R_{2}^{2}-R_{1}^{2}}{2}, \\
\cos \alpha_{2}=\frac{1}{R_{2}}, \\
-\gamma=\frac{\pi-\sigma}{2}, \\
R_{2}^{2}=1+4 I_{O N}^{2}, \\
\frac{\pi}{\omega_{S N}}=\sigma-\gamma+\pi-\eta+\alpha_{2}-\alpha_{1} .
\end{array}\right.
$$

The boundary trajectory between mode II and mode IV can be described by equations

$$
\left[\begin{array}{l}
\cos \sigma=1-2 I_{O N}^{2} \\
\frac{\pi}{\omega_{S N}}=\pi+\frac{\sigma}{2}+\theta
\end{array}\right.
$$

For $\omega_{S N} \leq \omega_{2}$, two boundary trajectories between mode II and mode IV exist, and one boundary trajectory between mode I and mode III exists.

For $\omega_{2}<\omega_{S N} \leq \omega_{1}$, one boundary trajectory between mode II and mode IV exists, while no boundary trajectory exists between mode I and mode III.

For $\omega_{1}<\omega_{S N}$, no boundary trajectory exists between mode II and mode IV, while one boundary trajectory between mode I and mode III exists.

From Figure C.2.4, as $\beta_{S}$ is further decrcases, the transitions of the converter's operating mode can be determined as follows. 
For the sequence in (a),

(a).l if $\omega_{M} \leq \omega_{S N}$, the converter's operation transits from mode III to mode $\mathrm{V}$ since no boundary trajectory exists between mode III and mode IV; the boundary $\beta_{S}$ angle between mode III and mode $\mathrm{V}$ is $\beta_{35}=2 I_{O N} \times \omega_{S N}$;

(a).2 if $\omega_{M}>\omega_{S N}$, the converter's operation transits from mode III to mode IV back to mode III to mode V since two boundary trajectories between mode III and mode IV exists; two sets of solutions, $(R, \sigma, \ell)$ and $\left(R^{\prime}, \sigma^{\prime}, \ell^{\prime}\right)$, can be obtained from equation (C.2.4); the boundary $\beta_{S}$ angles between mode III and mode IV are $\beta_{34 A}=(\sigma+\ell) \times \omega_{S N}$ and $\beta_{34 B}=\left(\sigma^{\prime}-\ell^{\prime}\right) \times \omega_{S N} ;$ the boundary $\beta_{S N}$ angle between mode III and mode $\mathrm{V}$ is calculated as in (a). $I$.

For the sequence in (b),

(b).l if $\omega_{M} \leq \omega_{S N}$, the converter's operation transits from mode III to mode $\mathrm{V}$ since no boundary trajectory exists between mode III and mode IV; the boundary $\beta_{S}$ angle between mode III and mode $\mathrm{V}$ is calculated as in (a).l;

(b).2 if $\omega_{M}>\omega_{S N}$, the converter's operation transits from mode III to mode IV back to mode III to mode V since two boundary trajectories between mode III and mode IV exists; the boundary $\beta_{S}$ angles between mode III and mode IV, and the boundary $\beta_{S N}$ angle between mode $\mathrm{III}$ and mode $\mathrm{V}$, are calculated as in $(a) .2$.

For the sequence in $(c)$, (c).I if $\omega_{L B} \geq \omega_{2}$ and $\omega_{L B} \leq \omega_{S N}$, the converter's operation transits from mode IV to mode III to mode V since one boundary trajectory between mode III and mode IV exists; the boundary $\beta_{S}$ angle between mode III and mode IV, and the boundary $\beta_{S N}$ angle between mode III and mode V, are calculated as in (a).2; 
(c).2 if $\omega_{L B} \geq \omega_{2}$ and $\omega_{L B}>\omega_{S N}$, the converter's operation transits from mode IV to mode $\mathrm{V}$ since no boundary trajectory exists between mode III and mode IV; the boundary $\beta_{S}$ angle between mode IV and mode $\mathrm{V}$ is $\beta_{45}=2 I_{O N} \times \omega_{S N}$;

(c).3 if $\omega_{L B}<\omega_{2}$, the converter's operation transits from mode IV to mode III to mode $\mathrm{V}$ since one boundary trajectory between mode III and mode IV exists; the boundary $\beta_{s}$ angles are calculated as in $(a) .2$.

The case for the sequence in $(d)$ is special since two boundary trajectories between mode II and mode IV exist.

(d).I If $\omega_{L B} \geq \omega_{2}$, the converter's operation transits from mode III to mode IV to mode II back to mode IV to mode V since one boundary trajectory between mode III and mode IV and two boundary trajectories between mode II and mode IV exist. The boundary $\beta_{S}$ angle between mode III and mode IV is calculated as in (a).2. The boundary $\beta_{S N}$ angle between mode IV and mode $V$ is calculated as in (c).2. The boundary $\beta_{S N}$ angles between mode II and mode IV are $\beta_{24 A}=\omega_{S N} \times \cos ^{-1}\left(1-2 I_{\delta_{N}}^{2}\right)$ and $\beta_{24 B}=\omega_{S N} \times\left(2 \pi-\cos ^{-1}\left(1-2 I_{O N}^{2}\right)\right)$.

(d).2 If $\omega_{L B}<\omega_{2}$ and $\omega_{L B} \leq \omega_{S N}$, the converter's operation transits from mode III to mode IV to mode II back to mode IV to mode III to mode V since two boundary trajectories between mode III and mode IV and two boundary trajectories between mode II and mode IV exist. The boundary $\beta_{S}$ angle between mode III and mode IV and the boundary $\beta_{S N}$ angle between mode III and mode $\mathrm{V}$ is calculated as in (a).2. The boundary $\beta_{S N}$ angles between mode II and mode IV are calculated as in (d).I.

(d).3 If $\omega_{L B}<\omega_{2}$ and $\omega_{L B}>\omega_{S N}$, the converter's operation transits from mode III to mode IV to mode II back to mode IV to mode V since one boundary trajectory between mode III and mode IV and two boundary trajectories between mode II and mode IV exist. The boundary $\beta_{S}$ angles are calculated as in $(d) 1$ 


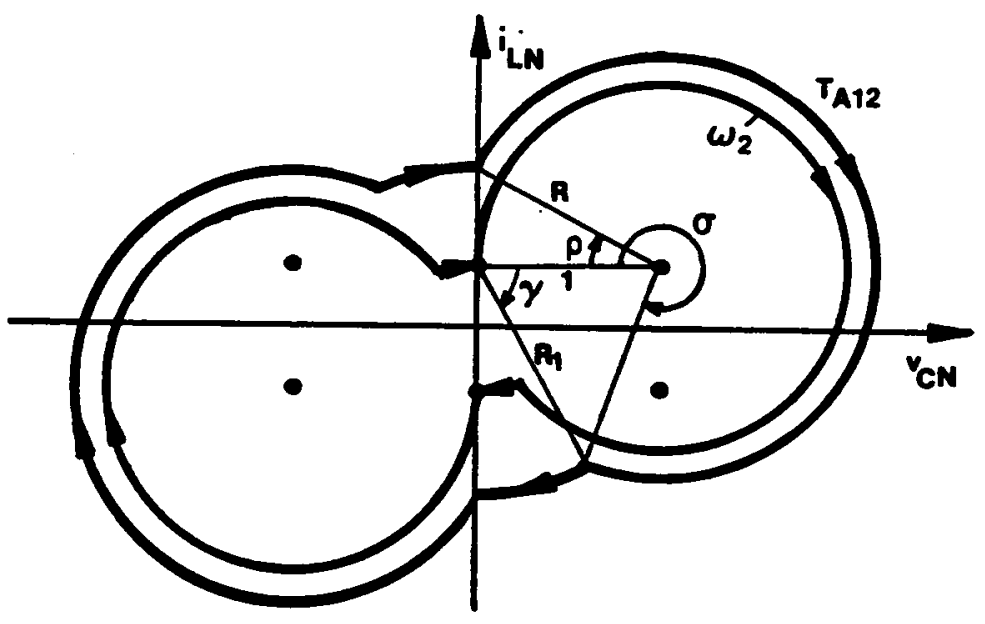

(a) $\sigma \geq \pi(\gamma \geq 0)$

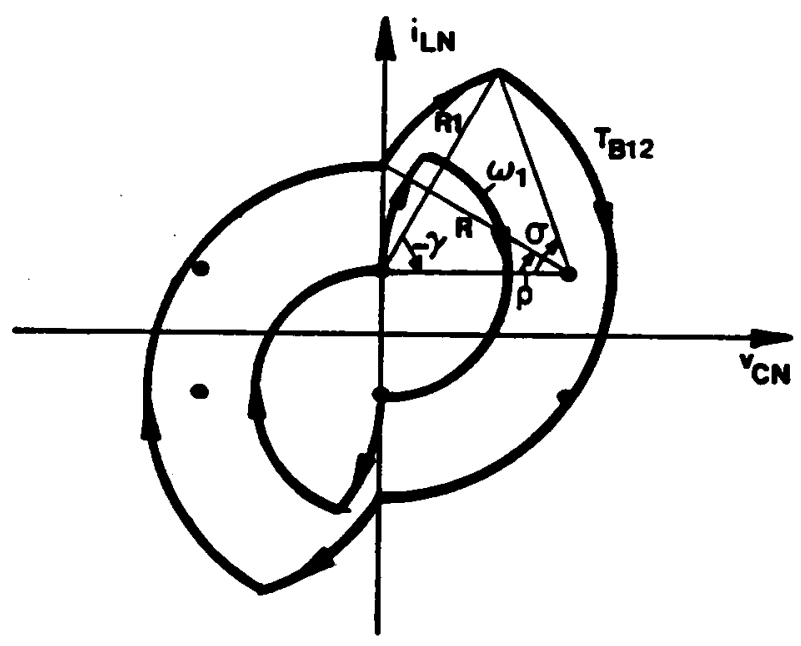

(b) $\sigma<\pi(\gamma<0)$

Figure C.2.1 Boundary Trajectories Between Mode I and Mode II 


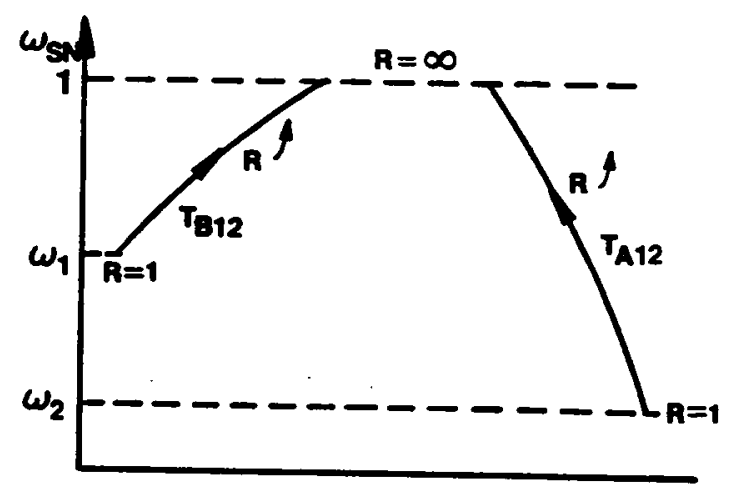

(a) $I_{O N} \leq 0.5$

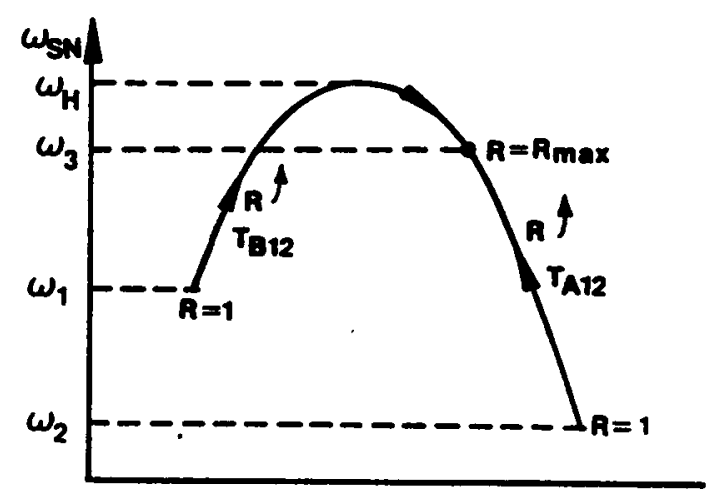

(b) $I_{O N}>0.5$

Figure C.2.2 Corresponding Frequency for the Trajectories in Figure C.2.1 


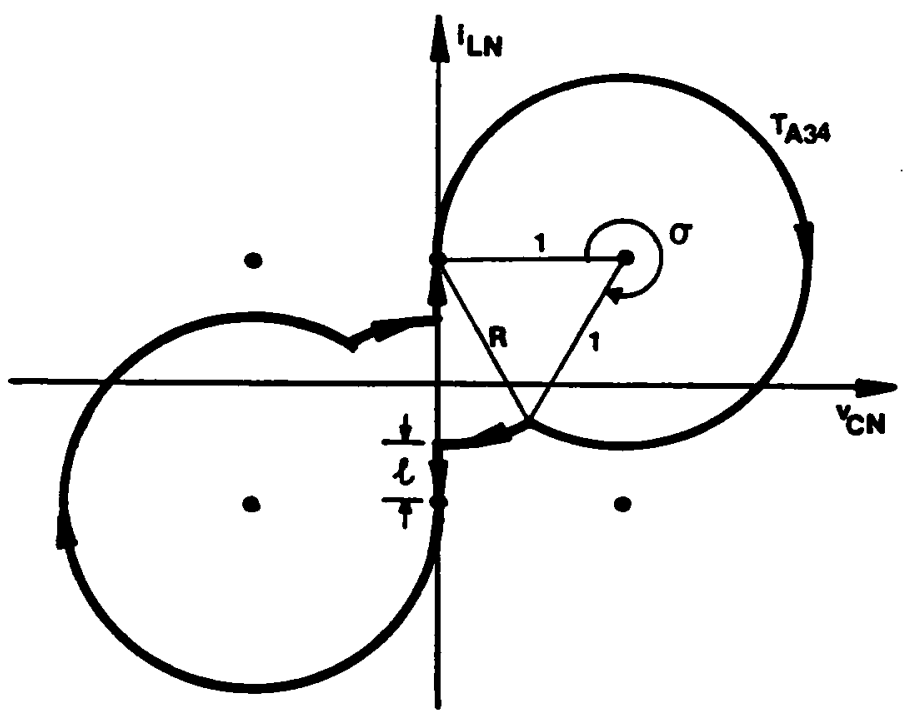

(a) $\sigma \geq \pi$

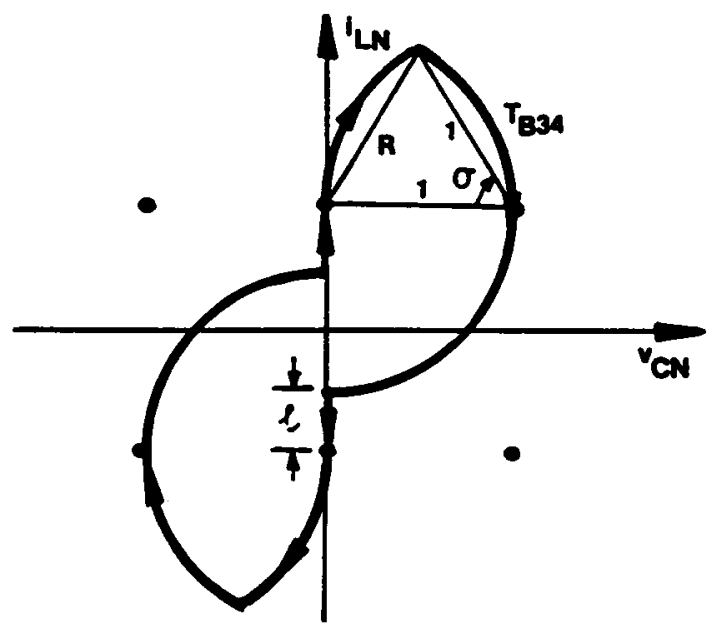

(b) $\sigma<\pi$

Figure C.2.3 Boundary Trajectories Between Mode III and Mode IV 


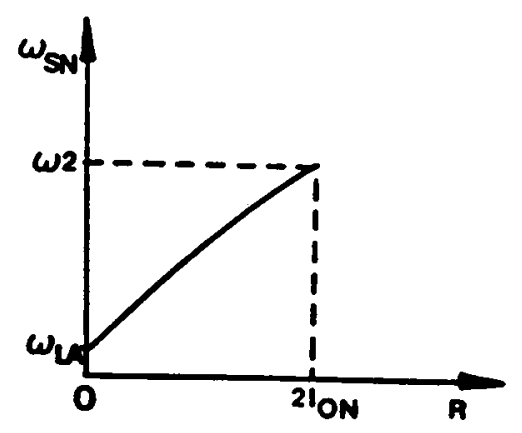

(a) Frequency for $T_{A 34}$
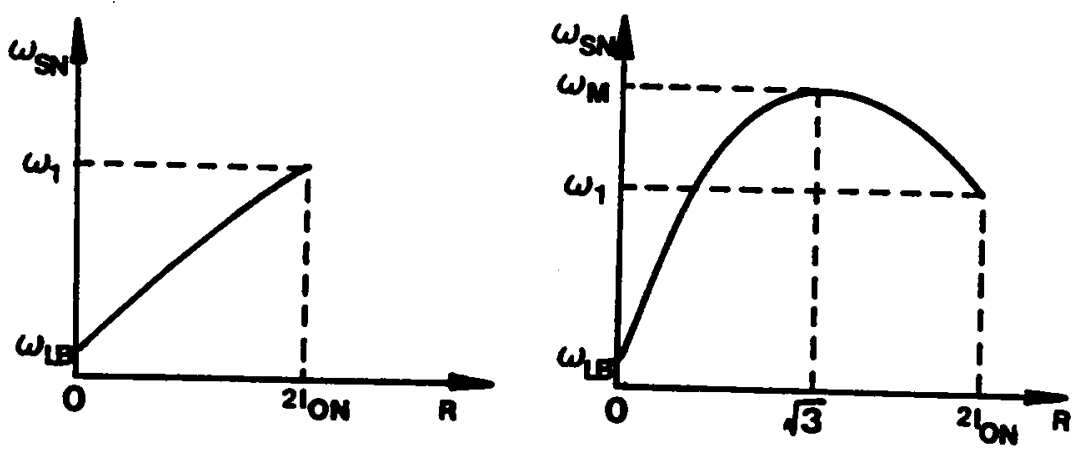

$$
I_{O N} \leq \frac{\sqrt{3}}{2}
$$

$I_{O N}>\frac{\sqrt{3}}{2}$

(b) Frequency for $T_{B 34}$

Figure C.2.4 Corresponding Frequency for the Trajectories in Figure C.2.3

332 


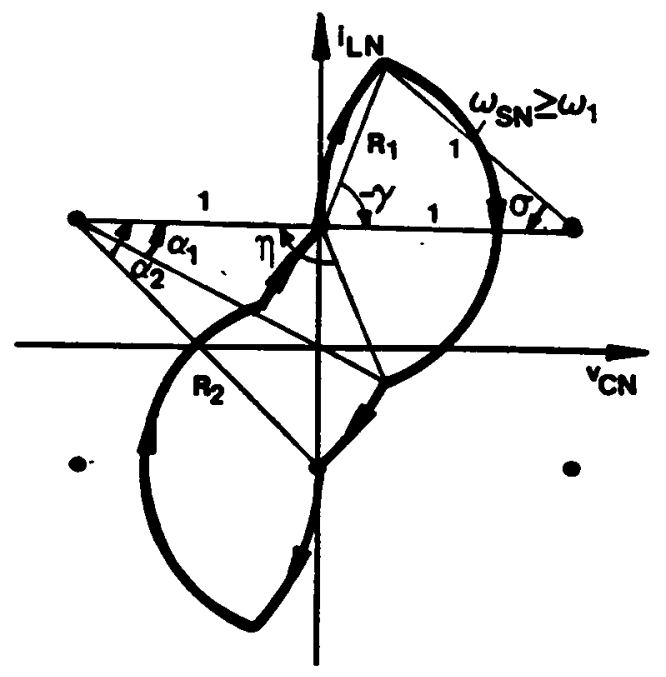

(a) Boundary between Mode I and Mode III

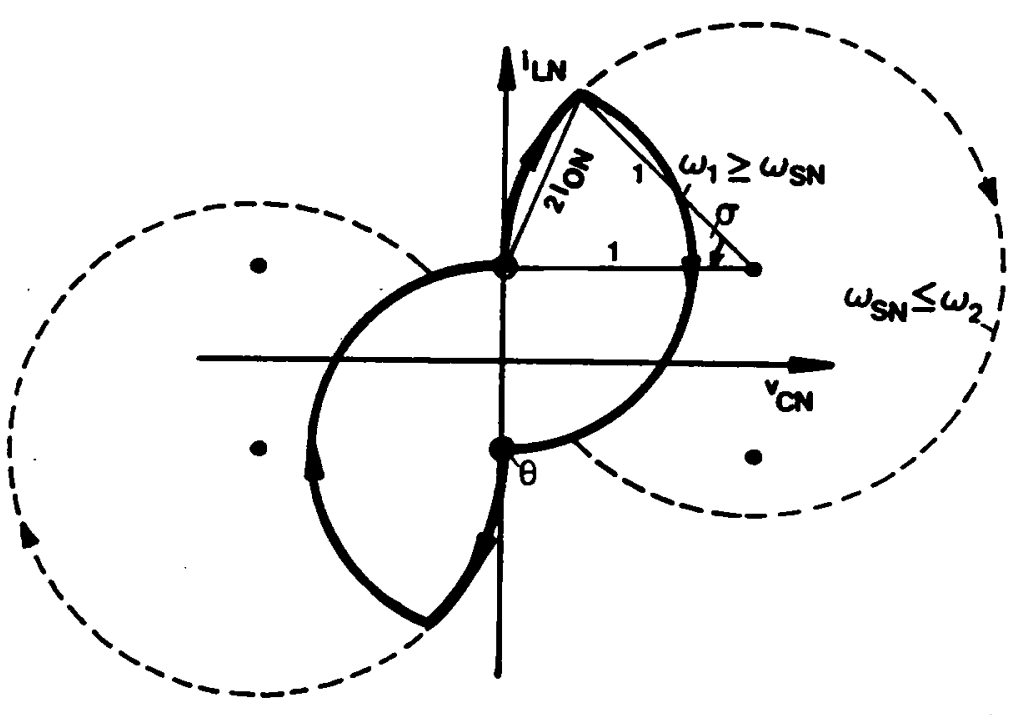

(b) Boundary between Mode II and Mode IV

Figure C.2.5 $\begin{gathered}\text { Boundary Trajectories for Discontinuous Capacitor Voltage } \\ \text { Waveform }\end{gathered}$ 


\section{APPENDIX C.3}

\section{PREDICTION OF MODE TRANSITIONS OF A CM-PRC OPERATING ABOVE RESONANT FREQUENCY}

Depending upon the switching frequency, the capacitor voltage waveform of a CM-PRC at $\beta_{s}=180^{\circ}$ can be continuous or discontinuous. The boundary frequency for continuous capacitor voltage waveform is

$$
\begin{gathered}
\omega_{n}=\frac{\pi}{\rho+\sigma+\gamma}, \\
\rho=\cos ^{-1}\left(1-I_{O N}^{2}\right), \sigma=\cos ^{-1}\left(\frac{1+I_{O N}^{2}}{\sqrt{1+4 I_{O N}^{2}}}\right), \quad \gamma=\cos ^{-1}\left(\frac{1}{\sqrt{1+4 I_{O N}^{2}}}\right) .
\end{gathered}
$$

If $\omega_{S N} \geq \pi / 2 I_{O N}$, the converter operates only in mode $\mathrm{V}$.

If $\omega_{D} \leq \omega_{S N}<\pi / 2 I_{O N}$, the converter first operates in mode III then transits to mode V. The boundary $\beta_{S}$ angle between mode III and mode $\mathrm{V}$ is $\beta_{3 S}=2 I_{O N} \times \omega_{S N}$.

If $\omega_{S N}<\omega_{D}$, the converter operates in mode I when $\beta_{S}$ is decreased from $180^{\circ}$ As $\beta_{s}$ is further decreased, the converter's operation may transit into mode IIA or mode III.

Consider the boundary trajectories, $T_{.121}$ and $T_{B 121}$, between mode I and mode IIA, as shown in Figure C.3.1. These trajectorics can be described by equations 


$$
\left[\begin{array}{l}
\cos \alpha_{1}=\frac{1+R_{3}^{2}-R_{2}^{2}}{2 R_{3}}, \\
\cos \alpha_{2}=\frac{1}{R_{3}}, \\
\cos \eta=\frac{1+R_{2}^{2}-R_{3}^{2}}{2 R_{2}}, \\
R_{3}^{2}=\left(R_{2}+2 I_{O N}\right)^{2}+1, \\
\frac{\pi}{\omega_{S N}}=\alpha_{2}-\alpha_{1}+\pi-\eta+\frac{\pi}{2},
\end{array}\right.
$$

and can be constructed only when $I_{O N} \leq 0.5$ and $R_{2} \geq R_{S}$, where $R_{S}=\frac{2 I_{O N}^{2}}{1-2 I_{O N}}$. The corresponding frequency for these trajectories are shown as a function of radius $R_{2}$ in Figure C.3.2, where $\omega_{S}$ is the frequency occurs at $R_{2}=R_{S}$ and $\omega_{M}$ is the highest possible frequency which can be calculated numerically. From Figure C.3.2, the transitions of the operating mode can be determined as follows.

(a). If $\omega_{S N} \geq \omega_{M}$, the converter's operation transits from mode I to mode III to mode $\mathrm{V}$ since no boundary trajectory exists between mode I and mode IIA. The boundary $\beta_{S}$ angle between mode I and mode III and the boundary $\beta_{S}$ angle between mode III and mode $V$ are calculated as in $\Lambda$ ppendix C.2.

(b). If $\omega_{S N}<\omega_{M}$, the converter's operation transits from mode I to mode IIA back to mode I to mode $\mathrm{V}$ since two boundary trajectories between mode I and mode IIA exist. Two sets of solutions, $\left(R_{2}, R_{3}, \eta, \alpha_{1}, \alpha_{2}\right)$ and $\left(R_{2}{ }^{\prime}, R_{3}{ }^{\prime}, \eta^{\prime}, \alpha_{1}{ }^{\prime}, \alpha_{2}{ }^{\prime}\right)$, can be obtained from equation C.3.1. The boundary $\beta_{S}$ angles between mode I and mode II $\Lambda$ are $\beta_{121 A}=\left(\alpha_{2}-\alpha_{1}\right) \times \omega_{S N}$ and $\beta_{12 A B}=\left(\alpha_{2}^{\prime}-\alpha_{1}^{\prime}\right) \times\left(^{\prime \prime}{ }_{S N}\right.$. The boundary $\beta_{S}$ angle between mode I and mode III and the boundary $\beta_{s}$ between mode III and mode $V$ are calculated as in Appendix C.2.

A Fortran program for determining the mode transitions and mode boundaries in this frequency range is included in the following. The program requires inputs for $\omega_{S N}$ and 
$I_{O N}$ and generates outputs for four boundary angles, $B A 1, B A 2, B C 1$, and $B C 2$. When $\beta_{S} \geq B A 1$, the converter operates in mode I. When $B A 1>\beta_{S} \geq B A 2$, the converter operates in mode II $\Lambda$. When $B A 2>\beta_{S} \geq B C I$, the converter operates in mode I. When $B C 1>\beta_{S} \geq B C 2$, the converter operates in mode III. When $B C 2>\beta_{S}$, the converter operates in mode $\mathrm{V}$. 


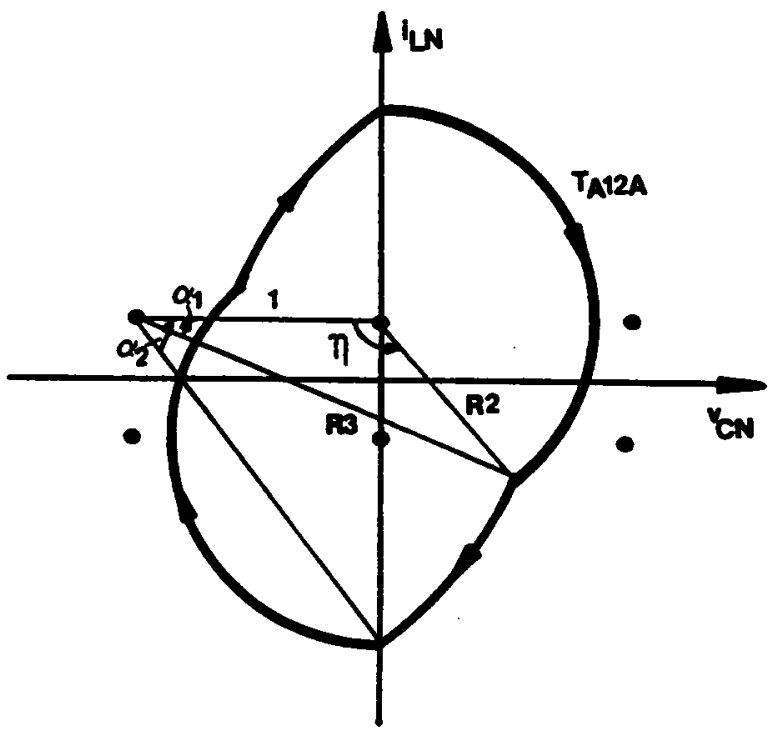

(a) $\eta \leq \pi\left(\alpha_{1} \geq 0\right)$

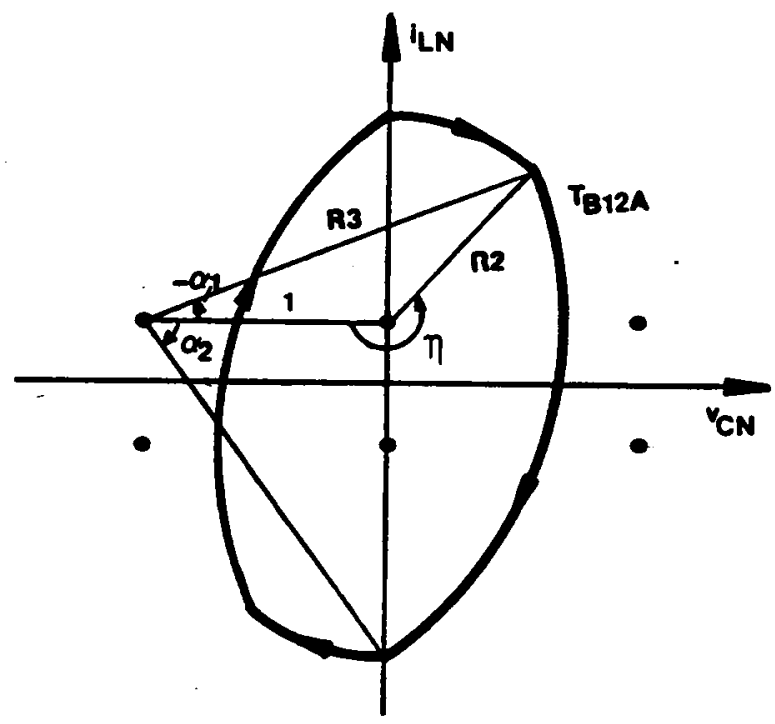

(b) $\eta>\pi\left(\alpha_{1}<0\right)$

Figure C.3.1 Boundary Trajectories Between Mode I and Mode IIA 


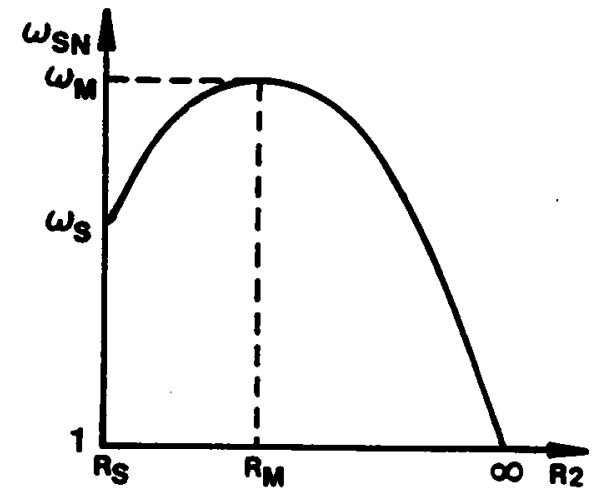

(a) Frequency for $T_{B 12 A}$

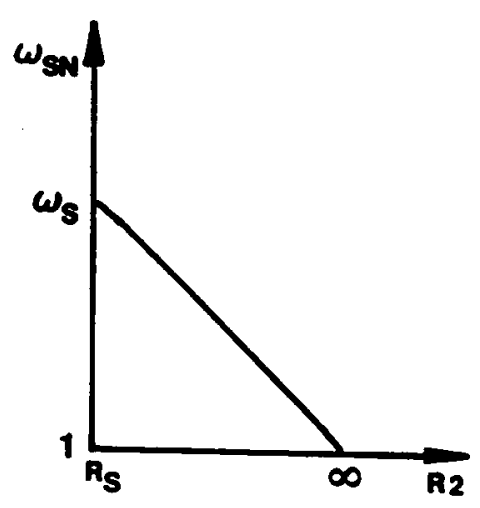

(b) Frequency for $T_{A 12 A}$

Figure C.3.2 Corresponding Frequency for the Trajectories in Figure C.3.1 
Fortran Program for Determining Mode Transitions and Mode Boundaries Above Resonant Frequency

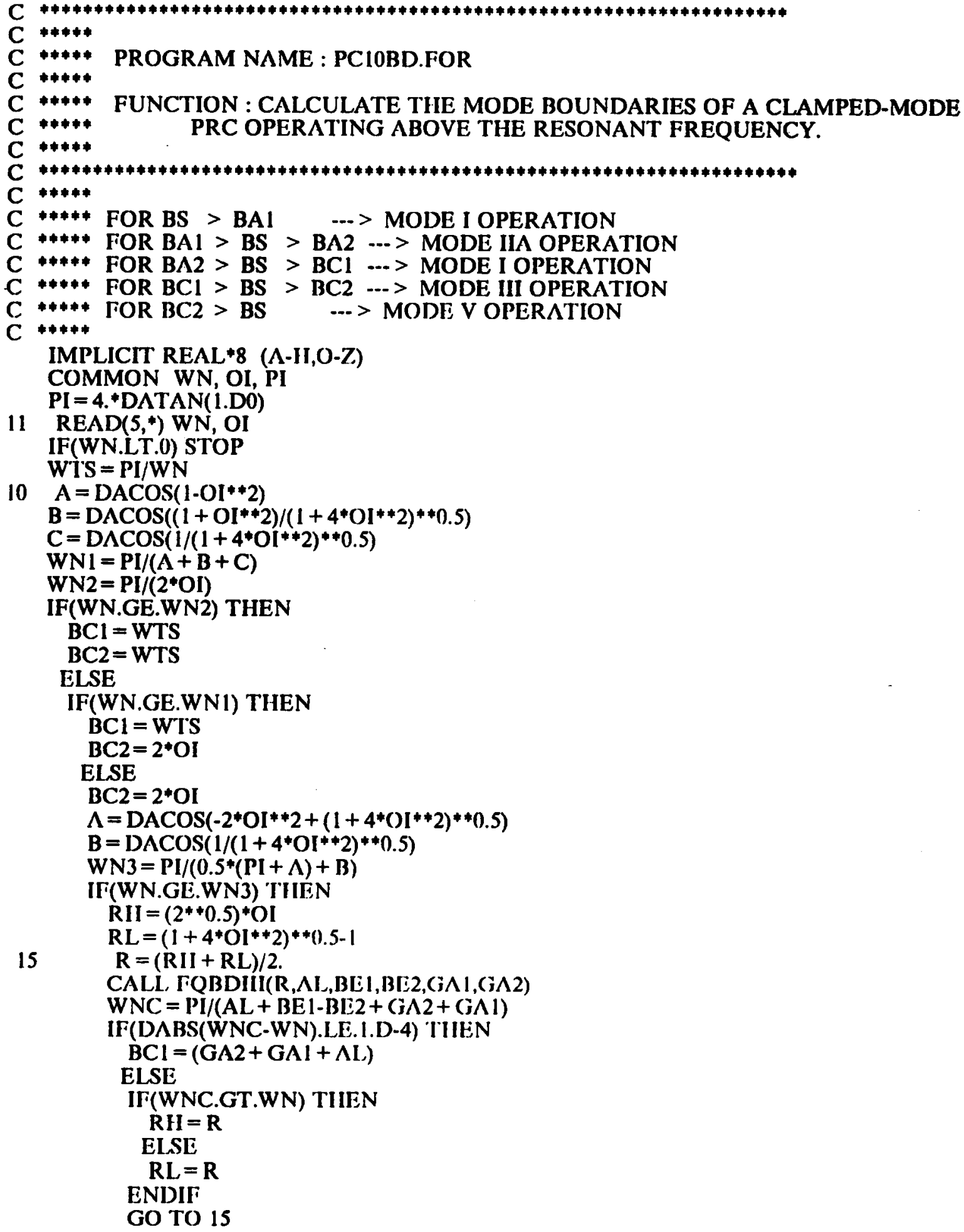




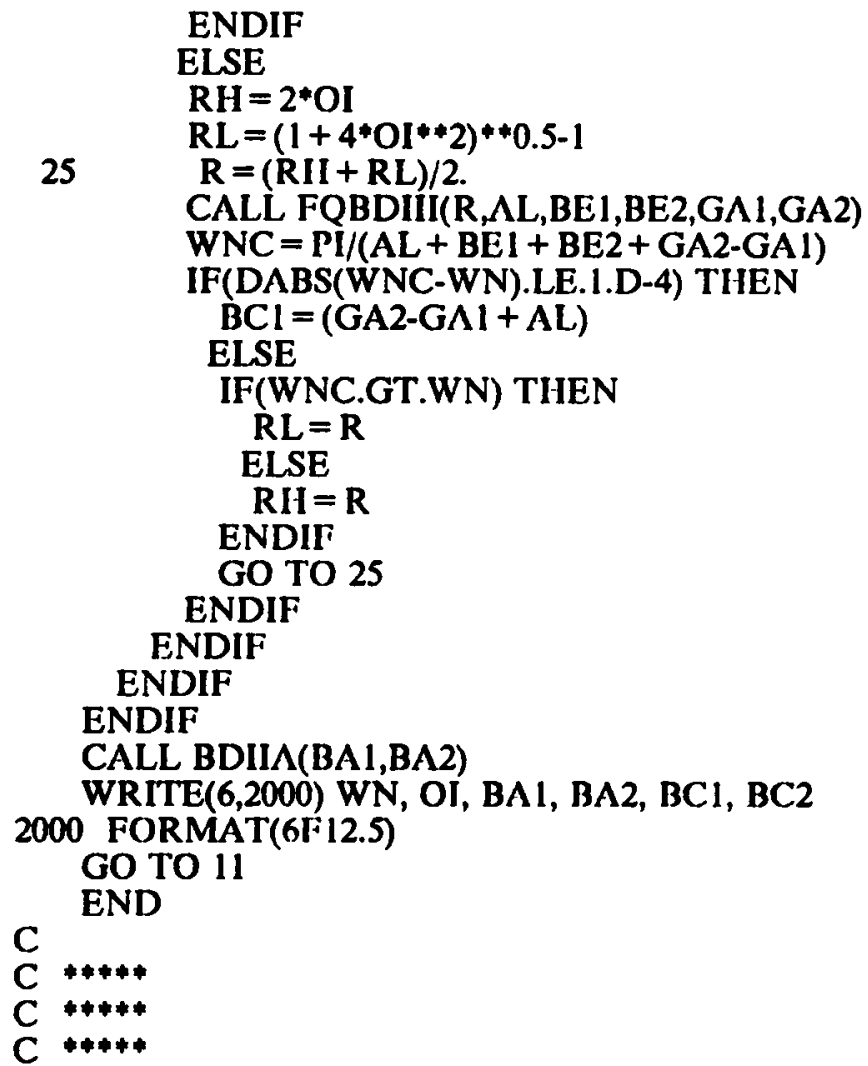


12 CALL FQIIA(R2,AL1,AL2,ET2,WNC1,WNC2)

IF(WNCI.GE.WA1) THEN

$\mathbf{R} 2=\mathbf{R} 2 * 2$.

GO TO 12

ENDIF

R2A2 $=$ R2

$\mathrm{WA} 2=\mathrm{WNC} 1$

RH $=\mathbf{R} 2 \mathrm{~A} 2$

$R \mathbf{L}=\mathbf{R} 2 \mathrm{Al}$

$14 R 2=(R H+R L) / 2$.

$R 2 P=R 2+1 . D-4$

CALL FQIIA(R2,AL1,AL2,ET2,WNC1,WNC2)

CALL FQIIA(R2P,AL1,AL2,ET2,WNCP1,WNCP2)

IF(DABS(RH-RL).LE.1.D-4) TIIEN

R2AMAX $=$ R2

WAMAX $=$ WNCl

ELSE

IF(WNC1.LE.WNCP1) THEN

$\mathbf{R L}=\mathbf{R} 2$

ELSE

RH = R2

ENDIF

GO TO 14

ENDIF

IF(WN.GE.WAMAX) THEN

BAI $=$ WTS

B $\Lambda 2=$ WTS

RETURN

ENDIF

IF(WN.GE.WA1) THEN

$\mathbf{R H}=\mathbf{R} 2 \mathrm{~A} 2$

$\mathrm{RL}=\mathrm{R} 2 \mathrm{AMAX}$

$22 \quad \mathrm{R} 2=(\mathrm{RH}+\mathrm{RL}) / 2$.

CALL FQII $\Lambda(R 2, A L 1, \Lambda L 2, E T 2, W N C 1, W N C 2)$

IF(DABS(RII-RL).LE.I.D-4) THEN

$B A 1=(A L 2+A L 1)$

ELSE

IF(WNC1.GT.WN) THEN

$\mathbf{R L}=\mathbf{R} 2$

ELSE

RH $=$ R2

ENDIF

GO TO 22

ENDIF

$\mathrm{RH}=\mathrm{R} 2 \Lambda \mathrm{M} \wedge \mathrm{X}$

$\mathrm{RI} .=\mathrm{R} 2 \Lambda \mathbf{I}$

$24 \mathrm{R} 2=(\mathrm{R} I I+\mathrm{RL}) / 2$.

CALL FQIIA(R2,AL,I,AL2,EI2,WNCI,WNC2)

IF(DABS(RII-RL).LE.1.D-4) TIIEN

$B A 2=(\Lambda L 2+A L 1)$

ELSE

IF(WNCI.GT.WN) TIIEN

RII = R2

ELSE

$R L=R 2$

ENDIF

GO TO 24

ENDIF 
ELSE

IF(WN.GE.WA2) TIIEN

$\mathrm{R} 2 \mathrm{~A} 3=\mathrm{R} 2 \mathrm{~A} 2$

ELSE

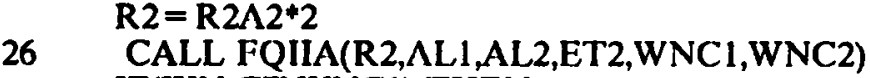
IF(WN.GT.WNC1) THIEN

$\mathrm{R} 2 \mathrm{~A} 3=\mathrm{R} 2$

ELSE

R2 $=\mathbf{R} 2 * 2$

GO TO 26

ENDIF

ENDIF

$\mathrm{RH}=\mathrm{R} 2 \mathrm{~A} 3$

$R L=R 2 A M A X$

$28 \quad R 2=(R H+R L) / 2$.

CALL FQIIA(R2, $\Lambda$ L1,AL2,ET2,WNC1,WNC2)

IF(DABS(RII-RL).LE.1.D-4) THIEN

$\mathrm{B} \Lambda \mathrm{I}=(\Lambda \mathrm{I}, 2+\mathrm{AI}, 1)$

ELSE

IF(WNCI.GT.WN) THEN

$\mathbf{R L}=\mathbf{R} 2$

ELSE

RII = R2

ENDIF

GO TO 28

ENDIF

$R 2=R 2 A 1+5$.

32 CALL FQIIN(R2,AL1,AL2,ET2,WNC1,WNC2)

IF(WNC2.LE.WN) THEN

$$
R 2 A 4=R 2
$$

\section{ELSE}

$\mathrm{R} 2=\mathrm{R} 2 * 2$.

GO TO 32

ENDIF

$\mathrm{R} I \mathrm{I}=\mathrm{R} 2 \Lambda 4$

$\mathrm{RL}=\mathrm{R} 2 \mathrm{Al}$

$34 \quad R 2=(R I I+R L) / 2$.

CALL FQIIA(R2,AL1,AL2,ET2,WNC1,WNC2)

IF(DABS(RII-RL).LE.1.D-4) TIIEN

$B \Lambda 2=(A L 2-A L 1)$

ELSE

IF(WNC2.GT.WN) TIIEN

$\mathrm{RL}=\mathrm{R} 2$

EISE

$\mathrm{RII}=\mathrm{R} 2$

ENDIF

GO TO 34

ENDIF

ENDIF

RETURN

C

END

C *****

C

C

SUBROUTINE FQII $(R 2, \Lambda L 1, \Lambda I, 2, E T 2, W N C 1, W N C 2)$ 
IMPLICIT REAL*8 (A-H,O-Z)

COMMON WN, OI, PI

$\mathrm{R} 3=((\mathrm{R} 2+2 * \mathrm{OI}) *+2+1) * *$.

$A L 2=\operatorname{DACOS}(1 / \mathrm{R} 3)$

$A L 1=D A C O S((1+R 3 * * 2-R 2 * * 2) /(2 * R 3))$

ET $1=2 *$ PI-DACOS $\left(\left(1+R 2 * * 2-R^{* *} 2\right) /(2 * R 2)\right)$

ET2 $=2 *$ PI-ET1

$\mathrm{WNCl}=\mathrm{PI} /(\mathrm{Al} \mathrm{L} 2+\mathrm{AL} 1+1.5 * \mathrm{PI}-\mathrm{ET} \mathrm{l})$

$W N C 2=P I /(A L 2-A L 1+1.5+P I-E T 2)$

RETURN

END 


\section{APPENDIX C.4 \\ CALCULATION OF TRAJECTORY PARAMETERS \\ OF A CM-PRC}

The parameters for the equilibrium trajectories of a CM-PRC can be obtained by solving sets of nonlinear equations. The equations are shown in Figures C.4.1 C.4.6.

There are different equilibrium trajectories shown for each operating mode. All the equilibrium trajectories can be described by the same sets of equations shown. However, all the trajectories must be considered when the circuit salient features are calculated. For example, the natural-commutation trajectory and the semi-commutation trajectories in an operating mode have different device conduction sequences. Thus, the expressions for calculating switch currents and diode currents are derived from different conduction intervals, which results in different expressions. The expressions for calculating peak capacitor voltage in the trajectories with the same commutation features are also different. $\Lambda s$ shown in Figure C.4.1(b) and Figure C.4.1(c), the peak capacitor voltage for the trajectories are $R_{1}+1$ and $R_{2}$, respectively.

The FORTR $\Lambda$ N programs for calculating trajectory parameters at frequencies below and above resonant frequency are included at the end of this section. The outputs generated by the programs are defined in the following:

- For mode-I trajectory in Iigure C.4.1, II $=1, \mathrm{X}(1)=R_{1}, \mathrm{X}(2)=R_{2}, \mathrm{X}(3)=R_{3}$, $\left.\mathrm{X}(4)=\rho, \mathrm{X}(5)=\sigma, \mathrm{X}(6)=\eta, \mathrm{X}(7)=\alpha_{1}, \mathrm{X}(8)=\alpha_{2} ; \mathrm{IDD}\right)=11$ for Trajectory C.4.1(a), IDD $=12$ for Trajectory C.4.1(b), IDI) $=13$ for Trajectory C.4.1(c), IDD = 14 for Trajectory C.4.1(d), IDD = 15 for Trajectory C.4.1(e).

- For mode-II trajectory in Figure $\mathrm{C.4.2,}$ II $=2, \mathrm{X}(1)=R_{1}, \mathrm{X}(2)=R_{2}, \mathrm{X}(3)=\rho, \mathrm{X}(4)=$ $\sigma, \mathrm{X}(5)=\alpha, \mathrm{X}(6) \sim \mathrm{X}(8)=$ dummy; IDI $=21$ for Trajectory $\mathrm{C} .4 .2(a), \mathrm{IDD}=22$ for 
Trajectory C.4.2(b), IDD $=23$ for Trajectory C.4.2(c), IDD $=29$ for Trajectory C.4.2(d).

- For mode-III trajectory in Figure C.4.3, ID $=3, \mathrm{X}(1)=R_{\mathrm{l}: \mathrm{ef}, \mathrm{X}} X(2)=: f . R_{2}, \mathrm{X}(3)=\sigma$, $\mathrm{X}(4)=\eta, \mathrm{X}(5)=\alpha_{1}, \mathrm{X}(6)=\alpha_{2}, \mathrm{X}(7) \sim \mathrm{X}(8)=$ dummy; IDD $=31$ for Trajectory C.4.3(a), IDD $=32$ for Trajectory C.4.3(b), IDD $=33$ for Trajectory C.4.3(c), IDD $=34$ for Trajectory C.4.3(d), IDD $=35$ for Trajectory C.4.3(e). IDD $=36$ for Trajectory C.4.3(f), IDD $=37$ for Trajectory C.4.3(g).

- For mode-IV trajectory in Figure C.4.4, ID $=4, \mathrm{X}(1)=R_{1}, \mathrm{X}(2)=\sigma, \mathrm{X}(3)=\theta$, $X(4) \sim X(8)=$ dummy ; IDD $=41$ for Trajectory C.4.4(a), IDD $=42$ for Trajectory C.4.4(b), IDD $=43$ for Trajectory C.4.4(c), IDI) $=44$ for Trajectory C.4.4(d).

- For mode-V trajectory in Figure C.4.5, ID =5, X(1) X(8)= dummy; IDD= 55 .

- For mode-II $\Lambda$ trajectory in Figure C.4.6, $\mathrm{ID}=21, \mathrm{X}(1)=R_{1}, \mathrm{X}(2)=R_{2}, \mathrm{X}(3)=R_{3}$, $\mathrm{X}(4)=\rho, \mathrm{X}(5)=\eta, \mathrm{X}(6)=\alpha_{1}, \mathrm{X}(7)=\alpha_{2}, \mathrm{X}(8)=$ dummy; IDD $=23$ for Trajectory C.4.6(a), IDD $=24$ for Trajectory C.4.6(b), IDD $=25$ for Trajectory C.4.6(c). 


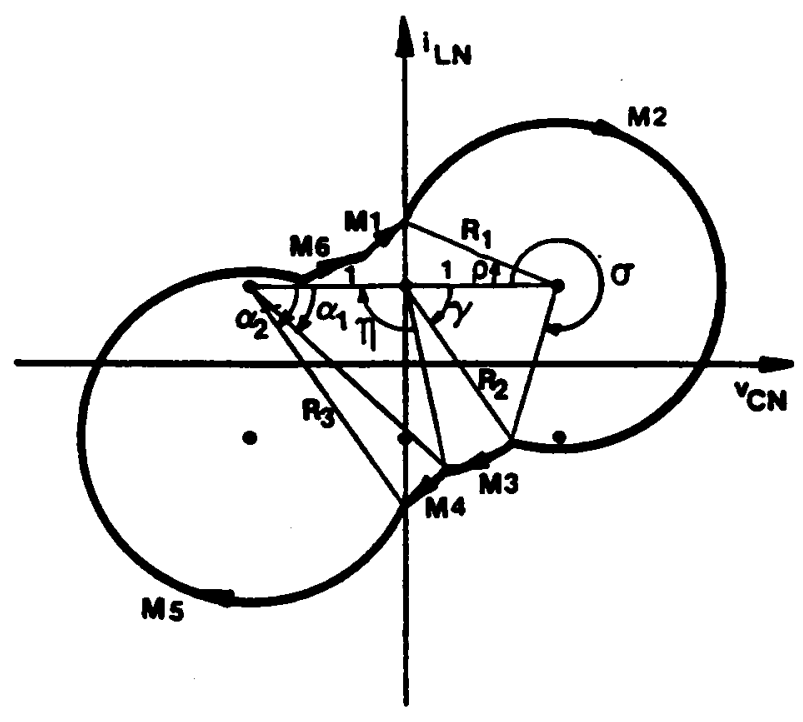

(a) Natural-commutation trajectory

$$
\begin{aligned}
\cos \rho & =\frac{1}{R_{1}}, \quad \cos \alpha_{2}=\frac{1}{R_{3}}, \quad R_{3}^{2}=1+\left(2 I_{O N}+\sqrt{R_{1}^{2}-1}\right)^{2}, \\
\cos \sigma & =\frac{1+R_{1}^{2}-R_{2}^{2}}{2 R_{1}}, \quad \cos \gamma=\frac{1+R_{2}^{2}-R_{1}^{2}}{2 R_{2}}, \\
\cos \eta & =\frac{1+R_{2}^{2}-R_{3}^{2}}{2 R_{2}}, \quad \cos \alpha_{1}=\frac{1+R_{3}^{2}-R_{2}^{2}}{2 R_{3}}, . \\
\frac{\beta_{S}}{\omega_{S N}} & =\alpha_{2}-\alpha_{1}+\sigma-\rho, \quad \frac{\pi}{\omega_{S N}}=\sigma-\rho+\pi-\gamma-\eta+\alpha_{2}-\alpha_{1} . \\
1 & \leq R_{1}, \quad 0 \leq R_{2}, \sqrt{1+4 I_{O N}^{2}} \leq R_{3}, \quad 0 \leq \alpha_{2}, \rho \leq \frac{\pi}{2}, \\
& -\frac{\pi}{2} \leq \alpha_{1}, \gamma \leq \frac{\pi}{2}, \quad 0 \leq \eta \leq \frac{3 \pi}{2}, 0 \leq \sigma \leq 2 \pi .
\end{aligned}
$$

Figure C.4.1 Mode-I Trajectories 


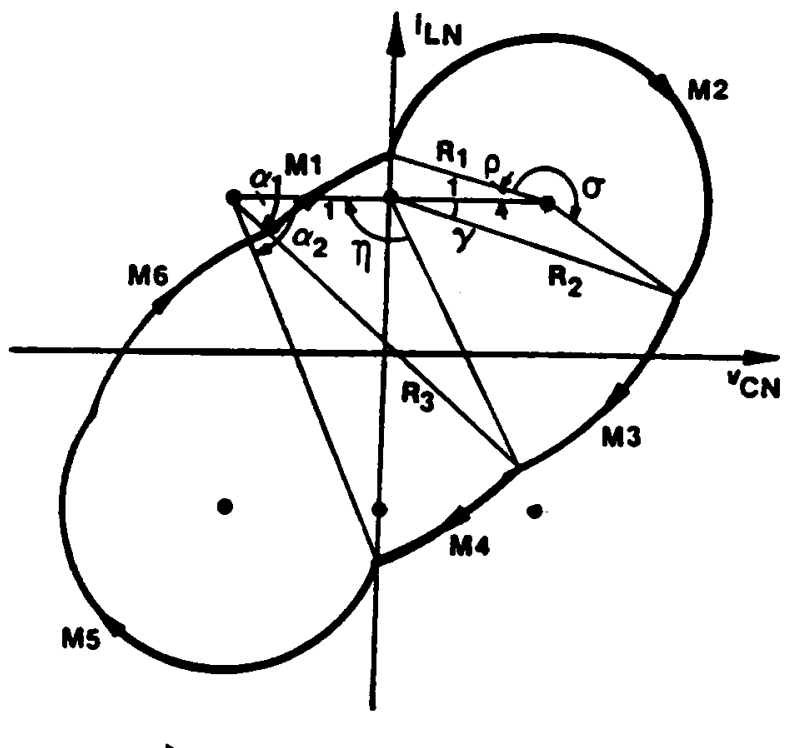

(b) Mixed-commutation trajectory $(\sigma \geq \pi)$

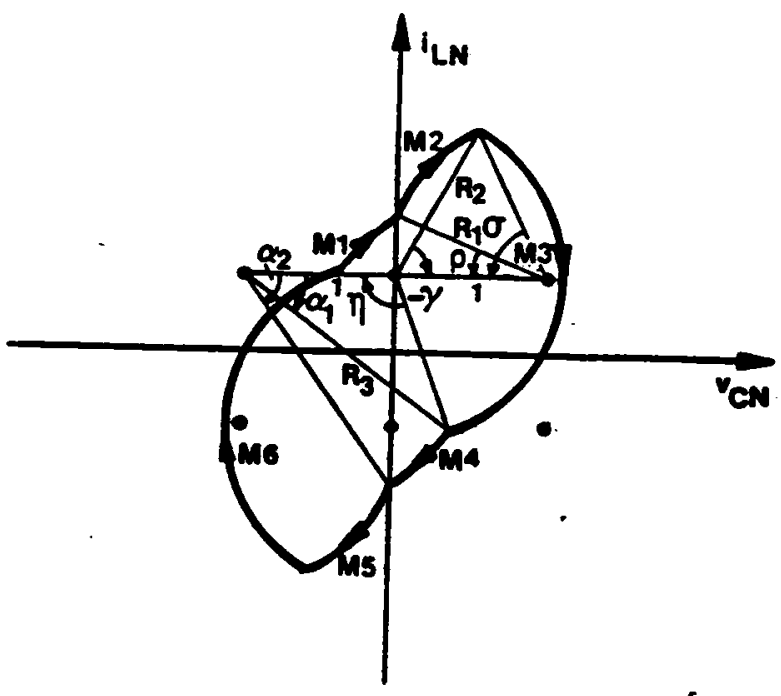

(c) Mixed-commutation trajectory $(\sigma<\pi)$

Figure C.4.1 Continued 


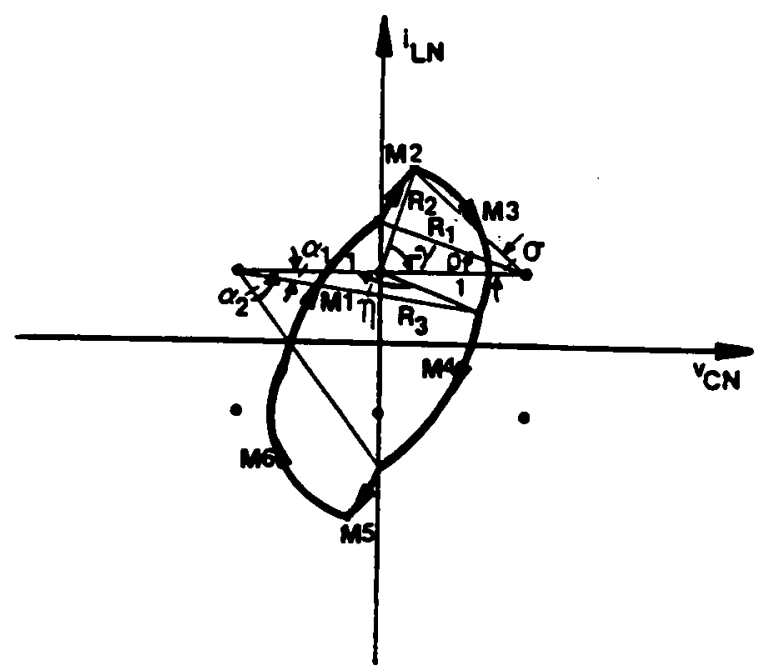

(d) Force-commutation trajectory $(\eta \leq \pi)$

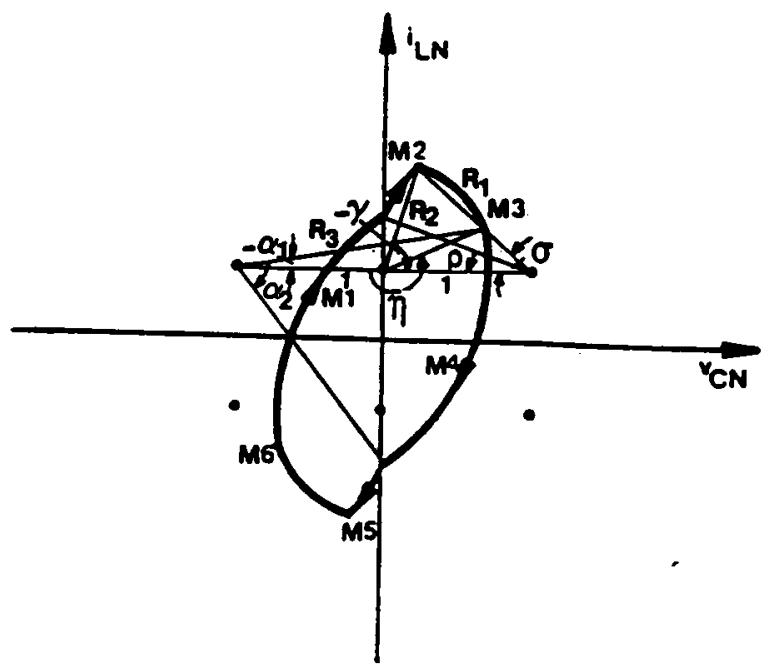

(e) Force-commutation trajectory $(\eta>\pi)$

Figure C.4.1 Continued 


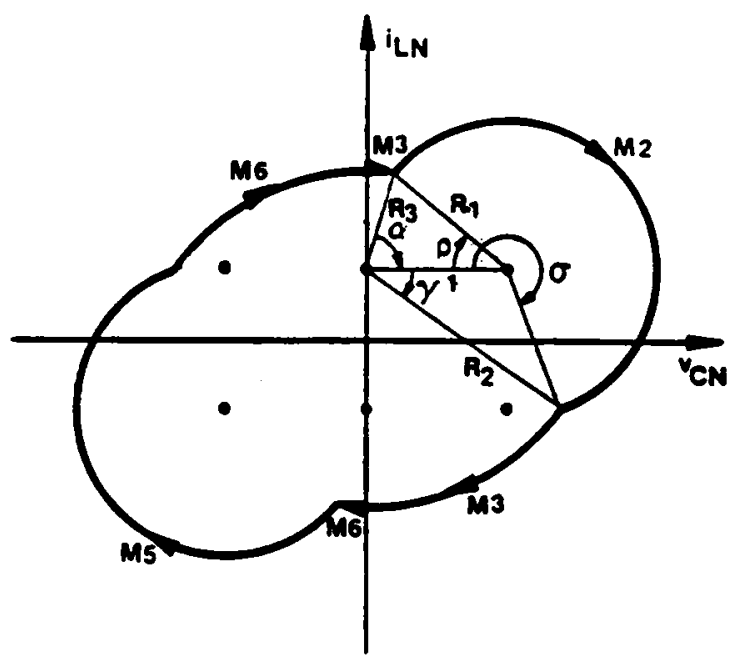

(a) Natural-commutation trajectory

$$
\begin{aligned}
& \cos \sigma=\frac{1+R_{1}^{2}-R_{2}^{2}}{2 R_{1}}, \quad \cos \gamma=\frac{1+R_{2}^{2}-R_{1}^{2}}{2 R_{2}}, \\
& \cos \rho=\frac{1+R_{1}^{2}-R_{3}^{2}}{2 R_{1}}, \quad \cos \alpha=\frac{1+R_{3}^{2}-R_{1}^{2}}{2 R_{3}}, \\
& \frac{\beta_{S}}{\omega_{S N}}=\sigma-\rho, \quad R_{3}=R_{2}-2 I_{O N}, \\
& \frac{\pi}{\omega_{S N}}=\sigma-\rho+\frac{\pi}{2}-\gamma+\frac{\pi}{2}-\alpha . \\
& 0 \leq R_{1}, R_{3}, \quad 2 I_{O N} \leq R_{2}, \quad-\frac{\pi}{2} \leq \alpha, \gamma, \rho \leq \frac{\pi}{2}, \quad 0 \leq \sigma \leq 2 \pi .
\end{aligned}
$$

Figure C.4.2 Mode-II Trajectories 


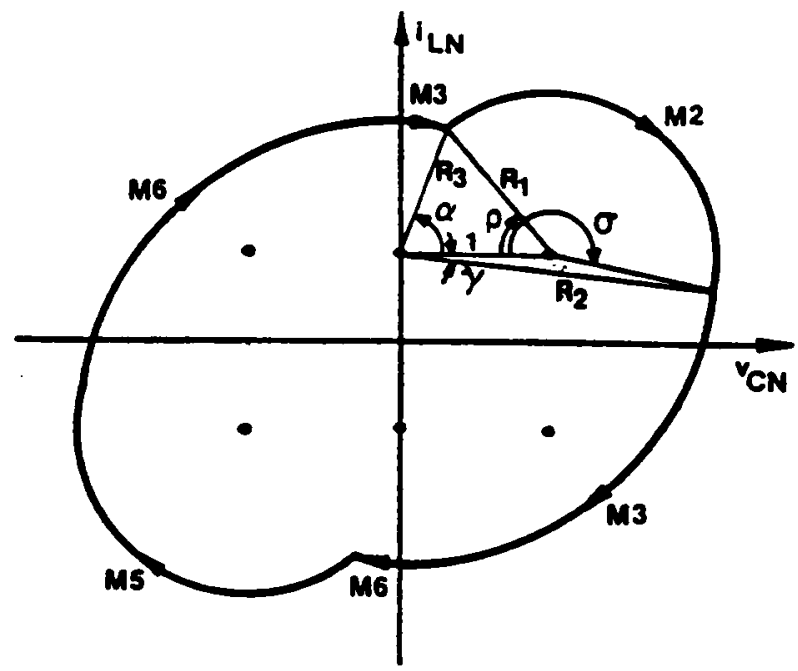

(b) Mixed-commutation trajectory $(\sigma \geq \pi)$

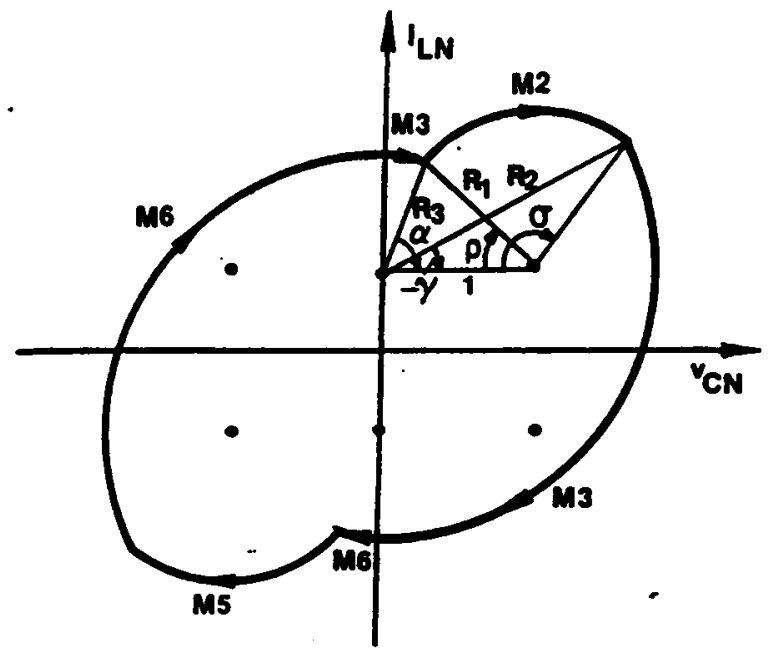

(c) Mixed-commutation trajectory $(\sigma<\pi)$

Figure C.4.2 Continued 


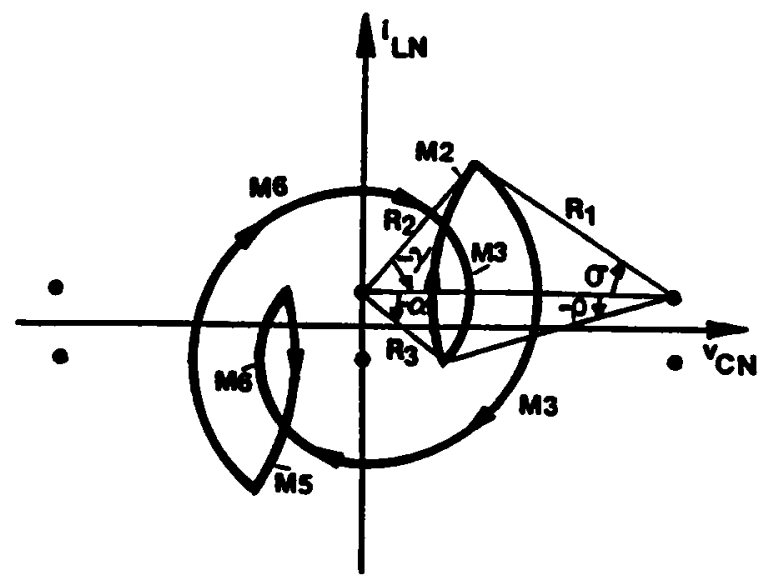

(d) Force-commutation trajectory (multiple conduction)

Figure C.4.2 Continued 


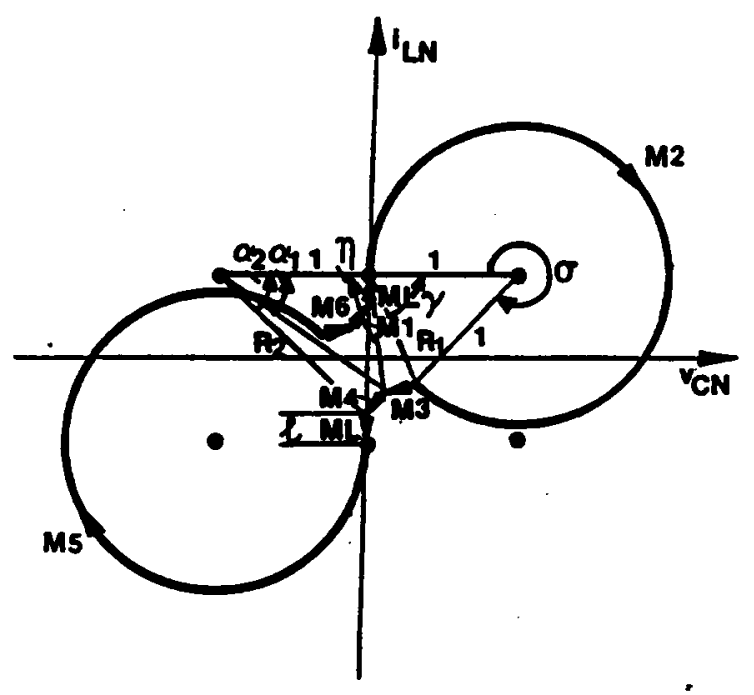

(a) Natural-commutation trajectory

$$
\begin{aligned}
& \cos \sigma=\frac{2-R_{1}^{2}}{2}, \quad \gamma=\frac{\sigma-\pi}{2}, \\
& \cos \eta=\frac{1+R_{1}^{2}-R_{2}^{2}}{2 R_{1}}, \quad \cos \alpha_{1}=\frac{1+R_{2}^{2}-R_{1}^{2}}{2 R_{2}}, \\
& \cos \alpha_{2}=\frac{1}{R_{2}}, \quad \sqrt{R_{2}^{2}-1}+\ell=2 I_{O N}, \\
& \frac{\beta_{S}}{\omega_{S N}}=\alpha_{2}-\alpha_{1}+\sigma+\ell, \quad \frac{\pi}{\omega_{S N}}=\sigma+\pi-\gamma-\eta+\alpha_{2}-\alpha_{1}+\ell . \\
& \quad 0 \leq R_{1}, \quad 1 \leq R_{2} \leq \sqrt{1+4 I_{O N}^{2}}, \quad 0 \leq \alpha_{2} \leq \frac{\pi}{2}, \\
& -\frac{\pi}{2} \leq \alpha_{1}, \gamma \leq \frac{\pi}{2}, \quad 0 \leq \eta \leq \frac{3 \pi}{2}, 0 \leq \sigma \leq 2 \pi, \quad 0 \leq \ell \leq 2 I_{O N} .
\end{aligned}
$$

Figure C.4.3 Mode-III Trajectories 


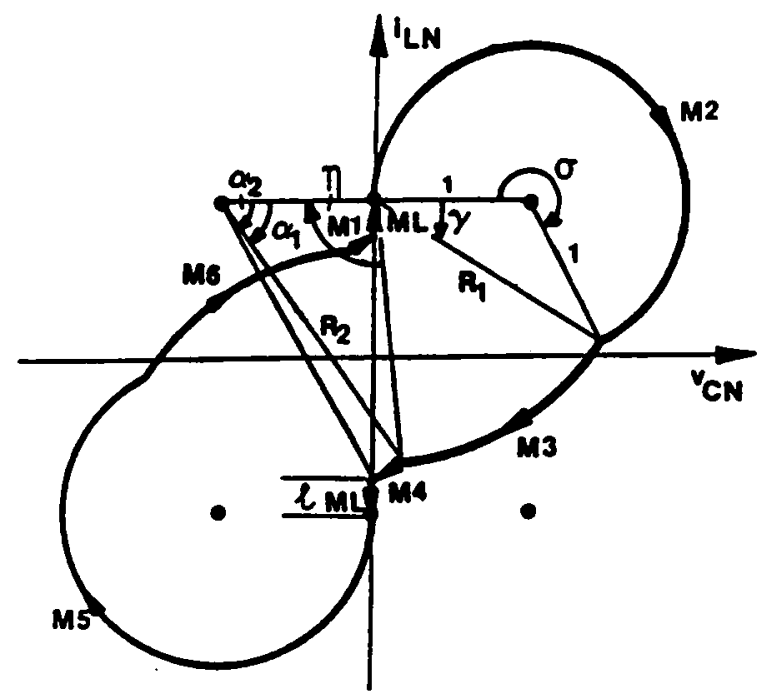

(b) Mixed-commutation trajectory $(\sigma \geq \pi)$

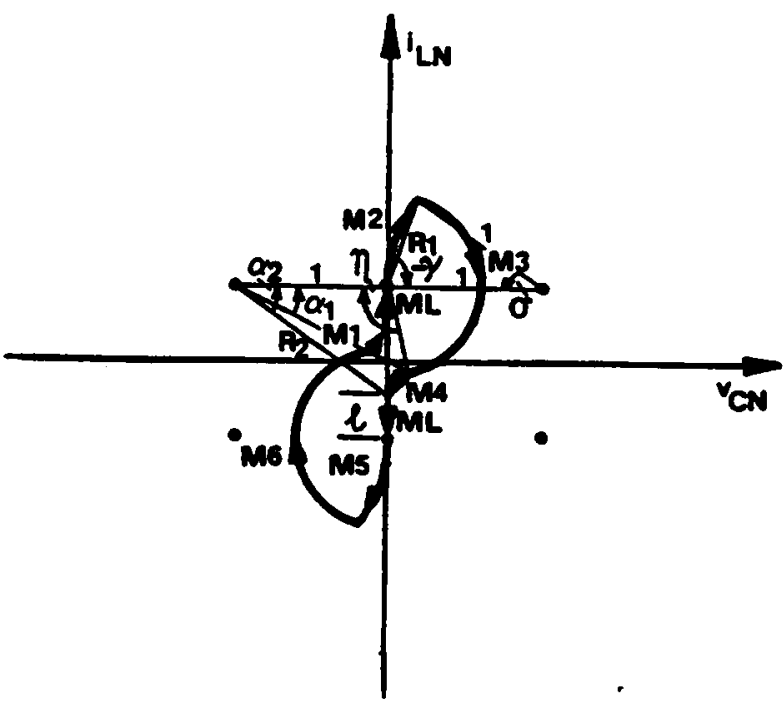

(c) Mixed-commutation trajectory $(\sigma<\pi)$

Figure C.4.3 Continued 


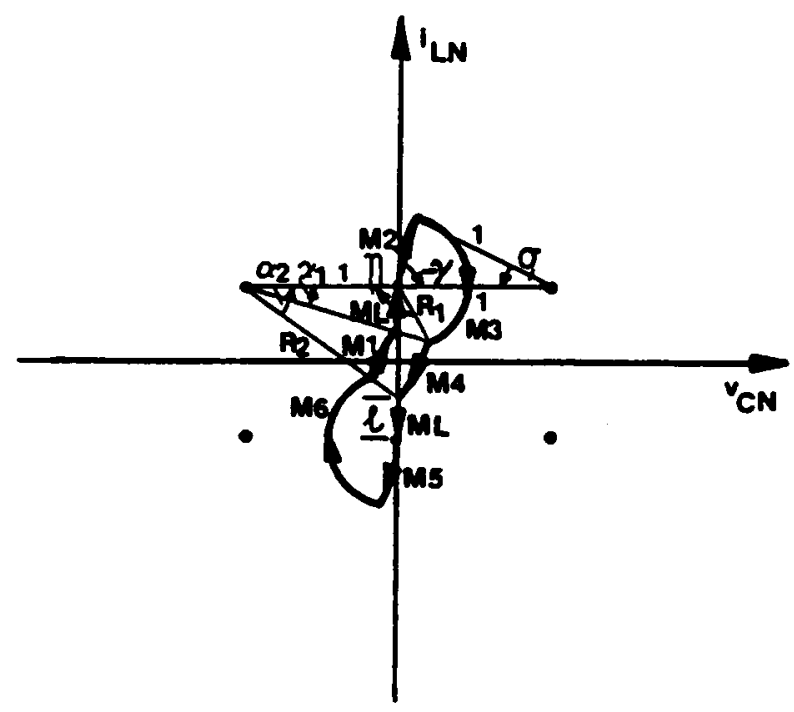

(d) Force-commutation trajectory ( $\eta \leq \pi, R_{2} \sin \alpha_{2} \geq I_{O N}$ )

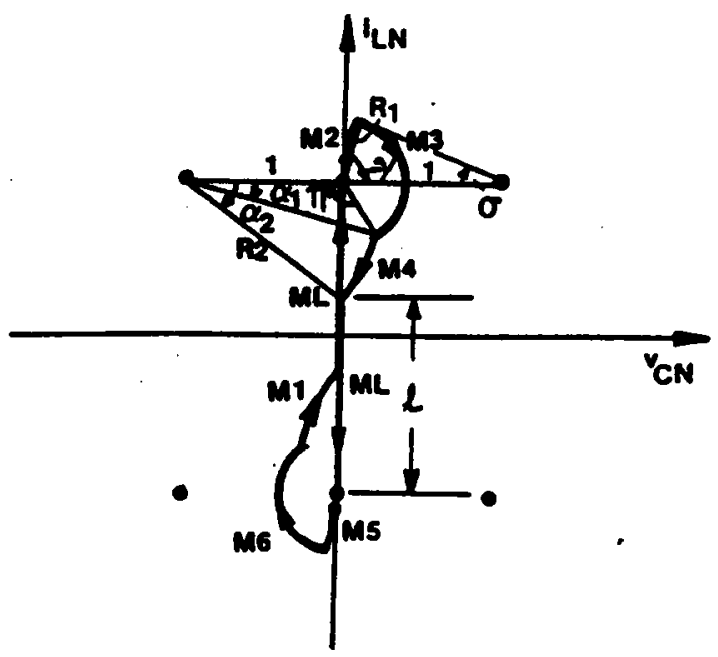

(e) Force-commutation trajectory ( $\left.\eta \leq \pi, R_{2} \sin \alpha_{2}<I_{O N}\right)$

Figure C.4.3 Continued 


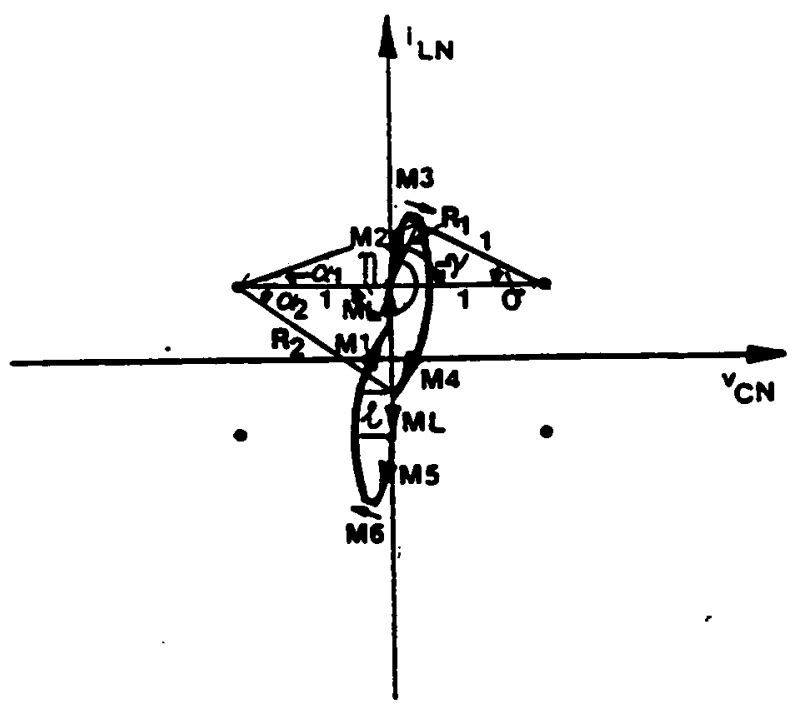

(f) Force-commutation trajectory ( $\left.\eta>\pi, \quad R_{2} \sin \alpha_{2} \geq I_{O N}\right)$

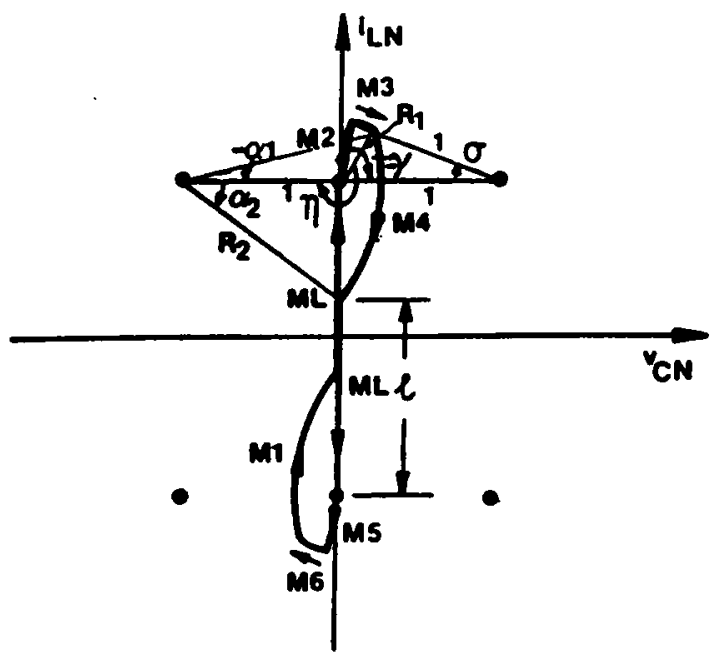

(g) Force-commutation trajectory $\left(\eta>\pi, R_{2} \sin \alpha_{2}<I_{O N}\right)$

Figure C.4.3 Continued 


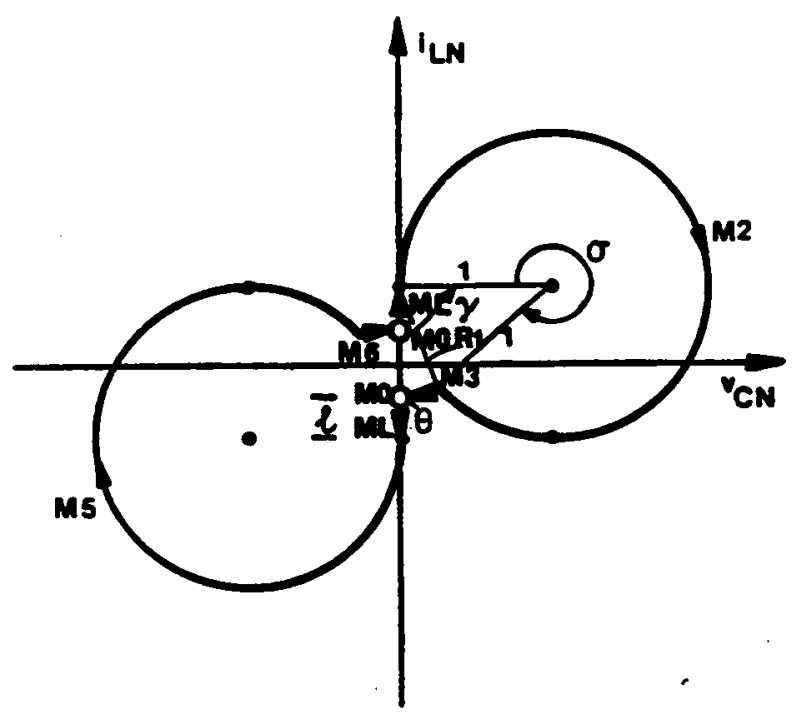

(a) Natural-commutation trajectory

$$
\begin{gathered}
\cos \sigma=\frac{2-R_{1}^{2}}{2}, \quad \gamma=\frac{\sigma-\pi}{2}, \\
\frac{\beta_{S}}{\omega_{S N}}=\sigma+\ell, \quad R_{1}=2 I_{O N}-\ell, \\
\frac{\pi}{\omega_{S N}}=\sigma+\frac{\pi}{2}-\gamma+\theta+\ell . \\
0 \leq R_{1} \leq 2 I_{O N},-\frac{\pi}{2} \leq \gamma \leq \frac{\pi}{2}, \quad 0 \leq \sigma \leq 2 \pi, \quad 0 \leq \ell \leq 2 I_{O N} .
\end{gathered}
$$

Figure C.4.4 Mode-IV·Trajectories 


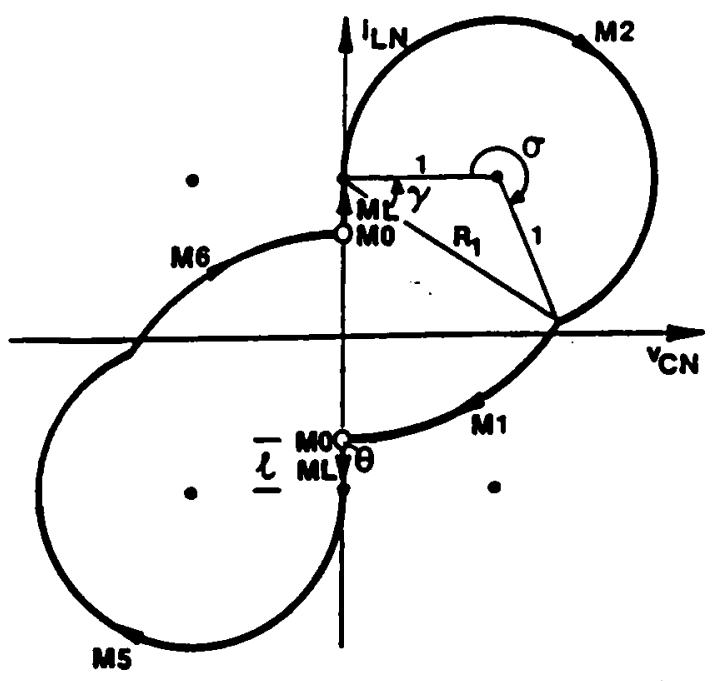

(b) Mixed-commutation trajectory $(\sigma \geq \pi)$

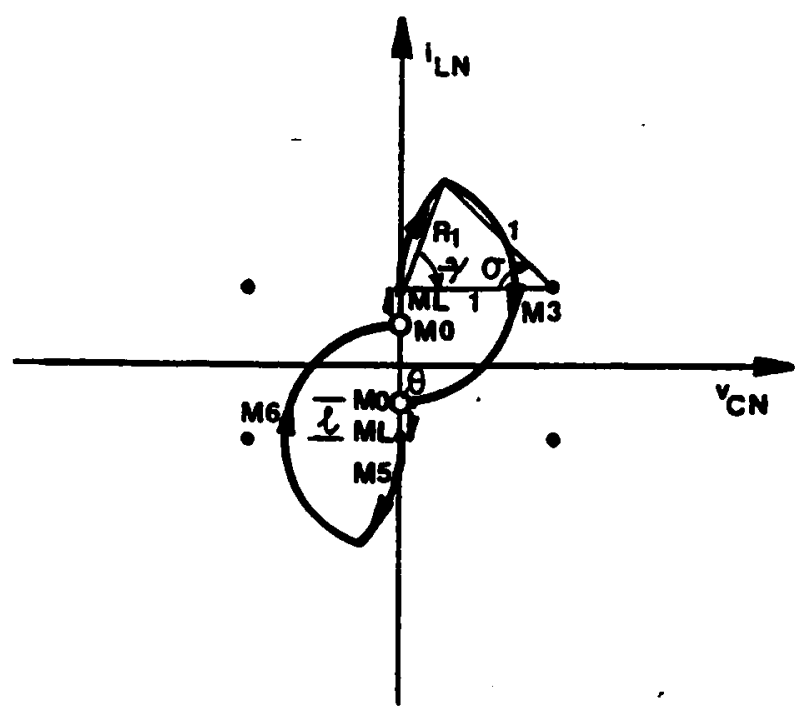

(c) Mixed-commutation trajectory $(\sigma<\pi)$

Figure C.4.4 Continued 


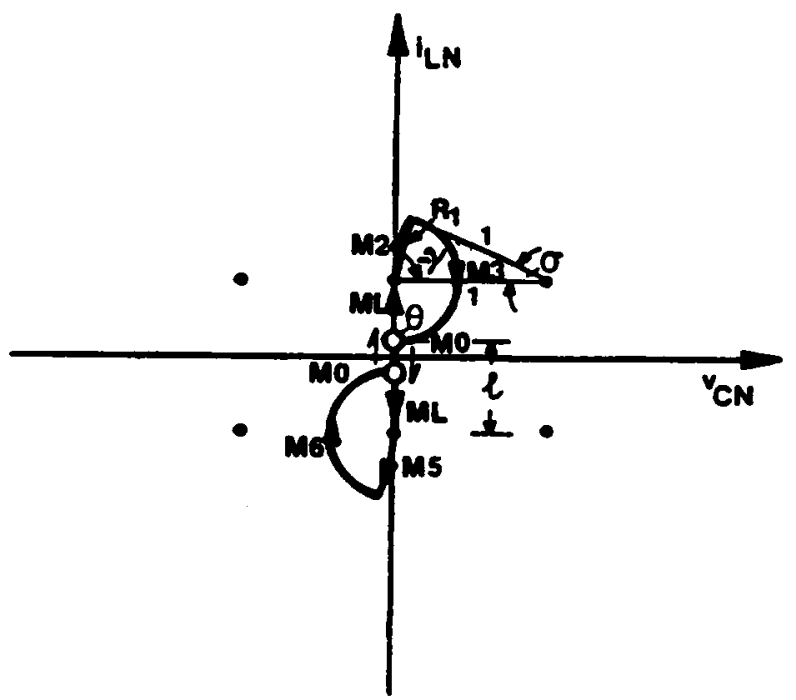

(d) Force-commutation trajectory

Figure C.4.4 Continued 


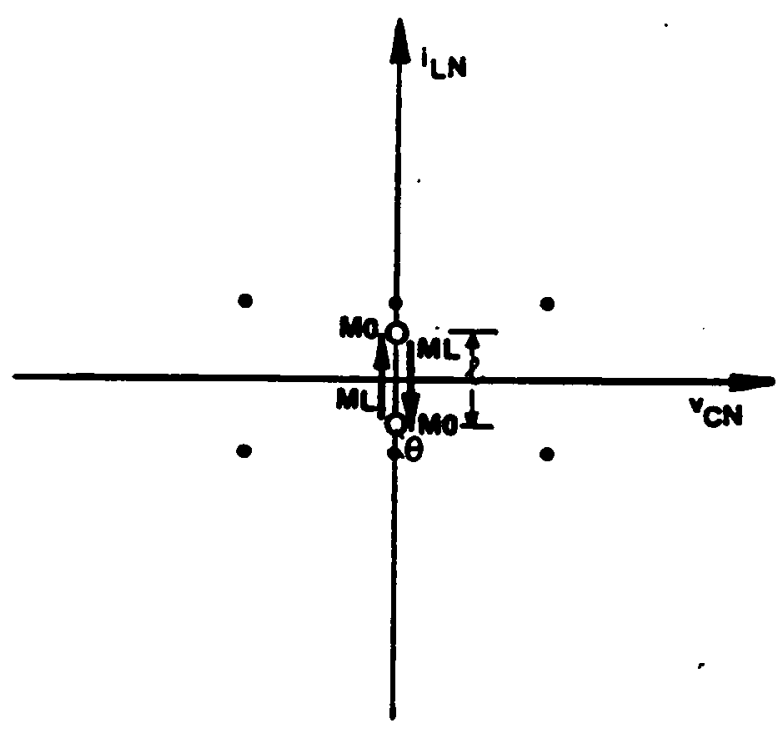

$$
\begin{gathered}
\frac{\beta_{S}}{\omega_{S N}}=\ell, \\
\frac{\pi}{\omega_{S N}}=\theta+\ell . \\
0 \leq \theta \leq 2 \pi, \quad 0 \leq \ell \leq 2 I_{O N} .
\end{gathered}
$$

Figure C.4.5 Mode-V Trajectory

359 


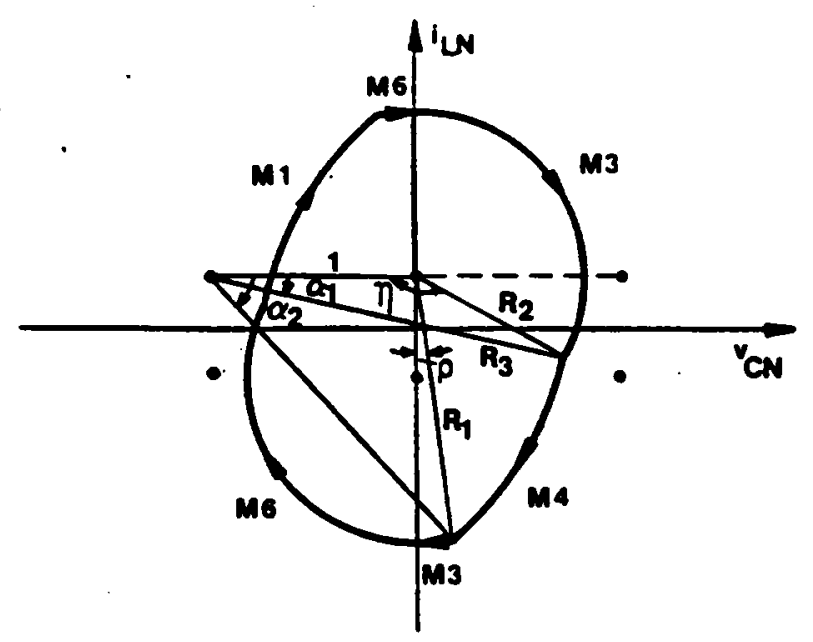

(a) Mixed-commutation trajectory

$$
\begin{aligned}
& \cos \left(\frac{\pi}{2}+\rho\right)=\frac{1+R_{1}^{2}-R_{3}^{2}}{2 R_{1}}, \quad \cos \alpha_{2}=\frac{1+R_{3}^{2}-R_{1}^{2}}{2 R_{3}}, \\
& \cos \eta=\frac{1+R_{2}^{2}-R_{3}^{2}}{2 R_{2}}, \quad \cos \alpha_{1}=\frac{1+R_{3}^{2}-R_{2}^{2}}{2 R_{3}}, \\
& \frac{\beta_{S}}{\omega_{S N}}=\alpha_{2}-\alpha_{1}, \quad R_{2}=R_{1}+2 I_{O N}, \\
& \frac{\pi}{\omega_{S N}}=\frac{3 \pi}{2}-\eta+\alpha_{2}-\alpha_{1}+\rho .
\end{aligned}
$$

$2 I_{O N} \leq R_{1}, \quad 0 \leq R_{2}, \quad 1 \leq R_{3}, \quad 0 \leq \alpha_{2}, \rho \leq \frac{\pi}{2}, \quad-\frac{\pi}{2} \leq \alpha_{1} \leq \frac{\pi}{2}, 0 \leq \eta \leq \frac{3 \pi}{2}$.

Figure C.4.6 Mode-IIA Trajectories 


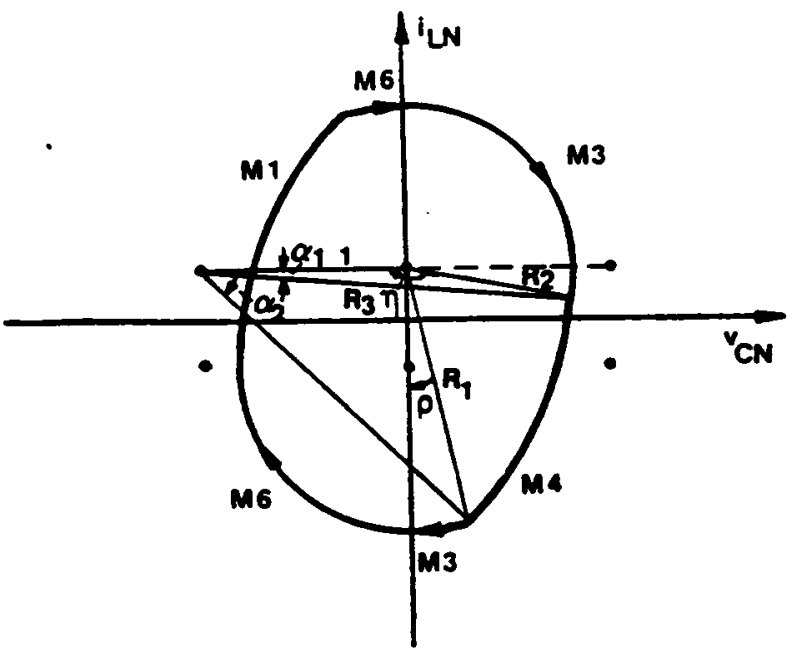

(b) Force-commutation trajectory $(\eta \geq \pi)$

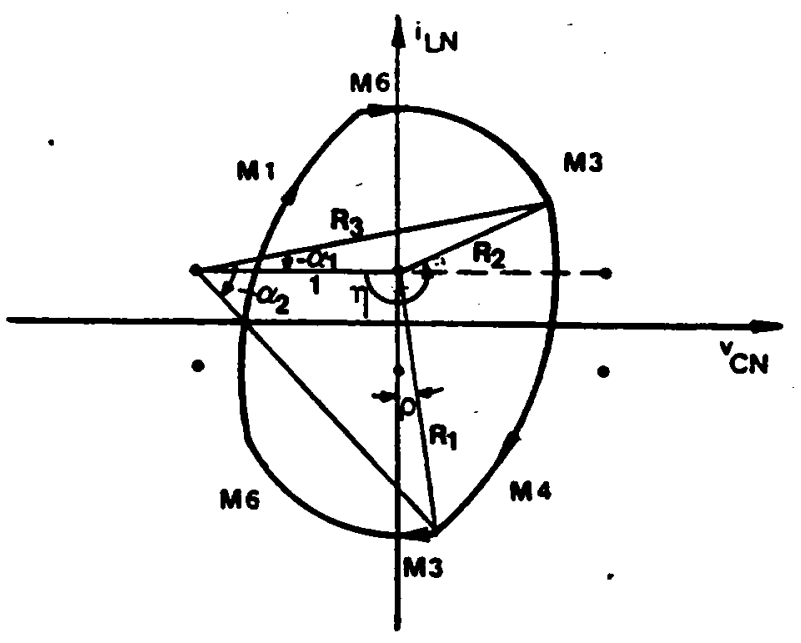

(c) Force-commutation trajectory $(\eta<\pi)$

Figure C.4.6 Continued 
FORTRAN Program for Calculating Trajectory Parameters Below Resonant Frequency

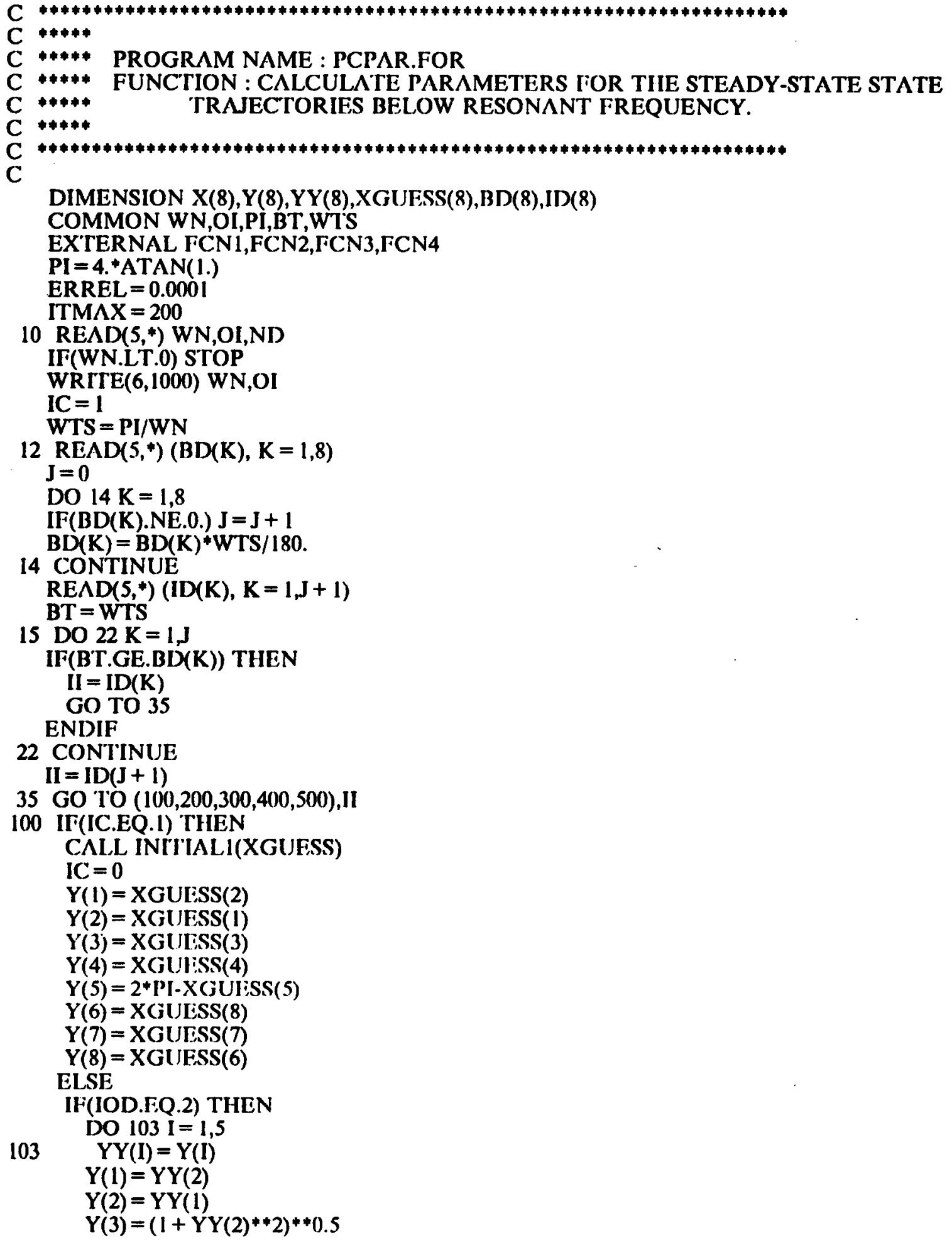




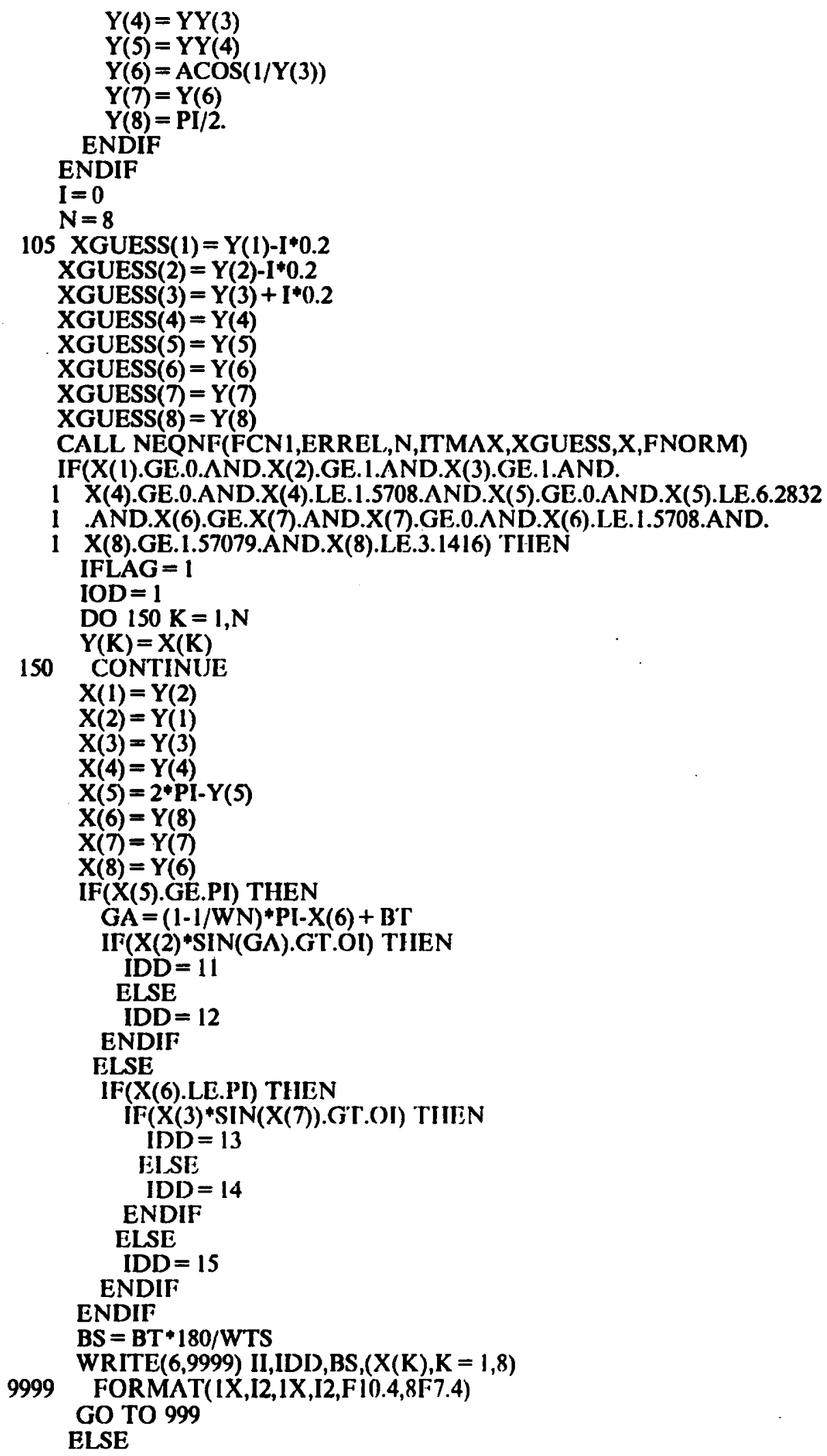




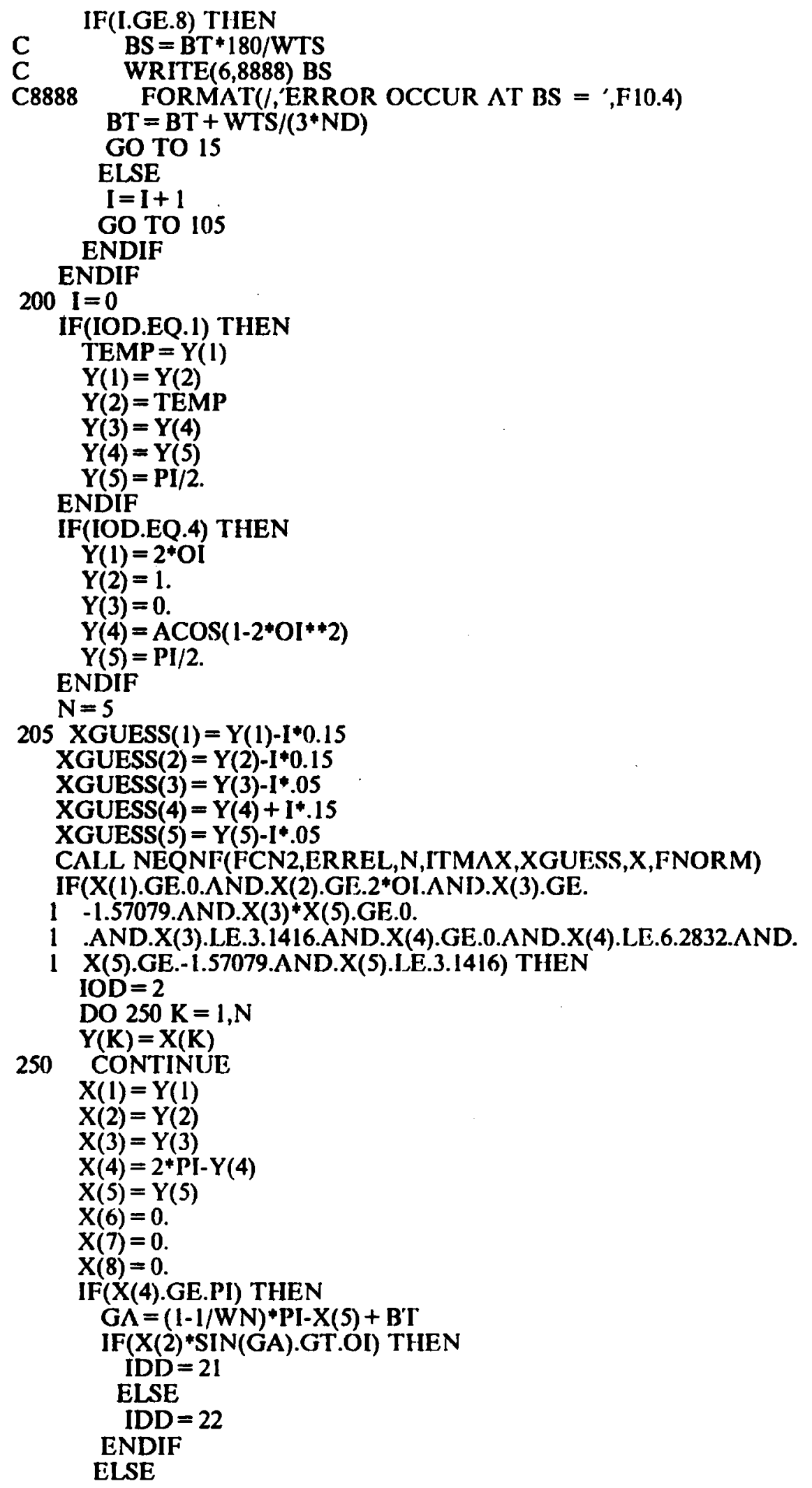




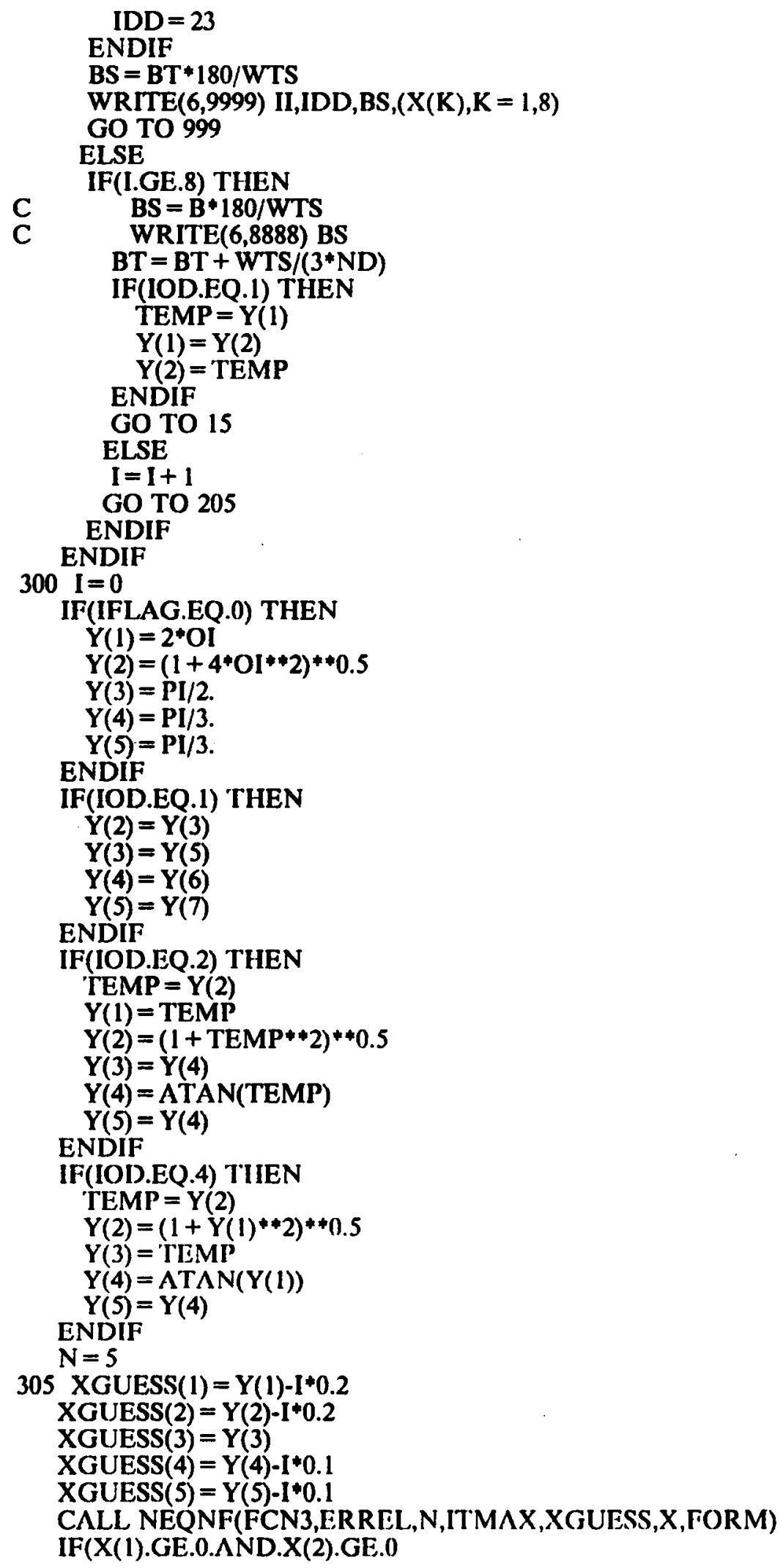




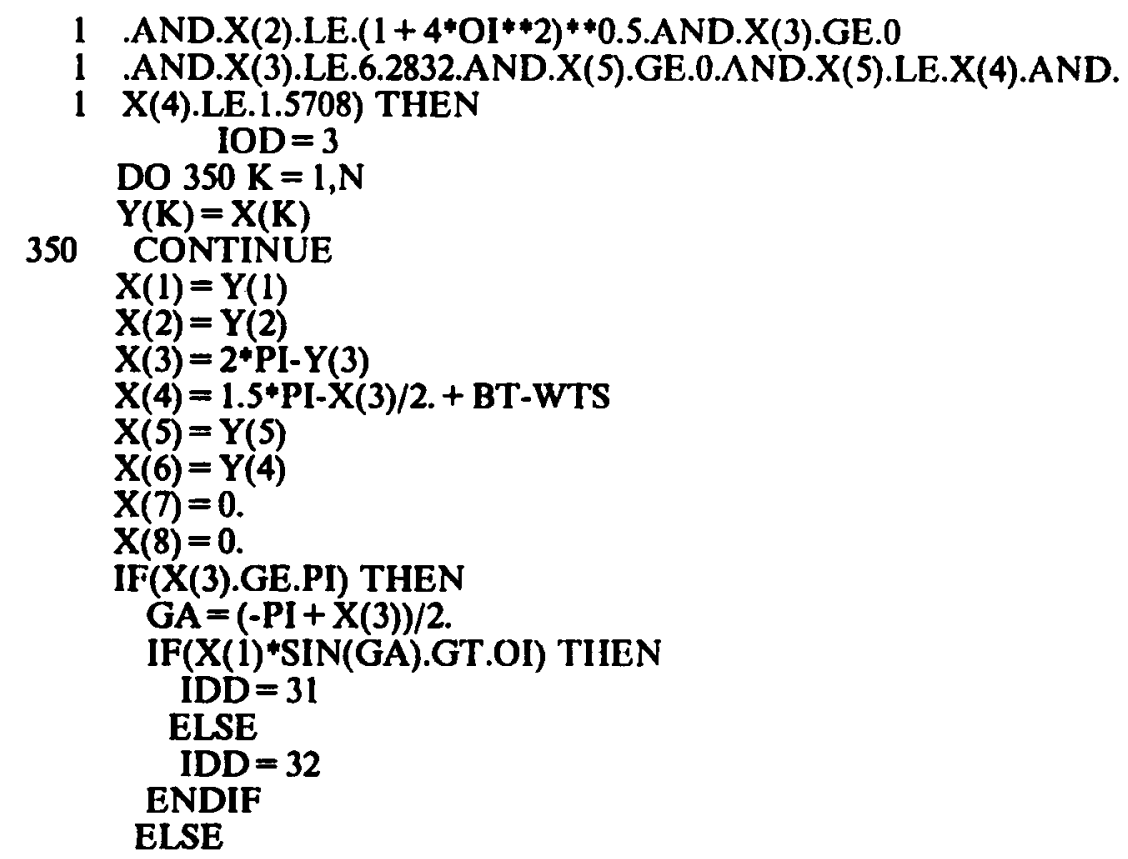


FORTRAN Program for Calculating Trajectory Parameters Above Resonant Frequency

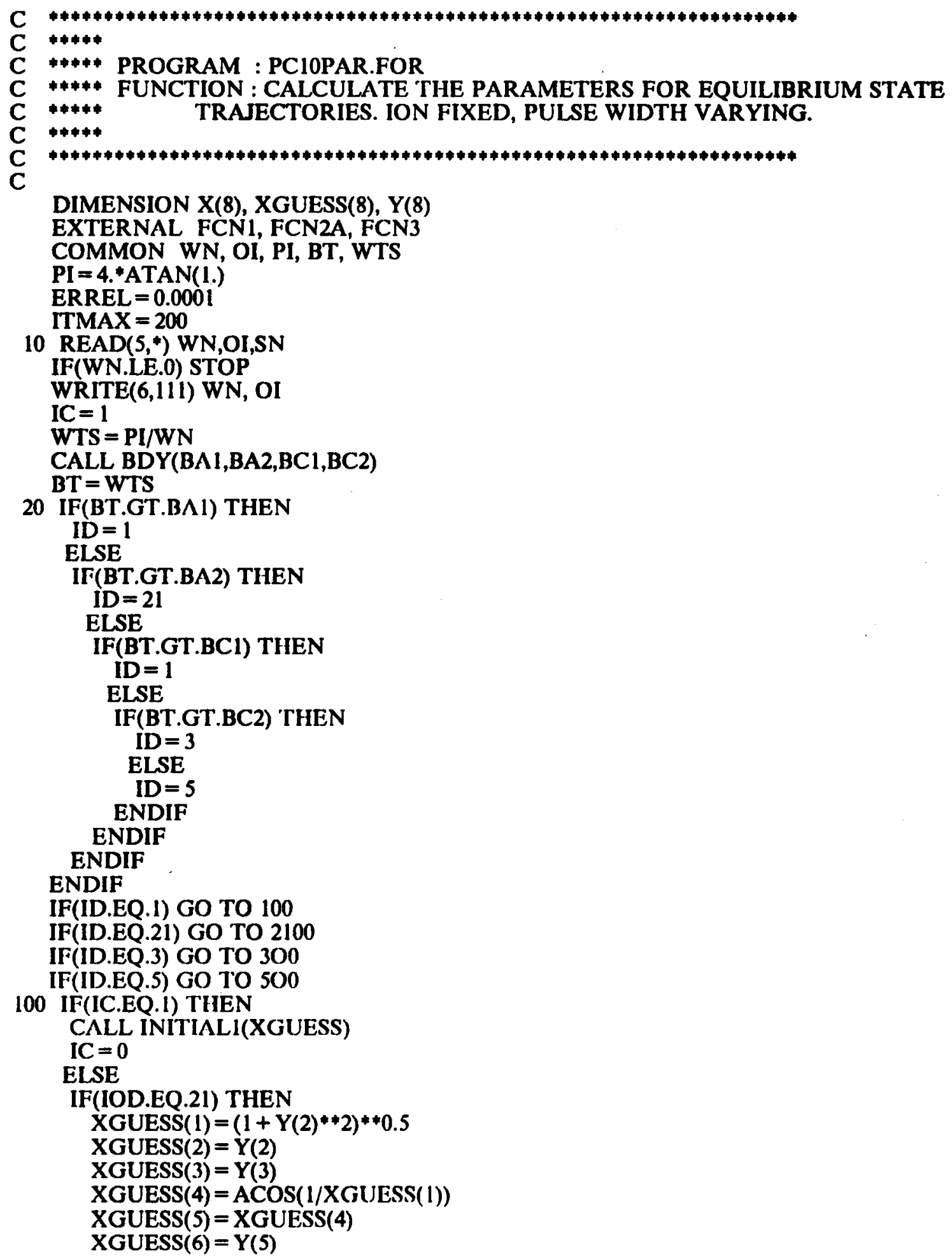




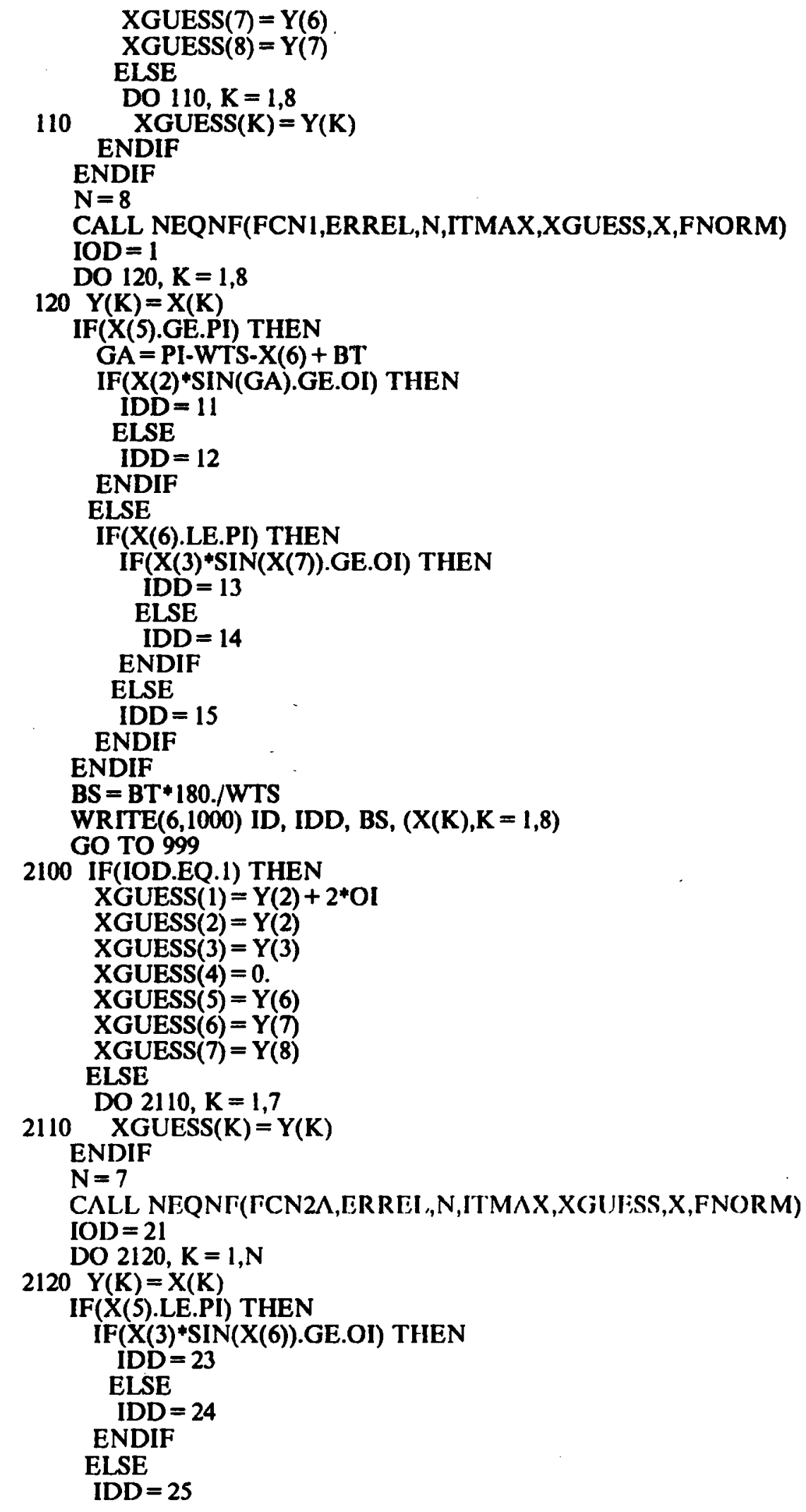




\section{ENDIF}

$\mathrm{BS}=\mathrm{BT} * 180 . / \mathrm{WTS}$

$\mathrm{X}(8)=0$.

WRITE $(6,1000)$ ID, IDD, BS, $(\mathrm{X}(\mathrm{K}), \mathrm{K}=1,8)$

GO TO 999

300 IF(IC.EQ.1) THEN

CALL INITIAL3(XGUESS)

IC $=0$

\section{ELSE}

IF(IOD.EQ.1) THEN

XGUESS $(1)=Y(2)$

XGUESS $(2)=Y(3)$

XGUESS $(3)=Y(5)$

XGUESS $(4)=Y(6)$

XGUESS $(5)=Y(7)$

XGUESS $(6)=Y(8)$

ELSE

IF(IOD.EQ.21) THEN

BT $=$ BT + WTS $/$ SN-0.005

GO TO 20

ENDIF

DO $310, K=1,6$

310

ENDIF

XGUESS $(K)=Y(K)$

ENDIF

$N=6$

CALL NEQNF(FCN3,ERREL,N,ITMAX,XGUESS,X,FNORM)

IOD $=3$

DO $320, K=1, N$

$320 \mathrm{Y}(\mathrm{K})=\mathrm{X}(\mathrm{K})$

IF(X (3).GE.PI) THEN

$\mathrm{GA}=(\mathrm{X}(3)-\mathrm{PI}) / 2$.

IF(X(I)*SIN(GA).GE.OI) THEN

IDD $=31$

ELSE

IDD $=32$

ENDIF

ELSE

IF(X(4).LE.PI) THEN

$\operatorname{IF}(X(2) * \operatorname{SIN}(X(5)) . G E . O I)$ THEN

IDD $=33$

ELSE

IF $(X(2) * \operatorname{SIN}(X(6))$.GT.OI) THEN IDD $=34$

ELSE

IDD $=36$

ENDIF

ENDIF

ELSE

$\operatorname{IF}(X(2) * \operatorname{SIN}(X(6))$.GT.OI) TIIEN

IDD $=35$

ELSE

IDD $=37$

ENDIF

ENDIF

ENDIF

$\mathrm{BS}=\mathrm{BT} * 180 . / \mathrm{WTS}$

$\mathrm{X}(7)=0$. 
$X(8)=0$.

WRITE $(6,1000)$ ID, IDD, BS, $(X(K), K=1,8)$

GO TO 999

500 IDD $=55$

$\mathbf{N}=\mathbf{2}$

$\mathrm{X}(1)=\mathrm{BT}$

$X(2)=$ WTS-BT

$\mathrm{BS}=\mathrm{BT} * 180 . / \mathrm{WTS}$

DO $505, K=3,8$

$505 \mathrm{X}(\mathrm{K})=0$.

WRITE(6,1000) ID, IDD, BS, (X(K), $K=1,8)$

999 IF(BT.EQ.0) GO TO 10

$\mathrm{BT}=\mathrm{BT}-\mathrm{WTS} / \mathrm{SN}$

IF(BT.LT.0) $\mathrm{BT}=0$

GO TO 20

111 FORMAT(1X,2F 10.5)

1000 FORMAT(IX,12,1X,I2,F 10.4,8F7.4)

END

C

C $* * * * * * * *$

C

C

SUBROUTINE BDY(BAI,BA2,BCl,BC2)

COMMON WN, OI, PI, BT, WTS

$10 \quad A=A \operatorname{COS}\left(1-O I^{* * 2}\right)$

$B=A C O S\left(\left(1+O I^{* * 2}\right) /\left(1+4 * O I^{* * 2}\right) * *(0.5)\right.$

$C=A \operatorname{Cos}\left(1 /\left(1+4^{*} O I^{* *} 2\right)^{* *} 0.5\right)$

$\mathrm{WNI}=\mathrm{PI} /(\mathrm{A}+\mathrm{B}+\mathrm{C})$

$\mathrm{WN} 2=\mathrm{PI} /(2 * \mathrm{OI})$

IF(WN.GE.WN2) THEN

$\mathrm{BCl}=$ WTS

$\mathrm{BC} 2=\mathrm{WTS}$

ELSE

IF(WN.GE.WNI) THIEN

$\mathrm{BCl}=\mathrm{W} \Gamma \mathrm{S}$

$\mathrm{BC} 2=2 * \mathrm{OI}$

ELSE

$\mathrm{BC} 2=2 * \mathrm{OI}$

$\mathrm{A}=\mathrm{ACOS}\left(-2^{*} \mathrm{OI}^{* * 2}+\left(1+4^{*} \mathrm{OI}^{* * 2}\right)^{* * 0.5}\right)$

$B=A C O S\left(1 /\left(1+4^{*} 0 I^{* *} 2\right) * * 0.5\right)$

$\mathrm{WN} 3=\mathrm{PI} /\left(0.5^{*}(\mathrm{PI}+\mathrm{A})+\mathrm{B}\right)$

IF(WN.GE.WN3) THEN

$\mathrm{RH}=\left(2^{* *} 0.5\right) * \mathrm{OI}$

$R L=(1+4 * 01 * * 2) * * 0.5-1$

$15 \quad \mathrm{R}=(\mathrm{RH}+\mathrm{RL}) / 2$.

C $\Lambda L L$ FQBDIII $(R, \Lambda L, B E 1, B E 2,(j \wedge I, G \Lambda 2)$

$W N C=P I /(A L+B E 1-B E 2+G \Lambda 2+G \Lambda 1)$

IF(ABS(WNC-WN).LE.1.E-4) TIIF,N

$\mathrm{BC} 1=(\mathrm{GA} 2+\mathrm{G} \Lambda 1+\Lambda \mathrm{L})$

ELSE

IF(WNC.GT.WN) THEN

RII $=$ R

EISE

$\mathbf{R L}=\mathbf{R}$

ENDIF

GO TO 15

ENDIF 


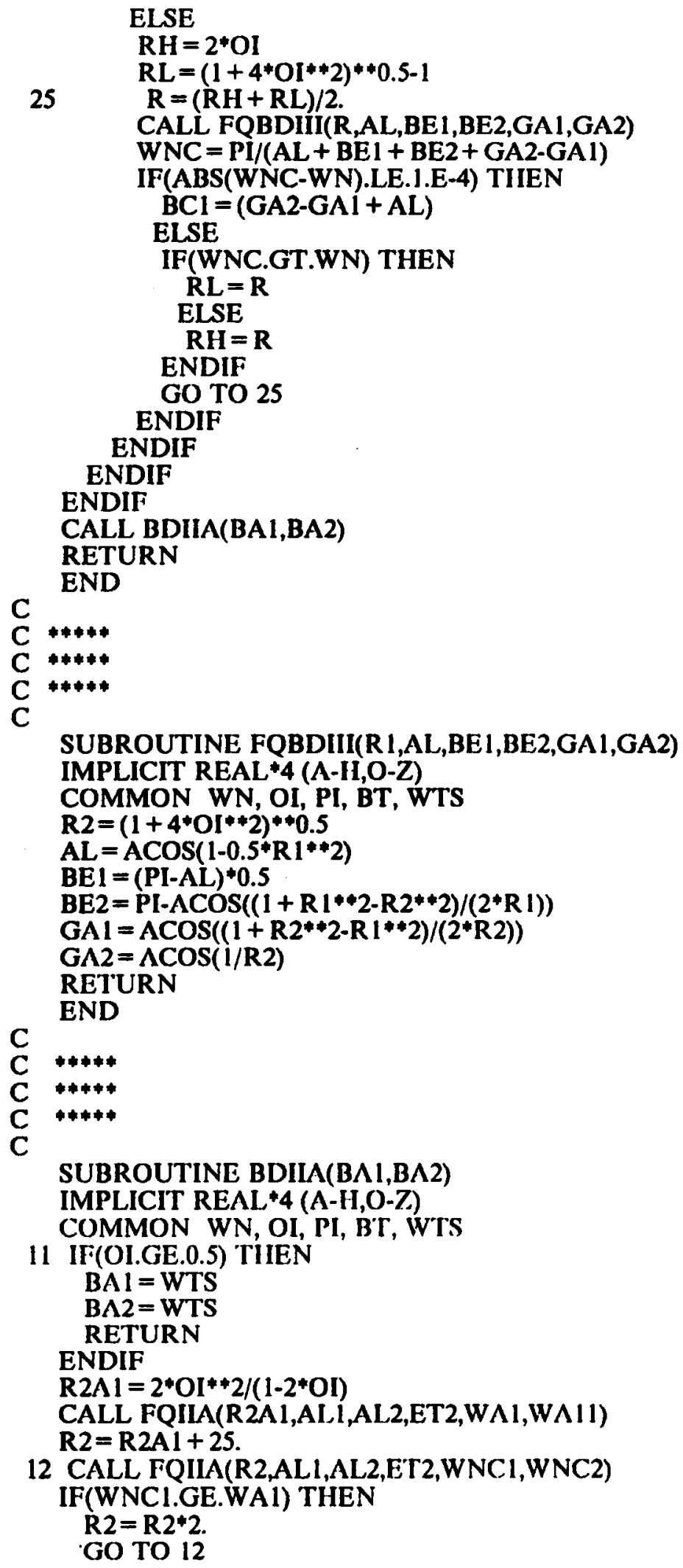

12 CALL FQIIA(R2,AL1,AL2,E'T2,WNC1,WNC2)

IF(WNCI.GE.WAI) THEN

$\mathrm{R} 2=\mathbf{R} 2 * 2$.

GO TO 12 


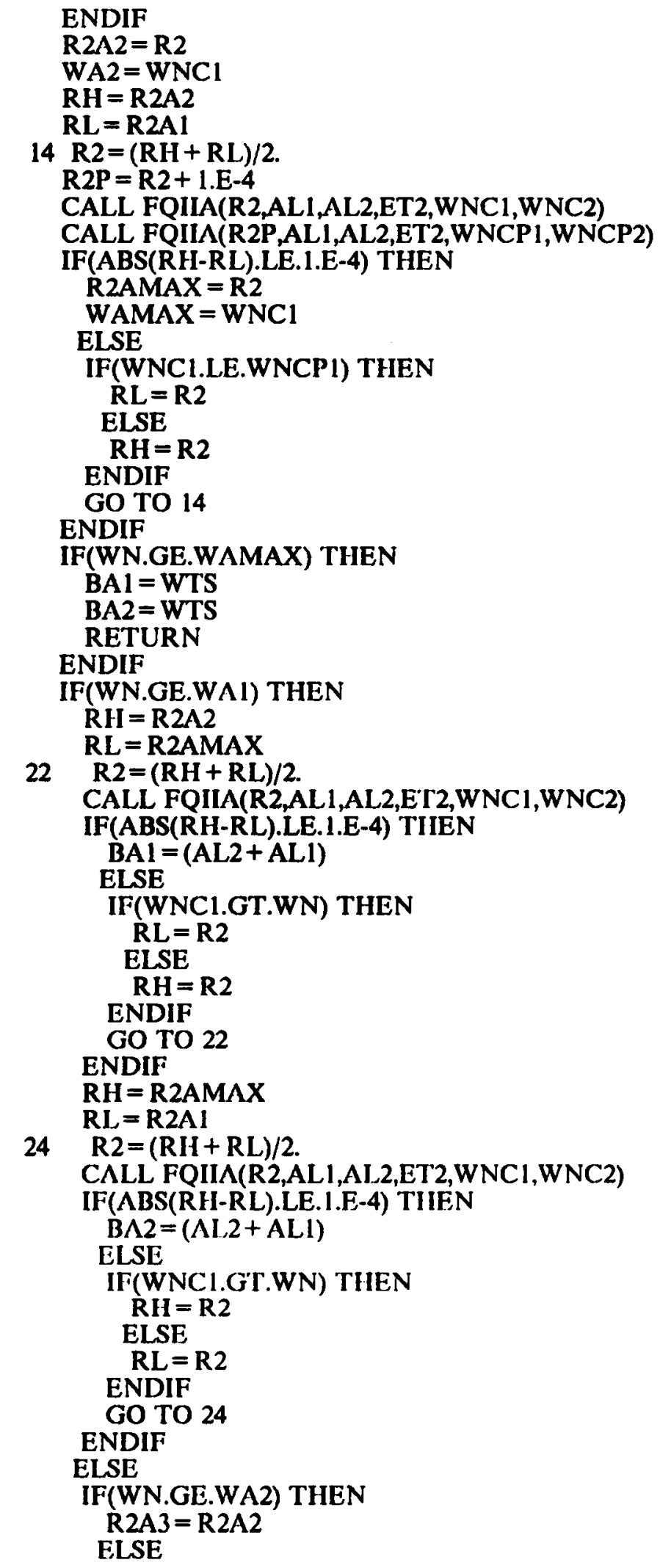




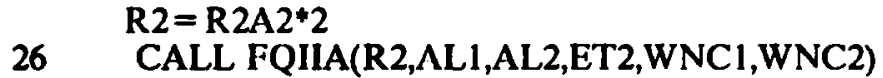
IF(WN.GT.WNCI) THEN

R2A3 = R2

ELSE

$\mathrm{R} 2=\mathrm{R} 2 * \mathbf{2}$

GO TO 26

ENDIF

ENDIF

RH $=$ R2A3

RL $=$ R2AMAX

$28 \quad \mathrm{R} 2=(\mathrm{RH}+\mathrm{RL}) / 2$.

CALL FQIIA(R2,AL1,AL2,ET2,WNCI,WNC2)

IF(ABS(RH-RL).LE.1.E-4) THIEN

$B A 1=(A L 2+A L 1)$

ELSE

IF(WNC1.GT.WN) THEN

$\mathbf{R L}=\mathbf{R} 2$

ELSE

$\mathrm{RH}=\mathrm{R} 2$

ENDIF

GO TO 28

ENDIF

R2=R2A1 + 5 .

32 CALL FQIIA(R2,AL1,AL2,ET2,WNC1,WNC2)

IF(WNC2.LE.WN) TIIEN

$$
\text { R2A4 = R2 }
$$

ELSE

$\mathrm{R} 2=\mathbf{R} 2 * 2$.

GO TO 32

ENDIF

RH $=$ R2A4

$\mathrm{RL}=\mathrm{R} 2 \mathrm{Al}$

$34 \quad \mathrm{R} 2=(\mathrm{RH}+\mathrm{RL}) / 2$.

CALL FQIIA(R2,AL1,AL2,ET2,WNC1,WNC2)

IF(ABS(RH-RL).LE.I.E-4) THEN

BA2 $=($ AL2-AL1)

\section{ELSE}

IF(WNC2.GT.WN) THEN

$\mathbf{R L}=\mathbf{R} 2$

ELSE

RH $=$ R2

ENDIF

GO TO 34

ENDIF

ENDIF

REIURN

C

END

C

$* * * * *$

C

SUBROUTINE FQIIA(R2,AL1,AL2,F:T2,WNC1,WNC2)

IMPLICIT REAL *4 (A-H,O-Z)

COMMON WN, OI, PI, B'T, WTS

$\mathrm{R} 3=((\mathrm{R} 2+2 * \mathrm{OI}) *+2+1) * *.(0.5$

$\mathrm{AL} 2=\mathrm{ACOS}(1 / \mathrm{R} 3)$ 


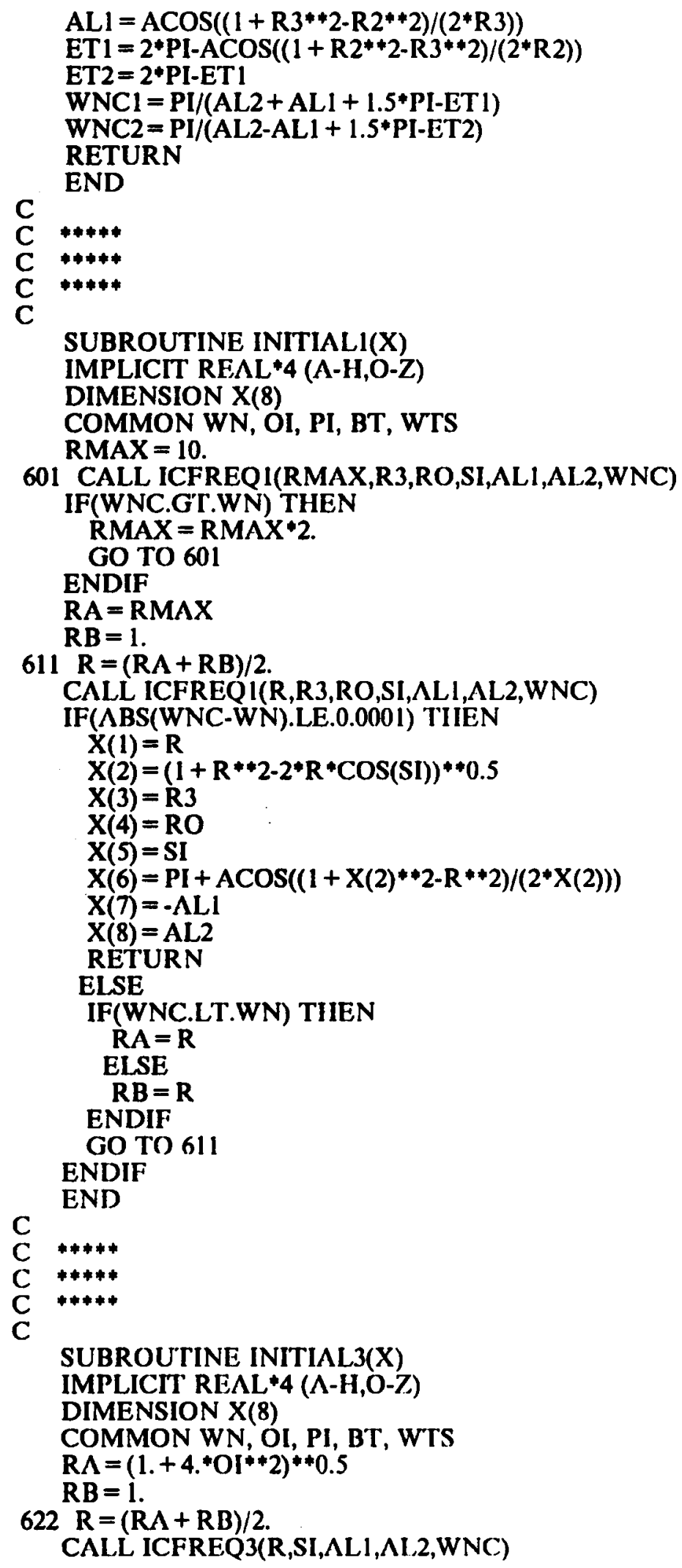




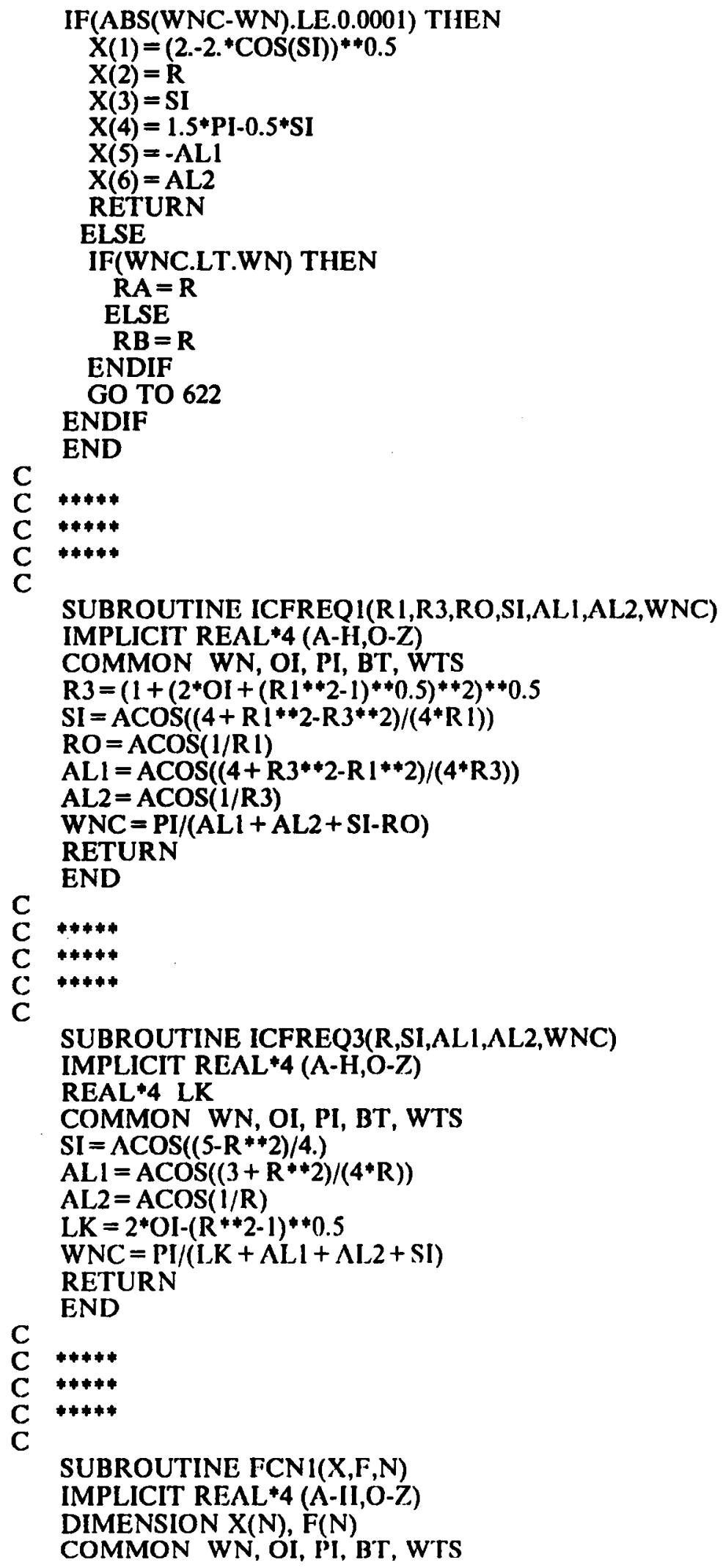




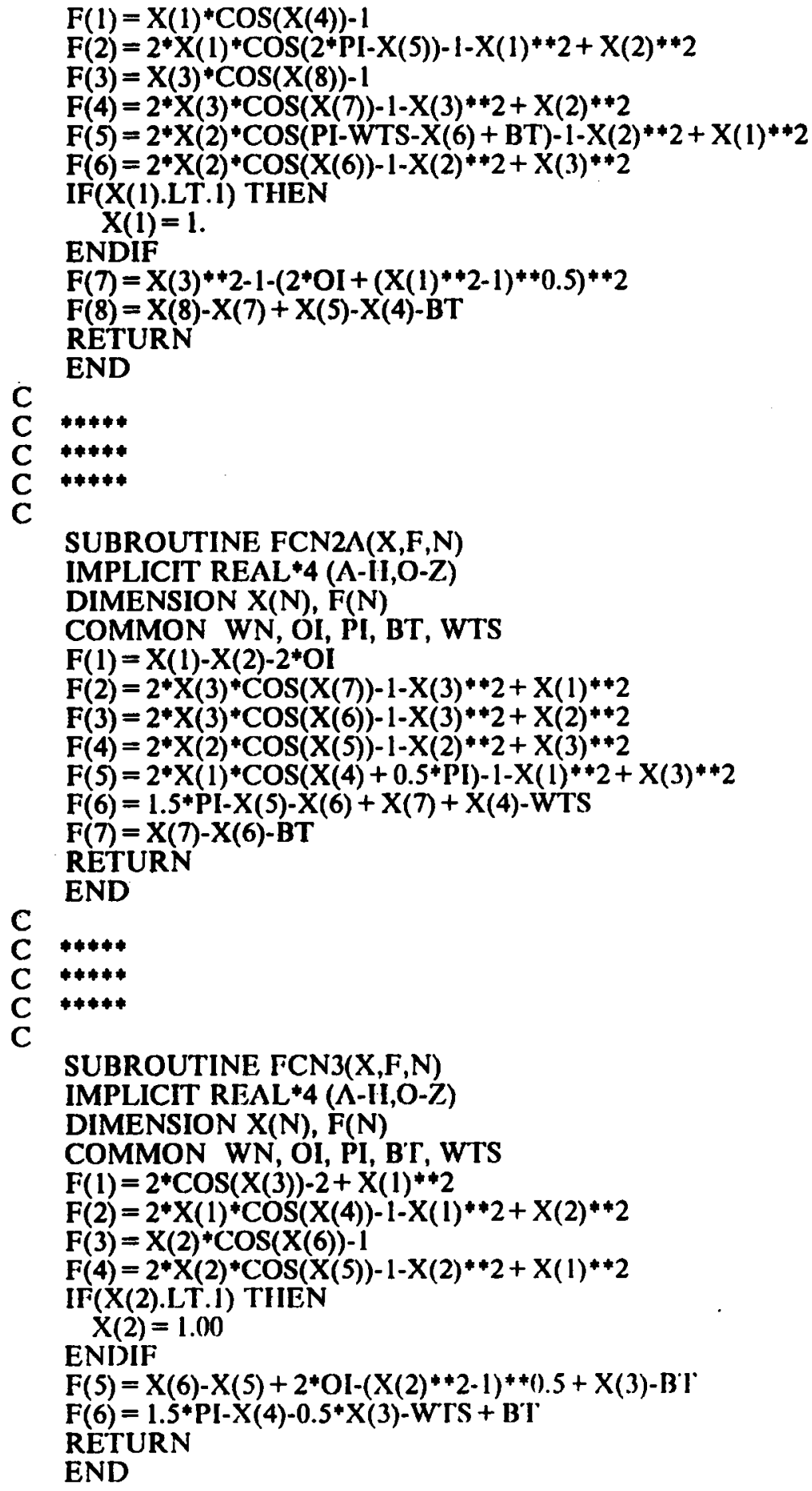




\section{APPENDIX C.5 \\ CALCULATION OF CIRCUIT SALIENT FEATURES \\ OF A CM-PRC}

Fortran Program for Galculating Circuit Salient Features of A CM-PRC (for Both Below and Above Resonant Frequency)

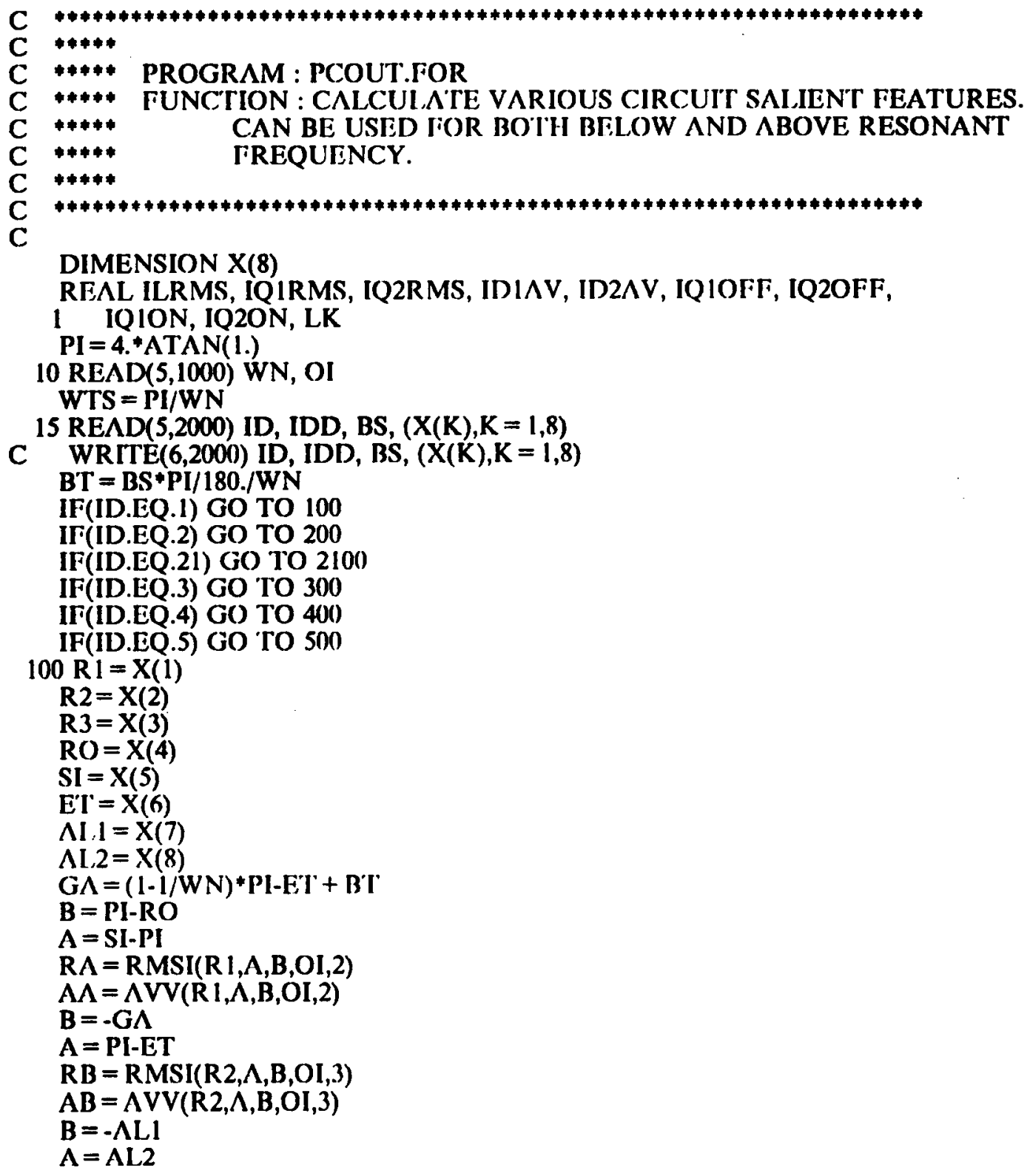




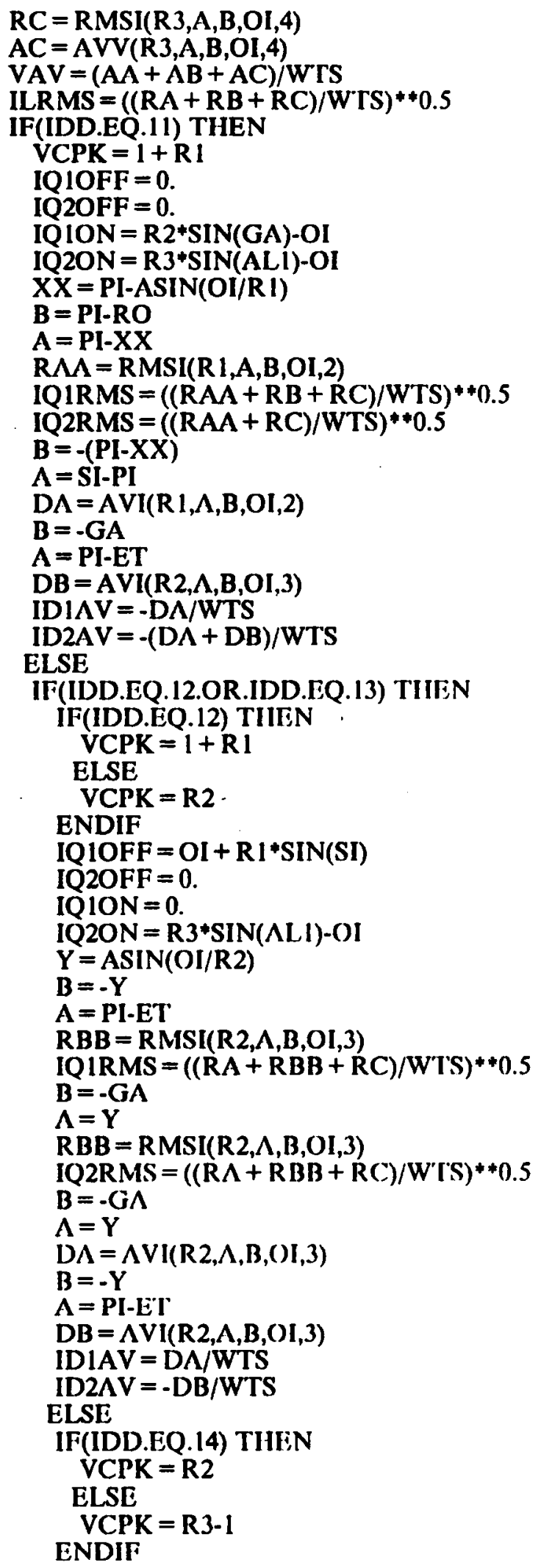




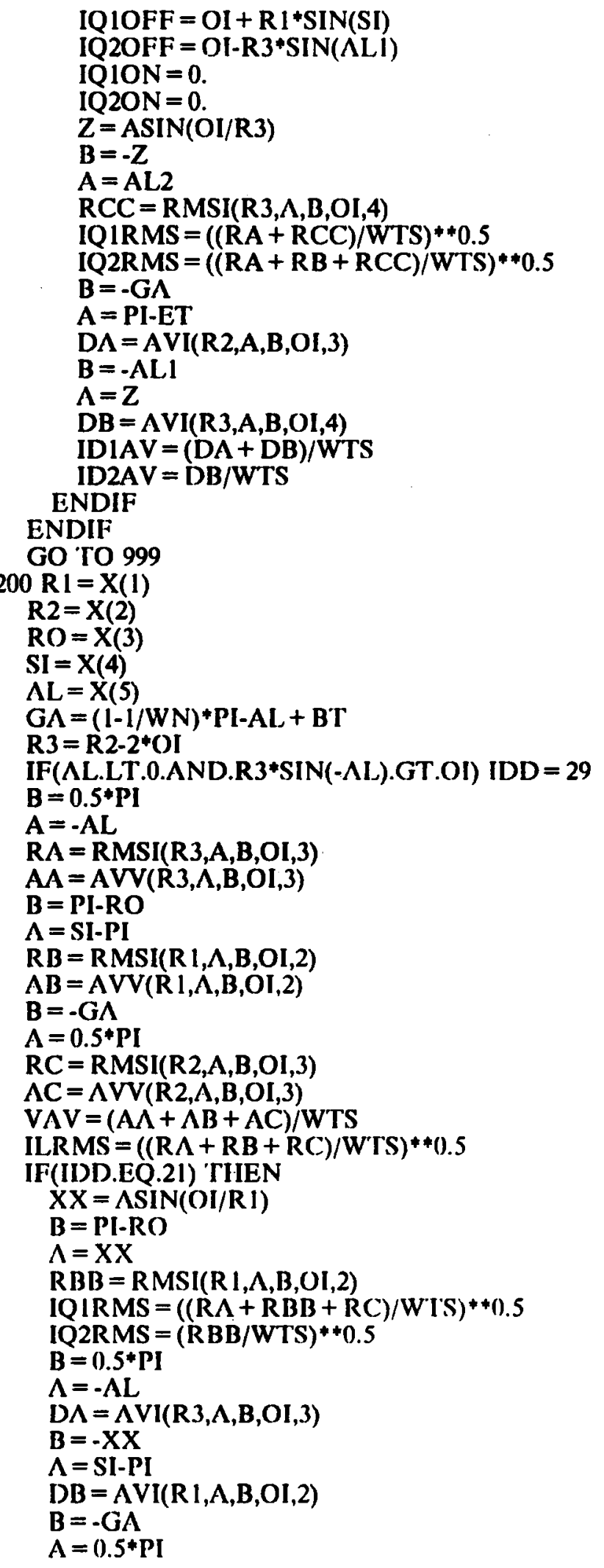




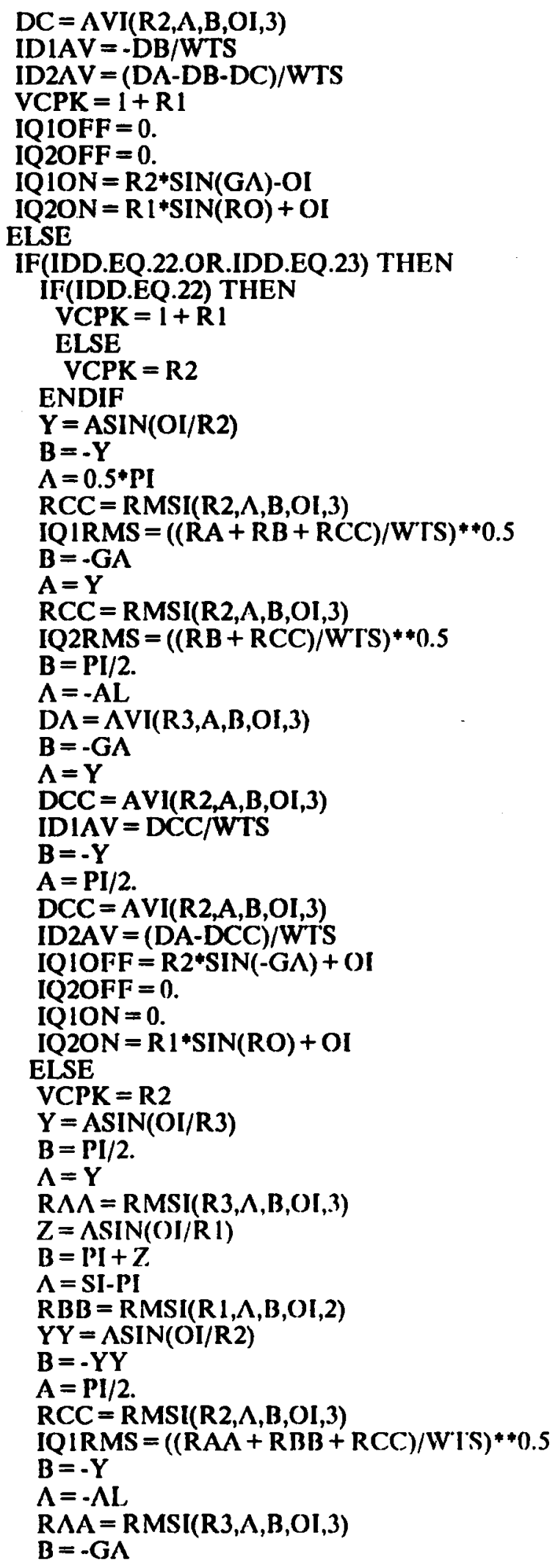




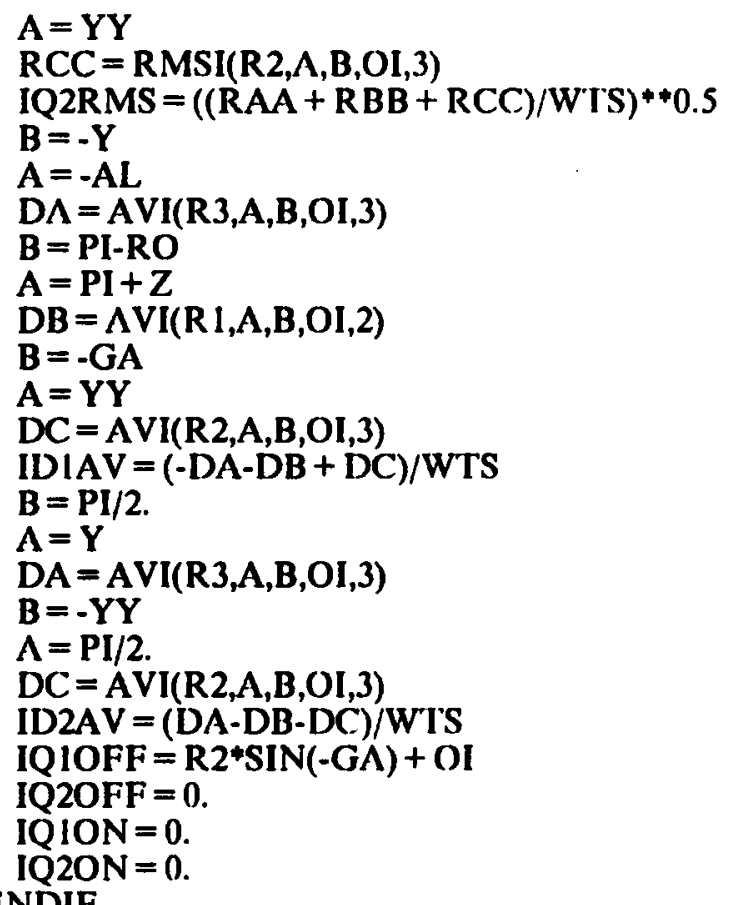

ENDIF

ENDIF

GO TO 999

$2100 \mathrm{R} 1=\mathrm{X}(1)$

$R 2=X(2)$

R3 $=$ X(3)

$\mathrm{RO}=\mathrm{X}(4)$

$E T=X(5)$

$\mathrm{AL} . \mathrm{l}=\mathrm{X}(6)$

$\mathrm{AL} 2=\mathrm{X}(7)$

$\mathrm{B}=0.5 * \mathrm{PI}$

$\Lambda=-(E T-P I)$

$\mathrm{RA}=\mathrm{RMSI}(\mathrm{R} 2, \mathbf{A}, \mathrm{B}, \mathrm{OI}, \mathbf{3})$

$A \Lambda=\Lambda V V(R 2, \Lambda, B, O I, 3)$

$B=-A L 1$

$A=\mathbf{A L 2}$

RB = RMSI(R3,A,B, OI,4)

$A B=\Lambda V V(R 3, \Lambda, B, O I, 4)$

$B=-(0.5 * P I-R O)$

$\Lambda=0.5 * \mathrm{PI}$

$\mathrm{RC}=\mathrm{RMSI}(\mathrm{R} I, \Lambda, \mathrm{B}, \mathrm{OI}, 3)$

$\Lambda \mathrm{C}=\Lambda \mathrm{VV}(\mathrm{R} I, \Lambda, \mathrm{B}, \mathrm{OI}, 3)$

$\mathrm{V} \Lambda \mathrm{V}=(\Lambda \mathrm{A}+\Lambda \mathrm{B}+\Lambda \mathrm{C}) / \mathrm{W} \mathrm{TS}$

IL.RMS $=((\mathrm{R} \Lambda+\mathrm{RB}+\mathrm{RC}) / \mathrm{WIS}) * * 0.5$

IF(IDD.EQ.23) THIEN

$\mathrm{XX}=\mathrm{ASIN}(\mathrm{OI} / \mathrm{R} 2)$

$B=\cdot X X$

$\Lambda=P I-E T$

$\mathrm{R} \Lambda \mathrm{A}=\mathrm{RMSI}(\mathrm{R} 2, \mathrm{~A}, \mathrm{~B}, \mathrm{OI}, 3)$

IQIRMS $=(($ RAA + RB $) /$ WTS $) * * 0.5$

$\mathrm{B}=0.5^{+} \mathrm{PI}$

$\mathrm{A}=\mathrm{XX}$

$\mathrm{RA} \Lambda=\mathrm{RMSI}(\mathrm{R} 2, \mathrm{~A}, \mathrm{~B}, \mathrm{OI}, 3)$

IQ2RMS $=((R \wedge A+R B+R C) /$ WTS $) *(0.5$ 


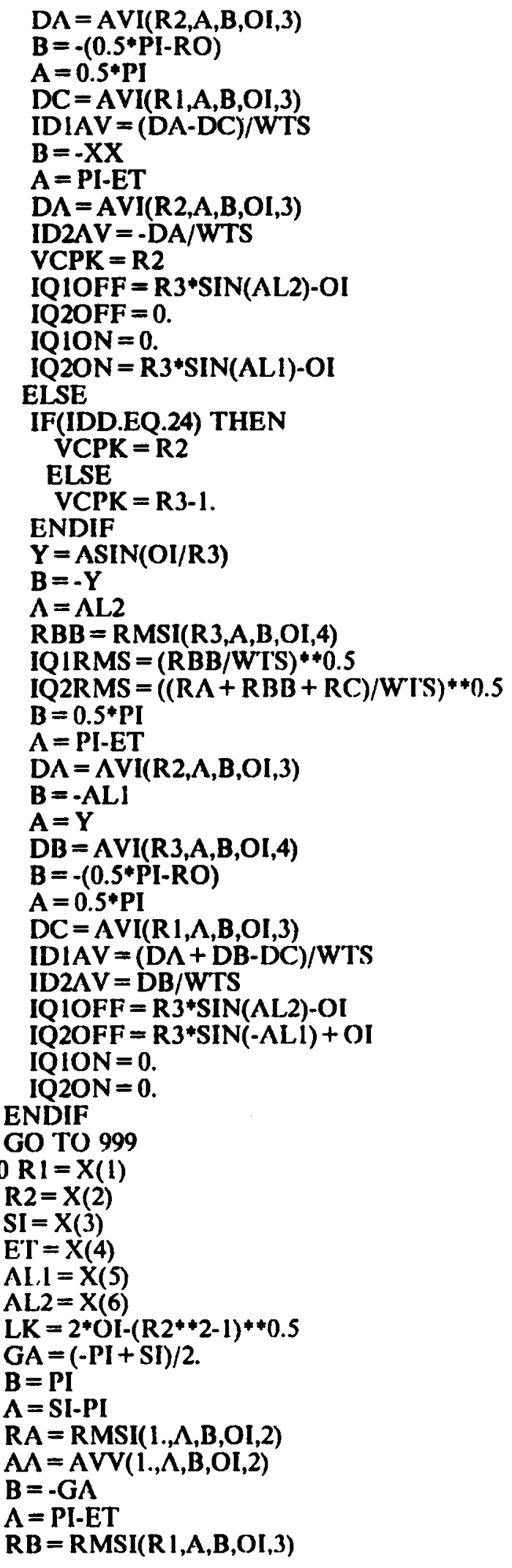




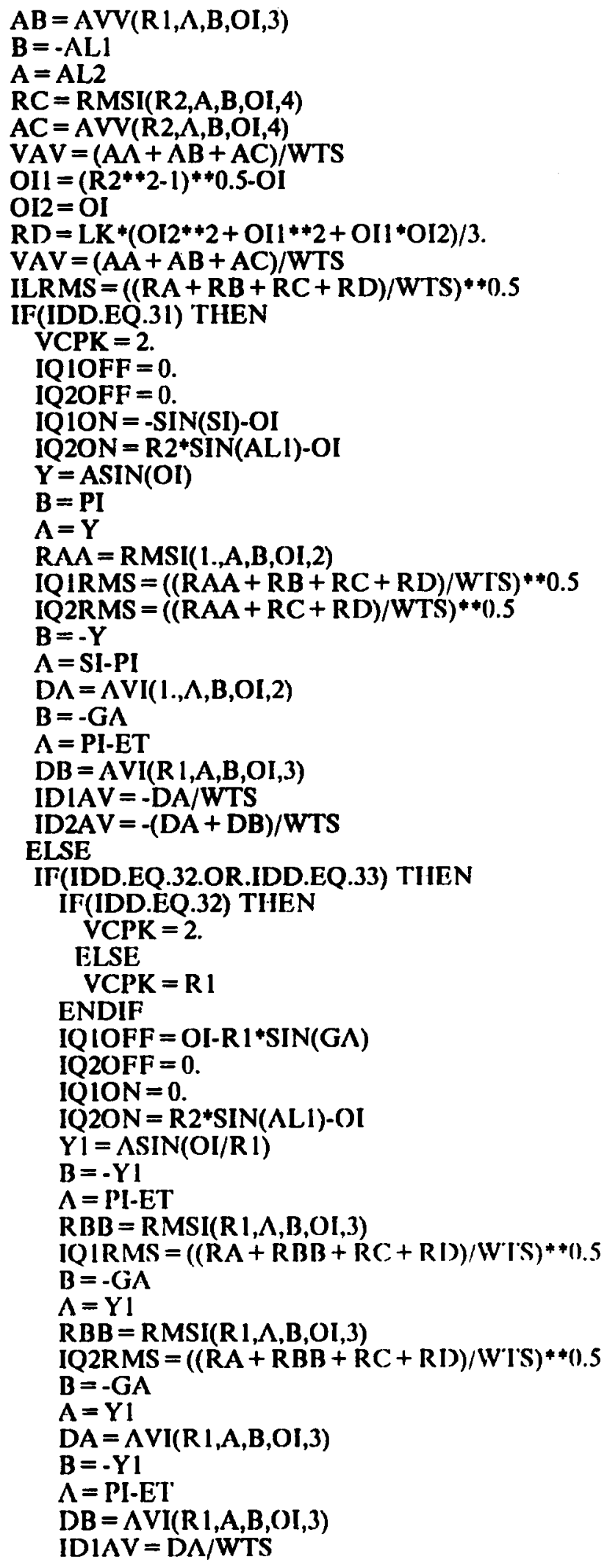




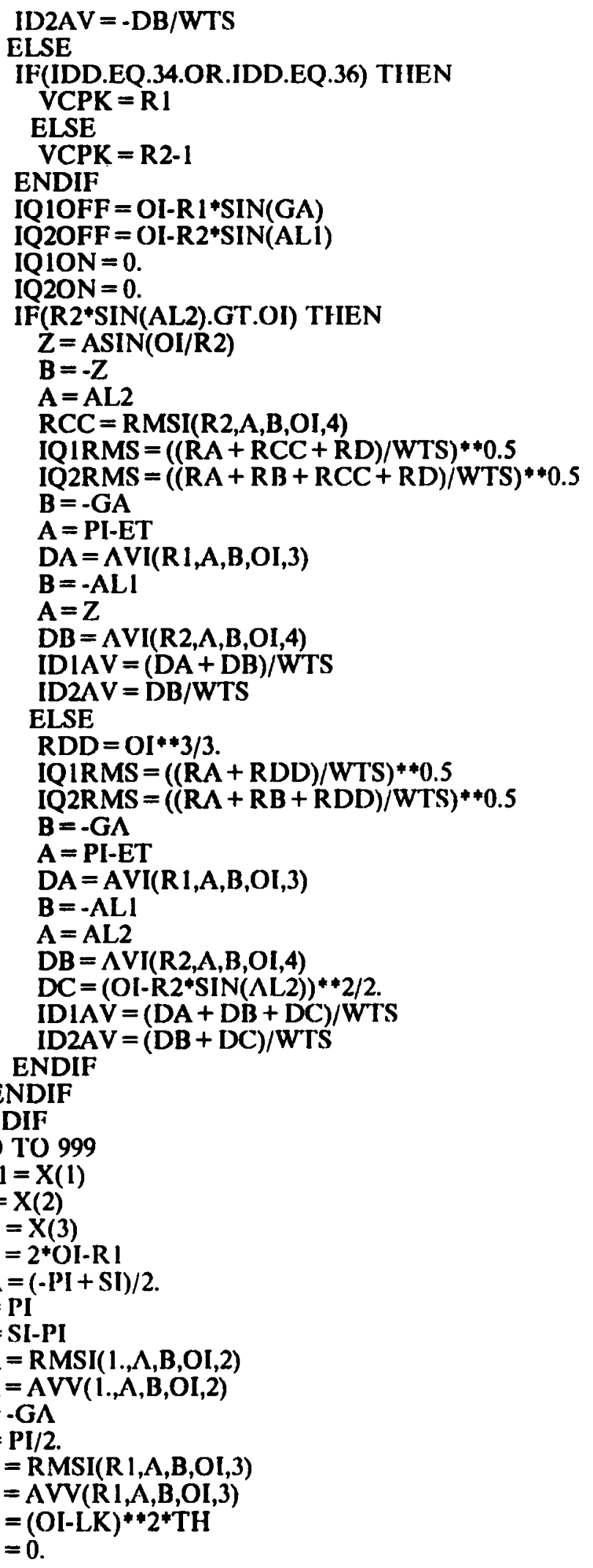




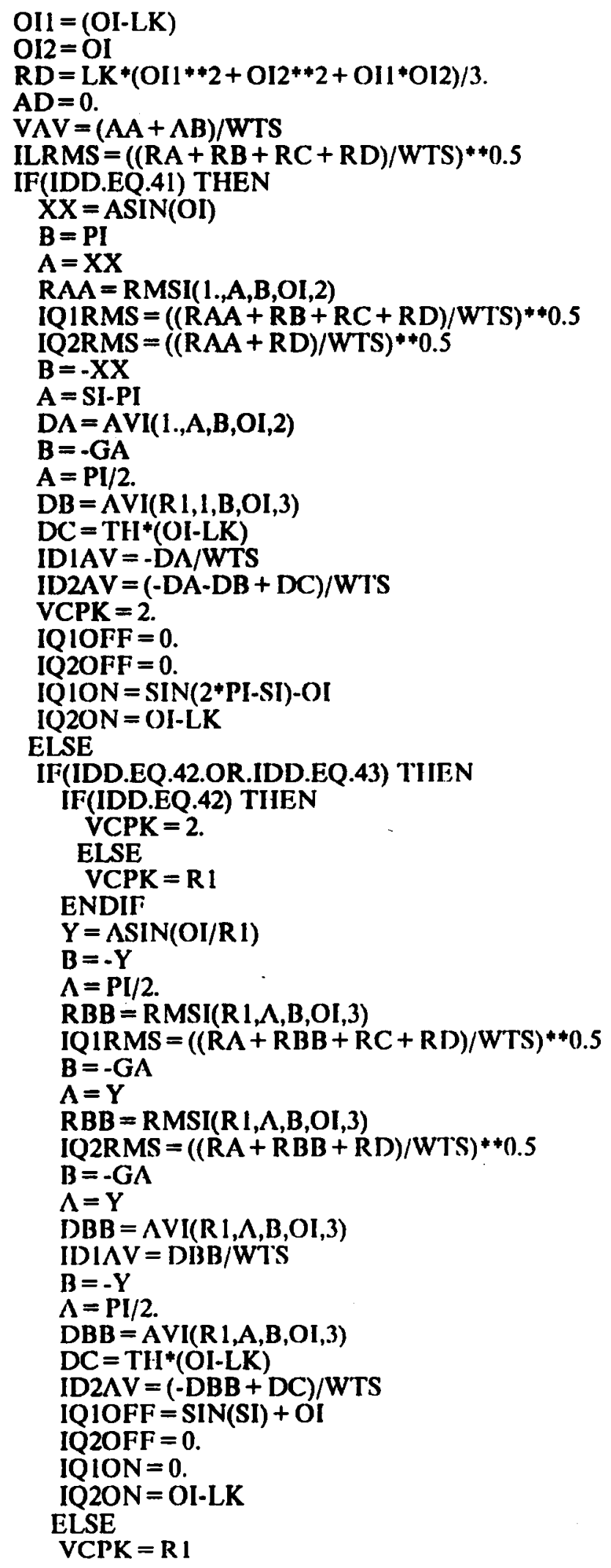




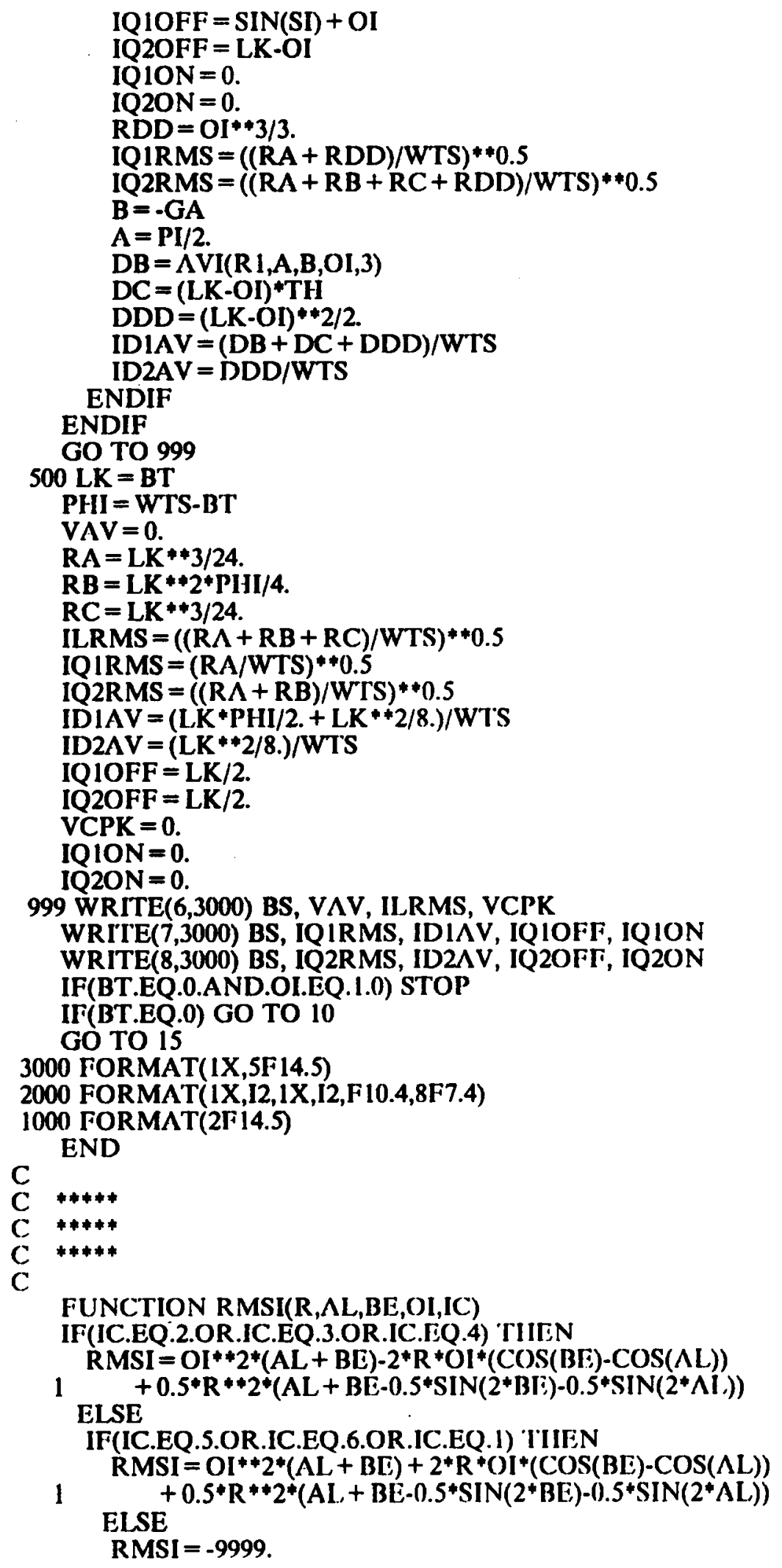




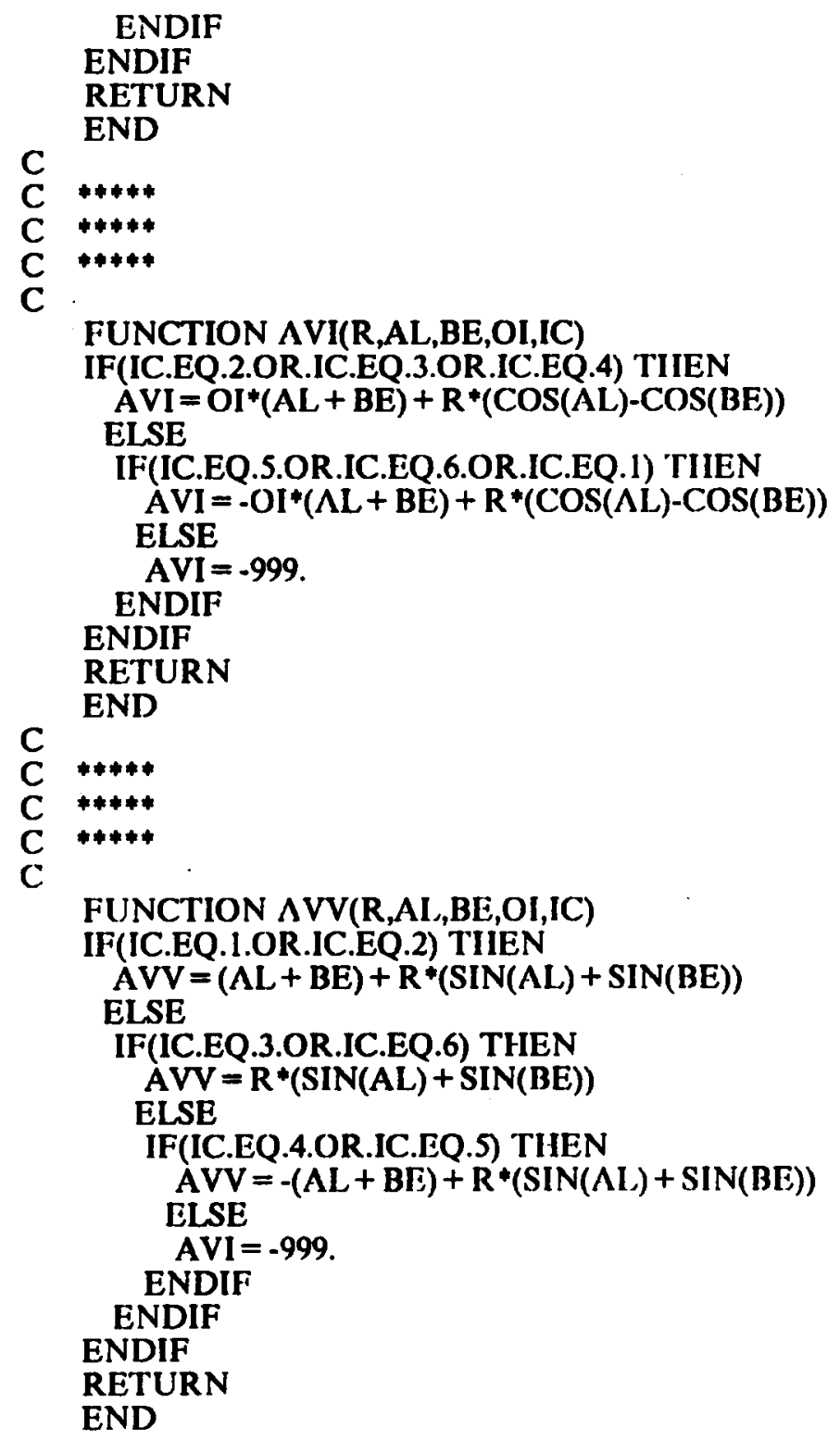




\section{APPENDIX C.6 \\ DC CHARACTERISTICS BELOW \\ RESONANT FREQUENCY}

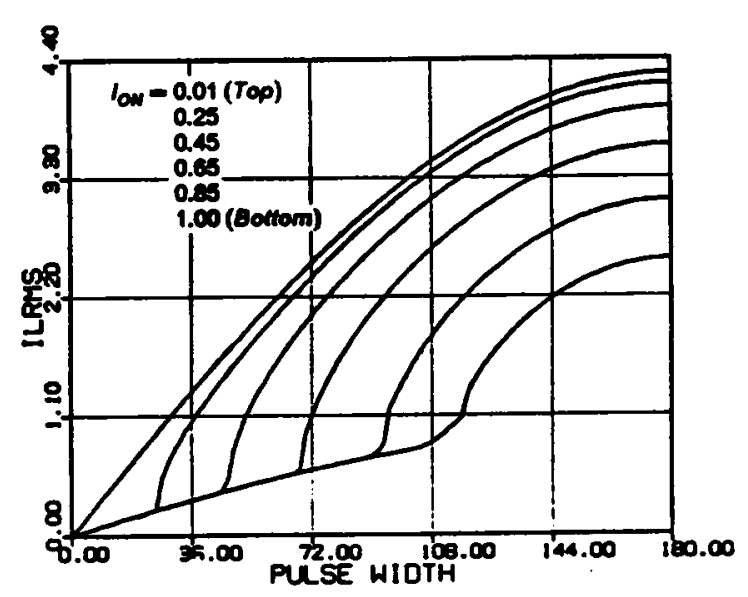

- RMS inductor current

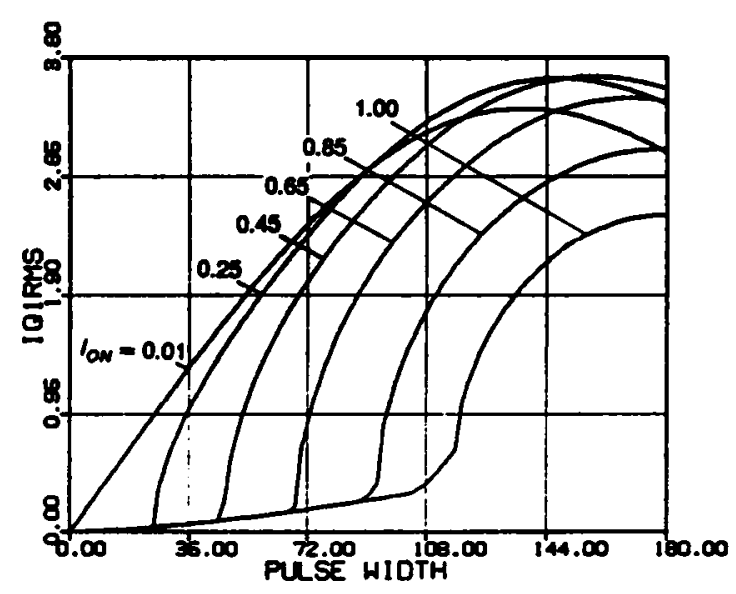

- RMS switch current (Q1,Q3)

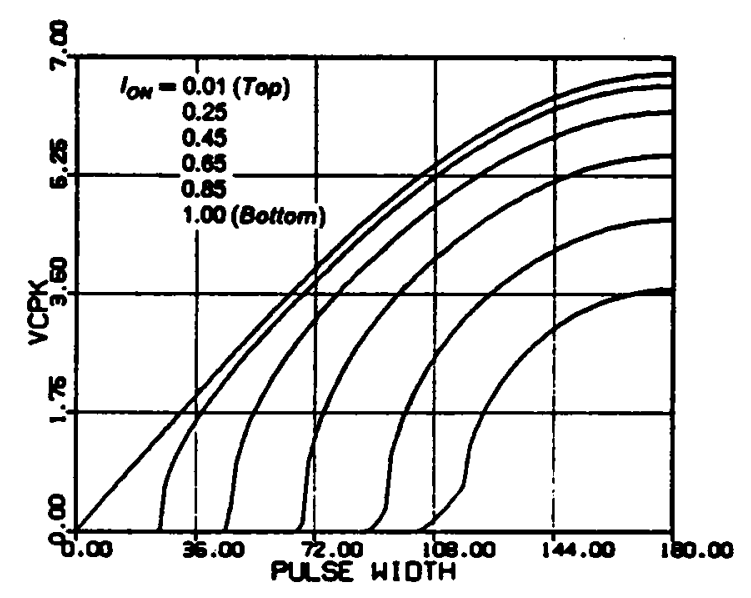

- Peak capacitor voltage

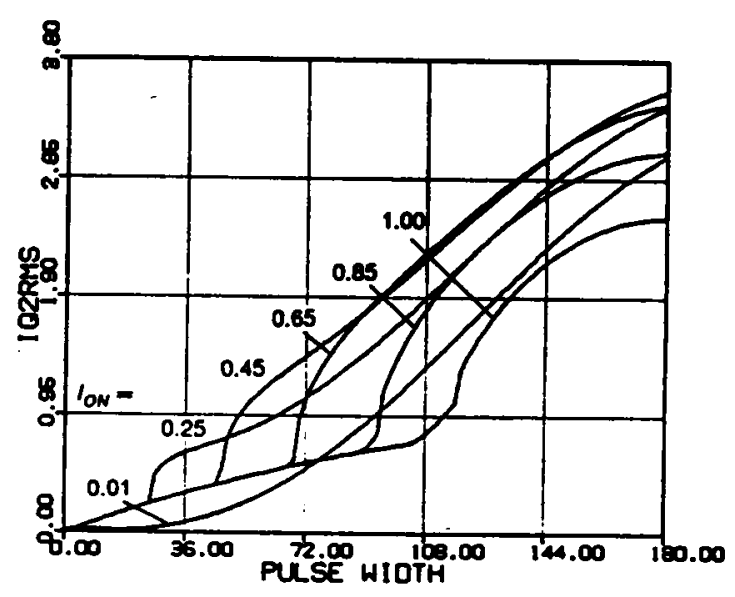

- RMS switch current $(Q 2, Q 4)$

Figure C.6.1 DC Characteristics for $\omega_{S N}=0.9$ 


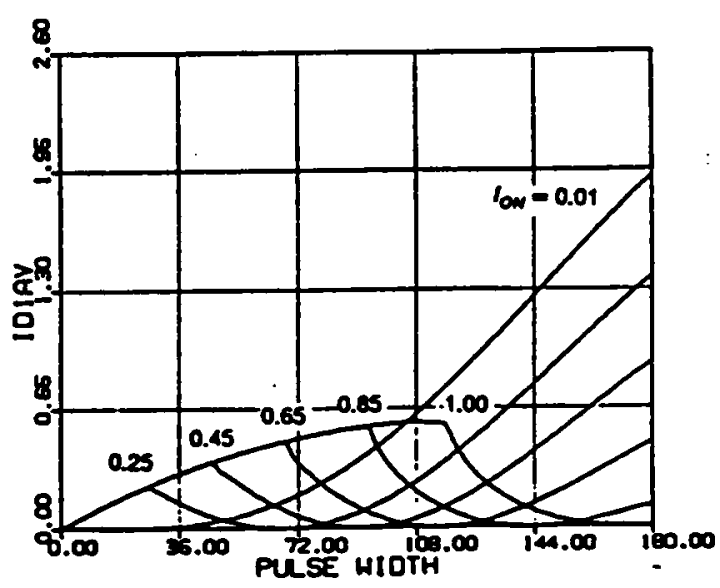

- Average diode current (DI.D3)

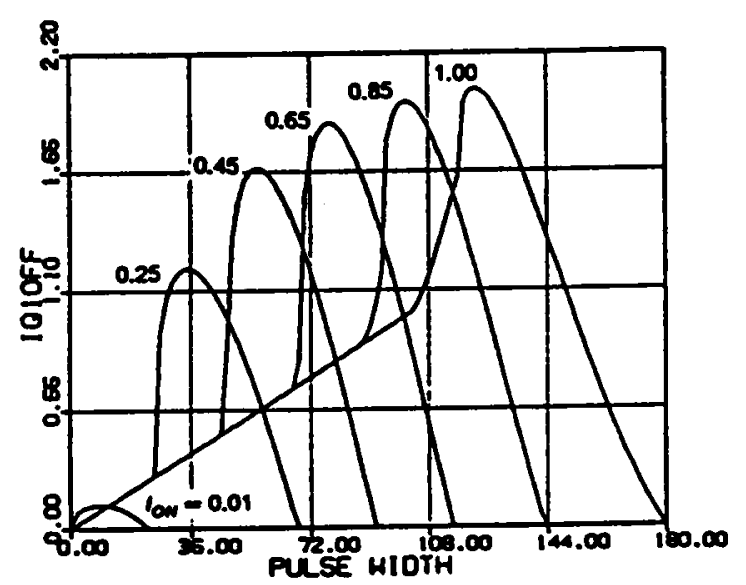

- Switch turn-off current (QI.Q3)

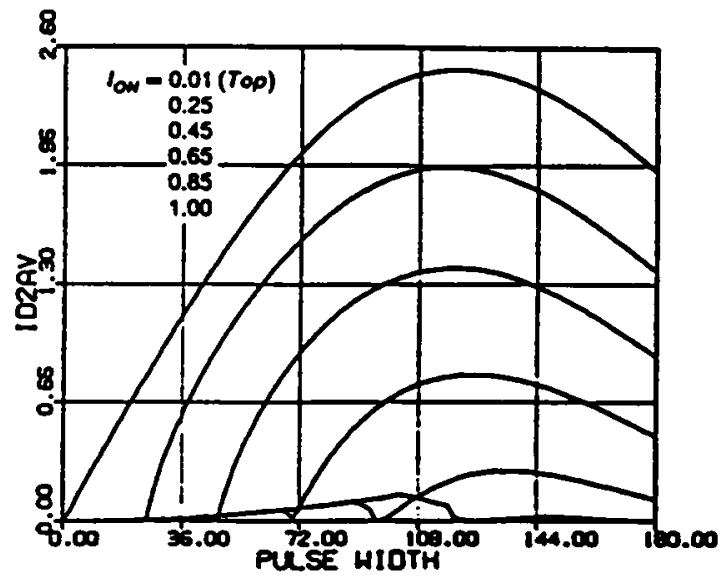

- Average diode current (D2.D4)

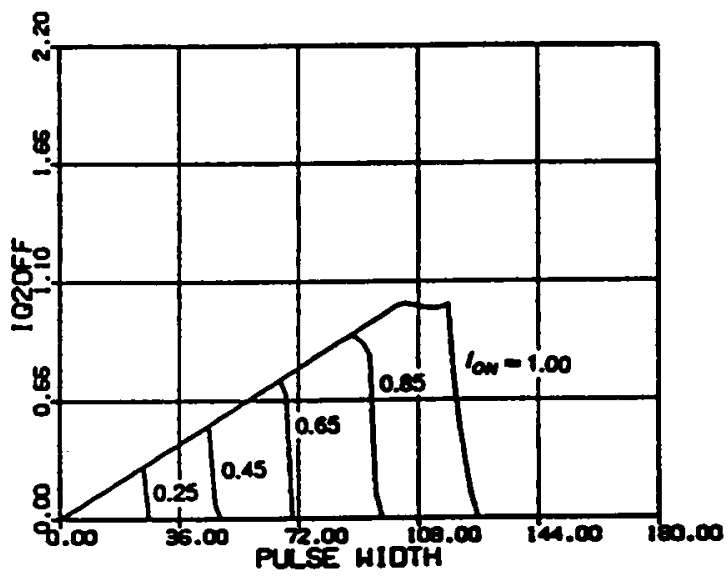

- Switch turn-off current (Q2.Q4)

Figure C.6.1 Continued 


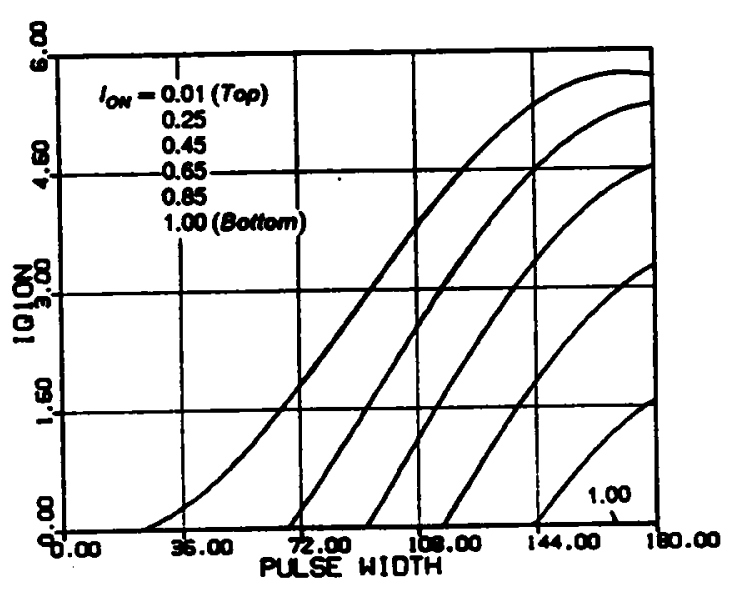

- Switch turn-on current $(Q 1, Q 3)$

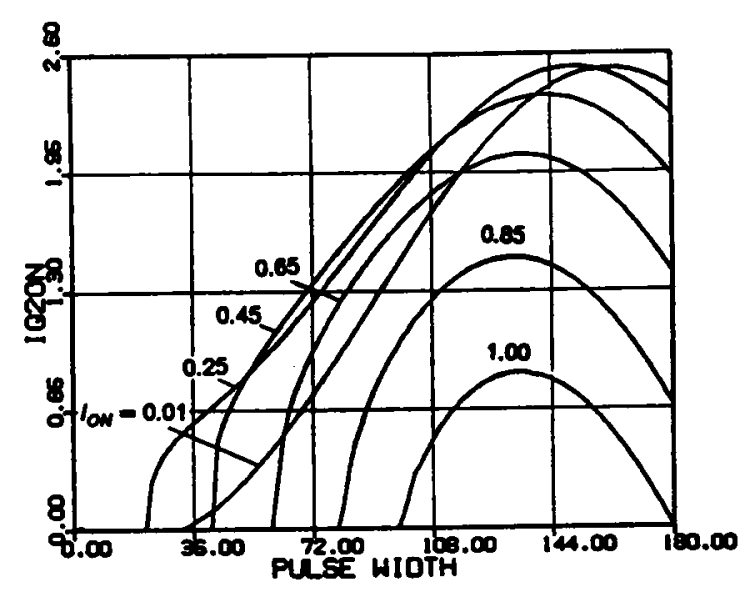

- Switch turn-on current (Q2.Q4)

Figure C.6.1 Continued 


\section{APPENDIX C.7}

\section{CHARACTERISTICS ABOVE \\ RESONANT FREQUENCY}

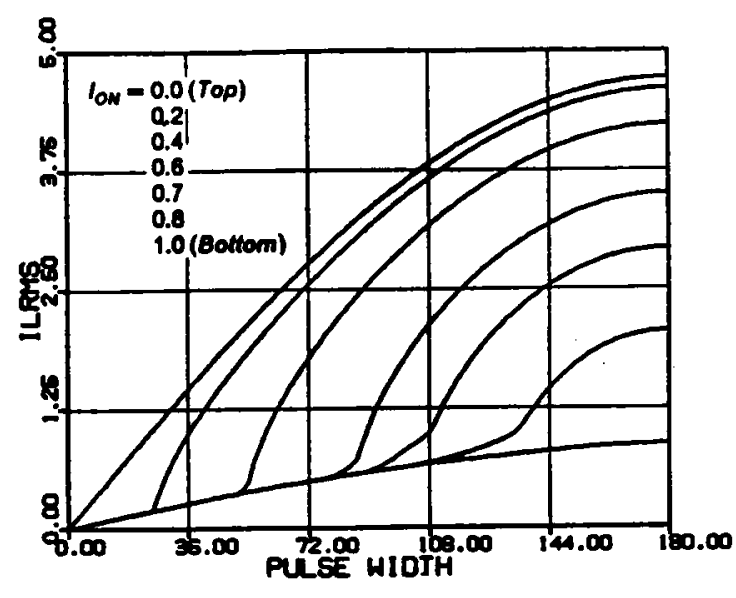

- RMS inductor current

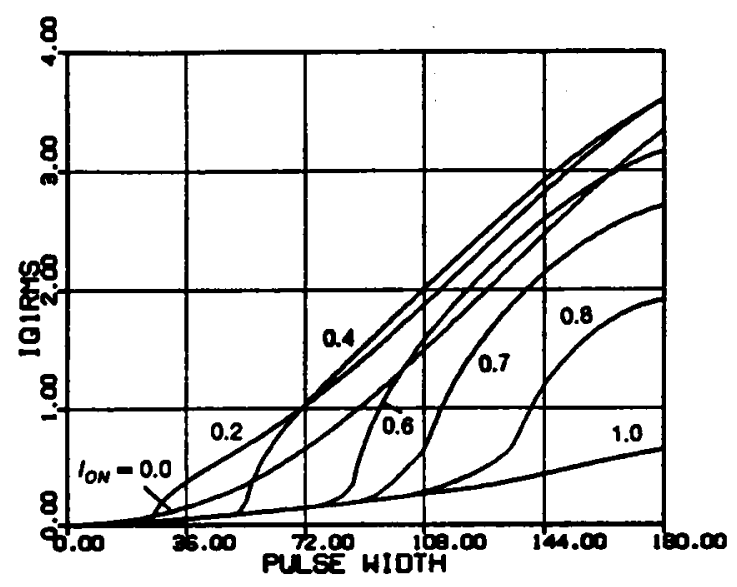

- RMS switch current $(Q 1, Q 3)$

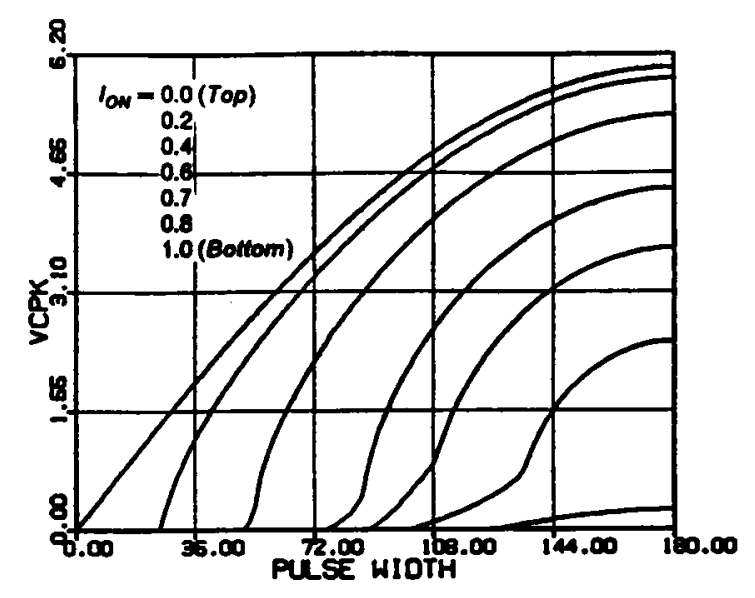

- Peak capacitor voltage

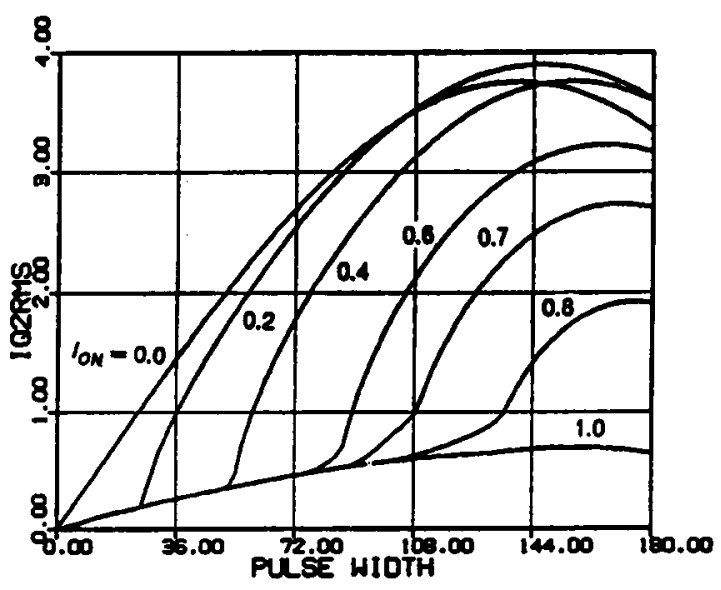

- RMS switch current (Q2,Q4)

Figure C.7.1 DC Characteristics for $\omega_{S N}=1.1$ 


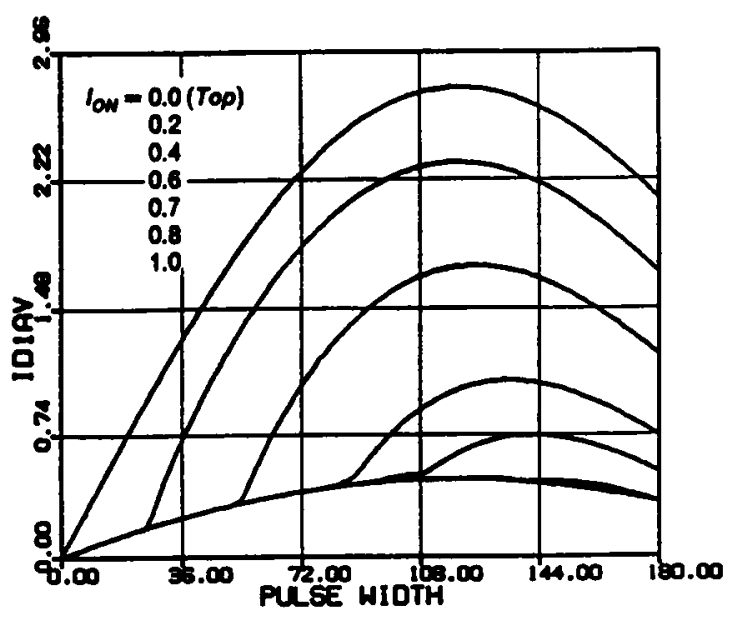

- Average diode current (DI,D3)

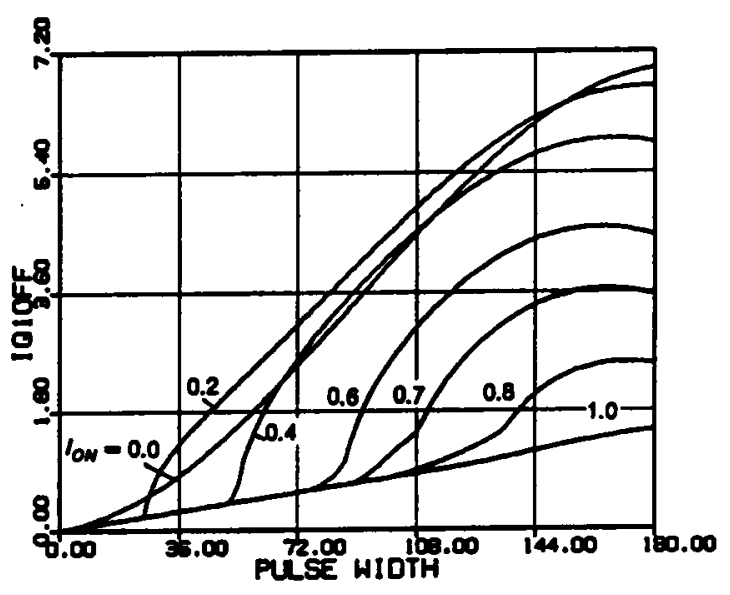

- Switch turn-off current (Q1,Q3)

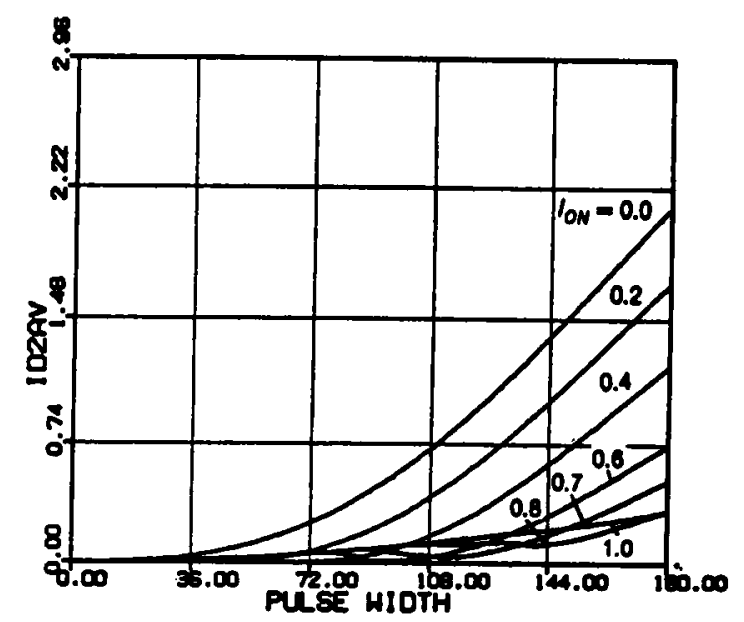

- Average diode current (D2,D4)

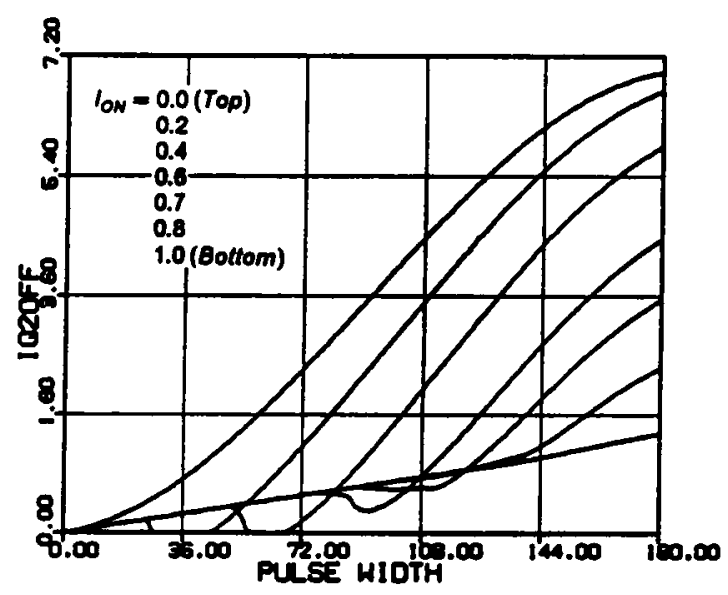

- Switch turn-off current $(Q 2, Q 4)$

Figure C.7.1 Continued 


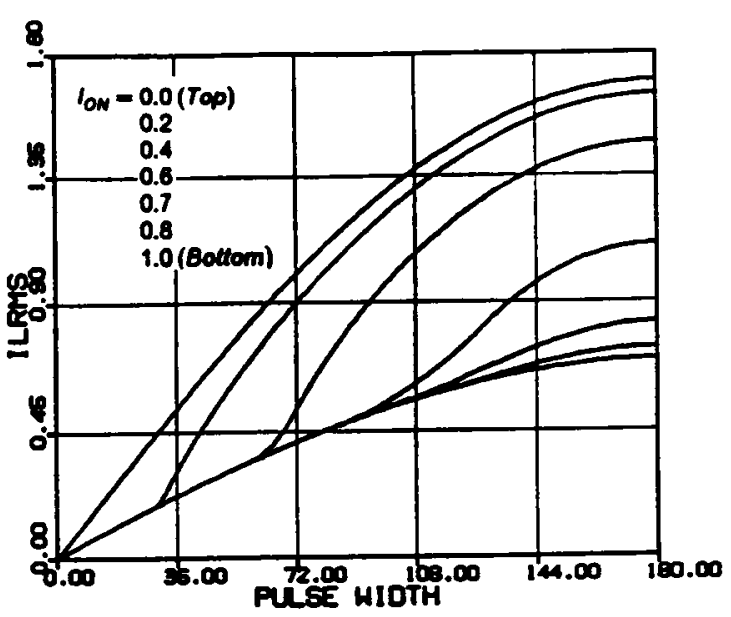

- RMS inductor current

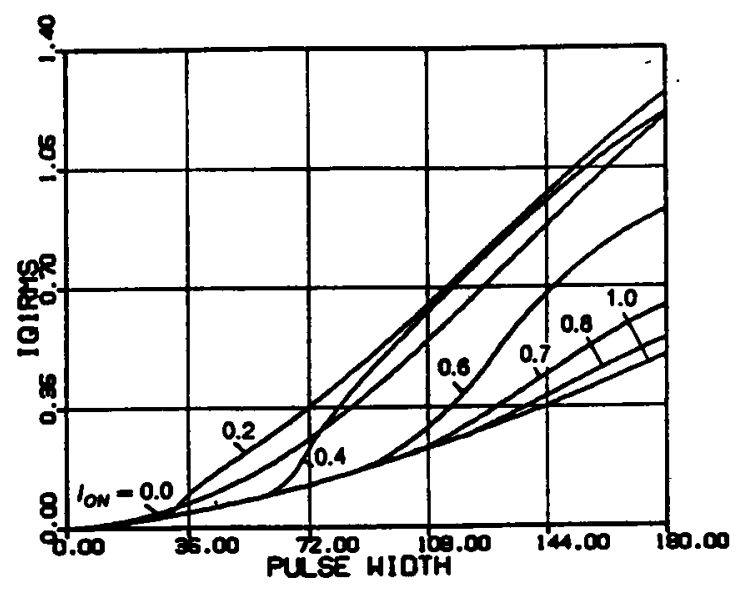

- RMS switch current (QI,Q3)

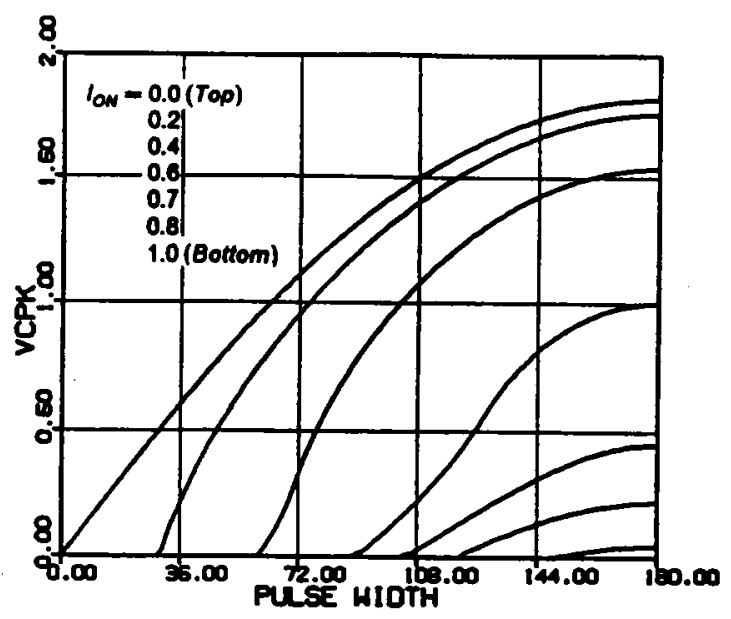

- Peak capacitor voltage

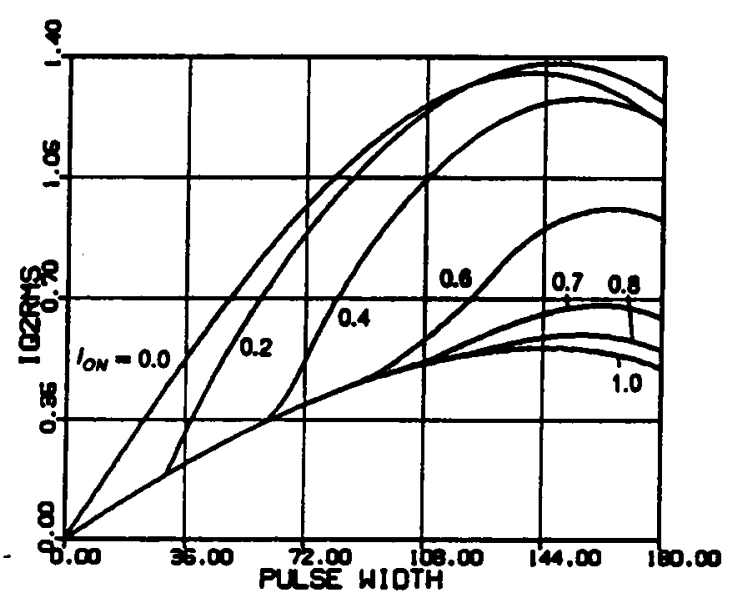

- RMS switch current (Q2,Q4)

Figure C.7.2 DC Characteristics for $\omega_{S N}=1.3$ 


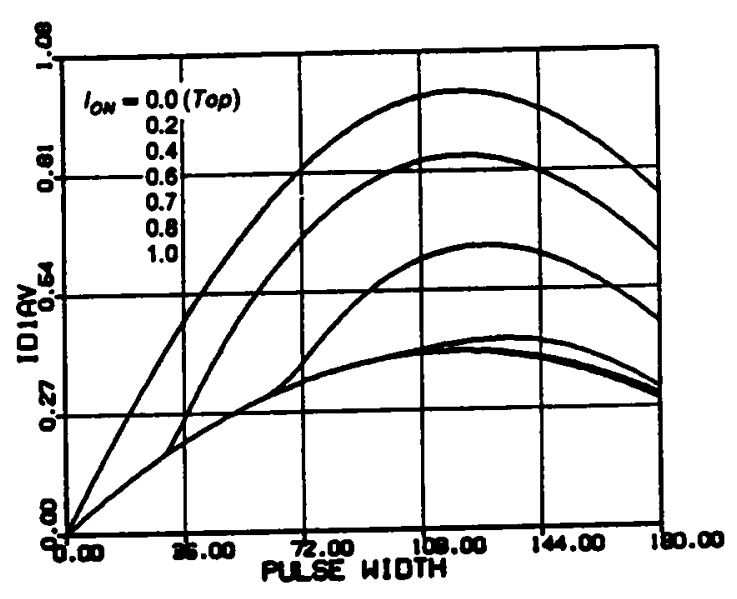

- Average diode current (DI,D3)

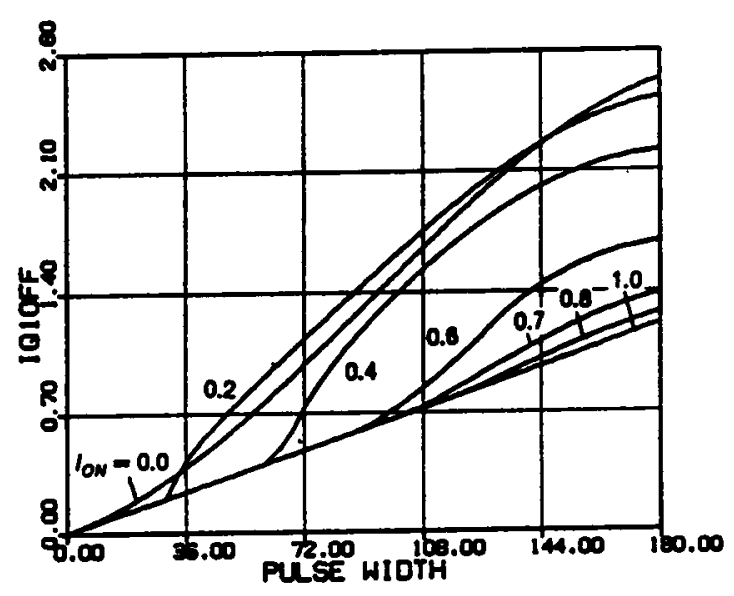

- Switch turn-off current ${ }^{\prime}(Q 1, Q 3$,

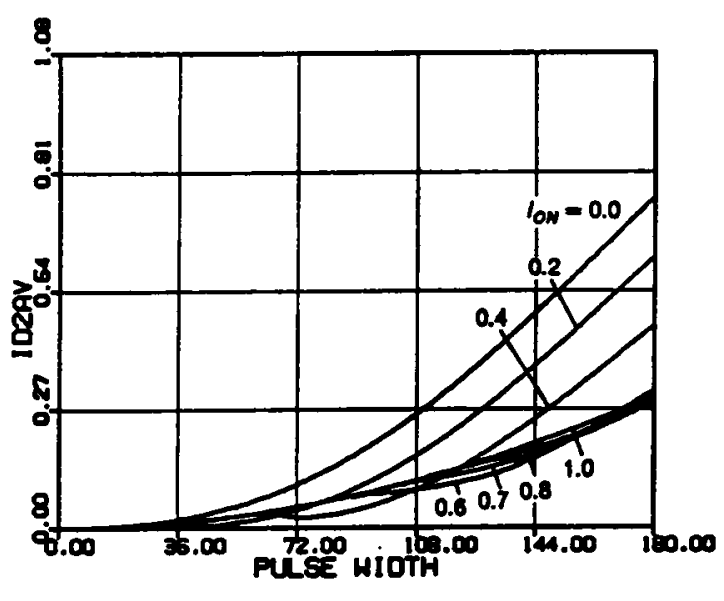

- Average diode current (D2,D4)

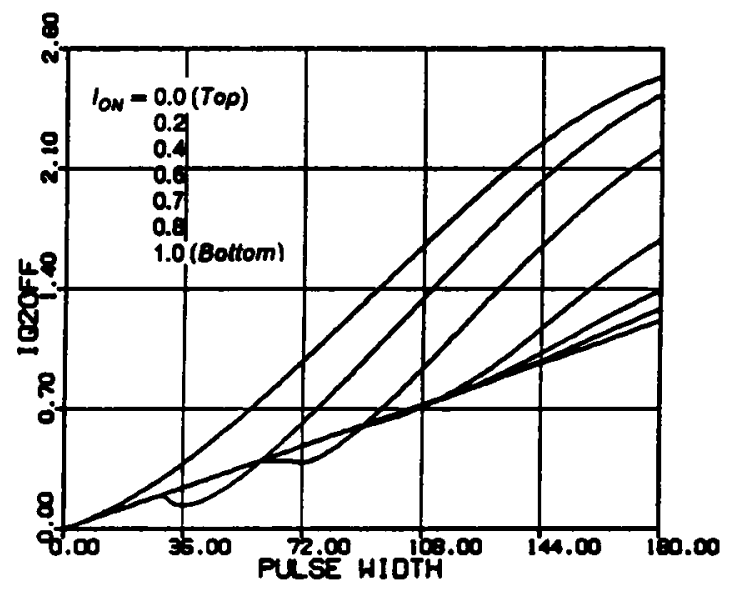

- Switch turn-off current $(Q 2, Q 4)$

Figure C.7.2 Continued 


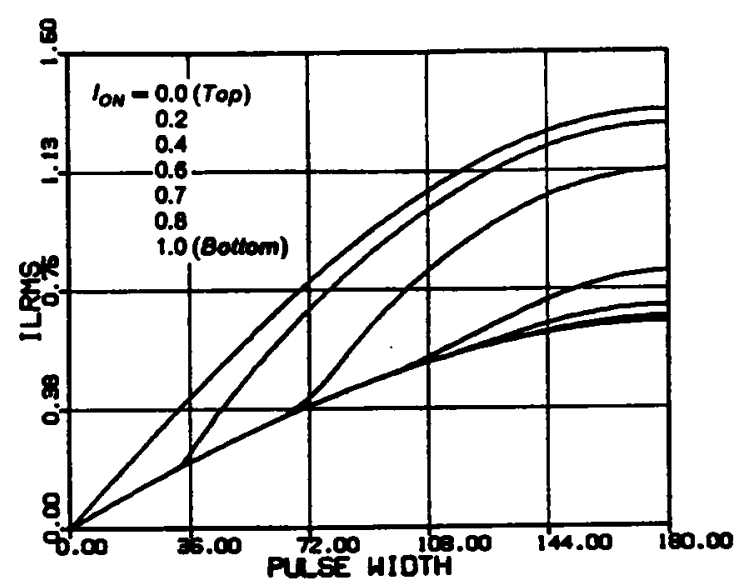

- RMS inductor current

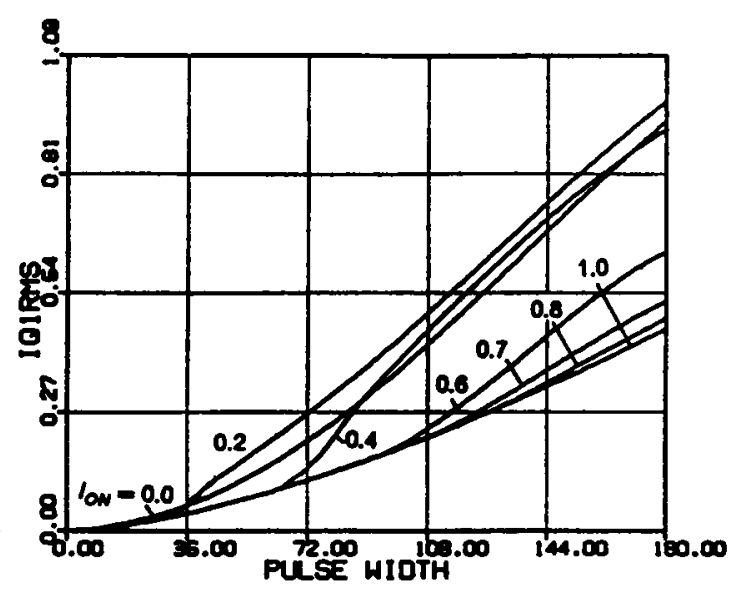

- RMS switch current (QI,Q3)

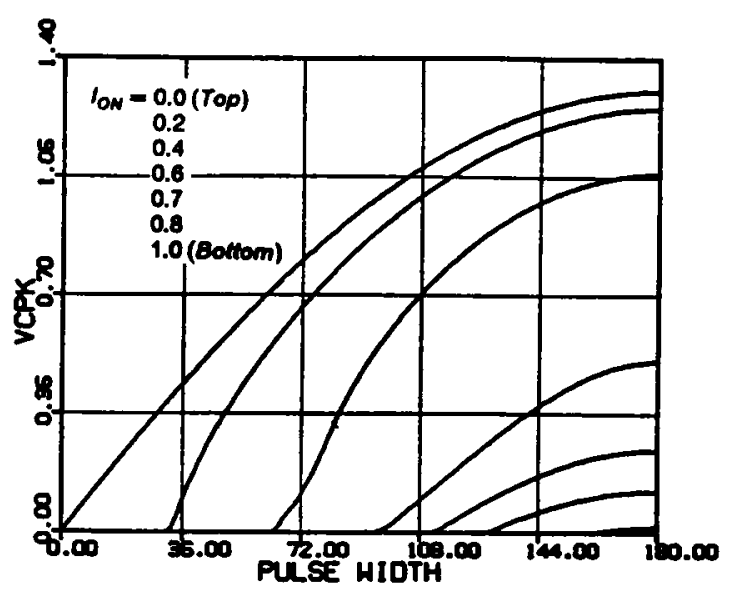

- Peak capacitor voltage

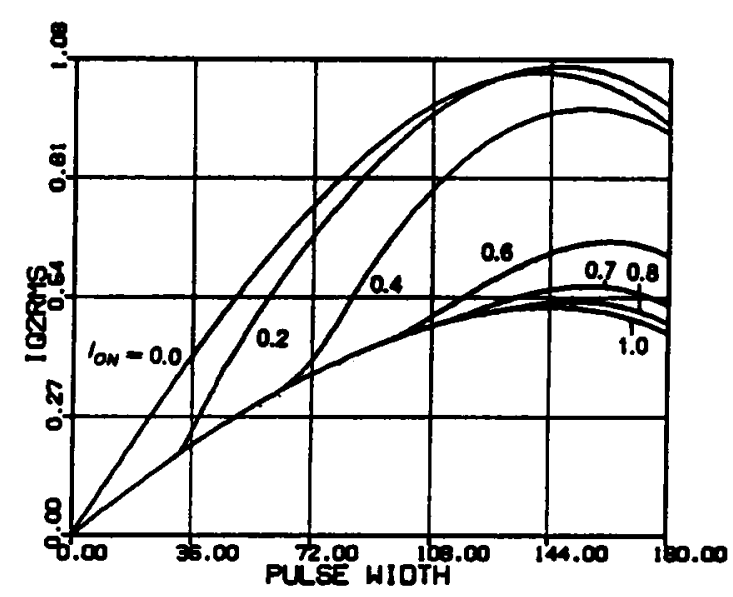

- RMS switch current (Q2,Q4)

Figure C.7.3 DC Characteristics for $\omega_{S N}=1.4$ 


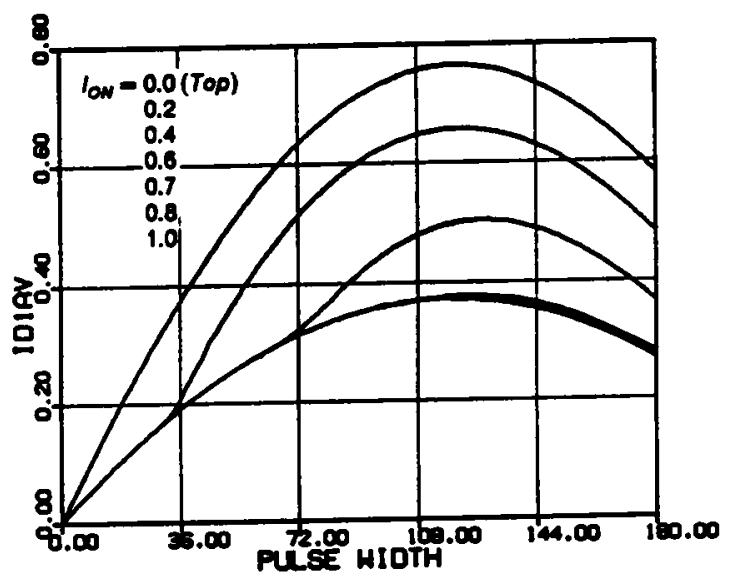

- Average diode current (DI,D3)

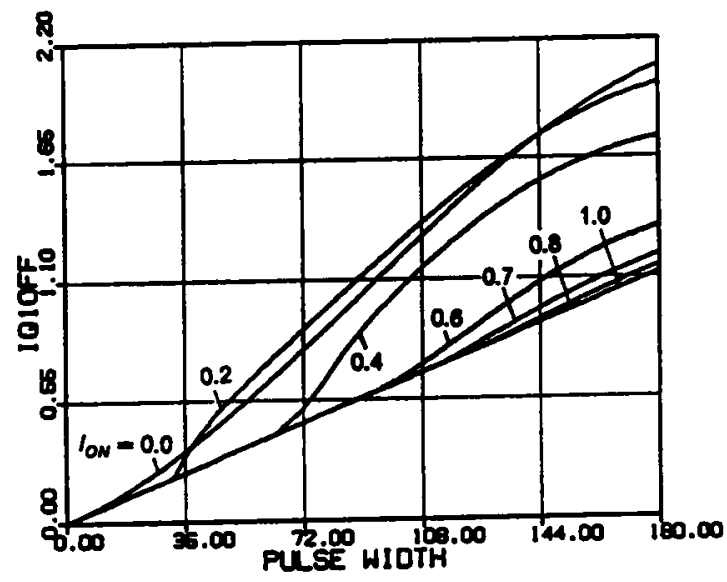

- Switch turn-off current $\left(Q I, Q^{3}\right)$

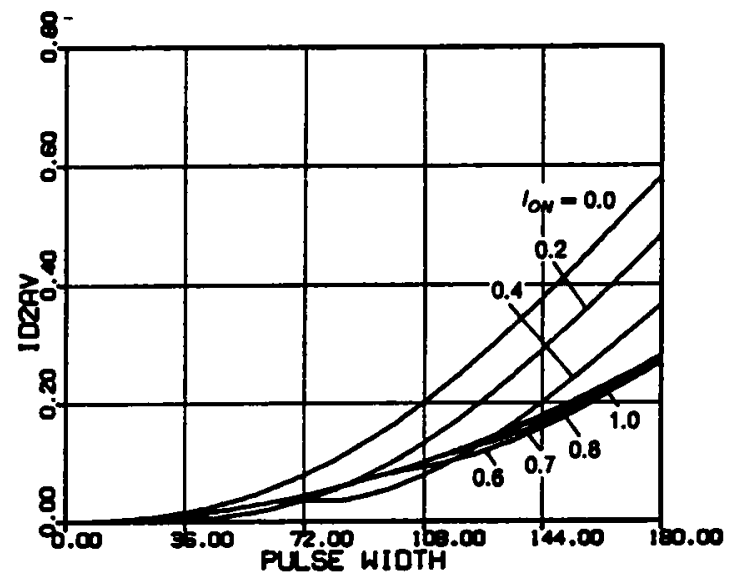

- Average diode current (D2,D4)

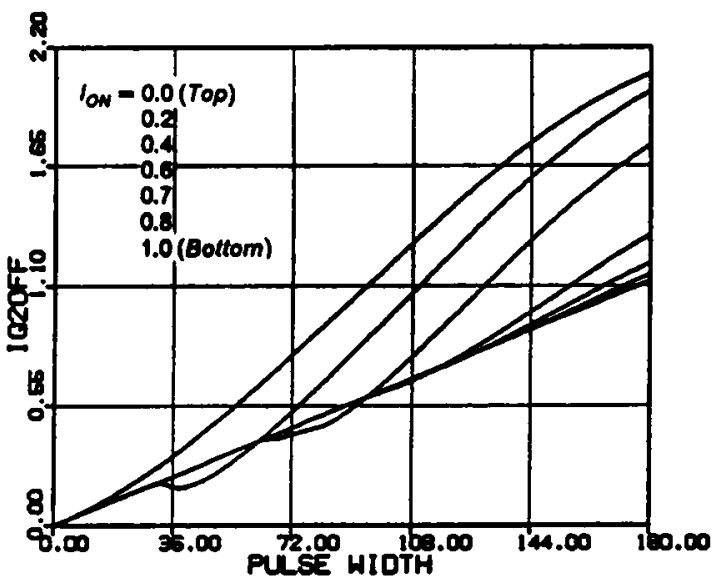

- Switch turn-off current $(Q 2, Q 4)$

Figure C.7.3 Continued 


\section{APPENDIX C.8}

\section{GENERATION OF CIRCUIT WAVEFORMS OF A CM-PRC}

Fortran Program for Generating Capacitor Voltage and Inductor Current Waveforms (for Both Below and Above Resonant Frequency)

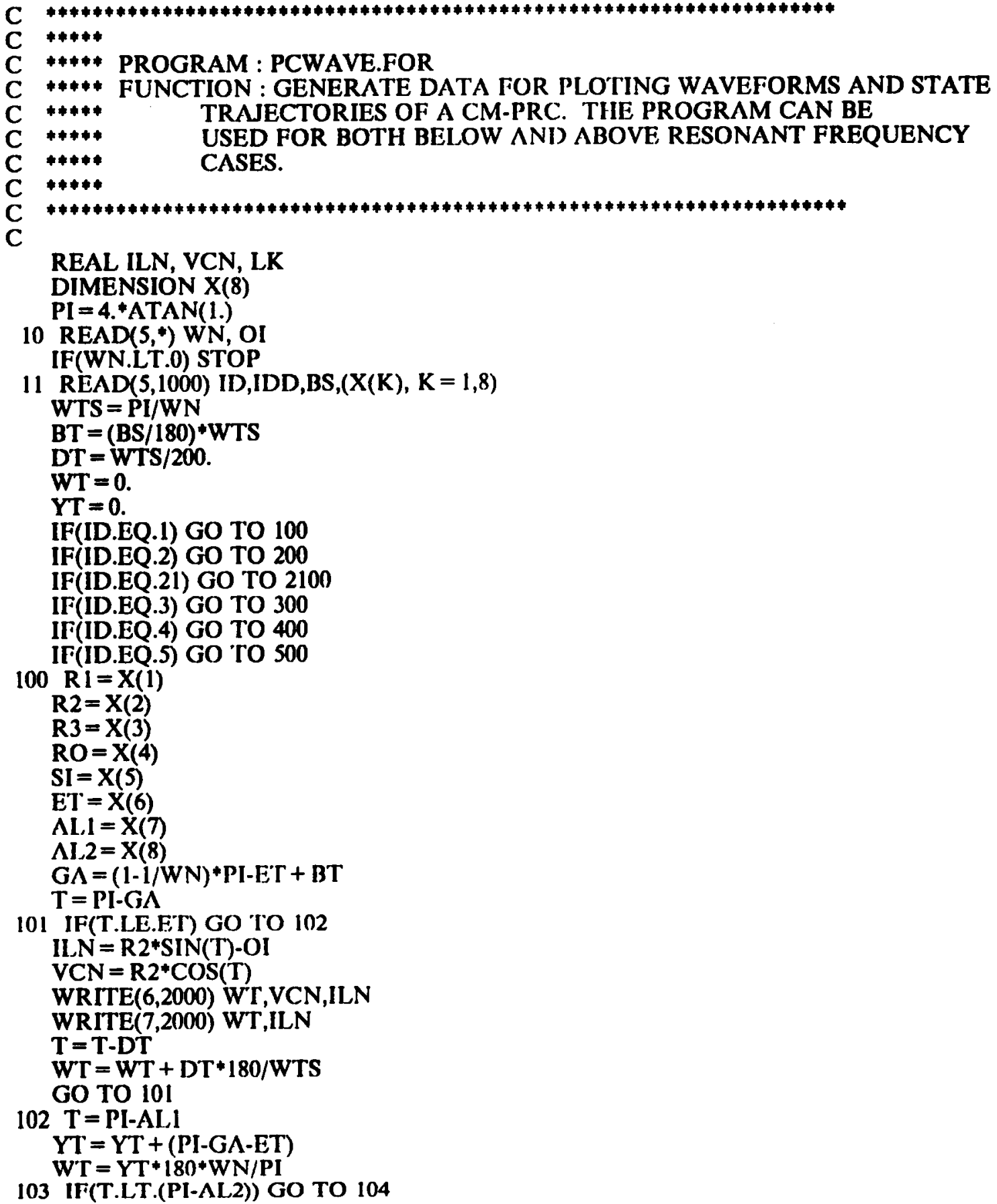


ILN $=$ R3*SIN(T)-OI

$\mathrm{VCN}=\mathrm{R} 3 * \operatorname{COS}(\mathrm{T})+1$

WRITE $(6,2000)$ WT,VCN,ILN

WRITE(7,2000) WT,ILN

$\mathrm{T}=\mathrm{T} \cdot \mathrm{DT}$

$W T=W T+D T * 180 / W T S$

GO TO 103

$104 \mathrm{~T}=\mathrm{PI}-\mathrm{RO}$

$Y T=Y T+(A L 2-A L 1)$

$W T=Y T * 180 * W N / P I$

105 IF(T.LT.(PI-SI)) GO TO 106

ILN $=$ RI*SIN(T) + OI

$\mathrm{VCN}=\mathrm{R} 1{ }^{*} \mathrm{COS}(\mathrm{T})+1$

WRITE $(6,2000)$ WT,VCN,ILN

WRITE $(7,2000)$ WT,ILN

$\mathrm{T}=\mathrm{T} \cdot \mathrm{DT}$

$W T=W T+D T * 180 / W T S$

GO TO 105

$106 \mathrm{~T}=-\mathrm{GA}$

$\mathrm{YT}=\mathrm{YT}+(\mathrm{SI}-\mathrm{RO})$

$\mathrm{WT}=\mathrm{YT} * 180 * \mathrm{WN} / \mathrm{PI}$

107 IF(T.LT.(-PI + ET)) GO TO 108

ILN $=$ R2*SIN(T) + OI

$\mathrm{VCN}=\mathrm{R} 2 * \operatorname{COS}(\mathrm{T})$

WRITE $(6,2000)$ WT, VCN,ILN

WRITE $(7,2000)$ WT,ILN

$\mathrm{T}=\mathrm{T}-\mathrm{DT}$

$\mathrm{WT}=\mathrm{WT}+\mathrm{DT} * 180 / \mathrm{WTS}$

GO TO 107

$108 \mathrm{~T}=-\mathrm{ALl}$

$Y T=Y T+(P I-G A-E T)$

$\mathrm{WT}=\mathrm{YT} * 180 * \mathrm{WN} / \mathrm{PI}$

109 IF(T.LT.-AL2) GO TO 110

ILN $=$ R3 $*$ SIN $(T)+$ OI

$\mathrm{VCN}=\mathrm{R} 3^{*} \mathrm{COS}(\mathrm{T})-1$

WRITE $(6,2000)$ WT,VCN,ILN

WRITE $(7,2000)$ WT,ILN

$\mathrm{T}=\mathrm{T} \cdot \mathrm{DT}$

$W T=W T+D T * 180 / W T S$

GO TO 109

$110 \mathrm{~T}=-\mathrm{RO}$

$\mathbf{Y T}=\mathrm{YT}+(\mathbf{A L 2}-\mathbf{A L} \mathbf{1})$

$\mathrm{WT}=\mathrm{YT} * 180 * \mathrm{WN} / \mathrm{PI}$

111 IF(T.LT.(-SI)) GO TO 112

ILN $=$ RI $* \operatorname{SIN}(T)-O I$

$\mathrm{VCN}=\mathrm{R} 1^{*} \mathrm{COS}(\mathrm{T})-1$

WRITE $(6,2000)$ WT,VCN,ILN

WRITE $(7,2000)$ WT,II.N

$\mathrm{T}=\mathrm{T} \cdot \mathrm{DT}$

$W T=W T+D T * 180 / W T S$

GO TO 111

$112 \mathrm{~T}=\mathrm{PI}-\mathrm{GA}$

$\mathrm{YT}=\mathrm{YT}+(\mathrm{SI}-\mathrm{RO})$

$\mathrm{WT}=\mathrm{YT} * 180^{*} \mathrm{WN} / \mathrm{PI}$

113 IF(T.LT.ET) GO TO 10

ILN $=$ R2*SIN $(T)-O I$

$\mathrm{VCN}=\mathrm{R} 2 * \mathrm{COS}(\mathrm{T})$

WRITE $(6,2000)$ WT,VCN,ILN

WRITE $(7,2000)$ WT,ILN

T $=$ T - DT

$\mathrm{WT}=\mathrm{WT}+\mathrm{DT} * 180 / \mathrm{WTS}$ 


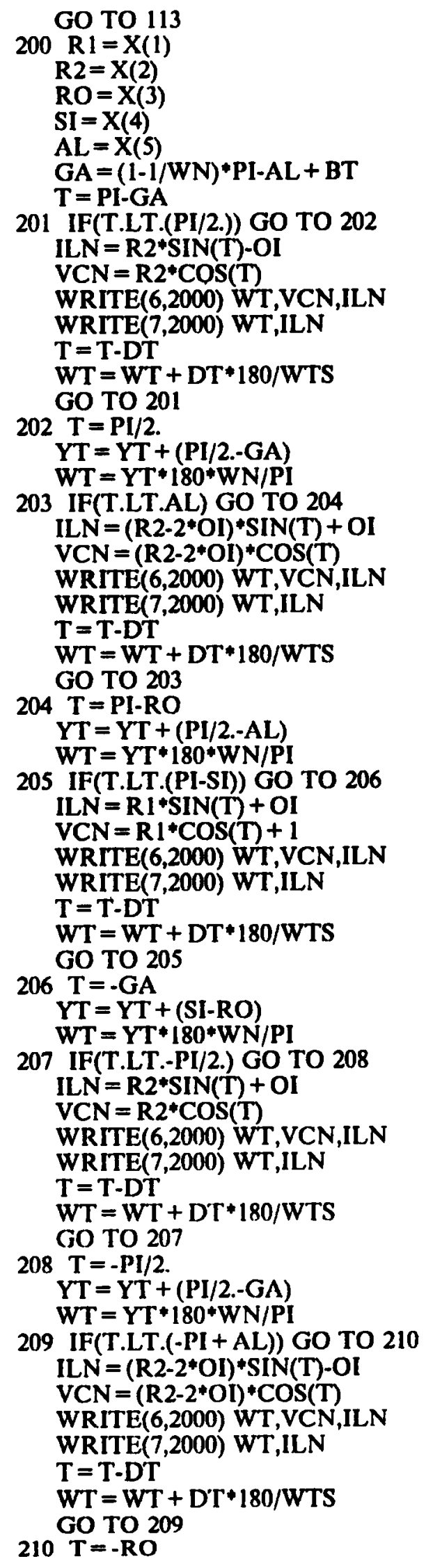


$\mathrm{YT}=\mathrm{YT}+(\mathrm{PI} / 2 .-\mathrm{AL})$

$\mathrm{WT}=\mathrm{YT} * 180 * \mathrm{WN} / \mathrm{PI}$

211 IF(T.LT.(-SI)) GO TO 212

ILN $=\mathrm{RI}$ *SIN(T)-OI

$\mathrm{VCN}=\mathrm{R} 1{ }^{*} \mathrm{COS}(\mathrm{T})-1$

WRITE $(6,2000)$ WT,VCN,ILN

WRITE $(7,2000)$ WT,ILN

$\mathrm{T}=\mathrm{T} \cdot \mathrm{DT}$

$\mathrm{WT}=\mathrm{WT}+\mathrm{DT} * 180 / \mathrm{WTS}$

GO TO 211

$212 \mathrm{~T}=\mathrm{PI}-\mathrm{GA}$

$\mathrm{YT}=\mathrm{YT}+(\mathbf{S I}-\mathbf{R O})$

$\mathrm{WT}=\mathrm{YT} * 180 * \mathrm{WN} / \mathrm{PI}$

213 IF(T.LT.(PI/2.)) GO TO 10

ILN $=R 2 * \operatorname{SIN}(T)-$ OI

$\mathrm{VCN}=\mathrm{R} 2 * \operatorname{COS}(\mathrm{T})$

WRITE $(6,2000)$ WT,VCN,ILN

WRITE $(7,2000)$ WT,ILN

$\mathbf{T}=\mathbf{T}-\mathrm{DT}$

$W T=W T+D T * 180 / W T S$

GO TO 213

$2100 \mathrm{R} 1=\mathrm{X}(1)$

$\mathrm{R} 2=\mathrm{X}(2)$

R3 $=X(3)$

$\mathrm{RO}=\mathrm{X}(\mathbf{4})$

$\mathrm{ET}=\mathrm{X}(5)$

$\mathrm{ALI}=\mathrm{X}(6)$

$\mathrm{AL2}=\mathrm{X}(\mathrm{T})$

$\mathrm{T}=-0.5 * \mathrm{PI}+\mathrm{RO}$

2101 IF(T.LT.(-PI/2.)) GO TO 2102

ILN $=$ RI*SIN(T) + OI

$\mathrm{VCN}=\mathrm{R} 1{ }^{*} \mathrm{COS}(\mathrm{T})$

WRITE $(6,2000)$ WT,VCN,ILN

WRITE $(7,2000)$ WT,ILN

$T=T-D T$

$\mathrm{WT}=\mathrm{WT}+\mathrm{DT} * 180 / \mathrm{WTS}$

GO TO 2101

$2102 \mathrm{~T}=-\mathrm{PI} / 2$

$\mathbf{Y} T=\mathbf{Y T}+\mathbf{R O}$

$\mathrm{WT}=\mathrm{YT} * 180^{*} \mathrm{WN} / \mathrm{PI}$

2103 IF(T.LT.(ET-2*PI)) GO TO 2104

ILN $=(\mathrm{R} 1-2 * \mathrm{OI}) * \mathrm{SIN}(\mathrm{T})-\mathrm{OI}$

$\mathrm{VCN}=(\mathrm{R} 1-2 * \mathrm{OI}) * \mathrm{COS}(\mathrm{T})$

WRITE $(6,2000)$ WT,VCN,ILN

WRITE $(7,2000)$ WT,ILN

$\mathrm{T}=\mathrm{T} \cdot \mathrm{D}^{\prime} \boldsymbol{\Gamma}$

$\mathrm{WT}=\mathrm{WT}+\mathrm{DT}+180 / \mathrm{WTS}$

GO TO 2103

$2104 \mathrm{~T}=-\mathrm{PI}-\mathrm{AL}, \mathrm{I}$

$\mathrm{YT}=\mathrm{YT}+(1.5 * \mathrm{PI}-\mathrm{ET})$

$\mathrm{WT}=\mathrm{YT} * 180 * \mathrm{WN} / \mathrm{PI}$

2105 IT'(T.LT.(-PI-AL2)) GO TO 2106

ILN $=$ R3*SIN(T)-OI

$\mathrm{VCN}=\mathrm{R} 33^{*} \mathrm{COS}(\mathrm{T})+1$

WRITE(6,2000) WT,VCN,ILN

WRITE(7,2000) WT,ILN

$\mathrm{T}=\mathrm{T}-\mathrm{DT}$

$\mathrm{WT}=\mathrm{WT}+\mathrm{DT} * 180 / \mathrm{WTS}$

GO TO 2105

$2106 \mathrm{~T}=0.5^{*} \mathrm{PI}+\mathrm{RO}$

$\mathrm{YT}=\mathrm{YT}+(\mathrm{AL2}-\mathrm{AL} 1)$ 
$\mathrm{WT}=\mathrm{YT} * 180 * \mathrm{WN} / \mathrm{PI}$

2107 IF(T.LT.PI/2.) GO TO 2108

ILN $=$ RI ${ }^{*} \operatorname{SIN}(\mathrm{T}) \cdot \mathrm{OI}$

$\mathrm{VCN}=\mathrm{R} 1^{*} \mathrm{COS}(\mathrm{T})$

WRITE $(6,2000)$ WT,VCN,ILN

WRITE(7,2000) WT,ILN

$\mathrm{T}=\mathrm{T}-\mathrm{DT}$

$\mathrm{WT}=\mathrm{WT}+\mathrm{DT} * 180 / \mathrm{WTS}$

GO TO 2107

$2108 \mathrm{~T}=\mathrm{PI} / 2$.

$\mathbf{Y T}=\mathbf{Y T}+\mathbf{R O}$

$\mathrm{WT}=\mathrm{YT} * 180 * \mathrm{WN} / \mathrm{PI}$

2109 IF(T.LT.(ET-PI)) GO TO 2110

ILN $=(R 1-2 * O I) *$ SIN $(T)+$ OI

$\mathrm{VCN}=(\mathrm{R} 1-2 * \mathrm{OI}) * \mathrm{COS}(\mathrm{T})$

WRITE $(6,2000)$ WT,VCN,ILN

WRITE $(7,2000)$ WT,ILN

$\mathrm{T}=\mathrm{T}-\mathrm{DT}$

$W T=W T+D T * 180 / W T S$

GO TO 2109

$2110 \mathrm{~T}=-\mathrm{ALl}$

$\mathrm{YT}=\mathrm{YT}+(1.5+\mathrm{PI}-\mathrm{ET})$

$\mathrm{WT}=\mathrm{YT} * 180^{*} \mathrm{WN} / \mathrm{PI}$

2111 IF(T.LT.(-AL2)) GO TO 2112

ILN $=$ R3 $*$ SIN $(T)+$ OI

$\mathrm{VCN}=\mathrm{R} 3^{*} \mathrm{COS}(\mathrm{T})-1$

WRITE $(6,2000)$ WT,VCN,ILN

WRITE $(7,2000)$ WT,ILN

$\mathrm{T}=\mathrm{T}-\mathrm{DT}$

$\mathrm{WT}=\mathrm{WT}+\mathrm{DT} * 180 / \mathrm{WTS}$

GO TO 2111

$2112 \mathrm{~T}=-0.5^{*} \mathrm{PI}+\mathrm{RO}$

$Y T=Y T+(A L 2-A L 1)$

$\mathrm{WT}=\mathrm{YT} * 180 * \mathrm{WN} / \mathrm{PI}$

2113 IF(T.LT.(-PI/2.)) GO TO 10

ILN $=$ RI + SIN (T) + OI

$\mathrm{VCN}=\mathrm{R} 1{ }^{*} \mathrm{COS}(\mathrm{T})$

WRITE $(6,2000)$ WT,VCN,ILN

WRITE(7,2000) WT,ILN

$\mathrm{T}=\mathrm{T} \cdot \mathrm{DT}$

$\mathrm{WT}=\mathrm{WT}+\mathrm{DT} * 180 / \mathrm{WTS}$

GO TO 2113

$300 \mathrm{R} 1=\mathrm{X}(1)$

$\mathrm{R} 2=\mathrm{X}(2)$

$\mathrm{SI}=\mathrm{X}(3)$

$\mathrm{ET}=\mathrm{X}(4)$

$\Lambda L 1=X(5)$

$A L 2=X(6)$

$\mathrm{GA}=0.5^{*}(-\mathrm{PI}+\mathrm{SI})$

$\mathrm{LK}=2 * \mathrm{OI}-(\mathrm{R} 2 * * 2-1) * * 0.5$

$\mathrm{T}=\mathrm{PI}-\mathrm{GA}$

301 IF(T.LT.ET) GO TO 302

ILN $=R I * \operatorname{SIN}(T)-O I$

$\mathrm{VCN}=\mathrm{R} 1{ }^{*} \mathrm{COS}(\mathrm{T})$

WRITE $(6,2000)$ WT,VCN,ILN

WRITE $(7,2000)$ WT,ILN

$\mathrm{T}=\mathrm{T}-\mathrm{DT}$

$\mathrm{WT}=\mathrm{WT}+\mathrm{DT} * 180 / \mathrm{WTS}$

GO TO 301

$302 \mathrm{~T}=\mathrm{PI}-\mathrm{\Lambda LI}$

$\mathbf{Y T}=\mathrm{YT}+(\mathrm{PI}-\mathrm{ET}-\mathrm{GA})$ 
$\mathrm{WT}=\mathrm{YT} * 180^{*} \mathrm{WN} / \mathrm{PI}$

303 IF(T.LT.(PI-AL2)) GO TO 304

ILN $=$ R2*SIN(T)-OI

$\mathrm{VCN}=\mathrm{R} 2 * \mathrm{COS}(\mathrm{T})+1$.

WRITE $(6,2000)$ WT, VCN,ILN

WRITE $(7,2000)$ WT,ILN

$\mathrm{T}=\mathrm{T}-\mathrm{DT}$

$\mathrm{WT}=\mathrm{WT}+\mathrm{DT} * 180 / \mathrm{WTS}$

GO TO 303

$304 \mathrm{~T}=0$.

$Y T=Y T+(A L 2-A L 1)$

$\mathrm{WT}=\mathrm{YT} * 180 * \mathrm{WN} / \mathrm{PI}$

305 IF(T.GT.LK) GO TO 306

ILN $=$ OI-LK + T

$\mathrm{VCN}=0$.

WRITE(6,2000) WT,VCN,ILN

WRITE $(7,2000)$ WT,ILN

$\mathbf{T}=\mathbf{T}+\mathbf{D T}$

$\mathrm{WT}=\mathrm{WT}+\mathrm{DT} * 180 / \mathrm{WTS}$

GO TO 305

$306 \mathrm{~T}=\mathrm{PI}$

$Y T=Y T+L K$

$\mathrm{WT}=\mathrm{YT} * 180 * \mathrm{WN} / \mathrm{PI}$

307 IF(T.LT.(PI-SI)) GO TO 308

ILN $=$ SIN $(T)+$ OI

$\mathrm{VCN}=\mathrm{COS}(\mathrm{T})+1$

WRITE $(6,2000)$ WT,VCN,ILN

WRITE $(7,2000)$ WT,ILN

$\mathrm{T}=\mathrm{T} \cdot \mathrm{DT}$

$W T=W T+D T * 180 / W T S$

GO TO 307

$308 \mathrm{~T}=-\mathrm{GA}$

$Y T=Y T+S I$

$\mathrm{WT}=\mathrm{YT} * 180 * \mathrm{WN} / \mathrm{PI}$

309 IF(T.LT.(ET-PI)) GO TO 310

ILN $=$ R $1 *$ SIN (T) + OI

$\mathrm{VCN}=\mathrm{R} 1{ }^{*} \mathrm{COS}(\mathrm{T})$

WRITE $(6,2000)$ WT,VCN,ILN

WRITE $(7,2000)$ WT,ILN

$\mathrm{T}=\mathrm{T} \cdot \mathrm{DT}$

$\mathrm{WT}=\mathrm{WT}+\mathrm{DT} * 180 / \mathrm{WTS}$

GO TO 309

$310 \mathrm{~T}=-\mathrm{ALI}$

$Y T=Y T+(P I-E T-G A)$

$\mathrm{WT}=\mathrm{YT} * 180 * \mathrm{WN} / \mathrm{PI}$

311 IF(T.LT.-AL2) GO TO 312

ILN $=\mathrm{R} 2 * \mathrm{SIN}(\mathrm{T})+\mathrm{OI}$

$\mathrm{VCN}=\mathrm{R} 2 * \operatorname{COS}(\mathrm{T})-1$

WRITE $(6,2000)$ WT,VCN,ILN

WRITE $(7,2000)$ WT,ILN

$\mathrm{T}=\mathrm{T} \cdot \mathrm{D} T$

$\mathrm{WT}=\mathrm{WT}+\mathrm{DT} * 180 / \mathrm{WTS}$

GO TO 311

$312 \mathrm{~T}=0$.

$Y T=Y T+(A L 2-A L 1)$

$W T=Y T * 180 * W N / P I$

313 IF(T.GT.LK) GO TO 314

ILN $=-0 I+L K-T$

$\mathrm{VCN}=0$.

WRITE(6,2000) WT,VCN,ILN

WRITE $(7,2000)$ WT,ILN 
$\mathrm{T}=\mathrm{T}+\mathrm{DT}$

$\mathrm{WT}=\mathrm{WT}+\mathrm{DT} * 180 / \mathrm{WTS}$

GO TO 313

$314 \mathrm{~T}=0$.

$\mathrm{YT}=\mathrm{YT}+\mathrm{LK}$

$\mathrm{WT}=\mathrm{YT} * 180 * \mathrm{WN} / \mathrm{PI}$

315 IF(T.LT.(-SI)) GO TO 316

ILN $=\operatorname{SIN}(\mathrm{T})-\mathrm{OI}$

$\mathrm{VCN}=\operatorname{COS}(\mathrm{T}) \cdot 1$

WRITE $(6,2000)$ WT,VCN,ILN

WRITE $(7,2000)$ WT,ILN

$\mathbf{T}=\mathbf{T} \cdot \mathbf{D T}$

$W T=W T+D T * 180 / W T S$

GO TO 315

$316 \mathrm{~T}=\mathrm{PI}-\mathrm{GA}$

$\mathbf{Y T}=\mathbf{Y T}+\mathbf{S I}$

$\mathrm{WT}=\mathrm{YT} * 180 * \mathrm{WN} / \mathrm{PI}$

317 IF(T.LT.ET) GO TO 10

ILN $=\mathrm{R} 1$ +SIN(T)-OI

$\mathrm{VCN}=\mathrm{R} 1^{*} \mathrm{COS}(\mathrm{T})$

WRITE $(6,2000)$ WT,VCN,ILN

WRITE(7,2000) WT,ILN

$\mathbf{T}=\mathbf{T} \cdot \mathbf{D T}$

$\mathrm{WT}=\mathrm{WT}+\mathrm{DT} * 180 / \mathrm{WTS}$

GO TO 317

$400 \mathrm{R} 1=\mathrm{X}(1)$

$\mathrm{SI}=\mathbf{X}(2)$

$\mathrm{TH}=\mathrm{X}(3)$

LK $=2 * O I-R 1$

$\mathrm{GA}=0.5^{+}(-\mathrm{PI}+\mathrm{SI})$

$\mathrm{T}=\mathrm{PI}-\mathrm{GA}$

401 IF(T.LT.PI/2.) GO TO 402

ILN $=\mathbf{R} 1+\operatorname{SIN}(\mathrm{T})-\mathrm{OI}$

$\mathrm{VCN}=\mathrm{R} 11^{*} \mathrm{COS}(\mathrm{T})$

WRITE $(6,2000)$ WT,VCN,ILN

WRITE $(7,2000)$ WT,ILN

$\mathbf{T}=\mathbf{T}-\mathrm{DT}$

$W T=W T+D T * 180 / W T S$

GO TO 401

$402 \mathrm{~T}=0$.

$\mathrm{YT}=\mathrm{YT}+(0.5 * \mathrm{PI}-\mathrm{GA})$

$W T=Y T * 180 * W N / P I$

403 IF(T.GT.TH) GO TO 404

ILN $=$ OI-LK

$\mathrm{VCN}=0$.

WRITE $(6,2000)$ WT,VCN,ILN

WRITE $(7,2000)$ WT,ILN

$\mathrm{T}=\mathrm{T}+\mathrm{DT}$

$\mathrm{WT}=\mathrm{WT}+\mathrm{DT} * 180 / \mathrm{WTS}$

GO TO 403

$404 T=0$.

$\mathbf{Y T}=\mathbf{Y T}+\mathbf{T H}$

$\mathrm{WT}=\mathrm{YT} * 180^{*} \mathrm{WN} / \mathrm{PI}$

405. IF(T.GT.LK) GO TO 406

ILN $=$ OI-LK + T

$\mathrm{VCN}=0$.

WRITE $(6,2000)$ WT,VCN,ILN

WRITE $(7,2000)$ WT,ILN

$\mathrm{T}=\mathrm{T}+\mathrm{DT}$

$W T=W T+D T * 180 / W T S$

GO TO 405 


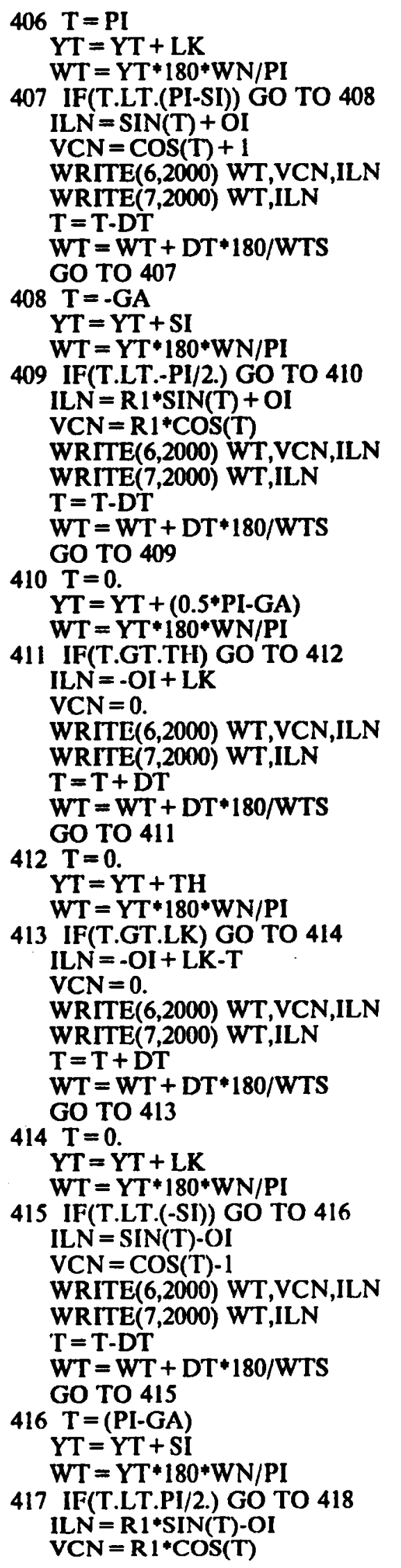


WRITE $(6,2000)$ WT,VCN,ILN

WRITE $(7,2000)$ WT,ILN

T $=$ T-DT

$W T=W T+D T * 180 / W T S$

GO TO 417

$418 \mathrm{~T}=0$.

$\mathrm{YT}=\mathrm{YT}+(0.5 *$ pi-GA $)$

$W T=Y T * 180 * W N / P I$

419 IF(T.GT.TH) GO TO 10

ILN $=$ OI-LK

$\mathrm{VCN}=0$.

WRITE(6,2000) WT,VCN,ILN

WRITE $(7,2000)$ WT,ILN

$\mathbf{T}=\mathbf{T}+\mathbf{D T}$

$W T=W T+D T * 180 / W T S$

GO TO 419

500 LK $=$ BT

TH $=\mathrm{PI} / \mathrm{WN}-\mathrm{BT}$

$T=0$.

501 IF(T.GT.TH) GO TO 502

ILN $=-\mathrm{LK} / 2$.

$\mathrm{VCN}=0$.

WRITE(6,2000) WT,VCN,ILN

WRITE $(7,2000)$ WT,ILN

$\mathbf{T}=\mathbf{T}+\mathbf{D T}$

$W T=W T+D T * 180 / W T S$

GO TO 501

$502 \mathrm{~T}=0$.

$\mathrm{YT}=\mathrm{YT}+\mathrm{TH}$

$\mathrm{WT}=\mathrm{YT} * 180 * \mathrm{WN} / \mathrm{PI}$

503 IF(T.GT.LK) GO TO 504

$\mathrm{ILN}=\mathrm{T} \cdot \mathrm{LK} / 2$.

$\mathrm{VCN}=0$.

WRITE(6,2000) WT,VCN,ILN

WRITE $(7,2000)$ WT,ILN

$T=T+D T$

$W T=W T+D T * 180 / W T S$

GO TO 503

$504 \mathrm{~T}=0$.

$\mathrm{YT}=\mathrm{YT}+\mathrm{LK}$

$\mathrm{WT}=\mathrm{YT} * 180^{*} \mathrm{WN} / \mathrm{PI}$

505 IF(T.GT.TH) GO TO 506

ILN $=\mathbf{L K} / 2$.

$\mathrm{VCN}=0$.

WRITE $(6,2000)$ WT,VCN,ILN

WRITE $(7,2000)$ WT,ILN

$\mathrm{T}=\mathrm{T}+\mathrm{DT}$

$W T=W T+D T * 180 / W T S$

GO TO 505

$506 \mathrm{~T}=0$.

$\mathbf{Y} \mathrm{T}=\mathrm{YT}+\mathrm{TH}$

$\mathrm{WT}=\mathrm{YT}+180^{*} \mathrm{WN} / \mathrm{PI}$

507 IF(T.GT.LK) GO TO 508

ILN $=$ LK/2.-T

$\mathrm{VCN}=0$.

WRITE $(6,2000)$ WT,VCN,ILN

WRITE $(7,2000)$ WT,ILN

$\mathrm{T}=\mathrm{T}+\mathrm{DT}$

$\mathrm{WT}=\mathrm{WT}+\mathrm{DT} * 180 / \mathrm{WTS}$

GO TO 507

$508 \mathrm{~T}=0$. 


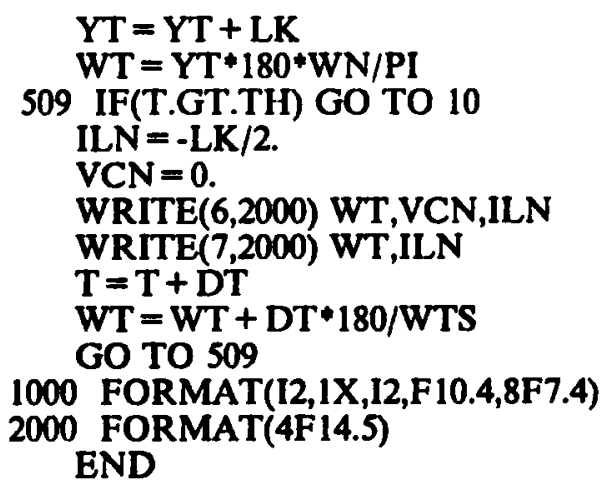

406 


\section{The vita has been removed from the scanned document}

